Министерство образования и науки Российской Федерации федеральное государственное бюджетное образовательное учреждение высшего профессионального образования

Московский государственный университет печати имени Ивана Федорова

\author{
В.К. Асташев, В.Л. Крупенин
}

\title{
НЕЛИНЕЙНАЯ ДИНАМИКА УЛЬТРАЗВУКОВЫХ ТЕХНОЛОГИЧЕСКИХ ПРОЦЕССОВ
}

\section{Учебник}

Рекомендовано федеральным государственным бюджетным образовательным учреждением высшего профессионального образования «Московский государственный университет печати имени Ивана Федорова» в качестве учебника для обучающихся по направлению «Машиностроение» уровней магистратуры и подготовки кадров высшей квалификации

Москва

2016 
A.М. Гуськов - доктор технических наук,

профессор кафедры «Прикладная механика» МГТУ им. Н.Э. Баумана

Л.Н. Лесневский - доктор технических наук,

профессор кафедры «Технология производства двигателей летательных аппаратов»

Московского авиационного института (НИУ)

\section{Асташев В.К., Крупенин В.Л.}

А 91 Нелинейная динамика ультразвуковых технологических процессов: учебник / В.К. Асташев, В.Л. Крупенин ; Моск. гос. ун-т печати имени Ивана Федорова. — М. : МГУП имени Ивана Федорова, 2016. - 372 с.

В книге систематически излагается современное состояние нелинейной теории ультразвуковых технологических процессов, а также её основные приложения. Указанная теория лежит в основе курса «Ультразвуковые технологические машины», читаемого студентам магистратуры и аспирантам кафедрой «Динамика машин-автоматов», созданной на базе ИМАШ РАН в Институте Принтмедиа и информационных технологий МГУП имени Ивана Федорова. В книге даются, как и ставшие традиционными, так и новейшие результаты в соответствующих разделах науки, а также высоких технологий в машиностроении.

Издание предназначено для студентов и аспирантов машиностроительных и физикоматематических специальностей, а также для инженеров и научных работников, интересующихся нелинейной динамикой.

Печатается в авторской редакции.

ISBN 978-5-8122-1325-1

УДК 621.7

ББК 32.873 


\section{ОГЛАВЛЕНИЕ}

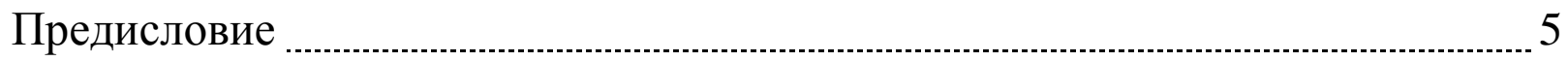

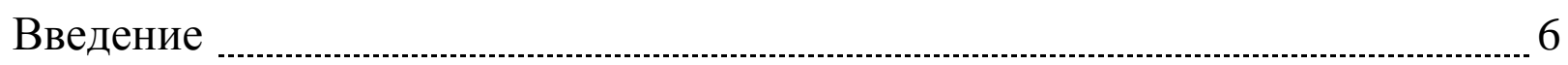

ГЛАВА 1 Нелинейные модели ультразвуковых $\quad$ технологических процессов

1. Ультразвуковые технологические процессы _..................................................... 14

2. Влияние ультразвука на процессы пластического деформирования ....... 27

3. Влияние ультразвука на процессы с сухим трением .................................... 46

ГЛАВА 2 Динамические характеристики у ультразвуковых

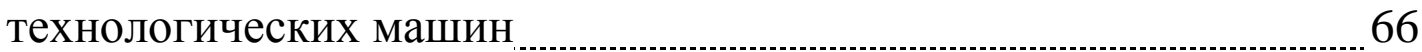

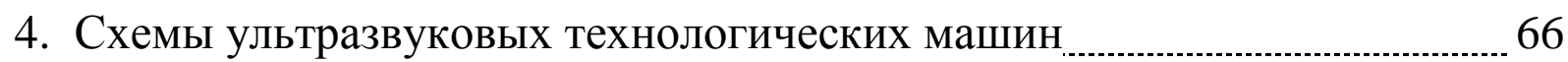

5. Продольные колебания ультразвуковых стержневых систем ................... 86

6. Изгибные колебания ультразвуковых стержневых систем...................... 120

7. Динамические характеристики электроакустических преобразователей

ГЛАВА 3 Нелинейные процессы в ультразвуковых технологических системах

8. Нелинейная технологическая нагрузка 164

9. Резонансные колебания ультразвуковых стержневых систем с нелинейной нагрузкой

10. Виброударные взаимодействия ультразвуковых стержневых систем

11. Вибрационное перемещение вязкоупругого стержня в среде с сухим трением

ГЛАВА 4 Резонансная настройка ультразвуковых технологических машин

12. Способы повышения эффективности ультразвуковых станков под нагрузкой 240

13. Нелинейная теория ультразвуковых концентраторов 267 
14. Авторезонансное возбуждение ультразвуковых технологических машин 281

15. Ультразвуковое резание как нелинейный (виброударный) процесс...... 307 Список литературы 332

Приложение 353 


\section{ПРЕДИСЛОВИЕ}

В основу этой книги положен курс лекций «Ультразвуковые технологические машины», прочитанный в 2015-2016 гг. для студентов магистратуры и аспирантов по направлению 15.06.01 - (Машиностроение (Уровень подготовки кадров высшей квалификации). В свою очередь, этот курс целиком опирается на оригинальные научные исследования в области нелинейной динамики ультразвуковых технологических машин и процессов, в течение многих лет проводимых в Институте машиноведения им. А.А. Благонравова РАН (ИМАШ РАН) под руководством профессора В.К.Асташева. Лекции читались кафедрой «Динамика машинавтоматов» (ДАМ), созданной на базе ИМАШ РАН в институте Принтмедиа и информационных технологий МГУП им. Ивана Федорова (МГУП).

Для чтения книги необходимы предварительные знания математики, физики и материаловедения в объеме, предлагаемом при изучении соответствующих дисциплин, изучаемых во МГУП. Её содержание достаточно подробно прокомментировано во Введении.

Авторы благодарны А.И. Винокуру и Г.О. Рытикову за помощь в становлении курса и выпуске этой книги, Г.В. Кожевникову за участие в организации кафедры ДАМ, а также В.Н. Перевезенцеву, Л.В. Колику и Н.А.Андрианову, совместно с которыми были выполнены исследования, резюмированные в Приложении.

В.К. Асташев, В.Л.Крупенин 


\section{ВВЕДЕНИЕ}

Многие современные материалы, разработанные главным образом для удовлетворения потребностей ряда новых прогрессивных отраслей промышленности, как правило, трудно поддаются обработке традиционными методами. К таким материалам относятся высокопрочные, жаропрочные и нержавеющие стали и сплавы, титан, керамические и другие неметаллические материалы и т.л. Обработка деталей из этих материалов обычными методами приводит к скалыванию или разрушению поверхностного слоя или всей детали и не позволяет получить необходимое качество изделия.

С другой стороны, при создании новых образцов часто возникают технологические проблемы, которые не могут быть решены в рамках традиционных технологий. В ряде случаев эти проблемы обусловлены конструкцией объекта и предъявляемыми к нему техническими требованиями. Например, при соединении деталей микроэлектроники часто недопустим нагрев соединяемых деталей, исключается присутствие постороннего промежуточного компонента и т.д. По этим причинам традиционные пайка и сварка не могут использоваться.

Эти и многие другие проблемы успешно решаются методами ультразвуковой технологии. Рабочий процесс ультразвуковой технологической машины осуществляется рабочим органом, которому кроме формообразующего движения подачи относительно обрабатываемого изделия сообщаются высокочастотные (ультразвуковые) колебания определенного направления, частоты и интенсивности. Данный процесс реализуется ультразвуковой технологической машиной. Конструкция машины и ее элементов определяется в значительной степени схемой технологического процесса, выполняемого рабочим органом. Колебания возбуждаются приводом, а передача их рабочему органу обеспечивается колебательной системой зачастую с изменением направления и амплитуды. 


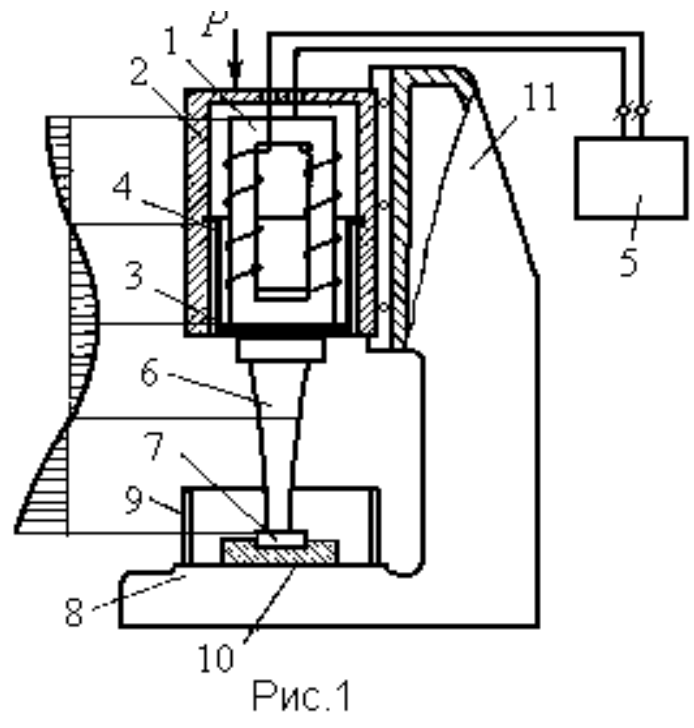

На рис.1 приведена схема ультразвукового станка для обработки хрупких материалов. Возбудитель колебаний - магнитострикционный преобразователь 1 закреплен в корпусе 2 акустической головки с помощью фланца 3 и тонкостенного стакана 4. Обмотка преобразователя питается переменным током от генератора 5 электрических

колебаний ультразвуковой частоты. Наводимое током переменное магнитное поле в сердечнике преобразователя вызывает его механические колебания, которые через волновод - концентратор 6 передаются инструменту 7 с увеличением амплитуды. Под инструментом на столе 8 в ванне 9 с абразивной суспензией помещено обрабатываемое изделие 10. Акустическая головка установлена в направляющих станины 11 и под действием статической силы Р продвигается по мере выработки материала в результате ударов инструмента по частицам абразива, оседающих на обрабатываемой поверхности. Во многом по подобной схеме строятся и другие ультразвуковые технологические машины.

Таким образом, ультразвуковые технологические машины относятся к общему классу вибрационных машин, однако они выделяются в отдельную группу по следующим основным причинам.

Первая определяется выявленными многочисленными экспериментами принципиальными особенностями поведения материалов и сред в ультразвуковом поле. Эти особенности проявляются в радикальном изменении наблюдаемых в эксперименте их упругопластических характеристик и реологических свойств, таких как хрупкость, пластичность и вязкость. Например, сухое трение в зоне контакта двух поверхностей под влиянием ультразвуковой вибрации преобразуется в вязкое. При деформировании образцов с наложением ультразвуковой вибрации 
наблюдается значительное снижение предела текучести. Эти эффекты многократно получены экспериментально, широко используются в разнообразных машинах для прессования и штамповки, волочения проволоки и труб, ультразвуковой размерной обработки хрупких материалов, ультразвукового точения, сварки металлов и пластмасс и многих других, но не получили должного теоретического объяснения. Попытка устранить этот пробел и дать необходимые описания - одна из целей этой книги. Объяснение экспериментально наблюдаемых эффектов проводится на основе построенных нелинейных моделей ультразвуковых технологических процессов.

Вторая причина обусловлена спецификой конструкции основных элементов машины, которые представляют собой стержневые колебательные системы, составленные, как правило, из неоднородных участков и работающих в режиме волноводов. По этой причине при описании колебаний основные элементы машины моделируются системами с распределенными параметрами и описываются дифференциальными уравнениями в частных производных. Взаимодействие рабочего органа с изделием приводит к существенной нелинейности колебательной системы в рабочих режимах.

В силу специфики конструкции колебательные системы обладают высокой добротностью. Поэтому ультразвуковая машина может эффективно работать только в резонансных режимах, позволяющих получить достаточные для проведения технологического процесса амплитуды колебаний рабочего органа. В практике построения и использовании ультразвуковых технологических машин резонансную настройку производят, как правило, на холостом ходу, предполагая, что влиянием технологической нагрузки в рабочих режимах можно пренебречь. Однако проведенные нами исследования установили, что указанное предположение далеко не справедливо. Показано, что технологическая нагрузка приводит не только к изменению резонансных частот колебательной системы, но и вызывает специфические нелинейные искажения еe амплитудно-частотных характеристик. Оценка влияния 
технологической нагрузки на динамические характеристики колебательных систем ультразвуковых машин - наша вторая цель.

Обе поставленные задачи решаются в работе на основе предложенных динамических моделей ультразвуковых технологических процессов. Эти модели позволяют не только построить ясную физическую картину наблюдаемых в эксперименте эффектов, но и, органично вписываясь в общую расчетную схему ультразвуковой машины, определить ее основные динамические свойства под нагрузкой. В результате основные параметры процесса, включая среднюю скорость его протекания, характеризующую производительность машины, ее КПД и показатели эффективности, удается связать с основными конструктивными параметрами машины.

И наконец, третья решаемая здесь задача состоит в разработке способов возбуждения и автоматического поддержания наиболее эффективных резонансных режимов работы ультразвуковых машин при изменении технологической нагрузки в широких пределах. Показано, что эта задача эффективно решается путем перехода к автоколебательному способу возбуждения, осуществляемого с помощью специально организованного контура положительной обратной связи.

Основное содержание книги изложено в 4-х Главах и Приложении, общее распределение материала по которым имеет следующий вид.

В Главе 1 рассматриваются физические основы ультразвуковых технологических процессов. Приводятся схемы ряда процессов, и дается обзор работ, посвященных их экспериментальному исследованию. Среди них ультразвуковая обработка хрупких материалов, поверхностное упрочнение металлов, пластическое деформирование изделий, процессы волочения прутков, ультразвуковое резание, сварка металлов и пластмасс и др. Обсуждаются эффекты, обнаруженные при экспериментальных исследованиях этих процессов. Отмечается, что наложение ультразвуковой вибрации приводит к 
существенному снижению статических сил, необходимых для пластического деформирования, разрушения и преодоления сил трения.

Далее предлагается подход к описанию технологических ультразвуковых процессов, основанный на использовании реологических моделей материалов, отражающих их реальные упругие, вязкие и пластические свойства. Рассматриваются вопросы влияния ультразвуковой вибрации на процессы пластического деформирования и сухого трения. Описываются возможные режимы взаимодействия колеблющегося инструмента с изделием. Показывается эффективность использования виброударных режимов взаимодействия. Проведенный анализ позволил выявить зависимость средней скорости процесса от действующих статических сил и параметров колебаний. Полученные результаты сопоставляются с известными экспериментальными данными. В результате построен достаточно ясный физический механизм описанных выше эффектов влияния ультразвука на процессы пластического деформирования и трения.

Глава 2 посвящена построению динамических характеристик ультразвуковых технологических машин различного назначения. Здесь приводятся схемы машин, описываются применяемые приводы магнитострикционные и пьезоэлектрические возбудители колебаний, концентраторы колебаний - волноводы, передающие колебания инструменту с увеличением амплитуды. Рассмотрена обобщенная модель машины, представляющая цепную электромеханическую систему связанных между собой элементов: возбудителя колебаний; механизма передачи колебаний; технологической нагрузки. Приводится общая схема анализа динамики машины с использованием передаточных функций возбудителя и операторов динамической податливости и жесткости волноводов и технологической нагрузки. Далее строятся динамические податливости ультразвуковых различных стержневых систем, совершающих продольные и изгибные колебания. С их помощью находятся резонансные и антирезонансные частоты и амплитуды систем, параметры концентраторов разных типов. 
Рассматриваются динамические характеристики магнитострикционных и пьезоэлектрических возбудителей колебаний. На основе анализа дается обоснование использования упрощенных динамических моделей.

В Главе 3 исследуются нелинейные процессы в ультразвуковых технологических системах. Строятся нелинейные динамические характеристики различных процессов. C помощью процедур метода гармонической линеаризации находятся упругие и диссипативные составляющие нелинейной технологической нагрузки. Предлагаются некоторые способы их упрощения.

Отыскиваются периодические движения систем, совершающих продольные и изгибные колебания и взаимодействующих с сосредоточенной нелинейной нагрузкой. Строятся амплитудно-частотные характеристики колебательных систем, выявляются специфические нелинейные динамические эффекты, исследуется устойчивость полученных решений.

Изучаются периодические виброударные процессы при взаимодействии двух волноводных систем, сочлененных с зазором, натягом или сжатых постоянной силой. Исследуются амплитудно-частотные характеристики систем. Показано, что нелинейность нагрузки вызывает специфические искажения резонансных кривых и приводит к появлению различных нелинейных эффектов, таких как возникновение областей неоднозначности амплитудно-частотных характеристик, затягивание колебаний в резонансные зоны по частоте и амплитуде, срывы колебаний при изменении частоты и жесткий запуск резонансных колебаний.

Рассматривается задача вибрационного перемещения вязкоупругого стержня в среде с сухим трением под действием постоянной силы. Резонансные колебания стержня возбуждаются силой, действующей в одном из сечений стержня, а сила сухого трения равномерно распределена по всей его длине. Отыскивается средняя скорость внедрения стержня в среду и ее зависимость от постоянной силы и параметров вибрации. Дается оценка 
взаимовлияния быстрых (колебательных) и медленных (со средней скоростью) движений стержня.

Глава 4 посвящена вопросам резонансной настройки ультразвуковых технологических машин под нагрузкой. Рассматриваются способы повышения эффективности ультразвуковых станков под нагрузкой. С помощью реологической модели процесса ультразвукового резания производительность станка связывается с его динамическими характеристиками и основными конструктивными параметрами. Полученные результаты сопоставляются с известными экспериментальными данными Рассматриваются предельные возможности различных способов настройки колебательной системы. Приводятся результаты экспериментов.

Излагается нелинейная теория ультразвуковых концентраторов, работающих на нелинейную упругодиссипативную нагрузку. Для процесса ультразвуковой обработки определены зависимости скорости резания, упругой и диссипативной составляющих нагрузки от параметров колебаний концентратора, рассчитанного с учетом нелинейной технологической нагрузки.

Показано, что существенное увеличение производительности при значительном снижении энергетических затрат может быть достигнуто за счет повышения добротности колебательной системы и резонансной настройки под нагрузкой. Принципиальную возможность полного использования динамических свойств машины дает создание авторезонансной системы возбуждения, осуществляемой с помощью цепи положительной обратной связи. Рассматриваются вопросы синтеза контура обратной связи, исследуется динамика автоколебательной системы возбуждения ультразвуковых технологических машин, проводится анализ условий самовозбуждения колебаний, исследуются вопросы существования и устойчивости периодических режимов. Показано, что авторезонансная система осуществляет автоматическое поддержание наиболее эффективных 
резонансных режимов при изменении параметров машины и технологической нагрузки в широких пределах.

В заключение этой главы ультразвуковое резание рассматривается как нелинейный (виброударный) процесс. Предлагается реологическая модель процесса. Объясняется экспериментально обнаруженный эффект снижения силы резания при ультразвуковой вибрации резца. Исследуется динамика устройства для ультразвукового резания, и строятся его амплитудночастотные характеристики. Приводятся результаты экспериментальных исследований. Дается описание авторезонансной системы возбуждения колебаний резца.

В Приложении изложены результаты исследований влияния авторезонансной ультразвуковой обработки на поверхность обрабатываемых образцов. После ультразвуковой обработки металлов возникает наноструктированные приповерхностные слои, отвечающие за макромеханические характеристики материала. Показаны результаты исследования ряда материалов, подвергнутых ультразвуковой токарной обработке, обеспечиваемой авторезонансным устройством. В частности, даётся описание, наноструктур, возникающих в материалах обрабатываемых образцов. Выясняется, что авторезонансная ультразвуковая обработка приводит к упрочнению поверхностных слоев образцов.

Список литературы составлен из источников, результаты которых используются или упоминаются в книге. По этой причине огромное число исследований, относящихся к разрабатываемой теме, не вошли в прилагаемый перечень. Попытка даже беглого их обзора привела бы к непомерному увеличению объема книги. Приложение снабжено собственным списком литературы.

В книге принята сквозная нумерация Параграфов и двойная нумерация формул и рисунков: первое число означает номер параграфа, второе - номер формулы или рисунка в данном Параграфе. 


\section{ГЛАВА I \\ НЕЛИНЕЙНЫЕ МОДЕЛИ \\ УЛЬТРАЗВУКОВЫХ ТЕХНОЛОГИЧЕСКИХ ПРОЦЕССОВ}

\section{1. Ультразвуковые технологические процессы}

1. Рабочий процесс ультразвуковой технологической машины осуществляется рабочим органом, взаимодействующим с обрабатываемым изделием или средой. Для этого рабочему органу сообщаются колебания определенного направления, частоты и интенсивности. Колебания возбуждаются приводом, а передача их рабочему органу обеспечивается колебательной системой зачастую с изменением направления и амплитуды.

Конструкция машины и ее элементов определяется в значительной степени схемой технологического процесса, выполняемого рабочим органом. Рассмотрим некоторые из таких процессов.

На рис.1.1, а показана схема ультразвуковой обработки [141-143, 173] твердых хрупких материалов.
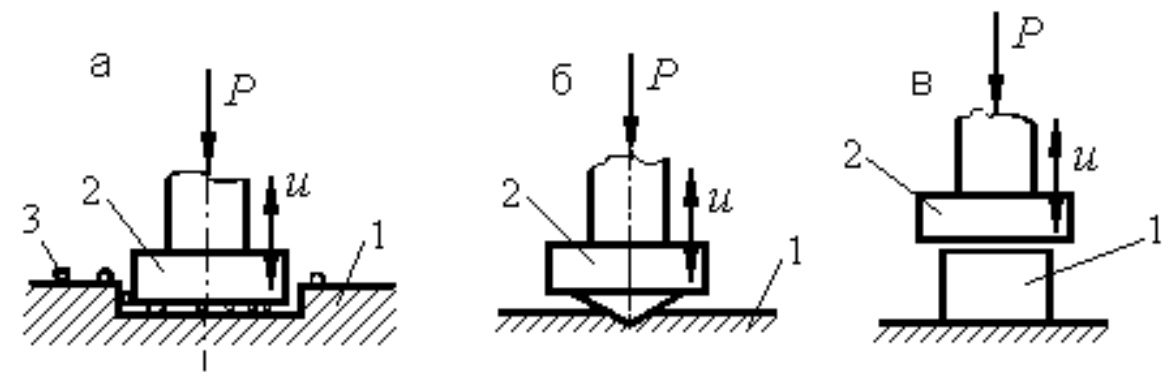

Pис.1.1

Обрабатываемое изделие 1 помещается под торец инструмента 2, которому сообщаются высокочастотные колебания в направлении, перпендикулярном к поверхности обработки. В рабочую зону, образованную торцом инструмента и поверхностью изделия, подается абразивная суспензия. Инструмент поджимается к изделию статическим усилием $P$ подачи. Под действием ударов инструмента по зернам абразива, оседающим из суспензии на обрабатываемой поверхности, 
происходит разрушение материала изделия и образуется полость, повторяющая форму инструмента.

Со времени изобретения [216] способ ультразвуковой обработки получил признание и широкое применение для резания трудно обрабатываемых традиционными методами материалов, таких как стекло, керамика, полупроводниковые материалы, феррит, алмаз, твердые сплавы и т.д. В этом существенную роль сыграли многочисленные экспериментальные исследования, посвященные главным образом оценке влияния различных факторов на скорость резания. Не вдаваясь в подробный обзор огромного числа работ, ограничимся здесь кратким изложением основных результатов, наиболее существенных для дальнейшего рассмотрения.

Сюда, прежде всего, следует отнести работы по выяснению физического механизма разрушения материала при ультразвуковой обработке. Экспериментами [2,3,171,172] с применением высокоскоростной киносъемки установлено, что разрушение материала происходит исключительно в результате соударений инструмента с обрабатываемой поверхностью через частицы абразива. Исследование возникающих при этом сил показало [53,109,211,236], что движение инструмента представляет собой периодический виброударный процесс, в котором соударения с изделием следуют с частотой колебаний инструмента, причем амплитуда силы удара является основным фактором, влияющим на скорость резания [110,235]. В свою очередь характер ударного взаимодействия - форма импульса, его продолжительность, максимальная величина силы, зависят от конкретных условий резания.

Скорость резания при прочих равных условиях возрастает с увеличением амплитуды и частоты колебаний инструмента, а также статического усилия подачи [218,219,233,237,238]. На производительность процесса ультразвукового резания оказывает влияние кроме того и ряд технологических факторов: механические свойства материала обрабатываемого изделия, инструмента и абразива; размер абразивных зерен и их концентрация в суспензии; размеры и 
форма инструмента; глубина обработки и т.д. Это обусловлено характером физической картины процесса резания. Ультразвуковой обработке лучше поддаются хрупкие материалы, причем скорость резания тем выше, чем меньше твердость материала [221]. При ударах инструмента разрушается не только материал изделия, но и абразивные зерна. Для стабилизации условий резания часто производят принудительную смену абразива в рабочей зоне [111].

Необходимо обратить внимание на важную особенность процесса ультразвукового резания. Для разрушения материала между инструментом и изделием необходимо создать силы, достаточные для получения в окрестности зерен абразива напряжений, превышающих предел прочности обрабатываемого материала. Эти силы могут достигать значительных величин. Вместе с тем процесс резания происходит даже при очень малых усилиях статического прижима инструмента к изделию. Таким образом, введение ультразвуковых колебаний в зону обработки позволяет существенно снизить статические усилия, необходимые для преодоления предельных нагрузок.

2. Отмеченный эффект снижения необходимых для проведения процесса статических сил является характерной особенностью большинства ультразвуковых технологических процессов, связанных с разрушением или пластическим деформированием обрабатываемой среды. На рис.1.1,б показана схема процесса поверхностного упрочнения $[143,144]$ детали 1 , происходящего в результате пластического деформирования поверхностного слоя металла при высокочастотных колебаниях наконечника 2. И в этом случае для поддержания технологического процесса требуется относительно малая статическая сила прижима $P$, как правило, на порядок меньшая необходимой для пластической деформации материала при отсутствии колебаний наконечника. Ультразвуковое поверхностное упрочнение протекает наиболее интенсивно, а снижение статического прижима наиболее значительно, если рабочий режим колебаний наконечника представляет собой, как и при ультразвуковом резании (рис.1.1,a), периодический виброударный процесс. 
По аналогичной схеме строится процесс осадки металлов (рис.1.1,в) с наложением ультразвуковых колебаний [178,179,202]. Остановимся на некоторых результатах по пластическому деформированию образца 1 , подвергаемого совместному воздействию статической нагрузки $P$ и высокочастотных колебаний инструмента 2. В [222,223] описан эксперимент, который выполнялся на установке, схематично изображенной на рис.1.2.

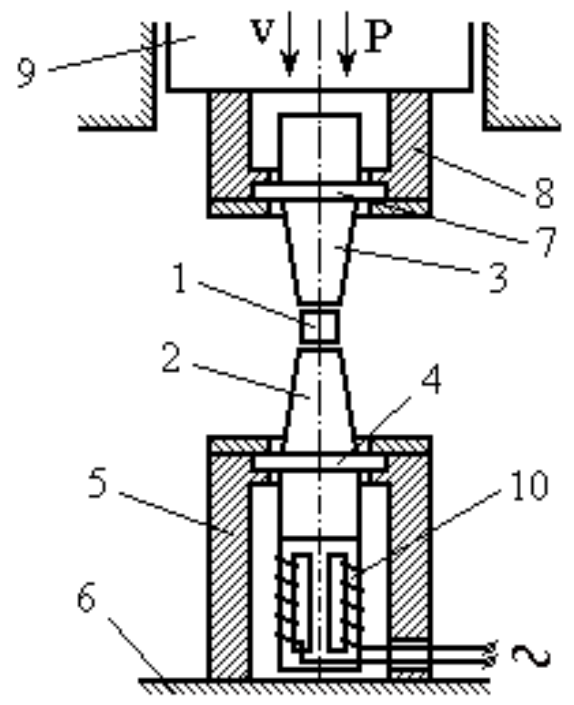

Рис.1.2

Испытуемый образец 1 помещен между торцами идентичных стержней 2 и 3. Стержень 2 с помощью фланца 4 связан с корпусом 5, установленным на станине 6 гидравлической испытательной машины. Стержень 3 через фланец 7 и корпус 8 соединен с поперечной траверсой 9 испытательной машины, перемещением которой с малой скоростью $\mathrm{v}$ производится деформация образца. Стержень 2 является волноводом, передающим в рабочую зону колебания от вибровозбудителя - магнитострикционного преобразователя 10 , получающего питание от генератора переменного тока. Установка снабжена устройствами регистрации статической нагрузки на образец и величины его деформации, а также системой контроля и стабилизации амплитуды колебаний стержня 2 при изменении усилия деформирования. 


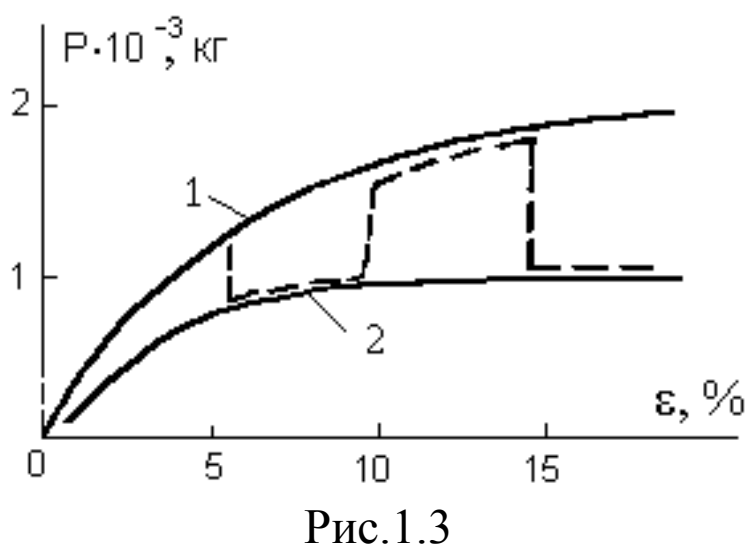

Результаты эксперимента [222] приведены на рис.1.3. Кривой 1 представлена диаграмма “деформация - сила”, полученная при статическом (без вибрации) сжатии алюминиевого образца диаметром $d=14$ мм и высотой $H=20$ мм. По оси абсцисс на рисунке отложена относительная деформация $\varepsilon=h / H$, где $h$ - укорочение образца. Кривой 2 показано изменение статического усилия $P$ при деформации аналогичного образца с одновременным подводом ультразвуковых колебаний в зону деформации. Из сопоставления кривых 1 и 2 ясно видно, что при ультразвуковом воздействии одна и та же величина деформации образца достигается при значительно меньшей статической нагрузке, хотя деформирование в обоих случаях производилось с постоянной скоростью $v=0,5$ мм/мин.

Отметим, что в экспериментах [222,223] возбуждались колебания с обычными для ультразвуковых технологических процессов частотами $f \approx 22$ кГц и амплитудами рабочего торца волновода $a \approx 10$ мкм. Колебания со столь высокими частотами и малыми амплитудами не различимы невооруженным глазом, и у наблюдателя создается впечатление, что под влиянием ультразвука происходит размягчение материала, изменение его упругопластических свойств. Существенно, что такое размягчение наблюдается только во время действия колебаний. Это подтверждается показанными на рис.1.3 штриховой линией результатами эксперимента по осадке образцов с периодическим наложением ультразвуковых колебаний. 
Описанные эффекты подтверждены многочисленными экспериментами, обобщенными в обзоре [202] и монографиях [177-179]. Отметим наиболее характерные особенности поведения материалов при деформациях с ультразвуковым воздействием. Для каждого материала существует определенное пороговое соотношение между статической нагрузкой и уровнем ультразвуковых колебаний, превышение которого приводит к возникновению остаточной пластической деформации. Введение ультразвуковых колебаний в зону деформации всегда приводит к снижению статического усилия, необходимого для пластического деформирования. Статическое усилие $P$ зависит от скорости $v$ нагружения. Податливость материала по отношению к статической силе возрастает с увеличением интенсивности колебаний. В отдельных экспериментах достигнуто снижение статических сил более чем на 85\% [202].

Аномальное по отношению к статической нагрузке поведение материалов в ультразвуковом поле породило ряд гипотез о механизмах воздействия ультразвука на материал [197]. В одних гипотезах предполагается влияние ультразвукового воздействия на внутреннюю структуру материала, распределение и подвижность дислокаций. В других повышение пластичности материалов в ультразвуковом поле связывают с тепловыми эффектами, вызванными рассеянием энергии при возбуждении высокочастотных колебаний образца. Однако гипотезы подобного рода не позволяют с достаточной степенью приближения объяснить наблюдаемые эффекты, и хотя указанные причины нельзя отвергать категорически, в рассматриваемых процессах они играют, по-видимому, второстепенную роль. Это утверждение основано на том, что обычно применяемые в ультразвуковых технологических процессах частоты порядка $10^{4}-10^{5}$ Гц слишком далеки от диапазона $10^{8}-10^{10}$ Гц, в котором возможно активное воздействие колебаний на кристаллические решетки и дислокационные линии [154]. Поэтому весьма вероятно, что наблюдаемое иногда при кристаллографических исследованиях образцов, подвергнутых ультразвуковому воздействию, изменение структуры материала [226] и плотности дислокаций [227] является не причиной аномального 
поведения материала в ультразвуковом поле, а следствием произведенной пластической деформации.

Относительно влияния тепловых эффектов отметим, что снижение статической силы под действием ультразвука наблюдается и в случае принудительного поддержания постоянной комнатной температуры [226]. Более того, экспериментальное сопоставление действия ультразвука и температуры показало, что для получения одинакового разупрочнения тепловой энергии требуется на несколько порядков больше, чем ультразвуковой. Поэтому в [222,226] указано, что тепловой эффект не может рассматриваться в качестве основного фактора снижения предела текучести материала под влиянием ультразвука.

Исходя из этих положений, в настоящей работе предлагается подход к описанию технологических ультразвуковых процессов, основанный на использовании реологических моделей материалов [169], отражающих их
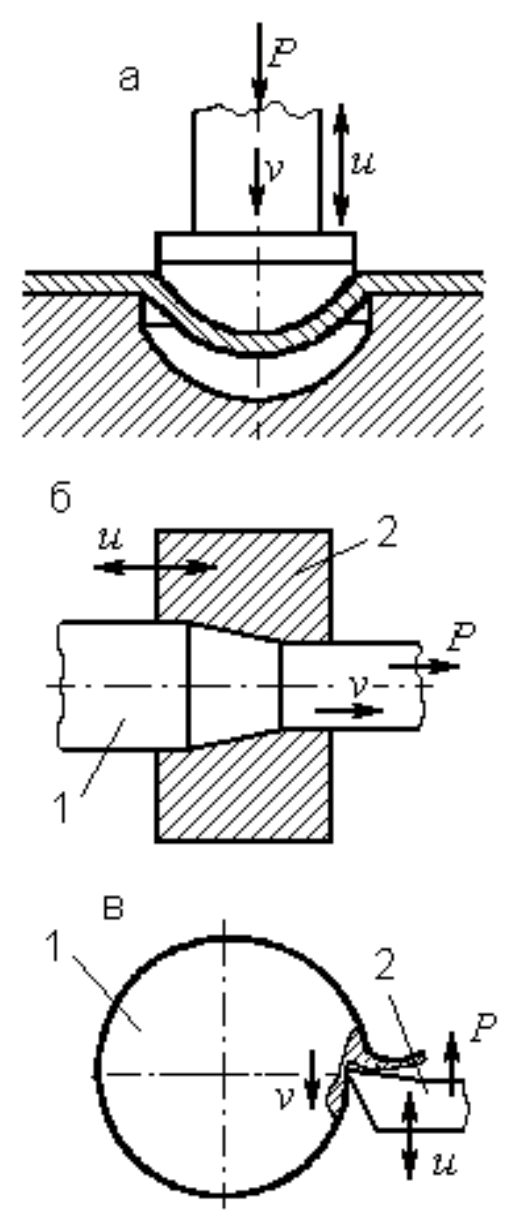

Рис. 1.4 реальные упругие, вязкие и пластические свойства. Различные аспекты такого подхода будут подробно рассмотрены ниже.

3. Описанные эффекты снижения статической нагрузки составляют основу применения ультразвука для интенсификации ряда технологических процессов, связанных с пластическим деформированием или разрушением материала. На рис.1.4, а показана схема процесса листовой штамповки [178] детали 1, в котором пуансону 2 сообщаются колебания в направлении действия статической силы $P$.

Эта схема аналогична приведенным ранее на рис.1.1,б, в. Особенность процесса листовой штамповки заключается в том, что по мере погружения пуансона изменяются условия 
пластического деформирования материала из-за изменения геометрии изделия. Кроме того, в течение технологического процесса помимо сил деформи-рования на пуансон действуют силы трения, направленные по касательной к его поверхности в сторону противоположную скорости пуансона относительно изделия. Силы трения могут составлять значительную долю в общем балансе сил, действующих на пуансон, особенно при штамповке трудно деформируемых материалов. Эксперименты показывают, что возбуждение колебаний пуансона приводит к снижению статических усилий, необходимых для преодоления сил трения.

Силы трения играют существенную роль в процессе волочения прутков, проволоки и труб. Схема процесса показана на рис.1.4,б. Заготовка 1 протягивается через отверстие волоки 2. В конической части отверстия происходит обжатие заготовки и еe пластическая деформация, цилиндрическая часть калибрует окончательный размер изделия. При протягивании заготовки через жестко закрепленную волоку в зоне деформации действуют силы сухого трения $G_{1}=v \cdot N_{1}, G_{2}=v \cdot N_{2}$, где $N_{1}, N_{2}-$ силы нормального давления, обусловленные упругопластическими свойствами материала; v - коэффициент трения.

Необходимая сила волочения

$$
P=N_{1} \cdot(\sin \theta+v \cdot \cos \theta)+v \cdot N_{2}
$$

где $\theta$ - угол конической части волоки.

Из соотношения (1.1) видно, что вклад того или иного слагаемого в величину силы $P$ зависит от свойств обрабатываемого материала, коэффициента трения и геометрии волоки.

Отмеченное обстоятельство наблюдается и при ультразвуковом волочении $[157,178,179,241]$, когда волоке сообщаются колебания в направлении про-тягивания заготовки. Как и во всех ранее рассмотренных технологических процессах, введение ультразвука в рабочую зону приводит к снижению статической силы волочения. Так уже в ранних экспериментах [241] 
по волочению стальной и медной проволоки было получено снижение статического усилия до 50\%. В работах [155,241] описаны эксперименты, цель которых заключалась в выяснении основной причины уменьшения силы волочения. В [241] такой причиной считается снижение сопротивления металла деформированию, в [155] - уменьшение внешнего трения. Ниже в п.3.4 мы покажем, что влияние каждого из этих факторов определяется в значительной степени их соотношением в (1.1), однако вклад каждого из слагаемых неравнозначен и зависит от конкретных условий деформирования.

Благодаря относительной простоте реализации, регулирования и регистрации основных параметров, ультразвуковое волочение - один из наиболее изученных экспериментально процессов деформирования с применением ультразвука. В результате экспериментов установлено [155,157], что ультразвуковое воздействие приводит к снижению статической силы лишь при скоростях волочения $v$ меньших амплитуды вибрационной скорости волоки $v<a \omega$, где $a$ - амплитуда, $\omega$ - угловая частота колебаний.

Наибольший эффект от применения ультразвука наблюдается при волочении высокопрочных материалов, которые в обычных условиях плохо поддаются пластическому деформированию из-за возникновения трещин и обрывов на выходе из волоки [195]. С другой стороны, вследствие существенного снижения статической силы при ультразвуковых колебаниях волоки удается увеличить степень обжатия и предотвратить обрывы проволоки из очень пластичных, подобных свинцу, металлов [114,202].

Отмеченные свойства характерны для всех технологических процессов с использованием ультразвуковых колебаний рабочего органа. Наблюдаются они и в процессах металлообработки с наложением ультразвуковых колебаний на режущий инструмент [106,130,141-143]. На рис.1.4,в изображена схема токарной обработки детали 1 резцом 2. Сила резания создается в основном за счет пластической деформации срезаемого слоя и преодоления сил внешнего трения на рабочих поверхностях инструмента. 
Возбуждение ультразвуковых колебаний резца вызывает снижение обеих основных составляющих силы резания. Наибольший эффект достигается в том случае, когда направление колебаний совпадает с направлением скорости резания, как это показано на рис.1.4,в. Аналогичный результат получается и при других видах металлообработки, например, при изготовлении зубчатых колес зубодолблением [102].

На рис.1.5 показана экспериментальная зависимость [106] силы резания $P$ от скорости $\mathrm{v}$ резания при традиционной токарной обработке (кривая 1) и точении с наложением ультразвуковых колебаний на режущий инструмент (кривая 2). Необходимо обратить внимание на "исчезновение" силы резания при малых, близких к нулю, скоростях $v$. Речь идет, конечно, о постоянной составляющей силы резания, которая оценивается либо по величине момента сил вращения заготовки, либо по смещению среднего уровня упругой деформации резца относительно его недеформированного состояния.

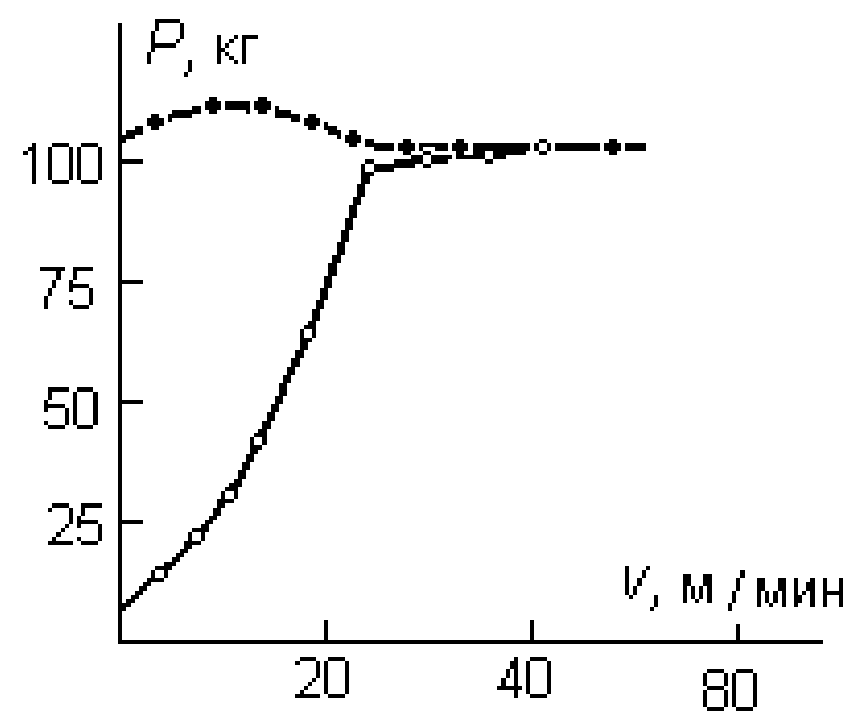

Рис.1.5

При увеличении скорости резания до значения $v=a \cdot \omega$ сила резания монотонно возрастает до ее величины при той же скорости в отсутствие колебаний. Такого вида связь постоянной силы и скорости направленного относительного перемещения характерна для систем с сухим трением при подводе колебаний в зону фрикционного контакта, для которых подобные 
зависимости экспериментально получены в [155,240]. Сказанное не означает, однако, что в рассматриваемом процессе именно силы трения играют решающую роль. Скорее, наоборот, силы пластического деформирования являются преобладающими. Отметим, что практически полное исчезновение статической силы при малых скоростях $v$ экспериментально наблюдается в процессах пластического деформирования с ультразвуком [202]. В дальнейшем физический механизм этого явления будет построен, исходя из реальных упругопластических свойств материала.

4. Если в описанных выше процессах силы трения, как правило, ухудшают нормальное течение процесса и являются вредным фактором, сопутствующим основной технологической нагрузке, то при ультразвуковой сварке металлов [119,129,152,153] трению принадлежит ведущая роль в образовании качественного сварного соединения [148,181].

На рис.1.6,а показана схема процесса ультразвуковой сварки металлов.
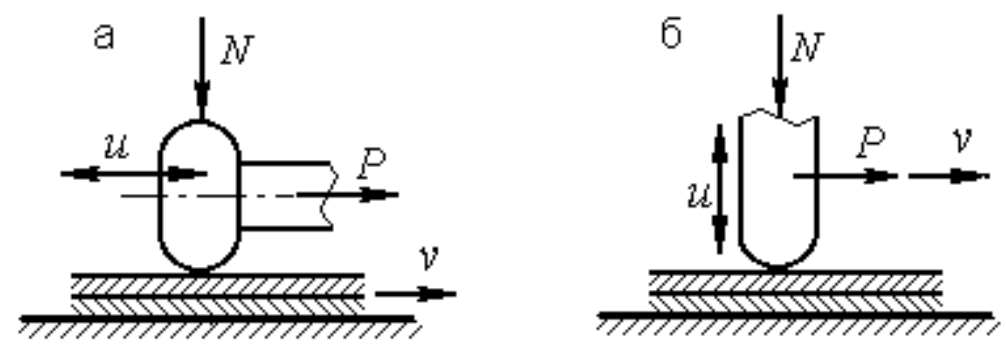

Рис. 1.6

Сварочному наконечнику 1, поджатому к соединяемым деталям постоянной силой $N$, сообщаются колебания в плоскости, перпендикулярной силе прижима. Благодаря вибрации, на границе деталей происходит сглаживание микронеровностей контактных поверхностей, их очистка от окисных пленок и сближение под действием нормальной сжимающей силы. Работа, затрачиваемая на преодоление сил трения, приводит к локальному разогреву деталей в зоне контакта до температуры, достигающей 0,4-0,6 от температуры плавления [204]. Все это создает благоприятные условия образования устойчивых металлических связей между свариваемыми деталями [204,243]. 
Механизм образования соединения при ультразвуковой сварке в основном аналогичен схватыванию металлов при сварке трением [180].

По мере образования сварного соединения производится подача наконечника в направлении, совпадающем с направлением колебаний (рис.1.6,a) или перпендикулярном к нему. В обоих случаях для подачи наконечника требуется статическая сила $P$, меньшая, чем при отсутствии колебаний $(P<v N)$. Таким образом, и здесь проявляется эффект снижения статической силы, необходимой для преодоления силы трения.

Ультразвуковая сварка применяется для соединения деталей из одинаковых или различных металлов, в том числе и таких, которые плохо поддаются сварке обычными методами. Разнообразные сочетания материалов, соединяемых с помощью ультразвуковой сварки, приведены в [195,204,213,214]. В полученном таким способом соединении металл почти не претерпевает структурных изменений и не изменяет своих физикомеханических свойств. Соединение отличает высокая прочность и пластичность, низкий уровень остаточных напряжений. Все это обеспечивает широкое применение ультразвуковой сварки в приборостроении, электронике, микроэлектронике.

По иной схеме (рис.1.6,б) строится процесс сварки пластмасс и синтетических тканей $[129,153,198]$. В этом случае сварочный наконечник 2 прижимается силой $P$ к поверхности соединяемых деталей 1. Направление колебаний наконечника, как и в рассмотренных ранее процессах упрочнения и пластического деформирования, совпадает с направлением действия силы прижима. При периодическом деформировании с частотой колебаний наконечника (обычно применяются частоты в диапазоне 20 - 100 кГц) происходит локальный разогрев соединяемых материалов. Разогрев вызывается потерями энергии на внутреннее трение и локализуется благодаря низкой теплопроводности свариваемых полимерных материалов. В результате происходит размягчение материалов в зоне сварки, их пластическая 
деформация и прочное сращивание. По мере образования сварного соединения материал подают под сварочный наконечник для получения непрерывного шва. И в этом случае усилие протягивания оказывается меньше силы трения при отсутствии вибрации.

Итак, мы рассмотрели некоторые примеры использования ультразвуковых колебаний в технологических процессах. Приведенные примеры не исчерпывают всех возможностей полезного использования энергии ультразвука [195]. Ультразвуковая кристаллизация и диспергирование, ультразвуковая очистка, дегазация и флотация $[1,89-91,93,196]$, ультразвуковая эхолокация, дефектоскопия и микроскопия $[65,136]$ - вот далеко не полный перечень использования ультразвука в металлургии и машиностроении, пищевой промышленности и приборостроении, в медицине и биологии.

Такое разнообразие приложений обусловлено, в первую очередь, специфическими физическими свойствами ультразвукового излучения. Сюда следует отнести простоту возбуждения ультразвуковых колебаний и их передачи с помощью волноводов обрабатываемой среде, возможность фокусировки и концентрации энергии ультразвука в узкой рабочей зоне, высокую направленность и проникающую способность ультразвукового излучения.

С другой стороны, разнообразны и механизмы воздействия ультразвука на твердые, жидкие и газообразные вещества и их смеси. Рассмотренные здесь процессы отличаются наиболее сильным взаимовлиянием источника ультразвуковых колебаний и обрабатываемой среды. Многочисленные аспекты такого взаимовлияния изучаются в данной книге. 


\section{2. Влияние ультразвука на процессы пластического деформирования}

1. В этой книге предлагается подход к описанию технологических ультразвуковых процессов, основанный на использовании реологических моделей [169] материалов, отражающих их реальные упругие, вязкие и пластические свойства. Такой подход позволяет построить достаточно ясный физический механизм [24] описанных выше эффектов влияния ультразвука на процессы пластического деформирования.

С этой целью обратимся к диаграмме “деформация - сила” (рис.2.1) при статическом сжатии некоторого образца.

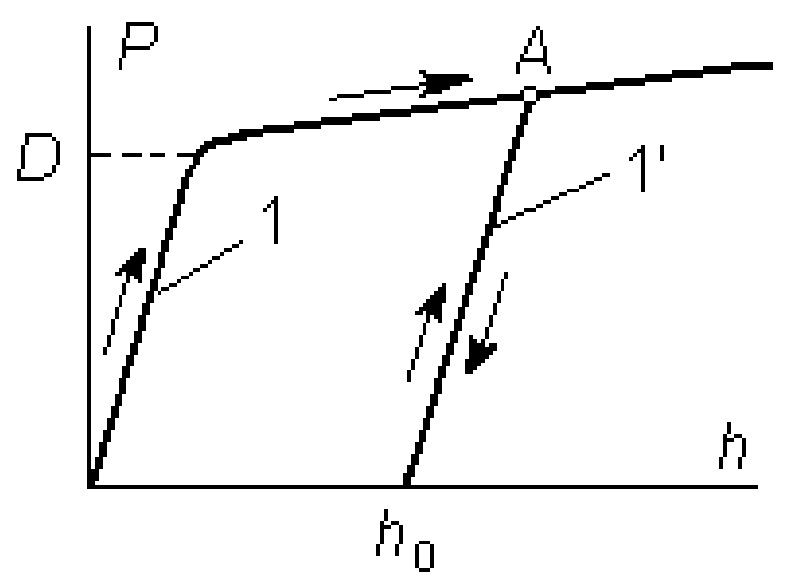

Рис.2.1

Здесь различаются области упругой и пластической деформации [168]. В упругой зоне при нагрузках $P$, не превышающих предел упругости $D$, сила $P$ и деформация $h$ связаны однозначной зависимостью $P=\varphi(h)$. Будем считать, что в этой зоне состояние материала подчиняется закону Гука

$$
P=k_{0} \cdot h=E \cdot S, h \leq D / k_{0}
$$

где $h$ - деформация образца; $k_{0}=E \cdot S / H$ - его статическая жесткость; $S, H$ площадь поперечного сечения и высота образца; $E$ - модуль упругости материала; $\varepsilon=h / H$ - относительная деформация. 
В зоне пластичности напряженно-деформированное состояние материала зависит от направления изменения деформации: при ее увеличении состояние отвечает кривой 1, а при уменьшении, например, от точки $A$ - линии 1', практически параллельной прямой 1 , как это показано стрелками на рис.2.1. После полной разгрузки обнаруживается остаточная пластическая деформация $h_{0}$. При повторном нагружении материал находится в упругом состоянии, определяемом линией 1', до момента достижения силой значения $P=D_{1}$, соответствующего точке $A$. Дальнейшее нагружение приводит к пластическому деформированию вдоль линии 1.

Показанная на рис.2.1 диаграмма нагружения является типичной для упругопластического материала с упрочнением, у которого усилие в зоне пластичности возрастает по мере деформирования. При циклическом деформировании такого материала его предел упругости возрастает от цикла к циклу и зависит от всей истории нагружения. (Именно этот эффект лежит в основе процесса вибрационного упрочнения (рис.1.1,б)). У идеального упругопластического материала пластическая деформация происходит при постоянной силе $P=D$ (штриховая линия на рис.2.1), и упрочнение отсутствует.

Поведение реального материала удобно описать, используя идеализированные модели, составленные из простейших элементов [145].

Так, модель (рис.2.2,a), содержащая последовательно соединенные упругий элемент $k_{0}$ и пару сухого трения $D$, дает при статическом нагружении характеристику идеальной упругопластической деформации (рис.2.2,б). Модель (рис.2.2,в), составленная из двух упругих элементов $k_{1}$, $k_{2}$ и пары трения $D$, приводит к характеристике упругопластической деформации с упрочнением (рис.2.2,г), где $k_{0}=k_{1}+k_{2}$. Отметим, что подобные модели успешно используются при исследовании колебаний упругопластических тел [157] и ударных явлений [161]. 

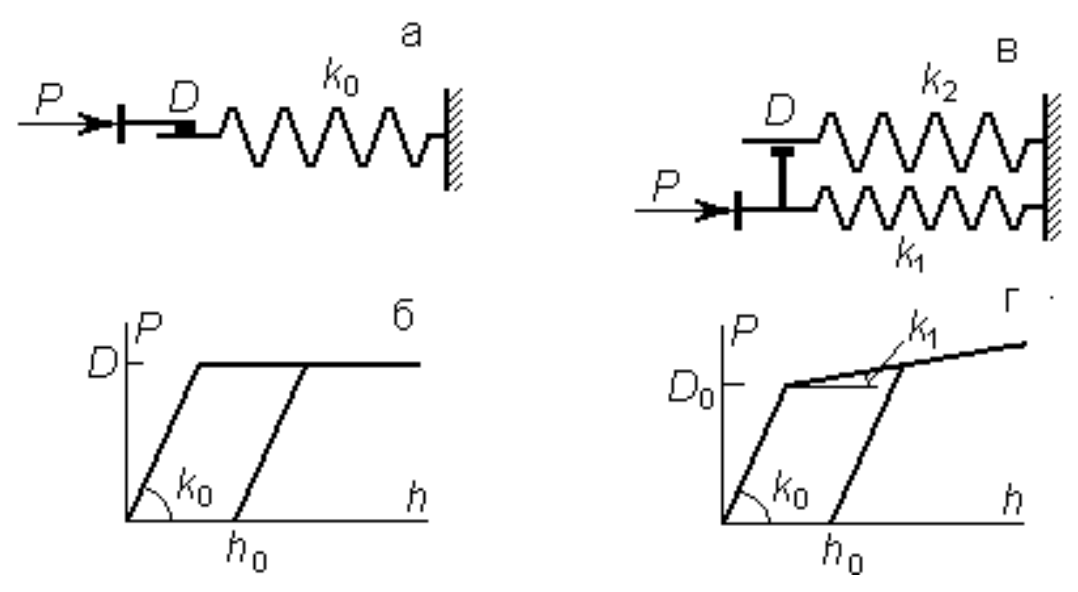

Рис. 2.2

2. С помощью приведенных моделей рассмотрим процесс деформации материала при вибрационном нагружении. Сначала оценим поведение идеального упругопластического материала.

Пусть деформация материала (рис.2.3,a) происходит в результате взаимодействия с инструментом 1 , приводимого в колебательное движение относительно каретки 2 по закону $u^{0}(t)=a \cdot \sin (\omega \cdot t)$, где $a$ амплитуда, $\omega$ - угловая частота.
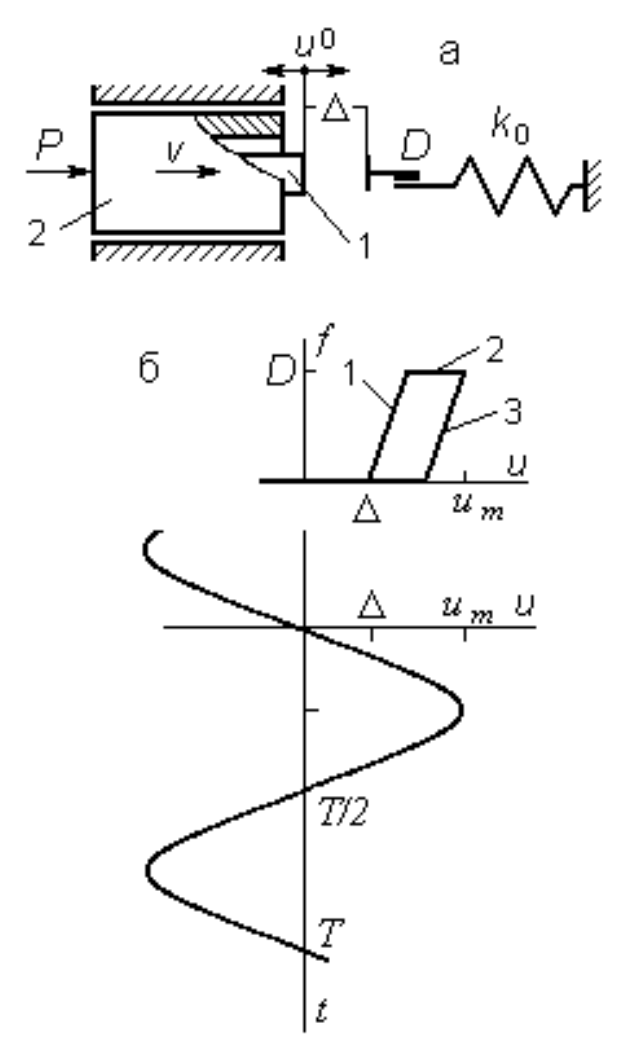

Рис.2.3

Каретка 2 нагружена постоянной статической силой $P$ и, в зависимости от условий деформирования, движется с некоторой постоянной скоростью $v$ или находится в состоянии равновесия $(v=0)$. В общем случае будем рассматривать полное движение инструмента вида $u(t)=v \cdot t+u^{0}(t)=v \cdot t+a \cdot \sin (\omega \cdot t)$

По аналогии со статическим нагружением проведем следующий эксперимент: медленно увеличивая силу $P$, будем следить за перемещением каретки. (Именно таким образом получены характеристики, показанные на 
рис.1.3.) Будем считать, что при каждом значении $P$ устанавливается периодический режим деформирования, поэтому можно ограничиться рассмотрением взаимодействия за один период колебаний инструмента. Обозначим (рис.2.3): $\Delta$ - расстояние средней линии колебаний инструмента от уровня недеформированного образца; $h=a-\Delta$ - смещение каретки относительно образца, равное максимальной деформации за период; $f$ - сила взаимодействия инструмента с образцом. Величины $\Delta>0$ соответствуют установке инструмента с предварительным зазором, а величины $\Delta<0$ - с предварительным натягом.

Будем считать, что деформированию подвергается короткий образец, такой, что время распространения вдоль него упругой волны много меньше периода нагружения, т.е. $H / c<<=2 \pi / \omega$, где $c$ скорость звука в материале. Поэтому нелинейная динамическая характеристика $f=f(u, \dot{u})$, устанавливающая связь силы $f$ c перемещением $u$ и скоростью $\dot{u}$ инструмента (рис.2.3,б), с точностью до обозначений и постоянного сдвига $\Delta$ совпадает с диаграммой статического нагружения (рис.2.2,б). Выражение для динамической характеристики запишем в виде

$$
f=f(u, \dot{u})= \begin{cases}0 & u \leq \Delta, \dot{u}>0 \\ k_{0}(u-\Delta) & \Delta \leq u \leq \Delta+\frac{D}{k_{0}}, \dot{u}>0 \\ D & \Delta+\frac{D}{k_{0}} \leq u \leq u_{m}, \dot{u}>0 \\ D+k_{0}\left(u-u_{m}\right) & u_{m}-\frac{D}{k_{0}} \leq u \leq u_{m}, \dot{u}<0 \\ 0 & u \leq u_{m}-\frac{D}{k_{0}}, \dot{u}<0\end{cases}
$$

где

$$
u_{m}=a\left[\sqrt{1-\left(\frac{v}{a \omega}\right)^{2}}+\frac{v}{a \omega} \arccos \left(-\frac{v}{a \omega}\right)\right]
$$

максимальное в течение одного периода значение функции (2.2). 
Принимая во внимание, что вследствие периодического характера исследуемых процессов сила взаимодействия

$$
F(t)=f[u(t), \dot{u}(t)]
$$

является периодической функцией времени с периодом $T=2 \pi / \omega$, с помощью теоремы импульсов получим следующее соотношение, связывающее постоянную силу $P$ с параметрами движения инструмента:

$$
P=\frac{1}{T} \int_{t_{1}}^{t_{1}+T} f(t) d t=\frac{1}{T} \int_{t_{1}}^{t_{1}+T} f[u(t), \dot{u}(t)] d t
$$

На рис.2.4 показаны возможные ситуации, возникающие при деформировании образца вибрирующим инструментом в случаях, когда деформация образца не выходит за пределы начального линейного участка динамической характеристики $\left(0<h \leq D / k_{0}\right)$, и в периодическом режиме каретка занимает некоторое положение динамического равновесия, a eе скорость $v=0$. Здесь на каждой позиции под динамической характеристикой $f(u, \dot{u})$ расположен график закона движения (2.2), а справа от нее построена временная развертка силы (2.5).
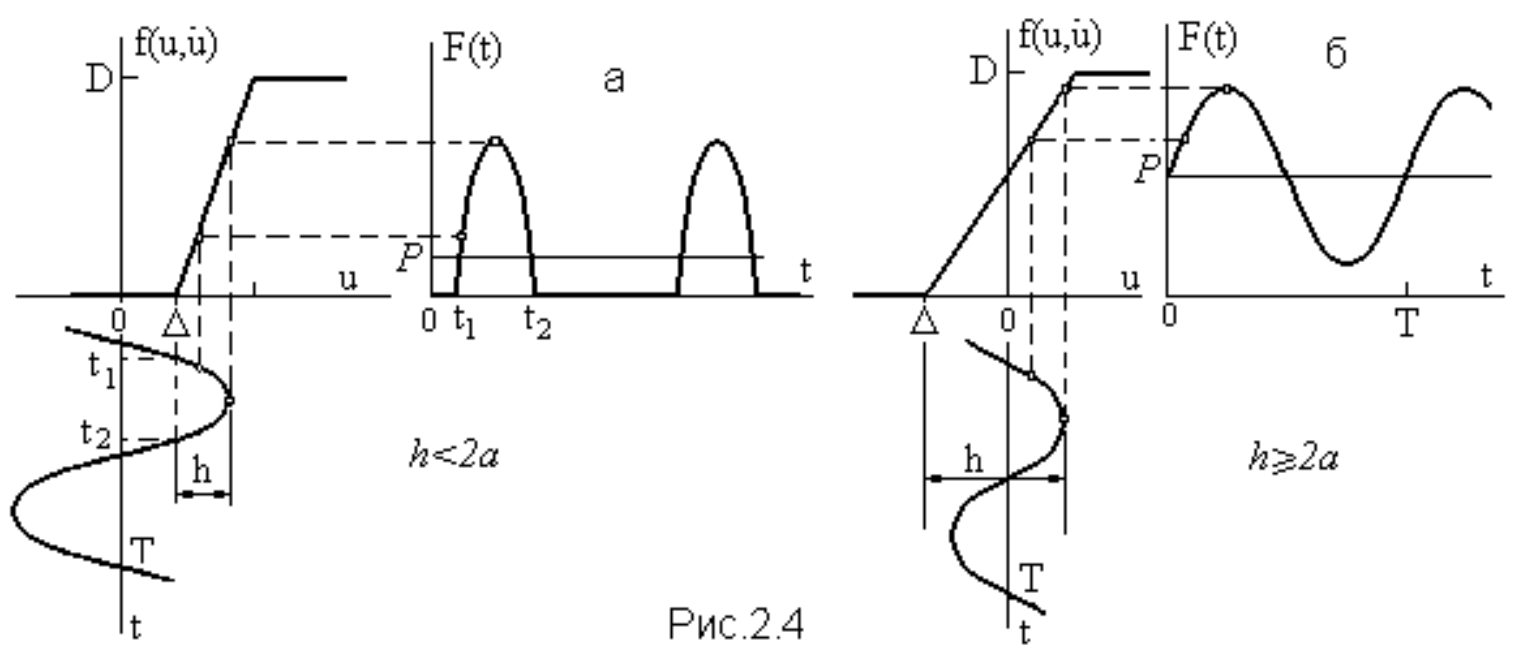

При $h<2 a$ (рис.2.4,a) воздействие инструмента на образец носит характер периодической последовательности силовых импульсов

$$
f(t)=k_{0}(a \sin \omega t-\Delta), t_{1} \leq t \leq t_{2},
$$

где $t_{1}=\frac{1}{\omega} \arcsin \frac{\Delta}{a}, t_{2}=\frac{\pi}{\omega}-t_{1}$. 
Подставив (2.7) в (2.6), с учетом равенства $\Delta=a-h$ получим

$$
P=\frac{a k_{0}}{\pi}\left[\sqrt{1-\left(1-\frac{h}{a}\right)^{2}}-\left(1-\frac{h}{a}\right) \arccos \left(1-\frac{h}{a}\right)\right]
$$

Если амплитуда колебаний $a>D / 2 k_{0}$, импульсный (виброударный) режим нагружения сохраняется во всей области упругого деформирования.

При амплитудах колебаний $a>D / 2 k_{0}$ и смещениях каретки $h \geq 2 a$ режим нагружения оказывается непрерывным (рис.2.4,б). Сила взаимодействия в области упругого деформирования описывается уравнением (2.7), в котором следует положить $t_{1}=-\frac{\pi}{2 \omega}$. В результате согласно (2.7) постоянная сила

$$
P=k_{0}(h-a)
$$

На рис.2.5 построены зависимости статической силы $P$ от смещения $h$ при различных величинах отношения $D / k_{0} a$. Штриховая прямая делит диаграмму на две области. В нижней области реализуются режимы импульсного (виброударного), в верхней - режимы непрерывного безударного деформирования.

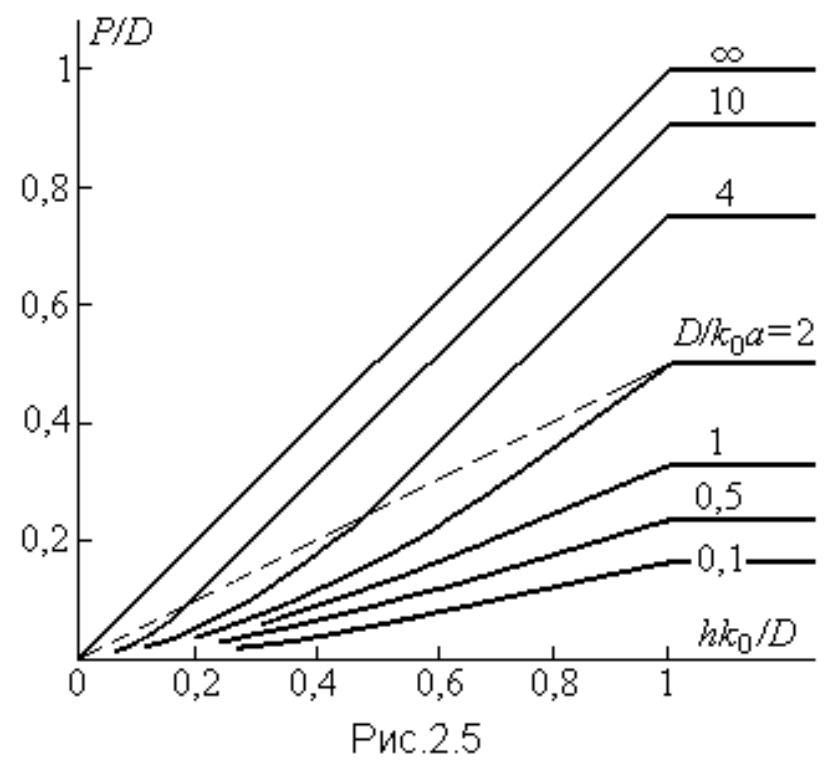


Уравнение границы перехода режимов $P=k_{0} h / 2$ получается из (2.8), (2.9) при $a=h / 2$. Здесь же для сравнения приведена диаграмма статического нагружения, получающаяся из (2.9) при $a=0$. Горизонтальные прямые отвечают значениям статических сил $P=D_{a}$,

$$
D_{a}=D \begin{cases}\left(1-\frac{k_{0} a}{D}\right) & \text { при } \quad a \leq \frac{D}{2 k_{0}} \\ \frac{1}{\pi}\left[\sqrt{\frac{2 k_{0} a}{D}-1}-\left(\frac{k_{0} a}{D}-1\right) \arccos \left(1-\frac{D}{k_{0} a}\right)\right] & \text { при } \quad a \geq \frac{D}{2 k_{0}}\end{cases}
$$

превышение которых приводит к появлению необратимых пластических деформаций. Эти значения находятся из соотношений (2.8), (2.9) при $h=D / k_{0}$.

Формула (2.10) определяет наблюдаемый предел текучести $\sigma_{a}=D_{a} / S$ (S - площадь сечения образца) материала при действии ультразвука. Его зависимость от амплитуды колебаний показана на рис.2.6.

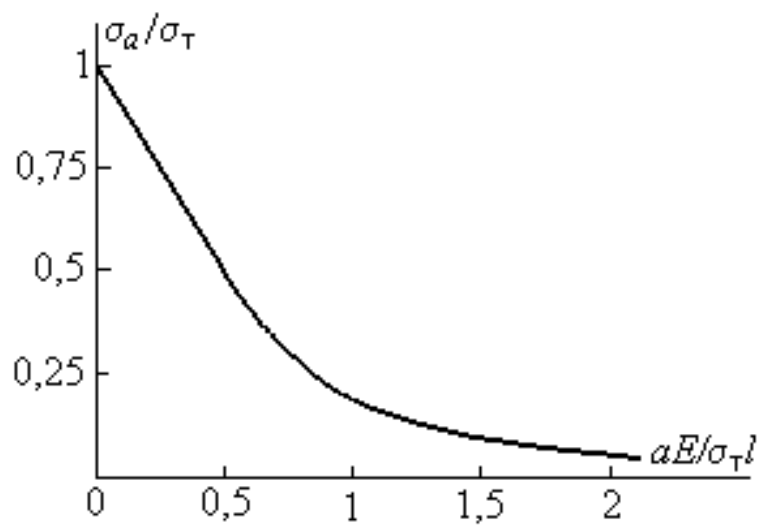

Рис.2.6

Здесь $\sigma_{\text {т }}=D / S, E=k_{0} l / S$ - предел текучести и модуль упругости материала; $l$ - длина испытуемого образца.

3. Перейдем к рассмотрению режимов, сопровождающихся выходом в область пластических деформаций $\left(h>D / k_{0}\right)$. Пусть в процессе деформирования каретка 2 (рис.2.3,a) движется с постоянной скоростью $v$ и, следовательно, полное движение инструмента 1 описывается уравнением (2.2). На рис.2.7 показаны возможные для этого случая ситуации деформирования. 

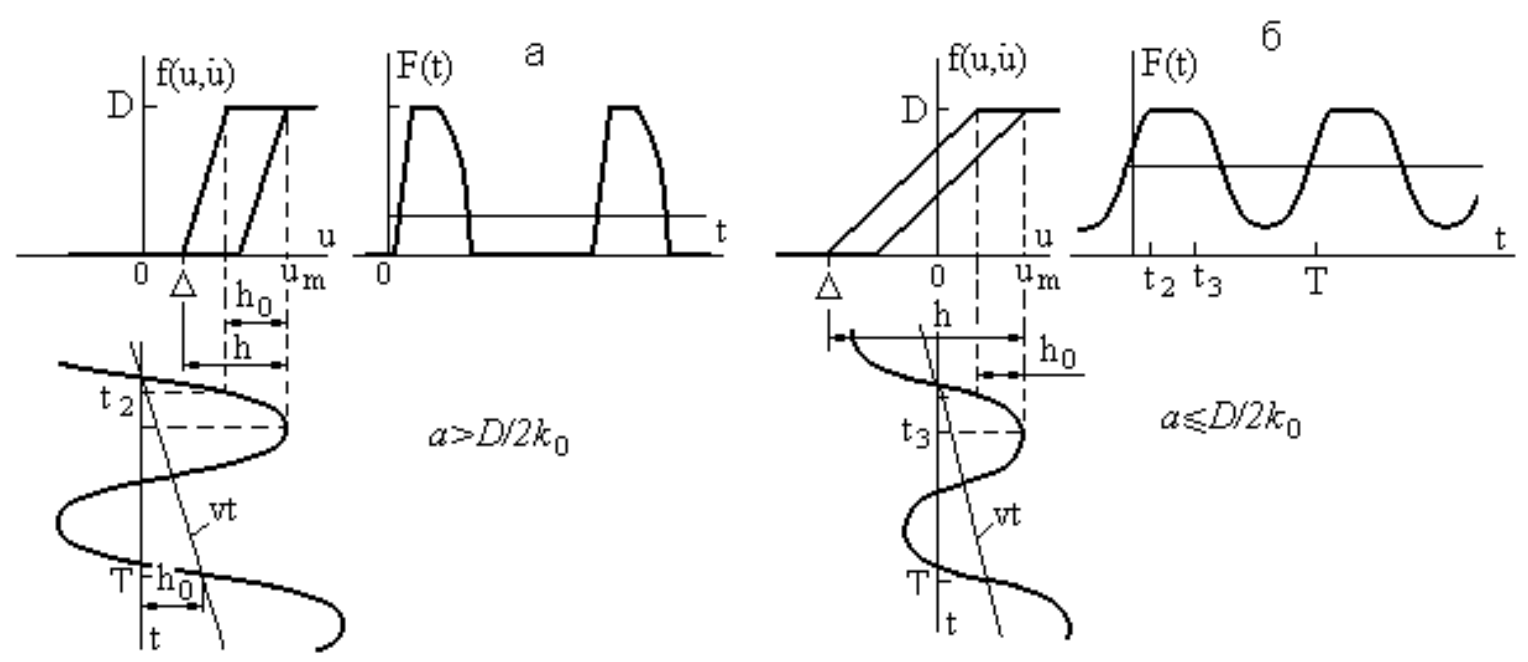

Рис. 2.7

Из рис.2.7 найдем величину остаточной деформации за один период колебаний $h_{0}=u_{m}-\Delta-D / k_{0}$. Тогда средняя скорость деформирования:

$$
v=\frac{h_{0}}{T}=\frac{u_{m} \omega}{2 \pi}\left(1-\frac{\Delta}{u_{m}}-\frac{D}{k_{0} u_{m}}\right)
$$

В режиме виброударного деформирования (рис.2.7,a), реализующемся при амплитудах колебаний $a>D / 2 k_{0}$, сила взаимодействия согласно (2.3), (2.2) имеет вид периодической последовательности импульсов

$$
f(t)=\left\{\begin{array}{cc}
k_{0}(v \cdot t+a \sin \omega \cdot t-\Delta) & t_{1} \leq t \leq t_{2} \\
D & t_{2} \leq t \leq t_{3} \\
D+k_{0}\left(v \cdot t+a \sin \omega \cdot t-u_{m}\right) & t_{3} \leq t \leq t_{4}
\end{array}\right.
$$

Здесь $t_{i},(i=1, \ldots, 4)$ - моменты переключения участков характеристики (2.3), определяемые следующими уравнениями:

$$
\begin{gathered}
\sin \tau_{1}=\frac{1}{a}\left(\Delta-\frac{v}{\omega} \tau_{1}\right), \sin \tau_{2}=\frac{1}{a}\left(\Delta+\frac{D}{k_{0}}-\frac{v}{\omega} \tau_{2}\right), \\
\tau_{3}=\arccos \left(-\frac{v}{a \omega}\right), \sin \tau_{4}=\frac{1}{a}\left(u_{m}-\frac{D}{k_{0}}-\frac{v}{\omega} \tau_{4}\right)
\end{gathered}
$$

где $\tau_{i}=\omega \cdot t_{i}$, причем $\tau_{12} \in\left[-\tau_{3}, \tau_{3}\right], \tau_{4} \in\left[\tau_{3}, 2 \pi+\tau_{1}\right]$; величина $u_{m}$ задается выражением (2.4). 
Подставив (2.12) в (2.6), получим

$$
P=\frac{k_{0}}{2 \pi}\left[\begin{array}{l}
\Delta \tau_{1}-\left(\Delta+\frac{D}{k_{0}}\right) \tau_{2}+u_{m} \tau_{3}-\left(u_{m}-\frac{D}{k_{0}}\right) \tau_{4}+ \\
+a\left(\cos \tau_{1}-\cos \tau_{2}+\cos \tau_{3}-\cos \tau_{4}\right)+ \\
+\frac{v}{2 \omega}\left(\tau_{2}^{2}-\tau_{1}^{2}+\tau_{4}^{2}-\tau_{3}^{2}\right)
\end{array}\right]
$$

Из (2.11) найдем

$$
\Delta=u_{m}-\frac{D}{k_{0}}-\frac{2 \pi \cdot v}{\omega}
$$

Равенство (2.14) после подстановки решений уравнений (2.13), полученных с учетом (2.15), дает зависимость статической силы $P$ от скорости $v$ пластического деформирования. Аналогичная зависимость находится для режимов непрерывного деформирования (рис.2.7,б), которые при амплитудах $a \leq D / 2 k_{0}$ реализуются во всем диапазоне изменения скорости $v$ каретки. Импульсные режимы также трансформируются в непрерывные при относительно больших скоростях $v$ после достижения в (2.13) предельных значений $\tau_{1}=-\tau_{3}$ и $\tau_{4}=2 \pi+\tau_{1}$. Для режимов непрерывного деформирования оказываются справедливыми те же соотношения (2.12) - (2.14), в которых следует положить

$$
\tau_{1}=-\tau_{3}=-\arccos \left(-\frac{v}{a \omega}\right), \tau_{4}=2 \pi+\tau_{1}
$$

Результаты расчетов приведены на рис.2.8, где построены зависимости статической силы $P$ от скорости пластического деформирования при различных значениях отношения $D / k_{0} a$. Штриховая линия, как и на рис.2.5, разделяет области вибро-ударного (нижняя) и безударного (верхняя) деформирования. При $v=0$ уравнения (2.12) - (2.16) приводят к величинам $P=D_{a}$, которые определяются выражениями (2.10). 


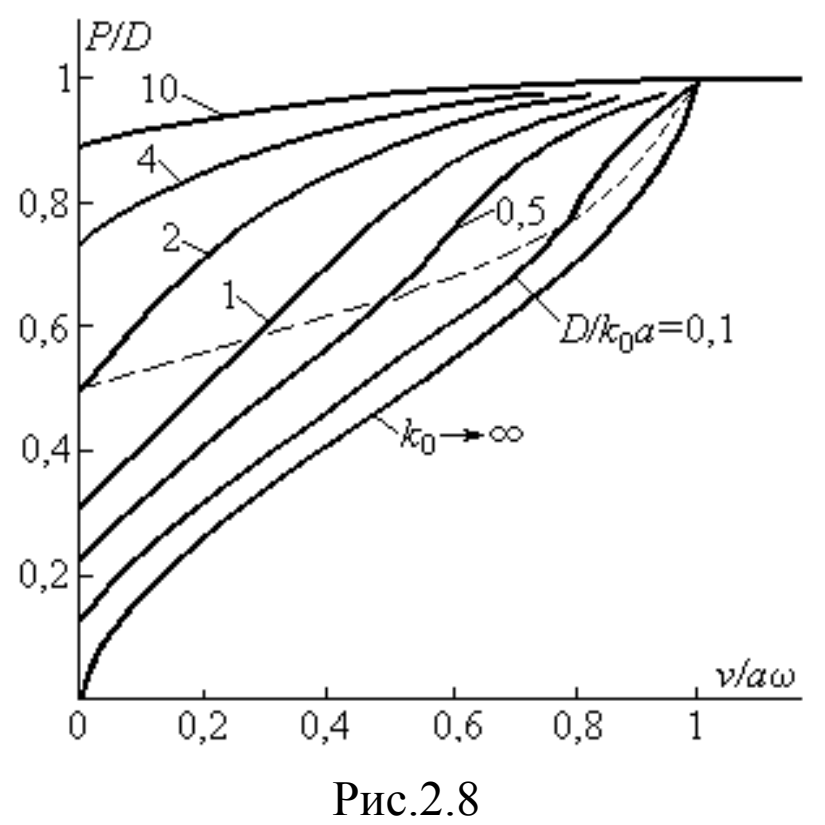

Согласно (2.4), (2.13), (2.16) найденные соотношения имеют действительные значения при скоростях деформирования $v \leq a \omega$. При $v=a \cdot \omega$. из (2.16), (2.13) находим $\tau_{1}=\tau_{2}=-\tau_{3}=-\tau_{4}=-\pi$, и после подстановки в (2.14) получаем $P=D$. Дальнейшее увеличение скорости $v$ не вызывает изменения статической силы $P$ пластического деформирования. Это отмечается во многих экспериментах [106,141, 158,179,202] по использованию ультразвука в технологических процессах (см. П.1.3 и формулу (1.2)). Заметим также, что как непрерывные, так и импульсные режимы неоднократно регистрировались в экспериментах $[53,109,172,202]$, причем импульсные режимы всегда оказываются более эффективными.

Многие ультразвуковые технологические процессы протекают с малыми скоростями $v<<a \omega$. В этом случае допустимо пренебречь влиянием малой скорости $v$ на величину силы взаимодействия (2.12) в течение одного периода колебаний, а также на моменты $t_{i}$ переключения участков характеристики (2.3). С учетом сделанных допущений из (2.13) находим

$$
\tau_{1}=\arcsin \frac{\Delta}{a}, \tau_{2}=\arcsin \left(\frac{\Delta}{a}+\frac{D}{k_{0} a}\right), \tau_{3}=\frac{\pi}{2}, \tau_{4}=\arcsin \left(1-\frac{D}{k_{0} a}\right),
$$


и после подстановки (2.12) при $v=0$ в (2.6) получим, принимая во внимание (2.15), следующие явные зависимости статической силы $P$ от скорости $v$ пластического деформирования:

$$
P=\frac{k_{0} a}{2 \pi}\left[\begin{array}{l}
\frac{\pi}{2}\left(\frac{2 D}{k_{0} a}-1\right)+\sqrt{1-\left(1-\frac{D}{k_{0} a}\right)^{2}}- \\
-\sqrt{1-\left(1-\frac{2 \pi \cdot v}{a \omega}\right)^{2}}+\sqrt{1-\left(1-\frac{D}{k_{0} a}-\frac{2 \pi \cdot v}{a \omega}\right)^{2}}+ \\
+\left(1-\frac{D}{k_{0} a}\right) \arcsin \left(1-\frac{D}{k_{0} a}\right)-\left(1-\frac{2 \pi \cdot v}{a \omega}\right) \arcsin \left(1-\frac{2 \pi \cdot v}{a \omega}\right)+ \\
+\left(1-\frac{D}{k_{0} a}-\frac{2 \pi \cdot v}{a \omega}\right) \arcsin \left(1-\frac{D}{k_{0} a}-\frac{2 \pi \cdot v}{a \omega}\right)
\end{array}\right]
$$

для режимов импульсного нагружения и

$$
P=\frac{k_{0} a}{2 \pi}\left[\begin{array}{l}
2 \pi\left(\frac{D}{k_{0} a}-1+\frac{\pi \cdot v}{a \omega}\right)-\sqrt{1-\left(1-\frac{2 \pi \cdot v}{a \omega}\right)^{2}}+ \\
+\left(1-\frac{2 \pi \cdot v}{a \omega}\right) \arccos \left(1-\frac{2 \pi \cdot v}{a \omega}\right)
\end{array}\right]
$$

для режимов непрерывного нагружения.

При $v=0$ выражения (2.18), (2.19) приводятся к величинам (2.10). Расчеты показывают, что полученными приближенными соотношениями допустимо пользоваться при скоростях деформирования, не превышающих величину $v=0,25 \cdot a \cdot \omega$.

4. Проведенное рассмотрение позволяет объяснить экспериментально наблюдаемый эффект “разупрочнения” материала эффект снижения статического усилия, необходимого для пластического деформирования при воздействии вибрации. Из (2.10) видно, что любая сколь угодно малая вибрация вызывает снижение статического усилия, приводящего к появлению остаточной деформации. С другой стороны это усилие может быть сделано сколь угодно малым путем увеличения амплитуды колебаний. 
Уменьшение статического усилия пластического деформирования с возрастанием амплитуды колебаний отмечается во всех экспериментах, связанных с технологическим использованием ультразвука [177179,195,202,222,223]. Рис.2.7 создает предельно ясную картину этого явления: в каждом цикле деформирования кратковременно преодолевается предел текучести материала, и возникает малая остаточная деформация, накапливающаяся от цикла к циклу. Если следить только за постоянной составляющей силы деформирования и медленным течением процесса, как это и делается в большинстве экспериментов, создается отчетливое впечатление о снижении предела текучести материала, его разупрочнении под действием ультразвуковых колебаний.

Следует еще раз подчеркнуть, что наблюдаемое явление - это кажущееся разупрочнение материала, фактические упругопластические свойства которого остаются неизменными; в каждом цикле деформирования сила воздействия на материал оказывается достаточной для преодоления предела текучести. Сделанное замечание необходимо, поскольку во многих работах (см., например, [195,202,222,223,226]) содержится утверждение именно о фактическом изменении свойств материала под действием ультразвука. Как правило, подобное утверждение основано на неверной трактовке экспериментальных данных и методических ошибках, подобных допущенным в $[222,226]$ при оценке сил, возникающих в очаге деформации. Так, например, в [222] ошибочно предполагается, что максимальная сила деформации не может превышать силы, возникающей в стержне 2 колебательной системы (рис.1.2) в узле смещений, расположенном в месте крепления мембраны 4.

Наряду с этим экспериментальные данные работы [223] достаточно хорошо согласуются с результатами проведенного выше анализа. По 
результатам деформирования образцов диаметром $d=14$ мм и высотой $H=15$ мм , выполненных из различных материалов, в [218] получена следующая эмпирическая зависимость наблюдаемого предела текучести б от амплитуды колебаний $a$

$$
\sigma=\sigma_{0}-K \cdot a,
$$

где $\sigma_{0}$ - истинный предел текучести, найденный при статическом деформировании; $K$ - постоянный коэффициент, зависящий от материала образца.

Из второго соотношения (2.10) получаем выражение для сил

$$
D_{a}=D-k_{0} \cdot a,
$$

которое, после деления на $S$, с помощью обозначений $D_{a} / S=\sigma, D / S=\sigma_{0}$, $k_{0} / S=E / H=K$, где $S, H$ - площадь поперечного сечения и высота образца, приводится к виду (2.20).

На рис.2.9 построены зависимости (2.21) для различных материалов.

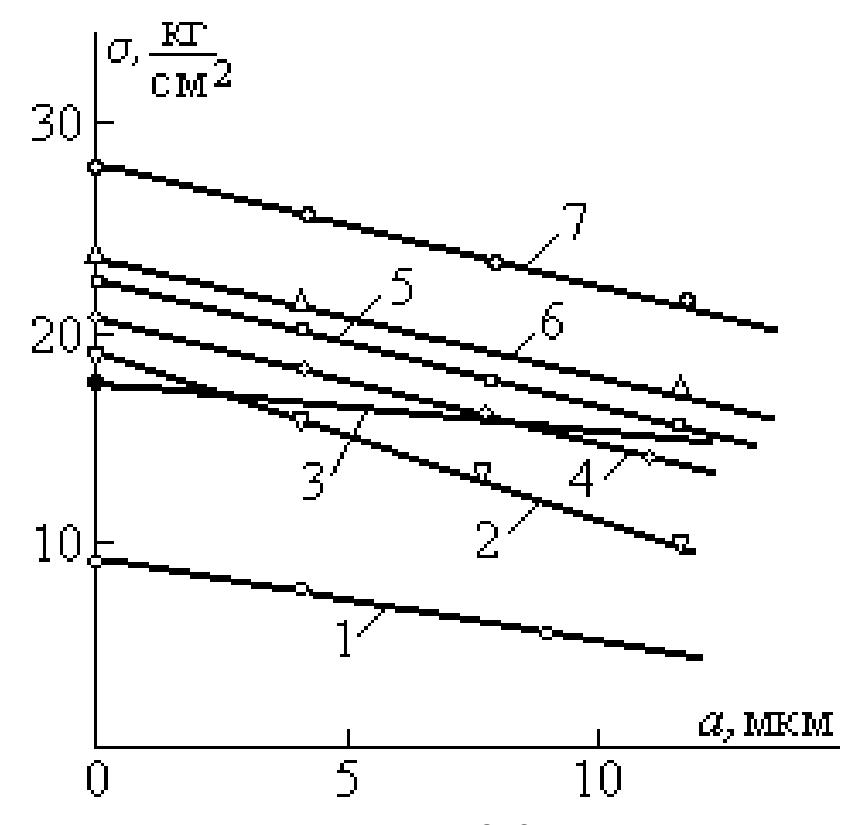

Рис. 2.9 
Принятые при расчете исходные данные приведены в таблице.

Таблица 2.1.

\begin{tabular}{|c|c|c|c|c|}
\hline № & \multicolumn{2}{|c|}{ Материал } & E, кг/ $\mathrm{MM}^{2}$ & $\sigma_{0}, \mathrm{\kappa} \Gamma / \mathrm{MM}^{2}$ \\
\hline 1. & \multicolumn{2}{|l|}{ Алюминий } & 7100 & 9,8 \\
\hline 2. & \multicolumn{2}{|l|}{ Медь } & 12500 & 19,2 \\
\hline 3. & \multicolumn{2}{|l|}{ Магний } & 4000 & 18,0 \\
\hline 4. & \multirow{4}{*}{$\begin{array}{l}\text { Латунь с } \\
\text { содержа- } \\
\text { нием Cu-Zn }\end{array}$} & $7-3$ & \multirow{4}{*}{10050} & 20,8 \\
\hline 5. & & $9-1$ & & 22,8 \\
\hline 6. & & $8-2$ & & 23,9 \\
\hline 7. & & $6-4$ & & 28,2 \\
\hline
\end{tabular}

Точками на рисунке отмечены экспериментальные данные работы [223]. Значения предела текучести $\sigma_{0}$ приняты в соответствии с результатами статического деформирования, показанными точками при $a=0$; значения модуля упругости $E$ выбраны согласно справочным данным из [191]. Заметим, что эксперименты проводились при колебаниях с малыми амплитудами, удовлетворяющими условиям реализации режимов непрерывного деформирования, для которых и выведено соотношение (2.21).

5. Из равенств (2.10) и рис.2.6 и 2.7 следует, что различные материалы неодинаково реагируют на вибрационное воздействие в процессе пластической деформации, причем более чувствительными к такому воздействию оказываются материалы с большим модулем упругости $E$ и образцы с большей статической жесткостью $k_{0}$. Достижимую степень снижения статического усилия пластического деформирования можно оценить, рассмотрев предельный случай $k_{0} \rightarrow \infty$, приводящий к характеристике идеального жесткопластического материала [145,168], показанной на рис.2.10. 


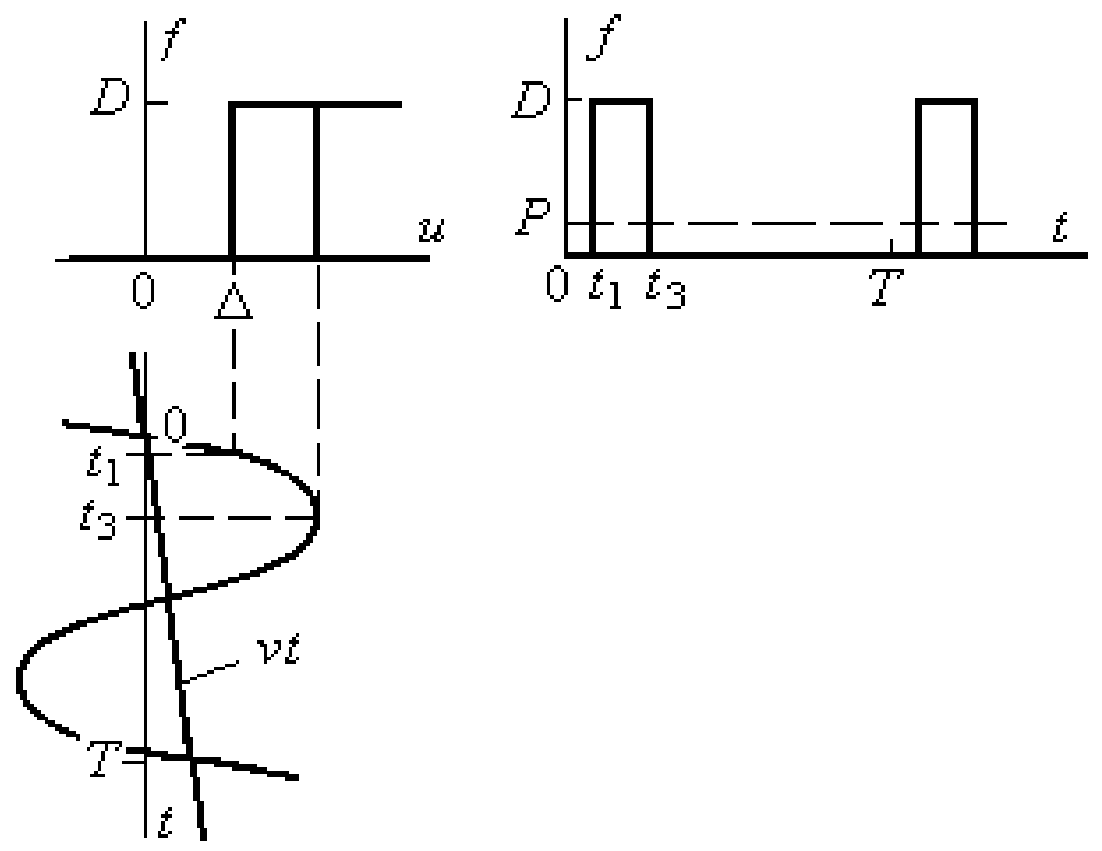

Рис. 2.10

Такой материал считается абсолютно жестким до достижения силой порогового значения $D$, при котором происходит необратимая пластическая деформация. Свойства жесткопластического материала по отношению к статической нагрузке при одновременном вибрационном воздействии определим предельным переходом $k_{0} \rightarrow \infty$ в отношениях (2.10), (2.13) - (2.15). В результате получаем $D_{a}=0$

$$
\begin{gathered}
\tau_{2}=\tau_{1}, \sin \tau_{1}=\frac{1}{a}\left(\Delta-\frac{v}{\omega} \tau_{1}\right), \tau_{4}=\tau_{3}=\arccos \left(-\frac{v}{a \omega}\right), \Delta=u_{m}-\frac{2 \pi \cdot v}{\omega}, \\
P=D \frac{\left(\tau_{3}-\tau_{1}\right)}{2 \pi} .
\end{gathered}
$$

На рис.2.8 с помощью этих уравнений построена кривая $k_{0} \rightarrow \infty$, дающая зависимость постоянной силы $P$ от скорости $v$ деформирования жесткопластического материала.

Используя допущения, принятые при выводе приближенных аналитических выражений (2.18), (2.19), т.е. полагая в (2.22) $\sin \tau_{1}=\Delta / a, \tau_{3}=\pi / 2, u_{m}=a$, из (2.23) получим

$$
P=\frac{D}{2 \pi} \arccos \left(1-\frac{2 \pi \cdot v}{a \omega}\right)
$$


Выражение (2.24) удобно записать в виде зависимости $P$

$$
v=\frac{a \omega}{\pi} \sin ^{2} \frac{\pi \cdot P}{D}
$$

Соотношением (2.24), как и (2.18), (2.19), можно пользоваться при значениях $\quad v<0,25 \cdot a \cdot \omega$; равенством (2.25) - при соответствующих указанному диапазону скоростей силах $P<0,35 \cdot D$.

Полученные соотношения и представленные на рис.2.8 кривые можно трактовать как некоторые приведенные характеристики поведения материала в ультразвуковом поле по отношению к действующим статическим нагрузкам.

Интересно отметить, что в условиях вибрации меняется характер поведения материала: упругопластический материал ведет себя подобно вязко-упругопластическому материалу с пониженным пределом текучести; жесткопластический материал подобно вязкой среде не держит статической нагрузки и течет при сколь угодно малой статической силе $P$. Это наглядно видно, если, положив $P<<D$, привести (2.25) к виду

$$
v=a \omega \pi(P / D)^{2}
$$

Полученное поведение материала в условиях ультразвукового воздействия является проявлением известного эффекта вибрационного сглаживания нелинейностей $[122,139]$, достаточно полно исследованного на примере колебаний систем с сухим трением [8-12, 70,71,73,85].

Однозначная связь статической силы $P$ и средней скорости $v$ деформирования составляет важную для технологических ультразвуковых процессов особенность поведения материалов в условиях вибрации, наблюдавшуюся экспериментально в [106,141,223]. Характеристики на рис.2.8, с одной стороны, позволяют выявить материалы, ультразвуковое воздействие на которые дает достаточно большое снижение статических сил и, следовательно, оказывается целесообразным. С другой стороны, эти характеристики дают возможность подобрать величину статической силы, 
амплитуду и частоту вибрации, обеспечивающие заданную производительность процесса, определяемую скоростью v.

6. Эффект снижения статических сил, вызывающих пластическую деформацию, мы описали с помощью реологических моделей идеального упруго- и жесткопластического материалов. Реальные металлы, используемые в экспериментах и технологических процессах, имеют характеристику с упрочнением в зоне пластичности. Реологическая модель и характеристика материала с упрочнением показаны на рис.2.2,в,г. Кроме того, в течение технологического процесса зачастую меняются условия деформирования изделия. При листовой штамповке, например, (рис.1.4,a) это происходит из-за изменения геометрии заготовки по мере погружения пуансона. Поэтому в динамической характеристике подобного рабочего процесса как сила пластического деформирования, так и жесткость образца в области упругой деформации зависят от всей истории нагружения и изменяются по мере накопления остаточной деформации, как это показано на рис.2.11,а.

Для описания подобных процессов можно воспользоваться
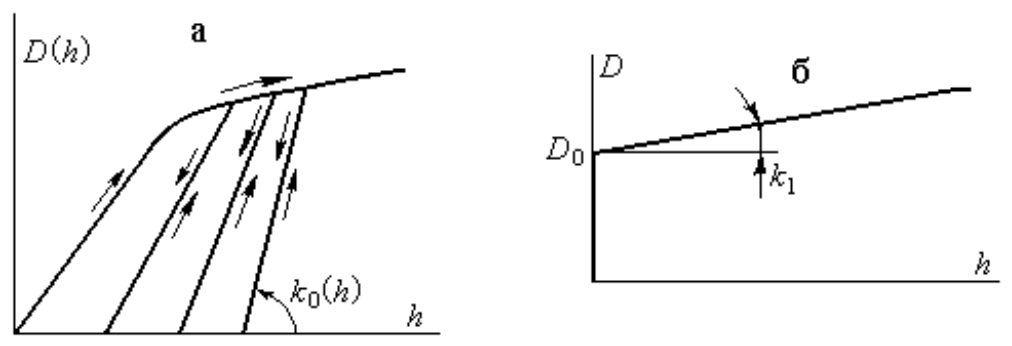

\section{Рис.2.11}

полученными выше результатами, считая предел текучести $D$ и статическую жесткость $k_{0}$ изделия функциями деформации $h$

$$
D=D(h), k_{0}=k_{0}(h),
$$

и пренебрегая малыми изменениями этих величин в пределах одного цикла нагружения. Так, например, изменение порога пластичности материала с линейным упрочнением (рис.2.2,в,г) в условиях 
вибрационного воздействия можно найти по соотношениям (2.10), в которых в соответствии с (2.27) и рис.2.2,г следует положить

$$
D=D_{0}+k_{1}\left(h-\frac{D_{0}}{k_{0}}\right) \text {. }
$$

Аналогичный прием применим и в том случае, когда диаграмма “деформация - сила” задана графически, например, после статических испытаний. Естественно, что и скорость пластического деформирования материала при постоянной силе (или статическая сила при постоянной скорости) будет изменяться по мере накопления остаточной деформации. Рассмотрим для примера процесс деформирования жесткопластического материала с упрочнением, характеристика которого показана на рис.2.11,б. Для нее имеем

$$
D=D_{0}+k_{1} h
$$

(Это выражение можно получить из (2.28) при $k_{0} \rightarrow \infty$ ).

Ограничимся рассмотрением деформации под действием малых статических сил $P<<D$. Подставляя (2.28) в (2.26) получим для скорости процесса

$$
v=\pi a \omega\left[P /\left(D_{0}+k_{1} h\right)\right]^{2}
$$

График зависимости (2.30) скорости деформирования от величины остаточной деформации при постоянной статической силе показан на

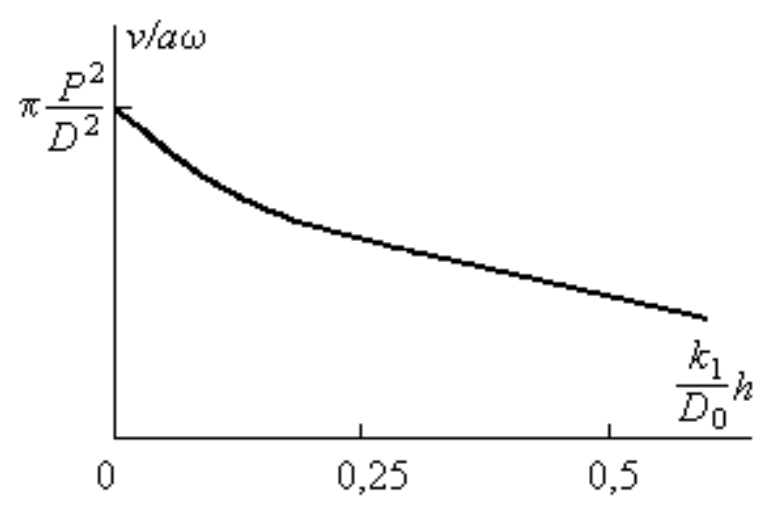

Рис. 2.12

При помощи (2.30) легко находится время $t$, необходимое для получения заданной величины $h_{0}$ остаточной деформации изделия, 


$$
t=\int_{0}^{h_{0}} \frac{d h}{v(h)}=\frac{h_{0}}{\pi \cdot a \omega}\left(\frac{D_{0}}{P}\right)^{2}\left[1+\frac{k_{1} h_{0}}{D_{0}}\left(1+\frac{3 k_{1} h_{0}}{D_{0}}\right)\right]
$$

В общем случае, когда величины $D$ и $k_{0}$ являются заданными функциями (2.27) деформации $h$, поведение материала в области пластического течения можно оценить, используя результаты, представленные на рис.2.8 следующим образом. Для текущего значения деформации $h$ определяются величины функций (2.27) и вычисляется параметр $D / k_{0} a$. На соответствующей этому параметру кривой (рис.2.8) отыскивается точка с ординатой, равной величине $P / D$. Абсцисса найденной точки определяет текущую скорость пластического деформирования. Таким образом строится зависимость $v=v(h)$ скорости деформирования от величины деформации при заданной статической силе $P$. Интеграл в (2.31) определяет время, а следовательно, и закон $h=h(t)$ деформирования.

При заданной скорости $v$ деформирования подобная процедура позволяет найти зависимость статической силы $P$ от деформации $h$.

Таким образом, предложенный подход позволяет описать разнообразные ультразвуковые технологические процессы, связанные с деформированием материала. Как было показано, получаемые при этом эффекты подтверждаются многочисленными экспериментами. Вместе с тем необходимо отметить, что многие экспериментальные данные отличаются большим разбросом и с трудом поддаются непосредственному сопоставлению с полученными в этом параграфе результатами. Это обусловлено, прежде всего, недостаточно четким описанием методики и условий проведения эксперимента. В ряде случаев разброс данных объясняется пренебрежением обратного влияния технологического процесса на условия возбуждения колебаний рабочего органа. В этих случаях трактовка экспериментальных результатов требует проведения анализа динамики ультразвуковой колебательной системы с учетом нелинейной нагрузки со стороны обрабатываемого изделия. С помощью такого подхода ниже в Параграфе 12, будут объяснены основные эффекты, наблюдаемые при ультразвуковой обработке хрупких материалов. 


\section{3. Влияние ультразвука на процессы с сухим трением}

1. В ультразвуковых технологических процессах встречаются разнообразные случаи возникновения сил трения при взаимодействии рабочего органа со средой, отличающиеся относительным расположением направления колебаний и медленного скольжения контактирующих тел.

На рис.3.1 показана модель системы с сухим трением, подобная приведенной ранее на рис.2.2.

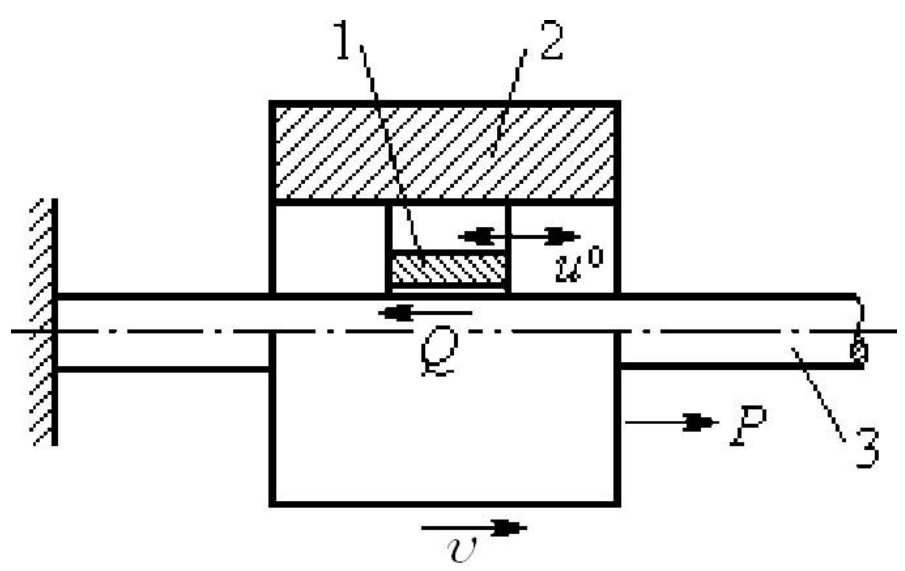

Рис.3.1

Пусть каретка 2 под действием статической силы $P$ движется со средней скоростью $v$ вдоль направляющей штанги 3 . Со штангой контактирует втулка 1, которая связана с кареткой и может совершать относительно нее колебания. В зоне контакта развивается сила сухого трения $Q$, направленная в сторону, противоположную скорости относительного скольжения втулки по штанге. Для поддержания равномерного движения при отсутствии колебаний необходимо статическое усилие $P=Q$.

Оценим эффект влияния колебаний в зоне контакта на величину статической силы, необходимой для преодоления сил трения. Наиболее характерным для ультразвуковых технологических процессов является случай, когда колебания совпадают по направлению со скоростью $v$ каретки. Так, например, при ультразвуковом резании (рис.1.1,a) возникают силы трения боковых поверхностей инструмента о стенки обрабатываемого 
отверстия. Аналогичная ситуация наблюдается в процессах волочения (рис.1.4,б), точения (рис.1.4,в), ультразвуковой сварки металлов с продольной подачей сварочного наконечника (рис.1.6,a).

Как и ранее в п.2.1, будем рассматривать установившееся движение втулки 1 (рис.3.1) вида

$$
u(t)=v t+u^{0}(t)=v t+a \sin (\omega \cdot t)
$$

Запишем выражение нелинейной динамической характеристики силы сухого трения $f(\dot{u})=Q \cdot \operatorname{sign}(\dot{u}) . \quad$ Вследствие периодичности рассматриваемого процесса (3.1) развиваемая в зоне контакта сила трения

$$
f(t)=f(\dot{u}(t))=Q \cdot \operatorname{sign}(v+a \omega \cdot \cos (\omega \cdot t))
$$

является периодической функцией периода $T=2 \pi / \omega$.

По аналогии с (2.6) запишем соотношение, связывающее статическую силу $P$ с параметрами движения системы

$$
P=\frac{1}{T} \int_{0}^{T} F(t) \mathrm{d} t=\frac{Q}{T} \int_{0}^{T} \operatorname{sign}(v+a \omega \cdot \cos (\omega t)) \mathrm{d} t
$$

На рис.3.2 показана характеристика силы трения $f(\dot{u})$.
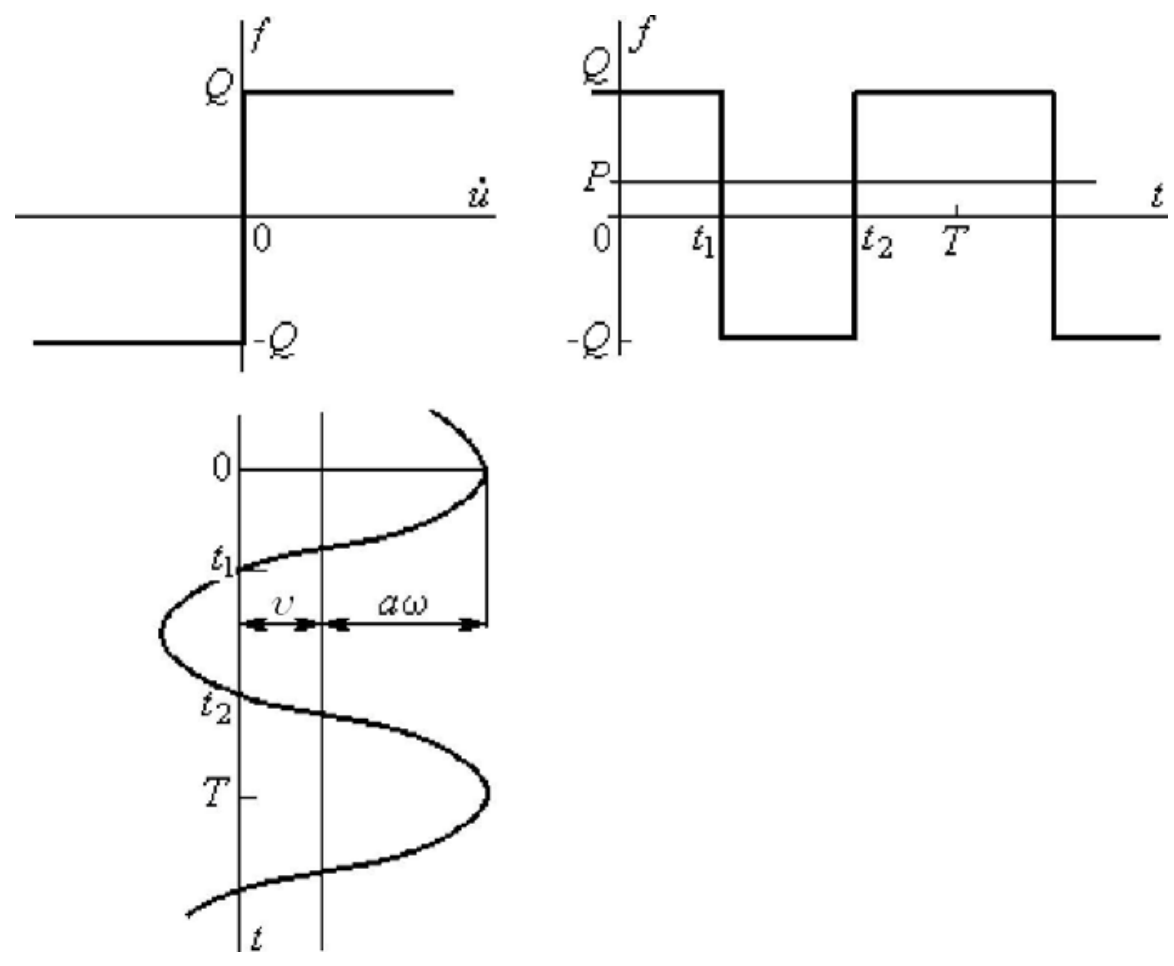

Рис.3.2 
Здесь же приведен график скорости $\dot{u}(t)$, и построена временная развертка силы трения $F(t)$ в зоне контакта. Интегрирование (3.3) дает

$$
P=\frac{Q}{\pi}\left[\pi-\left(\tau_{2}-\tau_{1}\right)\right]
$$

где

$$
\begin{aligned}
& \tau_{1}=\omega \cdot t_{1}=\arccos \left(-\frac{v}{a \omega}\right)=\frac{\pi}{2}+\arcsin \left(\frac{v}{a \omega}\right) \\
& \tau_{2}=\omega \cdot t_{2}=2 \pi-\tau_{1}
\end{aligned}
$$

$t_{1}, t_{2}$ - моменты перемены знака скорости $\dot{u}(t)$ (рис.3.2).

После подстановки величин (3.5) в равенство (3.4) получим окончательно

$$
P=\frac{2 Q}{\pi} \cdot \arcsin \left(\frac{v}{a \omega}\right)
$$

На рис.3.3 по соотношению (3.6) построена зависимость статической силы $P$ от отношения скоростей $v / a \omega$.

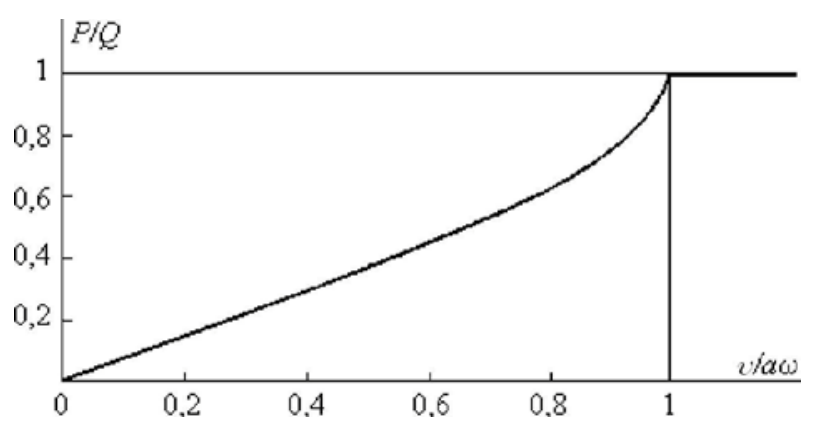

Рис.3.3

Отсюда видно, что вибрация в зоне контакта приводит к уменьшению необходимой для преодоления силы трения статической силы при скоростях скольжения $v<a \omega$. При очень малой скорости скольжения $v<<a \omega$ из (3.6) с точностью до величин первого порядка малости получаем

$$
P=\frac{2 Q}{\pi \cdot a \omega} v
$$

Таким образом, при малых скоростях $v$ поведение системы по отношению к статической силе в условиях вибрации аналогично ее движению в линейной вязкой среде. В этом заключается известный эффект вибрационного сглаживания нелинейностей, уже упоминавшийся в п.2.5. 
2. В практике ультразвуковых технологических процессов встречаются случаи, когда колебания совершаются в плоскости скольжения, но их направление перпендикулярно скорости медленного движения. Такому случаю отвечает описанная в п.1.4 схема процесса ультразвуковой сварки металлов с поперечной подачей сварочного наконечника. На модели (рис.3.1) это соответствует возбуждению крутильных колебаний втулки 1 вокруг направляющей штанги 3. Такая схема используется иногда в процессах ультразвукового волочения прутков и проволоки [164,179,202], главным образом, на операциях калибровки.

На рис.3.4 показана схема формирования силы трения для элемента, движущегося по плоскости П вдоль оси $x$ со скоростью $v$ и совершающего $T$-периодические колебания $\dot{u}(t)$ в направлении оси $y$.

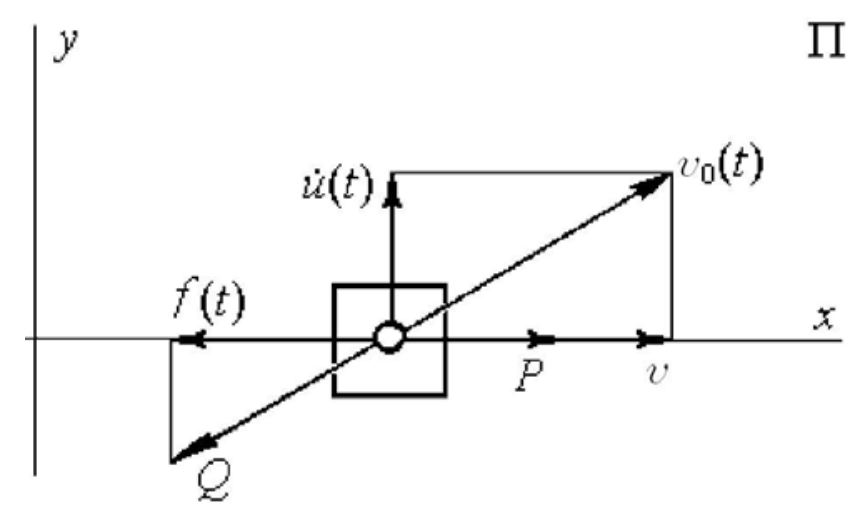

Рис.3.4

Постоянная по величине сила трения $Q$ направлена в сторону противоположную вектору скорости $v_{0}(t)$ скольжения по плоскости.

Проекция силы трения на ось $x$

$$
F(t)=Q \frac{v}{\sqrt{v^{2}+[\dot{u}(t)]^{2}}}
$$

является периодической функцией времени с периодом $T$.

Для преодоления силы трения в направлении постоянной скорости $v$ необходима постоянная сила

$$
P=\frac{1}{T} \int_{0}^{T} F(t) d t=\frac{Q}{T} \int_{0}^{T} \frac{v d t}{\sqrt{v^{2}+[\dot{u}(t)]^{2}}}
$$


Полагая в (3.9) скорость $\dot{u}(t)=a \cdot \omega \cdot \sin (\omega \cdot t)$ и обозначив $\omega \cdot t=\tau$, получим

$$
P=\frac{Q}{2 \pi} \int_{0}^{2 \pi} \frac{d \tau}{\sqrt{1+\left(\frac{a \omega}{v}\right)^{2} \sin ^{2} \tau}}=\frac{2 Q}{\pi} \int_{0}^{\pi / 2} \frac{d \tau}{\sqrt{1+\left(\frac{a \omega}{v}\right)^{2} \sin ^{2} \tau}}
$$

Интеграл в (3.10) не берется в элементарных функциях. Однако сразу видно, что подынтегральная функция

$$
F(\tau)=\left[1+\left(\frac{a \omega}{v} \sin \tau\right)^{2}\right]^{-1 / 2} \leq 1
$$

и, следовательно,

$$
\int_{0}^{\pi / 2} F(\tau) d \tau<\frac{\pi}{2}
$$

Таким образом, из (3.10) и (3.11) следует, что необходимая для преодоления трения статическая сила $P<Q$ при любых возможных значениях $\mathrm{v} / \mathrm{a \omega}$.

Для получения оценок силы $P$ сверху и снизу заменим в (3.10) $\sin \tau$ $(\tau \in[0, \pi / 2\rfloor)$ функциями $\Phi_{1}(\tau)$ и $\quad \Phi_{2}(\tau) \quad$ такими, что $\hat{O}_{1}(\tau) \leq \sin \tau \leq \hat{O}_{2}(\tau)$, $(\tau \in[0, \pi / 2])$. Тогда истинное значение силы $P$, определяемое выражением (3.10), окажется заключенным в интервале $P_{2} \leq P \leq P_{1}$, где

$$
P_{i}=\frac{2 Q}{\pi} \int_{0}^{\pi / 2} \frac{d \tau}{\sqrt{1+\left[\frac{a \omega}{v} \hat{O}_{i}(\tau)\right]^{2}}},(i=1,2)
$$

Выберем функции $\phi_{1}(\tau)$ и $\hat{O}_{2}(\tau)$ так, чтобы первая из них вписывалась, а вторая охватывала $\sin \tau$, как это показано на рис.3.5, т.е.

$$
\begin{aligned}
& \hat{O}_{1}(\tau)=\frac{3}{\pi} \tau-\left(\frac{3}{2 \pi} \tau-\frac{1}{4}\right) \eta\left(\tau-\frac{\pi}{6}\right) \\
& \hat{O}_{2}(\tau)=\tau-(\tau-1) \eta(\tau-1)
\end{aligned}
$$

где $\eta(\tau)$ функция единичного скачка. 


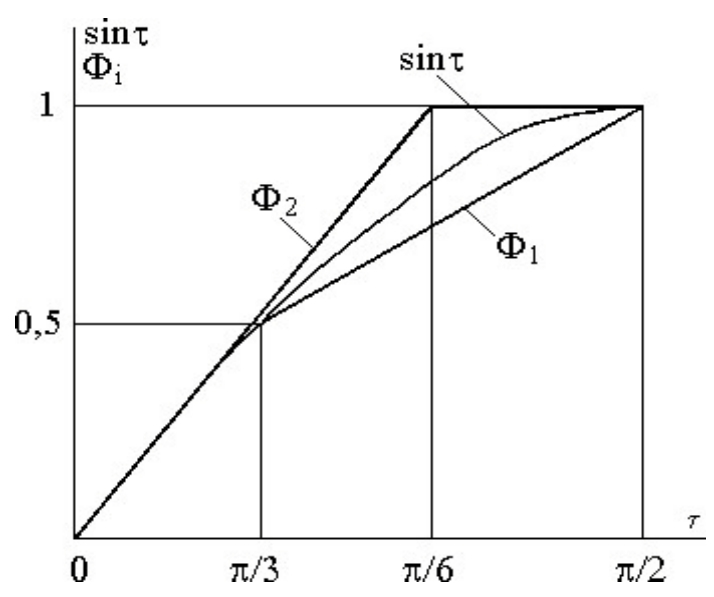

Рис.3.5

Отрезки кусочно-непрерывной функции $\hat{O}_{1}(\tau)$ проходят через точки с координатами $(0,0),(\pi / 6,1 / 2),(\pi / 2,1)$, принадлежащие $\sin \tau$. Отрезки функции $\Phi_{2}(\tau)$ являются касательными к $\sin \tau$ в точках $(0,0)$ и $(\pi / 2,1)$. Подставляя (3.13) в (3.12), получим после интегрирования

$$
\begin{gathered}
P_{1}=\frac{2}{3} Q \frac{v}{a \omega} \ln \left[\frac{2\left(1+\sqrt{\left(\frac{v}{a \omega}\right)^{2}+1}\right)^{2}}{\frac{v}{a \omega}\left(1+\sqrt{\left(\frac{2 v}{a \omega}\right)^{2}+1}\right)}\right] \\
P_{2}=\frac{2}{\pi} Q \frac{v}{a \omega}\left\{\ln \left[\frac{1+\sqrt{\left(\frac{v}{a \omega}\right)^{2}+1}}{\frac{v}{a \omega}}\right]+\frac{\frac{\pi}{2}-1}{\sqrt{\left(\frac{v}{a \omega}\right)^{2}+1}}\right\}
\end{gathered}
$$

На рис.3.6 по уравнениям (3.14), (3.15) построены верхняя $P_{1}$ и нижняя $P_{2}$ оценки статической силы $P$ в зависимости от отношения скоростей $v / a \omega$

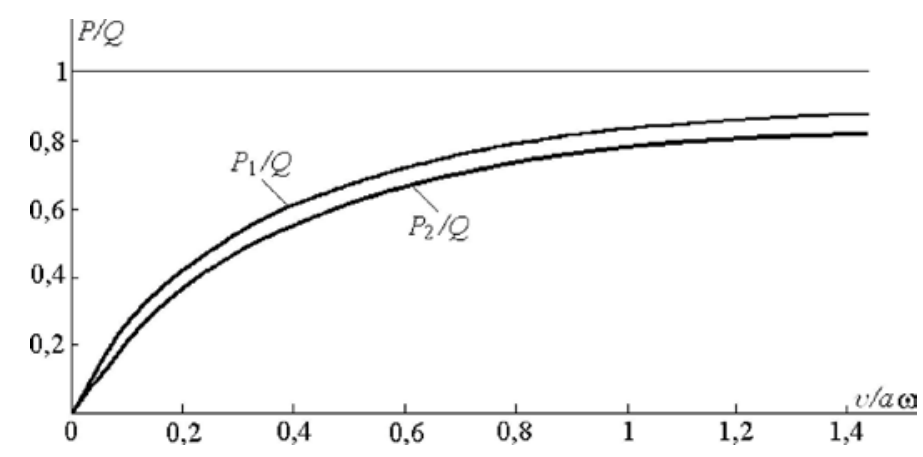

Рис.3.6 
Предельными переходами в (3.14), (3.15) легко показать, что величины $P_{i} \rightarrow 0$ при $(v / a \omega) \rightarrow 0$ и $P_{i} \rightarrow Q$ при $(v / a \omega) \rightarrow \infty$. Благодаря близости величин $P_{1}$ и $P_{2}$ отпадает необходимость вычисления уточненного значения статической силы $P$, требуемой для преодоления сил трения. В отличие от предыдущего случая (рис.3.3) введение в зону трения поперечных колебаний приводит к снижению статической силы при любой скорости скольжения $v$.

Сравнение рис.3.3 и рис.3.6 показывает, что при одной и той же колебательной скорости $a \omega$ и малых скоростях скольжения $v$ наибольший эффект снижения трения достигается при совпадении направлений колебаний и скольжения. Сказанное находит и экспериментальное подтверждение. В работе [240] приведены результаты экспериментов по оценке влияния ультразвука на уменьшение статической силы, необходимой для преодоления контактного трения.

Схема эксперимента показана на рис.3.7,а.

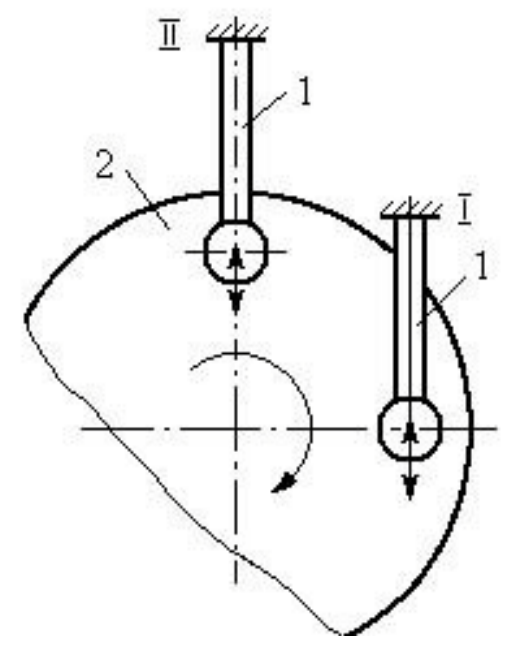

Рис.3.7,a

Сферический наконечник колебательной системы 1 поджимается к плоскости вращающегося с постоянной скоростью диска 2. Колебательная система устанавливается относительно диска так, что направление колебаний либо совпадает (позиция I) с направлением линейной скорости диска, либо перпендикулярно ему (позиция II). Об изменении статической силы судят по 
величине момента привода диска. В эксперименте возбуждались колебания наконечника с частотой $f=22$ кГц и амплитудой $a=12-18$ мкм.

Полученная в результате эксперимента зависимость статической силы $P$ от линейной скорости $v$ диска приведена на рис.3.7,б.

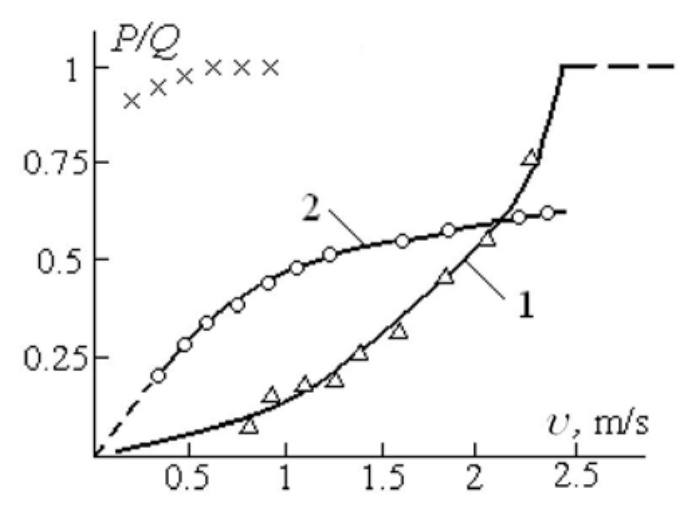

Рис.3.7,б

Кривая 1 соответствует случаю совпадения направлений колебаний наконечника и скорости диска (позиция I на рис.3.7,a); кривая 2 - их взаимно перпендикулярному расположению (позиция II). Сопоставление экспериментальных данных с результатами, показанными на рис.3.3 и рис.3.6, свидетельствует об их хорошем качественном и количественном соответствии.

3. В процессах ультразвукового упрочнения (рис.1.1,б) и сварки полимерных материалов (рис.1.6,в) колебания рабочего органа возбуждаются в направлении нормали к обрабатываемой поверхности, вдоль которой производится подача, приводящая к скольжению контактирующих поверхностей. При этом возникают противодействующие скольжению силы трения. Такая схема возбуждения колебаний используется также в процессах плющения проволоки и волочения изделий с применением ультразвука $[80,164,179,187,202]$. В модели, приведенной на рис.3.1, этому случаю соответствует возбуждение радиальных колебаний втулки, вызывающих периодическое сжатие и освобождение направляющей штанги. 
Эффект влияния ультразвука на процесс с сухим трением в зоне контакта по описанной схеме наложения колебаний удобно пояснить при помощи плоского аналога, показанного на рис.3.8.

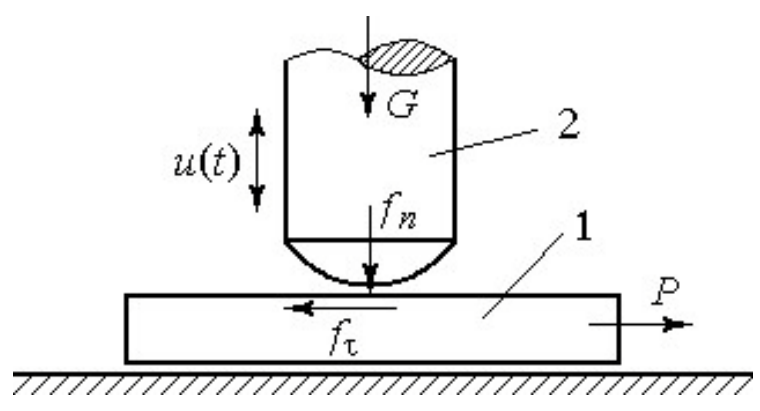

Рис.3.8

Пластина 1 протягивается постоянной силой $P$ под наконечником 2 , поджатым к пластине статической силой $G$ и совершающим периодические колебания $u(t)$ в направлении нормали к плоскости контакта. При взаимодействии наконечника с пластиной возникает нормальная реакция $f_{n}$, величина которой зависит от реологических свойств контакта и, как было показано в предыдущем параграфе, определяется нелинейной динамической характеристикой $f_{n}=f_{n}[u(t), \dot{u}(t)]$. Однако независимо от вида характеристики при установившихся колебаниях справедливо соотношение для импульсов сил $f_{n}$ и $G$

$$
\int_{0}^{T} f_{n}[u(t), \dot{u}(t)] d t=G T
$$

Одновременно в зоне контакта развивается сила сухого трения

$$
f_{\tau} \leq \vartheta \cdot f_{n},
$$

направленная в сторону, противоположную относительному скольжению контактирующих поверхностей.

Знак равенства в (3.17) принимается в том случае, если в процессе движения пластины не происходит ее остановок при торможении силами трения. Рассматривая установившееся движение без остановок под 
действием постоянной силы $P$ и периодической силы трения $f_{\tau}=\vartheta \cdot f_{n}[u(t), \dot{u}(t)]$, можно записать для импульсов этих сил

$$
\vartheta \int_{0}^{T} f_{n}[u(t), \dot{u}(t)] d t=P T
$$

Сопоставив выражения (3.16) и (3.18), найдем, что установившееся движение без остановок происходит при таком же соотношении сил

$$
P=\vartheta \cdot G
$$

как и при отсутствии колебаний наконечника.

Это подтверждается показанными крестиками на рис.3.7,б результатами экспериментов, описанных в работе [240].

Если статическая сила $P<\vartheta \cdot G$, то движение пластины сопровождается ее периодическими остановками, вызываемыми силами трения. Оценим такое движение при некоторых упрощающих допущениях о характеристике контактного взаимодействия наконечника с пластиной. Предположим сначала, что жесткость контактной области столь высока, что время взаимодействия оказывается пренебрежимо малым по сравнению с периодом $T$ колебаний. Таким образом, предполагается существование периодического виброударного процесса $[60,61,117,118]$, в результате которого возникают импульсы сил трения, приводящие к мгновенным остановкам пластины на каждом периоде колебаний. После отскока наконечника пластина движется с постоянным ускорением под действием силы $P$, приобретая к следующему удару скорость $v_{0}=P \cdot T / M$, где $M$ - масса пластины. Учитывая, что средняя скорость такого периодического движения $v=v_{0} / 2$, получим

$$
v=\frac{\pi}{\omega \cdot M} \cdot P
$$

или для статической силы

$$
P=\frac{\omega \cdot M}{\pi} \cdot v
$$


Таким образом, при скорости движения

$$
v<v^{*}=\frac{\pi}{\omega \cdot M} \cdot \vartheta \cdot G
$$

вибрационное воздействие приводит к снижению статической силы, необходимой для преодоления силы трения.

Интересно отметить, что в отличие от рассмотренных в п.П. 1 и 2 случаев величина статической силы не зависит от интенсивности вибрации. Благодаря возможности использования колебаний с малыми амплитудами такую схему целесообразно применять для снижения трения в кинематических парах, например, в опорах легких элементов приборов, т.е. там, где необходимо устранить нечувствительность, определяемую пороговыми значениями силы сухого трения. Один из способов снижения сухого трения с помощью высокочастотной вибрации предложен в [41].

Возвращаясь к рассмотрению ультразвуковых технологических процессов, оценим влияние реологических свойств контактирующих элементов на величину статической силы. Примем, что реакция $f_{n}$ описывается характеристикой жесткопластического материала (рис. 2.8) с пределом текучести $D$. Это означает, что нормальная сила в контакте $f_{n}=D$, а его продолжительность, согласно (3.16), $t_{k}=T \cdot G / D$. В течение времени $T-t_{k}$ под действием силы $P$ происходит разгон пластины, в результате которого она приобретает скорость $v_{0}=\left(T-t_{k}\right) \cdot P / M$ и проходит путь $s_{1}=v_{0} \cdot\left(T-t_{k}\right) / 2$.

Во время контактирования происходит торможение пластины силой $\vartheta D-P$ до полной остановки. Пройденный при этом путь $s_{2}=\frac{1}{2} v_{0}^{2} M /(\vartheta \cdot D-P)$. Вычисляя среднюю скорость движения $v=\left(s_{1}+s_{2}\right) / T$, получим

$$
v=\frac{\pi}{M \omega} P \frac{\vartheta \cdot(D-G)}{(\vartheta \cdot D-P)}
$$


Так как $P<\vartheta \cdot G$, легко показать, что при одной и той же статической силе $P$ скорость (3.23) меньше значения (3.20), причем в обоих случаях граничное значение скорости $v^{*}$ определяется выражением (3.22). (Величина $v^{*}$ получается из (3.20), (3.23) при $P=\vartheta \cdot G$.)

На рис.3.9 показаны зависимости статической силы $P$ от скорости движения $v$ пластины.

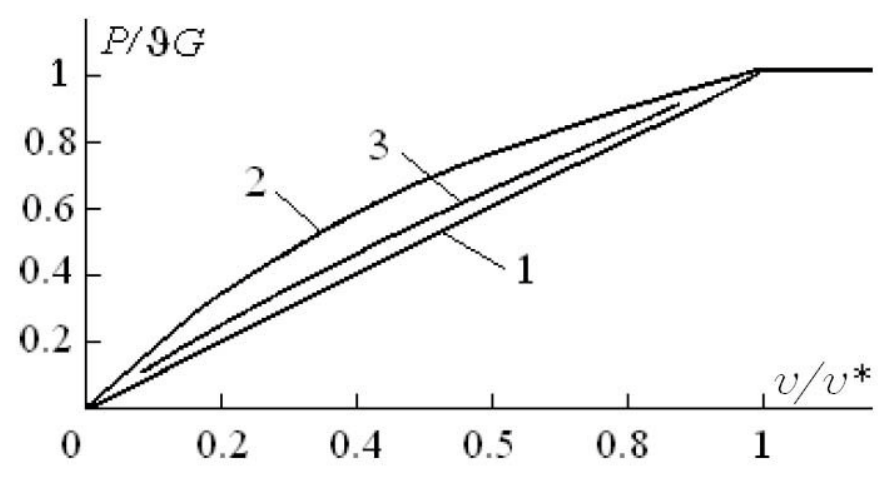

Рис.3.9

Линия 1 построена по уравнению (3.21). Кривые 2 и 3 построены с помощью (3.23) при значениях $D / G=2$ и $D / G=5$ соответственно. Как правило, ультразвуковые технологические процессы целесообразно использовать при $G<<D$. Поэтому для оценки эффекта снижения статической силы при действии нормальной к поверхности скольжения вибрации можно пользоваться простым соотношением (3.21), к которому приводится выражение (3.23) при $D \rightarrow \infty$.

Таким образом, и в этом случае ультразвуковые колебания в зоне контакта полностью устраняют зону нечувствительности к статической нагрузке, обусловленную сухим трением. Такой эффект экспериментально наблюдался в [217]. Качественное изменение характера трения при малых скоростях скольжения под действием 
ультразвуковых колебаний, нормальных к поверхности трения, отмечено также в экспериментах работы [15].

Здесь мы рассмотрели режимы колебаний, при которых происходит периодический разрыв контакта между трущимися поверхностями. Именно такие режимы характерны для ультразвуковых систем благодаря большим ускорениям вибрирующего элемента. (При частоте $f=20$ кГц и амплитуде $a=10$ мкм амплитуда виброскорости составляет $a_{\omega}=1,2 \mathrm{M} / \mathrm{c}$, a амплитуда виброускорения $a_{\omega}{ }^{2}=1,5 \cdot 10^{5} \mathrm{M} / \mathrm{c}^{2}$.) Влияние на сухое трение низкочастотных микроколебаний, при которых сохраняется контакт трущихся элементов, проанализировано в [82].

В заключение этого раздела отметим, что выявленные эффекты играют, по-видимому, существенную роль в процессах штамповки трудно деформируемых материалов. Сжатие изделия 1 (рис.3.10), помещенного в полость штампа 2, приводит к растеканию материала по полости.

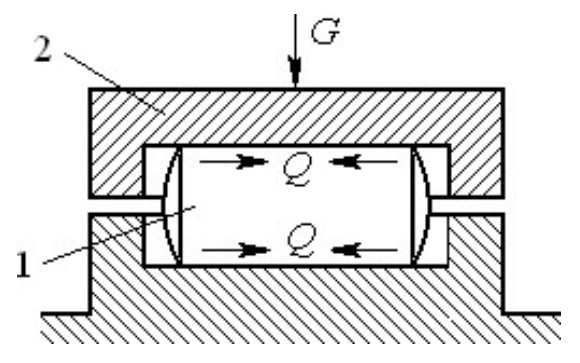

Рис.3.10

При этом на поверхностях контакта возникают силы трения $Q$, препятствующие деформированию. В результате суммарные напряжения в поверхностном слое материала могут оказаться достаточно высокими, чтобы привести к образованию трещин. Явление растрескивания поверхности характерно для трудно деформируемых материалов, обладающих большим пределом текучести. Ультразвуковое воздействие исключает возможность накопления касательных напряжений, практически полностью устраняя влияние сил трения на поверхностях контакта изделия и штампа.. Этот эффект, наряду с существенным 
снижением статического усилия деформирования, приводит к наблюдаемому экспериментально [195] качественному улучшению пластических свойств трудно деформируемых материалов под влиянием ультразвука.

4. Рассмотрим ситуацию, возникающую при волочении прутков, проволоки и труб. Схема сил, действующих на волоку при традиционном способе волочения (без вибрации), показана на рис.2.7. В п.3.1 была дана оценка влияния вибрации на величину статической силы, необходимой для преодоления трения на калибрующей поверхности волоки. В настоящем разделе оценим влияние вибрации на сопротивление, порождаемое в конической части волоки.

На рис.3.11 показана модель процесса волочения прутка 3 через волоку 1, совершающую колебания по гармоническому закону относительно каретки 2 и движущуюся вместе с ней с постоянной скоростью $v$ под действием статической силы $P$. Как и ранее, будем рассматривать движение волоки вида (3.1).

Перемещение волоки приводит к сближению конических поверхностей по нормали к образующей

$$
u_{n}(t)=u(t) \sin \theta=(v t+a \sin \omega \cdot t) \sin \theta
$$

и их относительному касательному смещению

$$
u_{\tau}(t)=u(t) \cos \theta=(v t+a \sin \omega \cdot t) \cos \theta,
$$

которые при контактировании этих поверхностей вызывают нормальную реакцию $f_{n}$ и силу сухого трения $f_{\tau}$.

По аналогии с (2.3) примем, что нормальная сила $f_{n}$ связана со смещением (3.24) характеристикой упругопластического материала 


$$
f_{n}\left(u_{n}, \dot{u}_{n}\right)= \begin{cases}0 & u_{n} \leq \Delta_{n}, \dot{u}_{n}>0 \\ k_{n}\left(u_{n}-\Delta_{n}\right) & \Delta_{n} \leq u_{n} \leq \Delta_{n}+\frac{D_{n}}{k_{n}}, \dot{u}_{n}>0 \\ D_{n} & \Delta_{n}+\frac{D_{n}}{k_{n}} \leq u_{n} \leq u_{n m}, \dot{u}_{n}>0 \\ D_{n}+k_{n}\left(u_{n}-u_{n m}\right) & u_{n m}-\frac{D_{n}}{k_{n}} \leq u_{n} \leq u_{n m}, \dot{u}_{n}<0 \\ 0 & u_{n} \leq u_{n m}-\frac{D_{n}}{k_{n}}, \dot{u}_{n}<0\end{cases}
$$

где $k_{n}, D_{n}$ - жесткость линейного участка характеристики и предел текучести; $\Delta_{n}$ - координата начала контактирования поверхностей; $u_{n m}$ максимальное в течение одного периода значение функции (3.24).

Характеристику силы сухого трения запишем в виде

$$
f_{\tau}=\vartheta \cdot f_{n}\left(u_{n}, \dot{u}_{n}\right) \operatorname{sgn} \dot{u}_{\tau}(t)=\vartheta \cdot f_{n}\left(u_{n}, \dot{u}_{n}\right) \operatorname{sgn} \dot{u}(t)
$$

Спроектировав силы (3.26), (3.27) на направление скорости $v$ (рис.3.11),

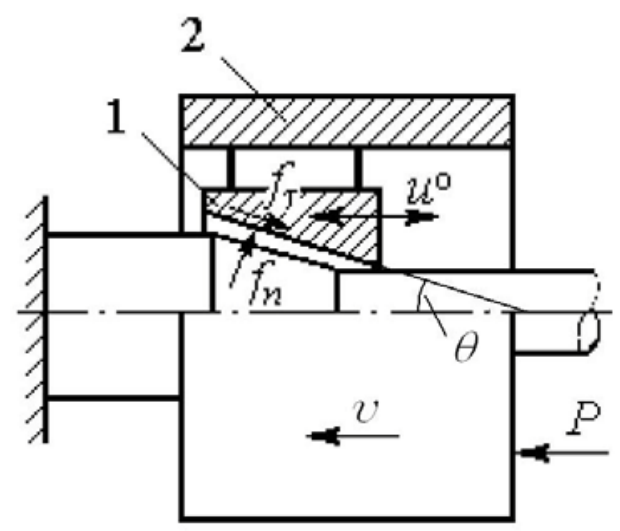

Рис.3.11

найдем суммарную силу, действующую в направлении движения в результате пластического деформирования изделия и сил трения на поверхностях контакта

$$
f_{v}=f_{n} \sin \theta+f_{\tau} \cos \theta=f_{n} \sin \theta[1+\vartheta \cdot \operatorname{ctg} \theta \cdot \operatorname{sgn} \dot{u}(t)]
$$

С учетом (3.24), (3.26) выражение (3.28) приведем к виду

$$
f_{v}\left(u_{n}, \dot{u}_{n}\right)=f(u, \dot{u})[1+\vartheta \cdot \operatorname{ctg} \theta \cdot \operatorname{sgn} \dot{u}]=f(u, \dot{u})+f_{1}(u, \dot{u}),
$$


где $f(u, \dot{u})$ - определяется выражением (2.3), в котором следует принять

$$
k_{0}=k_{n} \sin ^{2} \theta, D=D_{n} \sin \theta,
$$

а величины $\Delta=\Delta_{n} / \sin \theta$ и $u_{m}=u_{n m} / \sin \theta$ имеют тот же смысл, что и в (2.3).

Таким образом, равенство (3.29) можно рассматривать как приведенную динамическую характеристику взаимодействия волоки с изделием. Первое слагаемое в (3.29) описывает силы деформирования материала, второе - силы трения.

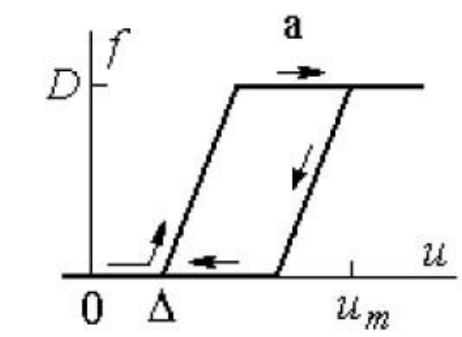

Эти составляющие показаны на рис.3.12,a,б; суммарная приведенная характеристика (3.29) построена на рис.3.12,в. Стрелками на диаграммах показаны направления нагружения и

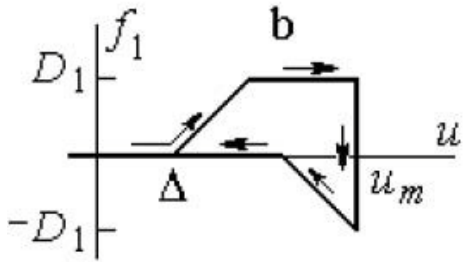
разгрузки в пределах одного цикла деформирования. На рис.3.12,б,в , кроме того, обозначено

$$
\begin{gathered}
D_{1}=D \vartheta \cdot \operatorname{ctg} \theta \\
D_{0}=D+D_{1}=D(1+\vartheta \cdot \operatorname{ctg} \theta)
\end{gathered}
$$

Величина (3.32) определяет усилие волочения без ультразвукового воздействия.

Статическая сила, необходимая для поддержания процесса ультразвукового волочения, определяется выражением

$$
P=\frac{1}{T} \int_{0}^{T} f_{v}[u(t), \dot{u}(t)] d t
$$

Вычисляя интеграл в (3.33) с учетом (3.29), (2.3) и (2.12), получим 


$$
P=\frac{k_{0}}{2 \pi}\left\{\begin{array}{l}
(1+\vartheta \operatorname{ctg} \theta)\left[\Delta \tau_{1}-\left(\Delta+\frac{D}{k_{0}}\right) \tau_{2}+a\left(\cos \tau_{1}-\cos \tau_{2}\right)+\frac{v}{2 \omega}\left(\tau_{2}^{2}-\tau_{1}^{2}\right)\right]+ \\
+(1-\vartheta \operatorname{ctg} \theta)\left[u_{m} \tau_{3}-\left(u_{m}-\frac{D}{k_{0}}\right) \tau_{4}+a\left(\cos \tau_{3}-\cos \tau_{4}\right)+\frac{v}{2 \omega}\left(\tau_{4}^{2}-\tau_{3}^{2}\right)\right]+ \\
+\frac{2 D}{k_{0}} \tau_{3} \vartheta \operatorname{ctg} \theta
\end{array}\right\}
$$

где значения $\tau_{i}(i=1, \ldots, 4)$ определяются из уравнений $(2.13)$, величина $\Delta$ связана со скоростью $v$ процесса равенством (2.15), а величины $k_{0}, D$ выражаются формулами (3.30).

На рис.3.13 построены зависимости статической силы $P$ от скорости $v$ волочения при различных значениях отношения $D / k_{0} a$.

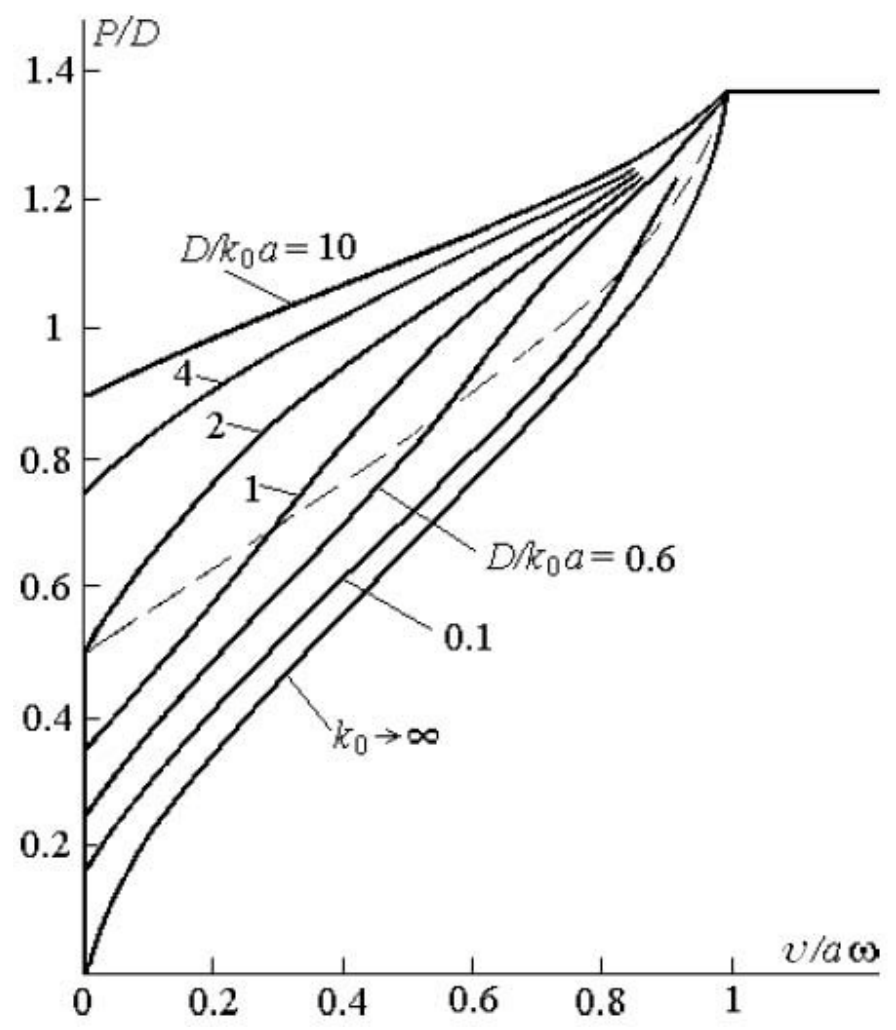

Рис.3.13

При расчетах принято: коэффициент трения $\vartheta=0,1$; угол волоки $\theta=15^{0}$. Как и в процессах пластического деформирования, рассмотренных ранее в Параграфе 2, при ультразвуковом волочении возможны режимы непрерывного и импульсного деформирования 
изделия. Для всех режимов справедливо соотношение (3.34), однако при расчете режимов непрерывного деформирования следует учитывать равенства (2.16). На рис.3.13 штриховая линия разделяет области импульсного (нижняя) и непрерывного (верхняя) деформирования.

Полученное соотношение (3.34) в силу (2.4), (2.13), (2.16) имеет действительные значения при скорости деформирования $v \leq a \omega$. При $v=a \omega$ из (2.16), (2.13) имеем $\tau_{1}=\tau_{2}=-\tau_{3}=-\tau_{4}=-\pi$ и после подстановки в (3.34) получаем $P=D_{0}$, где $D_{0}$ определяется формулой (3.32). Дальнейшее увеличение скорости волочения не изменяет величины статической силы.

$$
\begin{aligned}
& \text { При } v=0 \text { из (2.13) с учетом (2.15) находим } \\
& \qquad \tau_{1}=\arcsin (\Delta / a), \tau_{2}=\tau_{3}=\pi / 2, \tau_{4}=\pi-\tau_{1}
\end{aligned}
$$

Подставив значения (3.35) в (3.34), придем к выражениям (2.10), определяющим значения статических сил, превышение которых вызывает появление остаточных деформаций. Эти значения оказываются независящими от сил трения на контактирующих поверхностях. Легко показать, что ультразвуковое воздействие вообще устраняет влияние трения на процесс деформирования материала в пределах упругой зоны его характеристики. В этом случае приведенная динамическая характеристика (3.29) принимает вид, показанный на рис.3.14.

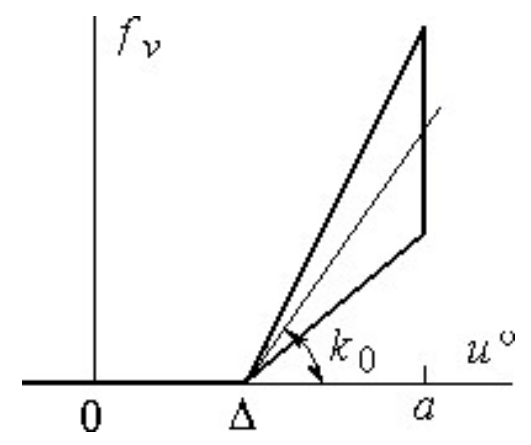

Рис.3.14

Здесь штриховой прямой показана составляющая $f(u)$, определяемая упругими силами. Подобного вида характеристики с треугольными 
петлями гистерезиса весьма характерны для систем с сухим трением [73,74]. Описывая по аналогии с п.2.2 поведение такого материала при вибрационном нагружении, находим, что статическая сила и деформация связаны теми же соотношениями (2.8) и (2.9), что и при деформировании чисто упругого материала, жесткость которого $k_{0}$ определяется формулой (3.30). Такой процесс деформирования описывается характеристиками, построенными на рис.2.6,a. В результате находим, что пластическое деформирование при волочении начинается при том же значении статической силы, что и при отсутствии трения. Это хорошо видно из сравнения рис.2.13 и рис.2.6.

Таким образом, снижение усилия волочения при ультразвуковом воздействии происходит как за счет снижения предела текучести материала, так и за счет уменьшения сил трения. Вклад в общий эффект той или иной составляющей зависит от соотношения между упругопластическими свойствами материала, коэффициентом трения, параметрами колебаний волоки и ее геометрическими характеристиками. При определенных соотношениях вклад одной из составляющих может оказаться преобладающим, как это часто и наблюдается в экспериментах $[156,202,241]$.

Наибольший эффект снижения статического усилия волочения достигается для жесткопластического материала, которому на рис.3.13 соответствует кривая, отмеченная индексом $k_{0} \rightarrow \infty$. Эта кривая строится с помощью полученных в п.2.5 уравнений (2.22), (2.23), в которых предел текучести $D$ следует заменить величиной $D_{0}$ из (3.32).

Предложенные в настоящем параграфе подходы могут быть использованы для описания разнообразных ультразвуковых технологических процессов, в которых наряду с силами деформирования материала существенную роль играют силы трения. Полученные здесь результаты позволяют объяснить многие экспериментально наблюдаемые 
эффекты. Так, например, графики на рис.3.13 достаточно хорошо отвечают воспроизведенной на рис.1.5 из работы [106] зависимости силы резания от скорости резания при токарной обработке изделия с наложением ультразвуковых колебаний на режущий инструмент. (См. также Параграф 15.) Те же результаты позволяют объяснить многократно наблюдаемые факты радикального улучшения свойств трудно обрабатываемых материалов под влиянием ультразвука в разнообразных технологических процессах [141-144, 177-179, 185, 195, 202].

Аналогичный подход применим также для описания эффекта вибрационного перемещения $[74,85,149]$, происходящего, в частности, и при ультразвуковом воздействии в случае организации надлежащей траектории вибровозбудителя, контактирующего с перемещаемым телом [14,63,240]. 


\section{ГЛАВА 2 \\ ДИНАМИЧЕСКИЕ ХАРАКТЕРИСТИКИ \\ УЛЬТРАЗВУКОВЫХ ТЕХНОЛОГИЧЕСКИХ МАШИН}

\section{4. Схемы ультразвуковых технологических машин}

1. В предыдущей Главе было показано, что производительность ультразвуковых технологических процессов возрастает с увеличением виброскорости $\omega \cdot a=2 \pi \cdot f \cdot a$ рабочего органа. Наиболее удобным для технической реализации высокочастотных колебаний большой интенсивности оказывается диапазон низких ультразвуковых частот $f=1,5 \cdot\left(10^{4}-10^{5}\right)$ Гц . По этой причине описанные выше технологические процессы и устройства получили названия ультразвуковых.

Рассмотрим типичные схемы ультразвуковых технологических машин и отметим особенности, выделяющие их в отдельную группу из общего класса вибрационных машин [85].

На рис.4.1,а приведена схема ультразвукового станка для обработки хрупких материалов [173,208]. Возбудитель колебаний магнитострикционный преобразователь 1 укреплен $\quad$ в $\quad$ корпусе 2 акустической головки $\mathrm{c}$ помощью промежуточного фланца 3 и тонкостенного опорного стакана 4. Обмотка преобразователя питается переменным током от генератора 5 электрических колебаний ультразвуковой частоты. Наводимое током переменное магнитное поле в сердечнике преобразователя, выполненном из магнитострикционного материала, преобразуется в механические колебания сердечника, которые через волновод - концентратор 6 сообщаются инструменту 7. Концентратор имеет вид стрежня переменного сечения, закон изменения которого выбирается так, чтобы обеспечить передачу колебаний от преобразователя к инструменту с увеличением амплитуды [146,147,191]. 
a
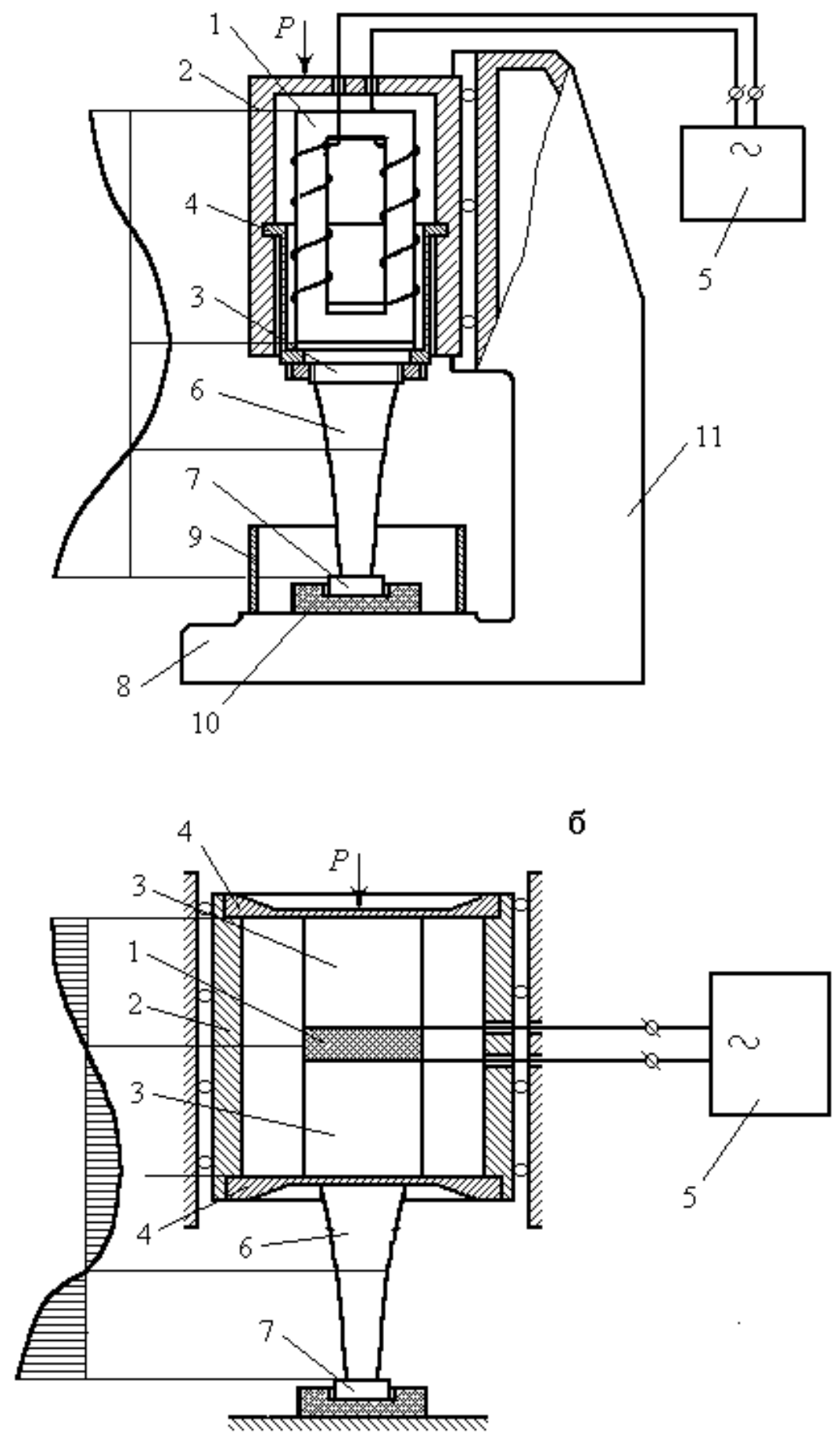

Рис. 4.1

Под инструментом на столе 8 в ванне 9 с абразивной суспензией помещено обрабатываемое изделие 10. Корпус акустической головки установлен в направляющих станины 11 и находится под действием статического усилия $P$, осуществляющего подачу инструмента в 
направлении обработки. Таким образом реализуется схема процесса ультразвукового резания хрупких материалов, показанная на рис.1.1,a.

Акустическая головка является основным узлом ультразвуковой технологической машины любого назначения. В п.1.2 уже приводилась схема установки для пластического деформирования образцов (рис.1.2), содержащей те же основные элементы. По аналогичной схеме строятся ультразвуковые устройства для процессов поверхностного упрочнения (рис.1.1,б), листовой штамповки (рис.1.4,a), сварки полимерных материалов (рис.1.6,б).

В зависимости от вида выполняемого технологического процесса используются колебательные системы мощностью от десятков ватт до десятков киловатт. Для возбуждения колебаний систем малой мощности часто применяются пьезоэлектрические преобразователи. Одна из конструкций такой системы [100] схематично показана на рис.4.1,б. Пьезоэлемент 1 установлен между накладками 3, связанными с корпусом 2 акустической головки упругими мембранами 4. Пьезоэлемент получает питание от электрического генератора 5 через торцевые обкладки. Возникающие за счет обратного пьезоэффекта механические колебания пьезоэлемента через нижнюю накладку и присоединенный к ней концентратор 6 передаются инструменту 7.

Для получения максимальной амплитуды колебаний инструмента, обеспечивающей эффективное проведение технологического процесса, производят резонансную настройку колебательной системы, возбуждая ее на частоте, совпадающей с одной из собственных частот. Настройку, как правило, осуществляют на холостом ходу, полагая несущественным влияние рабочего процесса на резонансные частоты. Это допущение справедливо, если рабочий процесс создает только диссипативную нагрузку на рабочий орган, вызывая снижение амплитуды колебаний. Так происходит, например, при ультразвуковой сварке металлов (рис.1.6,a), где на сварочный наконечник действуют лишь силы сухого трения. 
В процессах пластического деформирования и разрушения (рис.1.1, 1.4) существенную роль играют упругие силы. Проведенные в $[17,19,53]$ исследования характеристик ультразвуковых систем под нагрузкой показывают, что повышение усилия подачи приводит к уменьшению амплитуды колебаний не столько из-за диссипации энергии в рабочей зоне, сколько из-за изменения резонансной частоты системы вследствие существенных нелинейных искажений, обусловленных взаимодействием рабочего органа с изделием.

Разнообразные нелинейные эффекты, возникающие в ультразвуковых технологических системах при выполнении рабочего процесса, будут подробно рассмотрены в следующей Главе.

2. Характерная особенность ультразвуковых колебательных систем заключается в том, что их основные элементы являются волноводами, передающими энергию от источника к нагрузке, создаваемой рабочим процессом. Это связано с тем, что длина стержневых элементов

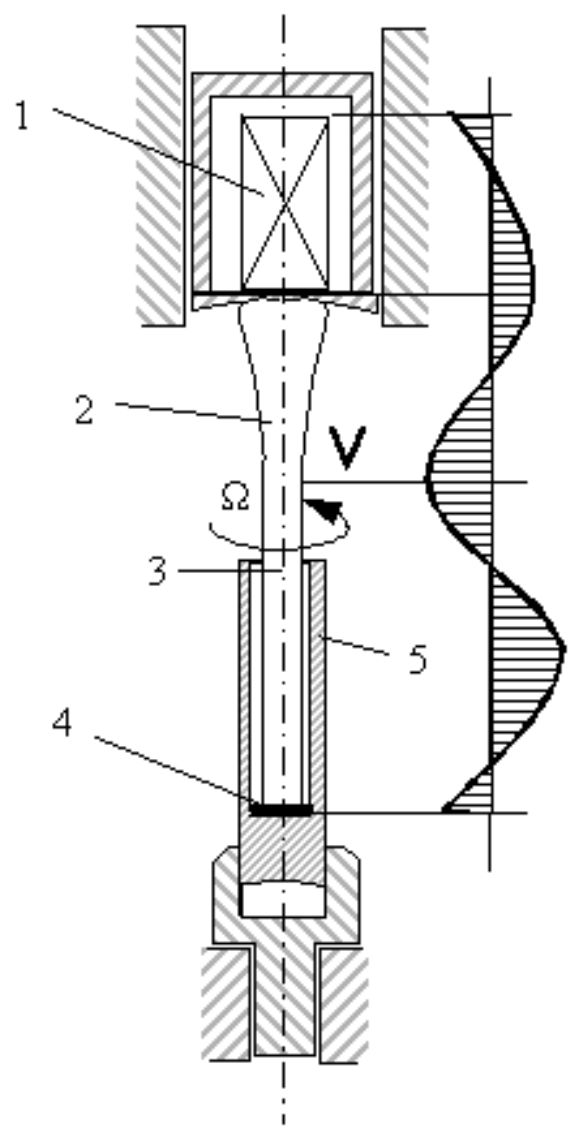

Рис. 4.2 колебательной системы соизмерима с длиной упругой волны в материале стержня. На рис.4.1 приведены эпюры амплитуд колебаний сечений колебательных систем. Здесь полная длина системы примерно равна длине волны, и колебательная система работает в режиме стоячей волны, при котором пучности и узлы смещений располагаются в определенных сечениях стержневой системы.

Волновой характер движения системы создает благоприятные условия для доставки энергии в рабочую зону, удаленную от возбудителя колебаний. С этой целью колебательную систему снабжают дополнительным волноводом. В 
станке для обработки глубоких отверстий $[163,205,206,228]$ колебания от преобразователя 1 (рис.4.2,a) передаются алмазному инструменту 4 через концентратор 2 и стержень - волновод 3. Обрабатываемому изделию 5 сообщается вращение с угловой скоростью $\Omega$ вокруг оси отверстия, а режущий инструмент вместе с акустической головкой принудительно подается с постоянной скоростью $v$ вдоль оси отверстия.

Обратим внимание на различие в способе подачи инструмента со случаем, приведенным на рис.4.1. В дальнейшем подачу, осуществляемую заданной силой прижима (рис.4.1), будем называть силовой, а принудительную подачу с заданной скоростью (рис.4.2) - кинематической. Ниже при рассмотрении обоих способов подачи (см. Гл.3) будет показано, что они обуславливают различные динамические свойства колебательной системы под нагрузкой, которые необходимо учитывать при настройке наиболее производительных резонансных режимов.

По аналогичной схеме построена ультразвуковая бурильная установка. Здесь волноводом, передающим энергию в рабочую зону, является погружаемая в скважину труба, которая своим верхним концом связана с возбудителем колебаний, а на нижнем конце несет турбобур. Продольная вибрация трубы приводит к снижению суммарной силы, необходимой для преодоления сил тре ния на боковой поверхности, и улучшает условия погружения трубы.

Естественной платой за такое улучшение является дополнительная затрата энергии, расходуемой не только на совершение полезного рабочего процесса, но и на компенсацию непроизводительных потерь, обусловленных внутренним трением в материале волновода. Задача о вибрационном погружении стержневой системы при действии распределенной силы сухого трения $[47,48]$ будет рассмотрена в Параграфе 11.

Высокая проникающая способность ультразвука предопределяет дополнительные утечки энергии через податливые места стыковки 
колебательной системы с корпусными деталями акустической головки и устройствами крепления обрабатываемых изделий. Источниками утечек являются переменные составляющие реакций, возникающих в точках крепления. Для их снижения применяют схемы крепления с акустической развязкой $[179,202]$. Идея акустической развязки заключается в том, что колебательную систему закрепляют в узлах стоячей волны, т.е. в тех сечениях, где амплитуда колебаний минимальна. На схеме, показанной на рис.4.3, колебания от электроакустического преобразователя 1 через концентратор 2 и обрабатываемое изделие 3 передаются отражателю 4, представляющему собой стержень полуволновой длины. В результате в концентраторе и отражателе устанавливается стоячая волна, эпюра которой показана на рис.4.3.

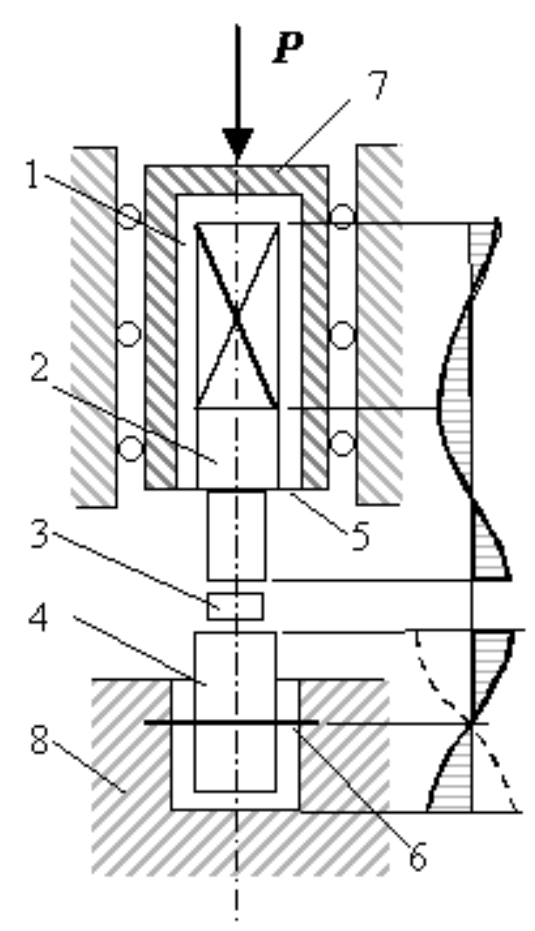

Рис. 4.3

Закрепление концентратора и отражателя мембранами 5, 6, расположенными в узлах стоячей волны, обеспечивает акустическую развязку корпуса акустической головки 7 и основания 8, которые воспринимают только статическую нагрузку, создаваемую силой $P$. По 
такой схеме построена описанная в П.1.2 ультразвуковая установка для пластического деформирования образцов (рис.1.2).

Основное преимущество применения схемы по рис.4.3 для процессов штамповки, прессования, сварки полимерных материалов [129,178,179,202,204] усматривают в том, что любые изменения статического усилия практически не влияют на величину амплитуды колебаний рабочего торца концентратора. Это действительно так, если колебательная система возбуждается на резонансной частоте холостого хода, как обычно принято в ультразвуковых технологических машинах. В этом случае присоединение полуволнового отражателя не нарушает режима, установившегося в колебательной системе преобразователь концентратор, которые вместе с отражателем совершают колебания, как единая стержневая система. В таком режиме рабочие торцы концентратора и отражателя движутся синфазно, и амплитуда их относительных смещений мала; поэтому эффективность ультразвукового воздействия на обрабатываемый материал незначительна.

В статье [28] обнаружен иной режим колебаний, при котором рабочие торцы концентратора и отражателя совершают встречные, противофазные движения. (Эпюра смещений сечений отражателя для этого случая показана на рис.4.3 штриховой линией.) Помещенное в рабочую зону изделие подвергается виброударному воздействию, интенсивность которого максимальна в резонансном состоянии, наступающем при определенном соотношении собственных свойств колебательной системы, статической силы прижима и частоты возбуждения. Именно в таком режиме ультразвуковое воздействие на обрабатываемый материал наиболее эффективно. Виброударные процессы, возникающие при взаимодействии волноводов, будут рассмотрены в Параграфе 10.

В ультразвуковых технологических машинах пассивным волноводом, отводящим часть энергии от активной колебательной системы через 
рабочую зону, является само обрабатываемое изделие. На рис.4.4 приведена схема ультразвуковой обработки отверстия в длинном стержне.

Колебания трубчатого концентратора 2 возбуждаются преобразователем 1. Абразивная суспензия принудительно подается в зону резания [109] через отверстия в концентраторе. Периодические соударения концентратора с обрабатываемой поверхностью через абразивные зерна приводят к выработке материала стержня 3 и одновременно вызывают его продольные колебания. И в этом случае настройка колебательной системы должна обеспечить поддержание резонансного виброударного процесса с наибольшей интенсивностью ударов. Очевидно, что по мере погружения концентратора условия резонансной настройки будут изменяться.

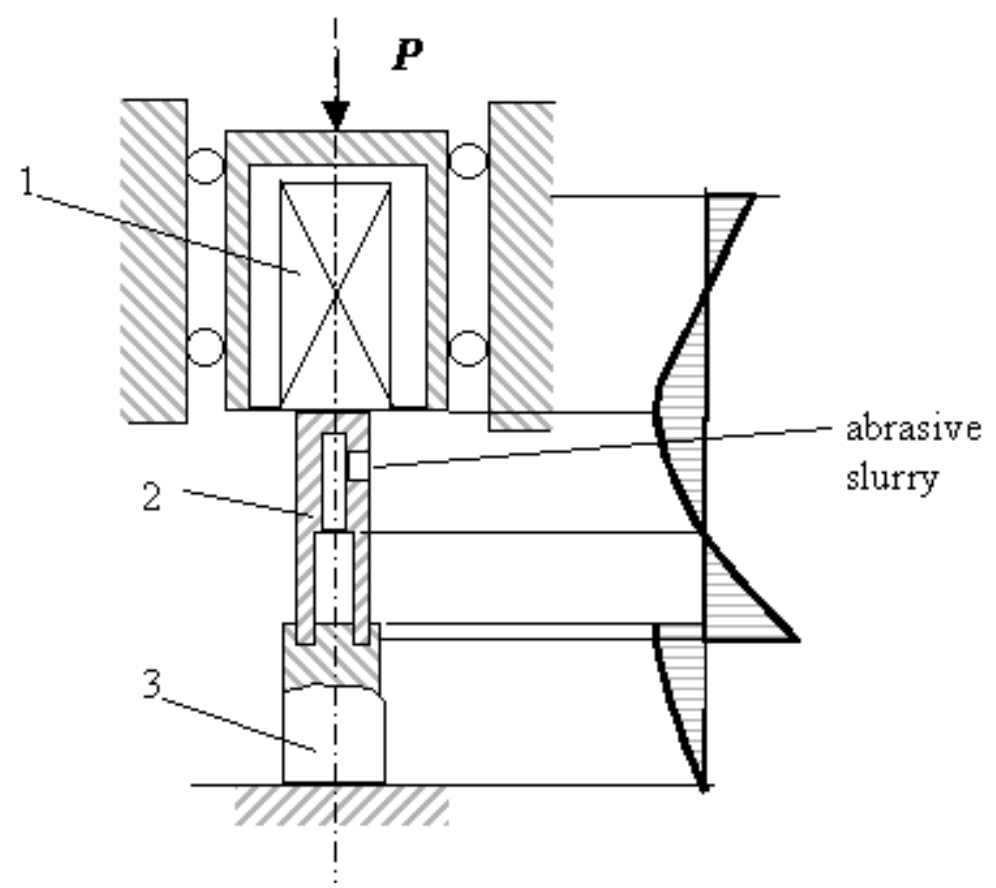

Рис. 4.4

Возбуждение колебаний в обрабатываемом изделии наблюдается и в процессе ультразвукового волочения [178,179,202]. На рис.4.5 показана схема ультразвуковой установки для волочения проволоки. Волока 3 укреплена на выходном конце концентратора 2, колебания которого возбуждаются электроакустическим преобразователем 1. Протягиваемая через волоку проволока 4 перематывается с питающего барабана 5 на приемную катушку 6. При значительном удалении барабанов от волоки в 
проволоке может возбудиться бегущая волна, постепенно затухающая по мере распространения от источника.

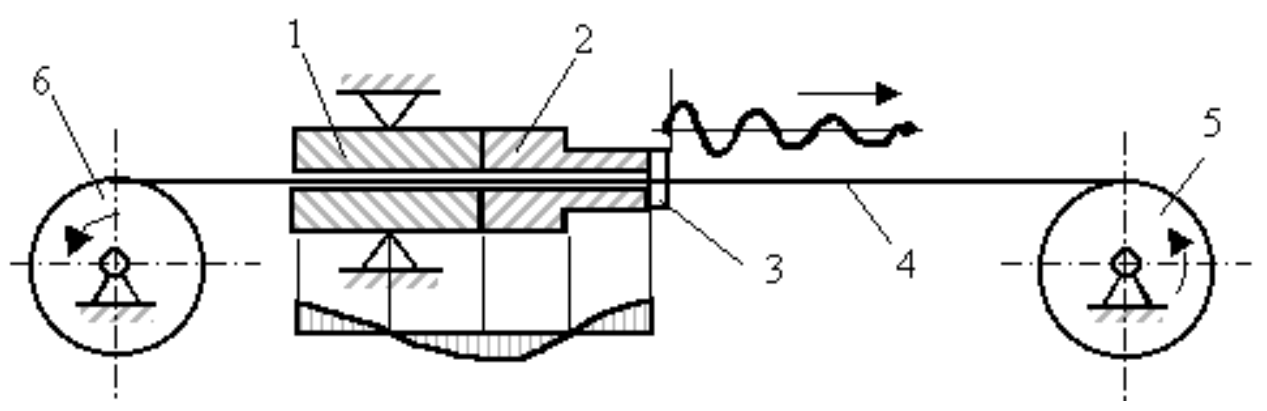

Рис.4.5

Случай виброударного возбуждения бегущей волны в пассивном волноводе будет рассмотрен в Параграфе 10.

3. В ряде ультразвуковых технологических машин используются стержневые элементы, совершающие изгибные колебания. На рис.4.6 показана схема колебательной системы для ультразвуковой сварки металлов [204].

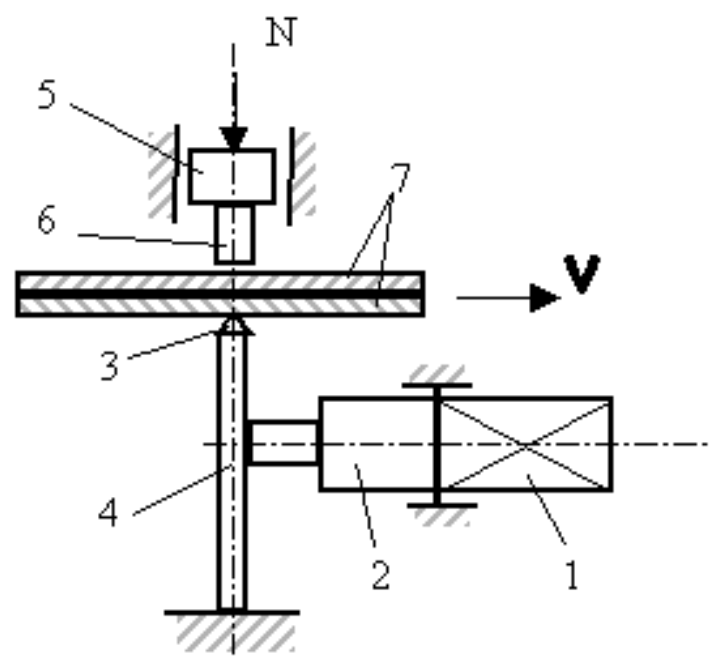

Рис.4.6

Сварочный наконечник 3 установлен на конце стержня 4, изгибные колебания которого возбуждаются преобразователем 1 и концентратором 2, совершающим продольные колебания. Каретка 5 через промежуточный стержень 6 поджимает свариваемые листы 7 к наконечнику статической силой $N$. 
Аналогичная схема используется в установке для точения трудно обрабатываемых материалов [130,141-143] с наложением ультразвуковых колебаний на режущий инструмент (рис.4.7). Резцу 3, установленному в резцедержателе 4, сообщаются изгибные колебания от преобразователя 1 через концентратор 2.

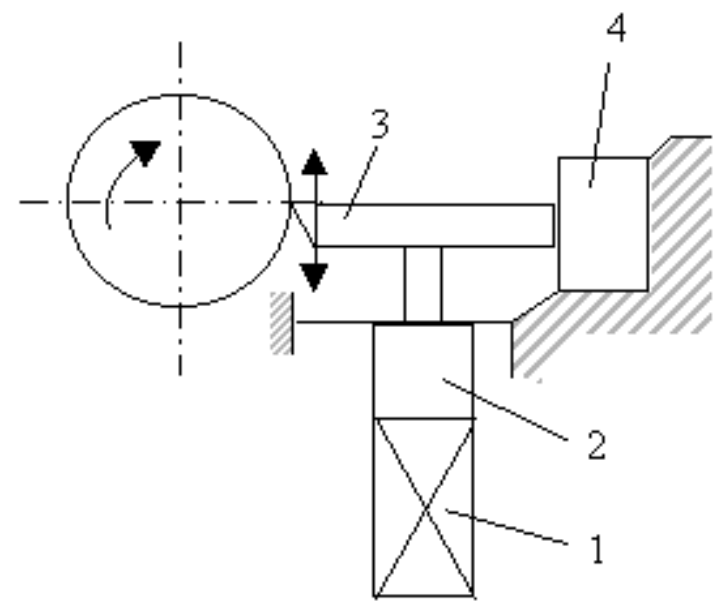

Рис.4.7

В станке для ультразвуковой обработки хрупких материалов (рис.4.8,a) колебания возбуждаются электроакустическим преобразователем 1 и передаются инструментам 3 с помощью крестообразно соединенных стержней 2, совершающих изгибные колебания.

Обрабатываемые изделия 4 установлены на четырех рабочих позициях стола 5. Стержни 2 имеют сужающееся к инструменту поперечное сечение и выполняют роль концентраторов, увеличивая амплитуду колебаний инструментов. 

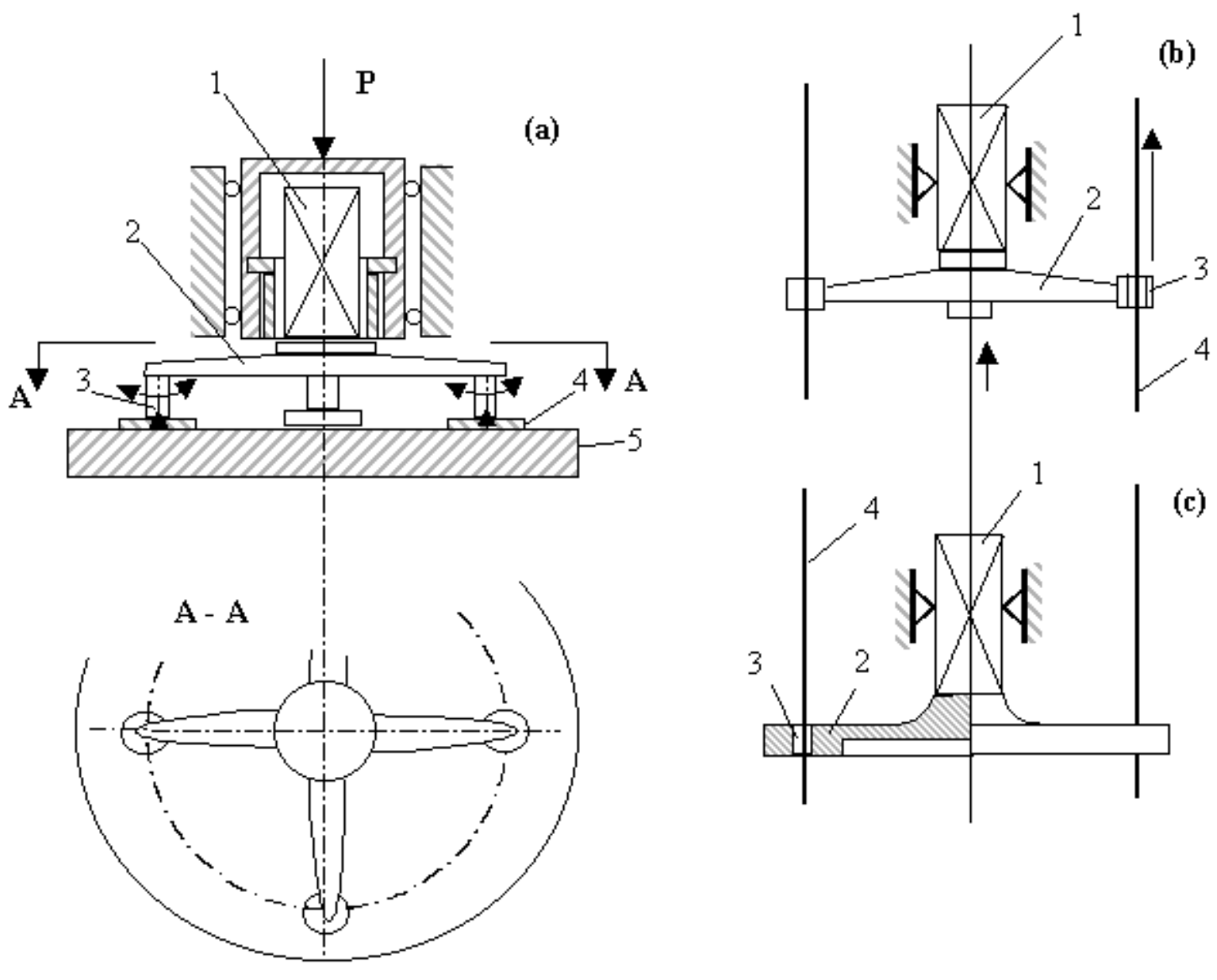

Рис.4.8

При такой схеме возбуждения инструменты, помимо поступательных колебаний в направлении резания, совершают еще и угловые колебания, как показано стрелками на рисунке. Поэтому эта колебательная система применяется лишь в случаях, не требующих высокой точности обработки. Вместе с тем, в Параграфе 6 будет показано, что определенным соотношением инерционных, упругих и геометрических параметров стержней и инструментов можно обеспечить строго поступательные движения инструментов, повысив таким образом точность обработки.

Подобная колебательная система может быть использована для различных технологических процессов. Так в устройстве [54] для ультразвукового волочения (рис.4.8,б) концентраторы 2, совершающие изгибные колебания, несут волоки 3, через которые протягивается обжимаемая проволока 4. Для исключения нежелательных угловых 
колебаний волок эта система может быть видоизменена, как показано на рис.4.8,в. Здесь преобразователь 1 возбуждает колебания мембраны 2, окантованной по периферии цилиндрической обечайкой. Последняя вместе с равномерно расположенными на ней волоками 3 совершает

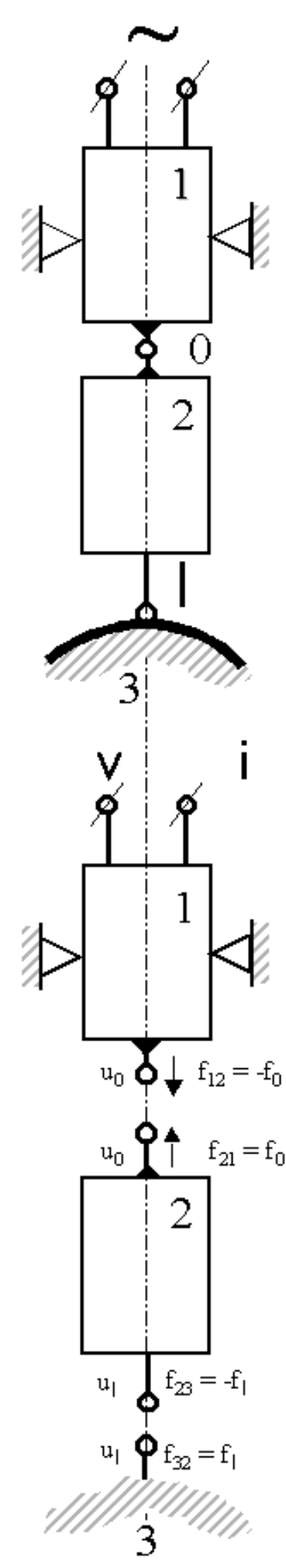

Рис.4.9 строго поступательные колебания вдоль протягиваемой проволоки 4.

Итак, мы рассмотрели основные схемы ультразвуковых технологических машин, конструкции которых подробно описаны в упомянутых выше работах.

4. В ультразвуковой технологической машине независимо от еe назначения и конструктивных особенностей можно выделить привод - возбудитель колебаний и колебательную систему, служащую для передачи колебаний обрабатываемому объекту или среде. Исходя из этого, представим машину в виде обобщенной схемы, показанной на рис.4.9, где колебательная система 2 связана в точке 0 с возбудителем колебаний 1, a взаимодействует с технологической нагрузкой 3 , создаваемой при выполнении рабочего процесса. Возбудитель колебаний производит преобразование электрических колебаний на его входе в механические колебания на выходе 0 возбудителя, который является входным элементом колебательной системы. Колебательная система трансформирует входные воздействия, создавая на выходе $l$ наиболее благоприятные условия выполнения технологического процесса. Таким образом, схема на 
рис.4.9,a представляет собой цепную систему соединенных между собой элементов $[99,138,190]$.

Расчленим цепную систему на составляющие подсистемы, выделив возбудитель колебаний 1 , колебательную систему 2 и технологическую нагрузку 3 и заменим их взаимодействие силами, приложенными к узловым точкам. Силы, действующие на смежные подсистемы в узловой точке, равны по величине и противоположны по направлению, т.е. отличаются только знаком

$$
f_{21}=-f_{12}, f_{23}=-f_{32}
$$

Здесь первый индекс обозначает номер подсистемы, на которую действует сила, второй индекс - номер подсистемы, со стороны которой приложена сила. Для упрощения дальнейших записей обозначим

$$
f_{21}=f_{0}, f_{23}=f_{l}
$$

Рассмотрим подсистему 2 , находящуюся под действием сил $f_{0}$ и $f_{1}$. Будем рассматривать гармонические колебания системы, представляя движение любого ее элемента $x$ величиной

$$
u_{X}(t)=m_{X}+u_{X}^{0}(t)=m_{X}+a_{X} e^{j\left(\omega \cdot t+\varphi_{X}\right)}
$$

где $m_{x}$ - постоянная составляющая; $a_{x}, \varphi_{x}$ - амплитуда и фаза периодической составляющей движения элемента $x$.

Периодическую составляющую в (4.3) удобно записать в иной форме

$$
u_{x}^{0}(t)=\bar{a}_{x} e^{j \omega t}
$$

где

$$
\bar{a}_{x}=a_{x} e^{j \varphi_{x}}=a_{x}\left(\cos \varphi_{x}+j \sin \varphi_{x}\right)
$$

- комплексная амплитуда колебаний.

Полагаем, что действующая на некоторый элемент $s$ системы сила $f_{s}(t)$, также является гармонической функцией времени

$$
f_{s}(t)=P_{s}+f_{s}^{0}(t)=P_{s}+F_{s} e^{j\left(\omega \cdot t+\theta_{s}\right)}
$$

где $P_{s}$ - постоянная составляющая; $F_{s}, \theta_{s}$ - амплитуда и фаза периодической составляющей силы. 
По аналогии с (4.4) периодическую составляющую силы (4.6) запишем в виде

$$
f_{s}^{0}(t)=\bar{F}_{s} \mathrm{e}^{j \omega \cdot t},
$$

где

$$
\bar{F}_{s}=F_{s} e^{j \theta_{s}}=F_{s}\left(\cos \theta_{s}+j \sin \theta_{s}\right)
$$

5. Будем считать колебательную систему линейной и стационарной, полагая, что ее движение описывается линейными дифференциальными уравнениями с постоянными коэффициентами. Для описания установившихся колебаний используем понятие динамической податливости $L_{s x}(j \omega)$ - комплексной величины, устанавливающей связь между силой $f_{s}$, действующей на элемент $s$ системы, и перемещением $u_{x}$ произвольного элемента $x$ [106] . С помощью этих представлений движение элемента $x$ колебательной системы описывается выражением

$$
u_{x}(t)=L_{0 x}(j \omega) \cdot f_{0}(t)-L_{l x}(j \omega) \cdot f_{l}(t)
$$

После подстановки в (4.9) выражений (4.4) - (4.7) и разделения постоянных и переменных составляющих получим

$$
\begin{gathered}
m_{x}=L_{0 x}(0) \cdot P_{0}-L_{l x}(0) \cdot P_{l} \\
\bar{a}_{x}=L_{0 x}(j \omega) \cdot \bar{F}_{0}-L_{l x}(j \omega) \cdot \bar{F}_{l}
\end{gathered}
$$

Равенство (4.10) связывает постоянную составляющую перемещения элемента $x$ с постоянными составляющими сил, действующих в узловых точках системы. Здесь величины $L_{s x}(0),(s=0, l)$, играют роль статических податливостей упругой системы, получаемых из динамических податливостей $L_{s x}(j \omega)$ при $j \omega=0$. Равенство (4.11) связывает комплексные амплитуды перемещений и сил. Из (4.11) для элементов $x=0$ и $x=l$ имеем

$$
\begin{aligned}
& \bar{a}_{0}=L_{00}(j \omega) \cdot \bar{F}_{0}-L_{l 0}(j \omega) \cdot \bar{F}_{l} \\
& \bar{a}_{l}=L_{0 l}(j \omega) \cdot \bar{F}_{0}-L_{l l}(j \omega) \cdot \bar{F}_{l}
\end{aligned}
$$

Величины динамических податливостей в выражениях (4.9) - (4.13) зависят от инерционных, упругих и диссипативных свойств колебательной системы. Их конкретные выражения для типичных элементов 
колебательных систем ультразвуковых технологических машин будут построены в следующих двух параграфах. Как следует из (4.11) - (4.13), динамическая податливость $L_{s x}(j \omega)$ определяется отношением комплексных амплитуд перемещения $\bar{a}_{x}$ элемента $x$ и действующей на элемент $s$

$$
L_{s x}(j \omega)=\bar{a}_{x} / \bar{F}_{s}
$$

В дальнейшем мы будем часто пользоваться понятием динамической жесткости $W_{s x}(j \omega)$ - величиной, обратной динамической податливости

$$
W_{s x}(j \omega)=L_{s x}^{-1}(j \omega)=\bar{F}_{s} / \bar{a}_{x}
$$

Заметим, что в акустике [134] для описания колебаний линейных систем обычно используют понятие импеданса $Z_{s x}(j \omega)$, представляющего собой отношение комплексных амплитуд силы $\bar{F}_{s}$ и виброскорости $j \omega \bar{a}_{x}$, т.е.

$$
Z_{s x}(j \omega)=\bar{F}_{s} / j \omega \bar{a}_{x}
$$

Из сравнения (4.15) и (4.16) видно, что динамическая жесткость и импеданс связаны соотношением

$$
W_{s x}(j \omega)=j \omega \cdot Z_{s x}(j \omega)
$$

В этой работе далее используются величины (4.14), (4.15), которые описывают не только периодические составляющие рассматриваемых процессов, но и дают связь постоянных составляющих типа (4.10). Эта связь, как будет показано ниже в Главе 3, оказывается необходимой и играет существенную роль при анализе нелинейных рабочих процессов.

Равенства (4.12), (4.13) представляют собой уравнения четырехполюсника [6,203], связывающие две входные и две выходные величины. Для полного описания четырехполюсника необходимо связать величины $\bar{F}_{0}, \bar{F}_{l}, \bar{a}_{0}, \bar{a}_{l}$ двумя дополнительными условиями. Эти условия диктуются физическим содержанием рассматриваемых процессов. Для их построения рассмотрим смежные подсистемы. 
6. Обратимся к подсистеме 1 , являющейся возбудителем колебаний. Эту подсистему также можно представить в виде четырехполюсника с электрической входной и механической выходной стороной. Пусть $v, q-$ мгновенные напряжение и заряд на входе возбудителя. Будем рассматривать установившиеся режимы при гармонически изменяющихся напряжении и заряде

$$
v=\bar{v}_{0} e^{j \omega \cdot t}, q=\bar{q}_{0} e^{j \omega \cdot t},
$$

где $\bar{v}_{0}, \bar{q}_{0}$ - комплексные амплитуды напряжения и заряда на входе четырехполюсника.

На механической выходной стороне четырехполюсника действует гармоническая сила с амплитудой $\bar{F}_{12}=-\bar{F}_{0}$, и возбуждаются колебания амплитуды $\bar{a}_{0}$. Между входными электрическими и выходными механическими величинами существуют два линейных соотношения [198].

$$
\begin{aligned}
& \tilde{F}_{12}=\alpha_{11}(j \omega) \tilde{a}_{0}+\alpha_{12}(j \omega) \cdot \tilde{q}_{0} \\
& \widetilde{v}_{0}=\alpha_{21}(j \omega) \tilde{a}_{0}+\alpha_{22}(j \omega) \cdot \widetilde{q}_{0}
\end{aligned}
$$

Комплексные коэффициенты $\alpha_{m n}(j \omega) \quad(m, n=1,2)$ зависят от параметров механической и электрической частей привода. Их конкретный вид для типичных электроакустических преобразователей будет построен в Параграфе 7. Физический смысл этих коэффициентов можно выяснить, если положить равной нулю одну из величин $\tilde{a}_{0}, \tilde{q}_{0}$ : $\alpha_{11}(j \omega)=\tilde{F}_{12} / \tilde{a}_{1 \mid \tilde{q}_{0}=0}-$ динамическая жесткость механической части привода при $\quad$ отсутствии $\quad$ тока; $\quad \alpha_{12}(j \omega)=\tilde{F}_{12} / \tilde{q}_{0 \mid \tilde{a}_{0}=0}, \quad \alpha_{21}(j \omega)=\tilde{v}_{0} / \tilde{a}_{0 \mid \tilde{q}_{0}=0} \quad-$ коэффициенты электромеханической связи, определяющие взаимовлияние электрической и механической частей привода, причем $\alpha_{12}(j \omega)= \pm \alpha_{21}(j \omega)$ в зависимости от типа преобразователя; $\alpha_{22}(j \omega)=\tilde{v}_{0} / \tilde{q}_{0 \mid \tilde{a}_{0}=0}-$ величина, обратная комплексной емкости электрической цепи привода при отсутствии смещений. Эту величину можно выразить через комплексное сопротивление (импеданс) $Z(j \omega)$ цепи 


$$
\alpha_{22}(j \omega)=j \omega \cdot \tilde{v}_{0} / \tilde{i}_{0}=j \omega \cdot Z(j \omega),
$$

где $\tilde{i}_{0}=j \omega \cdot \tilde{q}_{0}$ - комплексная амплитуда силы тока.

При отсутствии нагрузки на преобразователь, т.е. при $F_{12}=-F_{0}=0$, амплитуда колебаний $\tilde{a}_{0}^{*}$ согласно (4.15)

$$
\tilde{a}_{0}^{*}=\frac{\alpha_{12}(j \omega)}{\alpha_{12}^{2}(j \omega)-\alpha_{11}(j \omega) \alpha_{22}(j \omega)} \tilde{v}_{0}=\frac{\alpha_{12}(j \omega)}{\alpha_{11}(j \omega)} \widetilde{q}_{0}=\frac{\alpha_{12}(j \omega)}{j \omega \cdot \alpha_{11}(j \omega)} \tilde{i}_{0}
$$

Одна из электрических величин на входе четырехполюсника, как правило, задана. Это амплитуда напряжения $\tilde{v}_{0}$, если преобразователь питается от источника напряжения, или амплитуда силы тока $\tilde{i}_{0}=j \omega \cdot \tilde{q}_{0}$ при питании от источника тока. В любом случае величина амплитуды колебаний $\tilde{a}_{0}^{*}$ ненагруженного преобразователя характеризует уровень возбуждения системы. Из соотношений (4.15) с учетом (4.1), (4.2), (4.17) амплитуду колебаний преобразователя, нагруженного на механическую колебательную систему, удобно представить в виде

$$
\tilde{a}_{0}=\tilde{a}_{0}^{*}-L_{n}(j \omega) \cdot \tilde{F}_{0},
$$

где $L_{n}(j \omega)$ - динамическая податливость преобразователя, причем

$$
L_{n}(j \omega)=\alpha_{11}^{-1}(j \omega)
$$

при питании от источника тока и

$$
L_{n}(j \omega)=\left[\alpha_{11}(j \omega)-\frac{\alpha_{12}(j \omega) \cdot \alpha_{21}(j \omega)}{\alpha_{22}(j \omega)}\right]
$$

при питании от источника напряжения.

Равенство (4.18) связывает амплитуды перемещения $\tilde{a}_{0}^{*}$ и силы $\widetilde{F}_{0}$ на входе четырехполюсника (4.12), (4.13). Используя эту связь, из (4.12) находим

$$
\begin{gathered}
\bar{F}_{0}=\frac{\bar{a}_{0}^{*}-L_{l 0}(j \omega) \cdot \bar{F}_{l}}{L_{00}(j \omega)+L_{n}(j \omega)} \\
\bar{a}_{0}=\frac{\bar{a}_{0}^{*} L_{00}(j \omega)+L_{n}(j \omega) \cdot L_{l 0}(j \omega) \cdot \bar{F}_{l}}{L_{00}(j \omega)+L_{n}(j \omega)}
\end{gathered}
$$


Подставив (4.21) в (4.13), для амплитуды колебаний рабочего органа получим

$$
\bar{a}_{l}=\bar{a}_{l}^{*}-L_{M}(j \omega) \cdot \bar{F}_{l}
$$

где

$$
\bar{a}_{l}^{*}=\frac{L_{0 l}(j \omega)}{L_{00}(j \omega)+L_{n}(j \omega)} \bar{a}_{0}^{*}
$$

амплитуда колебаний рабочего органа машины на холостом ходу, т.е. при отсутствии технологической нагрузки $\left(\bar{F}_{l}=0\right)$;

$$
L_{M}(j \omega)=L_{l l}(j \omega)-\frac{L_{0 l}^{2}(j \omega)}{L_{00}(j \omega)+L_{n}(j \omega)}
$$

полная, приведенная к рабочему органу динамическая податливость колебательной системы и привода. При записи выражения (4.25) учтено равенство

$$
L_{s X}(j \omega)=L_{x s}(j \omega),
$$

справедливое для линейных систем согласно обобщенному принципу взаимности Рэлея [58,176,182,183].

7. Уравнение (4.23) определяет амплитуду колебаний рабочего органа ультразвуковой технологической машины под нагрузкой, создаваемой в результате взаимодействия рабочего органа с обрабатываемым изделием или средой. Как было показано в предыдущей Главе, сила взаимодействия рабочего органа с изделием описывается в общем случае нелинейной динамической характеристикой

$$
f_{l}=f_{l}\left[u_{l}(t), \dot{u}_{l}(t)\right]
$$

Характеристики типичных технологических процессов будут рассмотрены в Параграфе 8. При определенных допущениях относительно вида исследуемых процессов характеристика (4.27) позволяет получить связь комплексных амплитуд силы $\tilde{F}_{l}$ и перемещения $\tilde{a}_{l}$

$$
\tilde{F}_{l}=\tilde{F}_{l}\left(\tilde{a}_{l}\right)
$$

Соотношения (4.23), (4.28) образуют полную систему уравнений для 
отыскания неизвестных параметров процесса $\tilde{a}_{l}, \tilde{F}_{l}$. Эти задачи, составляющие основное содержание работы, будут подробно освещены в последующих главах.

В отдельных случаях характеристика технологической нагрузки может быть представлена линейной зависимостью силы $f_{l}$ от перемещения $u_{l}(t)$ рабочего органа

$$
f_{l}(t)=W_{H}(j \omega) \cdot u_{l}(t)=L_{H}^{-1}(j \omega) \cdot u_{l}(t)
$$

где $W_{H}(j \omega), \quad L_{H}(j \omega)$ динамические жесткость и податливость технологической нагрузки.

Так записывается, например, взаимодействие инструмента с изделием при непрерывном деформировании образца (рис.2.4,б), происходящем в пределах упругой зоны характеристики материала. Для линейного упруговязкого материала с учетом вида рассматриваемых процессов (4.4) можно записать

$$
f_{l}(t)=k_{0} u_{l}(t)+\beta_{0} \dot{u}_{l}(t)=\left(k_{0}+j \omega \cdot \beta_{0}\right) \cdot u_{l}(t)
$$

где $k_{0}, \beta_{0}$ - статическая жесткость образца и коэффициент сопротивления деформированию.

Из сравнения (4.29) и (4.30) находим

$$
W_{H}(j \omega)=k_{0}+j \omega \cdot \beta_{0}
$$

Для комплексной амплитуды силы (4.29) с учетом (4.4), (4.7) получаем

$$
\widetilde{F}_{l}=W_{H}(j \omega) \cdot \tilde{a}_{l}
$$

После подстановки (4.32) в (4.23) находим комплексную амплитуду колебаний рабочего органа при работе на линейную нагрузку

$$
\bar{a}_{l}=\bar{a}_{l}^{*}\left[1+\frac{W_{H}(j \omega)}{W_{M}(j \omega)}\right]^{-1}
$$

где $W_{M}(j \omega)=L_{M}^{-1}(j \omega)$ - приведенная к рабочему органу динамическая жесткость колебательной системы.

Из (4.33) следует, что нагружение колебательной системы изменяет 
амплитуду колебаний рабочего органа по отношению к амплитуде $\bar{a}_{l}^{*}$ холостого хода. Забегая вперед, отметим, что нагружение может привести как к уменьшению, так и к увеличению амплитуды колебаний в зависимости от соотношения динамических жесткостей колебательной системы и нагрузки. Это в полной мере относится и к машинам, работающим на нелинейную технологическую нагрузку. Различные характеристики технологической нагрузки будут рассмотрены в следующей Главе. Из построенных соотношений видно, что динамические свойства ультразвуковых технологических машин в значительной степени определяются динамическими характеристиками их отдельных элементов

- их динамическими податливостями и жесткостями. Перейдем к построению этих величин для типичных элементов ультразвуковых технологических машин. 


\section{5. Продольные колебания ультразвуковых стержневых систем}

1. Колебательные системы ультразвуковых технологических машин содержат разнообразные элементы, совершающие продольные колебания. Это, в первую очередь, стержневые волноводы с определенным распределением площади поперечного сечения вдоль оси стержня, который необходимо рассматривать как систему с распределенными параметрами. Строго говоря, в любой реальной системе инерционные, упругие и диссипативные свойства непрерывно распределены по всему ее объему. Однако при определенных условиях такое распределение можно не учитывать и идеализированно представить соответствующие элементы в виде точечной массы, сосредоточенной упругости и демпфера. (Условия идеализации будут получены ниже.) Сосредоточенными элементами можно считать присоединенные к концентратору рабочие инструменты, мембраны крепления колебательной системы в корпусе акустической головки, отдельные детали соединения концентратора с преобразователем и т.д.

Таким образом, колебательная система ультразвуковой технологической машины представляет собой комбинацию распределенных и сосредоточенных элементов. В настоящем параграфе основное внимание будет уделено отысканию динамических податливостей и жесткостей типичных ультразвуковых стержневых систем, совершающих продольные колебания. Рассмотрим стержень (рис.5.1) длиной $l$ с поперечным сечением, площадь которого изменяется по некоторому закону $S=S(x)$.

При описании колебаний предполагается, что поперечные сечения стержня остаются плоскими, а частицы, расположенные в этих сечениях, перемещаются только в направлении оси $x$. Смещение сечения $x$ от его положения в недеформированном стержне будем описывать функцией $u(x, t)$. 


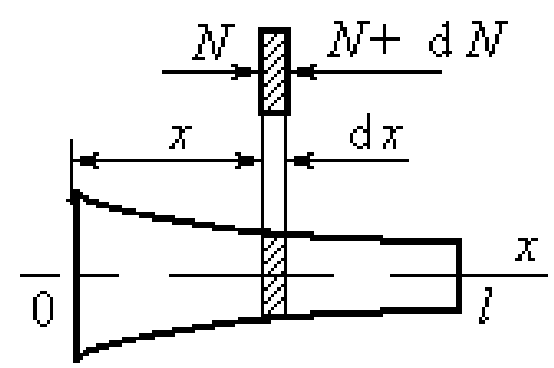

Рис.5.1

Рассматривая гармонические колебания вида

$$
u(x, t)=\bar{a}_{x} e^{j \omega \cdot t}=a_{x} e^{j\left(\omega \cdot t-\varphi_{x}\right)}
$$

$\left(\bar{a}_{x}=\bar{a}(x)\right.$ - комплексная амплитуда; $a_{x}, \varphi_{x}$ - амплитуда и фаза колебаний сечения $x$ ), запишем зависимость напряжения $\sigma$ от относительной деформации $\varepsilon$ в форме, предложенной Е.С. Сорокиным [184]:

$$
\sigma=\bar{E} \varepsilon=\bar{E} \cdot \partial u / \partial x
$$

где

$$
\widetilde{E}=E\left(1+j \frac{\psi}{2 \pi}\right),
$$

$E$ - модуль упругости; $\psi$ - коэффициент поглощения, характеризующий рассеяние энергии в результате внутреннего трения в материале $[83,159,166]$.

C учетом принятых допущений запишем уравнение движения элемента $\mathrm{dx}$ стержня (рис.5.1) $\rho \cdot S_{x} \frac{\partial^{2} u(x, t)}{\partial^{2}} d x=d N_{x}=\frac{\partial\left(S_{x} \cdot \sigma\right)}{\partial x} d x \quad, \quad$ из которого после подстановки (5.2) получим уравнение продольных колебаний стержня

$$
\rho \cdot \frac{d^{2} u(x, t)}{d t^{2}}=\bar{E}\left[\frac{\partial^{2} u(x, t)}{\partial x^{2}}+\frac{S_{x}^{\prime}}{S_{x}} \cdot \frac{\partial u(x, t)}{\partial x}\right],
$$

где $\rho$ - плотность материала стержня; $S_{x}=S(x)$ - площадь в сечении $x$; $S_{x}^{\prime}=d S(x) / d x$.

Будем считать, что один из концов стержня свободен, а ко второму приложена гармоническая сила. Пусть $x=s$ - сечение, в котором действует возбуждение $f_{s}(t)=F_{s} e^{j \omega \cdot t}$, причем $s=l$ при возбуждении правого конца и 
$s=0$ при возбуждении левого конца стержня. Координата свободного конца $x=l-s$. Решение уравнения (5.4) должно удовлетворять следующим граничным условиям:

$$
\begin{aligned}
& \left.\frac{\partial u(x, t)}{\partial x}\right|_{x=l-s}=0 \\
& \left.\bar{E} S_{s} \frac{\partial u(x, t)}{\partial x}\right|_{x=s}=(-1)^{(1-s / l)} F_{s} e^{j \omega \cdot t}
\end{aligned}
$$

Множитель $(-1)^{(1-s / l)}$ во втором условии выражает известное правило знаков $[83,196]$ сил, действующих на разные концы стержня. После подстановки искомого решения (5.1) получим вместо (5.4), (5.5)

$$
\begin{gathered}
\bar{E}\left[\bar{a}_{x}^{\prime \prime}+\left(s_{x}^{\prime} / s_{x}\right) \bar{a}_{x}^{\prime}\right]+\rho \cdot \omega^{2} \bar{a}_{x}=0 \\
\left.\bar{a}_{x}^{\prime}\right|_{x=l-s}=0,\left.\bar{E} S_{s} \bar{a}_{x}^{\prime}\right|_{x=s}=(-1)^{(1-s / l)} F_{s}
\end{gathered}
$$

Дальнейшее решение зависит от конкретной формы волновода, определяемой законом изменения площади $S_{x}$ поперечного сечения. Определив в результате решения комплексную амплитуду $\bar{a}_{x}, \mathrm{c}$ помощью выражений (4.14), (4.15) найдем динамические податливости $L_{s x}(j \omega)$ и жесткости $W_{s x}(j \omega)$.

Проведем такое решение для наиболее употребительных типов волноводов ультразвуковых технологических машин.

2. Рассмотрим стержень постоянного сечения $S_{x}=S=$ const . Уравнение (5.4) принимает вид $\bar{E} \bar{a}_{x}^{\prime \prime}+\rho \omega^{2} \bar{a}_{x}=0$. Отыскивая его решение, удовлетворяющее граничным условиям (5.7), и учитывая (4.14), получаем величину динамической податливости

$$
L_{s x}(j \omega)=(-1)^{s / l} \frac{\lambda}{\omega^{2} \rho \cdot S} \cdot \frac{e^{\lambda \cdot x}+e^{-\lambda \cdot x} e^{2 \lambda(l-s)}}{e^{\lambda \cdot s}-e^{-\lambda \cdot s} e^{2 \lambda(l-s)}}
$$

где

$$
\lambda=j \omega \sqrt{\frac{\rho}{\bar{E}}}=\frac{j \omega}{c \sqrt{1+j \psi / 2 \pi}}
$$

$c=\sqrt{E / \rho}$ - скорость звука в материале стержня. 
При действии возбуждающей силы на правый $s=l$ и левый $s=0$ концы стержня из (5.8) имеем соответственно

$$
\begin{gathered}
L_{l x}(j \omega)=-\frac{\lambda}{\rho \cdot S \omega^{2}} \cdot \frac{e^{\lambda \cdot x}+e^{-\lambda \cdot x}}{e^{\lambda \cdot l}-e^{-\lambda \cdot l}}=-\frac{\lambda}{\rho \cdot S \omega^{2}} \cdot \frac{\operatorname{ch}(\lambda \cdot x)}{\operatorname{sh}(\lambda \cdot l)} \\
L_{0 x}(j \omega)=-\frac{\lambda}{\rho \cdot S \omega^{2}} \cdot \frac{e^{\lambda(l-x)}+e^{-\lambda(l-x)}}{e^{\lambda \cdot l}-e^{-\lambda \cdot l}}=-\frac{\lambda}{\rho \cdot S \omega^{2}} \cdot \frac{\operatorname{ch}(\lambda(l-x))}{\operatorname{sh}(\lambda \cdot l)}
\end{gathered}
$$

В дальнейшем будем полагать, что диссипативная составляющая напряжений в (5.2) существенно меньше упругой, т.е. коэффициент поглощения $\psi$ - малая величина $(\psi<<1)$, и разложение величин, содержащих $\psi$, в ряд по малому параметру будем ограничивать линейными членами. В соответствии со сказанным равенство (5.9) приводим к виду

$$
\lambda=\left(j+\frac{\psi}{4 \pi}\right) \frac{\omega}{c}
$$

Рассмотрим предварительно два частных случая. Пусть длина стержня столь мала, что $|\lambda| \cdot l<<1$. Это условие с учетом эквивалентно следующему

$$
l<<\frac{c}{\omega}=\frac{\Lambda}{2 \pi}
$$

где $\Lambda$ - длина волны с частотой $\omega$.

Так как $0 \leq x \leq l$, то $|\lambda| \cdot l<<1$. Ограничивая разложение экспонент в (5.10), (5.11) в ряды по малому параметру величинами первого порядка малости, получаем

$$
L_{l x}(j \omega)=L_{0 x}(j \omega)=L(j \omega)=\left(-M \omega^{2}\right)^{-1}
$$

где $M=\rho \cdot l \cdot S$ - масса стержня.

Равенство (5.14) представляет собой динамическую податливость сосредоточенной массы $M$. Таким образом, короткий стержень, длина $l$ которого согласно (5.13) много меньше длины волны, можно идеализированно считать абсолютно жестким телом. 
Во втором частном случае рассмотрим полубесконечный стержень, возбуждаемый в начальном сечении $x=0$. Переходя в (5.11) к пределу при $l \rightarrow \infty$ и учитывая (5.12), получаем с принятой точностью

$$
L_{0 x}(j \omega)=\frac{1}{j \omega \cdot w} \exp \left[-\left(j+\frac{\psi}{4 \pi}\right) \frac{\omega \cdot x}{c}\right]
$$

где $w=S \sqrt{E \rho}$ - волновое сопротивление стержня.

Динамическая податливость в точке возбуждения $x=0$

$$
L_{00}(j \omega)=(j \omega \cdot w)^{-1}
$$

и, следовательно, по отношению к источнику возбуждения полубесконечный стержень эквивалентен сосредоточенному линейному сопротивлению с коэффициентом сопротивления равным волновому.

Если полубесконечный стержень возбуждается гармонической силой $f(t)=F_{0} e^{j \omega \cdot t}$, то колебания его сечений описываются функцией $u(x, t)=L_{0 x}(j \omega) F_{0} e^{j \omega \cdot t}$ или с учетом $(5.15)$

$$
u(x, t)=\frac{F_{0}}{j \omega \cdot w} \exp \left(-\frac{\psi \cdot \omega}{4 \pi \cdot c} x\right) \exp \left[j \omega\left(t-\frac{x}{c}\right)\right]
$$

Это выражение представляет собой уравнение бегущей гармонической волны $[116,117]$, амплитуда которой убывает вдоль стержня по экспоненциальному закону. Волна распространяется вдоль стержня со скоростью $c$.

На рис.5.2 построена форма бегущей волны в полубесконечном стержне; штриховой линией показана огибающая амплитуд

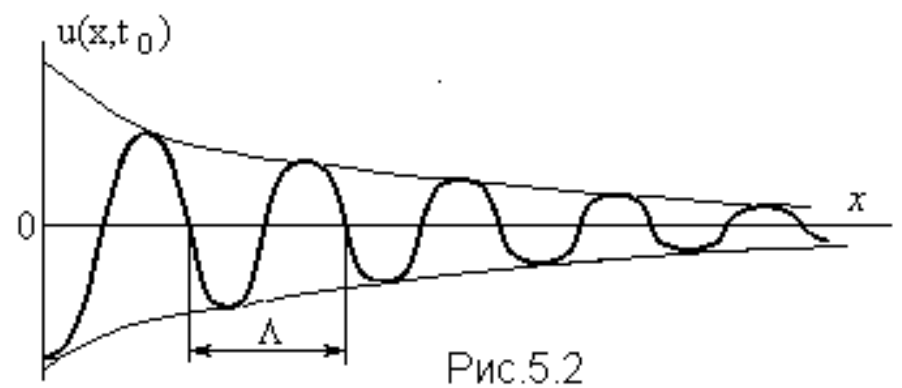


Отметим, что согласно (5.18)

$$
a_{x}=\frac{F_{0}}{\omega \cdot w} \exp \left(-\frac{\psi \cdot \omega}{4 \pi \cdot c} x\right)
$$

ограниченный стержень можно идеализированно представить полубесконечным при достаточно большой длине

$$
l>\frac{4 \pi \cdot c}{\psi \cdot \omega}=\frac{2 \Lambda}{\psi}
$$

При такой длине возвращающаяся к источнику отраженная от свободного конца стержня волна затухает не менее, чем в $e^{2}$ раз.

3. В ультразвуковых колебательных системах длина волноводов $l$, как правило, соизмерима с длиной упругой волны $\Lambda$. По аналогии с (5.17), используя (5.8), можно показать, что смещения сечений стержня ограниченной длины под действием гармонического возбуждения $f_{s}(t)$ образуются суперпозицией прямой $u_{1 x}(x, t)=\bar{a}_{1 x} \mathrm{e}^{j \omega(t-x / c)}$ и обратной $u_{2 x}(x, t)=\bar{a}_{2 x} \mathrm{e}^{j \omega(t+x / c)}$ волн, распространяющихся вдоль стержня.

Для удобства дальнейшего рассмотрения преобразуем выражения для динамических податливостей (5.10), (5.11), подставив значение (5.12) и ограничив разложение величин в числителе и знаменателе в ряды по малому параметру ж линейными членами. В результате из (5.10) получим

$$
L_{l x}(j \omega)=-\frac{l}{E S \xi} \cdot \frac{\cos \xi \frac{x}{l}-j \frac{\psi}{4 \pi}\left(\cos \xi \frac{x}{l}-\xi \frac{x}{l} \sin \xi \frac{x}{l}\right)}{\sin \xi-j \frac{\psi}{4 \pi} \xi \cos \xi}
$$

где $\xi=\omega \cdot l / c$. Из сравнения (5.10) и (5.11) видно, что выражение для $L_{0 x}(j \omega)$ можно получить из (5.20) заменой $x$ на $l-x$. Это выражение здесь не приводится. В дальнейшем нам потребуются, в основном, значения динамических податливостей для крайних сечений стержня. Из (5.10), (5.11) сразу следует

$$
L_{00}(j \omega)=L_{l /}(j \omega), L_{l 0}(j \omega)=L_{0 l}(j \omega)
$$


Первое из этих равенств является следствием симметрии однородного стержня; второе - отражает принцип взаимности перемещений [58, 176], справедливый для линейных систем.

При действии силы $f_{l}(t)=F_{l} e^{j \omega \cdot t}$ комплексные амплитуды колебаний стержня

$$
\bar{a}_{x}=F_{l} L_{l x}(j \omega)
$$

Для стержня без потерь $(\psi=0)$ с учетом (5.20) имеем

$$
\bar{a}_{x}=a_{x} e^{-j \varphi_{x}}=\bar{a}_{0} \cos \xi \frac{x}{l}=-\frac{F_{l} l \cos \xi \frac{x}{l}}{E S \xi \cdot \sin \xi}
$$

Равенство (5.23) определяет амплитудно-частотную и фазочастотную характеристики стержня без потерь. Уравнение амплитудночастотной характеристики для сечения $x$ будет

$$
a_{x}=\frac{F_{l} l}{E S}\left|\frac{\cos \xi \cdot \frac{x}{l}}{\xi \cdot \sin \xi}\right|
$$

Фаза колебаний находится из уравнения

$$
\cos \varphi_{x}=-\operatorname{sign}\left(\cos \xi \cdot \frac{x}{l} / \sin \xi\right)
$$

На рис.5.3,а,б штриховыми линиями показаны эти характеристики для крайнего сечения $x=l$ стержня.

На резонансных частотах

$$
\xi=\xi_{n}=\pi \cdot n,(n=0,1,2, \ldots)
$$

амплитуды колебаний $a_{x} \rightarrow \infty$. При этом колебания происходят по одной из собственных форм (рис.5.4,a), уравнения которых получаются из (5.23)

$$
a_{x}=a_{0} \cos \pi n x / 1
$$

Согласно (5.24) существуют такие “антирезонансные” частоты

$$
\xi=\xi_{n}^{*}=(2 n+1) \pi / 2
$$


при которых амплитуда колебаний точки возбуждения $a_{l}^{*}=0$. При этом амплитуда колебаний свободного конца

$$
a_{0}^{*}=\frac{2 F_{l} l}{\pi \cdot E S(n+1)}
$$

Антирезонансные формы колебаний показаны на рис.5.4,б. При прохождении частот (5.28) в точке действия возбуждения появляется узел смещений сечений стержня, который по мере увеличения частоты смещается вдоль стержня. При прохождении антирезонансной частоты происходит изменение фазы колебаний. Отметим, что “антирезонанс" является проявлением известного эффекта динамического гашения колебаний $[74,120,121]$.
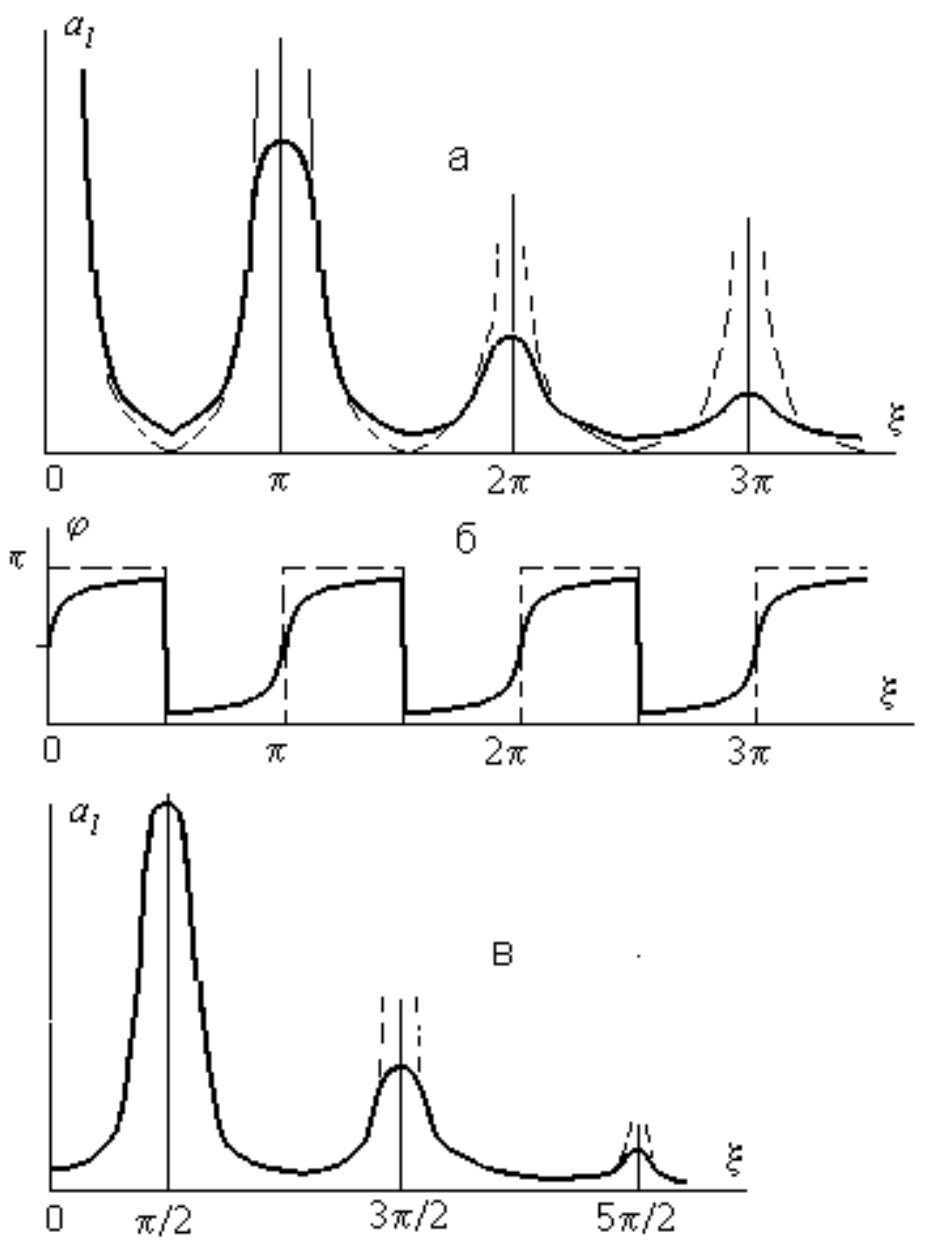

Рис. 5.3 
Сплошными линиями на рис.5.3,a,б показаны амплитудно- и фазочастотные характеристики при наличии рассеяния энергии $(\psi \neq 0)$, приводящего к ограничению амплитуд резонансных колебаний. Согласно (5.22), (5.20), (5.26) резонансные амплитуды крайних сечений

$$
a_{0}^{0}=a_{l}^{0}=\frac{4 \pi \cdot F_{l} l}{\psi \xi_{n}^{2} E S}=\frac{4 F_{l} l}{\pi \psi E S n^{2}}
$$

убывают пропорционально квадрату номера $n$ формы, причем диссипация слабо сказывается на виде формы колебаний (рис.5.4,a).

На “антирезонансных” частотах (5.28) амплитуды колебаний

$$
a_{l}^{*}=\frac{\psi \cdot F_{l} l}{4 \pi \cdot E S}, \quad a_{0}^{*}=\frac{\delta_{F}}{\xi_{n}^{*}}=\frac{2 F_{l} l}{\pi(n+1) E S}
$$
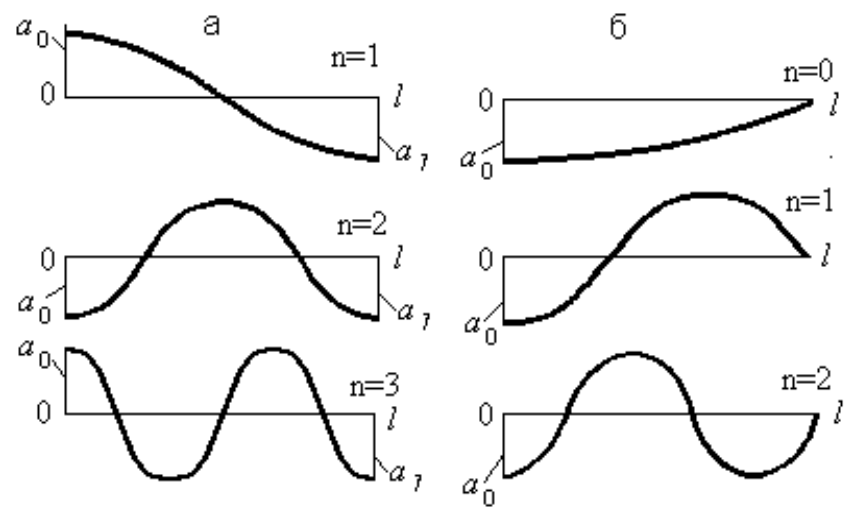

Pис.5.4

Интересно отметить, что при антирезонансе амплитуда точки возбуждения $a_{l}^{*}$ не зависит от частоты, а амплитуда свободного конца $a_{0}^{*}$ не зависит от коэффициента поглощения. Здесь, как и ранее, все вычисления ограничены величинами первого порядка малости. По мере увеличения частоты амплитуда $a_{0}^{*}$ убывает и при

$$
\xi \geq \frac{4 \pi}{\psi}
$$

оказывается $a_{0}^{*} \leq a_{l}^{*}$.

Как следует из (5.30), (5.31), на частотах, удовлетворяющих неравенству (5.32), антирезонансные амплитуды $a_{l}^{*}$ превышают резонансные $a_{l}^{0}$. Это означает, что колебательная система фактически 
перестает быть резонансной, и учет ограниченности стержня становится нецелесообразным. Принимая во внимание обозначение, $\xi=\omega \cdot l / c$, неравенство (5.32) приводим к виду

$$
l \geq \frac{4 \pi \cdot c}{\psi \cdot \omega}=\frac{2 \Lambda}{\psi}
$$

совпадающему с (5.19).

Отметим еще одну особенность неравенства (5.32). С этой целью из (5.20) найдем динамическую податливость в точке возбуждения $x=l$ на резонансных частотах (5.26)

$$
L_{l l}(j \omega)=\frac{4 \pi \cdot l}{j E S \psi \xi^{2}}=\frac{4 \pi \cdot c}{j \psi \cdot \omega \cdot E S \xi}=\frac{4 \pi}{j \psi \cdot \omega \cdot w \xi}
$$

Из (5.33) следует, что на границе (5.32) динамическая податливость $L_{l l}=(j \omega \cdot w)^{-1}$ равна динамической податливости полубесконечного стержня (5.16) в точке возбуждения.

4. По аналогии с (5.20) выражения для динамических податливостей элементов ультразвуковых технологических машин в предположении малости диссипативных потерь можно привести к виду

$$
L_{s X}(j \omega)=\frac{\mathrm{P}_{S X}(j \omega)}{Q(j \omega)}=\frac{\operatorname{Re}_{S X}(j \omega)+j \operatorname{Im} \mathrm{P}_{s X}(j \omega)}{\operatorname{Re} Q(j \omega)+j \operatorname{Im} Q(j \omega)},
$$

где $\mathrm{P}_{s x}(j \omega), Q(j \omega)$ - комплексные величины, причем последняя не зависит от координаты $x$ и точки возбуждения $s$.

Действительные составляющие (5.34) характеризуют упругие и инерционные свойства колебательной системы. Мнимые составляющие диссипативные свойства и в дальнейшем считаются малыми. В соответствии с этим условие резонанса, обеспечивающее достижение максимальных амплитуд, имеет вид

$$
\operatorname{Re} Q\left(j \omega_{n}\right)=0,
$$

где $\omega_{n}-$ резонансные частоты. 
Тогда для резонансных амплитуд получаем

$$
a_{x}^{0}=\frac{F_{s}\left|\mathrm{P}_{s X}\left(j \omega_{n}\right)\right|}{\left|\operatorname{Im} Q\left(j \omega_{n}\right)\right|}=F_{s} \frac{\sqrt{\operatorname{Re}^{2} \mathrm{P}_{s X}\left(j \omega_{n}\right)+\operatorname{Im}^{2} \mathrm{P}_{s X}\left(j \omega_{n}\right)}}{\left|\operatorname{Im} Q\left(j \omega_{n}\right)\right|}
$$

Равенство

$$
\operatorname{ReP}_{s x}(j \omega)=0
$$

при заданной частоте $\omega$ определяет координаты узловых точек формы колебаний, при заданной координате - частоту, при которой данная точка становится узловой. При $x=s$ уравнение (5.37) дает частоты “антирезонансов”, характеризующихся тем, что узловая точка формы колебаний совпадает с точкой возбуждения. С учетом (5.37) из (5.34) находим амплитуду колебаний узловых точек

$$
a_{x}^{*}=F_{s} \frac{\left|\operatorname{ImP}_{s x}(j \omega)\right|}{|Q(j \omega)|}=F_{s} \frac{\left|\operatorname{ImP}_{s x}(j \omega)\right|}{\sqrt{\operatorname{Re}^{2} Q(j \omega)+\operatorname{Im}^{2} Q(j \omega)}}
$$

Амплитуды колебаний узловых точек системы, находящейся в резонансном состоянии, согласно (5.35) - (5.38) будут

$$
a_{x}^{0 *}=F_{s} \frac{\left|\operatorname{ImP}_{s x}\left(j \omega_{n}\right)\right|}{\left|\operatorname{Im} Q\left(j \omega_{n}\right)\right|}
$$

Для точек, находящихся в пучностях смещений, из (5.36), пренебрегая малыми второго порядка, можно записать

$$
a_{x}^{0}=F_{S}\left|\frac{\operatorname{Re}_{s x}\left(j \omega_{n}\right)}{\operatorname{Im} Q\left(j \omega_{n}\right)}\right|
$$

Аналогично для узловых точек на частотах, далеких от резонансных,

$$
a_{x}^{*}=F_{s}\left|\frac{\operatorname{Im} P_{s x}(j \omega)}{\operatorname{Re} Q(j \omega)}\right|
$$

При рассмотрении ультразвуковых технологических машин, как было выяснено в гл.1, наибольший интерес представляют резонансные амплитуды (5.40) в пучностях форм колебаний, которые в $\sim \psi^{-1}$ раз превышают амплитуды (5.38) узловых точек собственных форм, и в $\sim \psi^{-2}$ раз - антирезонансные амплитуды (5.41). Поэтому, пренебрегая величинами второго порядка малости, для описания процессов близких к 
резонансным будем часто пользоваться следующей записью динамической жесткости, получаемой из (5.34):

$$
W_{s x}(j \omega)=L_{s x}^{-1}(j \omega)=U_{s x}(\omega)+j V_{s X}(\omega)
$$

где

$$
\begin{aligned}
& U_{s x}(\omega)=\operatorname{Re} W_{s x}(j \omega)=\operatorname{Re} Q(j \omega) / \operatorname{ReP}_{s x}(j \omega) \\
& V_{s x}(\omega)=\operatorname{Im} W_{s x}(j \omega)=\frac{\left[\operatorname{Im} Q(j \omega) \operatorname{ReP}_{s x}(j \omega)-\operatorname{ImP}_{s x}(j \omega) \operatorname{Re} Q(j \omega)\right]}{\operatorname{Re}^{2} \mathrm{P}_{s x}(j \omega)}
\end{aligned}
$$

Из (5.41) для резонансных частот и амплитуд вместо (5.35) и (5.40) получаем

$$
U_{s x}\left(\omega_{n}\right)=0 ; \quad a_{x}^{0}=F_{s} / V_{s x}\left(\omega_{n}\right)
$$

5. При расчетах ультразвуковых технологических установок часто можно считать, что колебательная система находится в условиях кинематического возбуждения, когда известно движение одного из ее элементов. Такой подход допустим, например, при рассмотрении концентраторов - неоднородных волноводов, присоединяемых к возбудителю колебаний, в случае, если влияние присоединенной системы на возбудитель пренебрежимо мало. Расчет таких систем может производиться с использованием тех же величин динамической податливости и жесткости, что и при силовом возбуждении.

Примем для определенности, что возбудитель колебаний сообщает принудительные колебания $u_{0}(t)=\bar{a}_{0} e^{j \omega \cdot t}$ с амплитудой $\bar{a}_{0}$ сечению $x=0$ стержневой колебательной системы (рис.5.5).

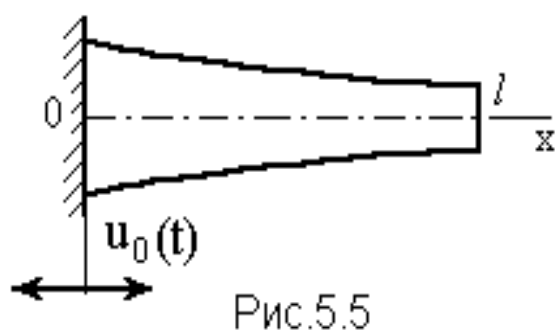


В точке стыка колебательной системы с возбудителем возникает периодическая реакция с амплитудой $\bar{F}_{0}$. Для амплитуд смещений сечений системы справедливо равенство

$$
\bar{a}_{x}=\bar{F}_{0} L_{0 x}(j \omega)
$$

Из (5.44) при $x=0$ находим амплитуду силы реакции

$$
\bar{F}_{0}=\bar{a}_{0} / L_{00}(j \omega),
$$

и для амплитуд колебаний получаем

$$
\bar{a}_{X}=\bar{a}_{0} \frac{L_{0 x}(j \omega)}{L_{00}(j \omega)}
$$

Уравнение (5.46) определяет амплитудно-частотную характеристику колебательной системы при кинематическом возбуждении.

Для однородного стержня выражение (5.46) после подстановки необходимых величин из (5.11) принимает вид

$$
a_{x}=a_{0} \frac{\operatorname{ch} \lambda(l-x)}{\operatorname{ch} \lambda l}
$$

или в предположении малости потерь

$$
a_{x}=a_{0} \frac{\cos \xi \cdot\left(1-\frac{x}{l}\right)+j \frac{\psi}{4 \pi} \xi \cdot\left(1-\frac{x}{l}\right) \sin \xi \cdot\left(1-\frac{x}{l}\right)}{\cos \xi+j \frac{\psi}{4 \pi} \xi \cdot \sin \xi} .
$$

На рис.5.3,в показана амплитудно-частотная характеристика сечения $x=l$ стержня при кинематическом возбуждении. Она описывается уравнением

$$
a_{l}=a_{0} / \sqrt{\cos ^{2} \xi+\left(\frac{\psi}{4 \pi} \xi \cdot \sin \xi\right)^{2}} .
$$

На частотах $\xi=(2 n-1) \pi / 2,(n=1,2, \ldots)$ достигается максимум амплитуд колебаний $a_{l}=8 a_{0} / \psi \cdot(2 n-1)$. На частотах $\xi=\pi \cdot n-$ минимальные амплитуды $a_{l}=a_{0}$.

Отметим, что сила (5.45) определяет нагрузку на возбудитель со стороны колебательной системы. С учетом (5.42) из (5.45) следует, что эта нагрузка будет минимальной при выполнении условия 


$$
U_{00}\left(j \omega_{n}\right)=\operatorname{Re} Q\left(j \omega_{n}\right)=0,
$$

совпадающего с (5.35).

При этом соотношение амплитуд колебаний на выходной $x=l$ и входной $x=0$ сторонах системы согласно (5.46), (5.42), (5.47)

$$
K=\frac{a_{l}}{a_{0}}=\left|\frac{V_{00}\left(\omega_{n}\right)}{V_{0 l}\left(\omega_{n}\right)}\right|=\left|\frac{\operatorname{ReP}_{0 l}\left(j \omega_{n}\right)}{\operatorname{Re} \mathrm{P}_{00}\left(j \omega_{n}\right)}\right|=\left|\frac{U_{00}\left(\omega_{n}\right)}{U_{0 l}\left(\omega_{n}\right)}\right|
$$

Настроенная таким образом колебательная система позволяет при минимальном нагружении возбудителя колебаний обеспечить необходимое увеличение амплитуды на выходном конце. Именно такую функцию выполняют концентраторы, представляющие собой стержни переменного сечения. Величину $K$, характеризующую степень трансформации интенсивности колебаний, передаваемых от возбудителя к рабочему органу, называют коэффициентом усиления концентратора $[146,191]$.

Отметим, что коэффициент усиления не зависит от потерь энергии в материале концентратора. В этом можно убедиться также, используя формулу (5.39). С помощью последней можно получить более общее выражение для коэффициента усиления

$$
K=\frac{a_{l}}{a_{0}}=\left|\frac{\operatorname{Re}_{s l}(j \omega)}{\operatorname{ReP}_{s 0}(j \omega)}\right|=\left|\frac{U_{s 0}(\omega)}{U_{s l}(\omega)}\right|, \quad(s=0, l)
$$

которая при $s=0$ приводится к (5.48).

Рассеяние энергии, однако, определяет нагрузку (5.45) на вибровозбудитель со стороны концентратора

$$
\bar{F}_{0}=j V_{00}(\omega) a_{0}=j \operatorname{Im} Q(j \omega) a_{0} / \operatorname{Re} P_{00}(j \omega),
$$

которая носит чисто диссипативный характер.

Если диссипация в концентраторе отсутствует, то $\bar{F}_{0}=0$, и оказывается, что в установившемся режиме не происходит нагружения вибровозбудителя со стороны присоединенной к нему колебательной системы. 
6. Рассмотрим некоторые типы концентраторов ультразвуковых технологических машин, формы которых приведены на рис.5.6.

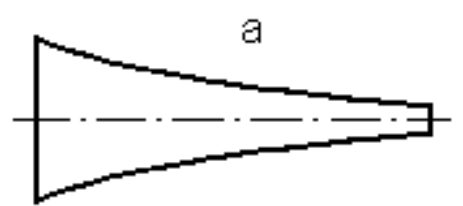

$\mathrm{B}$

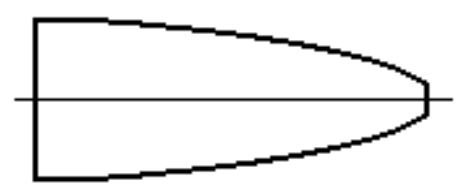

д

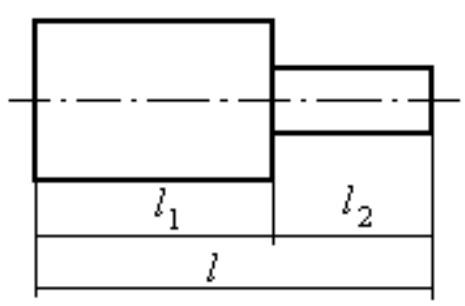

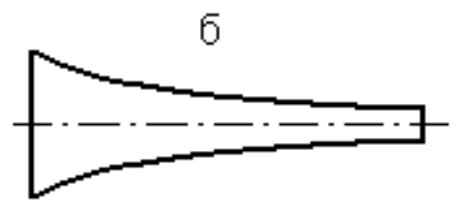

$\Gamma$

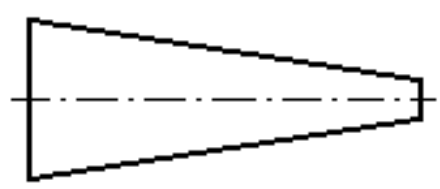

\section{Рис.5.6}

В широко применяемом экспоненциальном концентраторе (рис.5.6,a) площадь поперечного сечения изменяется по закону

$$
S_{x}=S_{0} \exp (-2 \gamma x / l)
$$

Форма катеноидального концентратора (рис.5.6,б) описывается уравнением

$$
S_{x}=S_{l} \operatorname{ch}^{2}[\gamma(x-l) / l]
$$

Можно построить косинусоидальный концентратор (рис.5.6,в) с поперечным сечением

$$
S_{x}=S_{0} \cos ^{2} \alpha x / l,(|\alpha|<\pi / 2)
$$

Сечение конического концентратора (рис.5.6,г) изменяется по закону

$$
S_{x}=S_{0}(1-\gamma x / 1)^{2} \quad(\gamma<1)
$$

При $\gamma=0$ формулы (5.51) - (5.54) описывают однородный волновод $S_{X}=S_{0}=$ const. 
На рис.5.6,д показан ступенчатый волновод, составленный из двух стержней с разной площадью поперечного сечения.

Отметим, что в [146] построена теория ультразвуковых концентраторов (5.51), (5.52), (5.54), исходя из анализа их свободных колебаний без учета потерь. Эта теория получила широкое практическое применение при расчете ультразвуковых систем различного технологического назначения [93,100,141-144,173,191]. В настоящем разделе результаты работы [146] будут получены как частный случай более общего подхода, при котором строятся динамические податливости неоднородных волноводов с учетом их диссипативных свойств. В дальнейшем найденные динамические податливости будут использованы при расчете концентраторов, работающих на нелинейную упругодиссипативную нагрузку, создаваемую технологическим процессом.

Для описания колебаний неоднородных волноводов (5.51)-(5.54) уравнение (5.6) с помощью подстановок

$$
S_{x}=r_{x}^{2}, \bar{a}_{x}=\bar{y}_{x} / r_{x}
$$

приводится к виду

$$
\bar{y}_{x}^{\prime \prime}+\bar{y}_{X}\left(\frac{\rho \cdot \omega^{2}}{\bar{E}}-\frac{r_{X}^{\prime \prime}}{r_{x}}\right)=0
$$

Его решение должно удовлетворять граничным условиям (5.7), в которых следует положить

$$
\bar{a}_{x}^{\prime}=\left(\bar{y}_{x} / r_{x}\right)^{\prime}=\left(y_{x}^{\prime} r_{x}-y_{x} r_{x}^{\prime}\right) / r_{x}^{2}
$$

Для экспоненциального волновода (5.51) уравнение (5.56) и граничные условия (5.7) принимают вид

$$
\begin{gathered}
\bar{y}_{x}^{\prime \prime}+\bar{y}_{x}\left[\frac{\rho \cdot \omega^{2}}{\bar{E}}-\left(\frac{\gamma}{l}\right)^{2}\right]=0 \\
\left(y_{x}^{\prime}+\frac{\gamma}{l} y_{x}\right) /\left.r_{x}\right|_{x=l-s}=0, \bar{E} S_{s}\left(y_{x}^{\prime}+\frac{\gamma}{l} y_{x}\right) /\left.r_{x}\right|_{x=s}=(-1)^{(1-s / l)} F_{s}
\end{gathered}
$$


Отыскивая решение уравнения (5.58), удовлетворяющее граничным условиям (5.59), и учитывая выражения (5.55) и (4.14), найдем величину динамической податливости экспоненциального волновода

$$
L_{s x}(j \omega)=(-1)^{s / l} \frac{\gamma \cdot\left(e^{\lambda \cdot x}-e^{-\lambda \cdot x} e^{2 \lambda(l-s)}\right)+\lambda \cdot l\left(e^{\lambda \cdot x}+e^{-\lambda \cdot x} e^{2 \lambda(l-s)}\right)}{\omega^{2} \rho \cdot S_{0} l\left(e^{\lambda \cdot s}-e^{-\lambda \cdot s} e^{2 \lambda(l-s)}\right)} e^{\gamma(x+s)}
$$

где $\lambda=j \sqrt{\frac{\rho \cdot \omega^{2}}{\bar{E}}-\left(\frac{\gamma}{l}\right)^{2}}$.

При $s=l$ и $s=0$ из (5.60) получаем

$$
\begin{gathered}
L_{l x}(j \omega)=\frac{\gamma \operatorname{sh} \lambda x-\lambda l \operatorname{ch} \lambda x}{\omega^{2} \rho S_{0} l \operatorname{sh} \lambda} e^{\gamma\left(1+\frac{x}{l}\right)} \\
L_{0 x}(j \omega)=\frac{\gamma \operatorname{sh} \lambda(x-l)-\lambda l \operatorname{ch} \lambda(x-l)}{\omega^{2} \rho S_{0} \operatorname{lsh} \lambda l} e^{\gamma \frac{x}{l}}
\end{gathered}
$$

Полагая в (5.3), (5.60) коэффициент поглощения $\psi$ малым, приведем выражения (5.61), (5.62) к виду (5.34), ограничивая разложение величин, содержащих малый параметр, линейными членами.

$$
L_{s x}(j \omega)=\mathrm{P}_{s x}(j \omega) / Q(j \omega), \quad(s=l, 0)
$$

где

$$
\begin{gathered}
Q(j \omega)=\frac{E S_{0}}{l}\left(\xi^{2}+\gamma^{2}\right) e^{-\gamma}\left[\sin \xi-j \frac{\psi}{4 \pi} \cdot \frac{\left(\xi^{2}+\gamma^{2}\right)}{\xi} \cos \xi\right] \\
\mathrm{P}_{l x}(j \omega)=e^{\gamma \cdot x / l}\left\{\begin{array}{l}
\gamma \cdot \sin \xi \frac{x}{l}-\xi \cos \xi \frac{x}{l}+ \\
+j \frac{\psi}{4 \pi} \cdot \frac{\left(\xi^{2}+\gamma^{2}\right)}{\xi}\left[\left(1-\gamma \frac{x}{l}\right) \cos \xi \frac{x}{l}-\xi \frac{x}{l} \sin \xi \frac{x}{l}\right] \\
\mathrm{P}_{0 x}(j \omega)=\mathrm{P}_{l(x-l)}(j \omega)
\end{array}\right.
\end{gathered}
$$

Здесь $\xi=\frac{\omega \cdot l}{c_{\Phi}}$,

$$
c_{\Phi}=\frac{c}{\sqrt{1-\left(\frac{\gamma \cdot c}{l \omega}\right)^{2}}}=c \frac{\sqrt{\xi^{2}+\gamma^{2}}}{\xi}
$$

- фазовая скорость звука в экспоненциальном волноводе. 
Отметим, что равенство (5.66) следует непосредственно из сравнения выражений (5.61) и (5.62), которое показывает, что последнее из них получается из первого заменой $x$ на $x-l$, т.е. $L_{0 x}(j \omega)=L_{l(x-l)}(j \omega)$.

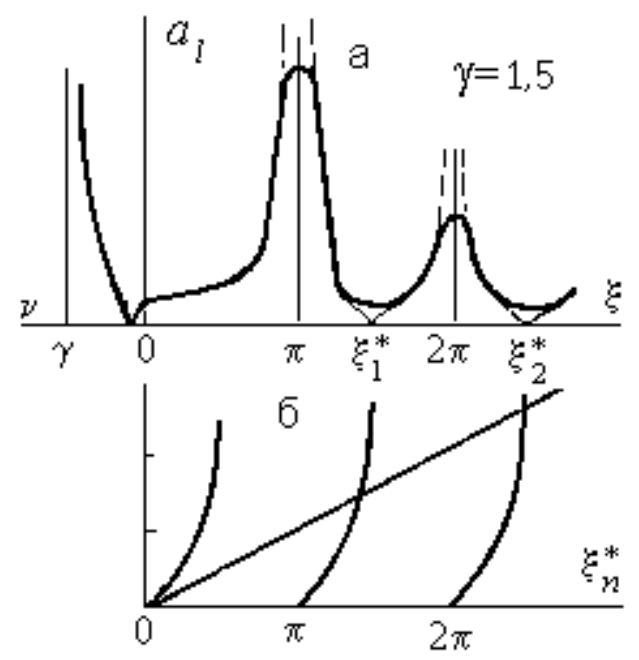

Pис.5.7

На рис.5.7,a с помощью выражений (5.63)-(5.65) построены амплитудно-частотные характеристики концевого сечения $x=l$ экспоненциального волновода при возбуждении его гармонической силой $f_{l}=F_{l} e^{j \omega \cdot t}$. Согласно (5.35) и (5.64) находим резонансные частоты стержня

$$
\xi_{n}=\pi \cdot n,(n=1,2, \ldots)
$$

На этих частотах достигаются максимальные амплитуды, определяемые из (5.39) с учетом (5.64), (5.65), (5.68), (5.51)

$$
a_{l}^{0}=\frac{4 l F_{l}}{\psi \cdot \pi \cdot E S_{l} n^{2}\left[1+(\gamma / \pi \cdot n)^{2}\right]^{2}}
$$

Антирезонансные частоты находятся из условия (5.36) при $x=s=l$, которое после подстановки (5.65) приводит к уравнению

$$
\operatorname{tg} \xi_{n}^{*}=\xi_{n}^{*} / \gamma
$$

Пример графического решения уравнения (5.70) показан на рис.5.7,б. Антирезонансные амплитуды вычисляются с помощью следующего выражения, полученного из (5.40), (5.64), (5.65), (5.70)

$$
a_{l}^{*}=\frac{\psi}{4 \pi} \cdot \frac{F_{l} l}{E S_{l}}\left(1-\frac{\gamma(1-\gamma)}{\xi_{n}^{2}}\right)
$$


Обратим внимание, что частота $\omega$ связана с безразмерной величиной $\xi$ соотношением

$$
\omega=\frac{c}{l} \sqrt{\xi^{2}+\gamma^{2}} .
$$

Полученные соотношения справедливы для значений $\xi>0$ и частот

$$
\omega \geq \omega_{k}=|\gamma| \cdot c / l,
$$

при которых фазовая скорость (5.67) является вещественной величиной. В диапазоне частот, лежащем ниже критического значения $\omega_{k}$, установившееся движение экспоненциального стержня перестает носить волновой характер. Однако это движение по-прежнему может быть описано уравнениями (5.63) - (5.67), (5.72), в которых следует положить

$$
\xi=j v,
$$

где $v \leq|\gamma|$ - действительное число.

Полагая $\psi=0$, из (5.63)-(5.65) получаем для частот $\omega<\omega_{k}$

$$
L_{l x}(j \omega)=-\frac{l e^{\gamma\left(1+\frac{x}{l}\right)}}{E S_{0}\left(\gamma^{2}-v^{2}\right)} \cdot \frac{v \cdot \operatorname{ch} v \frac{x}{l}-\gamma \cdot \operatorname{sh} v \frac{x}{l}}{\operatorname{sh} v}
$$

Ha рис.5.7,a амплитудно-частотная характеристика экспоненциального стержня достроена для частот $0<\omega<\omega_{k}$, которым отвечают значения $|\gamma| \geq v \geq 0$. Для амплитуды колебаний $a_{l}$ на критической частоте $\omega=\omega_{k}$ с помощью (5.63) - (5.65) при $\xi \rightarrow 0$ и (5.75) при $v \rightarrow 0$ получаем

$$
a_{l}=\frac{F_{l} l}{E S_{l}} \cdot \frac{(1+\gamma)}{\gamma^{2}}
$$

Интересно отметить, что амплитуда (5.76) не зависит от диссипации энергии в материале стержня.

Если экспоненциальный стержень используется в качестве концентратора, присоединяемого к возбудителю колебаний с частотой $\omega$, его параметры согласно (5.47) должны удовлетворять равенству (5.68). С 
учетом принятых обозначений (5.67) находим длину концентратора, настроенного на рабочую частоту

$$
l=\frac{\xi_{n} c_{\mathrm{TM}}}{\omega}=\frac{c}{\omega} \sqrt{(\pi \cdot n)^{2}+\gamma^{2}}
$$

Коэффициент усиления (5.48), (5.49) экспоненциального концентратора после подстановки соответствующих величин из (5.64), (5.66) вычисляется по формуле

$$
K=e^{\gamma}=\sqrt{S_{0} / S_{l}}
$$

Равенства (5.77), (5.78) совпадают с полученными в [146,191] иным путем. Они позволяют подобрать геометрические размеры концентратора, обеспечивающего заданное увеличение амплитуды колебаний.

Исследование колебаний неоднородных волноводов других типов проводится аналогично. Опуская подробности, приведем лишь основные результаты их анализа.

7. Для катеноидального стержня (5.52) уравнение (5.56) приводится к виду (5.58). Его решение, удовлетворяющее граничным условиям, получаемым из (5.7) с учетом (5.57), позволяет найти величины динамических податливостей

$$
\begin{aligned}
& L_{l x}(j \omega)=\frac{\lambda \cdot l \operatorname{ch} \lambda x-\gamma \cdot \operatorname{th} \gamma \cdot \operatorname{sh} \lambda x}{\lambda \cdot \bar{E} S_{l} \operatorname{ch} \gamma \cdot\left(1-\frac{x}{l}\right)(\lambda \cdot \operatorname{lsh} \lambda l-\gamma \cdot \operatorname{th} \gamma \cdot \operatorname{ch} \lambda l)} \\
& L_{0 x}(j \omega)=\frac{l \operatorname{ch} \lambda(l-x)}{\bar{E} S_{l} \operatorname{ch} \gamma\left(1-\frac{x}{l}\right)(\lambda \cdot l \operatorname{sh} \lambda l-\gamma \cdot \operatorname{th} \gamma \cdot \operatorname{ch} \lambda l) \operatorname{ch} \gamma}
\end{aligned}
$$

где $\lambda$ определяется равенством (5.60).

После приведения выражений (5.79), (5.80) к виду (5.34) получим

$$
Q(j \omega)=-\frac{\xi \cdot E S_{l}}{l}\left\{\begin{array}{l}
\xi \cdot \sin \xi+\gamma \cdot \operatorname{th} \gamma \cdot \cos \xi-j \frac{\psi}{4 \pi \cdot \xi^{2}} \cdot \times \\
\left.\cdot \times\left[\begin{array}{l}
\xi \cdot\left(\xi^{2}+\gamma^{2}\right)((1-\gamma \cdot \operatorname{th} \gamma) \sin \xi+\xi \cdot \cos \xi)- \\
-\left(\xi^{2}-\gamma^{2}\right)(\xi \cdot \sin \xi+\gamma \cdot \operatorname{th} \gamma \cdot \cos \xi)
\end{array}\right]\right\}
\end{array}\right.
$$




$$
\begin{aligned}
& \mathrm{P}_{l x}(j \omega)=\left\{\begin{array}{l}
\xi \cos \xi \frac{x}{l}-\gamma \mathrm{th} \gamma \cdot \sin \xi \frac{x}{l}-j \frac{\psi\left(\xi^{2}+\gamma^{2}\right)}{4 \pi \cdot \xi} \times \\
\times\left[(1-\gamma \cdot \operatorname{th} \gamma) \cos \xi \frac{x}{l}-\xi \frac{x}{l} \sin \xi \frac{x}{l}\right]
\end{array}\right\} / \operatorname{ch} \gamma\left(1-\frac{x}{l}\right) \\
& \mathrm{P}_{0 x}(j \omega)=\left[\begin{array}{l}
\xi \cdot \cos \xi \frac{l-x}{l}-j \frac{\psi\left(\xi^{2}+\gamma^{2}\right)}{4 \pi \cdot \xi}\left(\begin{array}{c}
\cos \xi \frac{l-x}{l}- \\
\left.-\xi \frac{l-x}{l} \sin \xi \frac{l-x}{l}\right)
\end{array}\right] / \operatorname{ch} \gamma \cdot \operatorname{ch} \gamma\left(1-\frac{x}{l}\right),
\end{array}\right.
\end{aligned}
$$

где $\xi$ определяется равенствами (5.67).

На рис.5.8,a приведена амплитудно-частотная характеристика. Резонансные и антирезонансные частоты катеноидального стержня при действии на него гармонического возбуждения в сечении $x=l$ находятся решением уравнений

$$
\begin{gathered}
\operatorname{ctg} \xi_{n}=-\xi_{n} / \gamma \operatorname{th} \gamma \\
\operatorname{tg} \xi_{n}^{*}=\xi_{n}^{*} / \gamma \operatorname{th} \gamma
\end{gathered}
$$

которые получены из условий (5.35), (5.36) после подстановки соответствующих величин из (5.81), (5.82).
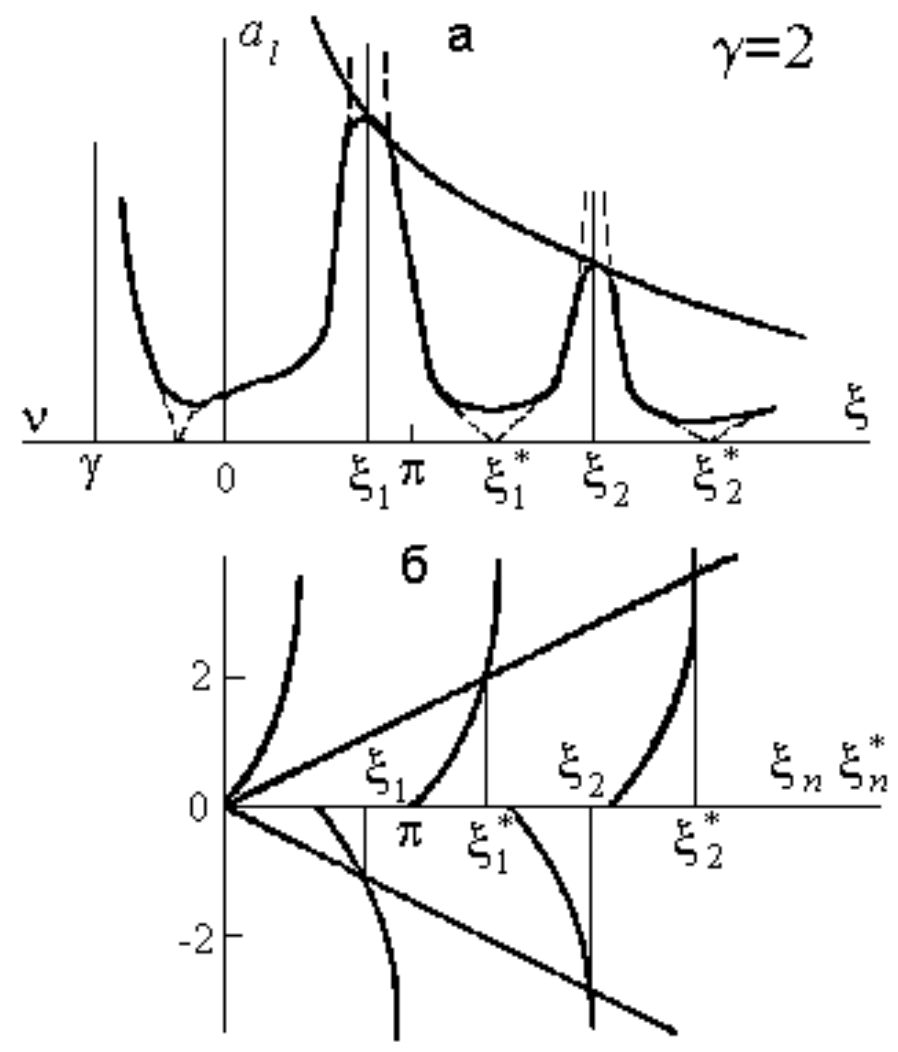

Pис 5.8 
Графическое решение уравнений (5.84), (5.85) показано на рис.5.8,б. Достигаемые при этом амплитуды колебаний определяются с помощью равенств (5.39), (5.40), которые с учетом (5.81), (5.82), (5.84), (5.85) приводятся к виду

$$
\begin{aligned}
& a_{l}^{0}=\frac{4 \pi \cdot F_{l} l\left(\xi_{n}^{2}+\gamma^{2} \mathrm{th}^{2} \gamma\right)}{\psi \cdot E S_{l}\left(\xi_{n}^{2}+\gamma^{2}\right)\left(\xi_{n}^{2}+\gamma^{2} \mathrm{th}^{2} \gamma-\gamma \cdot \operatorname{th} \gamma\right)} \\
& a_{l}^{*}=\frac{\psi}{4 \pi} \cdot \frac{F_{l} l}{E S_{l}} \cdot \frac{\left(\xi_{n}^{* 2}+\gamma^{2}\right)\left(\xi_{n}^{* 2}+\gamma^{2} \mathrm{th}^{2} \gamma-\gamma \cdot \operatorname{th} \gamma\right)}{\xi_{n}^{* 2}\left(\xi_{n}^{* 2}+\gamma^{2} \mathrm{th}^{2} \gamma\right)}
\end{aligned}
$$

На рис.5.8,a по уравнению (5.86) построена огибающая кривая, на которой лежат максимальные амплитуды резонансных кривых. При использовании катеноидального стержня в качестве концентратора, настроенного на частоту $\omega$, его длина по аналогии с (5.77) выбирается по формуле

$$
l=\frac{c}{\omega} \sqrt{\xi_{n}^{2}+\gamma^{2}},
$$

а коэффициент усиления (5.48), (5.49)

$$
K=\frac{\operatorname{ch} \gamma}{\left|\cos \xi_{n}\right|}=\sqrt{\frac{S_{0}}{S_{l}}} \cdot \frac{1}{\left|\cos \xi_{n}\right|}
$$

где $\xi_{n}$ - решение уравнения (5.84). Соотношения (5.84), (5.87), (5.88) совпадают с полученными в [146].

8. Для косинусоидального волновода (5.53) уравнение (5.56) также приводится к виду (5.58), если положить

$$
\gamma=j \alpha
$$

В этом случае величины динамических податливостей можно найти из выражений (5.79), (5.80), в которых нужно поменять местами индексы $l$ и 0 и заменить $x$ на $l-x$. В результате имеем

$$
L_{l x}(j \omega)=\frac{l \operatorname{ch} \lambda x}{\bar{E} S_{0}(\lambda l \operatorname{sh} \lambda l+\alpha \cdot \operatorname{tg} \alpha \cdot \operatorname{ch} \lambda l) \cos \alpha \cdot \cos \alpha \frac{x}{l}}
$$




$$
L_{0 x}(j \omega)=\frac{\lambda \cdot l \operatorname{ch} \lambda(l-x)+\alpha \cdot \operatorname{tg} \alpha \cdot \operatorname{sh} \lambda(l-x)}{\lambda \cdot \bar{E} S_{0}(\lambda l \operatorname{sh} \lambda l+\alpha \cdot \operatorname{tg} \alpha \cdot \operatorname{ch} \lambda l) \cos \alpha \frac{x}{l}}
$$

Аналогично с помощью (5.81) - (5.83) записываются составляющие динамических податливостей в форме (5.34). С учетом обозначения (5.89) для косинусоидального волновода оказываются справедливыми соотношения (5.84) - (5.87). Эти выражения здесь не приводятся.

Коэффициент усиления и длина косинусоидального концентратора (5.53) рассчитываются по формулам

$$
K=\frac{1}{\cos \alpha \cdot\left|\cos \xi_{n}\right|}=\sqrt{\frac{S_{0}}{S_{l}}} \cdot \frac{1}{\left|\cos \xi_{n}\right|}, l=\frac{c}{\omega} \sqrt{\xi_{n}^{2}-\alpha^{2}}
$$

где $\xi_{n}$ - решение уравнения

$$
\operatorname{ctg} \xi_{n}=\xi_{n} / \alpha \operatorname{tg} \alpha
$$

9. Для конического стержня (5.54) уравнение (5.56) принимает вид $\bar{y}_{x}^{\prime \prime}+\frac{\rho \cdot \omega^{2}}{\bar{E}} \bar{y}_{x}=0$. Его решение, удовлетворяющее граничным условиям, полученным из (5.7) с учетом (5.57), позволяет найти динамические податливости

$$
\begin{aligned}
& L_{l x}(j \omega)=\frac{\lambda^{2} l(\gamma \cdot \operatorname{sh} \lambda x-\lambda \cdot l \operatorname{ch} \lambda x)}{\omega^{2} \rho \cdot S_{0}\left(1-\gamma \frac{x}{l}\right)\left\{\gamma^{2} \lambda l \operatorname{ch} \lambda l+\left[(\lambda l)^{2}(1-\gamma)-\gamma^{2}\right] \operatorname{sh} \lambda l\right\}} \\
& L_{0 x}(j \omega)=-\frac{\lambda^{2} l[\gamma \cdot \operatorname{sh} \lambda(l-x)+\lambda l(1-\gamma) \operatorname{ch} \lambda(l-x)]}{\omega^{2} \rho S_{0}\left(1-\gamma \frac{x}{l}\right)\left\{\gamma^{2} \lambda l \operatorname{ch} \lambda l+\left[(\lambda l)^{2}(1-\gamma)-\gamma^{2}\right] \operatorname{sh} \lambda l\right\}}
\end{aligned}
$$

где $\lambda=j \omega / \sqrt{\rho / \bar{E}}=j \omega / c \sqrt{1+j \psi / 2 \pi}$.

Приводя выражения (5.94) к виду (5.34), получим

$$
\left.Q(j \omega)=\frac{E S_{0}}{l}\left\{\begin{array}{l}
{\left[\xi^{2}(1-\gamma)+\gamma^{2}\right] \sin \xi-\gamma^{2} \xi \cdot \cos \xi-} \\
-j \frac{\psi}{4 \pi}\left[\xi\left(\xi^{2}(1-\gamma)+2 \gamma^{2}\right) \cos \xi+\gamma^{2}\left(\xi^{2}-2\right) \sin \xi\right.
\end{array}\right]\right\}
$$




$$
\begin{gathered}
\mathrm{P}_{l x}(j \omega)=\left\{\begin{array}{c}
\left.\gamma \cdot \sin \xi \frac{x}{l}-\xi \cdot \cos \xi \frac{x}{l}+j \frac{\psi \cdot \xi}{4 \pi}\left[\begin{array}{c}
\left(1-\gamma \frac{x}{l}\right) \cos \xi \frac{x}{l}- \\
-\xi \frac{x}{l} \sin \xi \frac{x}{l}
\end{array}\right]\right\} /\left(1-\gamma \frac{x}{l}\right) \\
\mathrm{P}_{0 x}(j \omega)=-\left\{\begin{array}{l}
\gamma \cdot \sin \xi \frac{l-x}{l}+\xi \cdot(1-\gamma) \cos \xi \frac{l-x}{l}- \\
-j \frac{\psi \cdot \xi}{4 \pi}\left[\left(1-\gamma \frac{x}{l}\right) \cos \xi \frac{l-x}{l}-(1-\gamma) \xi \frac{l-x}{l} \sin \xi \frac{l-x}{l}\right]
\end{array}\right] /\left(1-\gamma \frac{x}{l}\right)
\end{array}\right.
\end{gathered}
$$

где $\xi=\omega \cdot l / c$.

Резонансные $\xi_{n}$ и антирезонансные $\xi_{n}^{*}$ частоты находятся решением уравнений

$$
\begin{gathered}
\operatorname{tg} \xi_{n}=\frac{\gamma^{2} \xi_{n}}{\xi_{n}{ }^{2}(1-\gamma)+\gamma^{2}} \\
\operatorname{tg} \xi_{n}^{*}=\xi_{n}^{*} / \gamma
\end{gathered}
$$

Соответствующие этим частотам амплитуды

$$
\begin{gathered}
a_{l}^{0}=\frac{4 \pi \cdot F_{l} l\left(\xi_{n}^{2}+\gamma^{2}\right)}{\psi \cdot E S_{0} \xi_{n}^{2}\left[\xi_{n}^{2}(1-\gamma)^{2}+3 \gamma^{2}(1-\gamma)+\gamma^{4}\right]} \\
a_{l}^{*}=\frac{\psi}{4 \pi} \cdot \frac{F_{l} l}{E S_{0}} \cdot \frac{\left[\xi_{n}^{* 2}-\gamma \cdot(1-\gamma)\right]_{1}}{(1-\gamma)^{2}\left(\xi_{n}^{* 2}+\gamma^{2}\right)}
\end{gathered}
$$

При использовании конического стержня в качестве концентратора, настроенного на частоту $\omega_{n}$, его длина рассчитывается по формуле

$$
l=c \xi_{n} / \omega_{n},
$$

а коэффициент усиления (5.48), (5.49) с учетом (5.96) - (5.98) определяется выражениями

$$
K=\sqrt{\frac{S_{0}}{S_{l}}} \cdot\left|\frac{\xi_{n}(1-\gamma)}{\gamma \cdot \sin \xi_{n}+\xi_{n}(1-\gamma) \cos \xi_{n}}\right|
$$

или после преобразований с учетом (5.98)

$$
K=\sqrt{\frac{S_{0}}{S_{l}}}\left|\frac{\xi_{n} \cos \xi_{n}-\gamma \cdot \sin \xi_{n}}{\xi_{n}}\right|
$$

Соотношения (5.98), (5.102), (5.103) совпадают с полученными в [146] иным способом. В [191] выражение для коэффициента усиления 
приведено в форме (5.104). Учитывая (5.98) легко доказать тождественность равенств (5.103), (5.104).

10. В колебательных системах ультразвуковых технологических установок часто используются волноводы, составленные из участков с различными свойствами. Такие участки могут быть выполнены из различных материалов. Площадь поперечного сечения участков может изменяться по различным законам. Некоторые составные части могут быть представлены в виде сосредоточенных элементов и т.д. К таким системам относятся, в частности, разнообразные составные концентраторы [147,165,210], стержневые колебательные системы с присоединенными инструментами и прочие. При описании составной колебательной системы еe динамическую податливость целесообразно выразить через динамические податливости составляющих элементов.

Рассмотрим, например, волновод, составленный из двух ступеней длиной $l_{1}$ и $l_{2}$ (рис.5.9,a), причем $l_{1}+l_{2}=l$.
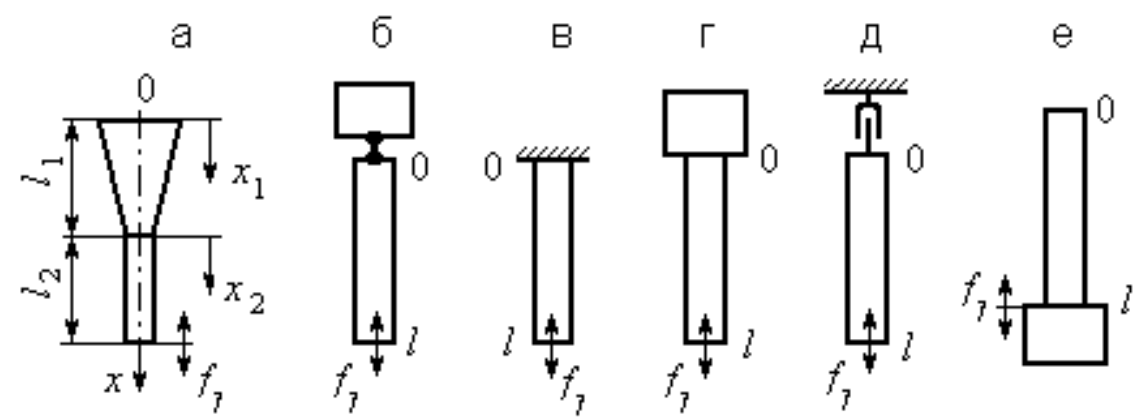

Рис.5. 9

Расчленяя волновод по месту стыка ступеней, представим взаимодействие частей реакцией $\mathrm{c}$ амплитудой $\bar{F}_{12}$. Введем новые координаты $x_{1}=x \quad\left(x_{1} \in\left[0, l_{1}\right]\right), x_{2}=x-l_{1}\left(x_{2} \in\left[0, l_{2}\right]\right)$ и запишем выражения для амплитуд смещений под действием приложенной к концу $x=l$ системы силы с амплитудой $\bar{F}_{l}$

$$
\begin{aligned}
& \bar{a}_{x_{1}}^{(1)}=\bar{F}_{12} L_{l_{1} x_{1}}^{(1)}(j \omega) \\
& \bar{a}_{x_{2}}^{(2)}=\bar{F}_{l} L_{l_{2} x_{2}}^{(2)}(j \omega)-\bar{F}_{12} L_{0 x_{2}}^{(2)}(j \omega)
\end{aligned}
$$

где верхний индекс в скобках обозначает номер подсистемы, к которой относится данная величина. 
Равенство $a_{l_{1}}^{(1)}=a_{0}^{(2)}$, выражающее условия неразрывности системы в сечении $x=l_{1}$, позволяет найти амплитуду реакции

$$
\bar{F}_{12}=\bar{F}_{l} L_{l_{2} 0}^{(2)}(j \omega) /\left[L_{l_{1} l_{1}}^{(1)}(j \omega)+L_{00}^{(2)}(j \omega)\right]
$$

После подстановки (5.106) в (5.105) с учетом (4.14) найдем динамическую податливость составной системы

$$
L_{l x}(j \omega)=\left\{\begin{array}{cc}
\frac{L_{l_{1} X_{1}}^{(1)}(j \omega) \cdot L_{l_{2} 0}^{(2)}(j \omega)}{L_{l_{1} l_{1}}^{(1)}(j \omega)+L_{00}^{(2)}(j \omega)} & 0 \leq x_{1}=x \leq l_{1} \\
L_{l_{2} X_{2}}^{(2)}(j \omega)-\frac{L_{0 x_{2}}^{(2)}(j \omega) \cdot L_{l_{2} 0}^{(2)}(j \omega)}{L_{l_{1} l_{1}}^{(1)}(j \omega)+L_{00}^{(2)}(j \omega)} & 0 \leq x_{2}=x-l_{1} \leq l_{2}
\end{array}\right.
$$

При $x_{2}=l_{2}$ выражение (5.107) с точностью до обозначений совпадает c (4.25). Аналогично отыскивается динамическая податливость составной системы при возбуждении в сечении $x=0$

$$
L_{0 x}(j \omega)=\left\{\begin{array}{cc}
L_{0 x_{1}}^{(1)}(j \omega)-\frac{L_{l_{1} x_{1}}^{(1)}(j \omega) \cdot L_{0 l_{1}}^{(1)}(j \omega)}{L_{l_{1} l_{1}}^{(1)}(j \omega)+L_{00}^{(2)}(j \omega)} & 0 \leq x_{1}=x \leq l_{1} \\
\frac{L_{0 x_{2}}^{(2)}(j \omega) \cdot L_{0 l_{1}}^{(1)}(j \omega)}{L_{l_{1} l_{1}}^{(1)}(j \omega)+L_{00}^{(2)}(j \omega)} & 0 \leq x_{2}=x-l_{1} \leq l_{2}
\end{array}\right.
$$

Рассмотрим некоторые примеры применения выведенных соотношений.

Найдем динамическую податливость однородного стержня (рис.5.9,б), к концу $x=0$ которого присоединена сосредоточенная нагрузка, характеризуемая динамической податливостью $L(j \omega)$. Подставляя в (5.107) соответствующие величины из (5.10), (5.11), получим

$$
L_{l x}(j \omega)=-\frac{\lambda}{\rho \cdot S \omega^{2}} \cdot \frac{\rho \cdot S \omega^{2} \operatorname{ch} \lambda x-\lambda \cdot L^{-1}(j \omega) \operatorname{sh} \lambda x}{\rho \cdot S \omega^{2} \operatorname{sh} \lambda l-\lambda \cdot L^{-1}(j \omega) \operatorname{ch} \lambda l}
$$

Переходя в (5.109) к пределу $\left|L^{-1}(j \omega)\right| \rightarrow \infty$, найдем динамическую податливость стержня с неподвижно закрепленным концом (рис.5.9,в)

$$
L_{l x}(j \omega)=-\frac{\lambda}{\rho \cdot S \omega^{2}} \cdot \frac{\operatorname{sh} \lambda x}{\operatorname{ch} \lambda l}
$$


Приводя выражение (5.110) с учетом (5.9) к виду (5.34), имеем

$$
L_{l x}(j \omega)=\frac{l}{E S \xi} \cdot \frac{\sin \xi \frac{x}{l}-j \frac{\psi}{4 \pi}\left(\sin \xi \frac{x}{l}+\xi \frac{x}{l} \cos \xi \frac{x}{l}\right)}{\cos \xi+j \frac{\psi}{4 \pi} \xi \sin \xi},
$$

где $\xi=\frac{\omega \cdot l}{c}$.

Отсюда с помощью (5.35), (5.39) находим резонансные частоты стержня

$$
\xi_{n}=(2 n-1) \cdot \pi / 2,
$$

где $n=1,2 \ldots$ - номер собственной формы, и амплитуды, например, сечения $x=l$.

$$
a_{l}^{0}=\frac{16 F_{l} l}{\pi \cdot \psi \cdot E S(2 n-1)^{2}}
$$

Если длина закрепленного стержня настолько мала, что $\xi<<1$, т.е. выполняется условие (5.13), то (5.111) при $x=l$ можно приближенно записать в виде

$$
L_{l l}(j \omega)=L(j \omega)=\frac{l}{\widetilde{E} S}=\frac{l}{E S(1+j \psi / 2 \pi)}=\frac{1}{k_{0}+j \omega \cdot b_{0}}
$$

где $k_{0}=E S / l \quad$ - статическая жесткость стержня; $b_{0}=\psi k_{0} / 2 \pi \omega \quad$ эквивалентный коэффициент сопротивления.

Равенство (5.114) представляет собой динамическую податливость сосредоточенного упруговязкого элемента [86, с.134].

Выражения (5.107), (5.108) позволяют учитывать все виды граничных условий на концах системы. Так, при нагружении стержня инерционной нагрузкой (рис.5.7,г) выражение (5.109) с учетом (5.14) приводится к следующему:

$$
L_{l x}(j \omega)=-\frac{\lambda}{\rho \cdot S \omega^{2}} \cdot \frac{\operatorname{ch} \lambda x+\mu \lambda l \operatorname{sh} \lambda x}{\operatorname{sh} \lambda l+\mu \lambda l \operatorname{ch} \lambda l}
$$

где $\mu=M / \rho \cdot S l ; M$ - масса присоединенного тела. 
Оценим влияние линейного вязкого сопротивления с податливостью $L(j \omega)=\left(j \omega \cdot b_{0}\right)^{-1}$ на характеристики стержня (рис.5.9,д), пренебрегая потерями энергии в его материале $(\psi=0)$. Для этого случая из $(5.109),(5.9)$ получим

$$
L_{l x}(j \omega)=-\frac{l}{E S \xi} \cdot \frac{\cos \xi \frac{x}{l}+j \frac{b_{0}}{w} \sin \xi \frac{x}{l}}{\sin \xi-j \frac{b_{0}}{w} \cos \xi}
$$

где $w=\rho \cdot c \cdot S$ - волновое сопротивление стержня.

Представляет интерес практически важный случай равенства сопротивления нагрузки $b_{0}$ и волнового сопротивления $w$. Учитывая принятые обозначения, из (5.116) имеем

$$
L_{l x}(j \omega)=\frac{1}{j \omega \cdot w} \cdot \frac{\cos \frac{\omega}{c} x+j \sin \frac{\omega}{c} x}{\cos \frac{\omega}{c} l+j \sin \frac{\omega}{c} l}=\frac{1}{j \omega \cdot w} e^{j \frac{\omega}{c}(x-l)}
$$

Из сравнения с (5.15) видно, что выражение (5.117) описывает форму бегущей гармонической волны, распространяющейся вдоль стержня от источника возбуждения. Это означает, что при такой нагрузке не происходит отражения волны от границы $x=0$, и ограниченный стержень находится в режиме бегущей волны [107,191].

11. Рассмотрим, наконец, систему, составленную из двух однородных стержней площадью $S_{1}$ и $S_{2}$ и длиной $l_{1}$ и $l_{2}$ (рис.5.6,д). Подставляя в (5.107) соответствующие величины из (5.10), (5.11) и полагая, что стержни выполнены из одинакового материала, получим

$$
L_{l x}(j \omega)= \begin{cases}-\frac{\lambda}{\rho \cdot \omega^{2}} \cdot \frac{\operatorname{ch} \lambda x}{S_{1} \operatorname{sh} \lambda l_{1} \operatorname{ch} \lambda l_{2}+S_{2} \operatorname{sh} \lambda l_{2} \operatorname{ch} \lambda l_{1}} & 0 \leq x \leq l_{1} \\ -\frac{\lambda}{S_{2} \rho \cdot \omega^{2}} \cdot \frac{S_{1} \operatorname{sh} \lambda\left(x-l_{1}\right) \operatorname{sh} \lambda l_{1}+S_{2} \operatorname{ch} \lambda\left(x-l_{1}\right) \operatorname{ch} \lambda l_{1}}{S_{1} \operatorname{sh} \lambda l_{1} \operatorname{ch} \lambda l_{2}+S_{2} \operatorname{sh} \lambda l_{2} \operatorname{ch} \lambda l_{1}} & l_{1} \leq x \leq l\end{cases}
$$

где $l=l_{1}+l_{2}$. 
Рассмотрим некоторые частные случаи, следующие из формулы (5.118). При $S_{1} \rightarrow \infty$ получаем динамическую податливость стержня с закрепленным концом (5.110). Пусть длина одного из стержней настолько мала, что $\left|\lambda \cdot l_{i}\right|<<1(i=1,2)$. При $i=1$, полагая $\rho S_{1} l_{1}=M, l_{2}=l$, приходим к формуле (5.115), определяющей динамическую податливость стержня с сосредоточенной массой на конце $x=0$ (рис.5.9,г). При $i=2$ аналогично найдем динамическую податливость стержня с массой в точке возбуждения (рис.5.9,e)

$$
L_{l x}(j \omega)=-\frac{\lambda}{\rho \omega^{2} S} \cdot \frac{\operatorname{ch} \lambda x}{\operatorname{sh} \lambda l+\mu \lambda l \operatorname{ch} \lambda l},
$$

где $\mu=M / \rho S l$.

Используя (5.118), проведем расчет ступенчатого концентратора [147, 191], широко применяемого в ультразвуковой технологической аппаратуре. С этой целью, пренебрегая потерями в материале концентратора, т.е. полагая $\psi=0$, и учитывая (5.9), запишем (5.118) в виде $L_{l x}(j \omega)= \begin{cases}-\frac{l}{E \xi} \cdot \frac{\cos \xi x / l}{S_{1} \sin \xi_{1} \cos \xi_{2}+S_{2} \sin \xi_{2} \cos \xi_{1}} & 0 \leq x \leq l_{1} \\ \frac{l}{E S_{2} \xi} \cdot \frac{S_{1} \sin \left(\xi x / l-\xi_{1}\right) \sin \xi_{1}-S_{2} \cos \left(\xi x / l-\xi_{1}\right) \cos \xi_{1}}{S_{1} \sin \xi_{1} \cos \xi_{2}+S_{2} \sin \xi_{2} \cos \xi_{1}} & l_{1} \leq x \leq l\end{cases}$

где $\xi=\omega \cdot l / c, \xi_{i}=\omega \cdot l_{i} / c=\xi \frac{l_{i}}{l},(i=1,2)$.

Из формул (5.34), (5.35), (5.120) получаем уравнение, определяющее собственные частоты ступенчатого концентратора

$$
\left(S_{1} / S_{2}\right) \operatorname{tg} \xi_{1}=-\operatorname{tg} \xi_{2},
$$

совпадающее с известным $[147,188]$.

Выражение для коэффициента усиления (5.49) принимает вид

$$
K=\left|\cos \xi_{1} \cos \xi_{2}-\left(S_{1} / S_{2}\right) \sin \xi_{1} \sin \xi_{2}\right|
$$

С учетом (5.121) выражение (5.122) приводится к известным [147, 191]

$$
K=\left|\frac{\cos \xi_{1}}{\cos \xi_{2}}\right|=\frac{S_{1}}{S_{2}}\left|\frac{\sin \xi_{1}}{\sin \xi_{2}}\right|=\frac{S_{1}}{S_{2}} \sqrt{\frac{1+\operatorname{tg}^{2} \xi_{2}}{\left(S_{1} / S_{2}\right)^{2}+\operatorname{tg}^{2} \xi_{2}}}
$$


Из (5.123) сразу следует, что максимальным коэффициентом усиления

$$
K=S_{1} / S_{2}
$$

обладают ступенчатые концентраторы при $\xi_{2}=(2 k+1) \pi / 2, \xi_{1}=(2 n+1) \pi / 2$, $(k, n=0,1,2, \ldots)$, т.е. концентраторы, имеющие длины ступеней

$$
l_{1}=(2 n+1) \pi c / 2 \omega, \quad l_{2}=(2 k+1) \pi c / 2 \omega
$$

Эти длины кратны нечетному числу четвертей длины волны $\Lambda=2 \pi c / \omega$ в стержне. В работе [75] показано, что такие концентраторы дают наибольшее усиление амплитуды среди всех известных типов концентраторов с одинаковым перепадом площадей поперечного сечения.

На рис.5.10 построены зависимости коэффициента усиления $K$ и длины $l$ от отношения площадей $S_{0} / S_{l}$ для различных типов концентраторов, показанных на рис.5.6.
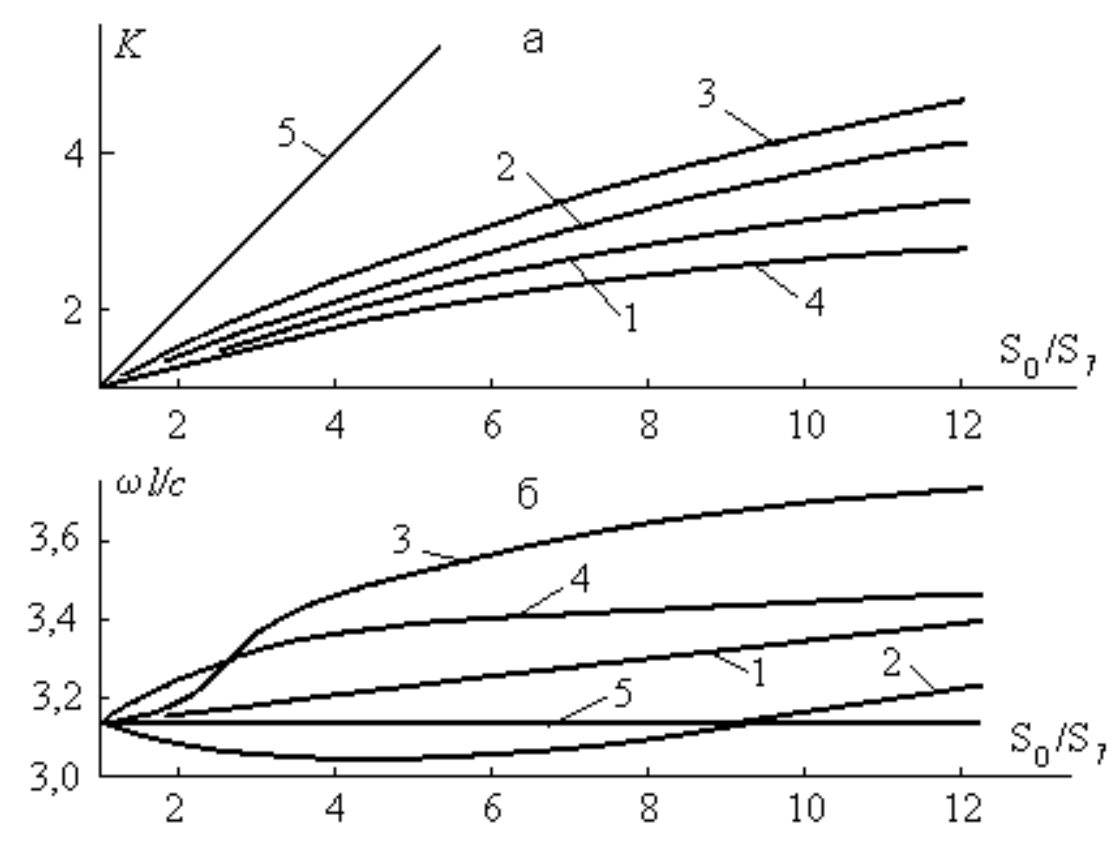

Pис. 5.10

Здесь кривые 1 относятся к экспоненциальному, 2 - к катеноидальному, 3 к косинусоидальному, 4 - к коническому, 5 - к ступенчатому (при $l_{1}=l_{2}$ ) концентраторам. 
12. При расчетах ультразвуковых колебательных систем часто встречаются случаи, когда действующие на систему силы приложены в некоторых промежуточных сечениях $x=s$ (рис.5.11,a).

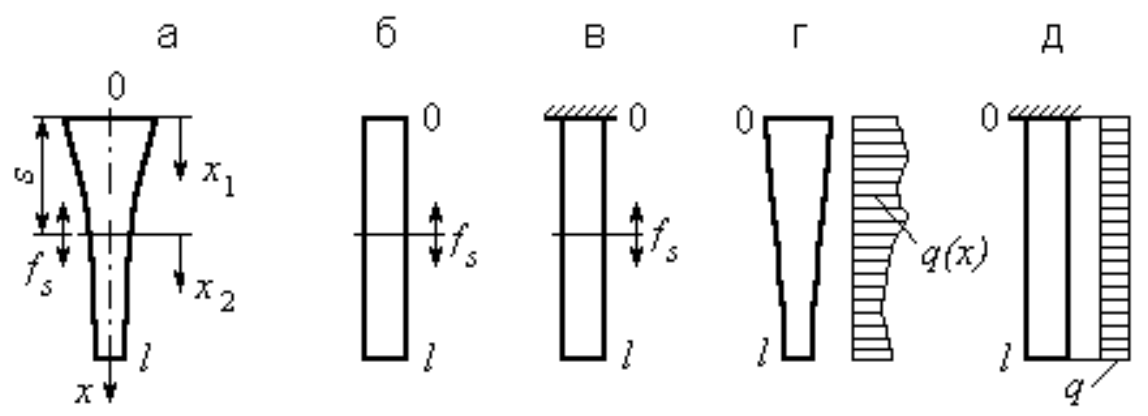

Pnc.5.11

Для описания такой системы расчленим ее по месту действия силы, относя силу к одной из частей и заменяя их взаимодействие реакцией с амплитудой $\bar{F}_{12}$.

По аналогии с (5.105), вводя координаты $x_{1}=x\left(x_{1} \in[0, s]\right), x_{2}=x-s$ $\left(x_{2} \in[0, l-s]\right)$, запишем

$$
\begin{aligned}
& \bar{a}_{x_{1}}^{(1)}=\left(\bar{F}_{s}+\bar{F}_{12}\right) L_{s x_{1}}^{(1)}(j \omega) \\
& \bar{a}_{x_{2}}^{(2)}=-\bar{F}_{12} L_{0 x_{2}}^{(2)}(j \omega) .
\end{aligned}
$$

Исключая с помощью очевидного равенства $\bar{a}_{s}^{(1)}=\bar{a}_{0}^{(2)}$ неизвестную реакцию $\bar{F}_{12}$ и обозначая $L_{s x}(j \omega)=\bar{a}_{x} / \bar{F}_{s}$, получим

$$
L_{s x}(j \omega)= \begin{cases}\frac{L_{s x_{1}}^{(1)}(j \omega) L_{00}^{(2)}(j \omega)}{L_{s S}^{(1)}(j \omega)+L_{00}^{(2)}(j \omega)} & 0 \leq x_{1}=x \leq s \\ \frac{L_{0 x_{2}}^{(2)}(j \omega) L_{s s}^{(1)}(j \omega)}{L_{s s}^{(1)}(j \omega)+L_{00}^{(2)}(j \omega)} & 0 \leq x_{2}=x-s \leq l-s\end{cases}
$$

Приведем некоторые примеры.

Найдем динамическую податливость однородного стержня со свободными концами (рис.5.11,б), возбуждаемого в сечении $x=s$. Подставив в (5.126) соответствующие величины из (5.10), (5.11), получим 


$$
L_{s x}(j \omega)= \begin{cases}-\frac{\lambda}{\rho \cdot S \omega^{2}} \cdot \frac{\operatorname{ch} \lambda x \operatorname{ch} \lambda(l-s)}{\operatorname{sh} \lambda l} & 0 \leq x \leq s \\ -\frac{\lambda}{\rho \cdot S \omega^{2}} \cdot \frac{\operatorname{ch} \lambda(l-x) \operatorname{ch} \lambda s}{\operatorname{sh} \lambda l} & s \leq x \leq l\end{cases}
$$

Из сравнения (5.127) при $x=l$ и (5.10) при $x=s$ следует равенство

$$
L_{s l}(j \omega)=L_{l s}(j \omega) \text {, }
$$

являющееся проявлением принципа взаимности.

Для амплитуд резонансных колебаний сечения $x=l$ стержня, возбуждаемого гармонической силой $f_{s}=F_{s} e^{j \omega \cdot t}$, приложенной в сечении $x=s$, с учетом $(5.20),(5.26),(5.128)$ имеем

$$
\bar{a}_{l}=\frac{4 F_{s} l}{\psi \pi E S n^{2}}\left[\cos \pi n \frac{s}{l}-j \frac{\psi}{4 \pi}\left(\cos \pi n \frac{s}{l}-\pi n \frac{s}{l} \sin \pi n \frac{s}{l}\right)\right]
$$

Если сила $f_{s}$ действует в одной из пучностей собственной формы (5.27), т.е. приложена к точке $s=l k / n$, где $k \leq n$ - целое число, выражение (5.126) приводит к формуле (5.30). При действии силы в одной из узловых точек $s=l(2 k+1) / 2 n$ собственной формы (5.27) из (5.129) получаем

$$
a_{l}=\frac{F_{s} l(2 k+1)}{2 \pi \cdot E S n^{2}}
$$

Амплитуда (5.130) на порядок меньше амплитуды (5.30). Поэтому при попадании возбуждающей силы в узловую точку формы колебаний соответствующий резонанс не проявляется и не обнаруживается экспериментально.

Аналогично из (5.126) с учетом (5.110), (5.111) найдем динамическую податливость стержня с закрепленным концом (рис.5.11,в) при его возбуждении в сечении $x=s$.

$$
L_{s x}(j \omega)= \begin{cases}-\frac{\lambda}{\rho \cdot S \omega^{2}} \cdot \frac{\operatorname{sh} \lambda x \cdot \operatorname{ch} \lambda(l-s)}{\operatorname{ch} \lambda l} & 0 \leq x \leq s \\ -\frac{\lambda}{\rho \cdot S \omega^{2}} \cdot \frac{\operatorname{ch} \lambda(l-x) \cdot \operatorname{sh} \lambda s}{\operatorname{ch} \lambda l} & s \leq x \leq l\end{cases}
$$

Отметим, что полученные иным путем выражения (5.127), (5.131) приведены в [77,78]. В [48] выражение (5.127) найдено решением 
исходного дифференциального уравнения с помощью преобразования Лапласа.

Используя полученные результаты, рассмотрим колебания стержневой системы под действием силы, распределенной вдоль системы по некоторому закону $q(x)$ (рис.5.11,г). Элементарная сила $q(s) d s$, действующая на элемент $d s$ в некотором сечении $s$, вызывает колебания сечения $x$ с амплитудой $d \bar{a}_{x}=L_{s x}(j \omega) q(s) d s$. Амплитуда этого сечения при действии распределенной нагрузки

$$
\bar{a}_{X}=\int_{0}^{l} L_{s X}(j \omega) q(s) d s
$$

В качестве примера рассмотрим колебания однородного стержня под действием равномерно распределенной по его длине силы возбуждения $q(x)=q=$ const $\quad$ (рис.5.11,д). После подстановки $(5.131)$ в (5.132) и интегрирования получим

$$
\bar{a}_{x}=\frac{q}{\rho \cdot S \omega^{2}} \cdot \frac{\operatorname{ch} \lambda(l-x)-\operatorname{ch} \lambda l}{\operatorname{ch} \lambda l}
$$

При малом $\psi$ выражение (5.133) с учетом (5.12) приводится к виду

$$
\bar{a}_{x}=\frac{q l^{2}}{E S \xi^{2}} \cdot \frac{\cos \xi\left(1-\frac{x}{l}\right)-\cos \xi+j \frac{\psi \cdot \xi}{4 \pi}\left[\left(1-\frac{x}{l}\right) \sin \xi\left(1-\frac{x}{l}\right)-\sin \xi\right]}{\cos \xi+j \frac{\psi}{4 \pi} \xi \sin \xi}
$$

где $\xi=\omega \cdot l / c$.

Равенство (5.134) позволяет найти амплитуды и фазы колебаний всех сечений стержня. Для амплитуд колебаний сечения $x=l$ на резонансных частотах (5.112), например, имеем

$$
a_{l}^{0}=\frac{32 q l^{2}}{\pi^{2} \psi \cdot E S(2 n-1)^{3}}
$$

Из сравнения (5.135) и (5.113) следует, что при одинаковой суммарной силе $F_{l}=q l$, возбуждение системы сосредоточенной силой 
более эффективно, чем распределенной, особенно при возбуждении высших форм колебаний.

Таким образом, изложенный в настоящем разделе подход позволяет описать колебания сложных систем, используя известные динамические характеристики их элементов. Аналогичный подход применим, конечно, для описания крутильных колебаний стержней и поперечных колебаний струн, описываемых теми же уравнениями. Полученные в этом параграфе общие соотношения могут быть использованы и при описании изгибных колебаний стержневых систем в том случае, когда взаимодействие подсистем сводится лишь к возникновению перерезывающих сил и не вызывает появления изгибающих моментов в месте стыка. В общем случае описание изгибных колебаний имеет ряд специфических особенностей, которые будут освещены в следующем Параграфе. 


\section{6. Изгибные колебания ультразвуковых стержневых систем.}

1. Рассмотрим изгибные колебания стержневой системы, возбуждаемой гармонически изменяющимися поперечными силами $f_{s}(t)=\widetilde{F}_{s} e^{j \omega \cdot t}$ и изгибающими моментами $r_{s}(t)=\widetilde{R}_{s} e^{j \omega \cdot t}$, действующими в некоторых сечениях $x=s$ (рис.6.1,a).

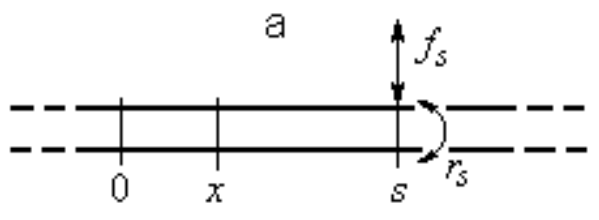

\section{Puc.6.1}

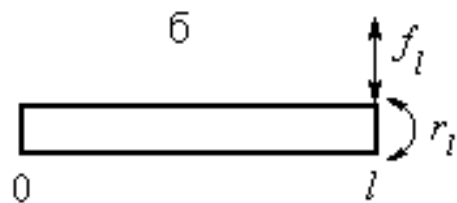

Движение произвольного сечения $x$ системы характеризуется смещением $u_{x}$ центра инерции сечения и его поворотом $\theta_{x}=\partial u_{x} / \partial x$. Будем рассматривать установившиеся движения вида (5.1). Амплитуды смещения и угла поворота связаны равенством

$$
\tilde{\theta}_{x}=d \tilde{a}_{x} / d x
$$

По аналогии с динамической податливостью $L_{s x}(j \omega)$ от действия поперечной силы, определяемой равенством (4.14), введем понятие динамической податливости от действия изгибающего момента

$$
\tilde{A}_{s x}(j \omega)=\tilde{a}_{x} / \tilde{R}_{s},
$$

связывающей комплексные амплитуды изгибающего момента в сечении $s$ и смещения сечения $x$. Найдем прежде всего динамические податливости $L_{s x}(j \omega)$ и $\ddot{a}_{s x}(j \omega)$ однородного стержня со свободными концами, возбуждаемого в своих крайних сечениях $s=l($ рис.6.1,б) или $s=0$.

С этой целью запишем уравнение изгибных колебаний однородного стержня $[68,83,160,192]$

$$
\rho \cdot \frac{\partial^{2} u(x, t)}{\partial \cdot t^{2}}+\widetilde{E}_{0} \mathrm{I} \frac{\partial^{4} u(x, t)}{\partial \cdot x^{2}}=0,
$$


где $\rho$ - масса единицы длины стержня; $\widetilde{E}_{0}=E_{0}(1+j \psi / 2 \pi) ; E_{0}-$ модуль упругости; ж - коэффициент поглощения; I - момент инерции поперечного сечения стержня.

При возбуждении стержня в сечении $x=l$ решение уравнения (6.3) должно удовлетворять следующим граничным условиям:

$$
\begin{gathered}
\left.\frac{\partial^{2} u(x, t)}{\partial x^{2}}\right|_{x=0}=0,\left.\frac{\partial^{3} u(x, t)}{\partial x^{3}}\right|_{x=0}=0 \\
\left.\frac{\partial^{2} u(x, t)}{\partial x^{2}}\right|_{x=l}=0,\left.\tilde{E}_{0} \mathrm{I} \frac{\partial^{3} u(x, t)}{\partial x^{3}}\right|_{x=l}=-f_{l}(t)
\end{gathered}
$$

при возбуждении силой и при возбуждении моментом

$$
\left.\tilde{E}_{0} \mathrm{I} \frac{\partial^{2} u(x, t)}{\partial x^{2}}\right|_{x=l}=r(t),\left.\frac{\partial^{3} u(x, t)}{\partial x^{3}}\right|_{x=l}=0
$$

После подстановки (5.1) в (6.3) - (6.6) получим уравнение относительно комплексной амплитуды $\tilde{a}_{x}$

$$
\tilde{a}_{x}-\frac{\tilde{E}_{0} \mathrm{I}}{\rho \cdot \omega^{2}} \cdot \frac{d^{4} \tilde{a}_{x}}{d x^{4}}=0
$$

с граничными условиями

$$
\begin{gathered}
\left.\frac{d^{2} \bar{a}_{x}}{d x^{2}}\right|_{x=0}=\left.\frac{d^{3} \bar{a}_{x}}{d x^{3}}\right|_{x=0}=0 \\
\left.\frac{d^{2} \bar{a}_{x}}{d x^{2}}\right|_{x=l}=0,\left.\quad \bar{E}_{0} \mathrm{I} \frac{d^{3} \bar{a}_{x}}{d x^{3}}\right|_{x=l}=-\widetilde{F}_{l} \\
\left.\bar{E}_{0} \mathrm{I} \frac{d^{2} \bar{a}_{x}}{d x^{2}}\right|_{x=l}=\widetilde{R}_{l},\left.\quad \frac{d^{3} \bar{a}_{x}}{d x^{3}}\right|_{x=l}=0
\end{gathered}
$$

В дальнейшем решении используются табулированные в

функции Крылова и функции частот, которые приводятся здесь в принятых в [5] обозначениях

$$
\begin{array}{rl}
S(z)=\frac{1}{2}(\operatorname{ch} z+\cos z) & T(z)=\frac{1}{2}(\operatorname{sh} z+\sin z) \\
U(z)=\frac{1}{2}(\operatorname{ch} z-\cos z) & V(z)=\frac{1}{2}(\operatorname{sh} z-\sin z) \\
A(z)=\operatorname{ch} z \sin z+\operatorname{sh} z \cos z & B(z)=\operatorname{ch} z \sin z-\operatorname{sh} z \cos z \\
C(z)=2 \operatorname{ch} z \cos z & S_{1}(z)=2 \operatorname{sh} z \sin z \\
D(z)=\operatorname{ch} z \cos z-1 & E(z)=\operatorname{ch} z \cos z+1
\end{array}
$$


Отыскивая решения уравнения (6.7), удовлетворяющие граничным условиям (6.8), найдем величины динамических податливостей (4.14), (6.2)

$$
\begin{array}{r}
L_{l x}(j \omega)=\frac{2 l^{3}}{E_{0} \mathrm{I}^{3} D(\tilde{\xi})}\left[U(\tilde{\xi}) T\left(\tilde{\xi} \frac{x}{l}\right)-V(\tilde{\xi}) S\left(\tilde{\xi} \frac{x}{l}\right)\right] \\
\Gamma_{l x}(j \omega)=\frac{2 l^{2}}{\widetilde{E}_{0} \mathrm{I} \tilde{\xi}^{2} D(\tilde{\xi})}\left[T(\tilde{\xi}) T\left(\tilde{\xi} \frac{x}{l}\right)-U(\tilde{\xi}) S\left(\tilde{\xi} \frac{x}{l}\right)\right] \\
\text { где } \tilde{\xi}=l \cdot 4 \sqrt[4]{\frac{\rho \cdot \omega^{2}}{\widetilde{E}_{0} \mathrm{I}}}=\xi \cdot\left(1+j \frac{\psi}{2 \pi}\right)^{-1 / 4}, \quad \xi=l \cdot \sqrt[4]{\frac{\rho \cdot \omega^{2}}{E_{0} \mathrm{I}}} .
\end{array}
$$

В силу геометрической симметрии однородного стержня (рис.6.1,б) можно сразу записать величины динамических податливостей при возбуждении в сечении $x=0$

$$
L_{0 x}(j \omega)=L_{l(l-x)}(j \omega), \Gamma_{0 x}(j \omega)=\Gamma_{l(l-x)}(j \omega),
$$

т.е. их выражения получаются из $(6.10),(6.11)$ заменой $x$ на $(l-x)$.

Согласно (6.1), (4.14), (6.2) амплитуда угла поворота сечения $x$ при возбуждении в сечении $s$ силой или моментом определяется равенствами

$$
\widetilde{\theta}_{x}=\widetilde{F}_{s} L_{s x}^{\prime}(j \omega), \widetilde{\theta}_{x}=\widetilde{R}_{s} \Gamma_{s x}^{\prime}(j \omega),(6.13)
$$

где штрихом обозначено дифференцирование по $x$.

Величины $L_{s x}^{\prime}(j \omega)$ и $\Gamma_{s x}^{\prime}(j \omega)$, также как и (6.10), (6.11), можно трактовать как динамические податливости, связывающие действующие в сечении $s$ силу и момент с угловым перемещением сечения $x$, вызываемым этими силовыми факторами. Непосредственной подстановкой функций (6.9) в выражения (6.10) - (6.13) легко убедиться в справедливости следующих равенств:

$$
L_{l l}^{\prime}(j \omega)=\Gamma_{l l}(j \omega), L_{00}^{\prime}(j \omega)=\Gamma_{00}(j \omega), L_{l 0}^{\prime}(j \omega)=-\Gamma_{l 0}(j \omega)
$$

Эти равенства означают, что единичная сила вызывает угловые перемещения крайних сечений, равные по величине линейным перемещениям тех же сечений под действием единичного момента.

Как видно из (6.10) - (6.13) любая из величин динамических податливостей записывается в виде (5.34), и поэтому анализ колебаний системы проводится с их помощью также, как изложено в п.4 
предыдущего раздела. Для примера приведем к виду (5.34) динамические податливости (6.10), (6.11) при $x=l$.

$$
\begin{gathered}
L_{l l}(j \omega)=\frac{l^{3}}{E_{0} I^{3}} \cdot \frac{B(\xi)-j \frac{\psi}{8 \pi}\left[3 B(\xi)-\xi \cdot S_{1}(\xi)\right]}{D(\xi)-j \frac{\psi}{8 \pi} B(\xi)} \\
\Gamma_{l /}(j \omega)=\frac{P^{2}}{2 E_{0} \xi^{2}} \cdot \frac{S_{1}(\xi)-j \frac{\psi}{8 \pi}\left[2 S_{1}(\xi)-\xi \cdot A(\xi)\right]}{D(\xi)-j \frac{\psi}{8 \pi} B(\xi)}
\end{gathered}
$$

Согласно (6.15), (6.16) резонансные частоты стержня определяются решениями уравнения $D(\xi)=0$, или с учетом обозначений (6.9)

$$
\operatorname{ch} \xi \cos \xi-1=0 \text {. }
$$

Ненулевыми решениями этого уравнения с высокой точностью будут числа

$$
\xi_{n}=\frac{\pi}{2}(2 n+1), \quad(n=1,2, \ldots)
$$

(В этом легко убедиться с помощью таблиц функций (6.9), приведенных в [6].)

Для резонансных амплитуд колебаний имеем:

$$
a_{l}=\frac{8 \pi \cdot F_{l} l^{3}}{\psi \cdot \xi_{n}^{4} E_{0} \mathrm{I}}=\frac{128}{\psi \cdot \pi^{3}(2 n+1)^{4}} \cdot \frac{F_{l} l^{3}}{E_{0} \mathrm{I}}
$$

при возбуждении силой и

$$
a_{l}=\frac{4 \pi \cdot R_{l} l^{2} S_{1}\left(\xi_{n}\right)}{\psi \cdot \xi_{n}^{3} E_{0} \mathrm{I} B\left(\xi_{n}\right)}=\frac{64}{\psi \cdot \pi^{2}(2 n+1)^{3}} \cdot \frac{R_{l} I^{2}}{E_{0} \mathrm{I}}
$$

при возбуждении моментом сил. В (6.20) учтено, что на частотах (6.18) с достаточной точностью $S_{1}\left(\xi_{n}\right) / B\left(\xi_{n}\right)=2$ (см., например, таблицы [6]).

Антирезонансные частоты при возбуждении силой и моментом находятся соответственно решением уравнений $B(\xi)=0$ и $S_{1}(\xi)=0$. Первое из них дает значения

$$
\xi_{n}^{*}=\frac{\pi}{4}(4 n+1),(n=1,2, \ldots)
$$

Bтороe -

$$
\xi_{n}^{*}=\pi \cdot n,(n=1,2, \ldots)
$$


На частотах (6.21) при возбуждении силой и (6.22) при возбуждении моментом практически отсутствуют колебания центра инерции сечения $x=l$, и это сечение совершает чисто угловые колебания, амплитуда которых может быть найдена с помощью равенств (6.13). Вместе с тем существуют частоты, на которых сечение совершает чисто поступательные колебания. Так при возбуждении силой поступательные колебания сечений $x=0$ и $x=l$ происходят на частотах (6.22). Это сразу видно, если воспользоваться равенствами (6.14). Из них , в частности, следует, что динамическая податливость (6.14) описывает угловые колебания сечения $x=l$ при воздействии на него силой $f(t)$, для которых частота (6.22) является антирезонансной. Здесь следует обратить внимание на принципиальную возможность реализации различных типов движения сечений колебательной системы. Такую возможность необходимо учитывать при рассмотрении движения рабочих элементов ультразвуковых систем. Мы уже упоминали об этом в п.4 (см., например, рис.4.8,a). В дальнейшем будет показана возможность эффективной реализации таких режимов путем определенного динамического согласования элементов колебательной системы.

Покажем, что на низких частотах возбуждения величины (6.10), (6.11) описывают колебания стержня как абсолютно жесткого тела. С этой целью, полагая $\xi \ll 1$, разложим числители и знаменатели выражений (6.10), (6.11) в ряды по малому параметру $\xi$. В результате, ограничивая разложение первыми значащими членами, получим

$$
\begin{gathered}
L_{l x}(j \omega)=\frac{2(1-3 x / l)}{\rho \cdot l \omega^{2}}=-\frac{1}{M \omega^{2}}-\frac{l}{2} \cdot \frac{x_{1}}{J \omega^{2}} \\
\tilde{A}_{l x}(j \omega)=\frac{6(1-2 x / l)}{\rho \cdot l^{2} \omega^{2}}=-\frac{x_{1}}{J \omega^{2}}
\end{gathered}
$$

где $M=\rho \cdot l$ - масса стержня; $J=\rho \cdot l^{3} / 12$ - его момент инерции относительно центра масс; $x_{1}=x-1 / 2$ - координата, отсчитываемая от центра масс. 
Равенство (6.24) описывает смещения сечений стержня при его угловых колебаниях под действием единичного момента. Динамическая податливость при угловых колебаниях

$$
\Gamma_{l x}^{\prime}(j \omega)=\Gamma^{\prime}(j \omega)=-\frac{1}{J \omega^{2}}
$$

Первое слагаемое в (6.23) описывает смещения при поступательных колебаниях тела под действием единичной силы; второе слагаемое описывает смещения точек тела при его угловых колебаниях относительно центра масс под действием момента, создаваемого единичной силой на плече $l / 2$.

Аналогично (6.10), (6.11) отыскиваются динамические податливости элементов при других условиях закрепления концов. Некоторые из них приведены в таблице 6.1 .

Таблица 6.1.

\begin{tabular}{|c|c|c|}
\hline $\begin{array}{l}\text { № } \\
\text { ПП }\end{array}$ & Схема элемента & Динамическая податливость \\
\hline 1. & $\chi_{0} \overbrace{}^{\mathrm{f}_{\mathrm{i}}}$ & $L_{l x}(j \omega)=\frac{2 l^{3}}{\widetilde{E}_{0} \mathrm{I}} \cdot \frac{T(\tilde{\xi}) \cdot U\left(\tilde{\xi} \frac{x}{l}\right)-S(\tilde{\xi}) \cdot V\left(\tilde{\xi} \frac{x}{l}\right)}{\tilde{\xi}^{3} E(\tilde{\xi})}$ \\
\hline 2. & 坊 & $\Gamma_{l x}(j \omega)=\frac{2 l^{2}}{\widetilde{E}_{0} \mathrm{I}} \cdot \frac{S(\tilde{\xi}) \cdot U\left(\tilde{\xi} \frac{x}{l}\right)-V(\tilde{\xi}) \cdot V\left(\tilde{\xi} \frac{x}{l}\right)}{\widetilde{\xi}^{2} E(\tilde{\xi})}$ \\
\hline 3. & $\stackrel{0}{q_{\pi}} \quad \uparrow^{f_{1}}$ & $L_{l x}(j \omega)=-\frac{l^{3}}{\widetilde{E}_{0} I} \cdot \frac{\operatorname{sh} \tilde{\xi} \cdot \sin \tilde{\xi} \frac{x}{l}+\sin \tilde{\xi} \cdot \operatorname{sh} \tilde{\xi} \frac{x}{l}}{\tilde{\xi}^{3} B(\tilde{\xi})}$ \\
\hline 4. & $\overbrace{1}^{0}$ & $\Gamma_{l x}(j \omega)=-\frac{l^{2}}{\widetilde{E}_{0} \mathrm{I}} \cdot \frac{\operatorname{ch} \tilde{\xi} \sin \tilde{\xi} \frac{x}{l}+\cos \tilde{\xi} \cdot \operatorname{sh} \tilde{\xi} \frac{x}{l}}{\tilde{\xi}^{2} B(\tilde{\xi})}$ \\
\hline 5. & $\stackrel{C_{1}}{r_{2}}$ & $\Gamma_{l x}(j \omega)=\frac{2 l^{2}}{\tilde{E}_{0} I} \cdot \frac{T(\tilde{\xi}) \cdot S\left(\tilde{\xi} \frac{x}{l}\right)-S(\tilde{\xi}) \cdot T\left(\tilde{\xi} \frac{x}{l}\right)}{\tilde{\xi}^{2} B(\tilde{\xi})}$ \\
\hline
\end{tabular}




\begin{tabular}{|c|c|c|}
\hline 6. & 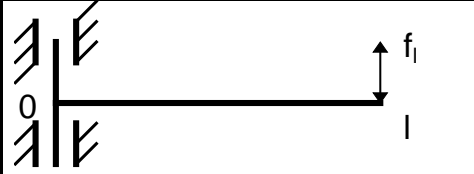 & $L_{l x}(j \omega)=-\frac{l^{3}}{\widetilde{E}_{0} \mathrm{I}} \cdot \frac{\operatorname{ch} \tilde{\xi} \cdot \cos \tilde{\xi} \frac{x}{l}+\cos \tilde{\xi} \cdot \operatorname{ch} \tilde{\xi} \frac{x}{l}}{\tilde{\xi}^{3} A(\tilde{\xi})}$ \\
\hline 7. & \begin{tabular}{ll}
$\lambda$ & \multicolumn{1}{l}{} \\
0 \\
$\lambda$
\end{tabular} & $\Gamma_{l x}(j \omega)=\frac{l^{2}}{\widetilde{E}_{0} \mathrm{I}} \cdot \frac{\sin \tilde{\xi} \cdot \operatorname{ch} \tilde{\xi} \frac{x}{l}-\operatorname{sh} \tilde{\xi} \cdot \cos \tilde{\xi} \frac{x}{l}}{\tilde{\xi}^{2} A(\tilde{\xi})}$ \\
\hline 8. & 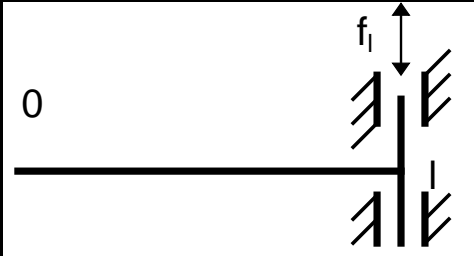 & $L_{l x}(j \omega)=\frac{2 \beta}{\widetilde{E}_{0} \mathrm{I}} \cdot \frac{V(\tilde{\xi}) \cdot T\left(\tilde{\xi} \frac{x}{l}\right)-S(\tilde{\xi}) \cdot S\left(\tilde{\xi} \frac{x}{l}\right)}{\tilde{\xi}^{3} A(\tilde{\xi})}$ \\
\hline 9. & $\uparrow^{f_{1}}$ & $L_{l x}(j \omega)=\frac{2 \beta}{\widetilde{E}_{0} \mathrm{I}} \cdot \frac{\psi(\tilde{\xi}) \cdot T\left(\tilde{\xi} \frac{x}{l}\right)-V(\tilde{\xi}) \cdot S\left(\tilde{\xi} \frac{x}{l}\right)}{\tilde{\xi}^{3} D(\tilde{\xi})}$ \\
\hline 10. & $\overbrace{1}^{r_{1}}$ & $\Gamma_{l x}(j \omega)=\frac{2 l^{2}}{\widetilde{E}_{0} \mathrm{I}} \cdot \frac{T(\tilde{\xi}) \cdot T\left(\tilde{\xi} \frac{x}{l}\right)-\psi(\tilde{\xi}) \cdot S\left(\tilde{\xi} \frac{x}{l}\right)}{\tilde{\xi}^{2} D(\tilde{\xi})}$ \\
\hline 11. & 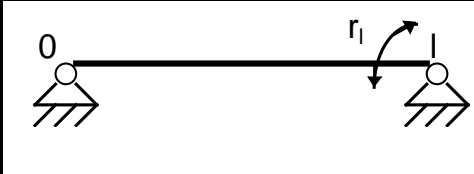 & $\Gamma_{l x}(j \omega)=\frac{l^{2}}{\widetilde{E}_{0} I} \cdot \frac{\sin \tilde{\xi} \cdot \operatorname{sh} \tilde{\xi} \frac{x}{l}-\operatorname{sh} \tilde{\xi} \cdot \sin \tilde{\xi} \frac{x}{l}}{\tilde{\xi}^{2} S_{1}(\tilde{\xi})}$ \\
\hline
\end{tabular}

2. Используя величины динамических податливостей элементов из табл.6.1, найдем динамические податливости $L_{s x}(j \omega), \Gamma_{s x}(j \omega)$ системы, возбуждаемой в некотором промежуточном сечении $x=s$. С этой целью расчленим систему по месту действия возбуждающего фактора, относя его к одной из частей и заменяя взаимодействие частей силой и моментом с амплитудами $\bar{F}_{12}$ и $\bar{R}_{12}$. Вводя координаты $x_{1}=x\left(x_{1} \in[0, s]\right)$ и $x_{2}=x-s$ $\left(x_{2} \in[0, l-s]\right)$, запишем

$$
\begin{aligned}
& \bar{a}_{x_{1}}^{(1)}=\bar{A}_{x_{1}}+\bar{F}_{12} L_{s x_{1}}^{(1)}(j \omega)+\bar{R}_{12} \tilde{A}_{s x_{1}}^{(1)}(j \omega) \\
& \bar{a}_{x_{2}}^{(2)}=-\bar{F}_{12} L_{0 x_{2}}^{(2)}(j \omega)-\bar{R}_{12} \tilde{A}_{0 x_{2}}^{(2)}(j \omega)
\end{aligned}
$$

где $\bar{A}_{x_{1}}=\bar{F}_{s} L_{s x_{1}}^{(1)}(j \omega)$ при возбуждении силой и $\bar{A}_{x_{1}}=\bar{R}_{s} \Gamma_{s x_{1}}^{(1)}(j \omega)$ при возбуждении моментом. 
С помощью очевидных равенств $\bar{a}_{s}^{(1)}=\bar{a}_{0}^{(2)} \quad$ и $\quad \bar{a}_{s}^{\prime(1)}=\bar{a}_{0}^{\prime(2)} \quad$ получим следующую систему уравнений относительно неизвестных реакций $\bar{F}_{12}$ и $\bar{R}_{12}$ :

$$
\begin{aligned}
& \alpha_{11} \bar{F}_{12}+\alpha_{12} \bar{R}_{12}=-\bar{A}_{s} \\
& \alpha_{21} \bar{F}_{12}+\alpha_{22} \bar{R}_{12}=-A_{s}^{\prime}
\end{aligned}
$$

где $\alpha_{11}=L_{s s}^{(1)}+L_{00}^{(2)}, \alpha_{12}=\tilde{A}_{s s}^{(1)}+\tilde{A}_{00}^{(2)}, \alpha_{21}=\alpha_{12}, \quad \alpha_{22}=\tilde{A}_{s s}^{\prime(1)}+\tilde{A}_{00}^{\prime(2)}$.

Решая (6.27), находим

$$
\bar{F}_{12}=\frac{\alpha_{12} A_{s}^{\prime}-\alpha_{22} A_{s}}{\alpha_{11} \alpha_{22}-\alpha_{12}^{2}}, \quad \bar{R}_{12}=\frac{\alpha_{12} A_{s}-\alpha_{11} A_{s}^{\prime}}{\alpha_{11} \alpha_{22}-\alpha_{21}^{2}}
$$

Подстановка (6.28) в (6.26) с учетом (4.14) и (6.2) дает искомые выражения динамических податливостей.

В качестве примера рассмотрим колебания однородной балки на шарнирных опорах, возбуждаемой в сечении $x=s$ силой (рис.6.2,a) и моментом (рис.6.2,б).

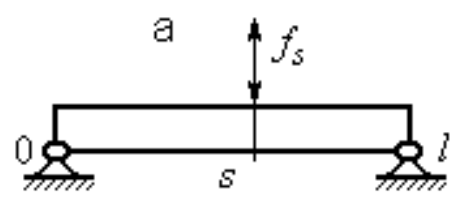

6

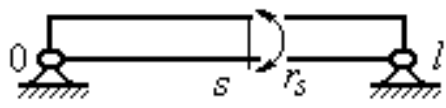

Pис.6.2

Используя величины динамических податливостей из табл.6.1 (строки 3, 4), выпишем коэффициенты уравнений (6.27).

$$
\begin{aligned}
& \alpha_{11}=-\frac{l^{3}}{\widetilde{E}_{0} \tilde{\xi}^{3}} \cdot\left[\frac{S_{1}\left(\tilde{\xi}_{1}\right)}{B\left(\tilde{\xi}_{1}\right)}+\frac{S_{1}\left(\tilde{\xi}_{2}\right)}{B\left(\tilde{\xi}_{2}\right)}\right], \\
& \alpha_{12}=-\frac{l^{2}}{\widetilde{E}_{0} \tilde{\xi}^{2}} \cdot\left[\frac{A\left(\tilde{\xi}_{1}\right)}{B\left(\tilde{\xi}_{1}\right)}-\frac{A\left(\tilde{\xi}_{2}\right)}{B\left(\tilde{\xi}_{2}\right)}\right], \\
& \alpha_{22}=-\frac{l}{\widetilde{E}_{0} I \tilde{\xi}^{2}} \cdot\left[\frac{C\left(\tilde{\xi}_{1}\right)}{B\left(\tilde{\xi}_{1}\right)}+\frac{C\left(\tilde{\xi}_{2}\right)}{B\left(\tilde{\xi}_{2}\right)}\right],
\end{aligned}
$$

где $\tilde{\xi}=/ \cdot \sqrt[4]{\frac{\rho \cdot \omega^{2}}{\tilde{E}}}, \tilde{\xi}_{1}=\tilde{\xi} \frac{s}{l}, \tilde{\xi}_{2}=\tilde{\xi} \frac{l-s}{l}$. 
С помощью формул (6.28) находим величины реакций

$$
\begin{aligned}
& \bar{F}_{12}=-\frac{F_{s}}{S_{1}(\tilde{\xi})} \cdot\left(\sin \tilde{\xi}_{1} \cdot \cos \tilde{\xi}_{2} \cdot \operatorname{sh} \tilde{\xi}+\operatorname{sh} \tilde{\xi}_{1} \cdot \operatorname{ch} \tilde{\xi}_{2} \cdot \sin \tilde{\xi}\right) \\
& \bar{R}_{12}=-\frac{F_{s} l}{\tilde{\xi} \cdot S_{1}(\tilde{\xi})} \cdot\left(\sin \tilde{\xi}_{1} \cdot \sin \tilde{\xi}_{2} \cdot \operatorname{sh} \tilde{\xi}+\operatorname{sh} \tilde{\xi}_{1} \cdot \operatorname{sh} \tilde{\xi}_{2} \cdot \sin \tilde{\xi}\right)
\end{aligned}
$$

при возбуждении силой и при возбуждении моментом

$$
\begin{aligned}
& \bar{F}_{12}=-\frac{\xi \cdot R_{s}}{I S_{1}(\tilde{\xi})} \cdot\left(\cos \tilde{\xi}_{1} \cdot \cos \tilde{\xi}_{2} \cdot \operatorname{sh} \tilde{\xi}+\operatorname{ch} \tilde{\xi}_{1} \cdot \operatorname{ch} \tilde{\xi}_{2} \cdot \sin \tilde{\xi}\right) \\
& \bar{R}_{12}=-\frac{R_{s}}{S_{1}(\tilde{\xi})} \cdot\left(\cos \tilde{\xi}_{1} \cdot \sin \tilde{\xi}_{2} \cdot \operatorname{sh} \tilde{\xi}+\operatorname{ch} \tilde{\xi}_{1} \cdot \operatorname{sh} \tilde{\xi}_{2} \cdot \sin \tilde{\xi}\right)
\end{aligned}
$$

После подстановки (6.29), (6.30) и соответствующих выражений из табл.6.1 в равенства (6.26) получаем искомые динамические податливости

$$
\begin{aligned}
& L_{s x}(j \omega)=\frac{l^{3}}{\widetilde{E}_{0} \tilde{\xi}^{3}} \begin{cases}\frac{\operatorname{sh} \tilde{\xi} \sin \tilde{\xi} \frac{l-s}{l} \sin \tilde{\xi} \frac{x}{l}-\sin \tilde{\xi} \operatorname{sh} \tilde{\xi} \frac{l-s}{l} \operatorname{sh} \tilde{\xi} \frac{x}{l}}{S_{1}(\tilde{\xi})} & 0 \leq x \leq s \\
\frac{\operatorname{sh} \tilde{\xi} \sin \tilde{\xi} \frac{s}{l} \sin \tilde{\xi} \frac{l-x}{l}-\sin \tilde{\xi} \operatorname{sh} \tilde{\xi} \frac{s}{l} \operatorname{sh} \tilde{\xi} \frac{l-x}{l}}{S_{1}(\tilde{\xi})} & s \leq x \leq l\end{cases} \\
& \Gamma_{s x}(j \omega)=\frac{l^{2}}{\widetilde{E}_{0} \tilde{\xi}^{2}} \begin{cases}\frac{-\operatorname{sh} \tilde{\xi} \cos \tilde{\xi} \frac{l-s}{l} \sin \tilde{\xi} \frac{x}{l}+\sin \tilde{\xi} \operatorname{ch} \tilde{\xi} \frac{l-s}{l} \operatorname{sh} \tilde{\xi} \frac{x}{l}}{\left.S_{1} \tilde{\xi}\right)} & 0 \leq x \leq s \\
\frac{\operatorname{sh} \tilde{\xi} \cos \tilde{\xi} \frac{s}{l} \sin \tilde{\xi} \frac{l-x}{l}-\sin \tilde{\xi} \operatorname{ch} \tilde{\xi} \frac{s}{l} \operatorname{sh} \tilde{\xi} \frac{l-x}{l}}{S_{1}(\tilde{\xi})} & s \leq x \leq l\end{cases}
\end{aligned}
$$

Выражение, аналогичное (6.31), приведено в [58,78]. Из (6.32) при $s=l$ получается динамическая податливость элемента, показанного в строке 11 табл. 6.1.

Аналогичным путем можно найти динамические податливости систем с иными условиями закрепления концов. Например, для однородного консольного стержня коэффициенты уравнений (6.27) имеют вид

$$
\alpha_{11}=\frac{I^{3}}{\widetilde{E}_{0} I \tilde{\xi}^{3}}\left[\frac{B\left(\tilde{\xi}_{1}\right)}{E\left(\tilde{\xi}_{1}\right)}+\frac{B\left(\tilde{\xi}_{2}\right)}{D\left(\tilde{\xi}_{2}\right)}\right],
$$




$$
\begin{gathered}
\alpha_{12}=\frac{I^{2}}{2 \widetilde{E}_{0} I \tilde{\xi}^{2}}\left[\frac{S_{1}\left(\tilde{\xi}_{1}\right)}{E\left(\tilde{\xi}_{1}\right)}-\frac{S_{1}\left(\tilde{\xi}_{2}\right)}{D\left(\tilde{\xi}_{2}\right)}\right], \\
\alpha_{22}=\frac{l}{\widetilde{E}_{0} \tilde{\xi}_{\xi}}\left[\frac{A\left(\tilde{\xi}_{1}\right)}{E\left(\tilde{\xi}_{1}\right)}+\frac{A\left(\tilde{\xi}_{2}\right)}{D\left(\tilde{\xi}_{2}\right)}\right]
\end{gathered}
$$

После подстановки коэффициентов (6.33) и соответствующих величин из строк 1, 2, 9, 10 табл.6.1 в выражения (6.28) и (6.26) найдем динамические податливости консольного стержня при его возбуждении силой и моментом в сечении $x=s$

$$
L_{s x}(j \omega)=\frac{\beta}{2 \widetilde{\xi}_{0} \mathrm{I} \tilde{\xi}^{3} E(\tilde{\xi})} \begin{cases}\chi_{1}\left(\tilde{\xi}_{1}, \tilde{\xi}_{2}\right) \cdot v\left(\tilde{\xi} \frac{x}{l}\right)-\chi_{2}\left(\tilde{\xi}_{1}, \tilde{\xi}_{2}\right) \cdot v\left(\tilde{\xi} \frac{x}{l}\right) & 0 \leq x \leq s \\ \chi_{3}\left(\tilde{\xi}_{1}, \tilde{\xi}_{2}\right) \cdot T\left(\tilde{\xi} \frac{I-x}{I}\right)-\chi_{4}\left(\tilde{\xi}_{1}, \tilde{\xi}_{2}\right) \cdot S\left(\tilde{\xi} \frac{I-x}{I}\right) & s \leq x \leq 1\end{cases}
$$

где $\quad \chi_{1}\left(\tilde{\xi}_{1}, \tilde{\xi}_{2}\right)=2 T\left(\tilde{\xi}_{1}\right) E\left(\tilde{\xi}_{2}\right)-2 U\left(\tilde{\xi}_{1}\right) B\left(\tilde{\xi}_{2}\right)-V\left(\tilde{\xi}_{1}\right) S_{1}\left(\tilde{\xi}_{2}\right)$,

$$
\begin{aligned}
& \chi_{2}\left(\tilde{\xi}_{1}, \tilde{\xi}_{2}\right)=2 S\left(\tilde{\xi}_{1}\right) E\left(\tilde{\xi}_{2}\right)-2 T\left(\tilde{\xi}_{1}\right) B\left(\tilde{\xi}_{2}\right)-U\left(\tilde{\xi}_{1}\right) S_{1}\left(\tilde{\xi}_{2}\right) \\
& \chi_{3}\left(\tilde{\xi}_{1}, \tilde{\xi}_{2}\right)=2 D\left(\tilde{\xi}_{1}\right) U\left(\tilde{\xi}_{2}\right)-2 B\left(\tilde{\xi}_{1}\right) N\left(\tilde{\xi}_{2}\right)-S_{1}\left(\tilde{\xi}_{1}\right) S\left(\tilde{\xi}_{2}\right) \\
& \chi_{4}\left(\tilde{\xi}_{1}, \tilde{\xi}_{2}\right)=2 D\left(\tilde{\xi}_{1}\right) V\left(\tilde{\xi}_{2}\right)-2 B\left(\tilde{\xi}_{1}\right) S\left(\tilde{\xi}_{2}\right)-S_{1}\left(\tilde{\xi}_{1}\right) T\left(\tilde{\xi}_{2}\right) \\
& \Gamma_{s x}(j \omega)=\frac{l^{2}}{2 \tilde{E}_{0} \tilde{\xi}^{2} E\left(\tilde{\xi}^{\prime}\right)} \begin{cases}\chi_{5}\left(\tilde{\xi}_{1}, \tilde{\xi}_{2}\right) U\left(\tilde{\xi} \frac{x}{l}\right)-\chi_{6}\left(\tilde{\xi}_{1}, \tilde{\xi}_{2}\right) N\left(\tilde{\xi} \frac{x}{l}\right) & 0 \leq x \leq s \\
-\chi_{7}\left(\tilde{\xi}_{1}, \tilde{\xi}_{2}\right) T\left(\tilde{\xi} \frac{l-x}{l}\right)+\chi_{8}\left(\tilde{\xi}_{1}, \tilde{\xi}_{2}\right) s\left(\tilde{\xi} \frac{l-x}{l}\right) & s \leq x \leq l\end{cases}
\end{aligned}
$$

где $\quad \chi_{5}\left(\tilde{\xi}_{1}, \tilde{\xi}_{2}\right)=2 V\left(\tilde{\xi}_{1}\right) A\left(\tilde{\xi}_{2}\right)+2 S\left(\tilde{\xi}_{1}\right) E\left(\tilde{\xi}_{2}\right)+U\left(\tilde{\xi}_{1}\right) S_{1}\left(\tilde{\xi}_{2}\right)$

$$
\begin{aligned}
& \chi_{6}\left(\tilde{\xi}_{1}, \tilde{\xi}_{2}\right)=2 U\left(\tilde{\xi}_{1}\right) A\left(\tilde{\xi}_{2}\right)+2 V\left(\tilde{\xi}_{1}\right) E\left(\tilde{\xi}_{2}\right)+T\left(\tilde{\xi}_{1}\right) S_{1}\left(\tilde{\xi}_{2}\right) \\
& \chi_{7}\left(\tilde{\xi}_{1}, \tilde{\xi}_{2}\right)=2 D\left(\tilde{\xi}_{1}\right) T\left(\tilde{\xi}_{2}\right)+2 A\left(\tilde{\xi}_{1}\right) S\left(\tilde{\xi}_{2}\right)+S_{1}\left(\tilde{\xi}_{1}\right) V\left(\tilde{\xi}_{2}\right) \\
& \chi_{8}\left(\tilde{\xi}_{1}, \tilde{\xi}_{2}\right)=2 D\left(\tilde{\xi}_{1}\right) U\left(\tilde{\xi}_{2}\right)+2 A\left(\tilde{\xi}_{1}\right) T\left(\tilde{\xi}_{2}\right)+S_{1}\left(\tilde{\xi}_{1}\right) S\left(\tilde{\xi}_{2}\right)
\end{aligned}
$$

В выражениях (6.34), (6.35) обозначения для величин $\tilde{\xi}_{,}, \tilde{\xi}_{1}, \tilde{\xi}_{2}$ совпадают с принятыми в (6.29). Нетрудно убедиться, что при $s=l$ выражения (6.34), (6.35) приводятся к виду, показанному в строках 1,2 табл.6.1. С помощью построенных динамических податливостей вида (6.31), (6.32) и (6.34), (6.35) могут быть рассмотрены изгибные колебания стержней, вызываемые распределенными по их длине силами. Такое рассмотрение аналогично изложенному ранее в п.5.12; при возбуждении 
распределенной силой колебания описываются формулой (5.132). Так, например, для однородного шарнирно закрепленного стержня, возбуждаемого равномерно распределенной нагрузкой с интенсивностью $q_{0}$, после подстановки (6.31) в (5.132) и интегрирования находим

$$
\tilde{a}_{x}=\frac{q_{0} l^{4}}{\widetilde{E}_{0} I^{4} \tilde{\xi}^{4}} \cdot \frac{\operatorname{ch} \frac{\tilde{\xi}}{2} \cos \left(\frac{\tilde{\xi}}{2}-\tilde{\xi} \frac{x}{l}\right)+\cos \frac{\tilde{\xi}}{2} \operatorname{ch}\left(\frac{\tilde{\xi}}{2}-\tilde{\xi} \frac{x}{l}\right)-2 C\left(\frac{\tilde{\xi}}{2}\right)}{C\left(\frac{\tilde{\xi}}{2}\right)}
$$

При возбуждении распределенным моментом в выражении (5.132) величину $L_{s x}(j \omega)$ следует заменить на $\Gamma_{s x}(j \omega)$, а под $q(s)$ понимать интенсивность распределенного момента.

3. При расчетах колебательных систем ультразвуковых технологических установок наибольший интерес представляет отыскание параметров движения рабочего органа, который, как правило, можно представить в виде абсолютно жесткого тела, присоединенного к стержневому элементу. Как уже отмечалось ранее, при изгибных колебаниях стержня присоединенное к нему тело совершает сложные движения в плоскости. В связи с этим рассмотрим одну из задач отыскания таких движений, решение которой будет использовано в дальнейшем при анализе нелинейных процессов в ультразвуковых колебательных системах.

Рассмотрим изгибные колебания балки с закрепленным на ее конце телом под действием приложенного к его центру масс $C$ внешнего гармонического возбуждения $f_{c}(t)=F_{c} \mathrm{e}^{j \omega \cdot t}$ (рис.6.3). (Решение этой задачи получено в [55].)

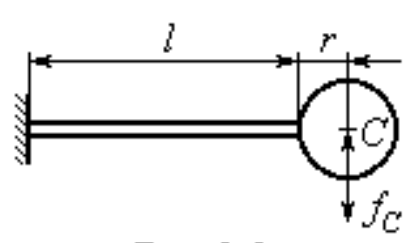

Рис.6.3

Смещение сечений балки от недеформированного состояния будем по прежнему описывать функцией $u_{x}(t)=\tilde{a}_{x} \mathrm{e}^{j \omega t}, \quad$ a движение присоединенного тела - координатой $u_{c}(t)=\tilde{a}_{c} \mathrm{e}^{j \omega t}$ его центра масс и углом 
поворота $\theta_{c}(t)=\tilde{\theta}_{c} \mathrm{e}^{j \omega \cdot t}$. При малых колебаниях эти величины связаны соотношениями

$$
\tilde{a}_{c}=\tilde{a}_{l}+r \tilde{\theta}_{c}, \quad \tilde{\theta}_{c}=\tilde{\theta}_{l}=\left.\frac{d \tilde{a}_{x}}{d x}\right|_{x=l}
$$

где $r$ - расстояние точки крепления тела от его центра масс.

Обозначив $\tilde{F}_{,}$и $\tilde{R}_{/}$амплитуды перерезывающей силы и изгибающего момента в сечении $x=l$ балки, с помощью динамических податливостей (5.14), (6.25), описывающих поступательные и угловые колебания твердого тела, запишем для их амплитуд

$$
-M \omega^{2} \tilde{a}_{c}=F_{c}-\tilde{F}_{l}, \quad-J \omega^{2} \tilde{\theta}_{c}=r \tilde{F}_{l}-\tilde{R}_{l}
$$

где $M, J$ - масса тела и его момент инерции относительно оси, проходящей через центр масс.

Движение балки опишем с помощью динамических податливостей $L_{l x}(j \omega)$ и $\Gamma_{l x}(j \omega)$

$$
\tilde{a}_{x}=L_{l x}(j \omega) \cdot \tilde{F}_{l}+\Gamma_{l x}(j \omega) \cdot \tilde{R}_{l}
$$

Подставляя величины $\tilde{F}_{/}$и $\widetilde{R}_{/}$из (6.38) в уравнение (6.39), получим

$$
\tilde{a}_{x}=L_{c x}(j \omega)\left(\tilde{F}_{l}+M \omega^{2} \tilde{a}_{c}\right)+\Gamma_{l x}(j \omega) \cdot J \omega^{2} \tilde{\theta}_{c}
$$

где $L_{c x}(j \omega)=L_{l x}(j \omega)+r \Gamma_{l x}(j \omega)$.

Из соотношения (6.40) после дифференцирования найдем при $x=l$ c учетом последнего равенства (6.37)

$$
\tilde{\theta}_{c}=\frac{\left(\tilde{F}_{l}+M \omega^{2} \tilde{a}_{c}\right) \cdot L_{c l}^{\prime}(j \omega)}{1-J \omega^{2} \Gamma_{l l}^{\prime}(j \omega)}
$$

где штрихом обозначена операция $d / d x$.

Прибавим величину $r \widetilde{\theta}_{c}$ к обеим частям равенства (6.40) при $x=l$ и подставим (6.41) в правую часть полученного уравнения. Из последнего, принимая во внимание первое соотношение (6.37), найдем амплитуду колебаний центра масс присоединенного тела

$$
\tilde{a}_{c}=F_{c} / W_{c}(j \omega),
$$


где динамическая жесткость колебательной системы в точке С задана выражением

$$
W_{c}(j \omega)=\left[L_{c l}(j \omega)+L_{c l}^{\prime}(j \omega) \frac{r+\omega^{2} J \Gamma_{l l}(j \omega)}{1-\omega^{2} J \Gamma_{l l}^{\prime}(j \omega)}\right]^{-1}-M \omega^{2}
$$

Амплитуды смещений сечений балки и угловых колебаний тела находятся теперь из соотношений (6.40) и (6.41). Для амплитуды угловых колебаний имеем

$$
\tilde{\theta}_{c}=\tilde{a}_{c} / K_{\theta}(j \omega)
$$

где

$$
K_{\theta}(j \omega)=\left\{L_{c l}(j \omega)+r L_{c l}^{\prime}(j \omega)+J \omega^{2} \Gamma_{l l}^{2}(j \omega)\left[\frac{L_{c l}(j \omega)}{\Gamma_{l l}(j \omega)}\right]^{\prime}\right\} / L_{c l}^{\prime}(j \omega)
$$

В качестве примера рассмотрим колебания консольного стержня с закрепленным на нем телом (рис.6.2). Из выражений, приведенных в строках 1 и 2 табл.6.1, находим при $x=1$ с учетом формул (6.9)

$$
\begin{array}{cc}
L_{l l}(j \omega)=\frac{l^{3}}{\widetilde{E}_{0} \widetilde{I}^{3}} \cdot \frac{B(\tilde{\xi})}{E(\tilde{\xi})}, & \Gamma_{l l}(j \omega)=\frac{l^{2}}{2 \tilde{E} \tilde{\xi}^{2}} \cdot \frac{S_{1}(\tilde{\xi})}{E(\tilde{\xi})} \\
L_{l l}^{\prime}(j \omega)=\Gamma_{l l}(j \omega), & \Gamma_{l l}^{\prime}(j \omega)=\frac{l}{\tilde{E} \mathrm{I} \tilde{\xi}} \cdot \frac{A(\tilde{\xi})}{E(\tilde{\xi})}
\end{array}
$$

где, как и ранее (см., например, (6.10), (6.11)),

$$
\tilde{\xi}=/ \cdot \sqrt[4]{\frac{\rho \cdot \omega^{2}}{\widetilde{E}_{0} \mathrm{I}}}=\xi \cdot\left(1+j \frac{\psi}{2 \pi}\right)^{-1 / 4}, \xi=/ \cdot \sqrt[4]{\frac{\rho \cdot \omega^{2}}{E_{0} \mathrm{I}}} .
$$

Подставляя (6.46) в соотношения (6.43), (6.45), получаем

$$
\begin{aligned}
& W_{c}(j \omega)=\frac{\tilde{E}_{0} I}{l^{3}}\left\{\frac{\tilde{\xi}^{3}\left[E(\tilde{\xi})-v \tilde{\xi}^{3} A(\tilde{\xi})\right]}{B(\tilde{\xi})+\eta \tilde{\xi} S_{1}(\tilde{\xi})+v \tilde{\xi}^{3} D(\tilde{\xi})+\eta^{2} \tilde{\xi}^{2} A(\tilde{\xi})}-\mu \cdot \xi^{4}\right\} \\
& K_{\theta}(j \omega)=\frac{2 l}{\tilde{\xi}} \cdot \frac{B(\tilde{\xi})+\eta \tilde{\xi} S_{1}(\tilde{\xi})+v \tilde{\xi}^{3} D(\tilde{\xi})+\eta^{2} \tilde{\xi}^{2} A(\tilde{\xi})}{S_{1}(\tilde{\xi})+2 \eta \tilde{\xi} A(\tilde{\xi})}
\end{aligned}
$$

где $\mu=M / \rho \cdot l ; . v=J / \rho \cdot l^{3} . \eta=r / l$.

Используя полученные результаты, построим амплитудно-частотные характеристики поступательных и угловых колебаний тела. С этой целью, полагая коэффициент поглощения $\psi$ малым и ограничивая разложение 
величин, содержащих $\psi$, линейными членами, приведем выражения (6.48) к виду, аналогичному (5.42)

$$
\begin{aligned}
& W_{c}(j \omega)=U_{c}(\omega)+j V_{c}(\omega) \\
& K_{\theta}(j \omega)=K_{1}(\omega)+j K_{2}(\omega)
\end{aligned}
$$

где $U_{c}(\omega)=\operatorname{Re} W_{c}(j \omega), . V_{c}(\omega)=\operatorname{Im} W_{c}(j \omega), K_{1}(\omega)=\operatorname{Re} K_{\theta}(j \omega), K_{2}(\omega)=\operatorname{Im} K_{\theta}(j \omega)$

Действительные составляющие величин (6.49) определяются выражениями (6.48) в которых, полагая $\psi=0$, согласно (6.47) следует принять $\tilde{\xi}=\xi, \widetilde{E}_{0}=E_{0}$. Для мнимых составляющих имеем

$$
\begin{aligned}
& V_{c}(\omega)=\left(\psi \xi^{3} E_{0} \mathrm{I} / 8 \pi l^{3}\right) \cdot\left[\begin{array}{l}
4 \xi \cdot T^{2}+B E+2 \eta \cdot \xi \cdot\left(4 \xi S T+E S_{1}\right)+ \\
+8 v \xi^{3} T(V-\xi U)+\eta^{2} \xi^{2}\left(4 \xi S^{2}+3 A E\right)+ \\
+v^{2} \xi^{6}(4 \xi U-A D)+v \eta \xi^{4}\left(A S_{1}-8 \xi S U\right)
\end{array}\right] \cdot \times \\
& \times \cdot\left(B+\eta \cdot \xi \cdot S_{1}+v \cdot \xi^{3} D+\eta^{2} \xi^{2} A\right)^{-2} \\
& K_{2}(\omega)=(\psi / / 16 \pi \xi) \cdot\left[\begin{array}{l}
B S_{1}-8 \xi \cdot T V+4 \eta \cdot \xi \cdot(A B-\xi \cdot V(2 \xi))+ \\
+2 v \xi^{3}(4 \xi \cdot U V-D S)+\eta^{2} \xi^{2}\left(A S_{1}-8 \xi S U\right)+ \\
+2 v \cdot \eta \cdot \xi^{4}\left(4 \xi \cdot U^{2}-A D\right)
\end{array}\right] \cdot \times \\
& \times \cdot\left(S_{1}+2 \eta \cdot \xi \cdot A\right)^{-2}
\end{aligned}
$$

(В формулах (6.50) опущен аргумент $\xi$ функций (6.9).)

На рис.6.4 приведены амплитудно-частотные характеристики колебаний центра масс тела (рис.6.4,a) и угловых колебаний тела (рис.6.4,б).

Уравнения резонансных кривых находятся из (6.42), (6.44) и с учетом (6.49) принимают вид

$$
a_{c}=\frac{F_{c}}{\left|W_{c}(j \omega)\right|}=\frac{F_{c}}{\sqrt{U_{c}^{2}(\omega)+V_{c}^{2}(\omega)}}, \theta_{c}=\frac{a_{c}}{\left|K_{\theta}(j \omega)\right|}=\frac{a_{c}}{\sqrt{K_{1}^{2}(\omega)+K_{2}^{2}(\omega)}}
$$



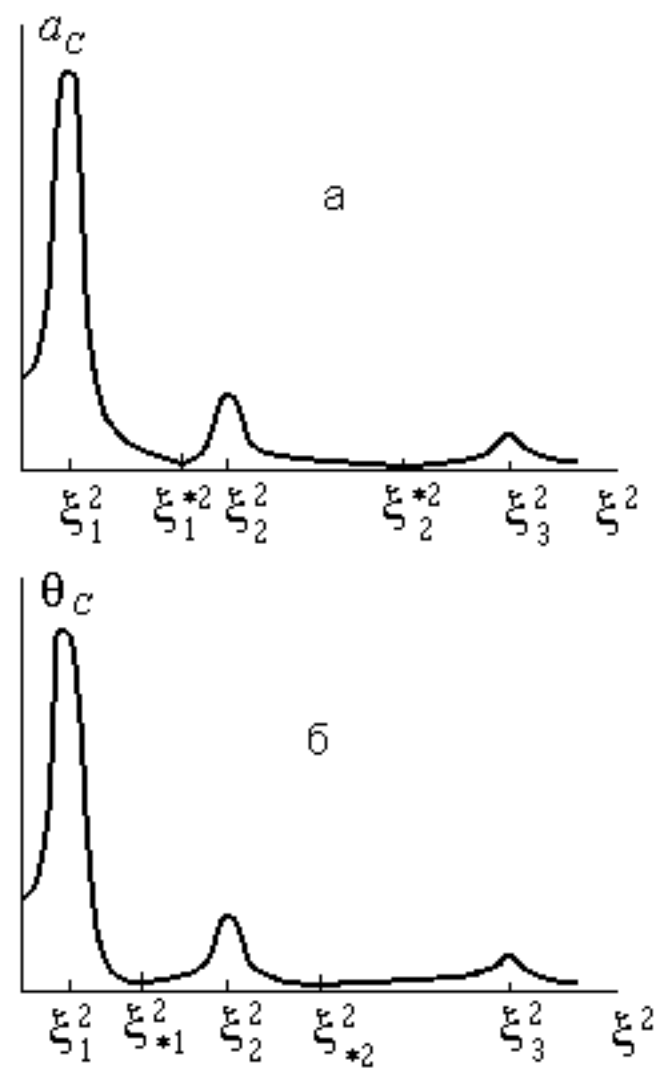

Pис.6.4

Согласно (6.51) резонансные частоты $\omega_{i}$ находятся из соотношения $U_{c}\left(\omega_{i}\right)=0$, которое после подстановки (6.48) дает следующее уравнение для определения собственных частот:

$$
E-v \cdot \xi_{i}^{3} A-\mu \cdot \xi_{i}\left(B+\eta \cdot \xi_{i} S_{1}+v \cdot \xi_{i}^{3} D+\eta^{2} \xi_{i}^{2} A\right)=0 .
$$

Амплитуда резонансных колебаний находится затем с помощью равенства $a_{c}=F_{c} / V_{c}\left(\omega_{i}\right)$, где $V_{c}\left(\omega_{i}\right)$ - значение (6.50), вычисленное после

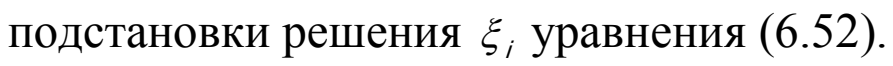

Для анализа зависимости резонансных частот $\xi_{i}^{2}$ от параметров $v, \eta, \mu$ уравнение (6.52) удобно привести к следующим соотношениям:

$$
\begin{gathered}
\mu=\frac{E-v \cdot \xi_{i}^{3} A}{\xi_{i}\left[B+\eta \cdot \xi_{i}\left(S_{1}+\eta \cdot \xi_{i} A\right)+v \cdot \xi_{i}^{3} D\right]} \\
v=\frac{E-\mu \cdot \eta \cdot \xi_{i}^{2}\left(S_{1}+\eta \cdot \xi_{i} A\right)-\mu \cdot \xi_{i} B}{\xi_{i}^{3}\left(\mu \cdot \xi_{i} D+A\right)} \\
\eta=\frac{-S_{1} \pm\left[S_{1}^{2}-4 A\left(B+v \cdot \xi_{i}^{3} D\right)+4 A\left(E-v \cdot \xi_{i}^{3}\right) / \mu \cdot \xi_{i}\right]^{1 / 2}}{2 \xi_{i} A}
\end{gathered}
$$


Аналогично равенство $U^{-1}(\omega)=0$ с учетом (6.48) приводит к уравнению, определяющему антирезонансные частоты $\xi_{i}^{*}$ колебаний центра масс тела

$$
\eta^{2} \xi_{i}^{* 2} A+\eta \cdot \xi_{i}^{*} S_{1}+v \cdot \xi_{i}^{* 3} D+B=0,
$$

которое можно записать в виде соотношений

$$
\begin{gathered}
v=-\frac{B+\eta \cdot \xi_{i}^{*}\left(S_{1}+\eta \cdot \xi_{i}^{*} A\right)}{\xi_{i}^{* 3} D} \\
\eta=\frac{-S_{1} \pm\left[S_{1}^{2}-4 A\left(B+v \cdot \xi_{i}^{* 3} D\right)\right]^{1 / 2}}{2 \xi_{i}^{*} A}
\end{gathered}
$$

Наконец, из (6.51) найдем выражение для антирезонансных частот $\xi_{* i}$ угловых колебаний тела $K_{1}^{-1}(\omega)=0$ или с учетом (6.48)

$$
\eta=-S_{1} / 2 \xi_{* i} A
$$

В формулах (6.52) - (6.59) опущены соответствующие аргументы $\xi_{i}, \xi_{i}^{*}, \xi_{* i}$ функций (6.9).

На рис.6.5,a построены зависимости (6.54) (кривые 1) и (6.57) (кривые 2) для трех низших форм колебаний при $\mu=0,5$ и $\eta=0,05$.

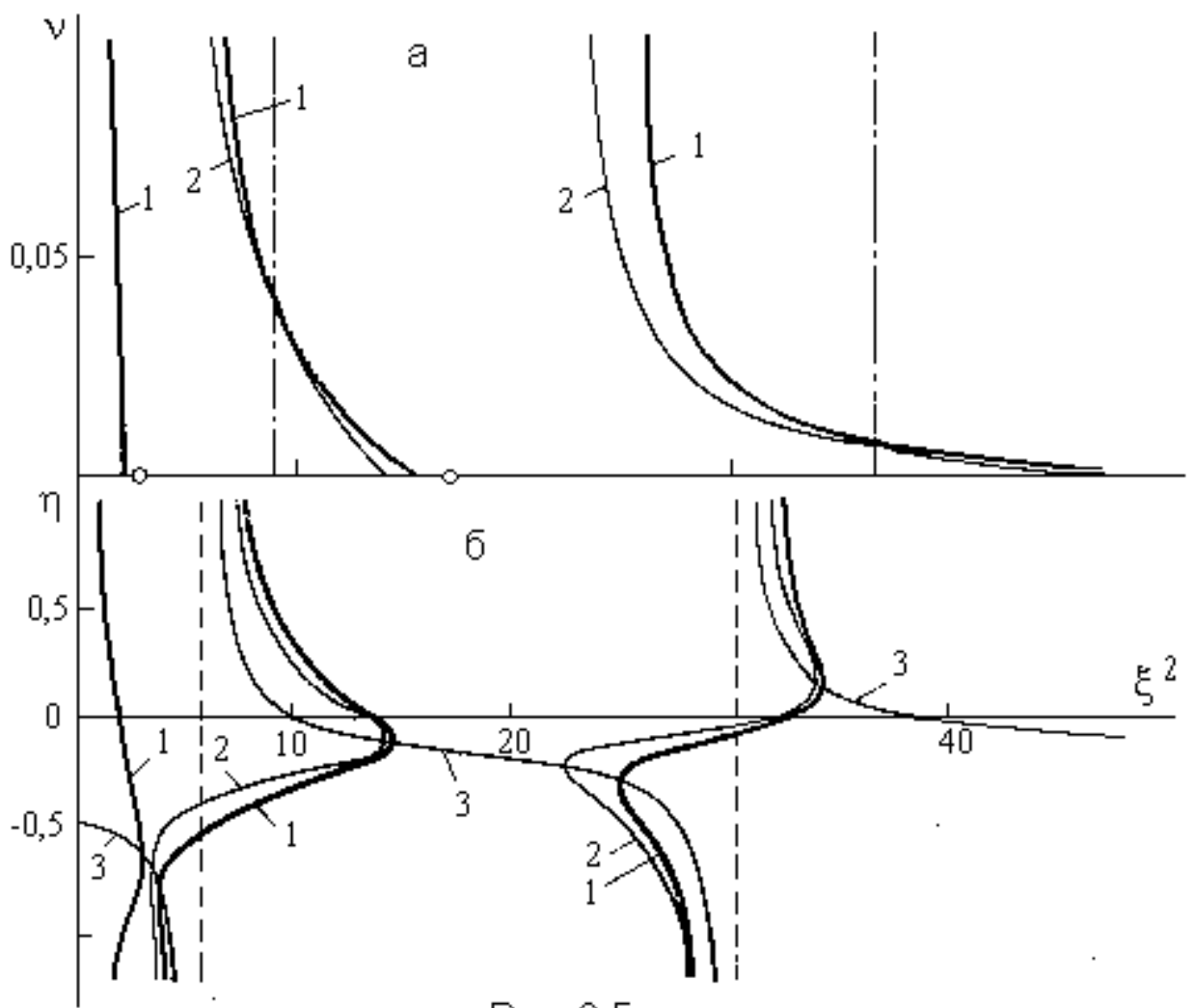

Pис.6.5 
Для сравнения на оси абсцисс кружками нанесены собственные частоты балки с точечной присоединенной массой, найденные из уравнения (6.51) при $v=\eta=0$. (В этом случае все полученные здесь результаты сводятся к формулам работ $[60,62]$, в которых не учитываются геометрические и инерционные параметры присоединенного тела при его угловых колебаниях.) Из рис.6.5,a видно, что влияние момента инерции тела на собственные свойства колебательной системы возрастает с увеличением номера формы.

Кривые 1 и 2 при $v \rightarrow \infty$ стремятся к асимпототам, являющимся соответственно квадратами корней уравнений

$$
\mu \cdot \xi \cdot D+A=0, \quad D=0
$$

Заметим, что при $v \rightarrow \infty$ из (6.48) имеем $\left|K_{\theta}(j \omega)\right| \rightarrow \infty$, и, следовательно, $\theta_{c} \rightarrow 0$, а динамическая жесткость

$$
W_{l}(j \omega)=-\frac{\tilde{E}_{0} \tilde{\xi}^{3}}{l^{3}}\left[\frac{A(\tilde{\xi})}{D(\tilde{\xi})}+\mu \cdot \tilde{\xi}\right]
$$

описывает систему, у которой присоединенное тело совершает поступательные колебания на всех частотах. Уравнения (6.60) определяют резонансные и антирезонансные частоты такой системы.

Рассматривая разность выражений (6.53) и (6.57), получим, что при

$$
A^{2} \xi_{i}^{2} \eta^{2}+A S_{1} \xi_{i} \eta+A B+E D=0
$$

значения этих выражений совпадают.

Отыскивая решение (6.62) и учитывая, что в соответствии с формулами (6.9) $S_{1}^{2} \equiv 4(A B+E D)$, получим

$$
\eta=\frac{-S_{1} \pm \sqrt{S_{1}^{2}-4(A B+E D)}}{2 \xi_{i} A}=-\frac{S_{1}}{2 \xi_{i} A}
$$

Таким образом, как следует из (6.59), (6.63), происходит касание кривых 1 и 2 на частотах, совпадающих с частотами антирезонансов $\xi_{* i}$ угловых колебаний, зависящих только от параметра $\eta$ и показанных на рис.6.5, а штрихпунктирными вертикальными прямыми. 
Функции (6.54), (6.58) определены на плоскости параметров $\left(\eta, \xi^{2}\right)$ соответственно при выполнении условий

$$
\begin{aligned}
& S_{1}^{2}-4 A\left(B+v \cdot \xi_{i}^{3} D\right)+4 A\left(E-v \cdot \xi_{i}^{3} A\right) / \mu \cdot \xi_{i} \geq 0 \\
& S_{1}^{2}-4 A\left(B+v \cdot \xi *_{i}^{3} D\right) \geq 0
\end{aligned}
$$

На рис.6.5,б построены зависимости (6.55) (кривые 1), (6.58) (кривые 2) и (6.59) (кривые 3) для трех низших форм колебаний при $\mu=0,5 ; v=0,01$ Как видно, графики всех трех функций имеют общие асимптоты при $\eta \rightarrow \pm \infty$, определяемые квадратами корней уравнения $A(\xi)=0$ и показанные на рис.6.5,б штриховыми линиями.

При выполнении равенств в условиях (6.64) зависимости (6.54), (6.58) принимают вид, совпадающий с уравнением (6.59) для антирезонансных частот угловых колебаний. Отсюда следует, что точки однозначности кривых 1 и 2 при любых значениях параметров $\mu, v$ расположены на линиях 3. Из (6.54), (6.58) следует, что при выполнении условия $E-v \cdot \xi_{i}^{3} A=0$ кривые 1 и 2 имеют две точки касания, расположенные симметрично относительно величины (6.59), как это показано на рис.6.5,б.

Проведенный анализ выявляет сильную зависимость резонансных и антирезонансных частот колебательной системы от инерционных и геометрических параметров присоединенного тела. Отметим, что на всех формах изгибных колебаний при определенных значениях параметров можно обеспечить совпадение резонансных частот с антирезонансами угловых колебаний тела. На этих частотах реализуются интенсивные поступательные колебания рабочего органа при его минимальных угловых колебаниях. О такой возможности мы уже упоминали в п.4.3. Таким образом, проведенные расчеты позволяют проводить синтез устройств, реализующих заданное движение рабочего органа ультразвуковой технологической системы. 
Мы подробно рассмотрели эту задачу, так как затронутые здесь вопросы недостаточно отражены в литературе. В известных работах, как правило, рассматриваются колебания систем с сосредоточенными массами [60,62,212]. В [194] обнаружено расхождение теоретических и экспериментальных результатов, возникающих из-за принятой идеализации присоединенного тела. В [220, 239] дается оценка влияния присоединенного тела, моделируемого сосредоточенной массой с эквивалентным моментом инерции, на собственные частоты и формы колебаний балки.

4. В заключение этого раздела кратко рассмотрим изгибные колебания стержневой системы при кинематическом возбуждении, используя известные величины динамических податливостей.

Пусть колебательная система кинематически возбуждается в некотором сечении $x=s$. Обозначив $\bar{F}_{s}, \bar{R}_{s}$ - амплитуды силы и момента в точке возбуждения, для амплитуды колебаний произвольного сечения $x$ запишем

$$
\bar{a}_{x}=L_{s x}(j \omega) \cdot \bar{F}_{s}+\Gamma_{s x}(j \omega) \cdot \bar{R}_{s}
$$

Отсюда для сечения $x=s$ с учетом (6.1) имеем

$$
\begin{aligned}
& \bar{a}_{s}=L_{s s}(j \omega) \cdot \bar{F}_{s}+\Gamma_{s s}(j \omega) \cdot \bar{R}_{s} \\
& \bar{\theta}_{s}=L_{s s}^{\prime}(j \omega) \cdot \bar{F}_{s}+\Gamma_{s s}^{\prime}(j \omega) \cdot \bar{R}_{s}
\end{aligned}
$$

Из (6.66) видно, что кинематическое возбуждение изгибных колебаний можно осуществить, сообщая сечению $s$ поступательные с амплитудой $\bar{a}_{s}$ или угловые с амплитудой $\bar{\theta}_{s}$ колебания. Если одна из этих величин задана, то для определения оставшихся трех неизвестных систему уравнений (6.66) необходимо дополнить соответствующим условием связи, которое, как правило, легко записывается , исходя из физического содержания задачи.

Обратимся к некоторым примерам. Рассмотрим колебания однородного стержня $(-l \leq x \leq l)$, среднему сечению $x=s=0$ которого сообщается возвратно-поступательное движение по закону $u_{0}(t)=a_{0} \mathrm{e}^{j \omega \cdot t} . \mathrm{K}$ 
такой схеме приводятся в силу симметрии системы, показанные ранее на рис.4.8,a,б.

В этом случае очевидно $\tilde{\theta}_{0}=0, \Gamma_{00}(j \omega)=0$, и из $(6.66),(6.65)$ находим при $s=0$

$$
\begin{gathered}
\tilde{F}_{0}=a_{0} / L_{00}(j \omega) \\
\tilde{a}_{x}=a_{0} L_{0 x}(j \omega) / L_{00}(j \omega)
\end{gathered}
$$

Подставляя в (6.68) соответствующие величины динамических податливостей из табл.6.1 (строка 8), получаем с учетом (6.9)

$$
\tilde{a}_{x}=2 a_{0} \frac{S(\tilde{\xi}) \cdot S\left(\tilde{\xi} \frac{x-l}{l}\right)-V(\tilde{\xi}) \cdot T\left(\tilde{\xi} \frac{x-l}{l}\right)}{E(\tilde{\xi})}
$$

Дальнейший анализ проводится по методике, аналогичной изложенной в П.5 предыдущего Параграфа. Так, например, максимальные амплитуды достигаются на частотах, определяемых из уравнения $E(\xi)=0$ и совпадающих с собственными частотами консольного стержня (см. строки 1 и 2 табл.6.1 и формулы (6.34), (6.35)). Для двух первых форм колебаний из таблиц справочника [5] находим

$$
\xi_{1}=1,87 ; \xi_{2}=4,69
$$

С достаточной для практических расчетов точностью для определения резонансных частот можно пользоваться соотношением

$$
\xi_{n}=(2 n-1) \pi / 2, \quad n=2,3, \ldots
$$

Считая по-прежнему коэффициент поглощения ж малым, из (6.69) найдем амплитуду резонансных колебаний сечения $x=l$

$$
a_{l}=\frac{16 \pi \cdot a_{0}}{\psi \cdot \xi_{n}}\left|\frac{S\left(\xi_{n}\right)}{B\left(\xi_{n}\right)}\right|
$$

Для двух первых форм колебаний с помощью таблиц [169] вычисляем $a_{l}=10,02 a_{0} / \psi$ и $a_{l}=5,45 a_{0} / \psi$. Для высших форм $(n>1)$ с учетом (6.9), (6.71) выражение (6.72) можно приближенно привести к виду

$$
a_{l}=8 \pi \cdot a_{0} / \psi \cdot \xi_{n}=16 a_{0} / \psi \cdot(2 n-1), \quad n=2,3, \ldots
$$


Заметим, что соотношения (6.67), (6.68) совпадают с выражениями (5.45), (5.46), описывающими продольные колебания стержней при кинематическом возбуждении. Поэтому в данном случае оказываются справедливыми выводы П.5.5, касающиеся, в частности, расчета концентраторов ультразвуковых колебательных систем.

При использовании стержня в качестве концентратора изгибных колебаний его параметры должны удовлетворять равенству (5.47), которое после подстановки соответствующих величин принимает вид $A(\xi)=0$ и с достаточной для практических расчетов точностью дает значения

$$
\xi_{n}=(4 n-1) \pi / 4, \quad n=1,2, \ldots
$$

С учетом принятых в (6.10), (6.11) обозначений находим длину концентратора, настроенного на рабочую частоту $\omega$

$$
I=\xi_{n} \cdot \sqrt[4]{\frac{E_{0} \mathrm{I}}{\rho \cdot \omega^{2}}}=\frac{(4 n-1) \pi}{4} \cdot \sqrt[4]{\frac{E_{0} \mathrm{I}}{\rho \cdot \omega^{2}}}
$$

Коэффициент усиления концентратора изгибных колебаний найдем с помощью (5.49) после подстановки соответствующих величин из строки 6 табл.6.1

$$
K=\frac{a_{1}}{a_{0}}=\frac{\operatorname{ch} \xi_{n} \cdot \cos \xi_{n}}{S\left(\xi_{n}\right)} \approx \sqrt{2}=1,41
$$

В заключение этого раздела рассмотрим изгибные колебания той же системы, сечению $x=s=0$ которой сообщаются угловые колебания $\Theta_{0}(t)=\theta_{0} e^{j \omega \cdot t}$. В этом случае $L_{00}(j \omega)=L_{00}^{\prime}(j \omega)=0$ и из (6.65), (6.66) находим при $s=0$

$$
\begin{aligned}
& \bar{R}_{0}=\theta_{0} / \Gamma_{00}^{\prime}(j \omega) \\
& \bar{a}_{x}=\theta_{0} \Gamma_{0 x}(j \omega) / \Gamma_{00}^{\prime}(j \omega)
\end{aligned}
$$

После подстановки в (6.77) соответствующих величин из табл.6.1 (строка 5) получаем

$$
a_{x}=2 \theta_{0} \frac{l}{\widetilde{\xi}} \cdot \frac{T(\tilde{\xi}) \cdot S\left(\tilde{\xi} \frac{l-x}{l}\right)-S(\tilde{\xi}) \cdot T\left(\tilde{\xi} \frac{l-x}{l}\right)}{E(\tilde{\xi})}
$$

Из (6.78) следует, что резонансные частоты системы определяются 
полученными выше значениями (6.70), (6.71). Для резонансных амплитуд $a_{l}$ конца $x=/$ стержня по аналогии с (6.72) находим

$$
a_{l}=\frac{16 \pi \cdot 1 \theta_{0}}{\psi \cdot \xi_{n}} \cdot\left|\frac{T\left(\xi_{n}\right)}{B\left(\xi_{n}\right)}\right|
$$

Для двух первых форм колебаний (6.70) с помощью таблиц [169] имеем $a_{l}=13,51 \cdot 1 \theta_{0} / \psi$ и $a_{l}=5,49 \cdot 1 \theta_{0} / \psi$. Для высших форм $(n>2)$ выражение (6.79) можно приближенно привести к виду (6.73), где следует положить $a_{0}=l \theta_{0}$. Полученные результаты будут использованы при исследовании резонансных колебаний ультразвуковых стержневых систем, работающих на нелинейную технологическую нагрузку (Параграф 10). 


\section{7. Динамические характеристики электроакустических}

преобразователей.

1. В рамках обобщенной схемы ультразвуковой технологической машины (рис.4.9) мы рассмотрели динамические характеристики типичных элементов ультразвуковых колебательных систем. Перейдем к построению величин, характеризующих динамические свойства возбудителей колебаний, ограничиваясь рассмотрением наиболее употребительных в ультразвуковой технике магнитострикционных и пьезоэлектрических преобразователей.

Рассмотрим магнитострикционный преобразователь [170] с сердечником, выполненным в виде замкнутого ярма, на котором размещено $N$ витков обмотки возбуждения (рис.7.1).

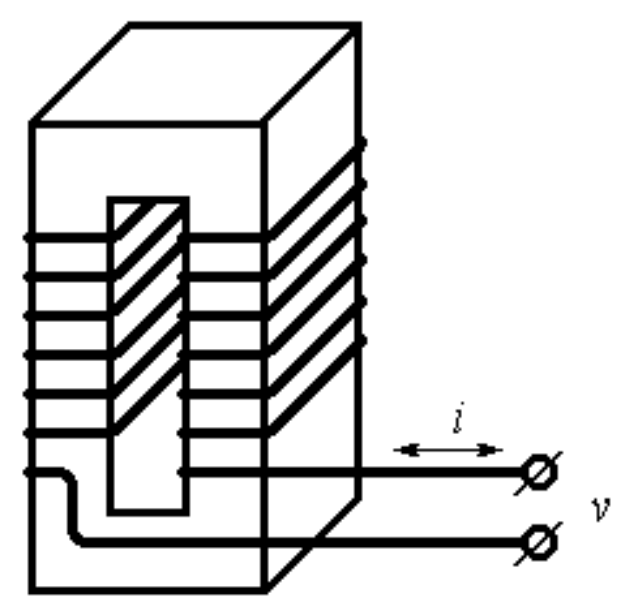

Pnc.7.1

Преобразователь питается от внешнего источника, создающего напряжение

$$
v=v_{m}+\widetilde{v}_{0} e^{j \omega \cdot t}
$$

В результате по обмотке возбуждения течет ток

$$
i=i_{m}+\tilde{i}_{0} e^{j \omega \cdot t}
$$

В (7.1), (7.2) величины $v_{m}, i_{m}$ - постоянные составляющие, $\widetilde{v}_{0}, \tilde{i}_{0}$ комплексные амплитуды переменных составляющих электрического напряжения и силы тока. 
Проходящий по обмотке возбуждения ток наводит в сердечнике магнитное поле напряженностью [207]

$$
H_{i}=\frac{N}{l} i,
$$

где $l$ - длина сердечника (рис.7.1).

Прямой магнитострикционный эффект заключается в том, что под действием магнитного поля в материале сердечника возникают механические напряжения

$$
\sigma_{H}=-\gamma \cdot B=-\gamma \cdot \widetilde{\mu} \cdot H
$$

где $B$ - магнитная индукция; $\gamma$ - магнитострикционная постоянная; $\tilde{\mu}$ комплексная магнитная проницаемость среды.

Равенство (7.4) означает, что магнитное поле приводит к появлению сжимающих напряжений в зажатом образце.

Обратный магнитострикционный эффект выражается в том, что изменение деформированного состояния сердечника порождает изменение его магнитного состояния. Изменение магнитного поля вследствие деформации $\varepsilon$ выражается равенством

$$
H_{\varepsilon}=\gamma \cdot \varepsilon
$$

Если материал сердечника одновременно подвергается механической и магнитострикционной деформации, то возникающее напряжение в материале

$$
\sigma=\widetilde{E}_{0} \varepsilon-\gamma \cdot \tilde{\mu} \cdot H,
$$

а суммарное поле в сердечнике, обусловленное проходящим по обмотке током и деформацией, согласно (7.3), (7.5)

$$
H=\frac{N}{l} i+\gamma \cdot \varepsilon
$$

Подставив (7.7) в (7.6), получим соотношение, связывающее механические напряжения и деформации сердечника магнитострикционного преобразователя с его магнитными и электрическими параметрами 


$$
\sigma=\widetilde{E}_{1} \varepsilon-\frac{\tilde{A}}{S} i
$$

где $\tilde{A}=\gamma \cdot \tilde{\mu} \cdot S N / /$ - коэффициент электромеханической связи;

$$
\tilde{E}_{1}=\widetilde{E}_{0}-\gamma^{2} \widetilde{\mu}=E_{1}\left(1+j \frac{\psi_{1}}{2 \pi}\right),
$$

$E_{1}=E_{0}-\gamma^{2} \mu$ - модуль упругости магнитострикционного материала при постоянном поле подмагничивания $H_{m}=\frac{N}{n} i_{m} ; \psi_{1}$ - приведенный коэффициент поглощения, учитывающий механический и магнитный гистерезис.

Из (7.8) с учетом вида рассматриваемых процессов легко получаются аналогичные соотношения для постоянных составляющих и комплексных амплитуд периодических составляющих входящих в (7.8) величин. Обратим внимание на то, что величины $\gamma$ и $\mu$ следует трактовать как некоторые линеаризованные значения в окрестности постоянного магнитного поля, обусловленного током подмагничивания $i_{m}$

Соотношение (7.8) описывает напряженное состояние элемента $d x$ сердечника. Изменение его магнитного состояния наводит в соответствующем элементе обмотки противоэлектродвижущую силу

$$
d e=-\frac{N}{l} S \frac{\partial \cdot B}{\partial \cdot t} d x=-\frac{N}{l} S \mu \frac{\partial \cdot H}{\partial \cdot t} d x
$$

или с учетом (7.7)

$$
d e=-\frac{\partial}{\partial \cdot t}\left(\frac{\tilde{L}}{l} i+\tilde{A} \varepsilon\right) d x
$$

где $\tilde{L}=\tilde{\mu} \cdot S \frac{N^{2}}{/}-$ индуктивность преобразователя.

Полную противоэлектродвижущую силу обмотки найдем, проинтегрировав (7.10) по всей длине преобразователя

$$
e=-\frac{d}{d t}\left[\tilde{L} i+\tilde{A}\left(u_{1}-u_{0}\right)\right]
$$

где $u_{l}, u_{0}$ - смещения конечных сечений сердечника. 
Теперь с учетом (7.11) и соотношения $i=d q / d t(q-$ электрический заряд) запишем уравнение электрической цепи преобразователя

$$
\widetilde{L} \frac{d^{2} q}{d t^{2}}+I_{0} \frac{d q}{d t}+\frac{1}{C} q+\tilde{A} \frac{d}{d t}\left(u_{l}-u_{0}\right)=v(t)
$$

где $r_{0}, C$ - сопротивление и емкость цепи.

Полученные уравнения (7.8) и (7.12) позволяют полностью описать колебания магнитострикционного преобразователя.

2. Рассмотрим некоторые характерные случаи. Пусть сердечник, длина которого мала по сравнению с длиной упругой волны в материале, т.е. удовлетворяет условию (5.13), закреплен в сечении $x=0$ и нагружен силой $f_{12}$ в сечении $x=l$ (рис.7.2).

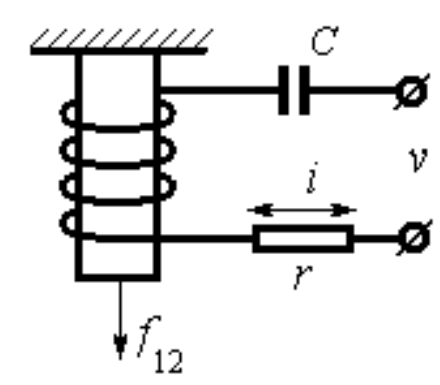

Pnc.7.2.

В этом случае $\sigma=f_{12} / S, . \varepsilon=u_{l} / l, u_{0}=0$

Учитывая характер рассматриваемых процессов, из (7.8), (7.12) получаем следующие уравнения для амплитуд:

$$
\begin{aligned}
& \tilde{F}_{12}=\tilde{k}_{1} \tilde{a}_{1}-j \omega \cdot \tilde{A} \tilde{q}_{0} \\
& \tilde{v}_{0}=j \omega \cdot \tilde{A} \tilde{a}_{1}+j \omega \cdot Z(j \omega) \tilde{q}_{0}
\end{aligned}
$$

где $\widetilde{k}_{1}=\widetilde{E}_{1} S / /$ - динамическая жесткость сердечника преобразователя при $\tilde{i}_{0}=0 \quad ; \quad Z(j \omega)=r+j\left\lfloor\omega \cdot L-(\omega \cdot C)^{-1}\right\rfloor \quad-\quad$ комплексное сопротивление электрической цепи при $\tilde{a}_{1}=0 ; r=r_{0}+r_{1} ; r_{1}=\frac{S N^{2} \omega^{2}}{/} \operatorname{Im} \tilde{\mu}$ - эквивалентное добавочное сопротивление, учитывающее магнитный гистерезис.

Уравнения (7.13) совпадают по форме с уравнениями четырехполюсника (4.15), коэффициенты которого

$$
\alpha_{11}=\widetilde{k}_{1}, \alpha_{12}=\alpha_{21}=j \omega \tilde{A}, \alpha_{22}=j \omega Z(j \omega)
$$


Подставив эти величины в соотношения (4.17), найдем амплитуду колебаний ненагруженного преобразователя $\left(\widetilde{F}_{12}=0\right)$ при питании от источника тока

$$
\tilde{a}_{1}^{*}=\tilde{A} \cdot i_{0} / \tilde{k}_{1}
$$

Пусть преобразователь возбуждает колебания механической системы с динамической жесткостью $W(j \omega)$. Амплитуду колебаний нагруженного таким образом преобразователя найдем, используя выражение (4.22), в котором следует положить $\widetilde{F}_{l}=0, L_{00}(j \omega)=W^{-1}(j \omega)$, $L_{n}(j \omega)=\widetilde{k}_{1}^{-1}$. В результате, учитывая (4.19) и (7.14), получим

$$
\tilde{a}_{1}=\frac{\tilde{i_{0}} \tilde{A}}{\tilde{k}_{1}+W(j \omega)}
$$

Из (7.16) видно, что возбуждаемая магнитострикционным преобразователем система с питанием от источника тока ведет себя подобно механическому элементу с приведенной динамической жесткостью $W_{0}(j \omega)=\widetilde{k}_{1}+W(j \omega)$ под действием периодической силы с амплитудой $\tilde{F}=\tilde{A} \tilde{i}_{0}$. Дальнейший анализ проводится по методике, изложенной в п.5.4.

Примем для определенности, что преобразователь возбуждает простейшую колебательную систему (рис.7.3)

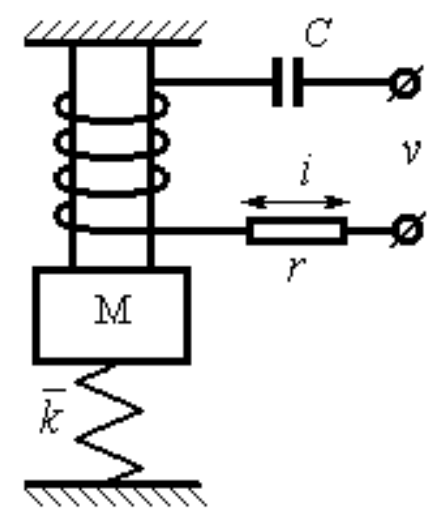

Puc.7.3

с динамической жесткостью

$$
W(j \omega)=\left(k_{2}-M \omega^{2}\right)+j \omega \cdot b_{2}
$$


где $M, k_{2}, b_{2}$ - масса, жесткость и коэффициент сопротивления системы.

Представив коэффициент $\tilde{A}$ в виде

$$
\tilde{A}=A\left(1-j \frac{\chi}{4 \pi}\right)
$$

будем, как и ранее, предполагать малыми величины $\psi_{1}, b_{2}, r, \mathcal{\chi}$, характеризующие диссипативные свойства механической, электрической и магнитной цепей системы. Из (7.16) с учетом (7.9), (7.17) и принятых в (7.13) обозначений имеем

$$
a_{1}=\frac{\tilde{i_{0}} A}{k} \cdot \frac{1-j \frac{\chi}{4 \pi}}{\left(1-\xi^{2}\right)+j \xi \cdot \eta}
$$

где $\xi=\omega / v ; v=\sqrt{k / M} ; \eta=b / \sqrt{k M} ; k=k_{1}+k_{2} ; b=b_{1}+b_{2}, b_{1}=\frac{k_{1} \psi_{1}}{2 \pi \cdot \omega}$.

Отсюда по аналогии с (5.35), (5.40) находим резонансную частоту и амплитуду

$$
\xi=1, a_{1}=i_{0} A / K \eta
$$

Подставив (7.19) во второе равенство (7.13), получим, ограничиваясь величинами первого порядка малости, напряжение питания преобразователя

$$
\widetilde{v}_{0}=\frac{\widetilde{i}_{0} L v}{j \xi\left[\left(1-\xi^{2}\right)+j \xi \cdot \eta\right]} \cdot\left\{\begin{array}{l}
\left(1-\xi^{2}\right)\left(\xi_{1}^{2}-\xi^{2}\right)-\xi_{M}^{2} \xi^{2}+ \\
+j \xi\left[\eta_{1}\left(1-\xi^{2}\right)+\eta \cdot\left(\xi_{1}^{2}-\xi^{2}\right)+\xi_{M}^{2} \xi \frac{\chi}{4 \pi}\right]
\end{array}\right\}
$$

где $\xi_{1}=1 / \sqrt{1 / v \cdot L C} ; \xi_{M}=H / \sqrt{k L} ; \eta_{1}=r / L v$.

На рис.7.4 показаны амплитудно-частотные характеристики колебаний массы $M$ и напряжения питания преобразователя при возбуждении от источника тока. Графики построены с помощью соотношений (7.19), (7.21) при $\eta=\eta_{1}=0,2, \chi=0, \xi_{M}=0,71$.

В общем случае $v_{1} \neq 0$ (кривая 1 на рис.7.4,б) напряжение питания $v_{0} \rightarrow \infty$ при $\xi \rightarrow 0$ и $\xi \rightarrow \infty$ из-за наличия емкости и индуктивности в цепи 
питания. Эта кривая имеет локальный максимум на частоте механического резонанса $(\xi=1)$ и два минимума на частотах, определяемых из уравнения

$$
\left(1-\xi^{2}\right)\left(\xi_{1}^{2}-\xi^{2}\right)-\xi_{M}^{2} \xi^{2}=0
$$

решения которого имеют вид

$$
\xi_{1,2}^{* 2}=\frac{1+\xi_{1}^{2}+\xi_{M}^{2}}{2} \pm \sqrt{\left(\frac{1+\xi_{1}^{2}+\xi_{M}^{2}}{2}\right)^{2}-\xi_{1}^{2}}
$$

При построении кривой 1 принято $\xi_{1}=2$.
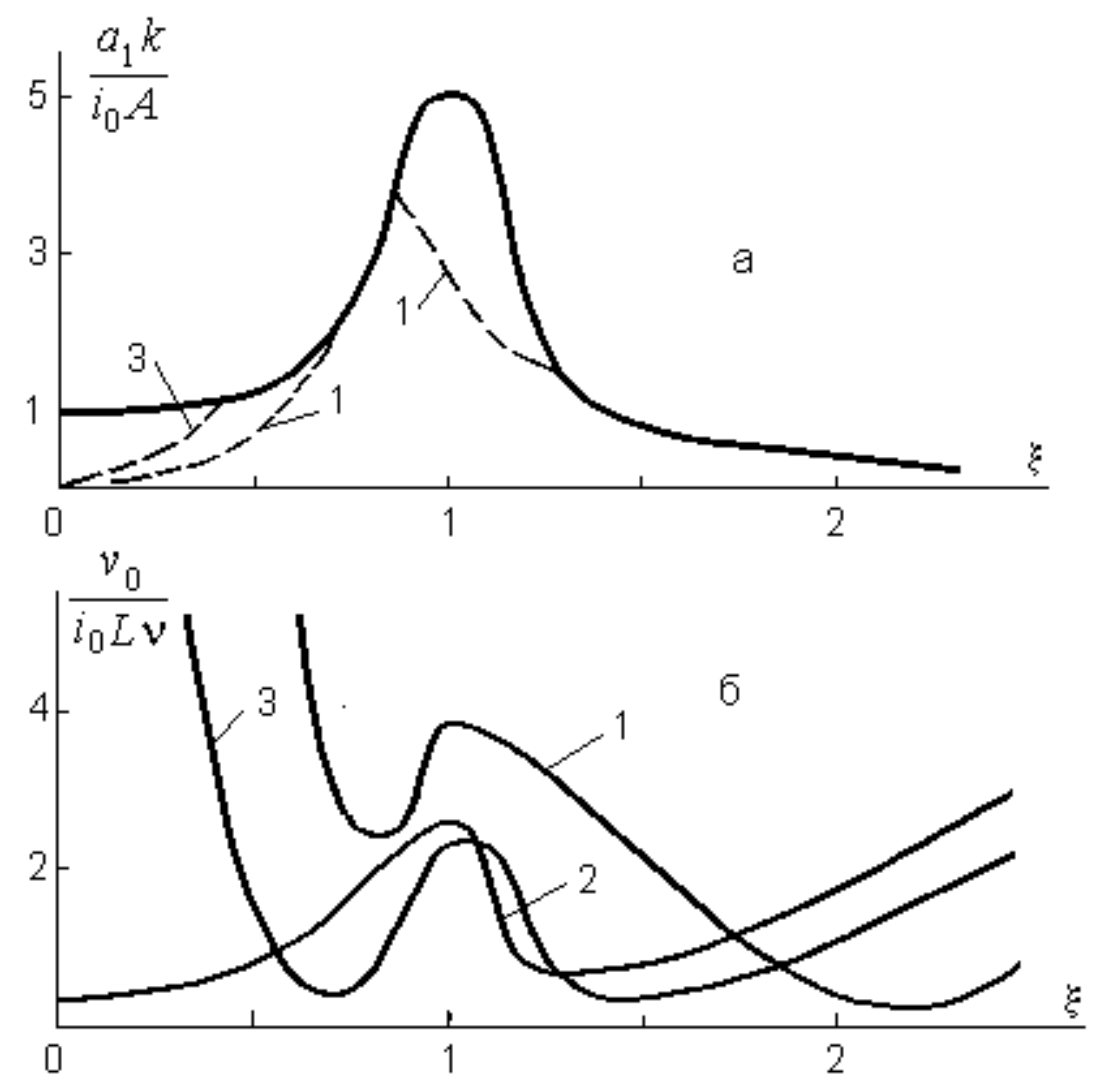

Pис.7.4

Кривая 2 на рис.7.4,б соответствует случаю $\xi_{1}=0$; кривая 3 - случаю $\xi_{1}=1$, когда равны собственные частоты парциальных механической и электрической систем [185], т.е. $\sqrt{k / M}=1 / \sqrt{L C}$. Можно показать, что такое согласование парциальных систем сводит к минимуму напряжения питания в окрестности резонанса $\xi=1$ и частот (7.22). Отметим, что при полном отсутствии диссипации $\left(\eta_{1}=\eta_{2}=\chi=0\right)$ имеем $a_{1}(1)=\infty, \quad v_{0}(1)=\infty$, $V_{0}\left(\xi_{1,2}^{*}\right)=0$. 
Отыскание параметров колебаний системы при ее возбуждении от источника напряжения $\left(v_{0}=\right.$ const $)$ целесообразно провести, используя соотношения (7.19) и (7.21). С этой целью из (7.21) выразим амплитуду силы тока

$$
\tilde{i}_{0}=\frac{j \xi \cdot \tilde{v}_{0}\left[\left(1-\xi^{2}\right)+j \xi \cdot \eta\right]}{v \cdot L\left\{\left(1-\xi^{2}\right)\left(\xi_{1}^{2}-\xi^{2}\right)-\xi_{M}^{2} \xi^{2}+j \xi \cdot\left[\eta_{1}\left(1-\xi^{2}\right)+\eta \cdot\left(\xi_{1}^{2}-\xi^{2}\right)+\xi_{M}^{2} \xi \frac{\chi}{4 \pi}\right]\right\}}
$$

и, подставив полученное выражение в (7.19), найдем амплитуду колебаний массы $M$ (рис.7.)

$$
\tilde{a}_{1}=\frac{j \xi \cdot \xi_{M}^{2} \tilde{v}_{0}\left(1-j \frac{\chi}{4 \pi}\right)}{A v \cdot\left\{\left(1-\xi^{2}\right)\left(\xi_{1}^{2}-\xi^{2}\right)-\xi_{M}^{2} \xi^{2}+j \xi\left[\eta_{1}\left(1-\xi^{2}\right)+\eta\left(\xi_{1}^{2}-\xi^{2}\right)+\xi_{M}^{2} \xi \frac{\chi}{4 \pi}\right]\right\}}
$$

На рис.7.5 приведены амплитудно-частотные характеристики колебаний массы $M$ и тока в обмотке возбуждения при питании от источника напряжения, построенные при тех же значениях параметров, что и рис.7.4.
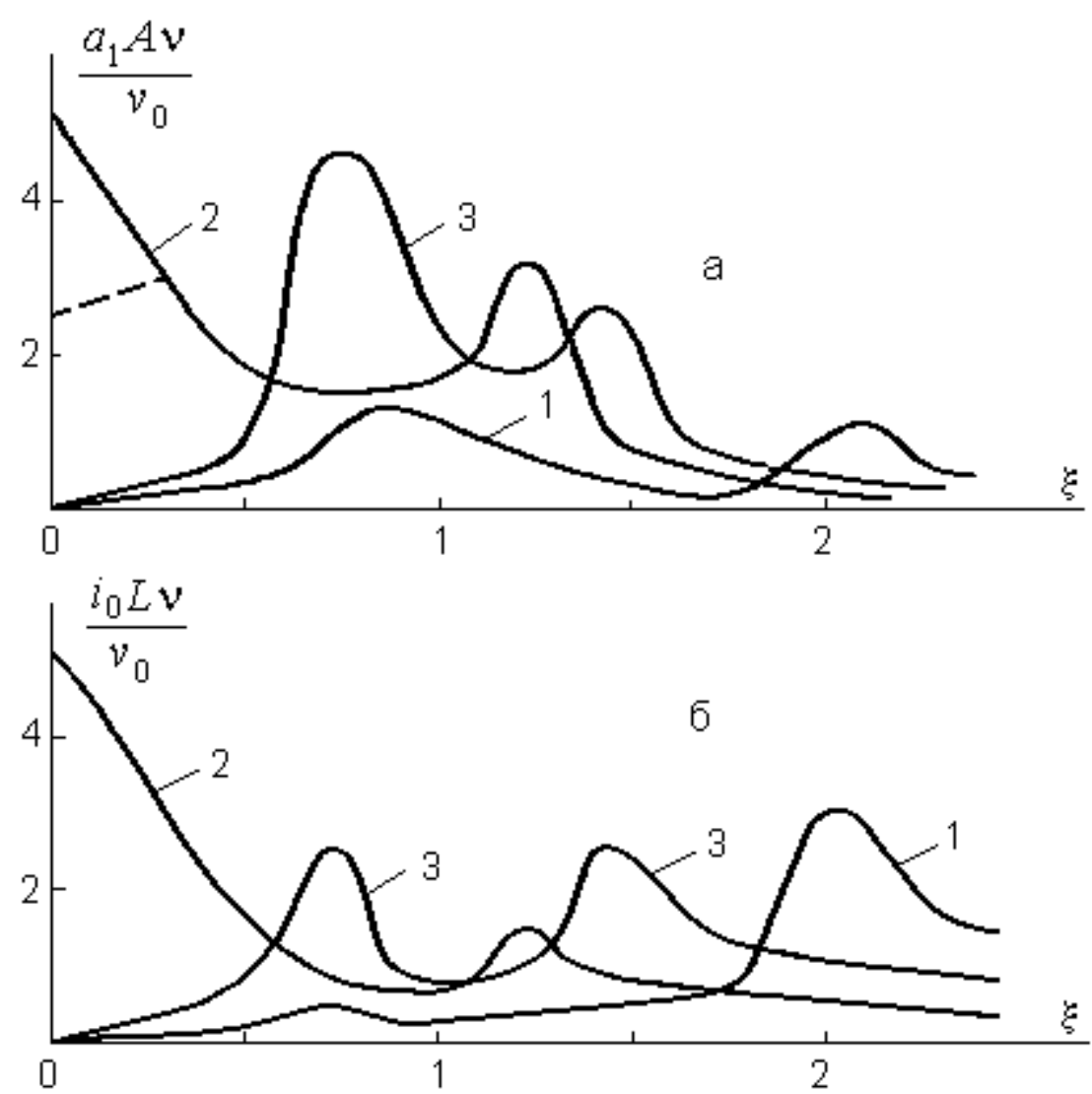

Pис.7.5 
Как следует из (7.24), (7.25), резонансные частоты, на которых достигается максимум амплитуд смещения и силы тока, определяются выражением (7.23). Легко показать, что минимум амплитуды (7.25) лежит в окрестности частоты, определяемой равенством $\xi=\sqrt{\left(1+\xi_{1}^{2}+\xi_{M}^{2}\right) / 2}$ и, следовательно, расположенной между резонансными частотами (7.23).

Уже из этих простых примеров видно, что свойства колебательной системы существенно зависят как от соотношения параметров механической и электрической парциальных систем, так и от способа питания магнитостриктора. Это аналогично известной в механике ситуации, возникающей при силовом и кинематическом возбуждении и рассмотренной в предыдущих разделах.

Полученные соотношения применимы и в том случае, когда генератор питания не является идеальным источником тока или напряжения. Если, например, генератор имеет ограничение напряжения или тока, как это показано штриховыми линиями на рис.7.4,б и рис.7.5,б, то на соответствующих участках амплитудно-частотных характеристик механических колебаний будут реализовываться ветви, изображенные штриховыми линиями на рис.7.4,a и рис.7.5,a.

3. Рассмотрим магнитострикционный преобразователь с сердечником, длина которого соизмерима или значительно превышает длину волны в его материале. Для определенности будем по-прежнему предполагать, что конец $x=0$ сердечника жестко закреплен, а к концу $x=$ $l$ приложена внешняя сила $f_{12}$. В этом случае деформация сердечника не является однородной и связана со смещением $u_{x}$ сечения $x$ соотношением $\varepsilon_{x}=\partial u_{x} / \partial x$. Записав уравнение движения элемента $d x$ сердечника $\rho S d x \frac{\partial^{2} u_{x}}{\partial \cdot t^{2}}=S \frac{\partial \cdot \sigma_{x}}{\partial \cdot x} d x$, получим с учетом (7.8) уравнение продольных колебаний

$$
\rho \frac{\partial^{2} u_{x}}{\partial \cdot t^{2}}-\tilde{E}_{1} \frac{\partial^{2} u_{x}}{\partial \cdot x^{2}}=0
$$


решение которого должно удовлетворять следующим граничным условиям

$$
\left.u_{x}\right|_{x=0}=0,\left.\tilde{E}_{1} S \frac{\partial \cdot u_{x}}{\partial \cdot x}\right|_{x=1}=f_{12}+\tilde{A} i=f_{12}+f_{l}
$$

Уравнение (7.26) с условиями (7.27) совпадает с уравнением (5.4) колебаний стержня с закрепленным концом под действием периодических сил, приложенных к свободному концу. Его решение запишем, используя операторы динамической податливости, полученные в разделе 5 этой главы. Для амплитуд колебаний сечений сердечника имеем

$$
\tilde{a}_{x}=L_{l x}(j \omega)\left(\tilde{F}_{12}+\widetilde{F}_{l}\right)=L_{l x}(j \omega)\left(\widetilde{F}_{12}+\tilde{A} \tilde{i}_{0}\right)
$$

Из (7.28) при $x=l$ и уравнения электрической цепи (7.12) с учетом (7.27) получим уравнения четырехполюсника вида аналогичного (7.13)

$$
\begin{aligned}
& \tilde{F}_{12}=W_{l l}^{(1)}(j \omega) \tilde{a}_{l}-j \omega \cdot \tilde{A} \tilde{q}_{0} \\
& \tilde{v}_{0}=j \omega \cdot \tilde{A} \tilde{a}_{l}+j \omega \cdot Z(j \omega) \tilde{q}_{0}
\end{aligned}
$$

Для этого четырехполюсника $\quad \alpha_{11}=W_{/ /}(j \omega), \quad$ a $\quad$ остальные коэффициенты совпадают с (7.14).

Амплитуда колебаний ненагруженного преобразователя $\left(F_{12}=0\right)$ при питании от источника тока

$$
\tilde{a}_{l}^{*}=\tilde{A} i_{0} / W_{l l}(j \omega)
$$

и при питании от источника напряжения

$$
\tilde{a}_{l}^{*}=\frac{j \omega \cdot \tilde{A} \tilde{v}_{0}}{j \omega \cdot Z(j \omega) W_{l l}(j \omega)-\omega^{2} A^{2}},
$$

где динамическая жесткость $W_{/ /}(j \omega)=L_{/ /}^{-1}(j \omega)$ определяется выражением (5.111).

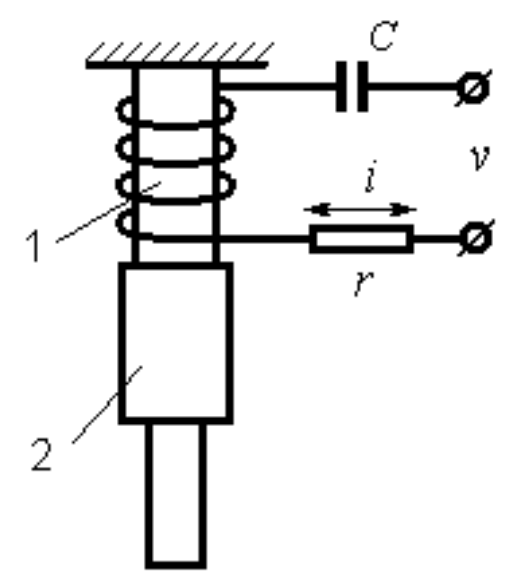

Pис.7.6 
Используя уравнения (7.29), опишем колебания системы (рис.7.6), в которой к магнитострикционному преобразователю 1 присоединен стержневой волновод 2.

Однотипные величины, относящиеся к преобразователю и волноводу, будем отмечать индексами 1 и 2, как мы это делали при рассмотрении составных стержневых систем в п.5.10. Для амплитуды колебаний торца преобразователя имеем

$$
\tilde{a}_{l}^{(1)}=\tilde{a}_{0}^{(2)}=\frac{\tilde{A} \tilde{i}_{0}}{W_{l l}^{(1)}(j \omega)+W_{00}^{(2)}(j \omega)}
$$

Принимая во внимание (5.66), найдем амплитуду колебаний свободного торца волновода

$$
\tilde{a}_{l}^{(2)}=\frac{L_{0 l}^{(2)}(j \omega)}{L_{00}^{(2)}(j \omega)} \cdot \frac{\tilde{A} \tilde{i}_{0}}{W_{l l}^{(1)}(j \omega)+W_{00}^{(2)}(j \omega)}
$$

Тот же результат получается непосредственно с помощью выражения (4.24).

После подстановки найденной амплитуды во второе из уравнений (7.29) получим напряжение источника питания магнитостриктора

$$
\tilde{v}_{0}=i_{0}\left[Z(j \omega)+j \omega \cdot A^{2} \frac{L_{0 l}^{(2)}(j \omega)}{L_{00}^{(2)}(j \omega)\left[W_{l l}^{(1)}(j \omega)+W_{00}^{(2)}(j \omega)\right]}\right]
$$

Характеристики преобразователя, получающего питание от источника напряжения, могут быть найдены с помощью выражений (7.33), (7.34), как это было сделано в предыдущем пункте.

В качестве примера рассмотрим колебательную систему, образованную магнитострикционным преобразователем и присоединенным к нему ступенчатым волноводом, выполненным из цилиндрических стержней равной длины. Будем пренебрегать потерями энергии в материале волновода, полагая их пренебрежимо малыми по сравнению с диссипацией в преобразователе. Для удобства выпишем 
соответствующие величины, входящие в (7.32), (7.33), используя (5.111) и $(5.120)$

$$
\begin{aligned}
& W_{l l}^{(1)}(j \omega)=\frac{E_{1} S_{1} \xi_{1}}{l_{1}} \cdot \frac{\cos \xi_{1}+j \frac{\psi_{1}}{4 \pi} \xi_{1} \sin \xi_{1}}{\sin \xi_{1}-j \frac{\psi_{1}}{4 \pi}\left(\sin \xi_{1}+\xi_{1} \cos \xi_{1}\right)} \\
& W_{00}^{(2)}(j \omega)=\frac{E_{2} S_{2} \xi_{2}}{2 l_{2}} \cdot \frac{(1+K) \sin \xi_{2}}{\sin ^{2} \frac{\xi_{2}}{2}-K \cos ^{2} \frac{\xi_{2}}{2}} \\
& L_{00}^{(2)}(j \omega)=1 / W_{00}^{(2)}(j \omega)^{;} L_{0 l}^{(2)}(j \omega)=-\frac{2 l_{2}}{E_{2} S_{2} \xi_{2}} \cdot \frac{K}{(1+K) \sin \xi_{2}}
\end{aligned}
$$

где $\xi_{i}=\omega \cdot I_{i} / c_{i}, i=1,2 ; K=S_{2} / S_{3} ; S_{2}, S_{3}$ - площади ступеней волновода.

Подставив (7.35) и (7.18) в (7.32) и (7.33) получим

$$
\begin{aligned}
& \tilde{a}_{l}^{(2)} / \tilde{a}_{0}^{(2)}=K /\left(K \cos ^{2} \frac{\xi_{2}}{2}-\sin ^{2} \frac{\xi_{2}}{2}\right) \\
& a_{l}^{(2)}=\frac{K \delta}{\xi_{1}} \cdot \frac{P(j \omega)}{Q(j \omega)}
\end{aligned}
$$

где $\delta=A i_{0} l_{1} / E_{1} S_{1}-$ статическая деформация сердечника преобразователя силой $F=A i_{0}$;

$$
\begin{gathered}
\mathrm{P}(j \omega)=\sin \xi_{1}-j \frac{1}{4 \pi}\left[\psi_{1} \xi_{1} \cos \xi_{1}+\left(\psi_{1}+\chi\right) \sin \xi_{1}\right] \\
Q(j \omega)=\cos \xi_{1}\left(K \cos ^{2} \frac{\xi_{2}}{2}-\sin ^{2} \frac{\xi_{2}}{2}\right)-\frac{w_{2}}{2 w_{1}}(1+K) \sin \xi_{1} \sin \xi_{2}+ \\
+j \frac{\psi_{1}}{4 \pi}\left[\xi_{1} \sin \xi_{1}\left(K \cos ^{2} \frac{\xi_{2}}{2}-\sin ^{2} \frac{\xi_{2}}{2}\right)+\frac{w_{2}}{2 w_{1}}(1+K) \sin \xi_{2}\left(\sin \xi_{1}+\xi_{1} \cos \xi_{1}\right)\right]
\end{gathered}
$$

$w_{i}=S_{i} \sqrt{E_{i} \rho_{i}}$ - волновые сопротивления сердечника преобразователя $(i=1)$ и волновода $(i=2)$ в точке их стыка.

Легко убедиться, что в частном случае при $w_{1}=w_{2}$ и $K=1$ выражения (7.36) описывают колебания однородного стержня с приведенной длиной $l=l_{1}+l_{2} c_{1} / c_{2}$, возбуждаемого в сечении $x=l_{1}$ силой с амплитудой $F=A i_{0}$ (см. п.5.12). Резонансные частоты такой системы определяются формулой (5.112)

$$
\xi=\xi_{1}+\xi_{2}=(2 n-1) \pi / 2, n=1,2 .
$$


а резонансные амплитуды свободного конца и торца преобразователя

$$
\begin{aligned}
& a_{0}^{(2)}=a_{l}^{(2)}\left|\cos \xi_{2}\right| \\
& a_{l}^{(2)}=\left|\frac{4 \pi \cdot \delta \cdot \sin \xi_{1}}{\psi_{1} \xi_{1}\left[\sin \xi_{1} \sin \xi_{2}-(-1)^{n} \xi_{1}\right.}\right|
\end{aligned}
$$

Если преобразователь выполнен резонансным, т.е. таким, что

$$
\xi_{1}=\left(2 n_{1}-1\right) \pi / 2, n_{1}=1,2, \ldots
$$

то резонанс (7.37) всей системы достигается при

$$
\xi_{2}=\xi-\xi_{1}=\left(n-n_{1}\right) \pi
$$

и равенства (7.38) дают

$$
a_{0}^{(2)}=a_{l}^{(2)}=\frac{16 \delta}{\pi \cdot \psi_{1}\left(2 n_{1}-1\right)^{2}}
$$

Таким образом, присоединение настроенного согласно (7.40) стержня не искажает резонансных колебаний преобразователя. Сказанное справедливо и в общем случае (7.36). Действительно, резонансные частоты этой системы находятся из получаемого в соответствии с условием (5.35) уравнения

$$
\frac{w_{1}}{w_{2}(1+K)} \operatorname{ctg} \xi_{1}=\frac{\operatorname{tg}\left(\xi_{2} / 2\right)}{K-\operatorname{tg}\left(\xi_{2} / 2\right)}
$$

В данном случае волновод работает в режиме ступенчатого концентратора, обеспечивая увеличение амплитуды колебаний, передаваемых от преобразователя (см.(5.124)). Здесь мы рассмотрели концентратор, не обладающий диссипативными потерями. Отметим, что присоединение к преобразователю любой колебательной системы без потерь не искажает режима его работы на частотах, равных собственным частотам присоединяемой системы, так как в этом случае $W_{00}^{(2)}(j \omega)=0$ и (7.32) совпадает с (7.30). Оценка влияния диссипации в концентраторе будет дана ниже в Главе 4.

Следует подчеркнуть, что значения (7.39), (7.40) определяют не все резонансные частоты системы, а лишь те, на которых волновод работает в 
режиме концентратора колебаний. Полный спектр резонансных частот дает уравнение (7.42). Пример его графического решения показан на рис.7.7 при $l_{2} c_{1} / l_{1} c_{2}=2$, т.е. $\xi_{2}=2 \xi_{1}$, причем функция в правой части (7.42) построена при различных значениях $K$, а функция в левой части при различных значениях параметра $\alpha=w_{1} / w_{2}(1+K)$.

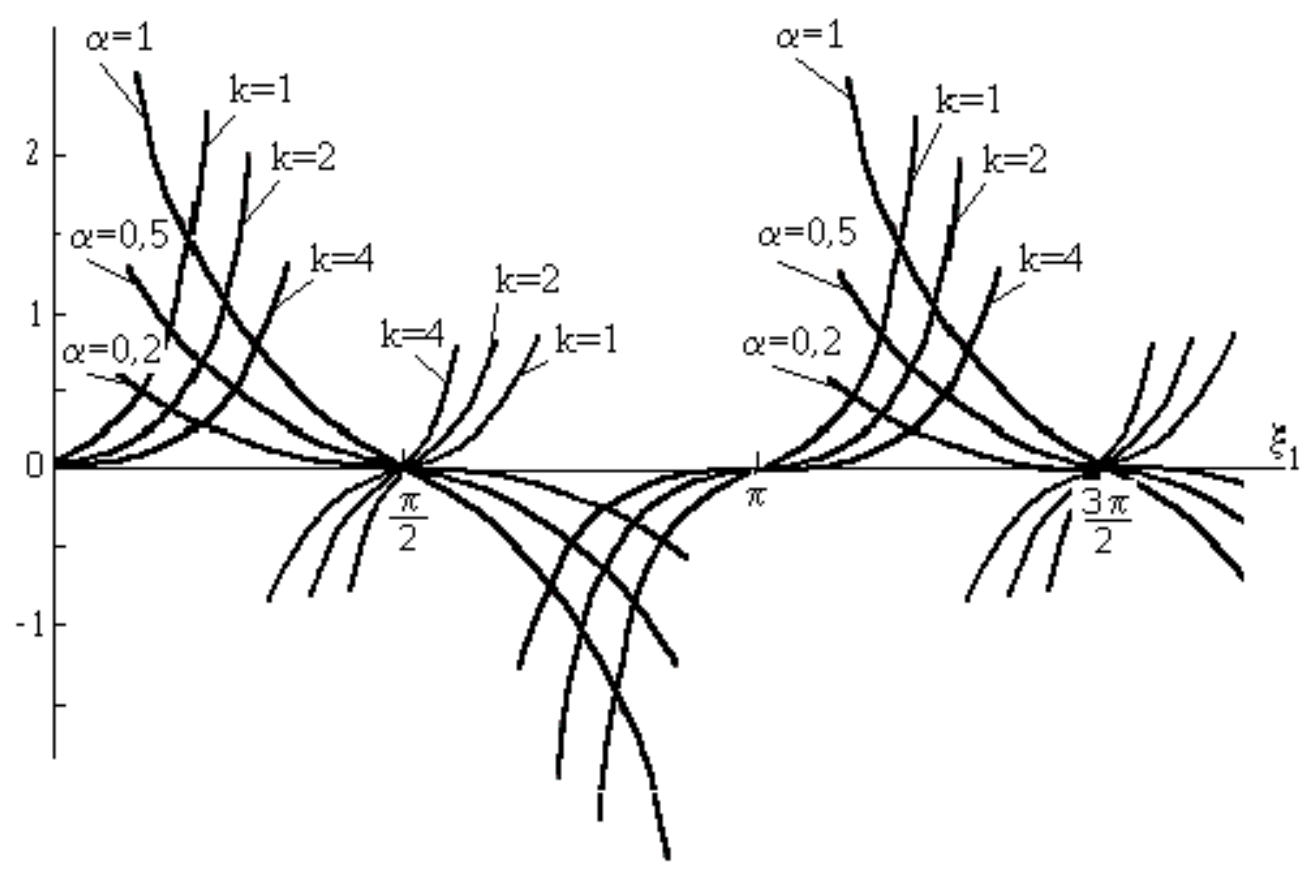

Pис.7.7

На рис.7.8 приведена амплитудно-частотная характеристика системы, построенная для свободного конца волновода при $K=4, \alpha=0,2, \psi_{1}=0,1$.

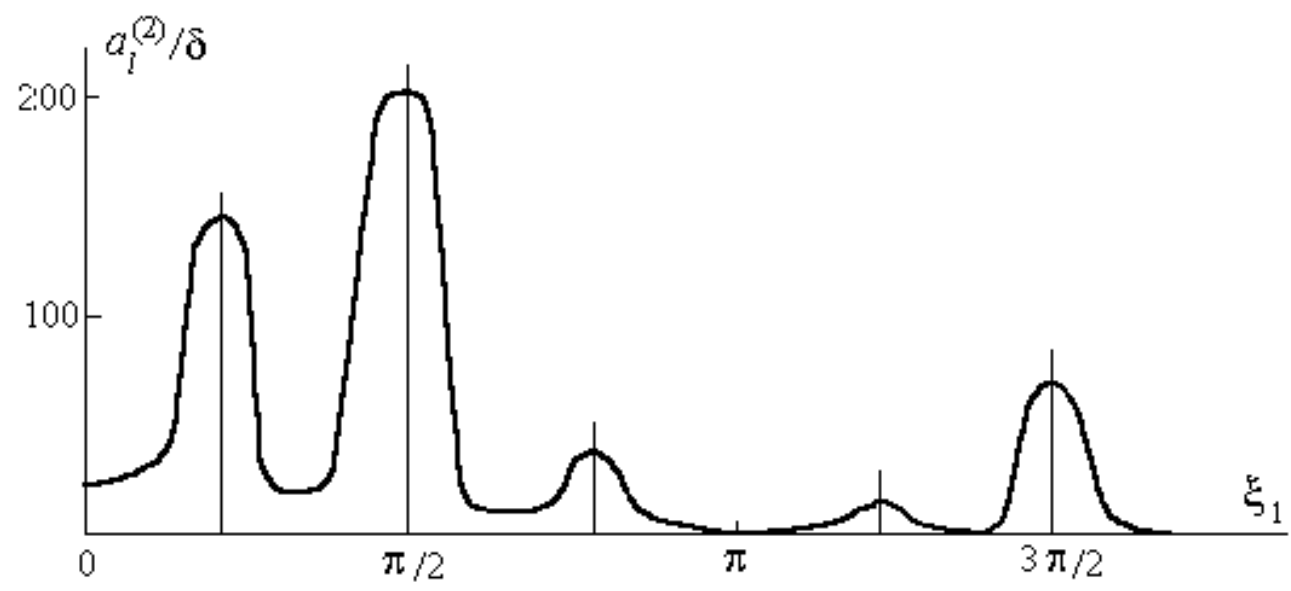

Pnc.7.8 
Достигаемые на резонансных частотах максимальные амплитуды находятся согласно (7.36), (7.42) из выражения

$$
a_{l}^{(2)}=\left|\frac{4 \pi \cdot K \delta \cdot \sin ^{2} \xi_{1}}{\Psi_{1} \xi_{1}\left(\xi_{1}+0,5 \sin 2 \xi_{1}\right)\left(K \cos ^{2} \xi_{2} / 2-\sin ^{2} \xi_{2} / 2\right)}\right|
$$

На резонансных частотах (7.39), (7.40) эта формула дает значения (7.41), (7.43) амплитуд колебаний крайних сечений концентратора.

Минимумы резонансных кривых системы достигаются, согласно (7.36), на частотах

$$
\xi_{1}=\pi \cdot n, n=1,2, \ldots
$$

при которых торец преобразователя оказывается в узле колебаний, и частотах

$$
\xi_{2}=(2 n-1) \frac{\pi}{2} \pm \operatorname{arctg} \sqrt{K}, n=1,2, \ldots
$$

при которых амплитуда точки крепления волновода к преобразователю $a_{l}^{(1)}=a_{0}^{(2)}=0$. Отметим, что частоты (7.46) определяют асимптоты функции, стоящей в правой части уравнения (7.42) и являются собственными частотами парциальной системы, образованной жестким закреплением конца $x=0$ ступенчатого волновода. Поэтому на этих частотах волновод играет роль динамического гасителя колебаний $[86,98,186]$ преобразователя. Амплитуды колебаний свободного торца волновода на частотах (7.45), (7.46) малы по сравнению с резонансными: на частотах (7.45) амплитуды

$$
a_{l}^{(2)}=\left|\frac{\psi_{1} K \delta}{4 \pi \cdot\left(K \cos ^{2} \xi_{2} / 2-\sin ^{2} \xi_{2} / 2\right)}\right|
$$

имеют порядок малой величины $\psi_{1}$, а на частотах (7.46) амплитуда

$$
a_{l}^{(2)}=\frac{\sqrt{K} A i_{0} l_{2}}{E_{2} S_{2} \xi_{2}}=\frac{\sqrt{K} w_{1}}{w_{2}} \cdot \frac{\delta}{\xi_{1}}-
$$

- величина порядка статической деформации $\delta$.

Наконец, при $\omega=0$, т.е. $\xi_{1}=\xi_{2}=0$, из (7.35) находим $\quad a_{l}^{(2)}=a_{0}^{(2)}=\delta$. 
Из рис.7.8 видна целесообразность использования неоднородного волновода в режиме концентратора на частотах (7.39). Полученные в этом примере результаты полностью отвечают общей теории концентраторов, изложенной в П.5.5. Вопросы взаимодействия концентратора, обладающего внутренними потерями, с возбудителем колебаний и нелинейной упруго-диссипативной нагрузкой будут подробно освещены в Главе 4.

4. Перейдем к отысканию динамических характеристик пьезоэлектрических преобразователей.

Типичный пьезопреобразователь (рис.7.9) представляет собой элемент 1, выполненный из пьезоактивного материала.

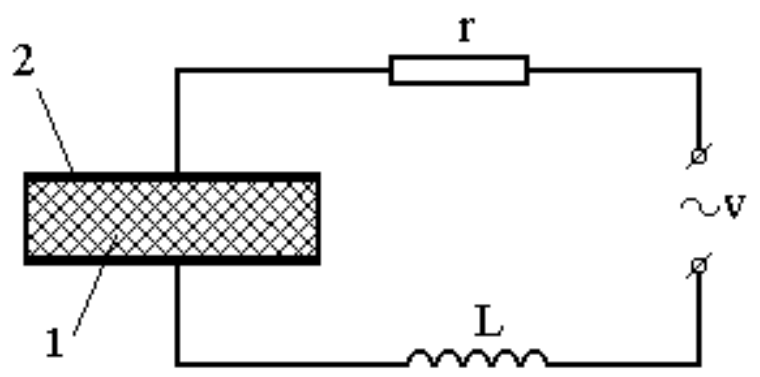

PUC. 7.9

К металлизированным обкладкам 2 пьезоэлемента подводится переменное электрическое напряжение $v$ от источника питания.

Рассмотрим случай продольных колебаний элемента в направлении, перпендикулярном обкладкам. Работа преобразователя основана на использовании пьезоэффекта [170, 197,203]. Прямой пьезоэлектрический эффект состоит в электрической поляризации кристаллов материала под действием механического напряжения $\sigma$. В результате поляризации возникает электрическое поле, электрическое смещение (индукция) $D$ которого связано с механическим напряжением $\sigma$ соотношением

$$
D /\left.\sigma\right|_{\mathrm{E}=0}=-d,
$$

где $d$ - пьезомодуль материала. Отношение в (7.47) берется при отсутствии внешнего электрического поля $(\mathrm{E}=0)$.

Обратный пьезоэлектрический эффект заключается в появлении 
деформации кристалла под действием электрического поля и при отсутствии механических напряжений $(\sigma=0)$ выражается равенством

$$
\varepsilon /\left.\mathrm{E}\right|_{\sigma=0}=-d
$$

где $\varepsilon$ - относительная деформация кристалла; Е - напряженность электрического поля.

С помощью (7.47), (7.48) запишем уравнения пьезоэлектрического преобразователя при совместном действии механических напряжений и электрического поля

$$
\begin{aligned}
& D=\xi \cdot \mathrm{E}-d \sigma \\
& \varepsilon=-d \mathrm{E}+\sigma / \widetilde{E}
\end{aligned}
$$

где $\xi$ - диэлектрическая постоянная кристалла при отсутствии механических напряжений; $\widetilde{E}$ - модуль упругости при отсутствии электрического поля.

Линейные соотношения (7.49) удобно записать в иной форме, выразив $\sigma$ и Е через $D$ и $\varepsilon$

$$
\begin{aligned}
& \sigma=E_{1} \varepsilon+\Phi D \\
& \mathrm{E}=\Phi \varepsilon+\xi_{1}^{-1} D^{\prime}
\end{aligned}
$$

где $E_{1}=E /\left(1-\lambda^{2}\right)$ - модуль упругости пьезоэлемента при отсутствии индукции $(D=0)$, т.е. при коротко замкнутых обкладках; $\lambda^{2}=d^{2} E / \xi<1$ [203]; $\Phi=\lambda^{2} / d\left(1-\lambda^{2}\right)$ - пьезоэлектрическая постоянная Мэсона $[231] ; \xi_{1}=\xi \cdot\left(1-\lambda^{2}\right)$ диэлектрическая постоянная в отсутствие деформации $(\varepsilon=0)$.

Учитывая, что свободные заряды $q$ могут появиться только на обкладках кристалла и, следовательно, электрическое смещение постоянно по его длине

$$
D=q / S
$$

из первого уравнения (7.50) получим соотношение, описывающее напряженное состояние кристалла

$$
\sigma=E_{1} \varepsilon+\frac{\Phi}{S} q
$$


Электрическое состояние пьезоэлемента опишем, используя равенства $\mathrm{E}=\frac{\partial \varphi_{x}}{\partial x}\left(\varphi_{x}\right.$ - потенциал в сечении $\left.x\right), v_{1}=\varphi_{1}-\varphi_{0}, \varepsilon=\partial u_{x} / \partial x$ и интегрируя второе уравнение из (7.50) по всей длине $l$ элемента

$$
V_{1}=\frac{q}{C_{1}}+\left(u_{l}-u_{0}\right) \Phi,
$$

где $C_{1}=\xi S / I-$ емкость пьезоэлемента в отсутствие деформации.

Если цепь питания преобразователя имеет сопротивление $r$ и индуктивность $L$ (рис.7.9),

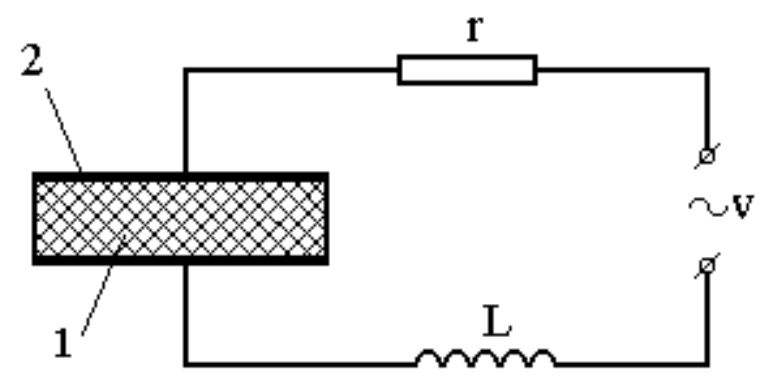

Puc. 7.9

то полное уравнение электрической цепи запишется в виде

$$
L \frac{d^{2} q}{d r^{2}}+r \frac{d q}{d t}+\frac{q}{C_{1}}+\left(u_{l}-u_{0}\right) \Phi=V
$$

Уравнения (7.52), (7.54) полностью описывают колебания пьезоэлектрического преобразователя. Эти уравнения имеют структуру, аналогичную уравнениям (7.8), (7.12), описывающим магнитострикционный преобразователь. Поэтому и уравнения четырехполюсника для пьезоэлектрического преобразователя можно сразу получить из (7.13) и (7.29) заменой $j \omega \cdot A \rightarrow \Phi$ и сменой знака перед последним членом первого уравнения.

5. Рассмотрим, например, колебания пьезоэлемента (рис.7.10,a), один конец которого $(x=0)$ жестко закреплен, а второй $(x=l)$ нагружен силой $f_{12}$. 

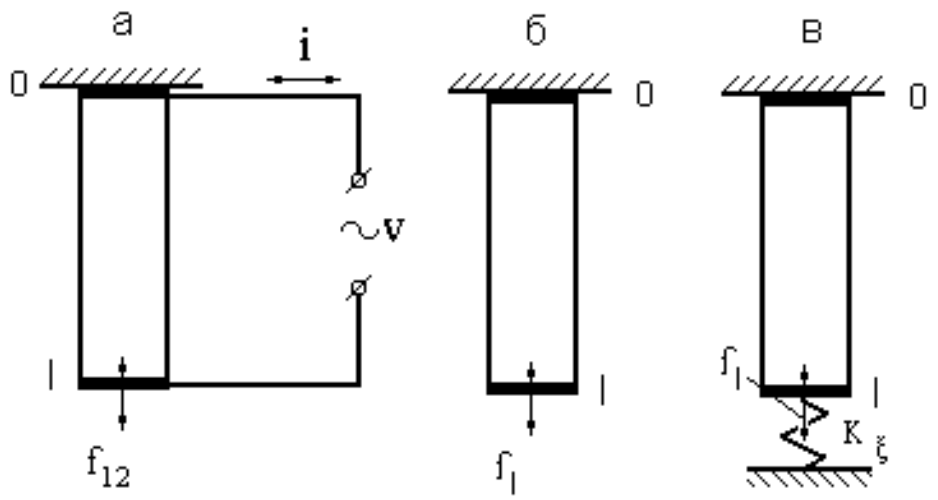

Pnc.7.10

Будем считать, что сопротивление и индуктивность электрической цепи пренебрежимо малы, и пьезоэлемент получает питание от источника тока или напряжения. В этом случае уравнения четырехполюсника принимают вид

$$
\begin{aligned}
& \tilde{F}_{12}=W_{l l}(j \omega) \tilde{a}_{l}+\hat{O} \tilde{q}_{0} \\
& \tilde{v}_{0}=\hat{O} \tilde{a}_{l}+\frac{1}{C_{1}} \tilde{q}_{0}
\end{aligned}
$$

Полагая здесь $\widetilde{F}_{12}=0$ (нагрузка на конце $x=l$ отсутствует), находим амплитуду колебаний холостого хода при питании от источника тока:

$$
\widetilde{a}_{l}^{*}=-\Phi q_{0} / W_{l l}(j \omega)
$$

и при питании от источника напряжения:

$$
\widetilde{a}_{l}^{*}=-\Phi C_{1} V_{0} /\left[W_{l l}(j \omega)-\Phi^{2} C_{1}\right]
$$

Из (7.56), (7.57) следует, что преобразователь можно описать с помощью моделей, первая из которых (рис.7.10,б) представляет собой однородный стержень, возбуждаемый на конце $x=l$ силой с амплитудой $F_{l}=-\Phi q_{0}$, а вторая (рис.7.10,в) - однородный стержень с фиктивной отрицательной жесткостью $K_{g}=-C_{1} \Phi^{2}$ на конце под действием силы $F_{l}=-\Phi C_{1} v_{0}$. Динамическая жесткость $W_{l /}(j \omega)$ в (7.55) - (7.57) определяется выражением (5.111), и резонансные частоты в обоих случаях оказываются различными.

Физический смысл этого различия можно оценить, рассмотрев случай малых частот возбуждения, когда динамическая жесткость $W_{/(}(j \omega)=E_{1} S / I$. Тогда равенство (7.56) принимает вид 


$$
a_{l}=-\Phi q_{0} l / E_{1} S,
$$

а равенство (7.57) с учетом принятых в (7.50) обозначений

$$
a_{l}=-\Phi C_{1} v_{0} l / E S
$$

Таким образом, при питании от различных источников пьезоэлектрические кристаллы ведут себя подобно элементам, выполненным из материалов с различным модулем упругости, под действием приведенных сил. Это кажущееся противоречие легко разрешается, если с помощью обозначений, принятых в (7.50), перейти в равенствах (7.58), (7.59) к исходным константам пьезоматериала.

В результате получаем

$$
a_{l}=\delta=-d q_{0} / C=-d v_{0},
$$

где $C=\xi \cdot S / l$ - емкость пьезоэлемента со свободными концами.

Эти равенства можно получить непосредственно из уравнений (7.49), учитывая (7.51), полагая $\sigma=0$ и принимая $\mathrm{E}=v / l$ и $\varepsilon=u_{l} / l$ вследствие однородности деформации. Интересно отметить, что деформация элемента вследствие пьезоэффекта не зависит от упругих свойств материала; происходит как бы кинематическое растяжение - сжатие кристалла. Это отражает процессы перестроения атомной структуры кристаллической решетки пьезоэлемента под влиянием электрического поля.

6. В заключение этого Параграфа

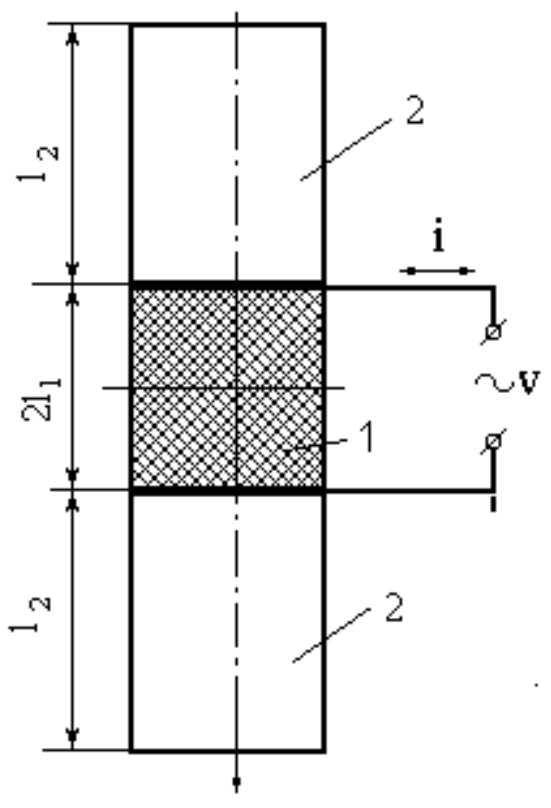

Puc.7.11 рассмотрим колебательную систему (рис.7.11), в которой пьезоэлемент 1 симметрично заключен между накладками 2, выполненными в виде однородных стержней. В силу симметрии допустимо рассмотреть колебания половины системы, имеющей узловую точку в среднем сечении преобразователя. Поэтому для описания колебаний можно воспользоваться соотношениями, полученными выше. Как и в п.7.3, однотипные величины, относящиеся к 
преобразователю и накладке, будем отмечать индексами 1 и 2. Тогда из первого уравнения системы (7.55) найдем амплитуду колебаний стыкового сечения при питании преобразователя от источника тока

$$
\tilde{a}_{l}^{(1)}=\tilde{a}_{0}^{(2)}=-\hat{O} \tilde{q}_{0} /\left[W_{l l}^{(1)}(j \omega)+W_{00}^{(2)}(j \omega)\right]
$$

Исключая величину $\widetilde{q}_{0}$ из второго уравнения (7.55) и равенства (7.61), имеем при питании от источника напряжения

$$
\widetilde{a}_{l}^{(1)}=\widetilde{a}_{0}^{(2)}=-\frac{C_{1} \Phi_{\widetilde{V}_{0}}}{W_{l l}^{(1)}(j \omega)+W_{00}^{(2)}(j \omega)-C_{1} \Phi^{2}}
$$

Амплитуду колебаний свободного торца накладки найдем с помощью (5.66)

$$
\widetilde{a}_{1}^{(2)}=\widetilde{a}_{0}^{(2)} L_{01}^{(2)}(j \omega) / L_{00}^{(2)}(j \omega)
$$

Величины динамических жесткостей и податливостей в (7.61) (7.63) определяются выражениями, полученными в разделе 5.

Рассмотрим характерные частные случаи. Пусть длины пьезоэлемента $l_{1}$ и накладки настолько малы, что удовлетворяют условию (5.13). Учитывая (5.114) и (5.14), имеем $\tilde{a}_{l}^{(1)}=\tilde{a}_{l}^{(2)}=\tilde{a}$ и выражения (7.61), (7.62) принимают вид

$$
\begin{gathered}
\widetilde{a}=-\frac{\Phi q_{0}}{\left(K_{1}-M \omega^{2}\right)+j \omega \cdot b_{1}} \\
\widetilde{a}=-\frac{C_{1} \Phi V_{0}}{\left(K-M \omega^{2}\right)+j \omega \cdot b}
\end{gathered}
$$

где $K_{1}=E_{1} S / l_{1}, b_{1}$ - статическая жесткость и коэффициент сопротивления короткозамкнутого преобразователя; $K=K_{1}-\Phi^{2} C_{1}=E S / I_{1}, \quad b \quad$ - то же разомкнутого преобразователя; $M$ - масса накладки.

Из (7.64) следует, что при питании от источника тока резонанс в колебательной системе достигается на частоте $\omega_{q}=\sqrt{K_{1} / M}$, а при питании от источника напряжения на частоте $\omega_{v}=\sqrt{K / M}$.

С учетом обозначений, принятых в (7.50) и (7.60), равенства (7.64) можно записать в иной форме

$$
\widetilde{a}_{l}=\frac{\delta}{\left(1-\omega^{2} / \omega_{0}^{2}\right)+j \eta_{0} \omega / \omega_{0}},
$$


где $\omega_{0}=\omega_{q}, \eta_{0}=b_{1} / \sqrt{K_{1} M}$ - при питании от источника тока; $\omega_{0}=\omega_{v}$, $\eta_{0}=b / \sqrt{K M}$ - при питании от источника напряжения.

Обратимся к случаю, когда длины пьезоэлемента и накладки соизмеримы или много больше длины звуковой волны в их материалах. Как правило, пьезоэлектрические материалы обладают очень малыми внутренними потерями, которыми можно пренебречь по сравнению с потерями в материале накладки. Подставляя в (7.61), (7.62) величины динамических жесткостей и податливостей (5.111), (5.20), (5.21) после приведения их к виду (5.42), получим

$$
\widetilde{a}_{l}^{(1)}=\widetilde{a}_{0}^{(2)}=\frac{F}{W_{0}(j \omega)-K_{g}}
$$

где $F=\Phi q_{0}, K_{g}=0$ - при питании от источника тока; $F=C_{1} \cdot \hat{O} \cdot v_{0}$, $K_{g}=C_{1} \Phi^{2}=\lambda^{2} E_{1} S / I$ - при питании от источника напряжения;

$$
\begin{aligned}
& W_{0}(j \omega)=W_{l l}^{(1)}(j \omega)+W_{00}^{(2)}(j \omega)= \\
& =\frac{E_{1} S_{1} \xi_{1}}{l_{1}}\left[\operatorname{ctg} \xi_{1}-\frac{W_{2}}{W_{1}}\left(\operatorname{tg} \xi_{2}-j \frac{\psi_{2}}{4 \pi} \cdot \frac{\xi_{2} \cos 2 \xi_{2}+0,5 \sin 2 \xi_{2}}{\cos ^{2} \xi_{2}}\right)\right]
\end{aligned}
$$

$\xi_{i}=\omega \cdot l_{i} / c_{i} ; c_{i}=\sqrt{E_{i} / \rho_{i}} ; w_{i}=\sqrt{E_{i} \rho_{i}} S_{i}$ - волновое сопротивление элементов; $i=1,2$.

Амплитуда колебаний (7.63) свободного торца накладки определяется выражением $\widetilde{a}_{1}^{(2)}=\widetilde{a}_{0}^{(2)} / \cos \xi_{2}$, совпадающим с (7.36) при $K=1$.

Положив в (7.67) $w_{1}=w_{2}=w$ и пренебрегая потерями энергии $\left(\psi_{2}=0\right)$, получим

$$
W_{0}(j \omega)=w \omega \frac{\cos \xi}{\sin \xi_{1} \cos \xi_{2}},
$$

где $\xi=\xi_{1}+\xi_{2}$.

К аналогичному виду приводит выражение (5.131) для динамической податливости однородного стержня под действием силы, сосредоточенной в промежуточном сечении, если положить $l=l_{1}+l_{2}, s=l_{1}$.

Таким образом, и в этом случае колебательная система может быть сведена к стержневой динамической модели, находящейся под действием приведенной силы возбуждения. Дальнейший анализ аналогичен проведенному в П.7.3. 


\section{ГЛАВА 3}

НЕЛИНЕЙНЫЕ ПРОЦЕССЫ

В УЛЬТРАЗВУКОВЫХ ТЕХНОЛОГИЧЕСКИХ СИСТЕМАХ

\section{8. Нелинейная технологическая нагрузка}

1. Взаимодействие рабочего органа ультразвуковой технологической машины с обрабатываемым изделием или средой при выполнении рабочего процесса создает технологическую нагрузку на колебательную систему. Как было показано в Гл.1, силу взаимодействия можно представить в виде силовой динамической характеристики $f_{l}\left(u_{l}, \dot{u}_{l}\right)$ рабочего процесса, связывающей действующую на изделие силу $f_{l}$ c перемещением $u_{l}$ и скоростью $\dot{u}_{l}$ рабочего органа.

В простейших случаях эта характеристика представляет собой линейную зависимость силы от перемещения

$$
f_{l}\left(u_{l}\right)=k_{0} u_{l}
$$

или скорости

$$
f_{l}\left(\dot{u}_{l}\right)=b_{0} \dot{u}_{l}
$$

Зависимость (8.1) описывает линейную упругую нагрузку, возникающую, например, при непрерывном деформировании образца с жесткостью $k_{0}$ в пределах упругой зоны характеристики материала (см.рис.2.4,б); зависимость (8.2) описывает линейную диссипативную нагрузку с коэффициентом сопротивления $b_{0}$, возникающую при колебаниях в вязкой жидкости без образования кавитации [197] или при излучении волны в неограниченную волноводную систему (см. П.5.2). 


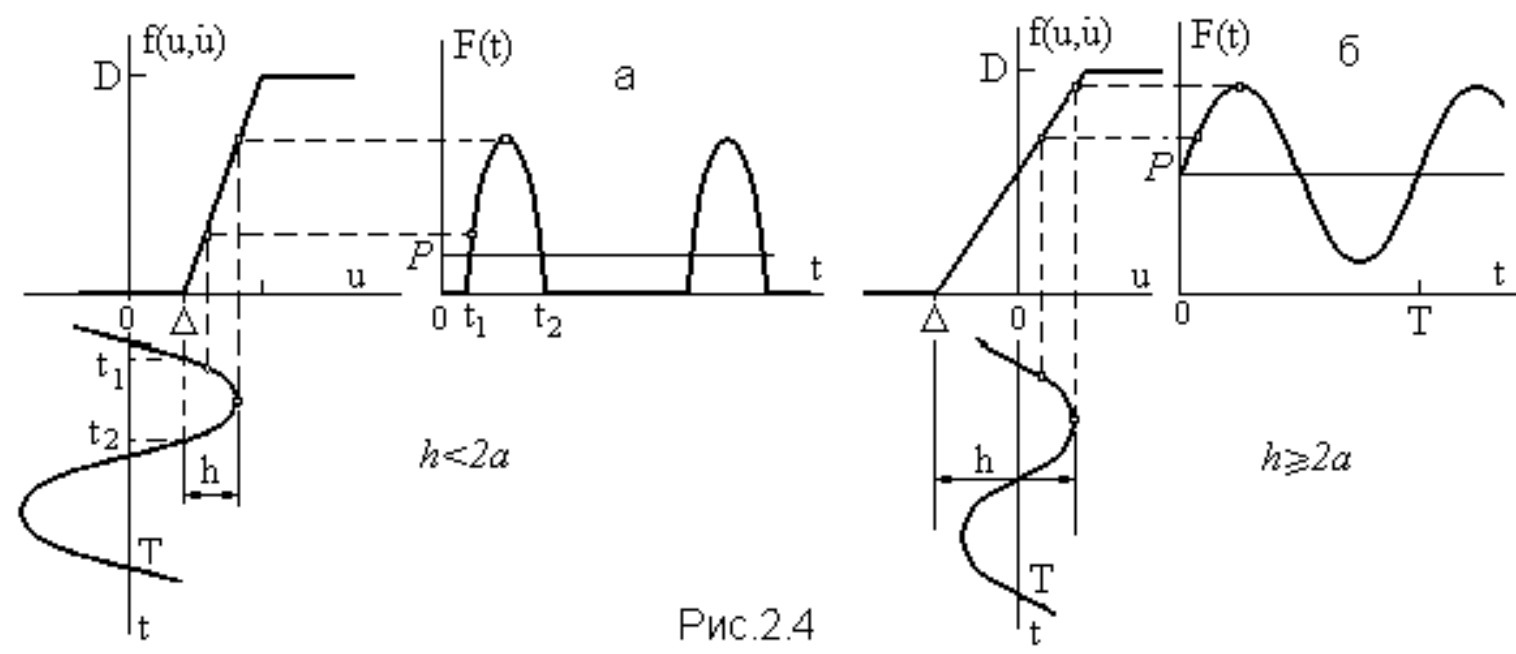

Суммарная характеристика

$$
f_{l}\left(u_{l}, \dot{u}_{l}\right)=k_{0} u_{l}+b_{0} \dot{u}_{l}
$$

описывает линейную упруго-диссипативную нагрузку, возникающую, например, при деформировании упруго-вязкого материала.

Учитывая характер рассматриваемых движений (4.4) и сил (4.7) в равенствах (8.1) - (8.3), можно перейти к комплексным амплитудам перемещения и силы. В частности, из (8.3) получим соотношение $\widetilde{F}_{l}\left(\widetilde{a}_{l}\right)=W_{H}(j \omega) \widetilde{a}_{l}$, связывающее комплексные амплитуды колебаний и сил с помощью динамической характеристики упруго-диссипативной нагрузки

$$
W_{H}(j \omega)=k_{0}+j \omega \cdot b_{0}
$$

При расчетах ультразвуковых технологических машин часто приходится учитывать инерционную нагрузку, создаваемую, например, массой $M$ сменного инструмента или присоединенного обрабатываемого материала. Характеристика инерционной нагрузки имеет вид $f_{l}\left(\ddot{u}_{l}\right)=M \ddot{u}_{l}$, а ее динамическая жесткость

$$
W_{H}(j \omega)=-M \omega^{2}
$$

Как было показано в П.4.7, при рассмотрении обобщенной схемы ультразвуковой технологической машины, амплитуда колебаний еe рабочего органа при работе на линейную нагрузку сразу определяется формулой (4.33) с помощью найденных в Параграфах 5 - 7 динамических характеристик колебательных систем и электроакустических преобразователей. 
2. Реальный рабочий процесс, реализуемый ультразвуковой технологической машиной, как правило, не удается представить с помощью линейной нагрузки и приходится обращаться к рассмотрению нелинейных моделей процесса, некоторые из которых были представлены в Гл.1. В этом случае сила взаимодействия рабочего органа с обрабатываемым изделием или средой описывается нелинейной динамической характеристикой $f_{l}=f_{l}\left(u_{l}, \dot{u}_{l}\right)$. Для отыскания периодических движений ультразвуковых систем с нелинейной нагрузкой мы будем использовать приближенный метод гармонической линеаризации, широко применяемый для анализа нелинейных систем автоматического управления [167], нелинейных виброзащитных [120,121], виброударных [60] и других систем.

Метод гармонической линеаризации основан на применении принципа гармонического баланса.

Предположим, как и ранее, существование периодического движения рабочего органа с частотой $\omega$ и представим его в виде ряда Фурье

$$
u_{l}(t)=m_{l}+\sum_{n=1}^{\infty}\left(A_{c n} \cos n \omega t+A_{s n} \sin n \omega t\right),
$$

который можно записать в следующей эквивалентной форме

$$
u_{l}(t)=m_{l}+\frac{1}{2} \sum_{n= \pm 1}^{ \pm \infty} \widetilde{a}_{\ln } \mathrm{e}^{n j \omega \cdot t},
$$

где $m_{l}$ - постоянная составляющ0ая; $\tilde{a}_{\mathrm{ln}}$ - комплексные амплитуды гармонических составляющих движения, связанные с коэффициентами ряда (8.6) равенствами

$$
\widetilde{a}_{\mathrm{ln}}=A_{c n}-j A_{s n} \operatorname{sgn} n,
$$

из которых следует, что амплитуды $\widetilde{a}_{\ln }$ при $n=k$ и $n=-k$ - комплексные сопряженные числа.

После подстановки периодической функции (8.7) в нелинейную 
динамическую характеристику получим периодическую функцию той же частоты $\omega$, разложение которой в ряд Фурье дает

$$
f_{l}\left[u_{l}(t), \dot{u}_{l}(t)\right]=P_{l}+\frac{1}{2} \sum_{n= \pm 1}^{ \pm \infty} \widetilde{F}_{\ln } e^{n j \omega \cdot t},
$$

где постоянная составляющая $P_{l}$ и комплексные амплитуды $\tilde{F}_{\text {In }}$ гармонических составляющих силы $f_{l}$ вычисляются по формулам

$$
\begin{gathered}
P_{I}=\frac{1}{T} \int_{0}^{T} f_{l}\left[u_{l}(t), \dot{u}_{l}(t)\right] d t=P_{l}\left(m_{l}, A_{c 1}, \ldots, A_{s 1}, \ldots\right) \\
\widetilde{F}_{\mathrm{ln}}=\frac{2}{T} \int_{0}^{T} f_{l}\left[u_{l}(t), \dot{u}_{l}(t)\right] e^{-n j \omega t} d t=\widetilde{F}_{\mathrm{ln}}\left(m_{l}, A_{c 1}, \ldots, A_{s 1}, \ldots\right)
\end{gathered}
$$

где $T=2 \pi / \omega-$ период рассматриваемых процессов.

Будем по-прежнему считать, что колебательная система и преобразователь ультразвуковой технологической машины представляют собой линейную систему, свойства которой полностью определяются величинами динамических податливостей, найденных в предыдущей главе. Тогда колебания этой системы при взаимодействии с нелинейной технологической нагрузкой могут быть описаны с помощью следующего уравнения:

$$
u_{l}(t)=u_{l}^{*}(t)-L_{M}(j \omega) \cdot f_{l}\left[u_{l}(t), \dot{u}_{l}(t)\right],
$$

где - установившиеся колебания с частотой $\omega$ на холостом ходу при отсутствии технологической нагрузки $\left(f_{l}=0\right) ; L_{M}(j \omega)$ - приведенная к рабочему органу динамическая податливость колебательной системы и преобразователя, определяемая формулой (4.25).

Движение на холостом ходу $u_{l}^{*}(t)$ находится методами, изложенными в предыдущей главе. Пусть для общности $u_{l}^{*}(t)$ - периодическая функция, разложение которой в ряд Фурье можно записать по аналогии с (8.7)

$$
u_{l}^{*}(t)=\frac{1}{2} \sum_{n= \pm 1}^{ \pm \infty} \widetilde{a}_{\mathrm{ln}}^{*} e^{n j \omega \cdot t},
$$

Комплексные амплитуды $\widetilde{a}_{\ln }^{*}$ определяются через динамические характеристики элементов колебательной системы при питании 
преобразователя от источника периодического напряжения или тока с помощью формул (4.17) и (4.24). Поэтому в уравнении (8.12) функция (8.13) играет роль заданного внешнего периодического возбуждения.

Подставим ряды (8.7), (8.9), (8.13) в уравнение (8.12)

$$
m_{l}+\frac{1}{2} \sum_{n= \pm 1}^{ \pm \infty} \widetilde{a}_{1 \mathrm{ln}} e^{n j \omega t}=\frac{1}{2} \sum_{n= \pm 1}^{ \pm \infty} \widetilde{a}_{\mathrm{ln}}^{*} e^{n j \omega t}-L_{M}(0) P_{l}-\frac{1}{2} \sum_{n= \pm 1}^{ \pm \infty} L_{M}(n j \omega) \widetilde{F}_{\mathrm{ln}} e^{n j \omega \cdot t}
$$

Приравнивая в левой и правой частях этого равенства постоянные составляющие и коэффициенты при экспонентах одинаковых степеней, получим уравнения

$$
\begin{gathered}
m_{l}=-L_{M}(0) P_{l}\left(m_{l}, A_{c \mid}, \ldots, A_{s 1}, \ldots\right) \\
\widetilde{a}_{\mathrm{ln}}=\widetilde{a}_{\mathrm{ln}}^{*}-L_{M}(n j \omega) \widetilde{F}_{\mathrm{ln}}\left(m_{l}, A_{c 1}, \ldots, A_{s 1}, \ldots\right),(n=1,2, \ldots),
\end{gathered}
$$

которые с учетом равенств (8.8) дают полную систему для отыскания неизвестных параметров движения $m_{l}, A_{c 1}, \ldots, A_{s 1}, \ldots$, или комплексных амплитуд $\tilde{a}_{\mathrm{ln}}$.

Отметим, что система уравнений, получающаяся из (8.14) при отрицательных $n$ ( $n=-1,-2, \ldots)$, эквивалентна системе (8.16), т.к. все входящие в эти уравнения одноименные комплексные числа оказываются сопряженными.

Получение решения системы бесконечного числа трансцендентных уравнений (8.15), (8.16) в общем случае невозможно, т.к. коэффициенты $P_{l}, \widetilde{F}_{\text {ln }}$ зависят от всех коэффициентов разложения функции $u_{l}(t)$ в ряд (8.16). Исключение составляет случай линейной нагрузки, описываемой, например, выражением (8.3). В этом случае из (8.11) после подстановки (8.7) и интегрирования получаем $\widetilde{F}_{\mathrm{ln}}=\widetilde{a}_{\mathrm{ln}}\left(k_{0}+n j \omega \cdot b_{0}\right)$ и система уравнений (8.16) с учетом (8.4) принимает вид $\tilde{a}_{\mathrm{ln}}=\tilde{a}_{\mathrm{ln}}^{*}-\tilde{a}_{\mathrm{ln}} L_{M}(n j \omega) W_{H}(n j \omega)$. Отсюда для комплексной амплитуды $\tilde{a}_{\mathrm{ln}}$ имеем выражение $\widetilde{a}_{\mathrm{ln}}=a_{\mathrm{ln}}^{*}\left[1+\frac{W_{H}(n j \omega)}{W_{M}(n j \omega)}\right]$, совпадающее с (4.33). 
В случае нелинейной технологической нагрузки приходится ограничивать число членов рядов (8.7), (8.9), отбрасывая остальные. При этом задача сводится к решению ограниченной системы трансцендентных уравнений, т.е. к отысканию приближенного решения. Как правило, при построении такого решения ограничиваются только постоянными составляющими и первыми гармониками периодических решений, т.е. отыскивается приближенное решение вида

$$
u_{l}(t) \approx m_{l}+\widetilde{a}_{l} e^{j \omega t},
$$

постоянная составляющая $m_{l}$ и комплексная амплитуда $\tilde{a}_{l}$ которого согласно (8.15), (8.16) находятся из уравнений

$$
\begin{gathered}
m_{l}=-L_{M}(0) \cdot P_{l}\left(m_{l}, a_{l}\right) \\
\widetilde{a}_{l}=\widetilde{a}_{l}^{*}-L_{M}(j \omega) \cdot \widetilde{F}_{l}\left(m_{l}, a_{l}\right)
\end{gathered}
$$

Метод отыскания периодических решений в такой форме называется методом гармонического баланса. Заметим, что уравнение (8.19) по форме совпадает с (4.23). Разница заключается в том, что в случае нелинейной нагрузки комплексная амплитуда силы взаимодействия является нелинейной функцией амплитуды $a_{l}$ и постоянной составляющей $m_{l}$ движения рабочего органа.

3. Используя (8.11) для первой гармоники

$$
\widetilde{F}_{l}=\frac{2}{T} \int_{0}^{T} f_{l}\left[u_{l}(t), \dot{u}_{l}(t)\right] e^{-j \omega t t} d t=\frac{2}{T} \int_{0}^{T} f_{l}\left[u_{l}(t), \dot{u}_{l}(t)\right](\cos \omega \cdot t-j \sin \omega \cdot t) d t,
$$

запишем выражение для комплексной амплитуды $\widetilde{F}_{/}$в иной форме

$$
\widetilde{F}_{l}=a_{l}(k+j \omega \cdot b),
$$

где $k=\frac{2}{T a_{l}} \int_{0}^{T} f_{l}\left[u_{l}(t), \dot{u}_{l}(t)\right] \cos \omega \cdot t \cdot d t, b=-\frac{2}{T \omega \cdot a_{l}} \int_{0}^{T} f_{l}\left[u_{l}(t), \dot{u}_{l}(t)\right] \sin \omega \cdot t \cdot d t$

Напомним, что при отыскании приближенного решения вида (8.17) принимается $f_{l}\left[u_{l}(t), \dot{u}_{l}(t)\right] \approx P_{I}+\widetilde{F}_{l} e^{j \omega t}$ или с учетом (8.20) и (8.17)

$$
f_{l}\left[u_{l}(t), \dot{u}_{l}(t)\right] \approx P_{l}+k u_{l}^{0}(t)+b \dot{u}_{l}(t),
$$

где $u_{l}^{0}(t)=\widetilde{a}_{l} e^{j \omega \cdot t}$. 
Таким образом, исходная нелинейная функция $f_{l}\left(u_{l}, \dot{u}_{l}\right)$ заменена линеаризованным выражением. Такая замена, отличающаяся от обычной линеаризации тем, что коэффициенты $P_{l}, k, b$ зависят от неизвестных параметров решения, называется гармонической линеаризацией $[120,167]$.

Удобный вид формул для вычисления коэффициентов гармонической линеаризации можно получить из (8.10) и (8.21), если принять

$$
u_{l}(t)=m_{l}+a_{l} \cos \omega \cdot t, \dot{u}_{l}(t)=-a_{l} \omega \cdot \sin \omega \cdot t
$$

и произвести замену переменных $\tau=\omega \cdot t$. В результате имеем

$$
\begin{gathered}
P_{l}=\frac{1}{2 \pi} \int_{0}^{2 \pi} f_{l}\left(m_{l}+a_{l} \cos \tau,-a_{l} \omega \cdot \sin \tau\right) d \tau=P_{l}\left(m_{l}, a_{l}\right) \\
k=\frac{1}{\pi a_{l}} \int_{0}^{2 \pi} f_{l}\left(m_{l}+a_{l} \cos \tau,-a_{l} \omega \cdot \sin \tau\right) \cos \tau \cdot d \tau=k\left(m_{l}, a_{l}\right) \\
b=\frac{1}{\pi a_{l} \omega} \int_{0}^{2 \pi} f_{l}\left(m_{l}+a_{l} \cos \tau,-a_{l} \omega \cdot \sin \tau\right) \sin \tau \cdot d \tau=b\left(m_{l}, a_{l}\right)
\end{gathered}
$$

Величина $P_{l}\left(m_{l}, a_{l}\right)$ определяет постоянную составляющую нелинейной нагрузки. Сравнивая (8.20) и (8.4), переменную составляющую нелинейной нагрузки удобно записать с помощью ее линеаризованной динамической жесткости

$$
W_{H}\left(j \omega, m_{l}, a_{l}\right)=k\left(m_{l}, a_{l}\right)+j \omega \cdot b\left(m_{l}, a_{l}\right)
$$

Коэффициенты $k\left(m_{l}, a_{l}\right), b\left(m_{l}, a_{l}\right)$, зависящие от неизвестных параметров движения, характеризуют эквивалентные упругую и диссипативную составляющие нелинейной нагрузки.

С помощью замены нелинейной динамической характеристики рабочего процесса гармонически линеаризованным представлением (8.22) уравнение для комплексной амплитуды $a_{l}$ приводится к виду $\widetilde{a}_{l}=\widetilde{a}_{l}^{*}-L_{M}(j \omega) W_{H}\left(j \omega, m_{l}, a_{l}\right) \widetilde{a}_{l}$, откуда получаем соотношение

$$
\widetilde{a}_{l}=\widetilde{a}_{l}^{*}\left[1+\frac{W_{H}\left(j \omega, m_{l}, a_{l}\right)}{W_{M}(j \omega)}\right],
$$

совпадающее по форме с полученным ранее для линейной нагрузки выражением (4.33). Их отличие заключается в том, что (8.27) является 
уравнением, которое совместно с уравнением (8.18), связывающим постоянную составляющую и амплитуду колебаний, дает полную систему уравнений для отыскания неизвестных параметров $m_{l}, a_{l}$ решения $u_{l}(t)$.

В дальнейшем, при анализе колебательных систем с нелинейной нагрузкой мы будем пользоваться методом гармонической линеаризации в изложенной здесь форме.

4. Приведем выражения для коэффициентов гармонической линеаризации динамических характеристик некоторых ультразвуковых технологических процессов, которые будут использованы в дальнейшем.

В п.2.2 было показано, что при деформировании упругого образца вибрирующим инструментом возможно установление периодического импульсного режима нагружения (рис.2.4,a). Такой процесс описывается с помощью характеристики одностороннего упругого ограничителя (рис.8.1,a), уравнение которой имеет вид

$$
f_{l}\left(u_{l}\right)=k_{0}\left(u_{l}-\Delta\right) \eta\left(u_{l}-\Delta\right),
$$

где $k_{0}$ - статическая жесткость ограничителя; $\eta(u)$ - функция единичного скачка.
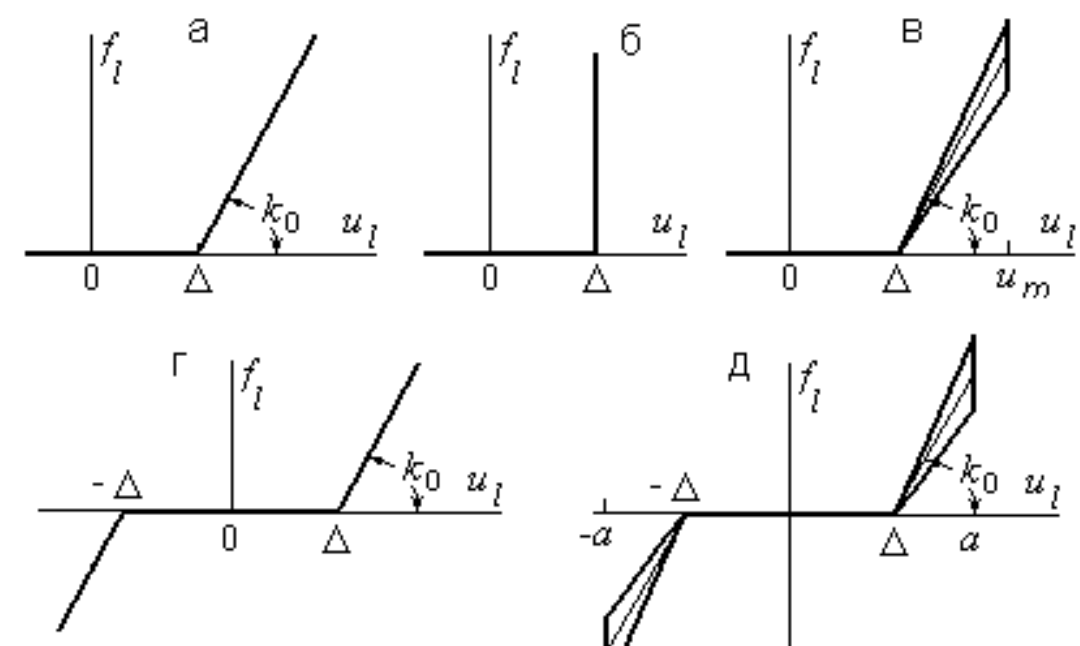

Pис.8.1

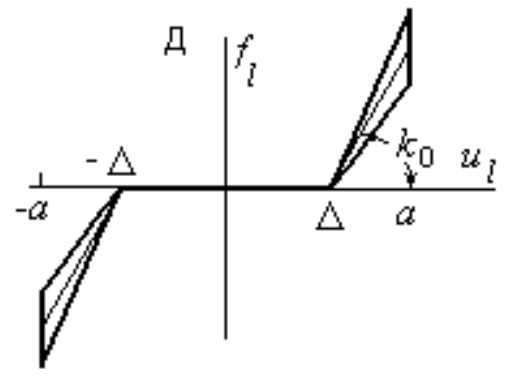

Подставив (8.28) в (8.23) и (8.24), имеем

$$
P_{l}=\frac{1}{\pi} \int_{0}^{\tau_{1}}\left(m_{l}-\Delta+a \cos \tau\right) d \tau, k=\frac{2 k_{0}}{\pi \cdot a_{l}} \int_{0}^{\tau_{1}}\left(m_{l}-\Delta+a \cos \tau\right) \cos \tau \cdot d \tau,
$$

где $\tau_{1}=\arccos \left(\Delta-m_{l}\right) / a_{1}$. 
После интегрирования находим

$$
\begin{gathered}
P_{l}=\frac{k_{0} a_{l}}{\pi}\left(\sqrt{1-\left(\frac{\Delta-m_{l}}{a_{l}}\right)^{2}}-\frac{\Delta-m_{l}}{a_{l}} \arccos \frac{\Delta-m_{l}}{a_{l}}\right) \\
k=\frac{k_{0}}{\pi}\left(\arccos \frac{\Delta-m_{l}}{a_{l}}-\frac{\Delta-m_{l}}{a_{l}} \sqrt{1-\left(\frac{\Delta-m_{l}}{a_{l}}\right)^{2}}\right)
\end{gathered}
$$

Выражение (8.25) дает для диссипативной составляющей нагрузки $b=0$. Обозначив

$$
\left(\Delta-m_{l}\right) / a_{l}=\alpha,-1 \leq \alpha \leq 1,
$$

из (8.29), (8.30) выведем приближенную формулу для коэффициента линеаризации $k\left(a_{l}\right)$.

С этой целью на рис.8.2,a,б построены зависимость $k(\alpha)$ по формуле (8.30) и зависимость $a_{l}(\alpha)$ по полученному из (8.29) уравнению

$$
a_{l}=\pi \frac{P_{l}}{k_{0}}\left(\sqrt{1-\alpha^{2}}-\alpha \cdot \arccos \alpha\right)^{-1}
$$

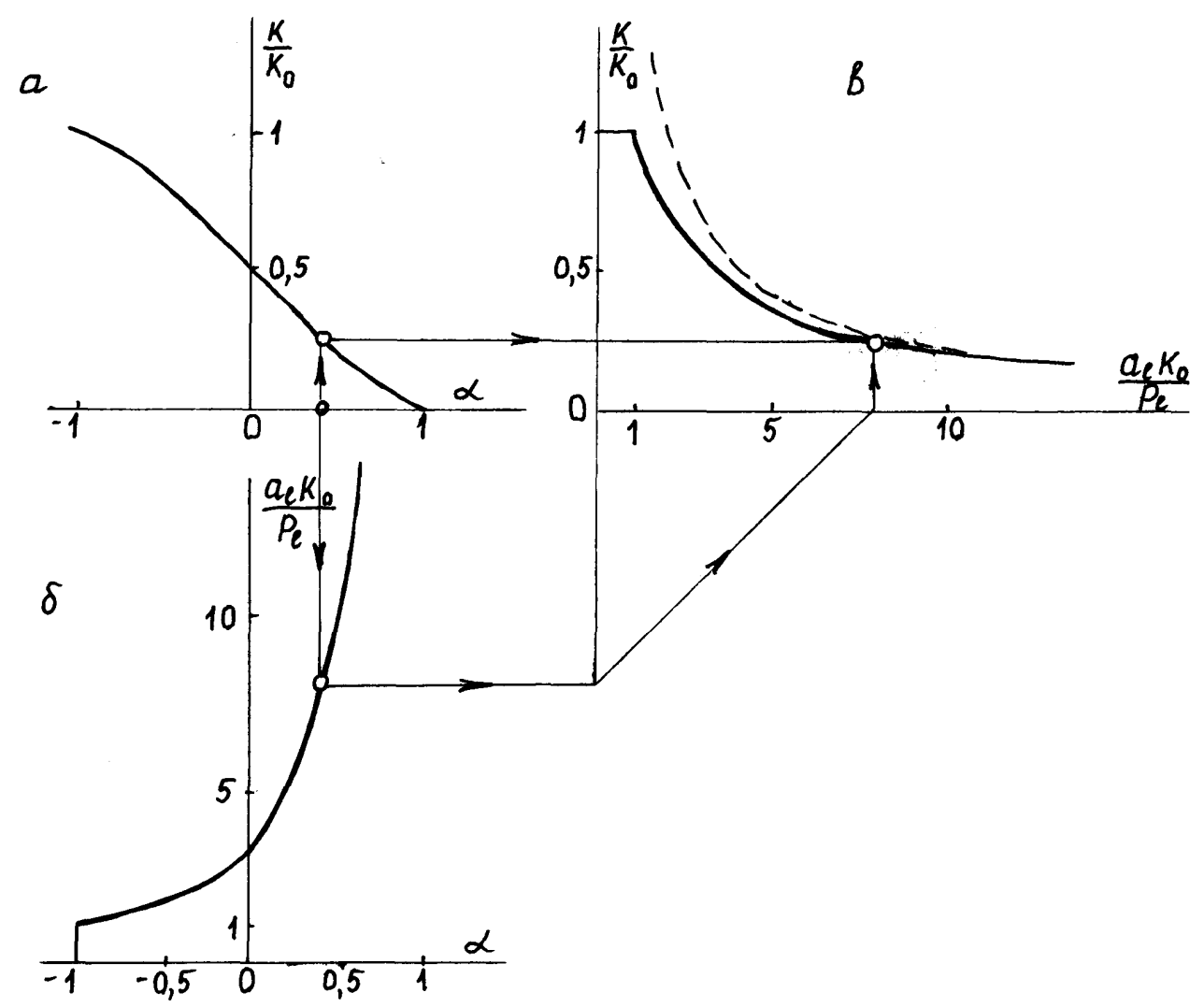

Рис.8.2

На рис.8.2,в с помощью этих кривых построена зависимость коэффициента $k$ от амплитуды $a_{l}$, которая достаточно хорошо 
аппроксимируется уравнением

$$
k=\frac{2 P_{l}}{a_{1}+P_{l} / k_{0}}
$$

(График (8.33) практически совпадает с кривой на рис.8.2,в.)

Заметим, что при амплитудах $a_{l} \leq P_{l} k_{0}$ колебания происходят в пределах упругой деформации ограничителя, поэтому нагрузка линейна и paвна $k=k_{0}$.

Найденные коэффициенты (8.29), (8.30), (8.33) будут в дальнейшем использоваться для анализа виброударных процессов, играющих большую роль в ультразвуковых технологических системах. Эти коэффициенты описывают, однако, лишь упругую составляющую нагрузки. Для нахождения диссипативной составляющей следует использовать иные модели ударного взаимодействия.

В основе простейшей модели лежит стереомеханическая теория удара $[60,117,118,137]$. Эта теория полностью исключает рассмотрение процесса удара из-за его малой продолжительности и дает оценку результирующих кинематических характеристик соударяющихся тел с помощью общих теорем механики. В рамках стереомеханической теории эффект соударения оценивается величиной коэффициента восстановления скорости $R(0 \leq R \leq 1)$, равной отношению относительных скоростей соударяющихся тел после и до удара. Так, тело с массой $M$, движущееся со скоростью $\dot{и}_{-}$, после удара о неподвижную преграду приобретает скорость

$$
\dot{u}_{+}=-R \dot{u}_{-}
$$

При $R=1$ равенство (8.34) описывает абсолютно упругий удар, при $R=0$ чисто пластический.

Удар характеризуется импульсом $J=\int_{0}^{\tau} f(t) d t$ сил $f(t)$ возникающих в контактной зоне за время $\tau$ удара. Действие импульса предполагается мгновенным. Используя теорему об изменении количества движения и 
учитывая (8.34), находим

$$
J=M\left(\dot{u}_{+}-\dot{u}_{-}\right)=-M \dot{u}_{-}(1+R)
$$

В результате удара происходит частичная потеря энергии, величина которой

$$
A=\frac{M}{2}\left(\dot{u}_{-}^{2}-\dot{u}_{+}^{2}\right)=1 / 2 M \dot{u}_{-}^{2}\left(1-R^{2}\right)
$$

Стереомеханическая теория описывает удар тела с абсолютно жестким ограничителем, характеристика которого показана на рис.8.1,б. Переходя в (8.33) к пределу при $k_{0} \rightarrow \infty$, получим коэффициент линеаризации характеристики абсолютно жесткого ограничителя (штриховая линия на рис.8.2,в)

$$
k=2 P_{l} / a_{l}
$$

Отметим, что это выражение можно получить непосредственно из формул (8.29), (8.30). Для этого достаточно заметить, что при $k_{0} \rightarrow \infty$ параметр $\alpha=\left(\Delta-m_{l}\right) / a_{l} \rightarrow 1$. Исключим в (8.29), (8.30) величину $k_{0}$, поделив первое равенство на второе

$$
\frac{P_{I}}{k}=a_{I} \frac{\sqrt{1-\alpha^{2}}-\alpha \cdot \arccos \alpha}{\arccos \alpha-\alpha \cdot \sqrt{1-\alpha^{2}}}
$$

Переходя в полученном отношении к пределу при $\alpha \rightarrow 1$ и раскрывая неопределенность, находим $P_{l} / k=a_{l} / 2$, откуда следует соотношение (8.37).

Для учета потерь энергии при ударах построим коэффициент линеаризации диссипативной части характеристики удара, исходя из энергетических соображений. Если $P_{l}$ - среднее за период значение силы ударного взаимодействия, то полная величина ударного импульса, действующего на тело при периодических соударениях

$$
J=-\frac{2 \pi}{\omega} P_{I}
$$

Приравнивая величины импульса, задаваемые равенствами (8.35) и (8.39), находим скорость тела при подходе к ограничителю $\dot{u}_{-}=\frac{2 \pi \cdot P_{I}}{M \omega \cdot(1+R)}$ 
и с помощью (8.36) потерю энергии при ударе

$$
A=\frac{1-R}{1+R} \cdot \frac{2 \pi^{2} P_{l}^{2}}{M \omega^{2}}
$$

Энергия, теряемая за период $T$, в эквивалентном линейном сопротивлении

$$
A=1 / 2 b\left(a_{l} \omega\right)^{2} T=\pi \cdot b \omega \cdot a_{l}^{2}
$$

Приравнивая величины (8.40) и (8.41), получим диссипативную составляющую коэффициента линеаризации ударных сил

$$
b=2 \pi \frac{1-R}{1+R} \cdot \frac{P_{l}^{2}}{M \omega^{3} a_{l}^{2}}
$$

С помощью (8.37) это выражение можно записать в иной форме

$$
b=\frac{\pi \cdot k^{2}(a)}{2 M \omega^{3}} \cdot \frac{1-R}{1+R}
$$

5. В случаях, когда временем ударного взаимодействия нельзя пренебречь, стереомеханическая модель удара оказывается неприменимой и приходится использовать иные модели. Одна из них построена в п.3.4 (рис.3.14); ее динамическая характеристика (рис.8.1,в) определяется уравнением

$$
f_{l}\left(u_{l}, \dot{u}_{l}\right)=k_{0}\left(u_{l}-\Delta\right) \eta\left(u_{l}-\Delta\right)\left(1+\theta \operatorname{sgn} \dot{u}_{l}\right)
$$

Характеристики такого рода в общем случае можно представить в виде

$$
f_{l}\left(u_{l}, \dot{u}_{l}\right)=f_{l}^{(1)}\left(u_{l}\right)+f_{l}^{(2)}\left(u_{l}, \dot{u}_{l}\right),
$$

где первое слагаемое $f_{l}^{(1)}\left(u_{l}\right)$ определяет упругие силы, a второе, удовлетворяющее условию $f_{l}^{(2)}\left(u_{l}, \dot{u}_{l}\right)=-f_{l}^{(2)}\left(u_{l},-\dot{u}_{l}\right) \quad, \quad$ описывает диссипативные силы. Легко показать, что формулы (8.23) - (8.25) для вычисления коэффициентов линеаризации характеристики типа (8.45) упрощаются и принимают вид [120]

$$
\begin{gathered}
P_{l}=\frac{1}{2 \pi} \int_{0}^{2 \pi} f_{l}^{(1)}\left(m_{l}+a_{l} \cos \tau\right) \cdot d \tau=P_{l}\left(m_{l}, a_{l}\right) \\
k=\frac{1}{\pi \cdot a_{l}} \int_{0}^{2 \pi} f_{l}^{(1)}\left(m_{l}+a_{l} \cos \tau\right) \cos \tau \cdot d \tau=k\left(m_{l}, a_{l}\right)
\end{gathered}
$$




$$
b=-\frac{1}{\pi \cdot \omega \cdot a_{l}} \int_{0}^{2 \pi} f_{l}^{(2)}\left(m_{l}+a_{l} \cos \tau,-a_{l} \omega \cdot \sin \tau\right) \sin \tau \cdot d \tau=b\left(m_{l}, a_{l}\right)
$$

Преобразованием последней формулы

$$
b=\frac{1}{\pi \cdot \omega \cdot a_{l}^{2}} \int_{0}^{2 \pi} f_{l}^{(2)}\left(m_{l}+a_{l} \cos \tau,-a_{l} \omega \cdot \sin \tau\right) \cdot d\left(a_{l} \cos \tau\right)=\frac{1}{\pi \cdot \omega \cdot a_{l}^{2}} \oint f_{l}\left(u_{l}, \dot{u}_{l}\right) d u=\frac{A}{\pi \cdot \omega \cdot a_{l}^{2}}
$$

коэффициент линеаризации $b$ выражается через рассеиваемую за цикл энергию $A$, равную площади петли гистерезиса характеристики $f_{l}\left(u_{l}, \dot{u}_{l}\right)$. Поэтому при исследовании колебаний, близких к гармоническим, форма петли гистерезиса не играет существенной роли.

Для характеристики (8.44) из ее сравнения с (8.28) следует, что коэффициенты линеаризации $P_{l}$ и $k$ определяются выражениями (8.29) и (8.30) или их аппроксимацией (8.33). Для диссипативной составляющей (8.47) находим

$$
b=\frac{k_{0} \vartheta}{\pi \cdot \omega}\left(1-\frac{\Delta-m_{l}}{a_{l}}\right)^{2}=\frac{k_{0} \vartheta}{\pi \cdot \omega}(1-\alpha)^{2}
$$

При исследовании виброударных процессов часто приходится рассматривать двусторонние ударные взаимодействия [60], динамические характеристики которых показаны на рис.8.1,г,д. Характеристика на рис.8.1,д описывается уравнением

$$
f_{l}\left(u_{l}\right)=k_{0}\left(\left|u_{l}\right|-\Delta\right) \eta \cdot\left(\left|u_{l}\right|-\Delta\right) \operatorname{signu}_{l}\left(1+\vartheta \cdot \operatorname{signu}_{l}\right),
$$

а на рис.8.1,г - тем же уравнением при $\vartheta=0$.

При рассмотрении симметричных процессов, таких что $u_{l}(t+T / 2)=-u_{l}(t)$, постоянные составляющие процессов $m_{l}=0$ и $P_{l}=0 . \mathrm{B}$ этом случае из сравнения (8.50) и (8.43) видно, что коэффициенты гармонической линеаризации динамической характеристики (8.50) получаются удвоением найденных ранее величин (8.30) и (8.49) при $m_{l}=0$ и имеют следующий вид:

$$
k=\frac{2 k_{0}}{\pi}\left(\arccos \frac{\Delta}{a_{l}}-\frac{\Delta}{a_{l}} \sqrt{1-\left(\frac{\Delta}{a_{l}}\right)^{2}}\right)
$$




$$
b=\frac{2 k_{0} \vartheta}{\pi \cdot \omega}\left(1-\frac{\Delta}{a_{1}}\right)^{2}
$$

На рис.8.3,а,б построены графики зависимостей коэффициентов (8.51), (8.52) от амплитуды $a_{l}$.

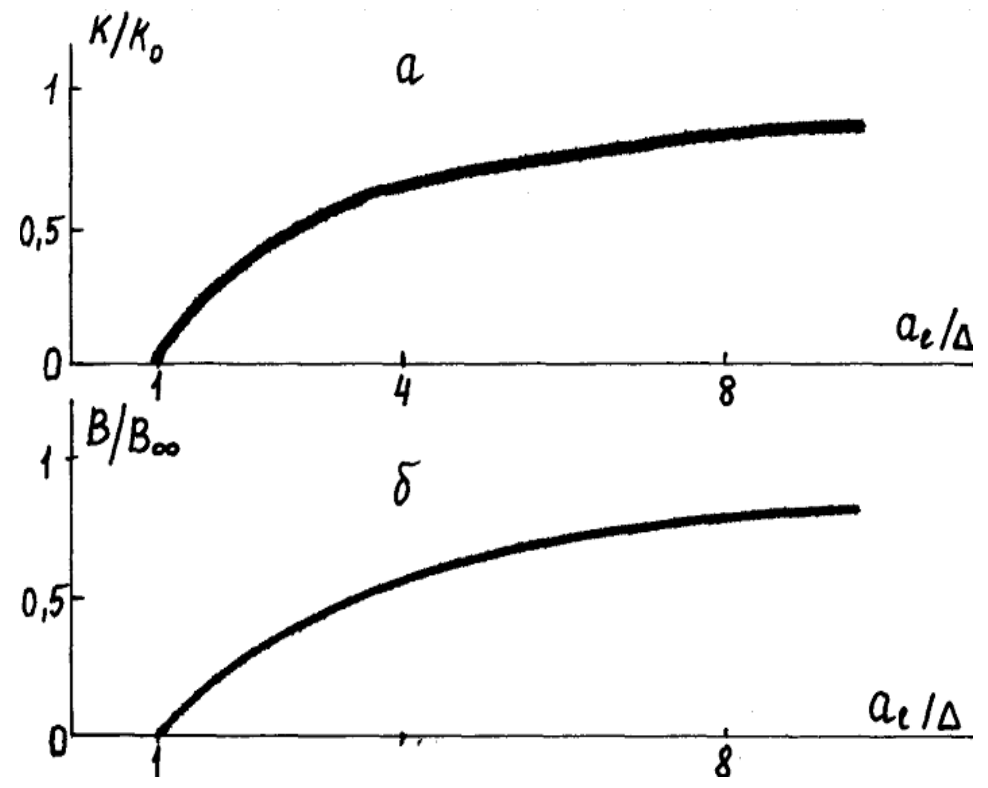

Рис. 8.3

При колебаниях в пределах зазора $\left(a_{l}<\Delta\right)$ нагрузка на колебательную систему отсутствует. При амплитуде $a_{l} \rightarrow \infty$ упругая нагрузка $k \rightarrow k_{0}$, а диссипативная $b \rightarrow b_{\infty}=2 k_{0} \vartheta / \pi \cdot \omega$.

6. В Гл.1 были рассмотрены модели технологических процессов, допускающие медленное, направленное в среднем, движение под действием приложенной к рабочему органу постоянной силы. Это движение определяет скорость проведения технологического процесса. Такие модели описывают процессы в системах с сухим трением, процессы пластического деформирования или разрушения материалов вибрирующим инструментом и другие. Динамические характеристики некоторых из этих процессов показаны на рис.8.4.

При рассмотрении таких процессов будем искать приближенное решение вида

$$
\begin{aligned}
& u_{l}(t) \approx v t+u_{l}^{0}(t)=v t+a_{l} \cos \omega \cdot t \\
& \dot{u}_{l}(t) \approx v+\dot{u}_{l}^{0}(t)=v-a_{l} \omega \cdot \sin \omega \cdot t
\end{aligned},
$$

где $v$ - скорость направленного движения; $u_{l}^{0}$ - периодическая 
составляющая решения с амплитудой $a_{l}$ и частотой $\omega$, а нелинейную динамическую характеристику $f_{I}\left(u_{l}, \dot{u}_{l}\right)$ заменим по аналогии с (8.22) линейной функцией

$$
f_{l}\left(u_{l}, \dot{u}_{l}\right) \approx P_{l}+k u_{l}^{0}+b \dot{u}_{l}^{0}
$$

Коэффициенты линеаризации в (8.54) вычисляются по формулам, подобным (8.32) - (8.25):

$$
\begin{gathered}
P_{l}=\frac{1}{2 \pi} \int_{0}^{2 \pi} f_{l}\left(\frac{v}{\omega} \tau+a_{l} \cos \tau, v-a_{l} \omega \cdot \sin \tau\right) d \tau=P_{l}\left(v, a_{l}\right) \\
k=\frac{1}{\pi \cdot a_{l}} \int_{0}^{2 \pi} f_{l}\left(\frac{v}{\omega} \tau+a_{l} \cos \tau, v-a_{l} \omega \cdot \sin \tau\right) \cos \tau \cdot d \tau=k\left(v, a_{l}\right) \\
b=\frac{1}{\pi \cdot a_{l} \omega} \int_{0}^{2 \pi} f_{l}\left(\frac{v}{\omega} \tau+a_{l} \cos \tau, v-a_{l} \omega \cdot \sin \tau\right) \sin \tau \cdot d \tau=b\left(v, a_{l}\right)
\end{gathered}
$$

В Гл.1 было показано, что колебания рабочего органа оказывают существенное влияние на статические силы, необходимые для преодоления предельных сопротивлений. Такое влияние отражается равенством (8.55). Соотношения (8.56), (8.57) показывают, что существует и обратное влияние медленного движения на коэффициенты гармонической линеаризации $k$ и $b$ и, следовательно, на параметры колебаний.

На рис.8.4,а показана характеристика нелинейного элемента типа «сухое трение», уравнение которой имеет вид

$$
f_{l}\left(\dot{u}_{l}\right)=Q \operatorname{sgn} \dot{u}
$$
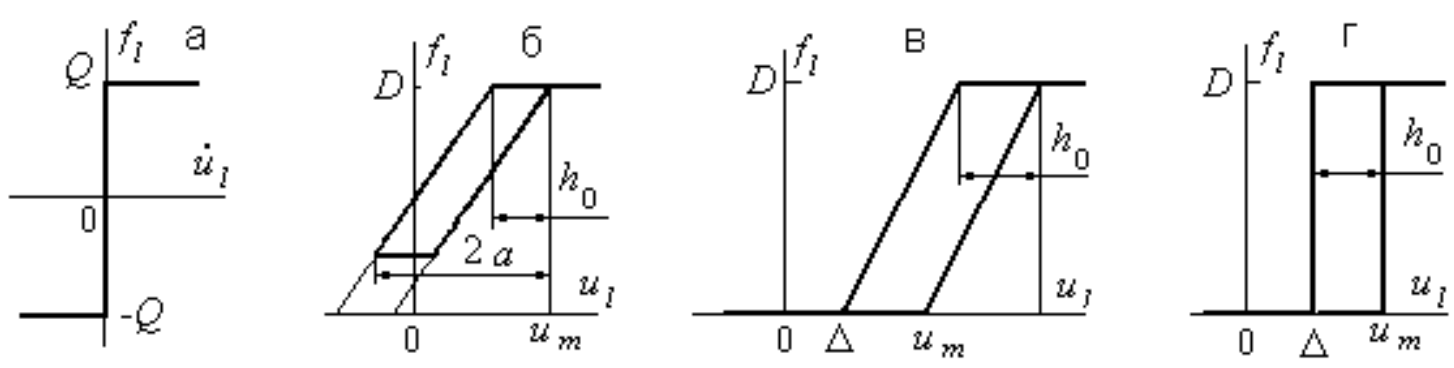

Рис.8.4

После подстановки (8.58), (8.53) в (8.55) - (8.57) и интегрирования получаем

$$
P=\frac{2 Q}{\pi} \arcsin \frac{V}{a_{l} \omega},
$$




$$
k=0, b=\frac{4 Q}{\pi \cdot \omega \cdot a_{l}} \sqrt{1-\left(\frac{V}{\omega \cdot a_{l}}\right)^{2}}
$$

Равенство (8.59) совпадает с (3.6) и отражает эффект вибрационного сглаживания нелинейности (рис.3.3).

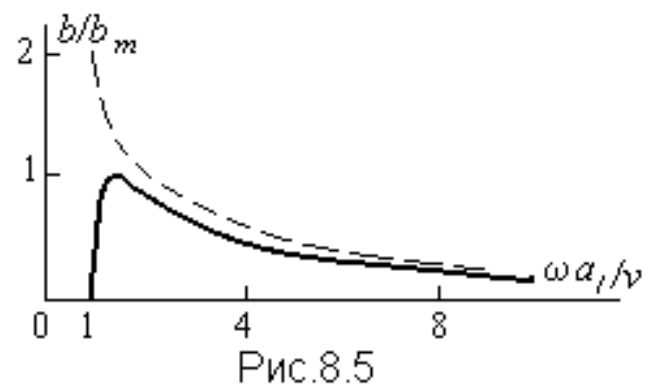

На рис.8.5 сплошной линией построена зависимость эквивалентного коэффициента сопротивления (8.60) от амплитуды колебательной скорости $\omega a_{1}$. Она достигает максимального значения $b_{m}=2 Q / \pi v$ при $\omega a_{l}=\sqrt{2} v$. Формулы (8.59), (8.60) справедливы при скоростях $v<a_{l} \omega . \mathrm{B}$ противном случае $P=Q$ и $b=0$, т.е. колебания не снижают сил сухого трения, а трение, в свою очередь, не оказывает влияния на колебания. При очень малой скорости $v<<\omega \cdot a_{l}$ из (8.60), пренебрегая величиной второго порядка малости, находим

$$
b=4 Q / \pi \omega a_{l}
$$

Зависимость (8.61) построена на рис.8.5 штриховой линией. Как показано в Гл.1 и следует из (8.59), применение ультразвука для снижения статических сил целесообразно именно при малых скоростях $v<<\omega \cdot a_{l}$. Поэтому при практических расчетах колебаний допустимо использовать коэффициент линеаризации в форме (8.61).

На рис.8.4,б,в показаны динамические характеристики процессов пластического деформирования материала в непрерывном (см. рис.2.5) и импульсном (рис.2.7) режимах. Стрелками показано направление деформирования и разгрузки. Приведем выражения для коэффициентов гармонической линеаризации этих характеристик при малой средней скорости деформирования $\left(v<<\omega \cdot a_{l}\right)$. Эти формулы получены в рамках допущений, принятых при выводе равенств (2.18) и (2.19), определяющих 
постоянные составляющие $P_{l}$ нагрузки.

Для характеристики на рис.8.5,б имеем

$$
\begin{gathered}
k=\frac{k_{0}}{2 \pi}\left[2 \pi-\arccos \left(1-\frac{2 \pi \cdot V}{a_{l} \omega}\right)+\left(1-\frac{2 \pi \cdot V}{a_{l} \omega}\right) \sqrt{1-\left(1-\frac{2 \pi \cdot V}{a_{l} \omega}\right)^{2}}\right] \\
b=\frac{2 k_{0}}{\pi \cdot \omega}\left[1-\left(1-\frac{\pi \cdot V}{a_{l} \omega}\right)^{2}\right]
\end{gathered}
$$

Обратим внимание на то, что в режиме непрерывного пластического деформирования коэффициенты (8.62), (8.63) не зависят от предела текучести $D$ материала. Это связано с тем, что они определяются величиной превышения предела текучести над статической силой $P_{l}$, т.е. разностью $D-P_{l}$, которая согласно (2.19) зависит лишь от соотношения колебательной $\omega \cdot a_{l}$ и медленной $v$ скоростей, амплитуды $a_{l}$ и статической жесткости $k_{0}$ изделия.

При $v=0$ формулы (8.62), (8.63) дают $k=k_{0}, b=0$, т.е. деформирование происходит в пределах упругой области динамической характеристики, а предел текучести достигается лишь в точке с максимальным отклонением рабочего органа.

Для характеристики на рис.8.5,в имеем

$$
\begin{aligned}
& k=\frac{k_{0}}{2 \pi}\left[\begin{array}{l}
\arccos \left(1-\frac{D}{k_{0} a_{l}}-\frac{2 \pi \cdot v}{a_{l} \omega}\right)+\arccos \left(1-\frac{D}{k_{0} a_{l}}\right)- \\
\arccos \left(1-\frac{2 \pi \cdot v}{a_{l} \omega}\right)-\left(1-\frac{D}{k_{0} a_{l}}-\frac{2 \pi \cdot v}{a_{l} \omega}\right) \sqrt{1-\left(1-\frac{D}{k_{0} a_{l}}-\frac{2 \pi \cdot v}{a_{l} \omega}\right)}- \\
-\left(1-\frac{D}{k_{0} a_{l}}\right) \sqrt{1-\left(1-\frac{D}{k_{0} a_{l}}\right)^{2}}+\left(1-\frac{2 \pi \cdot v}{a_{l} \omega}\right) \sqrt{1-\left(1-\frac{2 \pi \cdot v}{a_{l} \omega}\right)^{2}}
\end{array}\right] \\
& b=\frac{2 v D}{\left(a_{l} \omega\right)^{2}}
\end{aligned}
$$

Последняя формула легко выводится из энергетических соображений. Действительно, работа деформирования за цикл нагружения равна площади петли гистерезиса $A=D h_{0}$, где величина остаточной деформации $h_{0}$ связана со средней скоростью деформирования соотношением (2.11) и равна $h_{0}=v T=2 \pi \cdot v / \omega$. Подстановка этих величин в 
(8.48) приводит к формуле (8.65). Отметим, что при $v=0$ выражение (8.64) приводится к (8.30), где $\left(\Delta-m_{l}\right)=a_{l}-D / k_{0} \quad$, т.е. ограничитель деформируется на всю величину упругой зоны характеристики материала. Если к тому же $a_{1} \rightarrow D / 2 k_{0}$, то $k \rightarrow k_{0}$ и режим импульсного упругого деформирования переходит в гармонический режим непрерывного деформирования.

На рис.8.4,г показана динамическая характеристика деформирования жесткопластического материала. При малых скоростях деформирования $\left(v<<\omega \cdot a_{l}\right)$ коэффициенты линеаризации этой нелинейной характеристики имеют вид

$$
\begin{gathered}
P=\frac{D}{\pi} \arcsin \sqrt{\frac{\pi \cdot v}{a_{l} \omega}} \\
k=\frac{D}{\pi \cdot a_{l}} \sqrt{1-\left(1-\frac{2 \pi \cdot v}{a_{l} \omega}\right)^{2}} \\
b=\frac{2 D V}{\left(a_{l} \omega\right)^{2}}
\end{gathered}
$$

Выражения (8.66), совпадающее с (2.24), и (8.67) могут быть получены предельным переходом при $k_{0} \rightarrow \infty$ из (2.18) и (8.64), а равенство (8.68) совпадает с (8.65). Для удобства практических расчетов этим формулам можно придать иную форму. С этой целью из (8.66) выразим скорость $v$ через остальные величины

$$
v=\frac{a_{l} \omega}{\pi} \sin ^{2} \frac{\pi \cdot P}{D}
$$

Подставив полученную величину в (8.67) и (8.68), найдем

$$
\begin{aligned}
& k=\frac{D}{\pi \cdot a_{l}} \sin \frac{2 \pi \cdot P}{D} \\
& b=\frac{2 D}{\pi \cdot a_{l} \omega} \sin ^{2} \frac{\pi \cdot P}{D}
\end{aligned}
$$

Ряд ультразвуковых технологических процессов протекает при малых статических силах $P<<D$. В этом случае выражения (8.69), (8.70) упрощаются и приобретают вид 


$$
\begin{gathered}
V=\pi \cdot a_{l} \omega \cdot(P / D)^{2} \\
k=2 P / a_{l}, b=\frac{2 \pi}{a_{l} \omega} \cdot \frac{P^{2}}{D}
\end{gathered}
$$

Обратим внимание на то, что выражение (8.72) для коэффициента линеаризации $k$ не зависит от предела текучести $D$ жесткопластического материала и совпадает с формулой (8.37), полученной для характеристики абсолютно жесткого одностороннего ограничителя.

Итак, для ряда ультразвуковых технологических процессов мы нашли коэффициенты, характеризующие нелинейную технологическую нагрузку. Типичной особенностью этих коэффициентов является их зависимость от параметров колебаний рабочего органа. Такая зависимость неоднократно наблюдалась экспериментально при непосредственном измерении сопротивления нагрузки различных технологических процессов. В [197] обнаружена зависимость сопротивления нагрузки от амплитуды колебаний при излучении в жидкость после прохождения порога кавитации. В [204] такая зависимость выявлена при изучении процесса ультразвуковой сварки, а в [88] - процесса ультразвуковой обработки хрупких материалов. 


\section{Резонансные колебания ультразвуковых стержневых систем}

\section{с нелинейной нагрузкой.}

1. Нелинейность технологической нагрузки вызывает специфические эффекты при резонансных колебаниях ультразвуковых стержневых систем. Технологическая нагрузка существенно изменяет резонансные свойства стержневой системы, приводит к нелинейным искажениям амплитудно-частотных характеристик, зависящим от параметров системы, способа ее закрепления, упругих и диссипативных свойств нагрузки. Эти эффекты рассмотрим на примере стержневых систем, совершающих продольные и изгибные колебания.

Рассмотрим стержневую систему длиной $l$, колебания которой возбуждаются силой $f_{s}(t)=F_{s} \mathrm{e}^{j \omega \cdot t}$, действующей в сечении с координатой $x=s$. Смещение произвольного сечения $x$ будем описывать функцией $u_{x}(t)$. В процессе колебаний концевое сечение $x=l$ взаимодействует с технологической нагрузкой, динамическая характеристика которой записывается в функции смещения $u_{l}$ конца $x=l$ системы

$$
f_{l}=f_{l}\left(u_{l}, p u_{l}\right),(p=\partial / \partial \cdot t)
$$

Колебания стержневой системы опишем, используя понятие оператора динамической податливости $L_{s x}(p)$, устанавливающего связь между силой, действующей в сечении $s$, и вызываемым этой силой перемещением $x$. Смещение сечений системы определяется с помощью выражения

$$
u_{x}(t)=L_{s x}(p) \cdot F_{s} \mathrm{e}^{j \omega \cdot t}-L_{l x}(p) \cdot f_{l}\left[u_{l}(t), p u_{l}(t)\right]
$$

Приближенное периодическое решение уравнения отыскиваем в виде

$$
u_{x}(t) \approx m_{x}+u_{x}^{0}(t), u_{x}^{0}(t)=\widetilde{a}_{x} \mathrm{e}^{j \omega \cdot t}=a_{x} \mathrm{e}^{j\left(\omega \cdot t-\varphi_{x}\right)}
$$


Учитывая характер отыскиваемых решений, проведем гармоническую линеаризацию функции (9.1)

$$
f_{l}\left(u_{l}, p u_{l}\right) \approx P_{l}\left(m_{l}, a_{l}\right)+\left[k\left(m_{l}, a_{l}\right)+p b\left(m_{l}, a_{l}\right)\right] \cdot u_{l}^{0},
$$

где $P_{l}\left(m_{l}, a_{l}\right)$ - постоянная составляющая; $k\left(m_{l}, a_{l}\right), b\left(m_{l}, a_{l}\right)$ - эквивалентные упругая и диссипативная составляющие нагрузки, зависящие от искомых параметров решения и вычисляемые по методике, изложенной в Параграфе 8 .

Подставляя (9.4) в (9.2) и разделяя постоянные и периодические составляющие, с учетом (1.3) получаем

$$
\begin{gathered}
m_{X}=-L_{l x}(0) P_{l}\left(m_{l}, a_{l}\right) \\
u_{x}^{0}=L_{s x}(p) F_{s} \mathrm{e}^{j \omega \cdot t}-L_{l x}(p)\left[k\left(m_{l}, a_{l}\right)+p b\left(m_{l}, a_{l}\right)\right] u_{l}^{0}
\end{gathered}
$$

Из (9.5), (9.6) найдем для сечения $x=l$

$$
\begin{gathered}
m_{l}=-L_{l l}(0) P_{l}\left(m_{l}, a_{l}\right) \\
u_{l}^{0}=L_{s l}(p) F_{s} \mathrm{e}^{j \omega \cdot t}-L_{l l}(p)\left[k\left(m_{l}, a_{l}\right)+p b\left(m_{l}, a_{l}\right)\right] u_{l}^{0}
\end{gathered}
$$

Из (9.3) при $x=l$ запишем равенство

$$
\mathrm{e}^{j \omega \cdot t}=\frac{u_{l}^{0}}{a_{l}} \mathrm{e}^{j \varphi_{l}}=\frac{u_{l}^{0}}{a_{l}}\left(\cos \varphi_{l}+j \sin \varphi_{l}\right)
$$

Подставив (9.9) в уравнение (9.8), преобразуем его к эквивалентному однородному [186]

$$
\left\{1+\left[k\left(m_{l}, a_{l}\right)+p b\left(m_{l}, a_{l}\right)\right] \cdot L_{l l}(p)-\frac{F_{s}}{a_{l}}\left(\cos \varphi_{l}+j \sin \varphi_{l}\right) \cdot L_{s l}(p)\right\} \cdot u_{l}^{0}=0
$$

Условием существования периодических решений (9.3) уравнения (9.10) является наличие пары чисто мнимых корней $p= \pm j \omega$ его характеристического уравнения. Подставив в последнее $p=j \omega$, получим

$$
\frac{F_{s}}{\widetilde{a}_{l}}=\frac{F_{s}}{a_{l}}\left(\cos \varphi_{l}+j \sin \varphi_{l}\right)=W\left(j \omega, m_{l}, a_{l}\right),
$$

где $W\left(j \omega, m_{l}, a_{l}\right)=\left\{1+\left[k\left(m_{l}, a_{l}\right)+j \omega \cdot b\left(m_{l}, a_{l}\right)\right] \cdot L_{l l}(j \omega)\right\} / L_{s l}(j \omega)$ - приведенная к сечению $l$ суммарная динамическая жесткость колебательной системы и технологической нагрузки. 
По аналогии с (5.42) выражение для динамической жесткости можно привести к виду

$$
W\left(j \omega, m_{l}, a_{l}\right)=U\left(\omega, m_{l}, a_{l}\right)+j V\left(\omega, m_{l}, a_{l}\right),
$$

где $U\left(\omega, m_{l}, a_{l}\right)=\operatorname{Re} W\left(j \omega, m_{l}, a_{l}\right) ; V\left(\omega, m_{l}, a_{l}\right)=\operatorname{Im} W\left(j \omega, m_{l}, a_{l}\right)$.

После подстановки (9.12) в (9.11) и разделения вещественной и мнимой частей найдем

$$
\begin{aligned}
& \cos \varphi_{l}=a_{l} U\left(\omega, m_{l}, a_{l}\right) / F_{s} \\
& \sin \varphi_{l}=a_{l} V\left(\omega, m_{l}, a_{l}\right) / F_{s}
\end{aligned}
$$

Исключив из этих равенств тригонометрические функции, получим для амплитуды колебаний

$$
a_{l}=F_{s} / \sqrt{U^{2}\left(\omega, m_{l}, a_{l}\right)+V^{2}\left(\omega, m_{l}, a_{l}\right)}=F_{s} /\left|W\left(j \omega, m_{l}, a_{l}\right)\right|
$$

Соотношения (9.7) и (9.15) образуют систему уравнений относительно неизвестных параметров $m_{l}, a_{l}$ движения сечения $x=l$. Величины $m_{l}$ и $a_{l}$ через уравнения (9.13) и (9.14) определяют фазу $\varphi_{\text {, }}$, а при помощи уравнений (9.5) - (9.6) - параметры периодического движения остальных сечений стержневой системы.

Для исследования устойчивости полученных решений по отношению к малым возмущениям воспользуемся предложенным в $[59,60]$ приемом, основанным на идеях метода медленно меняющихся амплитуд. В силу непрерывной зависимости корней характеристического уравнения (9.10) от параметров системы малые возмущения движения приводят к малому уходу корней уравнения от мнимой оси. Учитывая это, полагаем, что в окрестности установившегося периодического решения (1.3) возмущенное движение имеет вид

$$
u_{x}^{*}(t)=m_{x}^{*}(t)+a_{x}^{*}(t) \exp j\left[\omega \cdot t-\varphi_{x}^{*}(t)\right]
$$

где $m_{x}^{*}(t), a_{x}^{*}(t), \varphi_{x}^{*}(t)$ - медленно меняющиеся функции времени.

Диссипативные свойства системы полностью учитываются функцией $V\left(\omega, m_{l}, a_{l}\right)$ в выражении (9.11) для динамической жесткости. Поэтому равенство (9.14) является приведенным условием баланса энергий диссипативных и вынуждающих сил на исследуемом стационарном движении. Используя (9.11), сформулируем условие 
устойчивости этого движения по отношению к малым отклонениям амплитуды в следующем виде:

$$
\frac{\partial}{\partial a^{*}}\left[V\left(\omega, m_{l}^{*}, a_{l}^{*}\right)-\frac{F_{s}}{a_{l}^{*}} \sin \varphi_{l}^{*}\right]_{a_{l}^{*}=a_{l}}>0
$$

Неравенство (9.17) означает, что при отклонении амплитуды $a_{l}^{*}(t)$ от стационарного значения $a_{l}=$ const баланс энергии нарушается таким образом, чтобы скомпенсировать это отклонение.

Вводя в (9.16) три неизвестные функции вместо одной, их можно связать двумя произвольными условиями. Положим, что в возмущенном движении функции $m_{l}^{*}(t)$ и $a_{l}^{*}(t)$ связаны равенством (9.7), а $\varphi_{l}^{*}(t)$ и $a_{l}^{*}(t)$ равенством (9.13). Дифференцируя (9.17) с учетом этих связей, имеем

$$
\left[\frac{\partial V}{\partial a_{l}^{*}}+\frac{F_{s}}{a_{l}^{* 2}}\left(\sin \varphi_{l}^{*}-a_{l}^{*} \frac{\partial \varphi_{l}^{*}}{\partial a_{l}^{*}} \cos \varphi_{l}^{*}\right)\right]_{a_{l}^{*}=a_{l}}>0
$$

Производную $\partial \varphi_{1}^{*} / \partial a_{1}^{*}$ найдем дифференцированием равенства (9.13) как неявной функции $\varphi_{I}\left(a_{1}\right)$

$$
\frac{\partial \cdot \varphi_{l}^{*}}{\partial a_{l}^{*}}=-\frac{1}{F_{s} \sin \varphi_{l}^{*}}\left(U+a_{l}^{*} \frac{\partial U}{\partial a_{l}^{*}}\right)
$$

Подставляя (9.19) в неравенство (9.18) и учитывая выражения (9.13) и (9.14), получаем окончательно условие устойчивости

$$
\left[U\left(U+a_{l}^{*} \frac{\partial U}{\partial a_{l}^{*}}\right)+V\left(V+a_{l}^{*} \frac{\partial V}{\partial a_{l}^{*}}\right)\right]>0
$$

При отсутствии в системе диссипативных потерь $V=0$ условие (9.20) дает следующие границы области устойчивости:

$$
U\left(\omega, m_{l}, a_{l}\right)=0, U\left(\omega, m_{l}, a_{l}\right)+a_{l} \frac{\partial \cdot U\left(\omega, m_{l}, a_{l}\right)}{\partial \cdot a_{l}}=0,
$$

где величины $m_{l}$ и $a_{l}$ связаны соотношением (9.7).

2. В качестве примера рассмотрим продольные колебания однородного стержня (рис.9.1,a), верхний конец $x=0$ которого жестко закреплен, а нижний $x=l$ взаимодействует с упругим ограничите-лем, установленным с зазором $\Delta$ по отношению к положению статического равновесия стержня. Отрицательная величина $\Delta$ соответствует уста-новке стержня с предварительным натягом. 


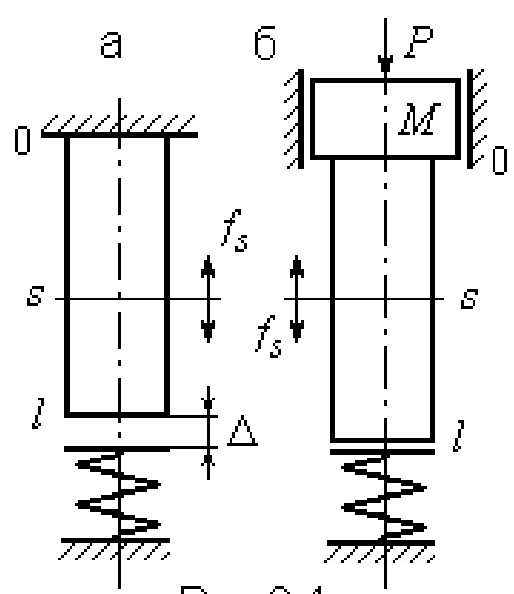

Рис.9.1

Коэффициенты гармонической линеаризации характеристики упругого ограничителя даются (8.29), (8.30). Выражение для динамической жесткости в (9.11) запишем в виде

$$
W\left(j \omega, m_{l}, a_{l}\right)=\left[W_{l l}(j \omega)+k\left(m_{l}, a_{l}\right)\right] \cdot K(j \omega)
$$

где $K(j \omega)=W_{s l}(j \omega) / W_{l l}(j \omega)$.

Используя динамические податливости (5.131) закрепленного стержня с учетом (5.12) и ограничивая разложения гиперболических функций, содержащих малый параметр $\psi$, линейными членами, запишем

$$
\begin{gathered}
W_{I I}(j \omega)=\frac{E S}{l}\left[\xi \cdot \operatorname{ctg} \xi+j \frac{\psi \cdot \xi}{4 \pi} \cdot \frac{(\xi+0,5 \sin 2 \xi)}{\sin ^{2} \xi}\right] \\
K(j \omega)=\frac{\sin \xi}{\sin \chi \cdot \xi}+j \frac{\psi \cdot \xi}{4 \pi} \cdot \frac{(\cos \xi \cdot \sin \chi \cdot \xi-\chi \cdot \sin \xi \cdot \cos \chi \cdot \xi)}{\sin ^{2} \chi \cdot \xi}
\end{gathered}
$$

где $\chi=s / l(0 \leq \chi \leq 1)$.

Заметим, что при действии возбуждения в крайнем сечении $s=l(\chi=1)$, коэффициент $K(j \omega)=1$. Величина (9.24) отражает влияние места приложения вынуждающей силы на амплитуду и фазу колебаний сечения $x=l$.

После подстановки (9.22), (9.23) уравнение (9.15) принимает вид

$$
a_{l}=\frac{\delta}{K(\xi)}\left\{(\xi \cdot \operatorname{ctg} \xi+q)^{2}+\left[\frac{\psi \cdot \xi}{4 \pi} \cdot \frac{(\xi+0,5 \sin 2 \xi)}{\sin ^{2} \xi}\right]^{2}\right\}^{-1 / 2},
$$

где $\delta=\frac{F_{s} l}{E S}, q=\frac{k\left(m_{l}, a_{l}\right) \cdot l}{E S}, K(\xi)=|K(j \xi)|$. 
Для определения параметров движения нижнего конца стержня к уравнению (9.25) следует добавить равенство (9.7), которое с учетом (8.29) и (9.23) приводится к виду

$$
a_{l}=\Delta \cdot\left[\alpha+\left(\alpha \cdot \arccos \alpha-\sqrt{1-\alpha^{2}}\right) q_{0} / \pi\right]^{-1}
$$

и выражение для коэффициента (8.30)

$$
q=\left(\arccos \alpha-\alpha \cdot \sqrt{1-\alpha^{2}}\right) q_{0} / \pi,
$$

где $q_{0}=k_{0} l / E S, \alpha=\left(\Delta-m_{l}\right) / a_{l}(-1 \leq \alpha \leq 1)$.

Используя полученные выражения, построим амплитудно-частотные характеристики для нижнего сечения стержня. Согласно (9.25) равенство

$$
\xi_{0} \operatorname{ctg} \xi_{0}+q=0,
$$

где $\xi_{0}=\omega_{0} / / c$, определяют конфигурацию скелетных кривых, отражающих зависимость частоты $\omega_{0}$ свободных колебаний системы от амплитуды.

Для построения скелетных кривых воспользуемся графическим способом, показанным на рис.9.2,а-в, где по формулам (9.26) - (9.28) соответственно построены графики зависимостей $a_{l}(\alpha), \quad q(\alpha)$ и $q\left(\xi_{0}\right)$. Задаваясь на рис.9.2,а значением амплитуды $a_{l}$ и осуществляя построение, показанное стрелками, находим с помощью рис.9.2,в собственную частоту $\xi_{0}$ и наносим на рис.9.2,г соответствующую точку скелетной кривой.

Отметим, что каждому значению амплитуды $a_{l}$ соответствует счетное множество собственных частот $\xi_{0}$, относящихся к различным формам колебаний. На рис.9.2,г показаны скелетные кривые для двух низших форм. Из-за нелинейности нагрузки частота колебаний по каждой форме зависит от амплитуды. При этом для системы с зазором $(\Delta>0)$ частота возрастает с ростом амплитуды, и система обладает скелетной кривой жесткого типа. Для системы с натягом $(\Delta<0)$ собственная частота убывает с ростом амплитуды, и система обладает скелетной кривой мягкого типа [120]. 


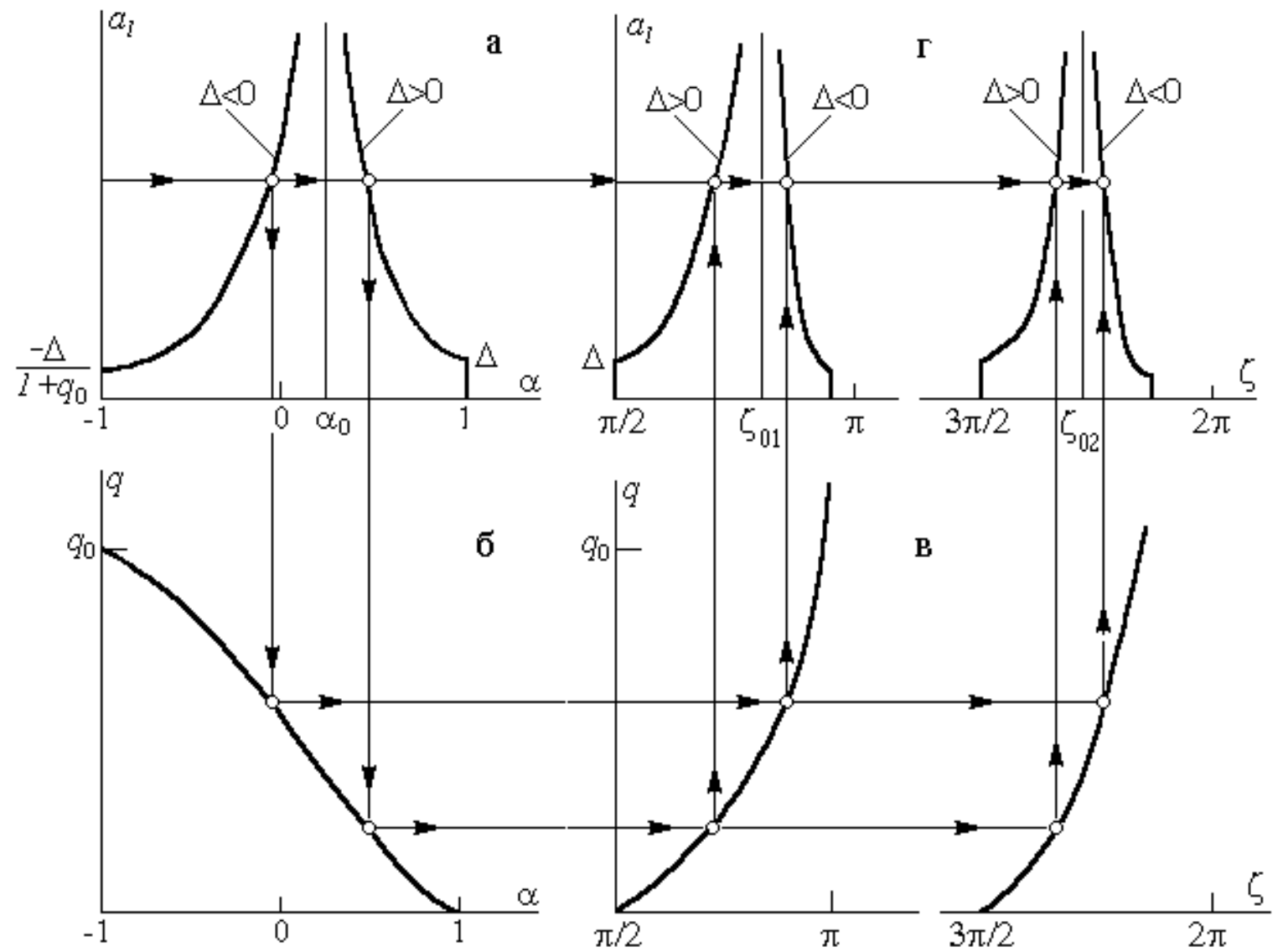

Puc. 9.2

Вместе с тем в обоих случаях могут реализоваться и линейные колебания. При $\Delta>0$ - это колебания в пределах зазора с амплитудой $a_{l}<\Delta$ и частотой собственных колебаний стержня со свободным нижним концом. При $\Delta<0$ возможны колебания в пределах предварительного натяга без отрыва нижнего сечения от ограничителя с амплитудой $a_{l} \leq-\Delta /\left(1+q_{0}\right)$ и частотой свободных колебаний стержня с упруго закрепленным нижним концом. Линейным колебаниям на рис.9.2,г соответствуют вертикальные отрезки скелетных кривых.

При $\Delta=0$ система обладает спектром постоянных, не зависящих от амплитуды, собственных частот, отличающихся от линейных.

Таким образом, скелетные кривые определены в диапазонах

$$
\begin{array}{ccc}
\xi_{n} \leq \xi_{0}<\xi_{0 n} & \text { при } & \Delta>0 \\
\xi_{0}=\xi_{0 n} & \text { при } & \Delta=0 \\
\xi_{0 n} \leq \xi_{0}<\xi_{H n} & \text { при } & \Delta<0
\end{array}
$$

где $\xi_{n}, \xi_{\text {нп }}$ - собственные частоты стержня со свободным и связанным с 
линейной упругой нагрузкой $k_{0}$ нижним концом, определяемые из уравнения (9.28) при $q=0$ и $q=q_{0}$ соответственно; $\xi_{0 п}$ - собственные частоты нелинейной системы при $\Delta=0$; индекс $n=1,2, \ldots$ обозначает номер собственной формы. Частоты $\xi_{0 п}$ также находятся из уравнения (9.28), в которое подставляется значение $q$, вычисленное по формуле (9.27) при $\alpha=\alpha_{0} \quad$, являющееся согласно (9.26) решением трансцендентного уравнения

$$
\alpha_{0}+\left(\alpha_{0} \arccos \alpha_{0}-\sqrt{1-\alpha_{0}^{2}}\right) q_{0} / \pi=0
$$

3. Амплитуды нижнего сечения $x=l$ стержня при вынужденных колебаниях находятся совместным решением системы уравнений (9.25)-(9.27), которое можно провести следующим графическим способом. Выражение (9.27) для коэффициента линеаризации $q$ подставляется в уравнение амплитудно-частотной характеристики (9.25) и полученная зависимость $a_{l}(\alpha)$ при различных частотах $\xi=$ const наносится на кривую (9.26), уже использовавшуюся при построении скелетных кривых. Точки пересечения указанных кривых определяют значения параметра $\alpha$ и амплитуды колебаний $a_{l}$, соответствующие заданной частоте $\xi$.

В результате таких построений, выполненных на рис.9.3,a,в, на рис.9.3,б,г получены возможные конфигурации резонансных кривых системы с предварительным зазором $(\Delta>0)$. Цифрой 1 обозначены скелетные кривые (рис.9.3,б,г) и их отображения (9.26) на плоскость $\left(a_{1}, \alpha\right)$ (рис.9.3,а,в). Параметром $\xi_{i}(i=1,2)$ на рис.9.3,а,в обозначены кривые, построенные по уравнениям (9.25), (9.27) при заданном значении частоты $\xi=\xi_{i}$. Получаемые решения отмечены точками. Из рис.9.3,а,в видно, что рассматриваемая система уравнений имеет либо одно, либо три решения. В последнем случае (рис.9.3,a) одно из решений определяется пересечением кривой $\xi_{i}$ с вертикальной прямой $\alpha=1$ и соответствует 
линейным колебаниям в пределах зазора с амплитудой $a_{1} \leq \Delta$. На рис.9.3,б,г, наряду с этими реализуемыми ветвями, штриховыми линиями показаны также нереализуемые $\left(a_{1}>\Delta\right)$ участки резонансных кривых линейных систем, определяемых уравнением (9.25) при $q=0$. Другие решения определяют разделенные скелетной линией ветви резонансных кривых, соответствующие нелинейным колебаниям с амплитудами $a_{l}>\Delta$. Из уравнений (9.25) - (9.27) при $\alpha=1$ и построений на рис.9.3 видно, что линейные и нелинейные ветви резонансных кривых сходятся в точках пересечения линейных ветвей уровнем ограничителя $a_{l}=\Delta$.

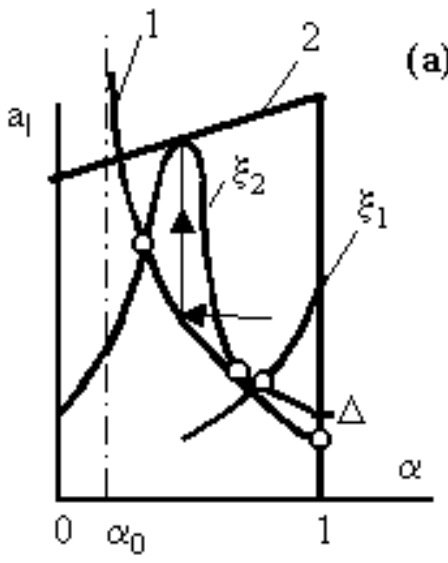

(a)
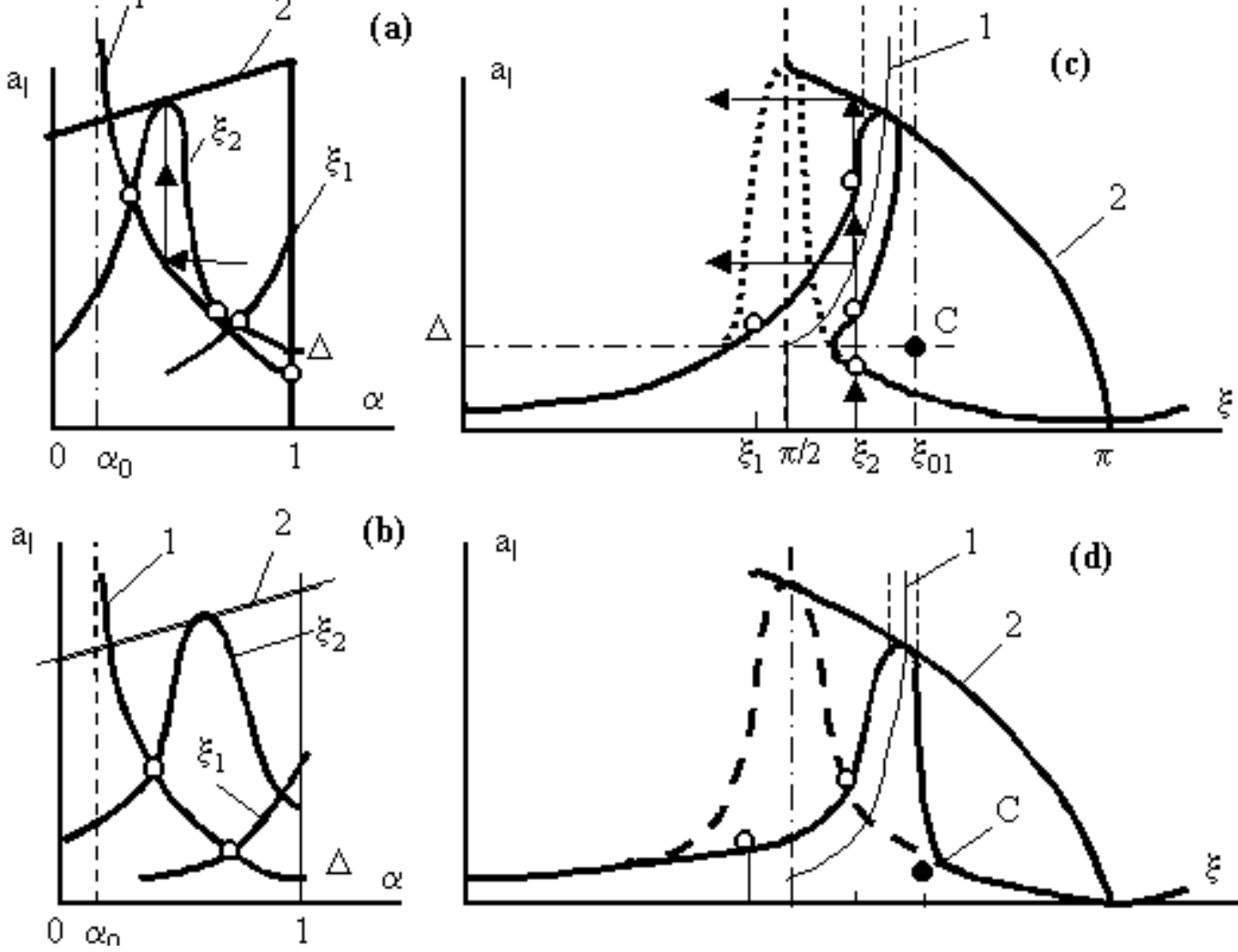

(b)

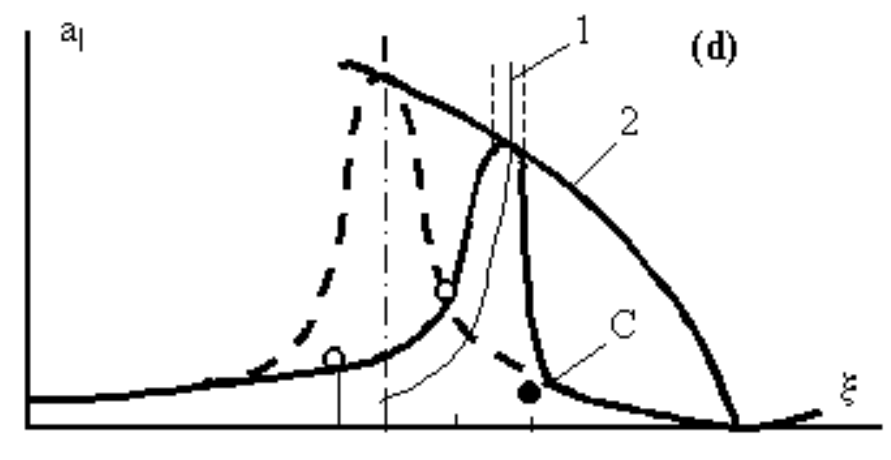

Рис.9.3

Из приведенных построений видно, что необходимым условием появления двузначности резонансной кривой (рис.9.3,б) является расположение зарезонансной ветви амплитудно-частотной характеристики линейной системы ниже точки $C$ пересечения уровня ограничения $a_{l}=\Delta \mathrm{c}$ асимптотой скелетной кривой. В противном случае (рис.9.3,г) резонансная кривая однозначна, причем нелинейная ветвь расположена в области 
частот между точками пересечения амплитудно-частотной характеристики линейной системы уровнем $a_{l}=\Delta$.

Полагая в уравнении (9.25) амплитудно-частотной характеристики $\xi=\xi_{0}$ и учитывая равенство (9.28), получим, оставляя в знаменателе величины первого порядка малости,

$$
a_{l}=\frac{4 \pi \cdot \delta}{\psi \cdot \xi_{0}} \cdot\left|\frac{\sin \xi_{0} \sin \chi \cdot \xi_{0}}{\xi_{0}+0,5 \sin 2 \xi_{0}}\right|
$$

Уравнение (9.31) определяет линию (2) предельных амплитуд (рис.9.3,б,г), которая пересекает скелетную кривую 1 в той же точке, что и резонансная. Линия предельных амплитуд является геометрическим местом точек пересечения резонансных и скелетных кривых, причем, как следует из сравнения (9.25) и (9.31), все точки резонансных кривых располагаются ниже линии (9.31). С помощью графических построений, показанных для частоты $\xi_{2}$ (рис.9.3,б) стрелками, на рис.9.3,a,в получено отображение 2 линии предельных амплитуд на плоскость $\left(a_{l}, \alpha\right)$. На этой линии располагаются максимумы кривых $\xi_{i}$, достигаемые при значениях $\alpha$, соответствующих скелетным кривым.

На рис.9.4 аналогичными построениями получены возможные конфигурации резонансных кривых системы с предварительным натягом $(\Delta<0)$.

Здесь в пределах упругой деформации ограничителя реализуются ветви резонансных кривых линейной системы, работающей на линейную упругую нагрузку $k_{0}$ и совершающей колебания с амплитудами $a_{l} \leq-\Delta /\left(1+q_{0}\right) \quad$ (нереализуемая часть резонансной кривой показана штриховой линией). Эти ветви описываются уравнением (9.25) при $q=q_{0}$. На рис.9.4,а,б показан случай неоднозначности резонансной кривой, нелинейные ветви которой охватывают скелетную кривую 1. Резонансная кривая, построенная на рис.9.4,в,г , однозначна во всем частотном диапазоне. 

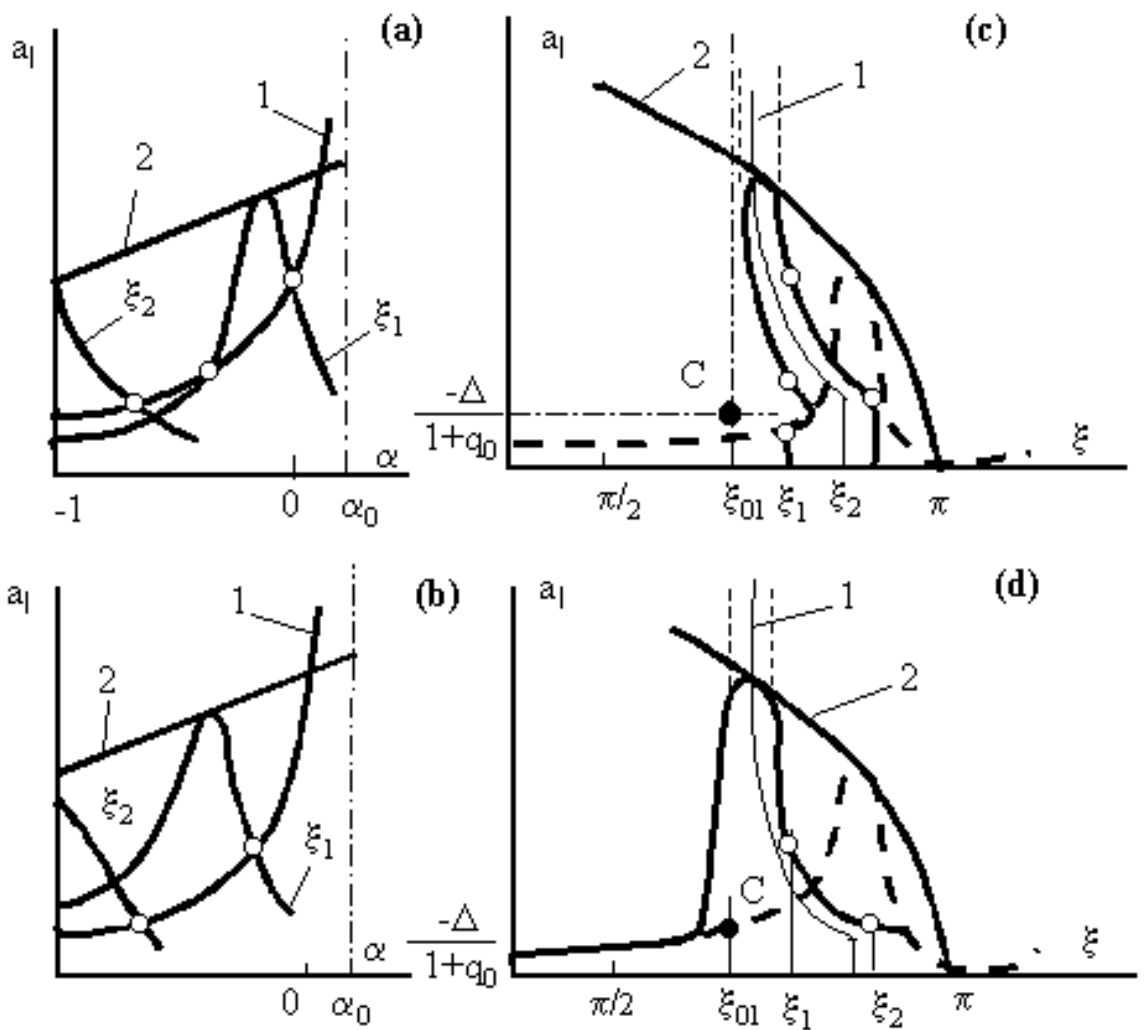

Рис.9.4

Из приведенных построений видно, что неоднозначность проявляется, если дорезонансная ветвь линейной системы проходит ниже точки $C$ пересечения асимптоты скелетной кривой с уровнем $a_{l} \leq-\Delta /\left(1+q_{0}\right)$ упругой деформации ограничителя. Высшие точки резонансных кривых определяются, как и ранее, пересечением скелетных кривых 1 и линий 2 предельных амплитуд (рис.9.4,б,г), описываемых уравнением (9.31).

На рис.9.5 приведено построение амплитудно-частотной характеристики системы с нулевым зазором $(\Delta=0)$.
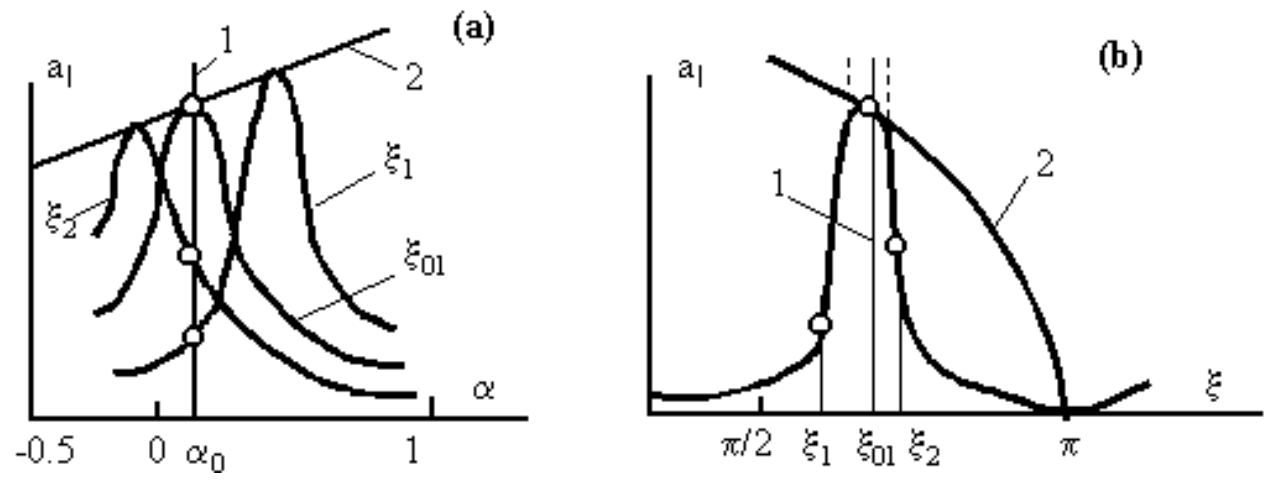

Рис.9.5 
В этом случае виброударные режимы существуют во всем диапазоне частот, причем максимальные амплитуды колебаний достигаются на частотах $\xi_{\text {оп }}$ (см.(9.29)) свободных колебаний системы с упругим ограничителем при $\Delta=0$ и определяются точкой пересечения скелетных линий 1 и линией 2 предельных амплитуд. Из построений на рис.9.5,a видно, что при любой частоте $\xi$ возбуждения всегда существует единственный режим колебаний.

Отметим, что при отсутствии диссипации энергии $(\psi=0)$ все резонансные кривые неограниченно продолжаются вдоль скелетных кривых, как показано пунктирными линиями на рис.9.3,б,г и рис.9.5,б. Здесь показаны примеры построения резонансных кривых в окрестности первой формы колебаний линейной системы. Аналогично строятся амплитудночастотные характеристики в окрестности высших форм колебаний.

4. Степень проявления нелинейных свойств системы увеличивается по мере ужесточения ограничителя. В предельном случае абсолютно жесткого ограничителя $\left(k_{0} \rightarrow \infty\right)$ коэффициент линеаризации $k(a)$ упругой характеристики связан с постоянной составляющей $P_{l}$ реакции ограничителя зависимостью (8.37). Из уравнения (9.7) связи постоянных составляющих сил и смещений с Рис.9.5 помощью равенства (8.31) найдем, учитывая, что $\alpha=\left(\Delta-m_{l}\right) / a_{l} \rightarrow 1$ при $k_{0} \rightarrow \infty$,

$$
P_{l}=\left(a_{l}-\Delta\right) L_{l l}^{-1}(0)
$$

Подставив это равенство в формулу (8.37), получим зависимость коэффициента линеаризации от одного неизвестного параметра $a_{l}$

$$
k\left(a_{l}\right)=\frac{2}{L_{I l}(0)} \cdot\left(1-\frac{\Delta}{a_{l}}\right)
$$

Для рассматриваемой системы согласно (9.23) статическая жесткость $W_{l l}(0)=L_{l l}^{-1}(0)=E S / 1$, и выражение (9.33) с учетом принятых в (9.25) обозначений принимает вид

$$
q\left(a_{l}\right)=2\left(1-\Delta / a_{l}\right)
$$


Подставляя коэффициент линеаризации (9.34) в равенство (9.25) и разрешая полученное уравнение относительно неизвестной амплитуды $a_{l}$, найдем следующее выражение для амплитудно-частотной характеристики системы:

$$
a_{l}=\frac{2 \Delta \cdot C(\xi) \pm \sqrt{[\delta(\xi) \cdot C(\xi)]^{2}+\Psi^{2}(\xi) \cdot\left[\delta^{2}(\xi)-4 \Delta^{2}\right]}}{C^{2}(\xi)+\Psi^{2}(\xi)}
$$

где $\delta(\xi)=\delta / K(\xi) ; C(\xi)=\xi \cdot \operatorname{ctg} \xi+2 ; \Psi(\xi)=\frac{\psi \cdot \xi}{4 \pi} \cdot \frac{\xi+0,5 \sin 2 \xi}{\sin ^{2} \xi}$.

На рис.9.6 9.7 показаны резонансные кривые для двух первых форм колебаний систем с нулевым зазором $(\Delta=0)$, предварительным зазором $(\Delta>0)$ и предварительным натягом $(\Delta<0)$.

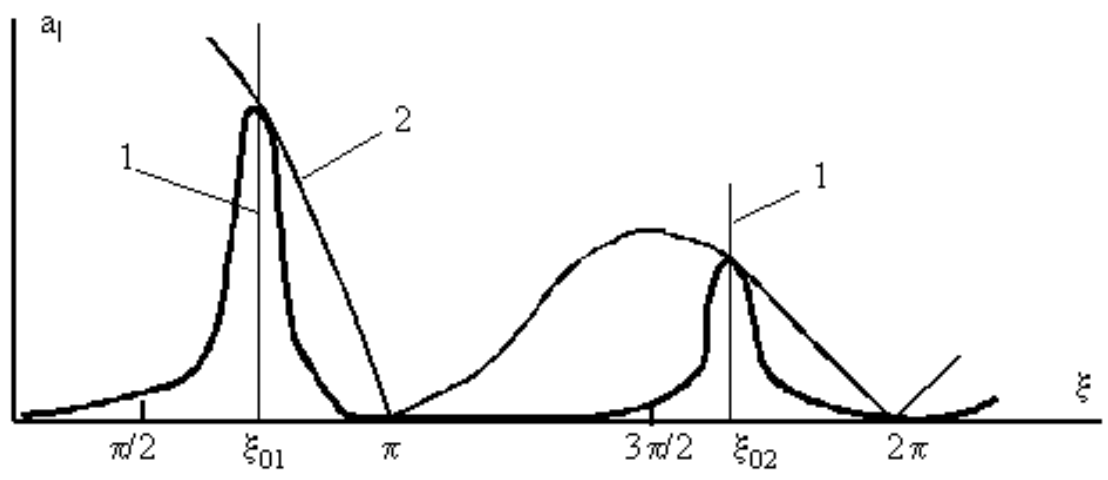

Рис.9.6
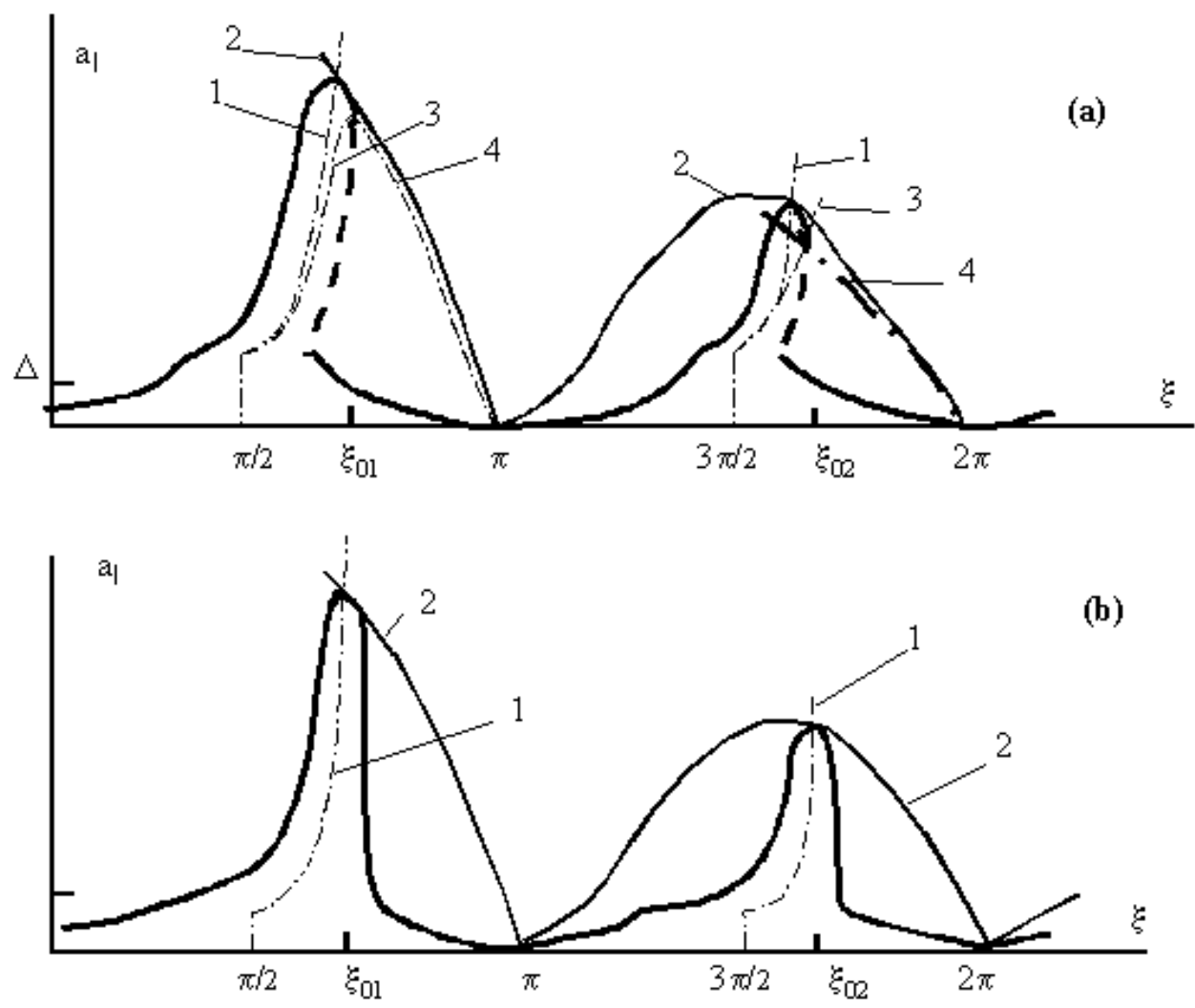

Рис.9.7 

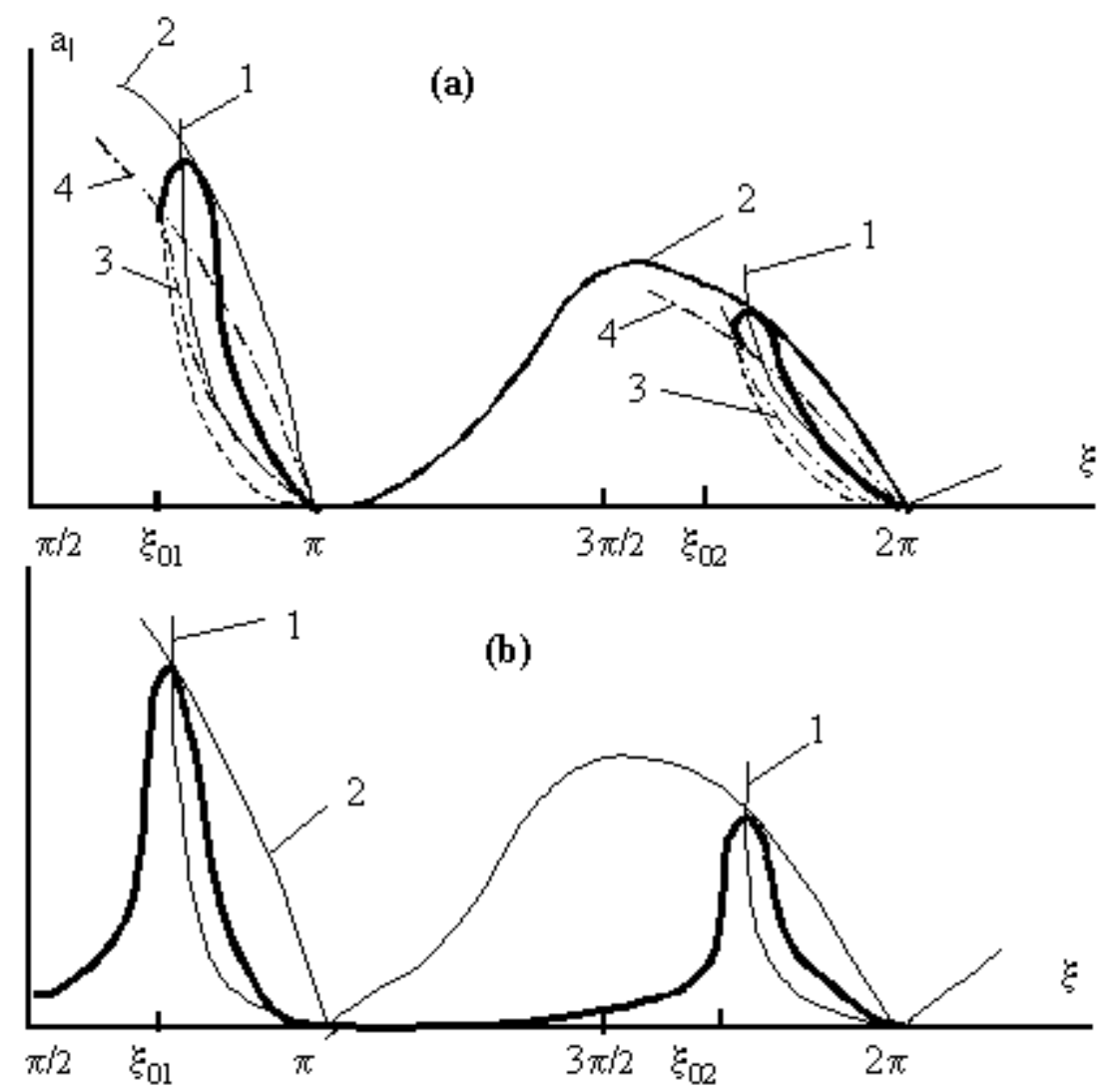

Рис.9.8

На этих рисунках индексом 1 обозначены скелетные кривые, уравнение которых

$$
a_{l}=\frac{2 \Delta}{C(\xi)}=\frac{2 \Delta}{\xi \cdot \operatorname{ctg} \xi+2}
$$

получается из (9.35) при $\delta=0, \psi=0$.

Области определения скелетных кривых описываются множествами (9.29), где следует положить $\xi_{n}=\pi \cdot(2 n-1) / 2 ; \xi_{H n}=\pi \cdot n$, а $\xi_{0 n}$ являются решениями уравнения $\xi \cdot \operatorname{ctg} \xi=-2$, первые четыре корня которого равны: $\xi_{01}=2,289 ; \xi_{02}=5,087 ; \xi_{03}=8,096 ; \xi_{04}=11,173$.

Из выражений (9.35), (9.36) получаем уравнение (9.31) линии 2 предельных амплитуд, пересечение которых со скелетными кривыми дает высшие точки амплитудно-частотных характеристик.

При $\Delta=0$ уравнение (9.35) принимает вид $a_{l}=\delta(\xi) / \sqrt{C^{2}(\xi)+\Psi^{2}(\xi)}$ и резонансные кривые однозначны во всем диапазоне частот (рис.9.6). Подчинив (9.35) необходимому условию существования режимов с 
соударениями $a_{l}>0$, найдем, что для систем с зазором (рис.9.7,a) и натягом (рис.9.8,a) резонансные кривые в областях определения скелетных кривых имеют зоны двузначности, если $\delta(\xi)<2|\Delta|$. При этом верхняя (сплошная линия) и нижняя (штриховая линия) ветви резонансной кривой сходятся в точке, для которой равно нулю подкоренное выражение в (9.35), т.е. при выполнении равенства

$$
C(\xi)=\Psi(\xi) \cdot \sqrt{\left(\frac{2 \Delta}{\delta(\xi)}\right)^{2}-1}
$$

Из (9.35) и (9.37) для нахождения граничной точки области двузначности резонансных кривых получаем систему уравнений

$$
\begin{gathered}
a_{1}=\frac{2 \Delta \cdot C(\xi)}{C^{2}(\xi)+\Psi^{2}(\xi)} \\
a_{1}=\frac{\delta(\xi)}{\Psi(\xi)} \cdot \sqrt{1-\left(\frac{\delta(\xi)}{2 \Delta}\right)^{2}}
\end{gathered}
$$

На рис.9.7,а и рис.9.8,a по уравнениям (9.38) и (9.39) штрихпунктирными линиями построены кривые 3 и 4. Из сравнения выражений (9.38) и (9.36) видно, что разделительная кривая 3 проходит ниже скелетной кривой и расположена между ветвями амплитудно-частотной характеристики в зоне двузначности. Согласно (9.39) и (9.31) кривая 4 проходит ниже линии предельных амплитуд 2 и в пересечении с разделительной линией 3 дает точку слияния верхней и нижней ветвей резонансной кривой.

При $\delta(\xi) \geq 2|\Delta|$ резонансные кривые как для систем с зазором (рис.9.7,б), так и для систем с натягом (рис.9.8,б) однозначны. Они описываются уравнением (9.35) со знаком плюс перед радикалом. Резонансные кривые на рис.9.7,а,б дополнены реализуемыми ветвями $a_{1} \leq \Delta$ линейной системы. 
Отметим, что при отсутствии демпфирования $(\psi=0)$ уравнение (9.35) с учетом (9.24) приводится к следующему простому выражению:

$$
a_{l}=\frac{2 \Delta \cdot \sin \xi \pm \delta \cdot \sin \chi \cdot \xi}{\xi \cdot \cos \xi+2 \sin \xi}
$$

При этом разделительная кривая (9.38) совпадает со скелетной кривой (9.36).

5. Используя условие (9.20), исследуем устойчивость полученных решений. Рассмотрим сначала случай абсолютно жесткого ограничителя $\left(k_{0} \rightarrow \infty\right)$.

Принимая во внимание выражения (9.22), (9.23), (9.33), имеем для производных в (9.20): $\partial V / \partial a_{l}=0 ; \quad \partial U / \partial a_{l}=\partial k\left(a_{l}\right) / \partial a_{l}=2 \Delta / a_{l}^{2} L_{l l}(0) . \quad$ После подстановки этих величин и соответствующих выражений из (9.22), (9.23), (9.33), условие (9.20) с учетом принятых в (9.35) обозначений принимает вид $\left[C(\xi)-\frac{2 \Delta}{a_{l}}\right] \cdot C(\xi)+\Psi^{2}(\xi)>0$. Разрешая это неравенство относительно амплитуды $a_{l}$, получаем окончательно

$$
a_{l}>\frac{2 \Delta \cdot C(\xi)}{C^{2}(\xi)+\Psi^{2}(\xi)}
$$

Правая часть условия устойчивости (9.41) совпадает с уравнением (9.38) разделительной кривой, поэтому показанные пунктиром на рис.9.7,a и 9.8,a нижние ветви резонансных кривых оказываются неустойчивыми. Из (9.41) и условий двузначности резонансных кривых следует, что режимы, соответствующие однозначным амплитудно-частотным характеристикам (рис.9.6, рис.9.7,б и рис.9.8,б), устойчивы во всей области существования.

Покажем, что и в общем случае при конечной жесткости упругих ограничителей неустойчивыми являются лишь режимы, соответствующие нижним ветвям областей двузначности резонансных кривых. Условие 
устойчивости (9.20) с учетом выражений (9.12), (9.22), (9.23) принимает вид

$$
C_{1}^{2}(\xi, \alpha)+\Psi^{2}(\xi)+a_{l} \frac{\partial q\left[\alpha\left(a_{l}\right)\right]}{\partial a_{l}}>0
$$

где $C_{1}(\xi, \alpha)=\xi \cdot \operatorname{ctg} \xi+q(\alpha)$.

Производную

$$
\frac{\partial q}{\partial a_{1}}=\frac{\partial q}{\partial \alpha} \cdot \frac{\partial \alpha}{\partial a_{l}}
$$

вычислим, используя соотношения (9.27) и (9.26),

$$
\begin{aligned}
& \frac{\partial q}{\partial \alpha}=-\frac{2 q_{0}}{\pi} \cdot \sqrt{1-\alpha^{2}} \\
& \frac{\partial \alpha}{\partial a_{l}}=-\frac{\Delta}{a^{2}} \cdot\left(1+\frac{q_{0}}{\pi} \arccos \alpha\right)^{-1}
\end{aligned}
$$

После подстановки (9.43), (9.44) условие устойчивости (9.42) запишется в виде

$$
a>-\frac{2 \Delta \cdot C_{1}(\xi, \alpha) \cdot \sqrt{1-\alpha^{2}}}{\left[C_{1}^{2}(\xi, \alpha)+\Psi^{2}(\xi)\right] \cdot\left(\pi / q_{0}+\arccos \alpha\right)}
$$

Неравенство (9.45) заведомо выполняется для всех режимов при $\Delta=0$ и режимов, соответствующих ветвям резонансных кривых, расположенных выше скелетных кривых при $\Delta \neq 0$, для которых

$$
\Delta \cdot C_{1}(\xi, \alpha)=\Delta \cdot[\xi \cdot \operatorname{ctg} \xi+q(\alpha)]>0 .
$$

На рис.9.9,а,б для систем с зазором $(\Delta>0)$ и натягом $(\Delta<0)$ показано графическое решение уравнений (9.25) (кривая 1) и (9.26) (кривая 2), дающее при заданной частоте $\xi$ два решения.
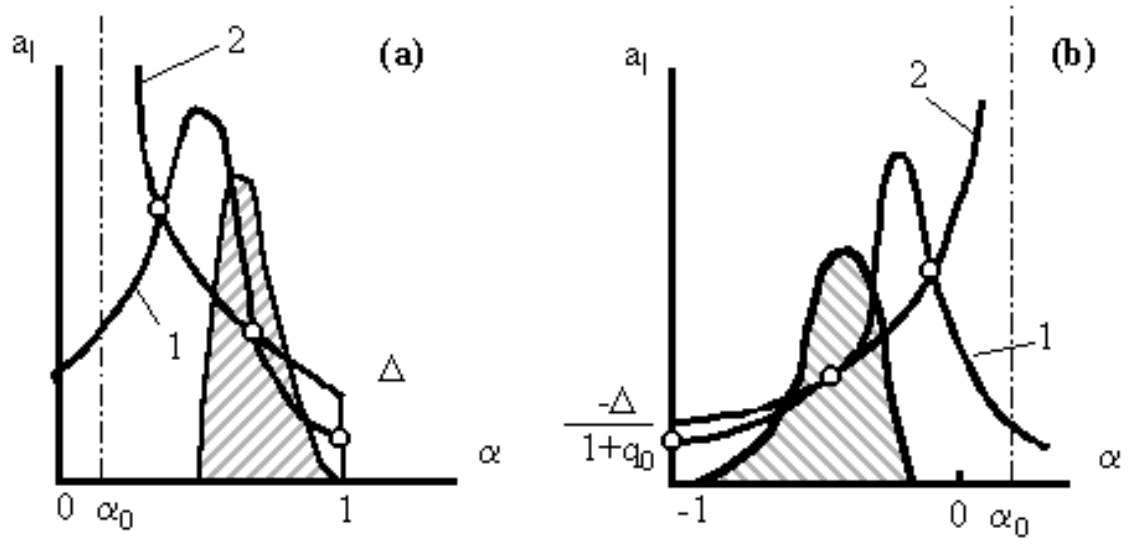

Рис.9.9 
Сюда же нанесена граница области устойчивости по уравнению в правой части неравенства (9.45). В зону неустойчивости, отмеченную штриховкой, попадает решение с меньшей амплитудой.

Из приведенных графических построений очевидно, что для точки слияния верхней и нижней ветвей резонансных кривых должно выполняться равенство как величин (9.25) и (9.26), так и их производных. Дифференцируя (9.25), с учетом (9.26) и (9.44) имеем

$$
\frac{d a_{1}}{d \alpha}=\frac{d a_{1}}{d q} \cdot \frac{d q}{d \alpha}=\frac{2 a_{1} C_{1}(\xi, \alpha) \cdot q_{0} \sqrt{1-\alpha^{2}}}{\pi \cdot\left[C_{1}^{2}(\xi, \alpha)+\Psi^{2}(\xi)\right]}
$$

Из (9.26) находим производную

$$
\frac{d a_{1}}{d \alpha}=-\frac{a_{1}^{2}}{\Delta} \cdot\left(1+\frac{q_{0}}{\pi} \arccos \alpha\right)
$$

Приравнивая (9.46) и (9.47), получаем, что для точки слияния должно выполняться равенство

$$
a_{l}=-\frac{2 \Delta \cdot C_{1}(\xi, \alpha) \cdot \sqrt{1-\alpha^{2}}}{\left[C_{1}^{2}(\xi, \alpha)+\Psi^{2}(\xi)\right] \cdot\left(\pi / q_{0}+\arccos \alpha\right)},
$$

которое совпадает с правой частью условия устойчивости (9.45). Таким образом, точка слияния верхних и нижних ветвей резонансных кривых принадлежит границе области устойчивости, и все режимы, соответствующие нижним ветвям зон двузначности, оказываются неустойчивыми.

На рис.9.10,а,б показаны резонансные кривые для систем с предварительным зазором $(\Delta>0)$ и натягом $(\Delta<0)$. Здесь сплошными линиями нанесены устойчивые, а штриховыми - неустойчивые ветви. Если частота возбуждения медленно изменяется, так что при каждом ее значении устанавливается режим вынужденных колебаний, то реализация той или иной ветви резонансной кривой зависит от направления изменения частоты, как это показано стрелками. 


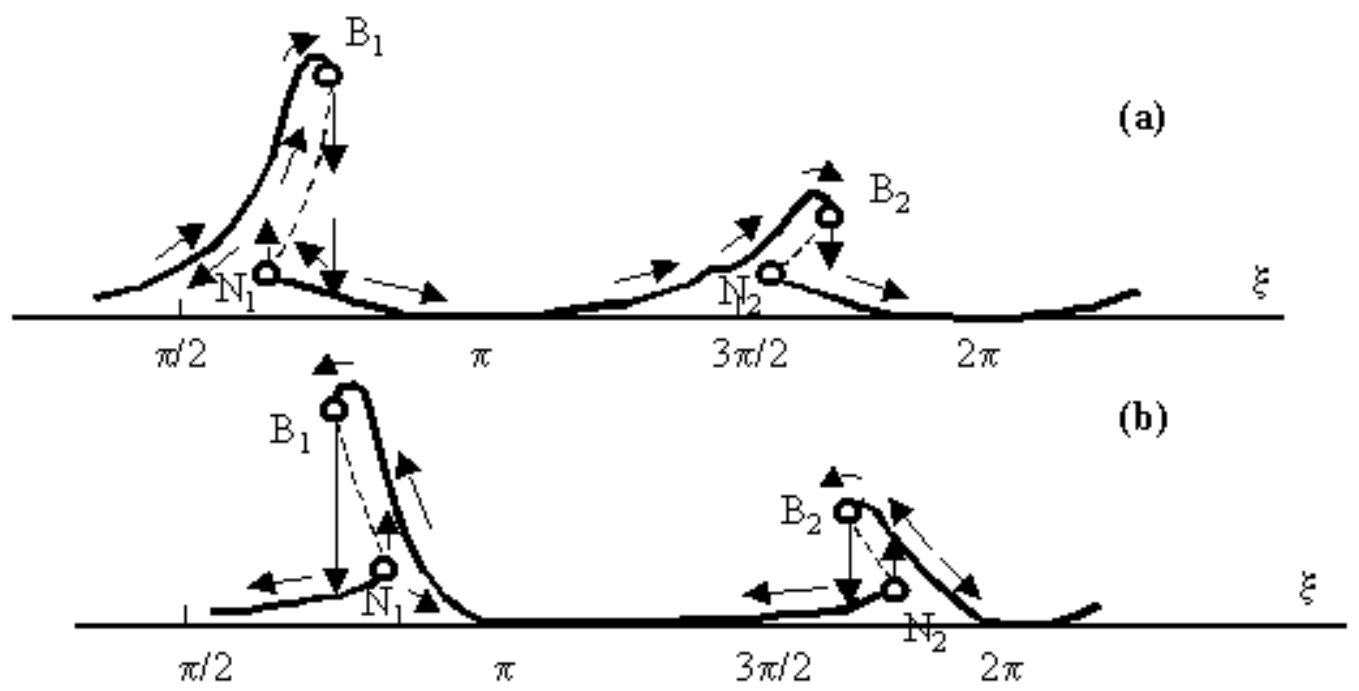

Рис.9.10

При перестройке частоты имеют место скачкообразные изменения амплитуды колебаний, причем один срыв происходит в точках $B_{i}(i=1,2, \ldots)$ слияния нелинейных ветвей, а другой - в точках $N_{i}$ слияния нижней неустойчивой ветви и ветви линейных режимов в пределах зазора (рис.9.10,a) или натяга (рис.9.10,б). Неустойчивые ветви физически нереализуемы.

6. Ультразвуковые стержневые системы, совершающие изгибные колебания и взаимодействующие $\mathrm{c}$ нелинейной технологической нагрузкой, сохраняют особенности поведения, выявленные при исследовании продольных колебаний. Покажем это на примере изгибных колебаний консольной балки с закрепленным на ее конце телом, взаимодействующим с односторонним ограничителем (рис.9.11).

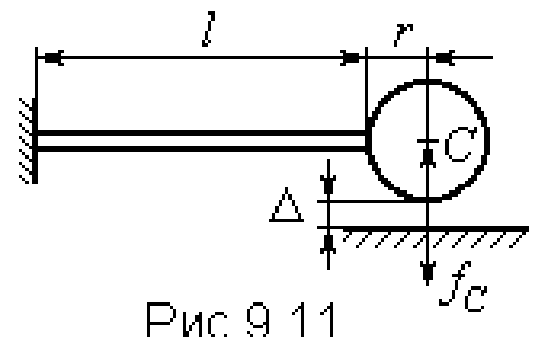

Система совершает колебания под действием гармонической вынуждающей силы $f_{c}(t)=F_{c} e^{j \omega t}$, приложенной в центре масс $C$ присоединенного тела. В этом случае смещение сечений балки от недеформированного состояния описывается функцией $u_{x}(t)$, a 
присоединенного тела - координатой $u_{c}$ его центра масс и углом поворота $\theta$. Динамические характеристики линейной системы построены в П.6.3, где получены динамические жесткости и податливости поступательных и угловых колебаний присоединенного тела.

Амплитуда смещений центра масс тела находится из уравнения (9.15), в котором индексы $l$ и $s$ следует заменить индексом $c$, а приведенная динамическая жесткость

$$
W\left(j \omega, m_{c}, a_{c}\right)=W_{c}(j \omega)+k\left(m_{c}, a_{c}\right)+j \omega \cdot b\left(m_{c}, a_{c}\right),
$$

где $W_{c}$ - динамическая жесткость линейной системы в точке $C$, определяемая соотношением (6.48).

Амплитуда $\theta_{c}$ угловых колебаний описывается равенством (6.51).

Ограничимся рассмотрением случая абсолютно жесткого ограничителя, установленного с зазором $\Delta$ относительно тела. Используя выражение для коэффициента гармонической линеаризации в форме (9.32), приведем уравнение (9.15) резонансных кривых системы при отсутствии диссипации $\left(V\left(a_{l}, \omega\right)=0\right)$ к виду

$$
a_{c}=\frac{2 \Delta \pm \delta}{2+L_{c}(0) \cdot U_{c}(\omega)},
$$

где $U_{c}(\omega)=\operatorname{Re}\left|W_{c}(j \omega)\right| ; \quad L_{c}(0)=U_{c}^{-1}(0) ; \quad \delta=F_{c} L_{c}(0)$.

Области существования решений находятся из условий $a \geq \Delta$ при $\Delta>0$ и $a>0$ при $\Delta \leq 0$. Положив в (9.50) $\delta=0 \quad\left(F_{c}=0\right)$, получаем уравнения скелетных кривых, определенных в диапазонах

$$
\begin{array}{ccc}
\omega_{n} \leq \omega<\omega_{0 n} & \text { при } & \Delta>0 \\
\omega=\omega_{0 n} & \text { при } & \Delta=0 \\
\omega_{0 n}<\omega \leq \omega_{n}^{*} & \text { при } & \Delta<0,
\end{array}
$$

где $\omega_{n}, \omega_{n}^{*}$ - резонансные и антирезонансные частоты линейной системы, определяемые соотношениями (6.53) - (6.56) и графиками на рис.6.5; $\omega_{0 п}$ собственные частоты виброударной системы при $\Delta=0$, которые являются решениями уравнения $2 U_{c}(0)+U_{c}(\omega)=0$ и зависят лишь от свойств линейной системы. 
Из (9.50) видно, что при $|\Delta| \leq \delta / 2$ резонансные кривые однозначны и существуют во всей частотной области. При $|\Delta|>\delta / 2$ резонансные кривые определены в диапазонах $\omega_{0 n}<\omega \leq \omega_{n}^{*}$ при $\Delta<0$ и в смежных с ними областях при $\Delta>0$, причем виброударные режимы могут существовать парами.

Амплитудно-частотные характеристики поперечных колебаний тела для трех низших форм построены на рис.9.12,a

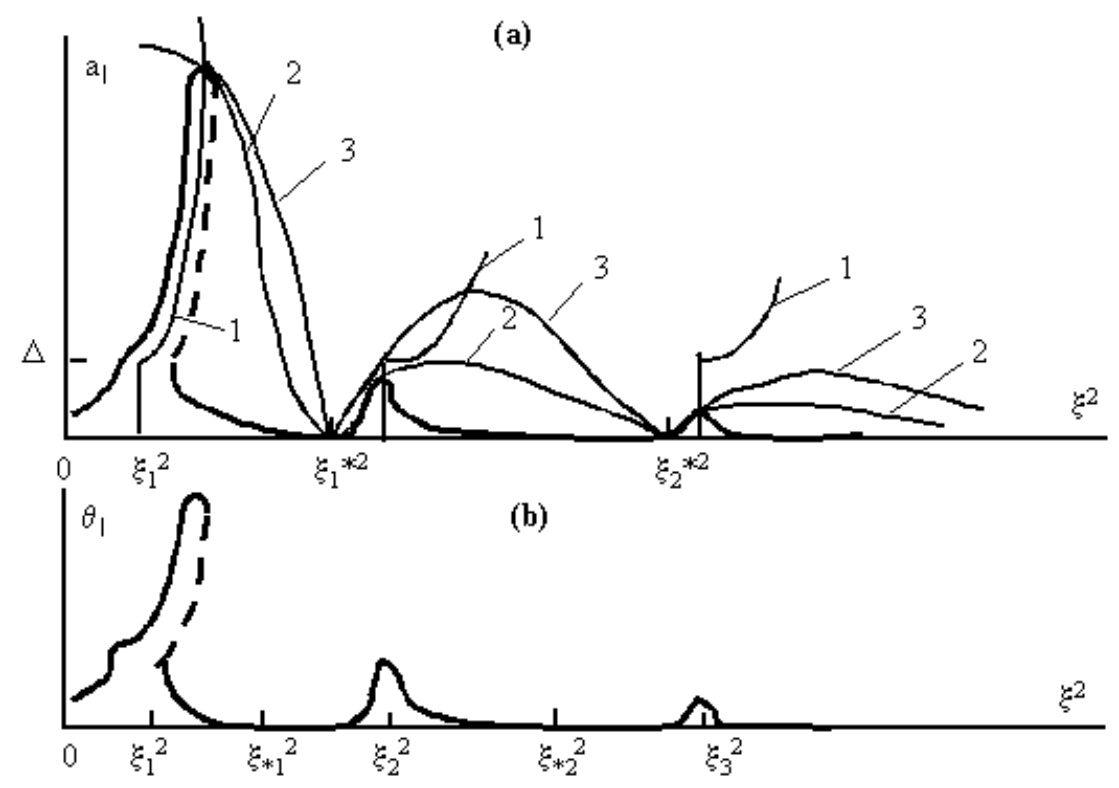

Рис.9.12

для системы с предварительным зазором $(\Delta>0)$ и на рис.9.13, а для системы с предварительным натягом $(\Delta<0)$.
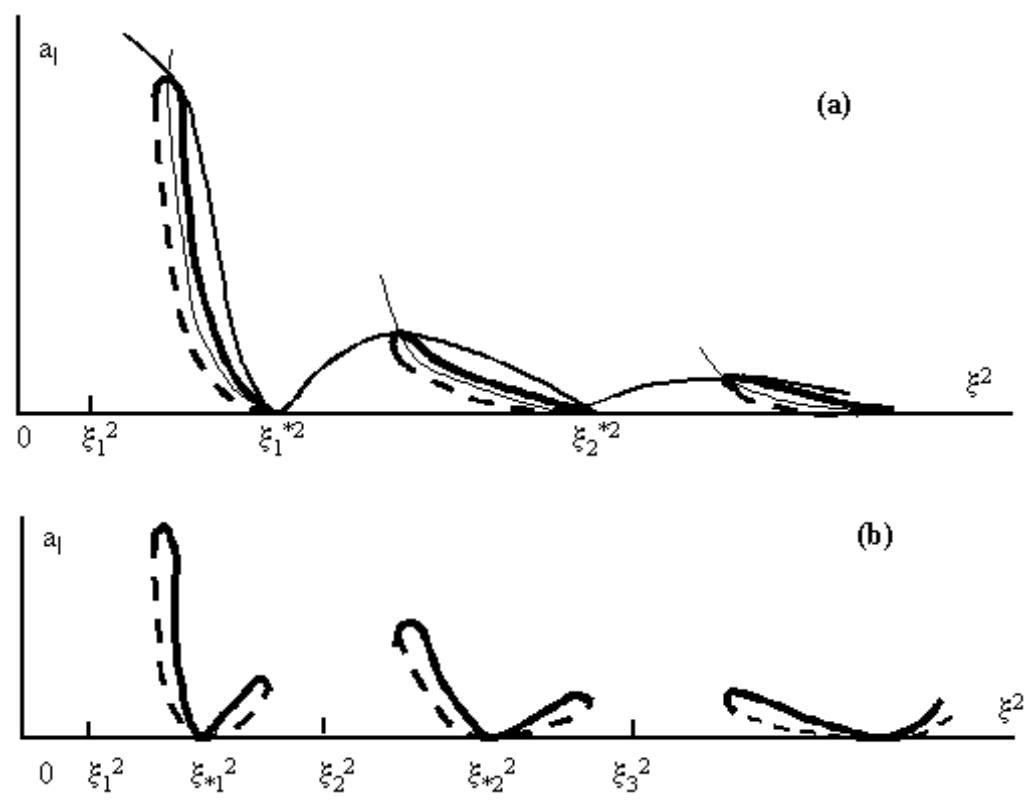

Рис.9.13 
Ветви резонансных кривых охватывают скелетные кривые 1 и при отсутствии диссипации неограниченно продолжаются вдоль последних, нигде их не пересекая. Ограничение амплитуд происходит вследствие затрат энергии на преодоление внутреннего трения в материале балки и диссипации при ударах об ограничитель.

Согласно (9.15) максимальные амплитуды определяются точкой пересечения скелетных кривых с линией предельных амплитуд, уравнение которой имеет вид

$$
a_{c}=F_{c} / V\left(a_{\omega}, \omega\right)=F_{c} /\left[V_{c}(\omega)+\omega \cdot b\left(a_{c}, \omega\right)\right],
$$

где $V_{c}(\omega)=\operatorname{Im} W_{c}(j \omega)$ дается формулой (6.50).

Полагая соударение присоединенного тела с ограничителем мгновенным и оценивая его эффект с помощью коэффициента восстановления $R$, примем для коэффициента $b(a, \omega)$ гармонической линеаризации диссипативной части характеристики удара выражение (8.43). Учитывая, что скелетная кривая определяется выражением

$$
U(\omega)=U_{c}\left(a_{c}, \omega\right)+k(a)=0,
$$

уравнение (9.52) линии предельных амплитуд приводится к виду

$$
a_{c}=F_{c}\left[V_{c}(\omega)+\frac{\pi}{2} \cdot \frac{1-R}{1+R} \cdot \frac{U_{c}^{2}(\omega)}{M \omega^{2}}\right]^{-1}
$$

На рис.9.12,а и рис.9.13,а линии предельных амплитуд, построенные по уравнению (9.54), отмечены индексом 2. Здесь же для сравнения изображены линии 3 предельных амплитуд при абсолютно упругом ударе $(R=1)$. Как видно, влияние ударной диссипации возрастает с увеличением номера формы колебаний. На рисунках штриховыми линиями показаны неустойчивые нелинейные ветви резонансных кривых. Показанные на рис.9.12,a резонансные кривые системы с зазором дополнены реализуемыми ветвями линейной системы с амплитудами $a \leq \Delta$.

На рис.9.12,б и 9.13,б построены резонансные кривые угловых колебаний тела. При этом использованы уравнения (6.44), (6.48). 
Нелинейные ветви характеристики угловых колебаний имеют те же частотные диапазоны, что и резонансные кривые поперечных колебаний. При антирезонансных частотах $\xi_{* \pi}$ угловых колебаний тело совершает поступательные колебания. При антирезонансных частотах $\xi_{\pi}^{*}$ линейных колебаний тело совершает чисто угловые колебания. Отметим, что амплитуды угловых колебаний виброударной системы могут значительно превосходить амплитуды угловых колебаний линейной системы. Это особенно заметно для ветвей, расположенных за антирезонансными частотами $\xi_{* \pi}$ системы с натягом (рис.9.13,б).

7. Используя результаты предыдущего пункта, рассмотрим частный случай простейшей виброударной системы, в которой масса балки считается пренебрежимо малой, а присоединенное тело моделируется материальной точкой с массой $M$.

Вопросы существования и устойчивости периодических движений осциллятора с неподвижным ограничителем исследовались точными методами (припасовывания, точечных отображений, методами частотновременного анализа) в ряде работ [61,66,118,135,175]. В [18] на основе точных решений построены амплитудно-частотные характеристики осциллятора, ударяющегося об ограничитель. Для той же системы в [60, 61] получены приближенные решения.

Положив в (6.48) величины $J=0, r=0$ и переходя в полученном выражении к пределу при $\rho \rightarrow 0$, найдем динамическую жесткость системы с сосредоточенными параметрами

$$
W_{c}(j \omega)=c+j \omega \cdot b-M \omega^{2},
$$

где $c=3 E \mathrm{I} / l$ - статическая жесткость консольной балки; $b=c \psi / 2 \pi \omega$ линеаризованный коэффициент сил внутреннего трения [74].

Подстановкой (9.55) в (9.50) получим уравнение амплитудночастотных характеристик системы при отсутствии диссипации

$$
a_{c}=\frac{2 \Delta \pm \delta}{3-\xi^{2}},
$$


где $\delta=F_{c} / c$ - статическая деформация упругой балки под действием силы $F_{c} ; \xi=\omega / \omega_{0} ; \omega_{0}=\sqrt{c / M}-$ собственная частота линейной системы.

На рис.9.14 показаны амплитудно-частотные характеристики виброударной системы для случаев установки ограничителя с предварительным зазором $\Delta>0$ (рис.9.14,a), с нулевым зазором $\Delta=0$ (рис.9.14,б) и предварительным натягом $\Delta<0$ (рис.9.14,в).
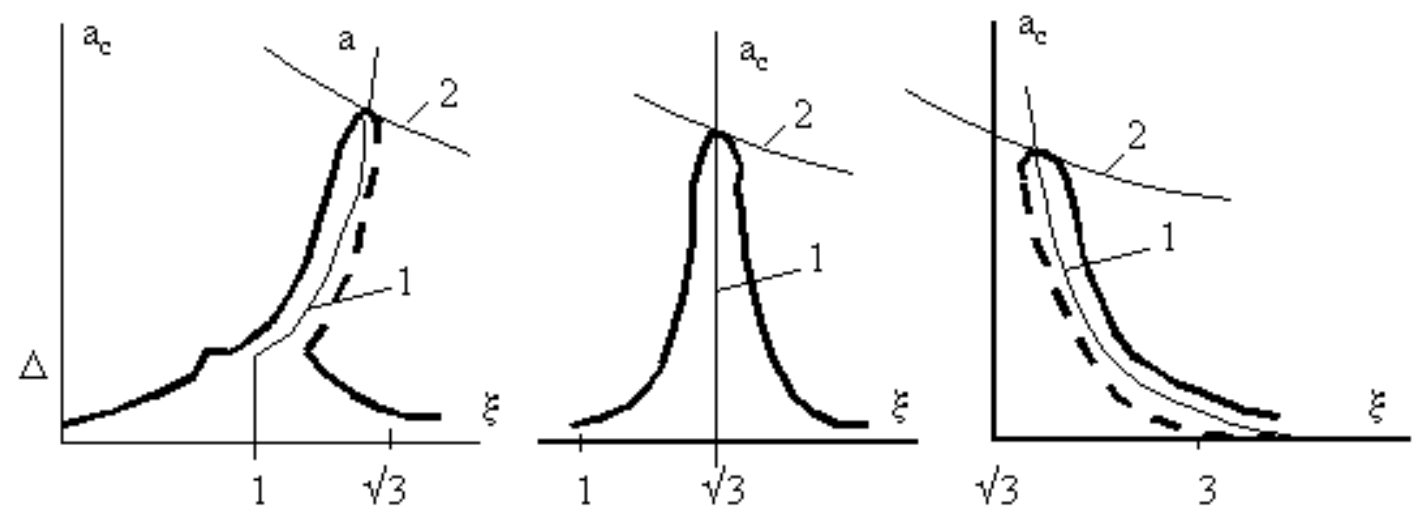

Рис.9.14

При отсутствии диссипации ветви резонансных кривых неограниченно продолжаются вдоль скелетных кривых (тонкие линии 1), уравнение которых получается из (9.56) при $\delta=0$

$$
a_{c}=\frac{2 \Delta}{3-\xi^{2}}
$$

Области существования виброударных режимов определяются из условия $a_{c} \geq \Delta$ при $\Delta>0$ и $a_{l}>0$ при $\Delta \leq 0$. Поэтому уравнение (9.56) при $\Delta \neq 0$ и малых силах возбуждения, таких что $\delta<2|\Delta|$, определяют две ветви резонансных кривых, разделенных скелетными кривыми (рис.9.14,а,в).

Нижние ветви, показанные штриховыми линиями, оказываются неустойчивыми. Система с нулевым зазором $(\Delta=0)$ имеет изохронную резонансную кривую во всем диапазоне существования. Аналогичный вид резонансных кривых имеют системы при $\Delta \neq 0$ и больших силах возбуждения $(\delta>2 \Delta)$.

В системе с предварительным зазором $(\Delta>0)$ реализуются также линейные гармонические колебания с амплитудой $a_{c} \leq \Delta$. Легко показать, 
что резонансные кривые (9.56) исходят из точек, где амплитудночастотные характеристики линейного осциллятора пересекают уровень ограничителя $a_{c}=\Delta$ (рис.9.14,a).

Диссипация энергии приводит к ограничению резонансных кривых с максимумом в точке пересечения скелетной кривой 1 и линии 2 предельных амплитуд, описываемой уравнением (9.54), которое после подстановки величин $V_{c}=\omega \cdot b$ и $U_{c}=c\left(1-\xi^{2}\right)$ из (9.55) принимает вид

$$
a_{c}=\frac{\delta}{\xi \cdot n+B \frac{\left(1-\xi^{2}\right)^{2}}{\xi^{2}},}
$$

где $n=b / \sqrt{c M} ; B=\frac{\pi}{2} \cdot \frac{1-R}{1+R}$.

В системе с нулевым зазором $(\Delta=0)$ максимум резонансной кривой согласно (9.56), (9.57) достигается на частоте $\xi=\sqrt{3}$, и резонансная амплитуда (9.58)

$$
a_{c}=\frac{\delta}{\sqrt{3} n+4 B / 3}
$$

При отсутствии вязкого трения $b_{0}=0(n=0)$ с учетом принятых обозначений имеем

$$
a_{c}=\frac{3}{2 \pi} \cdot \frac{F_{c}}{C} \cdot \frac{1+R}{1-R}
$$

Заметим, что в [18] для того же случая точными методами получена следующая формула для резонансной амплитуды

$$
a_{c}=\frac{1}{3} \cdot \frac{F_{c}}{C} \cdot \frac{1+R}{1-R}
$$

Сравнение (9.60) и (9.61) показывает, что они не только имеют одинаковую структуру, но и дают количественно близкие значения. Приведенные на рис.9.14 резонансные кривые сохраняют все особенности амплитудно-частотных характеристик, построенных в [18] в результате анализа точных решений.

Попутно отметим, что в перечисленных в начале этого пункта исследованиях виброударных систем, как правило, учитывается 
диссипация энергии, происходящая только в результате ударных взаимодействий. Полученные здесь приближенные решения позволяют оценить влияние любого вида рассеяния энергии. Так например, анализ показывает, что влияние вязкого и ударного демпфирования на резонансные режимы с максимальной амплитудой различно для систем с разной структурой. Действительно, положив в (9.58) последовательно $n=$ 0 и $B=0$ и приравняв результаты выражению (9.57), после решения полученных уравнений найдем частоты режимов с максимальными амплитудами: для системы с ударной диссипацией $(n=0)$

$$
\xi=\sqrt{\frac{3 \delta+4 \Delta B+\sqrt{(9 \delta+16 \Delta B) \cdot \delta}}{2(\delta+2 \Delta B)}} ;
$$

для системы с вязкой диссипацией $(B=0)$

$$
\xi=\frac{-\Delta \cdot n+\sqrt{(\Delta \cdot n)^{2}+3 \delta^{2}}}{\delta}
$$

На рис.9.15,а,б показаны зависимости частоты $\xi$ и амплитуды $a_{c}$ резонансных колебаний от величины зазора (натяга) $\Delta$ для систем с ударной (кривые 1) и вязкой (кривые 2) диссипацией.
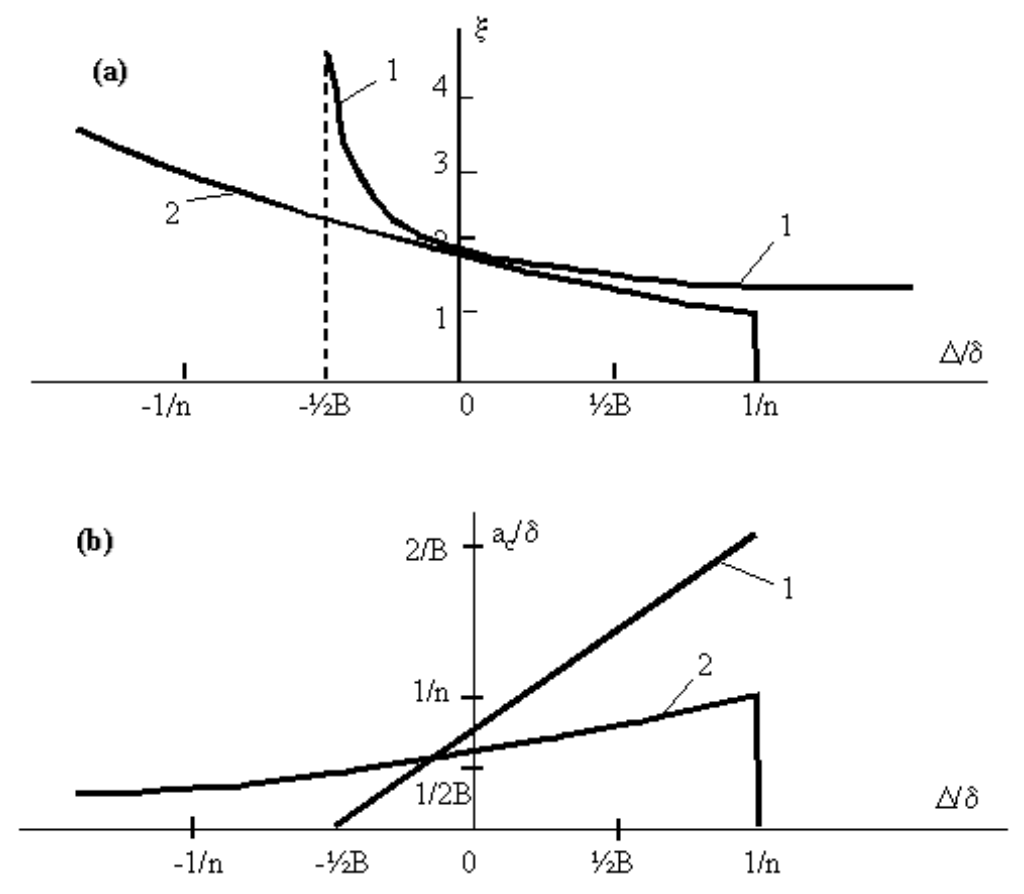

Рис.9.15 
Как видно, вязкое демпфирование ограничивает предельную величину зазора $\Delta=\delta / n$, достигаемую при частоте $\xi=1$ собственных колебаний линейной системы, но не ограничивает натяг $\Delta \rightarrow-\infty$ при $\xi \rightarrow \infty$ . Ударное демпфирование, наоборот, не ограничивает величину зазора $\Delta \rightarrow \infty$ при $\xi \rightarrow 1$, но ограничивает натяг величиной

$$
\Delta=-\frac{\delta}{2 B}=-\frac{\delta}{\pi} \cdot \frac{1-R}{1+R}
$$

при которой частота $\xi \rightarrow \infty$.

Найденный из (9.62) предельный натяг (9.64) совпадает с величиной (2.33) из книги [69], полученной в результате анализа точных решений.

И, наконец, отметим, что найденные приближенные решения позволяют оценить важную для виброударных систем величину импульса соударяющихся тел. С этой целью следует воспользоваться соотношением (8.39), которое после подстановки величин (9.32) и (9.56) приводит к уравнению импульсно-частотных характеристик $[71,193]$ осциллятора с ограничителем при отсутствии диссипации ( $\left.b_{0}=0, R=0\right)$ энергии

$$
J=\frac{2 \pi}{\xi} \cdot \sqrt{c M} \cdot \frac{\Delta\left(1+\xi^{2}\right) \pm \delta}{3-\xi^{2}}
$$

На рис.9.16 построены импульсно-частотные характеристики для систем с зазором $\Delta>0$ (рис.9.16,a), с нулевым зазором $\Delta=0$ (рис.9.16,б) и предварительным натягом $\Delta<0$ (рис.9.16,в).

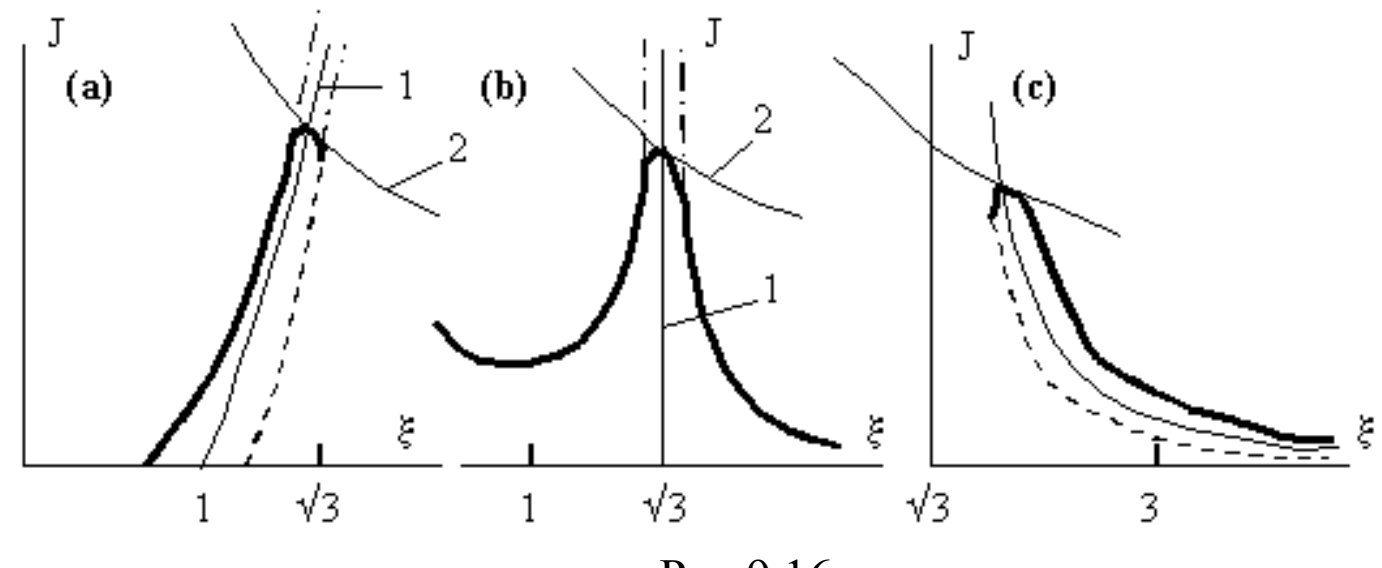

Рис.9.16 
При отсутствии диссипации характеристики неограниченно продолжаются (штрих-пунктирные линии) вдоль скелетных кривых 1, уравнение которых получается из (9.65) при $\delta=0$. Рассеяние энергии ограничивает характеристику в точке пересечения скелетной кривой и линии 2 предельных импульсов, уравнение которой найдем из (8.39), (9.32) и (9.58)

$$
J=\frac{2 \pi}{\xi} \cdot \sqrt{c M} \cdot\left[\frac{\delta}{\xi \cdot n+B \frac{\left(1-\xi^{2}\right)^{2}}{\xi^{2}}}-\Delta\right]
$$

В системе с нулевым зазором $(\Delta=0)$, например, максимальный импульс достигается при $\xi=\sqrt{3}$ и при $n=0$ равен

$$
J=\delta \cdot \sqrt{3 c M} \cdot \frac{1+R}{1-R}
$$

На основании точных решений в $[18,60,118,175]$ для того же случая получим следующее выражение для максимального импульса: $J=\frac{4}{3} \delta \cdot \sqrt{c M} \cdot \frac{1+R}{1-R}$, совпадающее с (9.67) по структуре и близкое с ним количественно.

Таким образом, полученные методом гармонической линеаризации приближенные решения позволяют выявить ясную качественную и достаточно точную количественную картину резонансных явлений даже в таких сильно нелинейных [61], как виброударная, системах.

8. В заключение этого Параграфа рассмотрим колебания системы, в которой предварительный натяг создается путем силового замыкания (рис.9.1,б). Она представляет собой стержень, своим верхним концом $x=0$ жестко связанный с бесконечно большой массой, колебаниями которой можно пренебречь. Вся система поджимается к ограничителю статической силой $P$. Особенность такой модели заключается в возможности смещения верхней массы, которая при колебаниях стержня занимает некоторое 
положение динамического равновесия. В установившемся режиме должно соблюдаться равенство $P_{l}\left(a_{l}, m_{l}\right)=P$, подстановка которого в $(8.29) \mathrm{c}$ учетом обозначений в (9.26), (9.27) приводит к уравнению

$$
a_{l}=\pi \cdot \delta_{P} / q_{0}\left(\sqrt{1-\alpha^{2}}-\alpha \cdot \arccos \alpha\right),
$$

где $\delta_{P}=P I / E S$.

Таким образом, задача сводится к решению системы уравнений (9.25), (9.27), (9.68), которое проводится способами, изложенными в П.2. На рис.9.17,а показан пример графического решения этих уравнений, а на рис.9.17,б - конфигурация резонансной кривой.
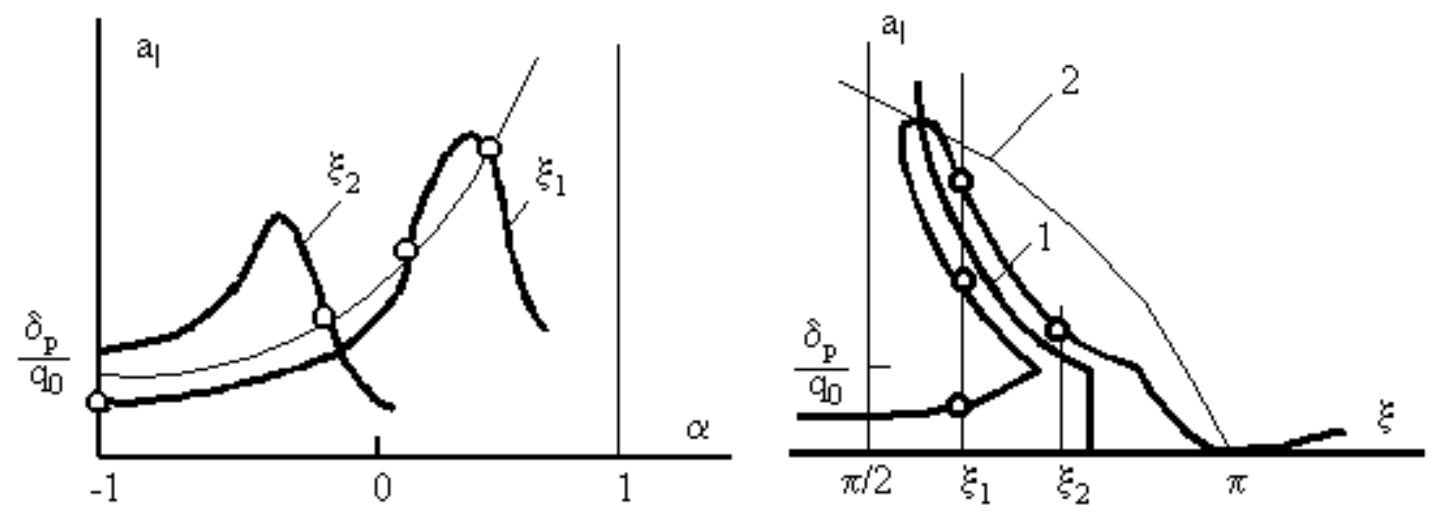

Рис.9.17

Как видно, и в этом случае возможно получение трех решений, одно из которых при $\alpha=-1$ отвечает линейным колебаниям в пределах предварительной деформации ограничителя $\left(a_{l} \leq \delta_{P} / q_{0}\right)$, а два других нелинейным колебаниям с отрывами от ограничителя. На рис. 9.17,б построены также скелетная кривая 1 и линия 2 предельных амплитуд, описываемая уравнением (9.31). Их пересечение дает высшую точку резонансной кривой.

Эту задачу можно решить и иным путем, если воспользоваться выражением (8.33) для коэффициента линеаризации, которое с учетом принятых обозначений запишем в виде

$$
q\left(a_{l}\right)=\frac{2 \delta_{p}}{a_{l}+\delta_{P} / q_{0}}
$$


После подстановки (9.69) в равенство (9.28) получим уравнение скелетной кривой

$$
a_{l}=-\delta_{P}\left(\frac{2}{\xi_{0} \operatorname{ctg} \xi_{0}}+\frac{1}{q_{0}}\right)
$$

Пересечение скелетной кривой 1 (рис.9.18,a) с линией предельных амплитуд 2 дает высшую точку резонансной кривой. Остальные точки находятся графическим решением системы уравнений (9.25), (9.69), пример которого показан на рис.9.18,б.

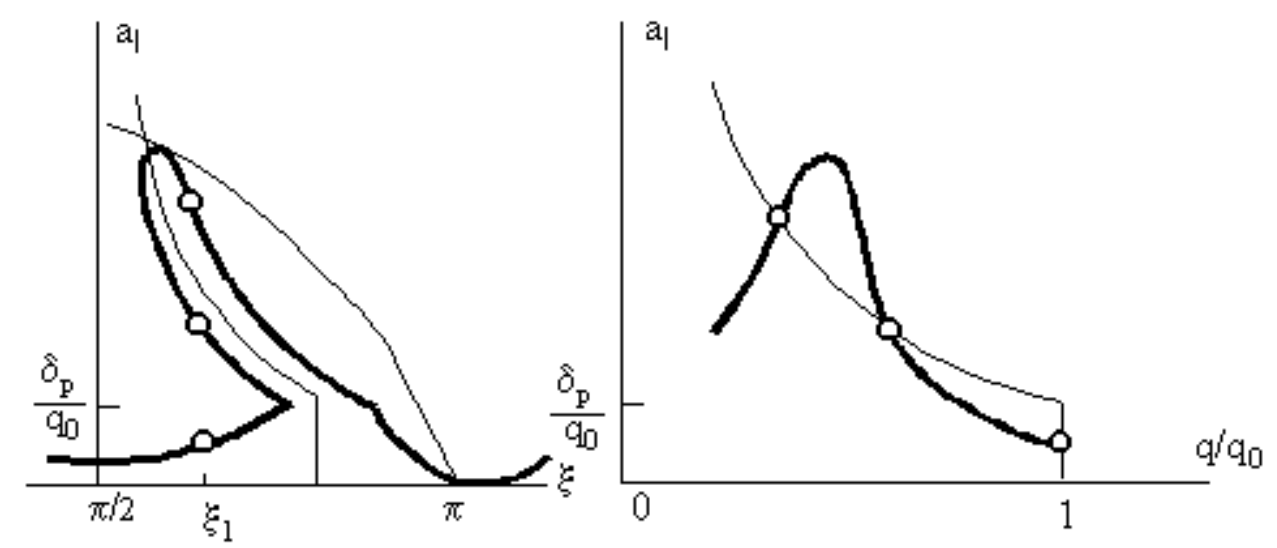

Рис.9.18

Рассматривая в этих уравнениях $q$ как независимый параметр, строятся графики зависимостей $a_{l}(q)$, точки пересечения которых и определяют искомые решения. Резонансные кривые дополнены линейными ветвями, соответствующими колебаниям в пределах предварительной деформации ограничителя $\left(a_{l} \leq \delta_{p} / q_{0}\right)$.

Остановимся на случае системы, взаимодействующей с абсолютно жестким ограничителем $\left(q_{0} \rightarrow \infty\right)$. В этом случае коэффициент линеаризации описывается выражением (8.37) или

$$
q\left(a_{1}\right)=2 \delta_{P} / a_{1}
$$

После подстановки коэффициента (9.71) в равенство (9.25) получим уравнение амплитудно-частотной характеристики, подобное (9.35) 


$$
a_{l}=\frac{-2 \delta_{P} \cdot C_{1}(\xi) \pm \sqrt{\left[\delta(\xi) \cdot C_{1}(\xi)\right]^{2}+\Psi^{2}(\xi) \cdot\left[\delta^{2}(\xi)-4{\delta_{P}}^{2}\right]}}{C_{1}^{2}(\xi)+\Psi^{2}(\xi)},
$$

где $C_{1}(\xi)=\xi \cdot \operatorname{ctg} \xi$.

На рис.9.19 показаны резонансные кривые для двух первых форм колебаний системы при различных значениях силы $P$ прижима.

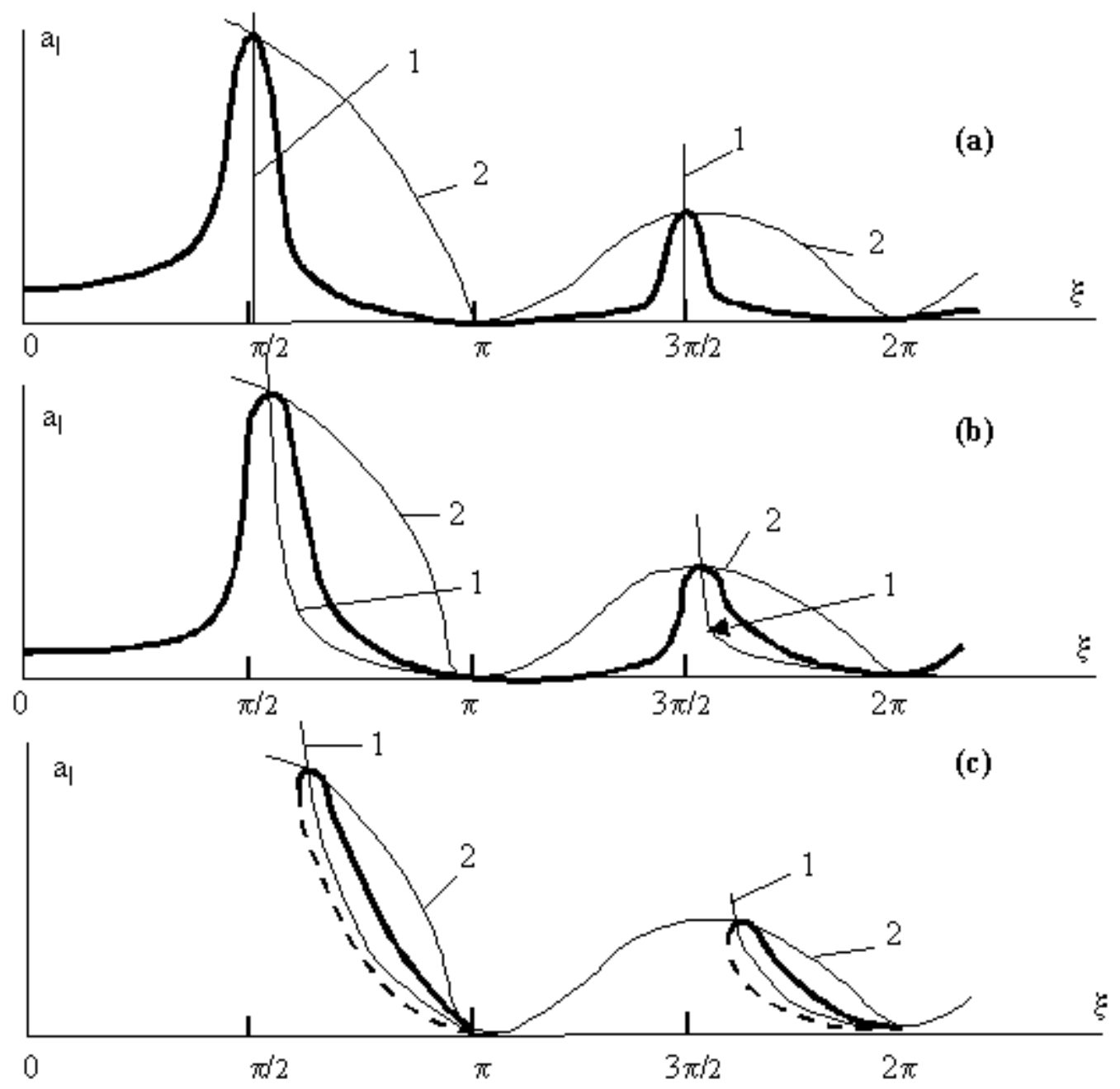

Рис.9.19

Индексом 1 обозначены скелетные кривые, уравнение которых

$$
a_{l}=-2 \delta_{P} / C_{1}(\xi)=-2 \delta_{P} / \xi \cdot \operatorname{ctg} \xi
$$

получено из (9.72) при $\delta=0, \psi=0$.

Пересечение скелетных кривых с линией 2 предельных амплитуд, уравнение которой (9.31) можно получить из (9.72), (9.73), дает высшие точки резонансных кривых. При отсутствии силы прижима $\left(\delta_{P}=0\right)$ система 
линейна, и ее резонансные кривые (рис.9.20,a) описываются уравнением (9.25) при $q=0$. Резонансные частоты системы $\xi_{n}=\frac{\pi}{2}(2 n-1), n=1,2, \ldots$

Из условия существования виброударных режимов $a_{l}>0$ следует, что при $\delta_{p} \leq \delta(\xi) / 2$ резонансные кривые (рис.9.19,б) однозначны во всем диапазоне частот. По мере увеличения усилия прижима резонансные частоты все более смещаются в сторону увеличения от собственных частот линейной системы. При $\delta_{p}>\delta(\xi) / 2$ резонансные кривые (рис.9.19,в) имеют зоны двузначности. Верхние (сплошные линии) и нижние (штриховые линии) ветви сходятся в точке, для которой подкоренное выражение в (9.72) обращается в нуль, т.е. $C_{1}(\xi)=\Psi(\xi) \cdot \sqrt{2 \delta_{P} / \delta(\xi)-1}$.

С учетом этого из (9.72) находим уравнения, решения которых определяют граничные точки областей двузначности резонансных кривых

$$
\begin{gathered}
a_{l}=-\frac{2 \delta_{P} C_{1}(\xi)}{C_{1}^{2}(\xi)+\Psi^{2}(\xi)} \\
a_{l}=\frac{\delta(\xi)}{\Psi(\xi)} \cdot \sqrt{1-\left(\frac{\delta(\xi)}{2 \delta_{P}}\right)^{2}}
\end{gathered}
$$

Кривая (9.74) разделяет ветви резонансных кривых и располагается ниже скелетной кривой (9.73). При отсутствии демпфирования $(\psi=0)$ уравнение (9.72) для резонансных кривых принимает вид

$$
a_{l}=\frac{-2 \delta_{P} \sin \xi \pm \delta \cdot \sin \chi \xi}{\xi \cdot \cos \xi},
$$

а разделительная кривая (9.74) совпадает со скелетной (9.73).

Устойчивость найденных решений исследуется, используя условие (9.20). После подстановки производных $\partial V / \partial a_{l}=0 ; \partial U / \partial a_{l}=\partial q / \partial a_{l}=-2 \delta_{P} / a_{l}^{2}$ условие устойчивости приводится к виду

$$
a_{l}>\frac{-2 \delta_{P} C_{1}(\xi)}{C_{1}^{2}(\xi)+\Psi^{2}(\xi)}
$$


Из (9.74) и (9.77) следует, что граница области устойчивости совпадает с разделительной кривой, поэтому показанные штриховыми линиями нижние ветви резонансных кривых неустойчивы (рис.9.19,в). Режимы, отвечающие однозначным амплитудно-частотным характеристикам (рис.9.19,б), устойчивы во всем диапазоне их существования.

Рассмотренный случай стержневых систем с силовым замыканием весьма важен для понимания динамических свойств ряда технологических ультразвуковых машин, в которых подача инструмента в процессе работы производится путем силового прижима (рис.4.1 - 4.4). 


\section{0. Виброударные взаимодействия}

\section{ультразвуковых стержневых систем}

1. В разделе 4.2 приведены схемы ультразвуковых технологических машин, в которых инструмент взаимодействует с пассивным волноводом. В системах, показанных на рис. 4.3 и рис.1.2, пассивный волноводотражатель предназначен для акустической развязки основания от динамической составляющей нагрузки. В ряде случаев (рис.1.4 и рис.4.5) пассивным волноводом является обрабатываемое изделие, длина которого соизмерима или значительно превышает длину звуковой волны в материале волновода.

В этом параграфе рассматриваются эффекты, обусловленные виброударным взаимодействием стержневых систем. Возникновение подобных эффектов возможно, конечно, не только в технологических ультразвуковых системах, но и в разнообразных вибропроводящих конструкциях, имеющих зазоры в сочленениях или стыки, допускающие раскрытие при пиковых нагрузках. Изучению отдельных аспектов теории колебаний конструкций подобного рода посвящены работы [51, 61, 67,112].

Рассмотрим две стержневые системы (рис.10.1), совершающие одномерные колебания. Смежные элементы систем, принятые за нулевые, установлены с зазором $\Delta$ (отрицательное $\Delta$ соответствует натягу) и м огут контактировать между собой без взаимопроникновения. Смещение

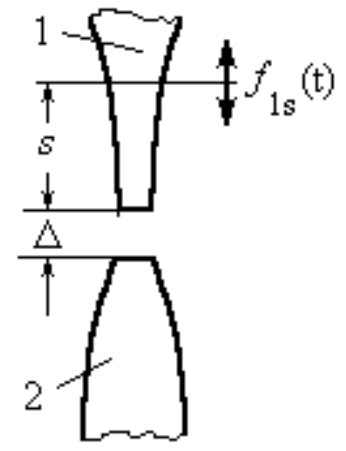

Рис.10.1 произвольного элемента $x$-й системы $(i=1,2)$ будем описывать функцией $u_{i x}(t)=u_{i}(x, t)$, отсчитываемой от недеформированного состояния. Колебания системы 1 возбуждаются вынуждающей периодической силой $f_{1 s}(t)=F_{1 s} \cdot e^{j \omega t}$, приложенной к некоторому элементу $\mathbf{s}$. Колебания второй системы возбуждаются в результате взаимодействия при контактировании элементов $x=0$. 
Обозначим

$$
V(t)=u_{10}(t)-u_{20}(t)
$$

относительное перемещение контактирующих элементов систем. Их силовое взаимодействие представим в виде нелинейной статической характеристики вида (8.28)

$$
f(v)=k_{0}(v-\Delta) \eta(v-\Delta),
$$

осуществляя в дальнейшем предельный переход $k_{0} \rightarrow \infty$, означающий отсутствие взаимопроникновения контактирующих элементов. Отражая основной эффект взаимодействия, такой подход лишает задачу известной неопределенности и дает простые расчетные соотношения.

Обозначив, по-прежнему, через $L_{s x}^{(i)}(p)$ оператор динамической податливости $i$-й системы, связывающий перемещение элемента $x$ с произвольной силой, приложенной к элементу $s$, запишем уравнения колебаний систем в виде

$$
\begin{aligned}
& u_{1 x}=L_{s x}^{(i)}(p) f_{1 s}(t)-L_{0 x}^{(i)}(p) f(v) \\
& u_{2 x}=L_{0 x}^{(2)}(p) f(v)
\end{aligned}
$$

Подставив (10.3) при $x=0$ в (10.1), получим уравнение относительного движения контактирующих элементов

$$
v(t)=L_{s x}^{(i)}(p) f_{1 s}(t)-L(p) f[v(t)]
$$

где $\quad L(p)=L_{00}^{(1)}(p)+L_{00}^{(2)}(p)$.

Отыскивая приближенное периодическое решение уравнения (10.4) в виде

$$
v(t)=m+v^{o}(t), \quad v^{o}(t)=a e^{j(\omega t-\varphi)}
$$

осуществим гармоническую линеаризацию функции (10.2)

$$
f(v)=P_{0}(m, a)+k(m, a) v^{0}
$$

Подставляя (10.5), (10.6) в (10.4) и разделяя постоянные и периодические составляющие, найдем при $p=j \omega$

$$
m=-L(0) P_{0}
$$




$$
[1+k L(j \omega)] \nu^{0}(t)=L_{s 0}^{(1)}(j \omega) F_{1 s} \mathrm{e}^{j \omega t}
$$

С учетом (10.5) получим из (10.8) выражения для амплитуды и фазы периодической составляющей относительного движения

$$
\begin{gathered}
a=F_{1 s}|W(j \omega, k)|^{-1}=F_{1 s}\left(U^{2}+V^{2}\right)^{1 / 2} \\
\cos \varphi=a U / F_{1 s}, \quad \sin \varphi=a V / F_{1 s}
\end{gathered}
$$

где

$$
W(j \omega, k)=[1+k L(j \omega)] / L_{s 0}^{(1)}(j \omega), \quad U=\operatorname{Re} W, \quad V=\operatorname{Im} W
$$

Выражения (10.7), (10.9, (10.12) совместно с выражениями для коэффициента $k=k(m, a)$ гармонической линеаризации образуют систему уравнений, позволяющую найти амплитуду $a$, и постоянную составляющую $m$ относительного движения и постоянную составляющую $P_{0}$ силы взаимодействия. Затем с помощью (10.10) отыскивается фаза $\varphi$, а из (10.3) параметры периодического движения стержневых систем 1 и 2.

Как было показано в Параграфе 8, предельный переход $k \rightarrow \infty$ в выражениях (8.29), (8.30) для коэффициентов гармонической линеаризации нелинейной функции (10.2) приводит к следующим соотношениям (см. П.8.4):

$$
\begin{gathered}
a=\Delta-m \\
k(a)=2 P_{0} / a
\end{gathered}
$$

Из (10.7), (10.12), (10.13) находим коэффициент линеаризации для стержневых систем установленных с предварительным зазором (натягом) $\Delta$

$$
k(a)=\frac{2}{L(0)}\left(1-\frac{\Delta}{a}\right)
$$

Приближенное периодическое решение уравнения абсолютного движения произвольного сечения $i$-го стержня ищем в виде

$$
u_{i x}(t) \approx m_{i x}+u_{i x}^{0}(t), u_{i x}^{0}(t)=a_{i x} \exp \left[j\left(\omega t-\varphi_{i x}\right)\right],(\mathrm{i}=1,2)
$$


Подставляя (10.15) и (10.6) в (10.3) и разделяя постоянные и периодические составляющие, получим с учетом (10.8)

$$
\begin{aligned}
m_{i x} & =(-1)^{\mathrm{i}} L_{0 x}^{(i)}(0) P_{0}, \\
u_{i x}^{o}(t) & =\frac{F_{1 s}}{W_{x}^{(i)}[j \omega, k(a)]} \mathrm{e}^{\mathrm{j} \omega t}
\end{aligned}
$$

где

$$
\begin{gathered}
W_{x}^{(1)}(j \omega, k)=\frac{1+k L(j \omega)}{[1+k L(j \omega)] L_{s x}^{(1)}+k L_{0 X}^{(2)}(j \omega) L_{s 0}^{(1)}(j \omega)} \\
W_{X}^{(2)}(j \omega, k)=\frac{1+k L(j \omega)}{k L_{0 X}^{(2)}(j \omega) L_{s 0}^{(1)}(j \omega)}
\end{gathered}
$$

Из (10.17) находим амплитуды и фазы периодических составляющих движения произвольного элемента $x$ i-й стержневой системы

$$
\begin{gathered}
a_{i x}=F_{1 s}\left|W_{i x}(j \omega, k)\right|^{-1}=F_{1 s}\left(U_{i x}^{2}+V_{i x}^{2}\right)^{1 / 2} \\
\cos \varphi=a U_{i x} / F_{1 s}, \sin \varphi=a V_{i x} / F_{1 s}
\end{gathered}
$$

где $U_{i x}=\operatorname{Re} W_{i x}, \quad V_{i x}=\operatorname{Im} W_{i x}$

Полученные соотношения позволяют найти все параметры колебаний двух соударяющихся систем произвольной структуры. Методику получения решений проиллюстрируем некоторыми характерными примерами

2. Рассмотрим колебания двух одинаковых соударяющихся стержней длиной $l_{i}=l(i=1,2)$. Для упрощения окончательных соотношений будем считать, что вынуждающая сила $f_{10}=f$ приложена к сечению $s=0$ стержня 1. В этом случае $L_{s x}^{(1)}(j \omega)=L_{s x}^{(2)}(j \omega)=L(j \omega)$, и выражения (10.11), (10.18), (10.19) приобретают вид

$$
\begin{gathered}
W(j \omega, k)=W_{00}(j \omega)+2 k(a) \\
W_{x}^{(1)}(j \omega, k)=W_{0 x}(j \omega) \frac{W_{00}(j \omega)+2 k(a)}{W_{00}(j \omega)+k(a)} \\
W_{x}^{(2)}(j \omega, k)=W_{0 x}(j \omega) \frac{W_{00}(j \omega)+2 k(a)}{k(a)}
\end{gathered}
$$


Динамическая жесткость однородного стержня с закрепленным концом при действии вынуждающей силы на свободный конец найдена в предыдущем параграфе, определяется выражением (9.23) и имеет вид

$$
W_{00}(j \omega)=\frac{E S}{l}\left[\xi \cdot \operatorname{ctg} \xi+j \frac{\psi \cdot \xi}{4 \pi} \cdot \frac{(\xi+0,5 \sin 2 \xi)}{\sin ^{2} \xi}\right]
$$

где $\xi=\omega l / c ; c=\sqrt{E / \rho}-$ скорость распространения звука в материале стержня; $E, \rho-$ модуль упругости и плотность материала; $S, l-$ площадь поперечного сечения и длина стержня; $\psi-$ коэффициент поглощения энергии в материале стержня.

После подстановки (10.22), (10.25) в (10.9) получим для амплитуды $a$ относительного движения

$$
a=\delta\left[\xi \operatorname{ctg} \xi+2 q+j \frac{\psi \xi}{4 \pi} \cdot \frac{(\xi+0,5 \sin 2 \xi)}{\sin ^{2} \xi}\right]^{-1} \mid
$$

где $\delta=F l / E S, q=q(a)=k(a) l / E S$

Аналогично из (10.17) с учетом (10.23)-(10.25) при $x=0$ находим амплитуды $a_{i 0}$ абсолютного движения контактирующих сечений стержней

$$
\begin{gathered}
a_{10}=\delta\left|\frac{\xi \operatorname{ctg} \xi+q+j \Psi}{\xi \operatorname{ctg} \xi(\xi \operatorname{ctg} \xi+2 q)+2 j \Psi(\xi \operatorname{ctg} \xi+q)}\right| \\
a_{20}=\delta q[\xi \operatorname{ctg} \xi(\xi \operatorname{ctg} \xi+2 q)+2 j \Psi(\xi \operatorname{ctg} \xi+q)]^{-1} \mid
\end{gathered}
$$

где $\Psi=\Psi(\xi)=\frac{\psi \xi}{4 \pi} \cdot \frac{(\xi+0,5 \sin \xi)}{\sin ^{2} \xi}$

Уравнения (10.26)-(10.28) совместно с выражениями (10.13), (10.14) для коэффициентов гармонической линеаризации позволяют построить амплитудно-частотные характеристики относительных и абсолютных колебаний сопряженных сечений стержней. 
3. Пусть стержни установлены с предварительным зазором (натягом) $\Delta$ (рис.10.2).

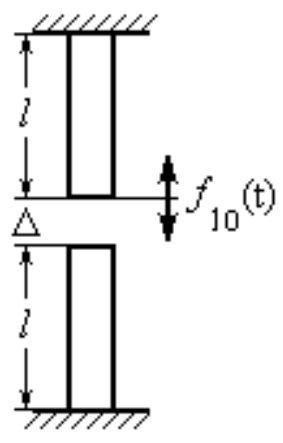

Pис.10.2

В принятых обозначениях выражение (10.14) для коэффициента гармонической линеаризации записывается в виде

$$
q=q(a)=1-\frac{\Delta}{a}
$$

Уравнение резонансной кривой относительных колебаний соударяющихся сечений находится из (10.26)

$$
a=\delta\left\{(\xi \operatorname{ctg} \xi+2 q)^{2}+\left[\frac{\psi \xi(\xi+0,5 \sin 2 \xi)}{4 \pi \sin ^{2} \xi}\right]^{2}\right\}^{-2}
$$

Заметим, что в рассматриваемом случае относительное движение смежных торцов стержней описывается уравнениями, эквивалентными уравнениям, описывающим движение конца стержня, соударяющегося с абсолютно жестким ограничителем. (См. П.8.4.) Поэтому все построения, проведенные в П.4 предыдущего параграфа, справедливы и при анализе относительного движения упругих стержней. Приведем здесь лишь ключевые моменты такого анализа.

Согласно (10.30) скелетные кривые амплитудно-частотных характеристик относительных движений соударяющихся сечений стержней описываются соотношением

$$
\xi \operatorname{ctg} \xi+2 q=0
$$

или после подстановки (10.29)

$$
a=-2 \Delta /(\xi \operatorname{ctg} \xi+2)
$$


Из (10.30) с учетом (10.32) найдем уравнение линии предельных амплитуд

$$
a=\frac{4 \pi \delta \sin ^{2} \xi}{\psi \xi(\xi+0.5 \sin 2 \xi)}
$$

Пересечение на плоскости ( $\xi, a)$ скелетных кривых и линии предельных амплитуд определяет, согласно (10.30), (10.31) высшую точку резонансной кривой. Остальные точки находятся с помощью построений, изложенных в предыдущем параграфе. При отсутствии потерь энергии на внутреннее трение в материале стержней $(\psi=0)$ из (10.30) и (10.29) получим следующее уравнение амплитудно-частотной характеристики относительного движения:

$$
a=\frac{2 \Delta \pm \delta}{\xi \operatorname{ctg} \xi+2}
$$

На рис.10.3,а,г показаны амплитудно-частотные характеристики относительного движения двух одинаковых соударяющихся стержней, контактирующие сечения которых установлены с предварительным зазором $(\Delta>0)$ и натягом $(\Delta<0)$.

$\Delta>0$
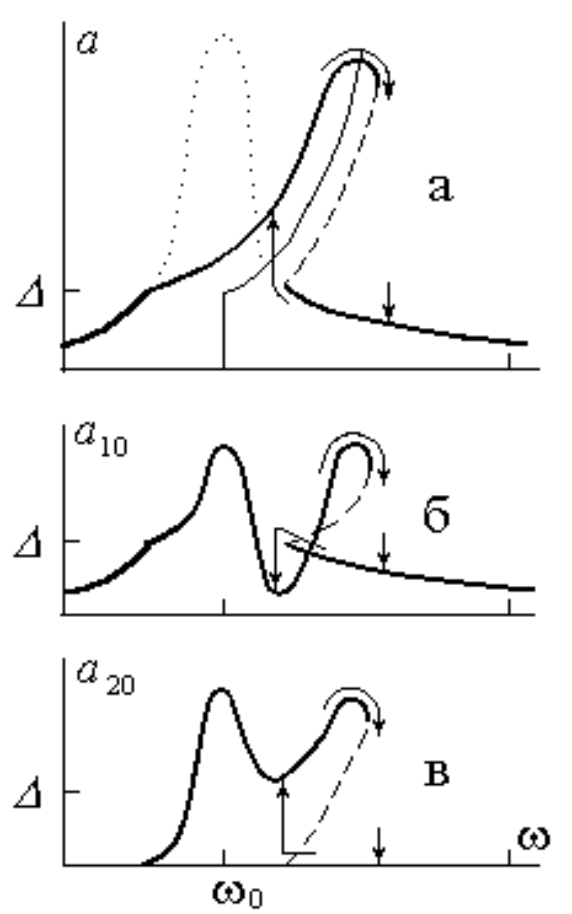

$\Delta<0$
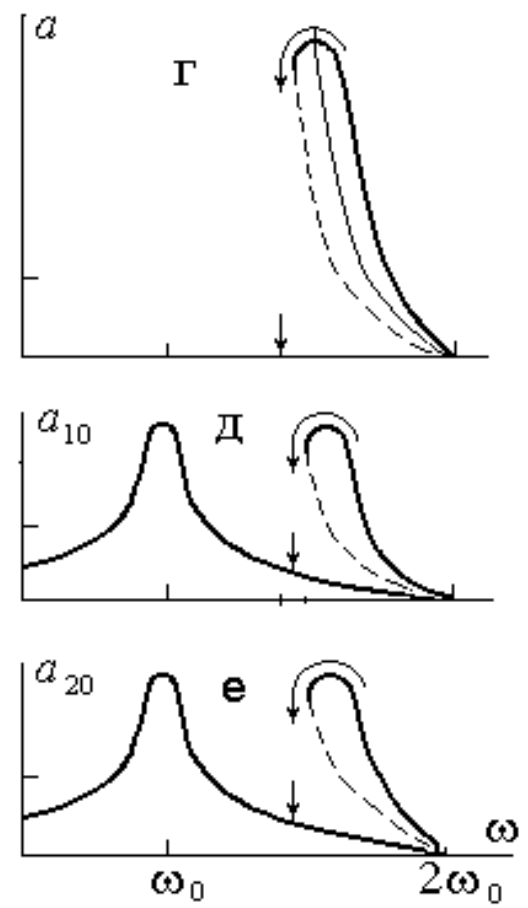

Рис. 10.3 
Из рис.10.3,а,г видно, что характер нелинейности зависит как от знака зазора: при установке стержней с зазором имеет место нелинейность жесткого, а при установке с натягом - мягкого типа. На приведенных рисунках и далее тонкими линиями построены скелетные кривые, а штриховыми - ветви резонансных кривых, соответствующие неустойчивым решениям. Пунктиром на рис.10.3,a показаны участки $a>\Delta$ резонансных кривых, которые реализуются только в отсутствие ограничителя; их всегда реализуемые пределах зазора ветви $a<\Delta$ построены жирными линиями.

При известной амплитуде $a$ относительного движения при помощи выражений (10.27)-(10.29) вычисляются амплитуды абсолютного движения контактирующих сечений стержней. При отсутствии диссипации $(\psi=0)$ уравнения амплитудно-частотных характеристик контактирующих сечений можно записать в виде

$$
a_{10}=\left|\frac{2 \Delta \xi \operatorname{ctg} \xi \pm \delta(\xi \operatorname{ctg} \xi+1)}{\xi \operatorname{ctg} \xi(\xi \operatorname{ctg} \xi+2)}\right| ; a_{20}=\left|\frac{-\Delta \xi \operatorname{ctg} \xi \pm \delta}{\xi \operatorname{ctg} \xi(\xi \operatorname{ctg} \xi+2)}\right|
$$

На рис.10.3,б,в приведены результаты построения амплитудночастотных характеристик абсолютного движения контактирующих сечений стержней, установленных с предварительным зазором $(\Delta>0)$, а на рис.10.3,д,е - стержней, установленных с предварительным натягом $(\Delta<0)$. Здесь кроме того построены ветви резонансных кривых, реализуемых при колебаниях с амплитудами $a<\Delta$ в пределах предварительного зазора (рис.3,б), и при совместных колебаниях контактирующих сечений с амплитудами $a<\mid \Delta / 2$ в пределах предварительного натяга (рис.3,д,е).

Приведенные характеристики выявляют аналогию между поведением виброударных систем сложной структуры с одной ударной парой и традиционных виброударных систем с сосредоточенными параметрами, с одной стороны, и обычными нелинейными колебательными системами, с другой стороны. Прежде всего здесь следует 
отметить наличие частотных областей двузначности резонансных кривых, устойчивая и неустойчивая ветви которых охватывают скелетные линии. Аналогичный приведенным амплитудно-частотным характеристикам вид имеют резонансные кривые в окрестности высших собственных частот линейных подсистем.

В обоих случаях относительного расположения стержней выход на резонансные режимы с максимальными амплитудами может быть осуществлен затягиванием колебаний по частоте из области низких частот для систем с предварительным зазором и из области высоких частот для систем с натягом и силовым прижимом, как это показано стрелкам на рис. 3. При дальнейшем изменении частоты после реализации максимальных амплитуд происходит срыв колебаний и прекращение виброударного процесса. При этом в системе с зазором устанавливаются линейные колебания активного стержня 1 в пределах зазора (рис.3,a,б). В системе с натягом (рис.3,г,д) возбуждаются совместные колебания стержней как единого стержня суммарной длины.

Здесь уместно отметить появление дополнительных резонансов при наблюдении абсолютных движений стержней, возбуждаемых на собственных частотах подсистем. Для системы с предварительным зазором - это резонанс (рис.10.3,б,в) при синфазных движениях подсистем с легкими соударениями, которые и приводят к раскачке пассивного стержня. Для системы с предварительным натягом - это резонанс (рис.10.3,д,е) стержня суммарной длины. В обоих случаях резонансы происходят на собственных частотах $\xi=\pi 2(r-1) / 2, \quad(r=1,2, \ldots)$. Следует обратить внимание на появление нелинейного антирезонанса в системы с предварительным зазором (рис.10.3,б,в) в частотном диапазоне между двумя резонансами. В этом режиме пассивный стержень выполняет роль виброударного динамического гасителя колебаний активного стержня, 
амплитуда которого оказывается малой и стремится к нулю при уменьшении диссипации энергии в материале стержней.

При возбуждении колебаний с фиксированной частотой, лежащей в областях двузначности резонансных кривых, выход на виброударный режим может быть выполнен путем жесткого запуска, заключающегося в принудительном сообщении системе дополнительной энергии, которой достаточно для выхода на верхнюю устойчивую ветвь характеристики. При реализации таких колебаний система допускает увеличение амплитуд путем затягивания по амплитуде, которое осуществляется увеличением зазора, натяга или силы прижима вплоть до достижения предельной для данной частоты амплитуды, определяемой энергетической границей области существования.

4. Построим амплитудно-частотные характеристики соударяющихся сечений двух одинаковых стержней, сжатых между собой продольной силой $P$ (рис.10.4).

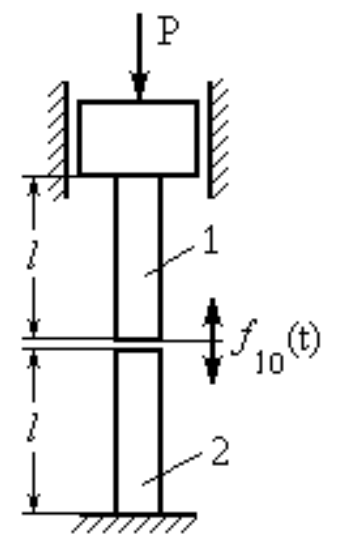

Рис.10.4

Предполагается, что пассивный стержень 2 жестко закреплен, а активный стержень 1 связан с достаточно большой массой, колебаниями которой можно пренебречь.

В этом случае выражение (10.13) для коэффициента гармонической линеаризации принимает вид

$$
q=q(a)=2 \delta_{P} / a, \quad(10.36)
$$


где $\delta_{P}=P I / E S$, а уравнение резонансной кривой относительных колебаний находится из (10.30)

$$
a=\delta\left\{\left(\xi \operatorname{ctg} \xi+4 \frac{\delta_{P}}{a}\right)^{2}+\left[\frac{\psi \xi(\xi+0,5 \sin 2 \xi)}{4 \pi \sin ^{2} \xi}\right]^{2}\right\}^{-2}
$$

Из (10.37) легко получается явное уравнение амплитудно-частотной характеристики

$$
a=\frac{-4 \delta_{P} \xi \operatorname{ctg} \xi \pm \sqrt{\delta^{2} \xi^{2} \operatorname{ctg}^{2}-\Psi^{2}(\xi)\left(16 \delta_{P}^{2}-\delta^{2}\right)}}{\xi^{2} \operatorname{ctg}^{2} \xi+\Psi^{2}(\xi)}
$$

где $\Psi(\xi)=\frac{\psi \cdot \xi}{4 \pi} \cdot \frac{(\xi+0,5 \sin 2 \xi)}{\sin ^{2} \xi}$

Согласно (10.37) (или (10.38) при $\delta=0, \psi=0$ ) скелетные кривые амплитудно-частотных характеристик относительных движений соударяющихся сечений стержней описываются соотношением

$$
a=-4 \delta_{P} / \xi \operatorname{ctg} \xi
$$

Уравнение линии предельных амплитуд по-прежнему определяется равенством (10.33)

На (рис.10.5,а,г) показаны амплитудно-частотные характеристики относительного движения контактирующих сечений двух одинаковых соударяющихся стержней, сжатых постоянной силой, в окрестности первой формы колебаний при различных соотношениях силы возбуждения и прижима.

Как видно, при малых силах прижима (рис.10.5,a) система ведет себя подобно линейной, однако с увеличением прижима резонансная частота уходит в область более высоких частот. При статических силах прижима $P>F / 4$ (рис.10.5,г) характер резонансной кривой радикально изменяется. Колебания с ударами могут существовать только парами в диапазонах частот от собственных частоты стержня до его антирезонансных частот.

Из сравнения рис.10.5,Г и рис.10.3,а,г видно, что характер нелинейности зависит не только от знака зазора, но и от структуры 
модели. В системе с силовым прижимом проявляется нелинейность только мягкого типа. На рис.10.5,a,г и далее тонкими линиями показаны скелетные кривые, а штриховыми - ветви резонансных кривых, соответствующие неустойчивым решениям.

При известной амплитуде $a$ относительного движения при помощи выражений (10.28), (10.29), (10.36) вычисляются амплитуды абсолютного движения контактирующих сечений стержней. При отсутствии диссипации $(\psi=0)$ уравнения амплитудно-частотных характеристик контактирующих сечений можно записать в виде

$$
a_{10}=\left|\frac{2 \delta_{P} \pm \delta}{\xi \operatorname{ctg} \xi}\right| ; a_{20}=\left|\frac{2 \delta_{P}}{\xi \operatorname{ctg} \xi}\right|
$$

На рис. 10.5,б,в,д,е приведены результаты построения амплитудночастотных характеристик абсолютного движения контактирующих сечений стержней, сжатых статической силой.
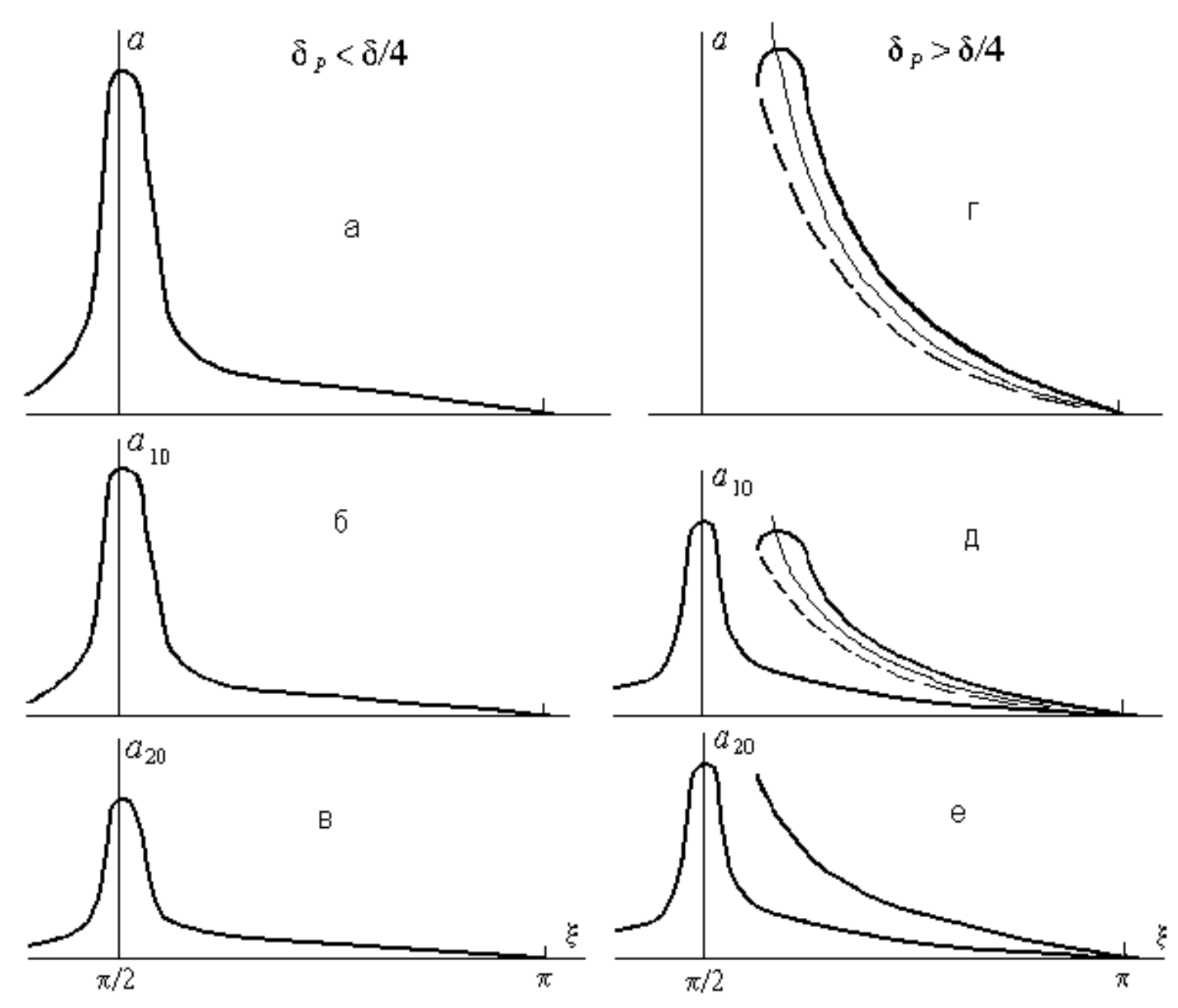

Pис. 10.5 
Здесь следует отметить, что в случае силового прижатия амплитуда колебаний пассивного стержня 2 в виброударных режимах не зависит от силы возбуждения, и поэтому скелетная кривая, устойчивая и неустойчивая ветви сливаются (рис. 10.5,e). Кроме того обратим внимание на появление дополнительных резонансов при наблюдении абсолютных движений стержней, возбуждаемых на собственных частотах подсистем. Как и для систем с гарантированным натягом (рис.10.3,г,Д) - это резонанс стержня суммарной длины.

5. Рассмотрим колебания-системы (рис. 10.6), в которой стержень 1 длиной $l$ соударяется с полубесконечным стержнем 2.

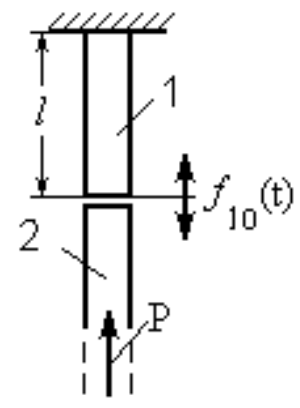

Рис. 10.6

Натяг создается статической силой $P$. Динамическая податливость полубесконечного стержня при возбуждении, действующем в сечении $x=0$ найдена в П.5.2. и имеет вид

$$
L_{0 x}^{(2)}(x, j \omega)=-j \frac{c_{2}}{\omega E_{2} S_{2}} \exp \left[-\left(j+\frac{\psi_{2}}{4 \pi}\right) \frac{\omega x}{c_{2}}\right]
$$

При $x=0$ получим для контактирующего сечения стержня 2

$$
L_{00}^{(2)}(0, j \omega)=-j \frac{c_{2}}{\omega E_{2} S_{2}}
$$

Коэффициент гармонической линеаризации $q(a)$ для рассматриваемой системы определяется формулой (10.36).

Построим частотные характеристики системы, пренебрегая потерями энергии в материале стержней, т.е. полагая $\psi_{1}=\psi_{2}=0$. Из (10.9), (10.11), (10.25), (10.36) и (10.42) получаем после преобразований для 
амплитуды относительного движения контактирующих сечений

$$
a=-\frac{2 \delta_{P} \pm \sqrt{\delta^{2}-4 \delta_{P}^{2} \frac{\operatorname{ctg}^{2} \xi}{W^{2}}}}{\xi \operatorname{ctg} \xi}
$$

где $\delta=F l_{1} / E_{1} S_{1}, \quad \delta_{P}=P I / E_{1} S_{1}, \quad W=W_{2} / w_{1}-$ отношение волновых сопротивлений $w_{i}=S_{i} \sqrt{E_{i} \rho_{i}}(i=1,2)$ стержней 1 и 2.

На рис. 10.7,a приведена амплитудно-частотная характеристика относительных колебаний контактирующих сечений стержней в окрестности первой собственной частоты активного стержня 1.

Аналогично по формулам (10.18)-(10.20) после подстановки соответствующих величин и преобразований найдем амплитуды колебаний контактирующих торцов стержней

$$
a_{10}=\left|\frac{\delta}{\xi \operatorname{ctg} \xi}\right| \sqrt{1+\left(\frac{2 \delta_{P}}{\delta}\right)^{2} \pm \frac{4 \delta_{P}}{\delta} \sqrt{1-\left(\frac{2 \delta_{P} \operatorname{ctg} \xi}{\delta W}\right)^{2}}} ; a_{20}=\frac{2 \delta_{P}}{\xi_{W}}
$$

На рис. 10.7,б,в приведены амплитудно-частотные характеристики
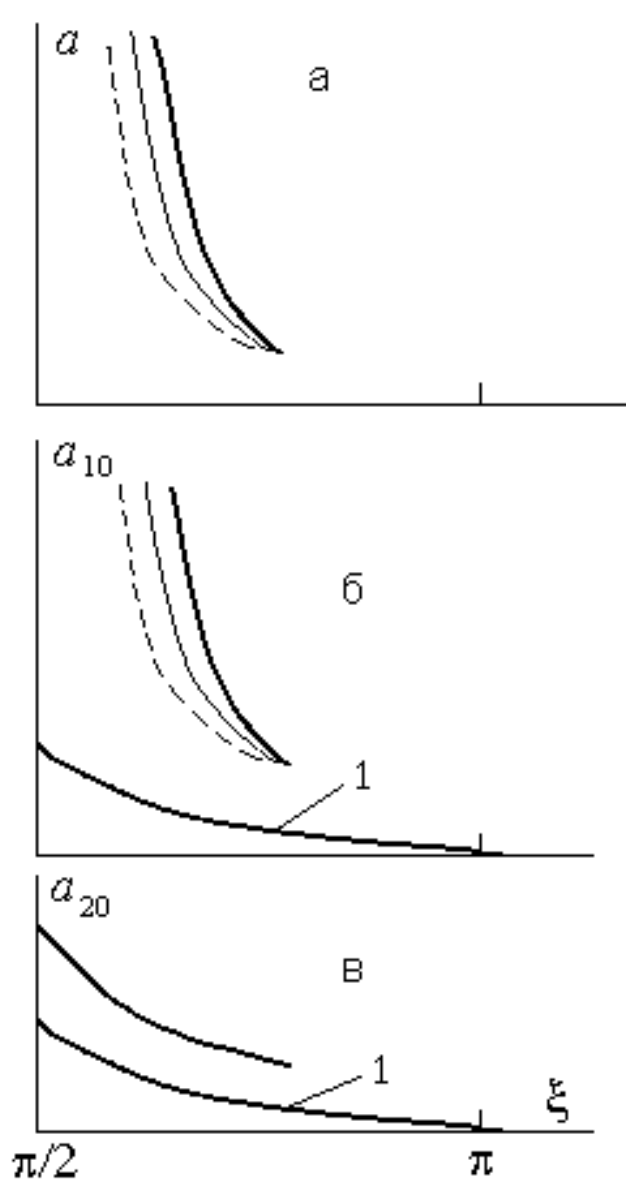

Рис. 10.7 контактирующих сечений стержней 1 и 2. Кривые 1 соответствуют безотрывным колебаниям, амплитуда которых находится предельным переходом $k \rightarrow \infty$ в выражениях (10.18), (10.19) при $X=s=0, \psi=0$

$$
a_{10}=a_{20}=\delta / \sqrt{(\xi \operatorname{ctg} \xi)^{2}+(\xi w)^{2}}
$$

Из рис. 10.7. видно, что наряду с безотрывными режимами, при которых стержни совершают колебания как единое целое, в определенном диапазоне частот могут существовать резонансные колебания активного стержня 1 большой амплитуды с соударениями в стыке. 
Согласно (10.43) виброударные режимы существуют при условии $\delta / \delta_{p}>2|\operatorname{ctg} \xi| w$. Двузначность амплитудно-частотных характеристик имеет место в диапазонах частот $(2 k-1) \pi / 2<\xi<k \pi(k=1,2, \ldots-$ номер собственной формы колебаний активного стержня 1) при $\delta<2 \delta_{p}$. Заметим, что диапазон существования виброударных режимов расширяется с увеличением относительного волнового сопротивления $w$ ограничителя, причем в пределе при $w \rightarrow \infty$, приходим к рассмотренному в предыдущем параграфе случаю соударения ограниченного стержня, поджатого постоянной силой к абсолютно жесткому ограничителю.

Как и в случае сжатых стержней конечной длины, в виброударных режимах амплитуда колебаний пассивного стержня, а следовательно и количество энергии, передаваемой через стык, не зависит от амплитуды возбуждения. Это связано с тем, что сила взаимодействия стержней при соударениях $f[r(t)] \approx P+2 P \exp j(\omega t-\varphi)$ не зависит от их относительного движения. Поэтому амплитудно-частотная характеристика пассивного стержня 2 (рис. 10.7, в) однозначна имеет однозначную ветвь, отвечающую виброударным режимам, и отвод энергии бегущей волной, распространяющейся вдоль волновода 2, не ограничивает амплитуду колебаний активного стержня при частотах $\xi \rightarrow(2 k-1) \pi / 2$.

В приведенном на рис. 10.7 примере виброударные режимы могут возникнуть лишь в результате «жесткого» возбуждения. Найдем условия «мягкого» раскрытия стыка. Очевидно, что стык не раскроется до тех пор, пока амплитуда переменной составляющей напряжения в стержне 2 в месте стыка при совместном движении стержней не превзойдет величину $P / S_{2}$, т.е.

$$
\left.E_{2} \frac{\partial u_{2 x}^{0}}{\partial x}\right|_{x=0}>\frac{P}{S_{2}}
$$


Функцию $u_{2 x}^{0}$, описывающую безотрывное движение, найдем из (10.17) при $k \rightarrow \infty$. Полагая $s=0$, имеем

$$
u_{2 x}^{0}(t)=F_{10} \frac{L_{0 x}^{(2)}(j \omega)}{1+L_{00}^{(2)}(j \omega) / L_{00}^{(1)}(j \omega)} \exp (j \omega t)
$$

Подставляя в (10.47) значения операторов динамической податливости (10.25), (10.42), получим при $\psi_{1}=\psi_{2}=0$

$$
u_{2 x}^{0}(t)=-F_{10} \frac{c_{2}}{E_{2} S_{2} \omega}\left(1-\mathrm{j} \frac{\operatorname{ctg} \xi}{w}\right) \exp \left(j \omega t-\frac{\omega X}{c_{2}}\right)
$$

С учетом (10.48) условие (10.46) принимает вид $F_{10}>P \sqrt{1+\left(\frac{\operatorname{ctg} \xi}{w}\right)^{2}}$.

Следует отметить, что полученные результаты имеют отношение не только к ультразвуковым системам, но и к многочисленным конструкциям, имеющим стыки и подверженным вибрационным воздействиям. В таких конструкциях предварительный натяг в стыке выбирают, как правило, таким образом, чтобы не происходило его раскрытия при амплитудном значении вибрационной силы. Однако из приведенных расчетов видно, что даже при натягах, выполненных с большим запасом, в результате жесткого запуска, например, в силу однократной случайной перегрузки может возникнуть виброударный процесс. Этот процесс может привести к серьезным неприятным, а иногда и к катастрофическим последствиям, связанным с разрушением элементов конструкции, ее разгерметизацией и т.д. 


\section{1. Вибрационное перемещение вязкоупругого стержня \\ в среде с сухим трением}

В П.4.2 приведены схемы ультразвуковых технологических машин для глубокого сверления и бурения, в которых существенную роль играют силы трения, действующие на боковые поверхности колеблющегося инструмента, медленно продвигающегося по мере проведения процесса. Исследование этих систем сводится к задаче вибрационного перемещения вибрирующего объекта в среде с сухим трением. Возникающий при этом эффект уменьшения силы сопротивления среды при вибрации вибрационное сглаживание нелинейностей [70,71,122] - используется при вибропогружении свай в строительстве, ультразвуковом резании, наложении вибраций на режущие инструменты, в вибротранспортировке и т.д. Анализ эффекта вибрационного сглаживания проводился только для систем с сосредоточенными параметрами [9-13,73,74,149]. Вопросы распространения вибрации в стержне при наличии сил сухого трения рассмотрены в $[152,188,189]$.

В данном параграфе рассматривается движение вязкоупругого стержня в среде с сухим трением под действием суммы сил — постоянной и гармонической с частотой собственных колебаний стержня. Анализ производится методом гармонической линеаризации с разделением движения на быстрые колебания упругого стержня и его медленное движение как жесткого тела. Исследуется их взаимное влияние в установившихся и переходных режимах.

На вязкоупругий стержень длины $I$ (рис. 11.1) действуют: гармоническая сила $f_{s}(t)=F_{s} \mathrm{e}^{\text {jot }}$ частоты $\omega$, приложенная в сечении $x=S$ и статическая сила $P$ в сечении $x=0$.

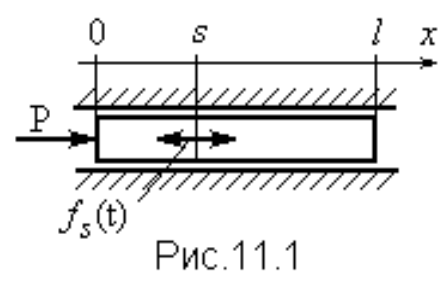


Сила сухого трения $91 \quad c$ интенсивностью $\vartheta$ равномерно распределена по всей длине стержня, причем $P<\vartheta 1$. Полное движение сечения $x$ стержня будем определять суммой переносного движения $y$ и относительного смещения $u_{x}(t)$, отсчитываемого от положения сечения в недеформированном стержне. Уравнение движения вязкоупругого стержня имеет вид

$$
\begin{gathered}
\rho S \frac{\partial^{2} u_{x}}{\partial t^{2}}-E S\left(\frac{\psi}{2 \pi \omega} \frac{\partial^{3} u_{x}}{\partial x^{2} \partial t}+\frac{\partial^{2} u_{x}}{\partial x^{2}}\right)=-\rho S \frac{d^{2} y}{d t^{2}}-\vartheta \operatorname{sgn}\left(\frac{\mathrm{d} y}{\mathrm{~d} t}+\frac{\partial u_{x}}{\partial t}\right)+ \\
+F_{s} \mathrm{e}^{j \omega t} \delta(x-s)+P \delta(x)
\end{gathered}
$$

где $E, \rho-$ модуль упругости и плотность материала стержня; $S-$ площадь поперечного сечения стержня; $\vartheta-$ коэффициент поглощения энергии в материале стержня ; $\delta(x)$ - функция Дирака.

Если скорость переносного движения мало изменяется за период колебаний, то в относительном движении можно положить $\mathrm{d} y / \mathrm{d} t=\dot{y}=$ const .

Отыскивая приближенно относительное движение сечения $x$ в виде

$$
u_{X} \approx a_{X} \exp j\left(\omega t-\varphi_{X}\right)
$$

где $a_{x}, \varphi_{x}$ - медленно меняющиеся амплитуда и фаза колебаний, проведем гармоническую линеаризацию силы сухого трения

$$
\vartheta \operatorname{sgn}\left(\dot{y}+\frac{\partial u_{x}}{\partial t}\right) \approx \vartheta\left(m_{x}+b_{x} \frac{\partial u_{x}}{\partial t}\right)
$$

где медленная составляющая $m_{x}$ и коэффициент $b_{x}$ при быстрой скорости определяются соотношениями

$$
\begin{gathered}
m_{x}=\frac{2}{\pi} \arcsin \frac{\dot{y}}{\omega\left|a_{x}\right|}, b_{x}=\frac{4}{\pi \omega\left|a_{x}\right|} \sqrt{1-\left(\frac{\dot{y}}{\omega a_{x}}\right)^{2}} \quad\left(\dot{y} \leq \omega a_{x}\right) \\
m_{x}=1, b_{x}=0 \quad\left(\dot{y} \geq \omega a_{x}\right)
\end{gathered}
$$

Подставляя (11.3) в (11.1) и разделяя быстро и медленно меняющиеся составляющие, для относительного движения получим

$$
\rho S \frac{\partial^{2} u_{x}}{\partial t^{2}}-E S\left(\frac{\psi}{2 \pi \omega} \frac{\partial^{3} u_{x}}{\partial^{2} x \partial t}+\frac{\partial^{2} u_{x}}{\partial t^{2}}\right)=F_{S} \mathrm{e}^{j \omega t}-\vartheta b_{x} \frac{\partial u_{x}}{\partial t}
$$


Интегрирование (11.1) по всей длине стержня с учетом (11.3), (11.6) дает уравнение его медленное движение как абсолютно твердого тела

$$
M \ddot{y}=P-\vartheta \int_{0}^{l} m_{x} \mathrm{~d} x
$$

где $M=\rho \cdot l \cdot S$ - масса стержня.

При $\dot{y} \geq \omega a_{x}$ уравнение (11.7) с учетом (11.5) принимает вид $M \ddot{y}=P-\vartheta I$ и быстрое (11.6) и медленное движения оказываются независимыми, причем сила трения 91 влияет только на переносное движение.

Подставляя в уравнение (11.7) величину $m_{. x}$ из (11.4), получим при $\dot{y} \leq \omega a_{x}$

$$
M \ddot{y}=P-\frac{2 \vartheta}{\pi} \int_{0}^{l} \arcsin \frac{\dot{y}}{\omega\left|a_{x}\right|} \mathrm{d} x
$$

Характер взаимного влияния быстрых и медленных движений в этом случае качественно можно оценить, если положить $\dot{y}<<\omega a_{x}$ и записать (11.8) в первом приближении в виде

$$
M \ddot{y}+\theta \dot{y}=P, \quad \theta=\frac{2 \vartheta}{\pi \omega} \int_{0}^{l} \frac{\mathrm{d} x}{\left|a_{x}\right|}
$$

Таким образом, при малых скоростях переносного движения вследствие действия вибрации происходит преобразование сухого трения, которое в среднем оказывает такое же воздействие, как вязкое с коэффициентом $\theta$, зависящим от распределения амплитуд вдоль стержня. С другой стороны, последнее слагаемое в (11.6) отражает обратное влияние медленного движения на колебания.

Найдем периодическое решение уравнения (11.6), представив его в виде

$$
u_{x}=\int_{0}^{l}\left[f_{s}(t) \delta(z-s)-\vartheta b_{z} \frac{\partial u_{z}}{\partial t}\right] L_{z x}(j \omega) \mathrm{d} z
$$

где $L_{z x}(j \omega)$ - оператор динамической податливости, связывающий перемещение сечения $x$ вязкоупругого стержня с гармонической силой, приложенной к сечению z. 
С учетом выражений (11.2), (11.4) из (11.9) получаем уравнение отоносительно амплитуд и фаз

$$
a_{x} \mathrm{e}^{-\mathrm{j} \varphi_{x}}=F_{s} L_{s x}(j \omega)-j \frac{4 \vartheta}{\pi} \int_{0}^{l} \mathrm{e}^{-\mathrm{j} \varphi_{z}} \sqrt{1-\left(\frac{\dot{y}}{\omega a_{z}}\right)^{2}} L_{z x}(j \omega) \mathrm{d} z
$$

Оператор динамической податливости $L_{s x}(j \omega)$ найден в П.5.12 (формула (5.127)) и имеет вид

$$
L_{s x}(j \omega)= \begin{cases}-\frac{\lambda}{\rho \cdot S \omega^{2}} \cdot \frac{\operatorname{ch} \lambda x \operatorname{ch} \lambda(I-s)}{\operatorname{sh} \lambda I} & 0 \leq x \leq s \\ -\frac{\lambda}{\rho \cdot S \omega^{2}} \cdot \frac{\operatorname{ch} \lambda(I-x) \operatorname{ch} \lambda s}{\operatorname{sh} \lambda I} & s \leq x \leq l\end{cases}
$$

где $\lambda=\left(j+\frac{\psi}{4 \pi}\right) \frac{\omega}{c}$ (см. (5.12)); $c$ - скорость звука в материале стержня.

В дальнейшем. будем рассматривать резонансные колебания стержня с частотами $\omega_{n}=n \pi c / l$, где $n=1,2, \ldots$ - номер формы колебаний.

Раскладывая гиперболические функции в (11.11) в ряды по малому параметру и ограничиваясь лишь линейными членами, получим следующее выражение для оператора динамической податливости в резонансном случае:

$$
L_{s x}\left(j \omega_{n}\right)=-j \frac{4 l}{\psi \pi n^{2} E S} \cos \frac{\pi n S}{l} \cos \frac{\pi n x}{l}
$$

Переходя к решению уравнения (11.10), заметим, что диссипативные силы слабо влияют на распределение собственных частот системы. Поэтому, ограничиваясь рассмотрением резонансных движений, предположим, что формы и фазы колебаний при наличии сухого трения близки к резонансным формам и фазам вынужденных колебаний вязкоупругого стержня без кулонова трения, определяемым соотношением (11.12), т. е.

$$
\begin{aligned}
& a_{x} \approx a_{0} \cos \frac{\omega x}{c}=a_{0} \cos \frac{\pi n x}{l} \\
& \varphi_{x} \approx \arcsin \left[\operatorname{sgn}\left(\cos \frac{\pi n x}{l}\right)\right]
\end{aligned}
$$

где $a_{0}$ - неизвестная величина. 
После подстановки (12)—(14) в уравнение (11.10) получаем следующее выражение для амплитуды $a_{0}$ :

$$
a_{0}=\frac{4 l}{\psi \pi n^{2} E S}\left[F_{s} \cos \frac{\pi n S}{l}-\frac{4 \vartheta}{\pi} \int_{0}^{l} \operatorname{sgn}\left(\cos \frac{\pi n z}{l}\right) \sqrt{1-\left(\frac{\dot{y}}{\omega_{n} a_{z}}\right)^{2}} \cos \frac{\pi n z}{l} \mathrm{~d} z\right]
$$

Для приближенного вычисления определенного интеграла в уравнении (11.15) разложим подкоренное выражение в степенной ряд

$$
\sqrt{1-v_{z}^{2}}=1-1 / 2 v_{z}^{2}-1 / 8 v_{z}^{4}-\ldots
$$

где $\quad v_{z}=\dot{y} / \omega_{n} a_{z}$.

При вычислении интеграла в (11.15) необходимо учитывать ограничение

$$
\dot{y} \leq \omega_{n} a_{z}
$$

следующее из условий на коэффициенты гармонической линеаризации (11.4), (11.5) и означающее, что в окрестности узлов форм абсолютная скорость сечений не меняет знака, и сухое трение, действующее на этих участках стержня, не оказывает влияния на пара-метры колебаний. С учетом (11.13) найдем длину отрезка (рис11.2), на котором выполняется условие (11.16)

$$
l_{1}=\frac{l}{\pi n} \arccos \frac{\dot{y}}{\omega_{n} a_{0}}
$$

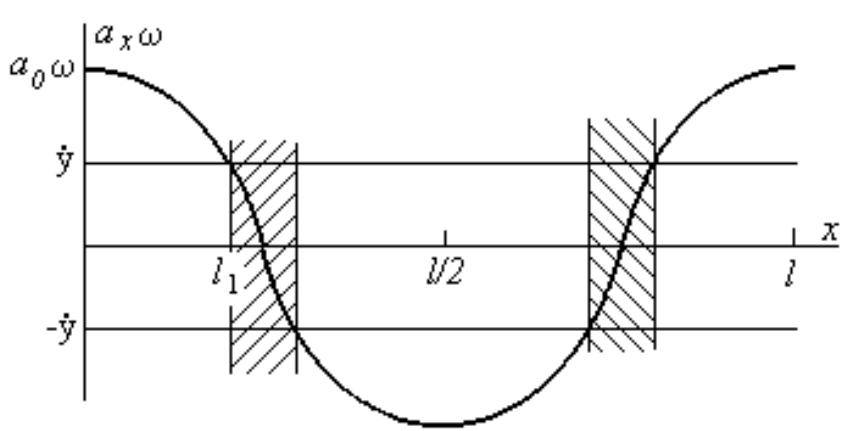

Pис.11.2

Вследствие симметрии форм колебаний при резонансе для интеграла в уравнении (11.15) имеем

$$
\int_{0}^{l} \operatorname{sgn}\left(\cos \frac{\pi n z}{l}\right) \sqrt{1-\left(\frac{\dot{y}}{\omega_{n} a_{z}}\right)^{2}} \cos \frac{\pi n z}{l} \mathrm{~d} z=2 n \int_{0}^{l} \sqrt{1-\left(\frac{\dot{y}}{\omega_{n} a_{z}}\right)^{2}} \cos \frac{\pi n z}{l} \mathrm{~d} z
$$


Подставляя (11.16)-(11.19) в правую часть уравнения (11.15) и ограничивая разложение (11.16) двумя первыми слагаемыми, получим после интегрирования и преобразований

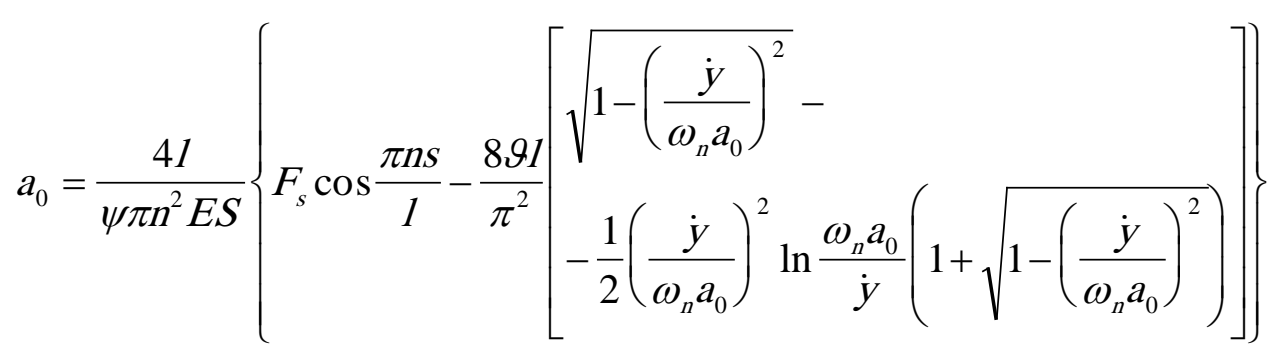

Из формулы (19) следует, что при отсутствии медленного движения $(\dot{y}=0)$ колебания возможны при условии

$$
F_{s} \cos \frac{\pi n s}{l}>\frac{8}{\pi^{2}} \vartheta I=0,81 \vartheta l
$$

Увеличение скорости медленного движения улучшает условия колебаний, снижая демпфирование, вносимое сухим трением. Если абсолютная скорость не меняет знака для всех сечений, т. е. скорость $\dot{y}>\omega_{n} a_{0}$, и следовательно условие (11.17) не выполняется по всей длине стержня, сухое трение вообще не влияет на колебания, амплитуда которых $a_{x}^{*}=\frac{4 F_{s} l}{\psi \pi n^{2} E S} \cos \frac{\pi n s}{l} \cos \frac{\pi n x}{l}$ совпадает с определяемой при помощи оператора (11.12) (См. также П. 5.12). Зависимость амплитуды колебаний $a_{0}$ сечения $x=0$ от отношения $v_{0}=\dot{y} / \omega_{n} a_{0}$ переносной $\dot{y}$ и колебательной $\omega_{\tau} a_{0}$ скоростей приведена на рис.11.3,а.
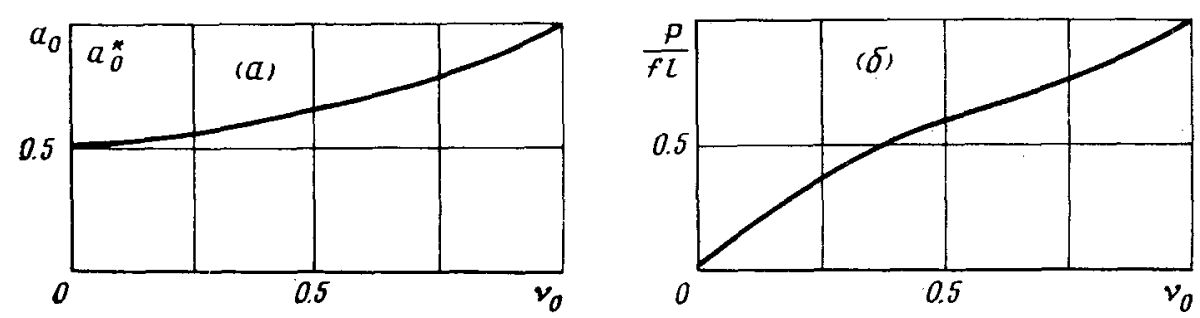

Рис.11.3

Величина медленной скорости $\dot{y}$ в уравнении (11.20) определяется из общего решения уравнения (11.8). Обозначим в нем

$$
G=\frac{2 \vartheta}{\pi} \int_{0}^{1} \arcsin \frac{\dot{y}}{\omega\left|a_{x}\right|} \mathrm{d} x
$$


Раскладывая в ряд входящую в (11.22) подынтегральную функцию

$$
\arcsin v=v+1 / 6 v^{3}+\ldots
$$

с учетом (5), (13), (18) получим после интегрирования

$$
G=\frac{2}{\pi} \vartheta\left[\frac{2}{\pi} \frac{\dot{y}}{\omega_{n} a_{0}} \ln \frac{\omega_{n} a_{0}}{\dot{y}}\left(1+\sqrt{1-\left(\frac{\dot{y}}{\omega_{n} a_{0}}\right)^{2}}\right)+\arcsin \frac{\dot{y}}{\omega_{n} a_{0}}\right]
$$

При вычислении (11.24) принят во внимание только первый член ряда (11.23), так как учет второго члена дает поправку, на порядок меньшую. Отметим, что второе слагаемое в (11.24) соответствует расположенным вблизи узлов колебаний участкам стержня длиной $I_{2}=I / n-2 I_{1}$, где вследствие малой скорости вибрации не выполняется условие (11.17), и величина силы трения остается неизменной с интенсивностью $\vartheta$.

Полагая в (11.24) $G=P$, согласно (8), находим соотношение, связывающее параметры быстрых и медленных движений в установившемся режиме $\dot{y}=$ const. Эта зависимость показана на рис.11.3,б. $C$ ее помощью по заданной величине $P / \vartheta 1$ определяется отношение $v_{0}=\dot{y} / \omega_{n} a_{0} \quad$ переносной $\dot{y} \quad$ и $\quad$ колебательной $\omega_{r} a_{0} \quad$ скоростей $\quad$ в установившемся движении, а затем по формуле (11.20) - амплитуду $a_{0}$ колебаний конца стержня, а следовательно и распределение амплитуд (11.13) по его длине.

Таким образом, при колебаниях распределенной системы в среде с сухим трением происходит вибрационное сглаживание нелинейной характеристики трения. Используем этот факт для построения процессов установления, принимая соотношение (11.24) в качестве приведенной характеристики трения в медленном движении. Учитывая близость кривой на рис.11.2,б к линейной, запишем уравнение (11.7) в виде

$$
M \ddot{y}+\frac{f l}{\omega_{n} a_{0}} \dot{y}=P
$$

Здесь согласно соотношению (11.20) (рис.11.2,a) величина $a_{0}$ является функцией $a_{o}=a_{o}(\dot{y})$. 
Разрешая совместно (11.20), (11.25), можно получить искомые переходные процессы $\dot{y}(t), a_{0}(t)$, происходящие в темпе медленного времени. На рис.11.4 показан переходный процесс $\dot{y}(t)$ и огибающие колебаний $a_{0}(t), \quad$ построенные при следующих параметрах системы: $f=2 \pi / \omega=20,4$ кГц $l=0,507 \mathrm{м} ; n=4 ; S=3,14 \mathrm{~cm}^{2} ; \psi=0,05 ; F_{0}=39$ кг; $P=20$ кг; $9 l=24$ кг; $a_{0}^{*}=5$ мкм.

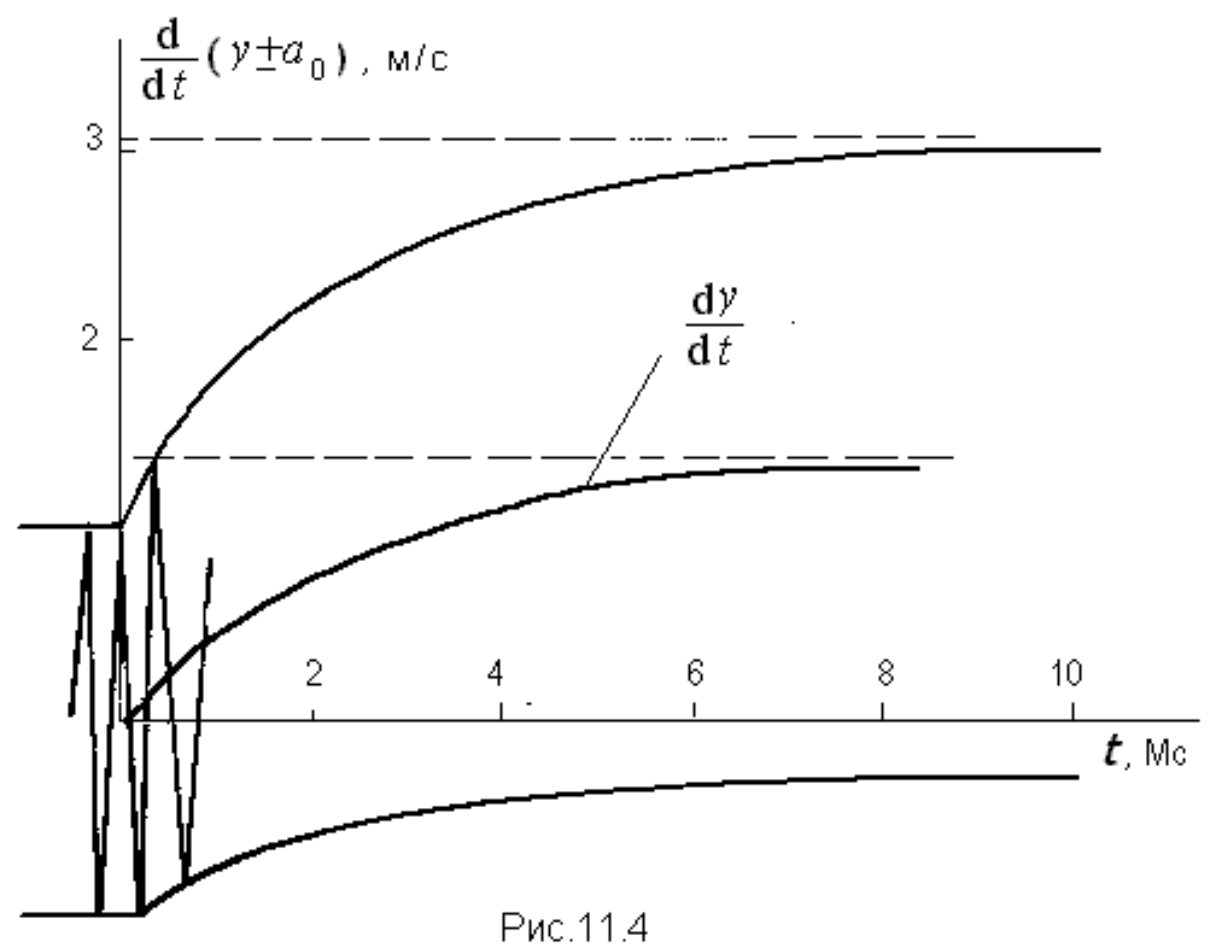

Вместе с тем предельные оценки переходного процесса можно получить, подставив в (11.25) постоянные значения амплитуды $a_{0}$, соответствующие начальной $\dot{y}=0$ и установившейся величине переносной скорости $\dot{y}$. Тогда решение (11.25) примет вид

$$
\dot{y}=\frac{P}{\vartheta l} \omega_{n} a_{a}(1-\exp b t)
$$

где $b=\frac{\vartheta I}{M \omega_{n} a_{0}}$ - коэффициент преобразованного за счет вибрации сопротивления среды движению стержня, как твердого тела.

Выражение (11.26) при указанных значениях амплитуды $a_{0}$ описывает граничные кривые, охватывающие реальный переходный процесс. 


\section{ГЛАВА 4 \\ РЕЗОНАНСНАЯ НАСТРОЙКА УЛЬТРАЗВУКОВЫХ ТЕХНОЛОГИЧЕСКИХ МАШИН}

\section{2. Способы повышения эффективности ультразвуковых станков}

\section{под нагрузкой}

1. Схема процесса ультразвуковой обработки подробно рассмотрена в П.1.1. Там же описаны экспериментально полученные основные закономерности процесса. Экспериментальными исследованиями [171] установлено, что ультразвуковая обработка происходит в результате ударов инструмента по частицам абразива, оседающим из суспензии на поверхности изделия. В $[111,141,171,233]$ собран обширный экспериментальный материал, позволяющий оценить влияние статической силы подачи и основных параметров колебаний инструмента на скорость резания. Выяснено, что для получения высокой производительности необходимо обеспечить большую амплитуду колебаний инструмента при значительных усилиях подачи.

Для возбуждения колебаний с максимальной амплитудой стремятся осуществить резонансную настройку колебательной системы. Однако такую настройку производят обычно на холостом ходу, считая, что взаимодействие инструмента с изделием не оказывает ощутимого влияния на работу системы. Вместе с тем исследование характеристик ультразвукового станка под нагрузкой [19,27,30,31] показывает, что повышение усилия подачи при работе станка на резонансной частоте холостого хода приводит к уменьшению амплитуды колебаний инструмента из-за существенных нелинейных искажений, обусловленных взаимодействием инструмента с изделием в процессе резания. Проявляющиеся при этом нелинейные эффекты выявлены в Параграфе 9. 
В данном разделе работы с помощью реологической модели процесса ультразвукового резания производительность станка связывается с его динамическими характеристиками и основными конструктивными параметрами. Рассматриваются предельные возможности различных способов настройки колебательной системы. Приводятся результаты экспериментов.

Полученные результаты сопоставляются с известными экспериментальными данными. Основные закономерности процесса резания получим из рассмотрения условно показанной на рис.12.1 колебательной системы, поджатой к обрабатываемому изделию статической силой $P$.

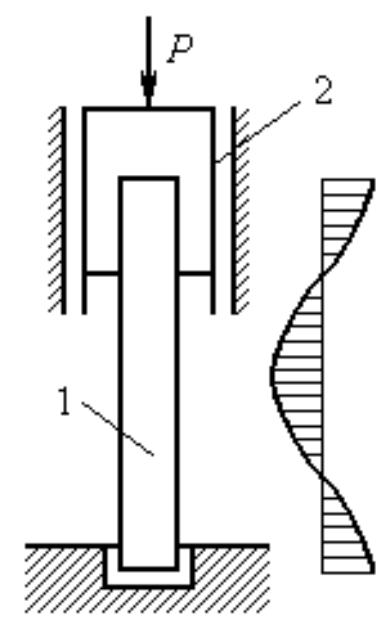

Pnc.12.1

Будем рассматривать движение инструмента вида

$$
u(t)=v t+u^{0}(t)=v t+a \exp j(\omega t-\varphi)
$$

где $v$ - малая постоянная скорость перемещения системы вследствие выработки материала изделия; $a, \omega, \varphi$ - амплитуда, частота и фаза периодической составляющей $u^{0}(t)$ движения инструмента.

Силовое взаимодействие инструмента с изделием опишем характеристикой жесткопластичекого материала (рис.12.2)

$$
f(u)=1 / 2 D \eta(u-\Delta)(1+\operatorname{sgn} \dot{u})
$$

и проведем ее гармоническую линеаризацию

$$
f(u) \approx f_{0}(a)+[k(a)+j \omega b(a)] \cdot u^{0} .
$$




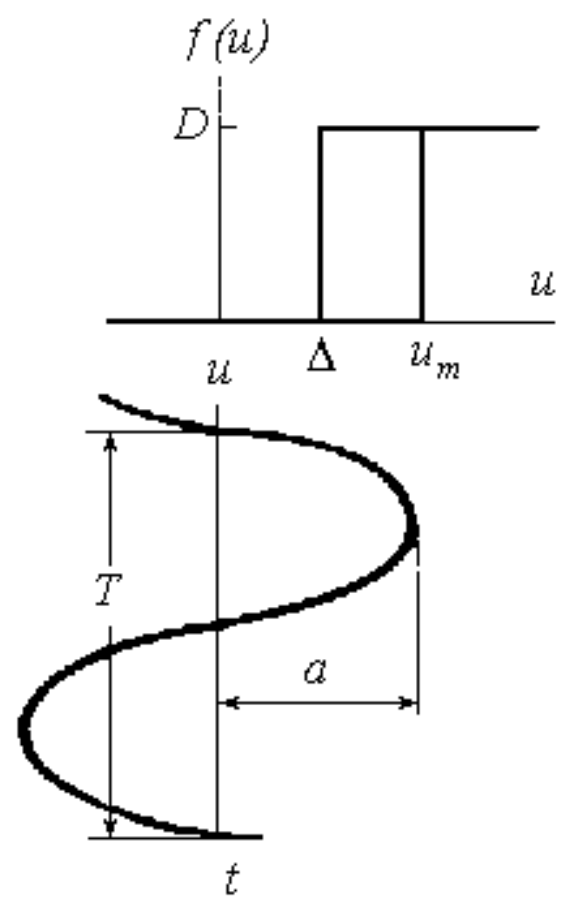

Pис. 12.2

В выражениях (12.1), (12.2) величина $D$ зависит от материала изделия, площади инструмента, вида абразивной суспензии и состояния абразива в зоне резания; $\Delta-$ значение координаты $u(t)$ в момент начала взаимодействия; $\eta(u)-$ функция единичного скачка; $f_{0}(a), \quad k(a), \quad b(a)$ - коэффициенты гармонической линеаризации. Эти коэффициенты вычислены в Параграфе 8.

Здесь мы для упрощения вывода применим следующий прием. Поскольку в рассматриваемом процессе постоянная скорость $v$ движения инструмента много меньше его колебательной скорости $a \omega$ т.е. $v<<a \omega$ при вычислении коэффициентов гармонической линеаризации медленным движением в течение одного цикла колебаний можно пренебречь, и, полагая $v=0$, воспользоваться формулами (8.23) (8.25). В результате получим

$$
\begin{gathered}
f_{0}(a)=\frac{D}{2 \pi} \arccos \frac{\Delta}{a}, \\
k(a)=\frac{D}{\pi a} \sqrt{1-\left(\frac{\Delta}{a}\right)^{2}}, \quad b(a)=\frac{D}{\pi a \omega}\left(1-\frac{\Delta}{a}\right) .
\end{gathered}
$$

Учитывая связь $f_{0}(a)=P$, из соотношения (12.3) находим

$$
\Delta / a=\cos (2 \pi P / D)
$$

и после подстановки (5) в (4) получаем

$$
k(a)=\frac{D}{\pi a} \sin \frac{2 \pi P}{D}, \quad b(a)=\frac{2 D}{\pi a \omega} \sin ^{2} \frac{\pi P}{D}
$$

Коэффициенты (12.6) определяют упругую и диссипативную составляющие нагрузки на колебательную систему в процессе резания. Их зависимость от амплитуды $a$ и статической силы прижима $P$ соответствует полученной экспериментально в [88]. 
Разность $a-\Delta$ представляет выработку обрабатываемого материала за один цикл (рис.12.1,a). Поэтому скорость резания $V=(a-\Delta) \omega / 2 \pi$ и с помощью (5) находим

$$
V=\frac{a \omega}{\pi} \sin ^{2} \frac{\pi P}{D}
$$

Заметим, что найденные соотношения (12.6), (12.7) совпадают с формулами (8.69), (8.70), полученными в П.8.6 из коэффициентов гармонической линеаризации полной динамической характеристики в предположении малости скорости обработки $v<<a \omega$.

Амплитуда $a$ колебаний инструмента в рабочем режиме существенно зависит от величины нагрузки. Пусть на холостом ходу $(P=0) \quad$ инструмент совершает колебания $u_{0}(t)=a_{0} \exp j \omega t$. Считая колебательную систему станка линейной, колебания инструмента под нагрузкой опишем следующим уравнением:

$$
u(t)=u_{0}(t)-W^{-1}(j \omega)[k(a)+j \omega b(a)] u(t),
$$

где $W(j \omega)$ - динамическая жесткость колебательной системы, связывающая перемещение инструмента с действующими на него силами. Обозначим

$$
W(j \omega)=U(\omega)+j V(\omega) .
$$

В режиме холостого хода амплитуда $a$ колебаний инструмента достигает своего максимального значения $a_{0}=a_{0}^{*}$ на резонансной частоте $\omega=\omega_{0}$, при которой $U\left(\omega_{0}\right)=0$. Величина $a_{0}^{*} V_{0}$, где $V_{0}=V\left(\omega_{0}\right)$, характеризует уровень возбуждения системы. Амплитуды холостого хода на частотах в окрестности резонанса

$$
a_{0}=a_{0}^{*} V_{0} /|W(j \omega)| .
$$

Из уравнения (8) с учетом (9), (10) для амплитуды колебаний под нагрузкой имеем

$$
a=\frac{a_{0}^{*} V_{0}}{\sqrt{[U(\omega)+k(a)]^{2}+[V(\omega)+\omega \cdot b(a)]^{2}}} .
$$


Рассмотрим некоторые возможные способы настройки ультразвукового станка под нагрузкой.

2. Настройка большинства современных ультразвуковых станков производится на резонансной частоте холостого хода $\omega=\omega_{0}$. Такая настройка позволяет вести обработку лишь при достаточно малых усилиях прижима $P<<D$. При этом, согласно (12.6), в первом приближении можно принять $k(a)=2 P / a$, а диссипативной составляющей нагрузки пренебречь вследствие ее малости по сравнению с потерями в колебательной системе $\left(\omega_{0} \beta<V_{0}\right)$. При таких допущениях из (12.11) находим амплитуду колебаний инструмента под нагрузкой

$$
a=a_{0}^{*} \sqrt{1-\left(\frac{2 P}{a_{0}^{*} V_{0}}\right)^{2}} .
$$

Ограничивая разложение (12.7) в ряд по малому параметру $P / D$ первым значащим членом, с учетом (12.12) получим выражение, определяющее скорость резания при работе на резонансной частоте холостого хода $\omega=\omega_{0}$

$$
V=\pi a_{0}^{*} \omega_{0}\left(\frac{P}{D}\right)^{2} \sqrt{1-\left(\frac{2 P}{a_{0}^{*} V_{0}}\right)^{2}}
$$

Из (12.13) следует известный экспериментальный факт существования оптимального усилия подачи $P=P_{0}$, при котором достигается максимум скорости резания [141,173,233]. Найдем его из условия $d v / d P=0$. В результате имеем

$$
P_{0}=0,4 a_{0}^{*} V_{0}
$$

Соответствующее подаче (14) максимальное значение скорости резания

$$
V_{0}=0,3 a_{0}^{* 3} \omega_{0}\left(V_{0} / D\right)^{2}
$$

Отметим, что пропорциональность оптимального усилия $P_{0}$ резонансной амплитуде $a_{0}^{*}$ холостого хода хорошо согласуется с экспериментальными 
данными, приведенными в $[141,173]$. В работе [111] можно найти экспериментальное подтверждение независимости силы $P_{0}$ от величины $D$, пропорциональной площади инструмента. В $[111,143,195]$ представлена эмпирическая формула, которая с учетом (12.14) приводит к подобной (12.15) зависимости скорости резания от амплитуды $a_{0}^{*}$ и частоты $\omega_{0}$.

На рис.12.3,а. по формуле (12.13) построены зависимости скорости резания от усилия прижима при различных значениях амплитуды $a_{0}^{*}$. (1-8; 2-11; 3- 16,5; 4- 20,5 мкм).
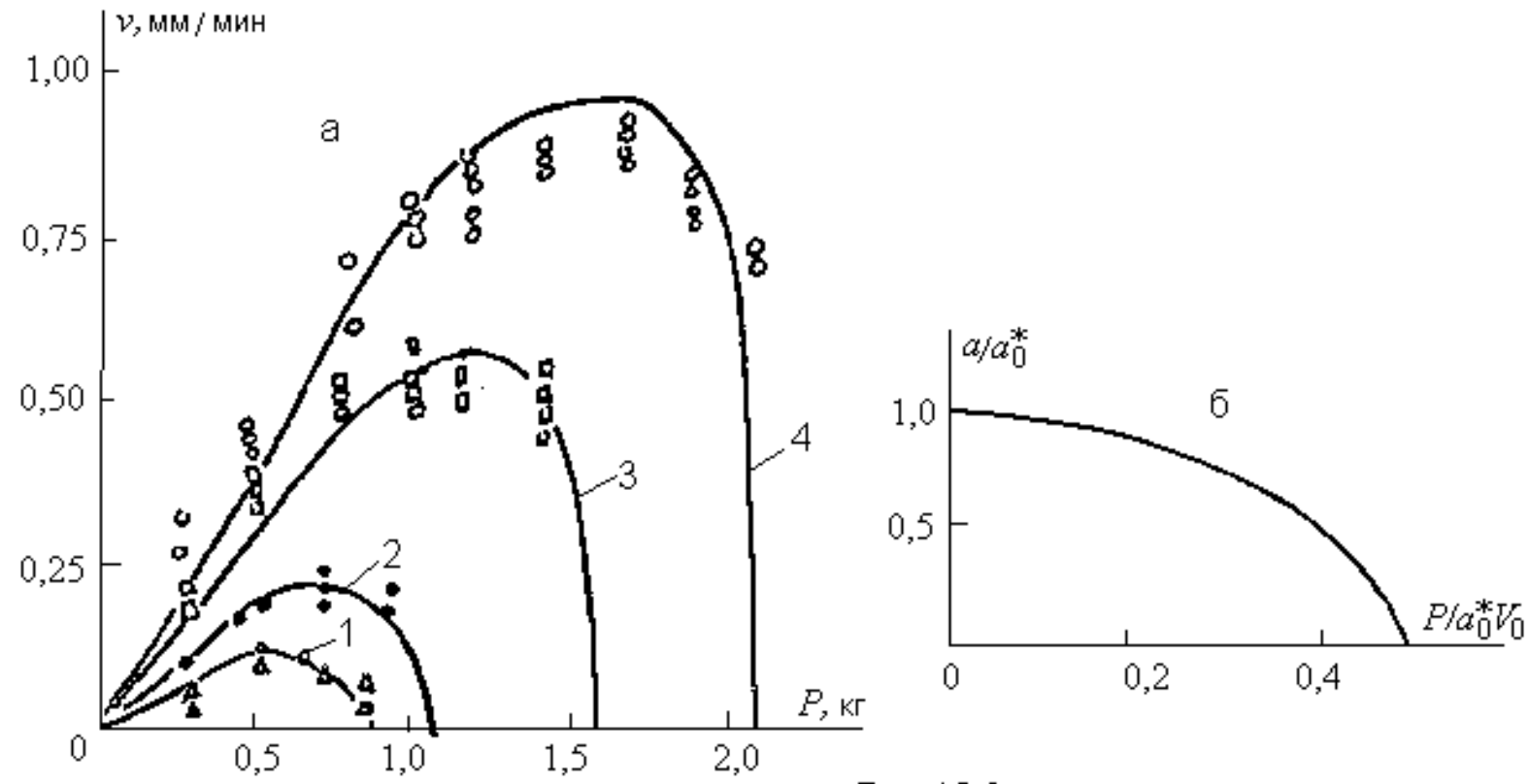

Pис. 12.3

Исходные данные для расчета и показанные точками результаты экспериментов заимствованы из работы [233]. При этом величины $V_{0}=1800$ Н/мм и $D=3700$ Н вычислены с помощью (12.14), (12.15) по одной экспериментальной точке, соответствующей оптимальной силе прижима. График на рис.12.3,б, построенный по формуле (12.12), иллюстрирует уменьшение амплитуды колебаний при возрастании силы прижима. Заметим, что при оптимальном значении $P_{0}$ силы прижима амплитуда

$$
a_{0} \approx 0,6 a_{0}^{*}
$$

Обратимся к формуле (12.15), определяющей максимальную производительность станка. При проектировании колебательной системы 
частоту возбуждения выбирают в области низких ультразвуковых частот $\left(f=\omega / 2 \pi=18-22\right.$ кГц). Предельное значение амплитуды $a_{0}^{*}$ ограничено условиями прочности волноводов. Поэтому величины $\omega_{0}$ и $a_{0}^{*}$ можно считать исходными. Параметр $V_{0}$ определяется диссипативными свойствами колебательной системы; рассеиваемая ею на холостом ходу мощность $N_{\mathrm{x}}=1 / 2 a_{0}^{* 2} \omega_{0} V_{0}$. Следовательно, повышение скорости резания при указанном способе настройки осуществляется главным образом за счет повышения характеризуемых величиной $V_{0}$ диссипативных потерь в колебательной системе и, следовательно, увеличения мощности. Это подтверждается данными по промышленным образцам ультразвуковых станков.

В табл. 12.1 приведены паспортные данные двух промышленных образцов ультразвуковых станков модели 4770 и 4772.

Таблица 12.1

\begin{tabular}{|c|c|c|c|c|}
\hline \multicolumn{2}{|c|}{ Модель станка } & 4770 & 4772 & AP \\
\hline \multicolumn{2}{|c|}{ Мощность генератора, кВт } & 0,25 & 1,5 & 0,15 \\
\hline \multicolumn{2}{|c|}{$\begin{array}{l}\text { Наибольшее усилие } P \\
\text { подачи, Н }\end{array}$} & 45 & 150 & 600 \\
\hline $\begin{array}{l}\text { Площадь } S \\
\text { обработки, } \\
\text { мм }^{2}\end{array}$ & $\begin{array}{c}\text { Усилие P } \\
\text { подачи, Н }\end{array}$ & \multicolumn{3}{|c|}{$\begin{array}{c}\text { Производительность, } \\
\text { мм³ } / \text { мин }^{2}\end{array}$} \\
\hline \multirow{3}{*}{80} & 30 & 280 & 430 & 480 \\
\hline & 100 & - & 2800 & 3900 \\
\hline & 200 & - & - & 9000 \\
\hline \multirow{5}{*}{800} & 30 & 28 & 48 & 48 \\
\hline & 100 & - & 280 & 470 \\
\hline & 150 & - & 720 & 1200 \\
\hline & 300 & - & - & 3800 \\
\hline & 600 & - & - & 9000 \\
\hline
\end{tabular}

Здесь показана производительность станков при различной площади $S$ обработки и различных усилиях $P$ подачи. 
Оценим теперь КПД ультразвукового станка при данном способе настройки колебательной системы. КПД определяется следующей формулой: $\quad \eta=N_{\mathrm{p}} /\left(N_{\mathrm{p}}+N_{\mathrm{k}}\right), \quad$ где $N_{\mathrm{p}}=D V \quad-\quad$ полезная мощность, затрачиваемая на процесс резания, $N_{\mathrm{k}}=1 / 2 a^{2} \omega_{0} V_{0}$. - мощность рассеиваемая в колебательной системе. При оптимальном для данной настройки усилии подачи $P_{0}$ с учетом формул $(12.15),(12.16)$ находим $\eta=\frac{1}{1+\left(0,6 D / a_{0}^{*} V_{0}\right)}$. При данных рассмотренного выше эксперимента (см. рис.12.3,a), например для амплитуды $a_{0}^{*}=20,5$ мкм, имеем $\eta \approx N_{\mathrm{p}} / N_{x}=0,017=1,7 \%$. Таким образом, при используемом обычно способе настройки мощность в основном рассеивается в колебательной системе за счет внутреннего трения в ее материале.

Зависимость величины $V_{0}$ от конструкции, размеров и материала колебательной системы оценим на примере модели (рис.12.1), в которой колебательная система представляет собой однородный упругий стержень 1 , возбуждаемый по одной из своих собственных форм и закрепленный в корпусе 2 в одном из узлов смещения. (На рис.12.1 приведена эпюра смещений сечений стержня.) Длина стержня с резонансной частотой $\omega_{0}$ определяется соотношением $l=\pi n c / 2 \omega_{0}, \quad$ где $c=\sqrt{E / \rho}-$ скорость распространения продольной волны; $E, \rho-$ модуль упругости и плотность материала стержня; $n$ - число четвертьволн, укладывающихся по длине стержня; нечетным $n$ отвечают конструкции, в которых стержень связан с корпусом своим верхним концом.

Динамическая жесткость (12.9) такой системы отыскивается способом, изложенным в Параграфе 5 , и может быть записана в виде

$$
W(j \omega)=w \omega\left[\operatorname{tg} \frac{\pi n}{2}\left(1-\frac{\omega}{\omega_{0}}\right)+j \frac{\psi}{4 \pi} \frac{\left(\pi n \omega / \omega_{0}\right)-(-1)^{n} \sin \left(\pi n \omega / \omega_{0}\right)}{1+(-1)^{n} \cos \left(\pi n \omega / \omega_{0}\right)}\right],
$$

где $w=S \sqrt{E \rho}$ —волновое сопротивление; $S$ —площадь сечения стержня; $\psi$ - коэффициент поглощения.

Из (12.17) при $\omega=\omega_{0}$ находим 


$$
V_{0}=\left|W\left(j \omega_{0}\right)\right|=1 / 8 \psi w \omega_{0} n
$$

После подстановки (12.18) в формулу (12.15) получим максимально достижимую скорость резания при настройке станка на холостом ходу

$$
V_{0}=3,75 \cdot 10^{-2}\left(a_{0}^{*} \omega_{0}\right)^{3}(\psi n w / D)^{2} .
$$

Из последнего соотношения следует, что повышение производительности обработки может быть достигнуто за счет увеличения коэффициента поглощения, т.е. снижения добротности колебательной системы, увеличения числа четвертьволн $n$, т.е. длины колебательной системы и работы на высших формах собственных колебаний, и, наконец, увеличения волнового сопротивления $w$ или площади поперечного сечения стержневой системы, модуля упругости и плотности ее материала. Все сказанное подтверждается опытом проектирования ультразвуковых станков.

3. Возвращаясь к выражениям (12.7) и (12.12), отметим, что уменьшение скорости резания при усилиях подачи, превышающих значение (12.14), вызывается резким снижением амплитуды колебаний инструмента (рис.12.2,б). Избежать этого можно, осуществляя резонансную настройку колебательной системы под нагрузкой. Согласно (12.11), максимальная амплитуда колебаний инструмента под нагрузкой достигается при выполнении следующих соотношений: $U(\omega)+k(a)=0$, $a=a_{0}^{*} V_{0} /[V(\omega)+\omega \beta(a)]$.

Подставив сюда коэффициенты (12.6), получим систему уравнений

$$
\begin{aligned}
& a=-\frac{D}{\pi \cdot U(\omega)} \sin \frac{2 \pi P}{D} \\
& a=a_{0}^{*} \frac{V_{0}}{V(\omega)}\left(1-\frac{2 D}{\pi a_{0}^{*} V_{0}} \sin ^{2} \frac{\pi P}{D}\right)
\end{aligned}
$$

решение которой определяет резонансную частоту $\omega^{*}$ и соответствующую амплитуду $a^{*}$ при действии статической силы прижима $P$. Первое из этих 
уравнений определяет скелетную кривую амплитудно-частотной характеристики, второе - линию предельных амплитуд.

Возможности такого способа настройки оценим на примере рассмотренной выше системы (рис.12.1,б). С учетом (12.9), (12.17), уравнения (12.19) принимают вид

$$
\begin{aligned}
& a=-\frac{D}{\pi \cdot w \omega} \sin \frac{2 \pi P}{D} \operatorname{ctg} \frac{\pi n}{2}\left(1-\frac{\omega}{\omega_{0}}\right), \\
& a=\frac{a_{0}^{*}}{2} \frac{\pi n \omega_{0}}{\omega}\left(1-\frac{2 D}{\pi \cdot a_{0}^{*} V_{0}} \sin ^{2} \frac{\pi P}{D}\right) \frac{1+(-1)^{n} \cos \left(\pi n \omega / \omega_{0}\right)}{\left(\pi n \omega / \omega_{0}\right)-(-1)^{n} \sin \left(\pi n \omega / \omega_{0}\right)} .
\end{aligned}
$$

На рис.12.4 показано графическое решение системы уравнений (12.20) при различных значениях $P$.

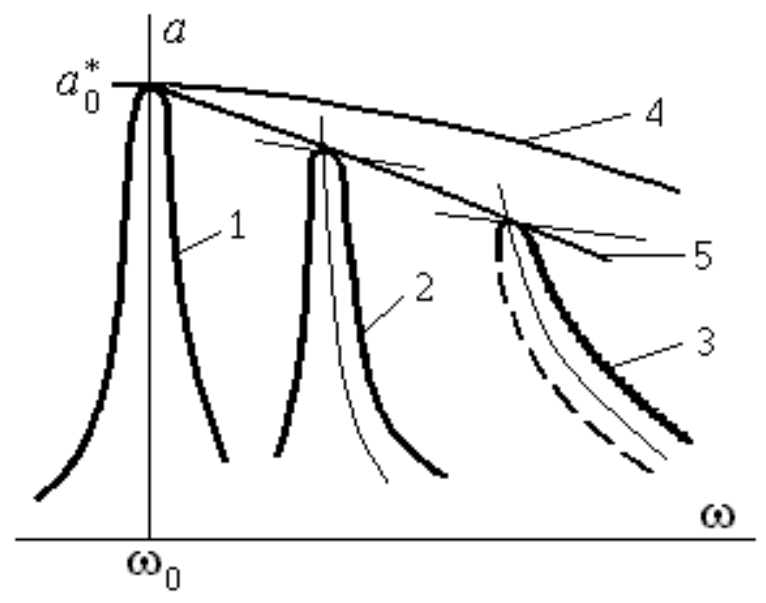

Pис. 12.4

Кривые, отвечающие уравнениям (12.20) показаны тонкими линиями. Здесь же приведены участки резонансных кривых системы. Они аналогичны подробно описанным в Параграфе 9. При относительно малых силах прижима $P \leq a_{0}^{*} V_{0} / 2$ вид резонансных кривых под нагрузкой (кривая 2) и на холостом ходу (кривая 1) одинаков, однако их максимум смещается в область более высоких частот по мере возрастания $P$. Дальнейшее увеличение силы прижима $\left(P>a_{0}^{*} V_{0} / 2\right)$ существенно изменяет характер резонансной кривой (кривая 3), приводя к появлению неустойчивой ветви, показанной штриховой линией.

Кривая 4 является линией предельных амплитуд системы с абсолютно жестким ограничителем (см. П.9.8); ее уравнение получается из 
второго уравнения (12.20) предельным переходом $D \rightarrow \infty$. Линия 5, образованная решениями системы уравнений (12.20), является огибающей резонансных кривых и определяет полную совокупность резонансных режимов при всевозможных значениях усилия подачи $P$.

На рис.12.5 приведены зависимости резонансных частот $\omega^{*}$ и амплитуд $a^{*}$, а также скорости резания $v$, достигаемой при резонансной настройке под нагрузкой, от усилия подачи $P$ и амплитуды $a_{0}^{*}$. Здесь
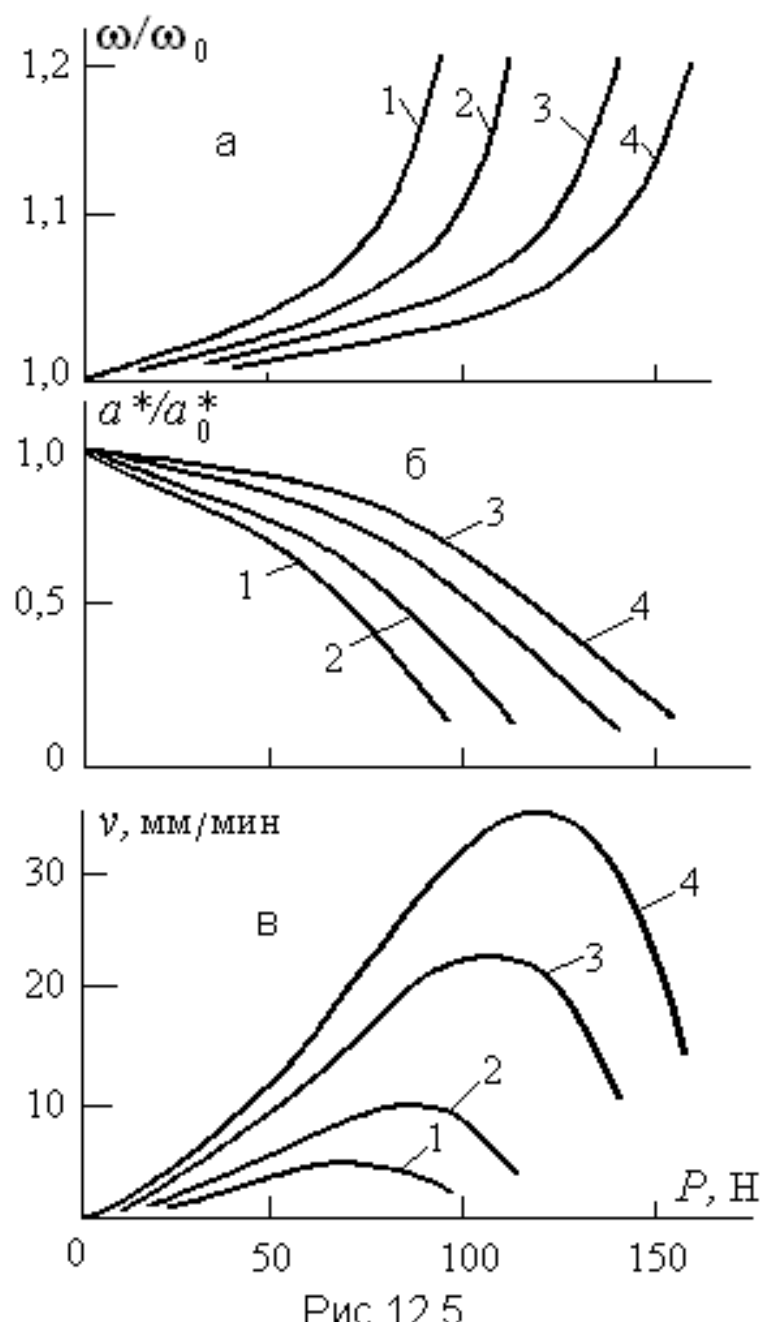
приняты те же обозначения кривых, что и на рис.12.3,а. Зависимости на рис.12.5,а,б построены в результате решения уравнений (12.20) при тех же значениях $V_{0}=1800 \mathrm{H} / \mathrm{Mм}$ и $D=3700 \quad$ Н, что и рис.12.3,a. Коэффициент поглощения принят равным $\psi=0,05$. Графики на рис.6,в построены с помощью формулы (12.7).Из рис.12.5,в видно, что при некотором значении усилия подачи скорость резания достигает максимума. В этом режиме амплитуда колебаний $a=a_{0}^{*} / 2$. Дальнейшее увеличение усилия подачи приводит к резкому

уменьшению амплитуды колебаний и уменьшению скорости резания.

Сопоставление рис.12.3, а и рис.12.5,в свидетельствует о высокой эффективности резонансной настройки станка под нагрузкой. Расчеты показывают, что КПД станка при такой настройке настройке достигает 30\%. Однако ее практическая реализация в режиме вынужденных колебаний связана с серьезными трудностями, обусловленными 
специфическими искажениями резонансных кривых (рис.12.3) вследствие нелинейности нагрузки.

Забегая вперед, заметим, что система автоматической настройки, которая будет подробно рассмотрена в Параграфе 14, позволяет избежать этих трудностей. В автоматическом режиме резонансные колебания являются единственным устойчивым состоянием системы.

В последнем столбце табл. 12.1 приведены данные разработанного в Лаборатории вибротехнических систем ИМАШ экспериментального образца ультразвукового станка, реализующего резонансную настройку колебательной системы под нагрузкой. Из сравнения данных этого устройства с серийно выпускаемыми станками видно, что при несколько меньшей мощности по сравнению со станком модели 4770 он допускает более чем 30-ти кратное увеличение производительности и при 10-ти кратном снижении мощности по сравнению со станком 4772 не только перекрывает возможности последнего, но и позволяет более чем в 10 раз увеличить производительность.

4. Рассмотрим, наконец. возможности еще одного способа настройки под нагрузкой, предложенного в [8]. Он состоит в том, что к инструменту присоединяют дополнительную массу $M$, добавление которой приводит к компенсации расстройки, вызванной взаимодействием инструмента с изделием. При такой компенсации резонансная настройка под нагрузкой достигается на частоте $\omega=\omega_{0}$. Величина компенсирующей добавочной массы определяется соотношением $M=k(a) / \omega_{0}^{2}$

Амплитуду колебаний под нагрузкой найдем из второго уравнения (12.19)

$$
a^{*}=a_{0}^{*}\left(1-\frac{2 D}{\pi a_{0}^{*} V_{0}} \sin ^{2} \frac{\pi P}{D} .\right)
$$

Подставив (12.21) в (12.7), получим скорость резания 


$$
v=\frac{a_{0}^{*} \omega}{\pi} \sin ^{2} \frac{\pi P}{D} \cdot\left(1-\frac{2 D}{\pi a_{0}^{*}} \sin ^{2} \frac{\pi P}{D}\right)
$$

Из условия $d v / d P=0$ найдем усилие прижима $P^{*}$, при котором скорость резания максимальна

$$
P^{*}=\frac{D}{\pi} \arcsin \sqrt{\frac{\pi a_{0}^{*} V_{0}}{4 D}} .
$$

В реальных условиях, как правило, $a_{0}^{*} V_{0}<<D$, и формула (22) принимает вид $P^{*}=\sqrt{a_{0}^{*} V_{0} D / 4 \pi}$.

Согласно (12.22), (12.23), максимальная скорость резания

$$
V^{*}=1 / 8 a_{0}^{* 2} \omega_{0} V_{0} / D
$$

а амплитуда (12.21) колебаний инструмента .в этом режиме

$$
a^{*}=a_{0}^{*} / 2
$$

Для рассмотренной выше системы (рис.3,a) расчет по формуле (12.24) при $a_{0}^{*}=20,5$ мкм дает $v^{*}=49$ мм/мин. Такая скорость резания достигается при усилии прижима $P^{*}=10^{4} \mathrm{H}$.

Интересно отметить, что в режиме с максимальной производительностью мощность $N_{p}$, затрачиваемая на разрушение материала, равна мощности $N_{k}$, рассеиваемой в колебательной системе, T.e.

$$
N_{p}=N_{k}=1 / 8 a_{0}^{* 2} \omega_{0} V_{0}
$$

Это легко показать, если, используя (12.6), (12.7), найти $N_{p}=1 / 2(a \omega)^{2} \beta(a)=D V$ и учесть, что $N_{k}=1 / 2 a^{* 2} \omega_{0} V_{0}$, после чего с учетом (12.24), (12.25) и находится равенство (12.26). Таким образом, рассматриваемая настройка обеспечивает наилучшее согласование колебательной системы как с упругой, так и с диссипативной составляющей нагрузки и позволяет реализовать предельные возможности ультразвукового станка. При этом его КПД составляет $\eta=50 \%$. 
Соотношениями (12.24), (12.26) удобно пользоваться при расчете колебательной системы станка с заданной производительностью.

При увеличении усилия подачи выше значения (12.23) КПД возрастает, однако скорость резания падает. Следовательно КПД не может являться показателем эффективности настройки. Эффективность той или иной настройки удобно оценивать коэффициентом эффективности $\phi=v / v^{*}$ [33], представляющим собой отношение получаемой скорости $v$ обработки к ее предельному значению $v^{*}$, определяемому равенством (12.24). Так, например, согласно формулам (12.15) и (12.24) коэффициент эффективности настройки на холостом ходу $\phi=V_{0} / V^{*}=2,4 a_{0}^{*} V_{0} / D$

Величина $a_{0}^{*} V_{0}$ характеризует уровень возбуждения системы и имеет порядок малых диссипативных сил. Для ультразвуковых колебательных систем мощностью 0,1-10 кВТ эта величина находится в пределах $a_{0}^{*} V_{0}=10 \div 10^{4} \mathrm{H}$. Значения величины $D$ лежат в пределах $D=10^{3} \div 10^{6} \mathrm{H}$.

Отсюда следует, что настройка на холостом ходу крайне не эффективна: для рассмотренного выше случая такой настройки $\varphi=0,024$. Это означает, что в данном случае используется всего лишь $\approx 2,5 \%$ потенциальных возможностей станка. В то же время резонансная настройка под нагрузкой позволяет получить коэффициент эффективности $\varphi \approx 0,7$, a введение компенсации позволяет довести коэффициент эффективности до значений близких к 1 (!).

Из сопоставления зависимостей (12.24) и (12.15) следует, что настройка под нагрузкой может оказаться особенно эффективной при обработке жестких изделий на стенках малой мощности, имеющих колебательные системы с высокой добротностью. (Под жесткими понимаются изделия с большой площадью обработки или выполненные из материалов, плохо поддающихся резанию.) Аналогичный вывод сделан в работе [171] на основании проведенных экспериментов. 
Заметим, что полученные здесь результаты могут быть использованы при оценке эффективности и настройке разнообразных технологических ультразвуковых установок [195] для поверхностного упрочения деталей, сварки пластмасс и синтетических тканей, возбуждения высокочастотных колебаний металлорежущего инструмента в устройствах для вибрационного резания (см. Параграф 15) и др.

5. Приведем результаты экспериментального исследования динамики колебательной системы ультразвукового станка под нагрузкой. Цель эксперимента состояла в определении основных динамических характеристик колебательной системы ультразвукового станка, проверка принятых при теоретическом анализе допущений, и оценка влияния нелинейности, вносимой в результате взаимодействия инструмента с изделием, на ее работу.

Эксперимент проводился на ультразвуковом станке, схема которого показана на рис.12.6. Основным узлом станка является акустическая головка, состоящая из корпуса 2, магнитострикционного преобразователя 2 и припаянного к торцу его сердечника ступенчатого концентратора 3 с
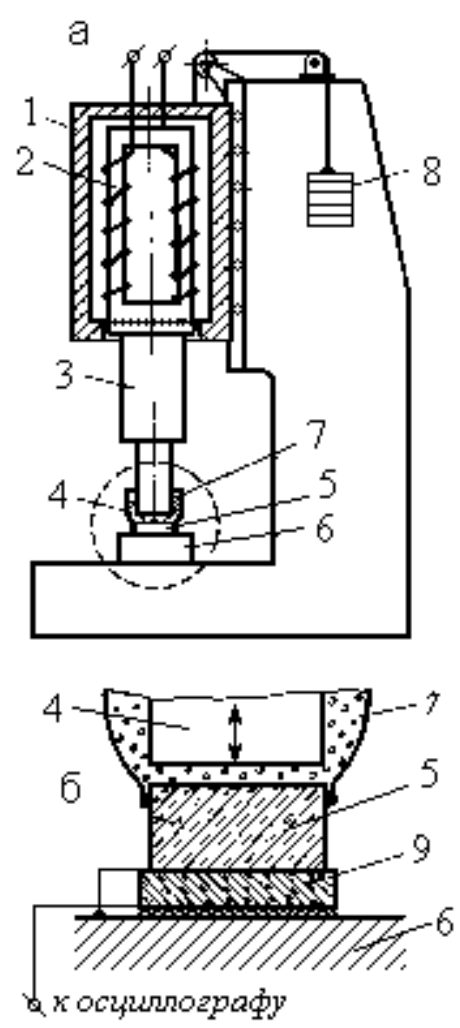

Рис 12.6

инструментом 4. Обмотка возбуждения магнитострикционного преобразователя получает питание от генератора мощностью до 1 кВт. Используемый генератор допускал регулирование частоты возбуждения и амплитуды колебаний сердечника преобразователя в широких пределах. Регулировка последней осуществлялась путем изменения тока, проходящего через обмотку возбуждения преобразователя.

Колебания от преобразователя посредством волновода-концентратора передаются с увеличением амплитуды инструменту. Под торцом 
инструмента установлено обрабатываемое изделие - стеклянная пластина 5 диаметром 12 мм и толщиной 5 мм, приклеенная к жесткому массивному основанию 6. Пластина 5 и инструмент 4 помещены в резервуар 7 с абразивной суспензией.

Непрерывный процесс резания обеспечивается тем, что инструмент поджимается к изделию статическим усилием подачи. Это усилие создается за счет разности веса головки и связанных с ней грузов 8. Для обеспечения плавности подачи головка установлена в шариковых направляющих станины.

В процессе резания регистрировалась сила взаимодействия между инструментом и обрабатываемым изделием. Схема датчика силы показана на рис.12.6,б. Пьезоэлемент 9 датчика вклеен между основанием 6 и обрабатываемой стеклянной пластиной 5. Нижняя обкладка пьезоэлемента изолирована от основания тонкой капроновой сеткой, выполняющей одновременно роль демпфера. Верхняя обкладка датчика заземлена, а с нижней снимается полезный сигнал, который подается на вход катодного осциллографа, с экрана которого и фотографировались осциллограммы. Такая конструкция обеспечивает хорошее демпфирование собственных колебаний датчика и его надежную защиту от электромагнитных помех и емкостных наводок от колеблющегося инструмента.

Для определения собственных свойств датчика были сняты осциллограммы ударов шариков, бросаемых на пластину датчика с различных высот. Одна из них приведена на рис.12.7. Верхняя кривая на этой осциллограмме представляет собой сигнал, возникающий при ударе и последующих собственных колебаниях датчика. Осциллограмма снималась на ждущей развертке с запуском от снимаемого с датчика сигнала. Поэтому плавная кривая исходит не из нуля, а от некоторого значения $u$, которое является уровнем запуска развертки. Нижняя кривая на осциллограмме сигнал с генератора с частотой 200 кГц, дающий масштаб времени. 


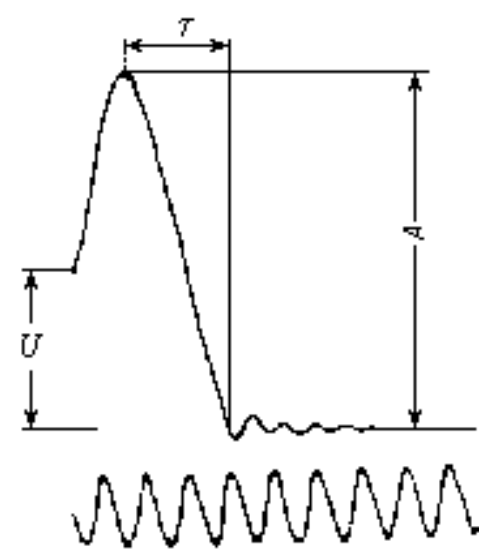

Pис. 12.7

Испытания показали, что датчик [52] обладает высокой собственной частотой, превышающей 300 кГц, и хорошими демпфирующими свойствами. Декремент собственных колебаний датчика равен 2. Обработка большого числа осциллограмм показала, что датчик обладает достаточной линейностью. Так, например, оказалось, что время удара не зависит от высоты $h$, падения шарика, а амплитуда снимаемого при ударе сигнала пропорциональна скорости шарика в момент начала удара. Отсюда следует, что датчик ведет себя как жесткая пружина с линейной упругой характеристикой. Это обстоятельство позволяет легко провести тарировку датчика.

Для тарировки воспользуемся теоремой импульсов. Для ударяющего тела, брошенного с высоты $H$ без начальной скорости

$$
m \sqrt{2 g H}(1+R)=\int_{0}^{2 \tau} F(t) d t
$$

где $m$ - масса шарика; $R=\sqrt{h / H}-$ коэффициент восстановления скорости при ударе; $h$ - высота отскока шарика; $F(t)$ - закон изменения силы удара; $2 \tau^{\sim}$ - время удара. Заметим, что в экспериментах величина коэффициента восстановления $R \approx 1$.

Считая , что датчик имеет линейную характеристику, примем $F(t)=k_{F} A \sin \frac{\pi}{2 \tau} t$ где А - амплитуда сигнала, снимаемого с датчика во время удара; kF- - масштабный коэффициент силы, и из (12.27) получим 
$A \tau=\frac{\pi m \sqrt{2 g H}}{4 k_{F}}(1+R)$

. Отсюда следует, что произведение $A \tau$ является линейной функцией величины $m \sqrt{H}$.

На рис.12.8 график этой функции построен в результате обработки большого числа осциллограмм ударов по датчику шариков различной массы, бросаемых с различной высоты.

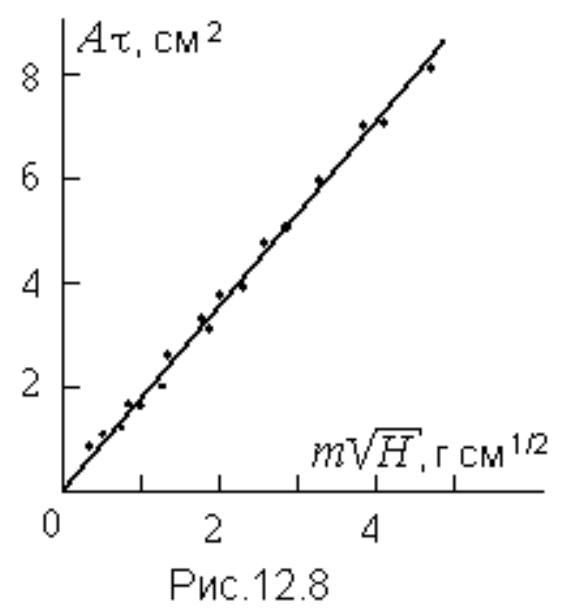

Как видно, все экспериментальные точки хорошо укладываются на прямой линии. Это означает, что датчик действительно обладает хорошей линейностью, и масштабный коэффициент является постоянной величиной, определяемой по формуле $k_{F}=\frac{\pi m \sqrt{2 g H}}{4 A \tau}(1+R)$.

6. В экспериментах исследовались динамические характеристики колебательной системы как под нагрузкой, так и на холостом ходу.

В режимах холостого хода снималась амплитудно-частотная характеристика колебательной системы. С этой целью к торцу инструмента приклеивался пьезоэлемент, выполняющий роль акселерометра. Внутренняя обкладка пьезоэлемента, с которой снимается полезный сигнал, изолирована от инструмента тонкой капроновой сеткой, а внешняя заземлена. Этим обеспечивается экранировка датчика от внешних электромагнитных полей магнитострикционного преобразователя. Датчик обладает хорошей линейностью. Его тарировка заключается в том, что под микроскопом измеряется амплитуда $a$ 
колебаний инструмента в одном из режимов с частотой $\omega$, которая сопоставляется с амплитудой $A$ снимаемого с датчика сигнала. Масштабный коэффициент вычисляется по формуле $k_{a}=a / A$.

Амплитуда $a^{*}$ резонансного режима холостого хода зависит от величины тока, проходящего через обмотку возбуждения магнитостриктора. В дальнейшем величиной $a^{*}$ будем характеризовать уровень возбуждения системы, т.е. независимо от исследуемого режима будем предполагать, что возбуждение соответствует первоначальной настройке на холостом ходу. Амплитудно-частотные характеристики колебательной системы на холостом ходу имеют вид, характерный для линейных систем. Резонансная частота системы не зависит от уровня возбуждения и равна $f_{p}=18$ кГц. При изменении частоты возбуждения в сторону ее увеличения и уменьшения экспериментальные точки ложатся на одну и ту же кривую. Для дальнейшего важно, что сама по себе колебательная система является линейной. Ее диссипативные свойства находятся с помощью полученных резонансных кривых. Для этого, прежде всего, найдем добротность $Q$ колебательной системы $Q=f_{p} /\left(f_{2}-f_{1}\right)$, где $f_{1}, f_{2}$ - частоты, определяемые точками пересечения резонансной кривой с горизонтальной прямой, проведенной на уровне $a / 2$ (рис.12.14), а затем логарифмический декремент $\mathrm{d}=\pi / Q$ и декремент $D=\mathrm{e}^{d}$ колебаний. Для исследуемой системы имеем $Q=97, d=0,324, D=1,03$.

7. Режимы работы станка, при которых происходит взаимодействие между инструментом и обрабатываемым изделием, будем называть рабочими. Это взаимодействие будем характеризовать амплитудой силы удара $F_{m}$, под которой понимается максимальное отклонение силы от нулевого значения. Эта величина измерялась на большом количестве осциллограмм, полученных при различных значениях амплитуды $a^{*}$ настройки , частоты возбуждения $f$ и статического усилия подачи $P$. 
Две характерные осциллограммы рабочих режимов приведены на

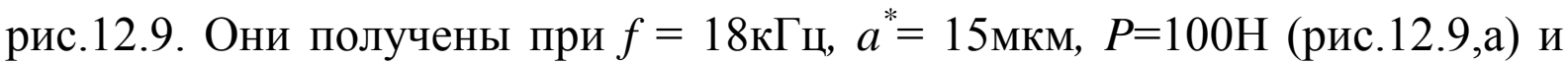
$P=160 \mathrm{H}$ (рис.12.9,б). Верхние кривые на этих осциллограммах представляют силу взаимодействия инструмента с обрабатываемым изделием, нижние - сигнал с генератора возбуждения магнитострикционного преобразователя. Из осциллограмм видно, что рабочие режимы являются периодическими с частотой, равной частоте возбуждения.
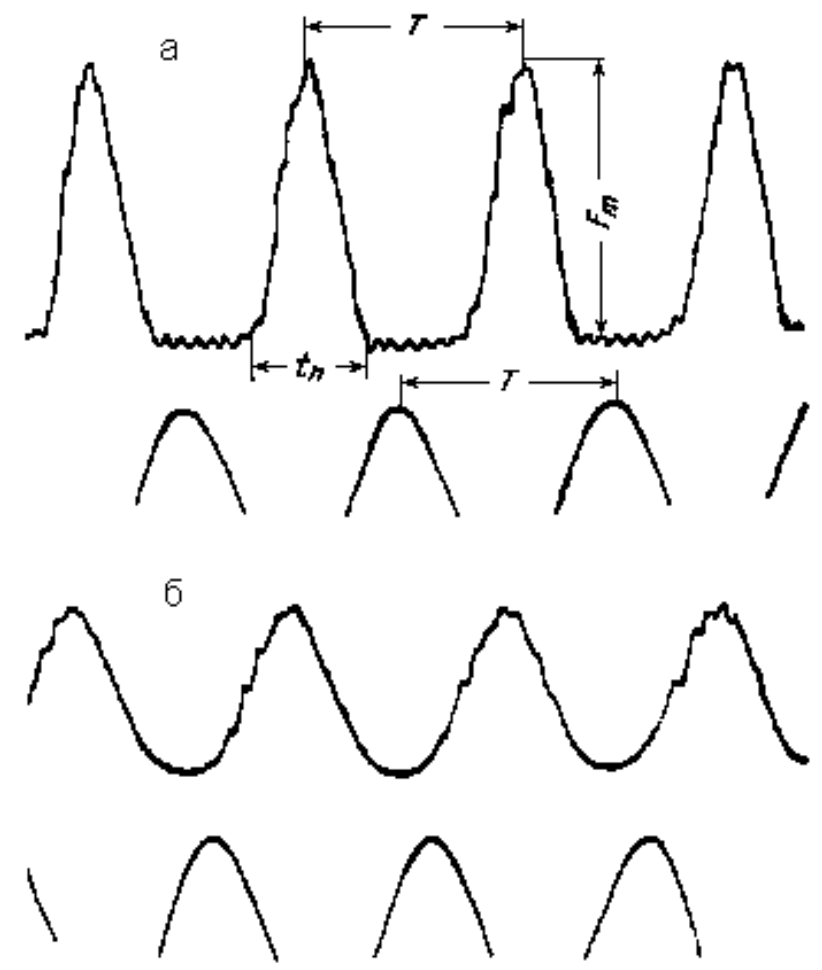

Pис. 12.9

Следует различать режимы двух типов. В режимах типа рис.12.9,а сила взаимодействия носит импульсный характер. Она действует в течение интервала $t_{n}$, а ее амплитуда равна $F_{m}$. На остальной части периода $T-t_{n}$ инструмент совершает колебание в отрыве от обрабатываемой поверхности, т. е. сила взаимодействия равна 0. Такие режимы будем называть ударными. Именно такие режимы отыскивались выше при теоретическом анализе работы колебательной системы под нагрузкой.

Режимы второго типа, один из которых представлен на (рис.12.9,б), отличаются тем, что в течение всего периода инструмент прижат к 
обрабатываемой детали и совершает малые колебания в пределах упругости ограничителя. Такие режимы будем называть безударными. Сопоставление осциллограмм, полученных при постоянных $f$ и $a^{*}$, показало, что по мере увеличения усилия подачи происходит постепенный переход от ударных режимов к безударным.

Рассмотрим основные динамические характеристики системы, полученные в результате обработки осциллограмм рабочих режимов. На рис.12.10 приведены зависимости амплитуды силы удара (рис.12.10,a) и продолжительности удара (рис.12.10,б) от статического усилия подачи. Эти зависимости получены при постоянной частоте возбуждения 18 кГц (собственная частота линейной системы) и различных значениях амплитуды $a^{*}$.
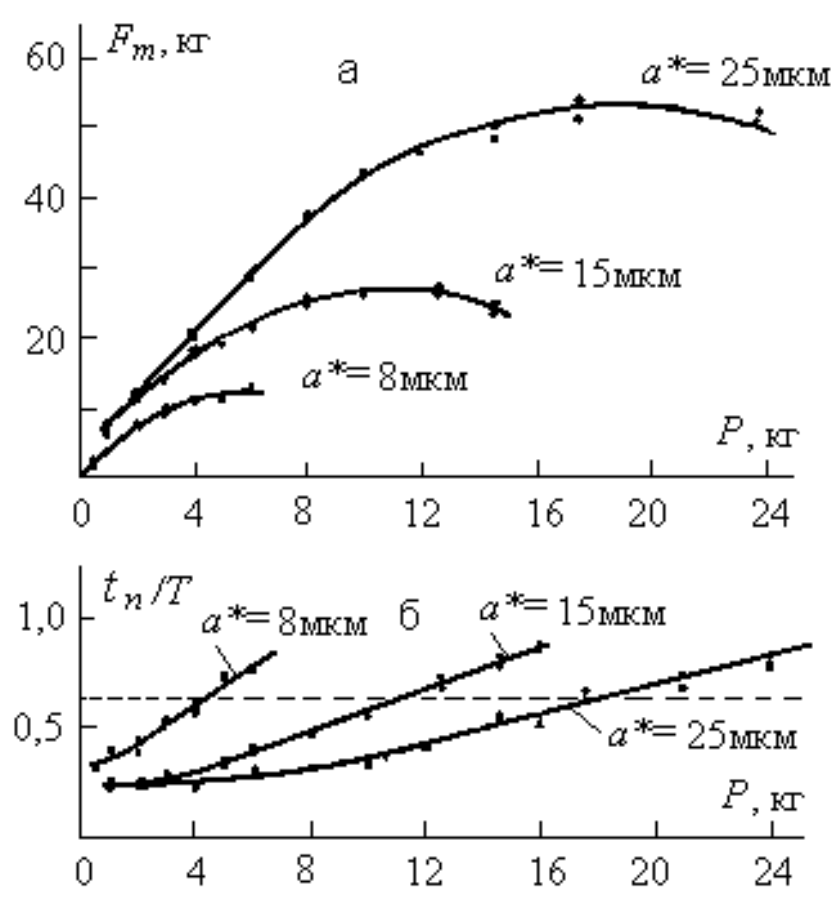

Рис. 12.10

Из графиков видно, что существует оптимальное усилие подачи, соответствующее максимуму силы удара. Величина оптимального усилия подачи пропорциональна амплитуде $a^{*}$. Увеличение подачи сверх оптимальной приводит к уменьшению силы взаимодействия, при этом время соударения возрастает, и режимы постепенно становятся 
безударными. Заметим, что в режимах с максимальной силой удара продолжительность соударения $t_{n}=0,6 T$ (штриховая линия на рис.12.9,б).

Полученные зависимости являются важными характеристиками ультразвукового станка. Дело в том, что амплитуда силы удара является основной величиной, определяющей производительность ультразвуковой обработки. В этом нетрудно убедиться, если сопоставить приведенные графики с зависимостями скорости ультразвуковой обработки от усилия подачи, экспериментально полученными в работе [233] и теоретически обоснованы в П.2 настоящего Параграфа (см. рис.12.3). Характер этих кривых совершенно аналогичен. Следовательно, максимальная производительность станка при работе на постоянной частоте с данной амплитудой будет иметь место при оптимальном усилии подачи, при котором сила удара достигает максимального значения.

8. Требования повышения производительности обработки заставляют искать пути интенсификации режима работы станка. В настоящее время обработка производится на постоянной частоте, равной резонансной частоте холостого хода. При этом, как было показано выше, единственным способом увеличения производительности является повышение усилия подачи, которое требует увеличения мощности колебательной системы. Поэтому этот путь, как правило, и применяемый в практике построения ультразвуковых технологических систем, крайне не эффективен.

В П.12.3 теоретически было показано, и это подтверждается экспериментально, что наилучшим способом повышения эффективности ультразвуковой технологической системы является еe резонансная настройка под нагрузкой.

На рис.12.11,a приведена типичная частотная характеристика системы. Эта зависимость амплитуды силы удара $F_{m}$. от частоты возбуждения $f$ получена при постоянных настроечной амплитуде $a^{*}=15$ 
мкм и усилии прижима $P=200$ Н. На рис.12.11,б показана полученная при тех же условиях эксперимента зависимость времени удара от частоты
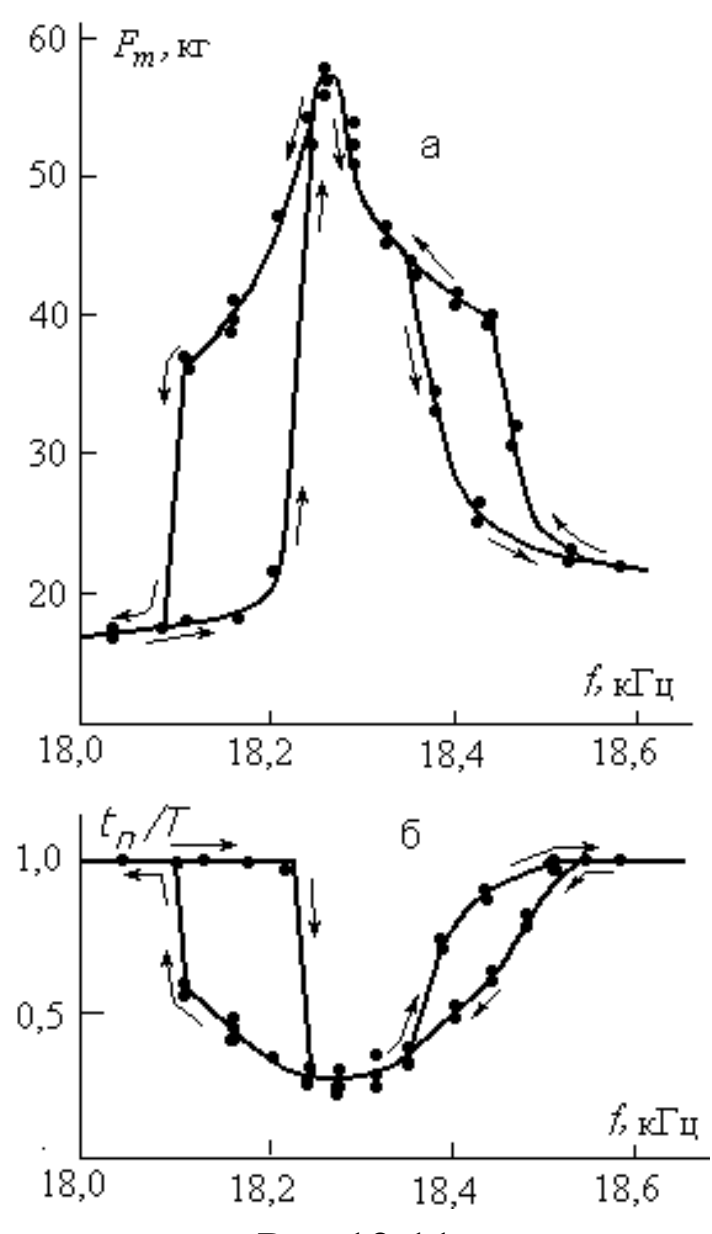

Рис.12.11

возбуждения.

Частотная

характеристика системы имеет ярко выраженный резонансный характер. Частоту $f_{\mathrm{p}}$, при которой сила удара достигает максимума, будем считать резонансной.

Интересной

особенностью системы является наличие областей двузначности частотной характеристики, расположенных слева и справа от резонанса. Выход на ту или иную ветвь характеристики определяется направлением изменения частоты, как показано стрелками на рисунке. В левой области двузначности нижней ветви характеристики соответствуют безударные режимы работы, а верхней ветви - ударные. Переход с одной ветви на другую в этой области происходит скачком. При этом здесь наблюдается ярко выраженный эффект затягивания виброударных режимов. Указанные эффекты предсказываются полученными выше теоретическими расчетами в рамках принятых моделей.

В правой области двузначности обеим ветвям соответствуют ударные режимы работы. Выход на нижнюю ветвь осуществляется при увеличении частоты из резонансной зоны. При этом амплитуда силы убывает, а режимы постепенно становятся безударными. Выход на 
верхнюю ветвь происходит при обратном изменении частоты возбуждения.

9. Здесь следует отметить, что появление в эксперименте второй зоны двузначности амплитудно-частотной характеристики явилось полной неожиданностью, поскольку в расчетах принятых моделей этот эффект не обнаруживается. Вместе с тем тот же эффект наблюдался и в аналогичных экспериментах, проведенных позднее учеными Витебского института физики твердого тела Белорусской Академии Наук под руководством В.В. Клубовича. Поэтому объяснение этого эффекта является принципиальным, поскольку в противном случае возникает сомнение в адекватности рассматриваемых моделей.

Для объяснения эффекта обратимся к модели на рис 12.1. Напомним, что вся система, включая каретку $M$, при поджатии к ограничителю постоянной силой как бы плавает над ограничителем, причем величина всплытия изменяется при изменении амплитуды колебаний таким образом, чтобы поддерживалась известная величина импульса силы удара $J=P T$. Поэтому каретка поднимается при увеличении амплитуды и опускается при уменьшении амплитуды колебаний инструмента,. Теперь заметим, что, хотя каретка и установлена в шариковых направляющих станины, в этих направляющих на каретку действует сила сухого трения $P_{\text {т }} \operatorname{sgn} \dot{a}$. Следовательно постоянная составляющая $f_{0}$ силы, действующей в контакте инструмента с изделием, в зависимости от характера изменения амплитуды колебаний инструмента изменяется в пределах

$$
P_{1}=\left(P-P_{\mathrm{T}}\right) \leq f_{0} \leq P_{2}=\left(P+P_{\mathrm{T}}\right)
$$

причем граничные значения устанавливаются при движении каретки вниз или вверх. При неподвижной каретке постоянная составляющая $f_{0}$ может принять любое значение в указанных пределах, и пока сила $f_{0}$ не достигнет одного из граничных значений система ведет себя как система с 
предварительным натягом, достигнутым в результате предыстории процесса.

На рис.12.11 по методике, изложенной в Параграфе 9, линиями 1 и 2 построены амплитудно-частотные характеристики системы при значениях постоянной составляющей $P_{1}$ и $P_{2}$ соответственно. Тонкими линиями 1 ' и 2' показаны их скелетные кривые. Вертикальный участок скелетных

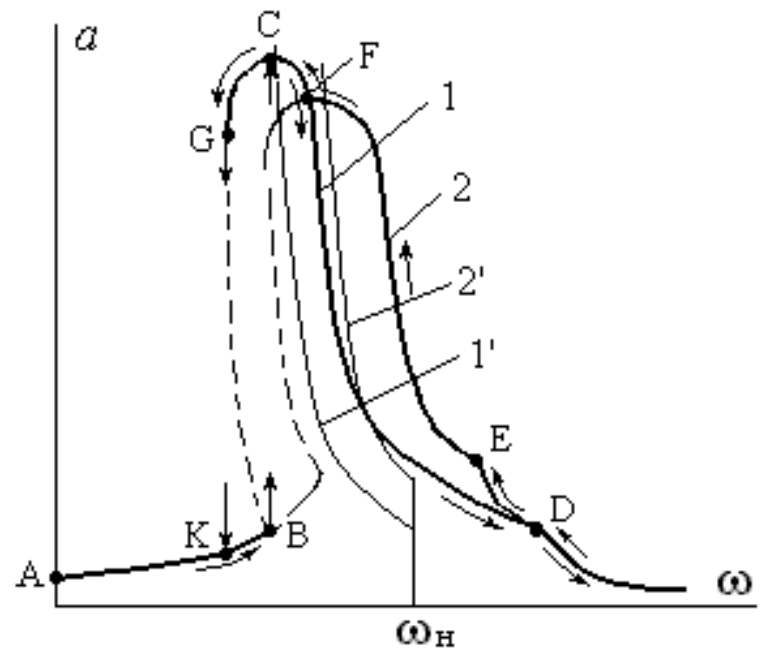

Рис.12.11

кривых соответствует собственной частоте, линейной системы при ее колебаниях в пределах упругой деформации ограничителя без отрывов. Здесь же показаны ветви линейных колебаний с амплитудами $a \leq P_{i} / k_{0}$, где $k_{0}$ - статическая жесткость изделия, $i=1,2$, в пределах упругости изделия.

Реализуемые участки этих характеристик показаны жирными, а нереализуемые - тонкими линиями. Штриховыми линиями изображены неустойчивые ветви. Стрелки указывают направление изменения частоты возбуждения.

Теперь можно проследить поведение системы при изменении частоты возбуждения. Пусть в начальный момент система поджимается к изделию и возбуждается с частотой, соответствующей точке А. При этом постоянная составляющая $f_{0}=P_{1}$. При увеличении частоты до значения, отвечающего точке В срыва колебаний амплитуда следует вдоль линейной ветви характеристики 1, и наблюдаются безударные колебания. После 
срыва колебаний в точку С при дальнейшем увеличении частоты происходит уменьшение амплитуды, изменение которой следует вдоль характеристики 1, переходя к безударным режимам в точке D. и следуя далее вдоль линейной ветви характеристик.

При изменении частоты возбуждения в обратном направлении амплитуда возрастает вдоль линейной ветви характеристики 1 до точки D. Здесь возникают ударные режимы, и вследствие возрастания амплитуды и изменения направления силы трения в направляющих происходит возрастание силы $f_{0}$, и до выполнения равенства $f_{0}=P_{2}$, изменение амплитуды следует вдоль участка DE, который принадлежит резонансной кривой системы с предварительным натягом. После прохождения точки Е реализуется ветвь характеристики 2. Дальнейшее ясно из рисунка.

Таким образом, все найденные экспериментально эффекты объясняются с позиций подходов, развиваемых в данной работе.

10. В результате эксперимента было установлено, что рабочая резонансная частота системы смещается по отношению к резонансной частоте холостого хода. Величина «ухода» резонанса видна из рис.12.12.

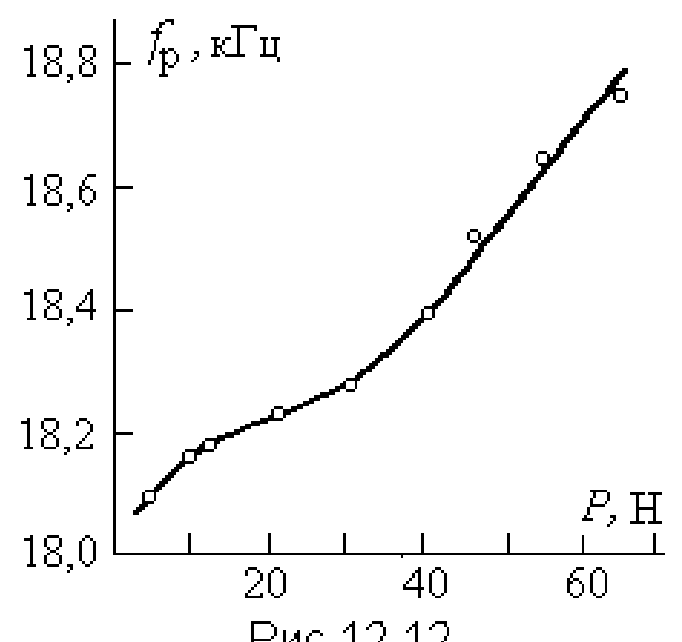

На этом рисунке приведена зависимость резонансной частоты $f_{\mathrm{p}}$ колебательной системы от статического усилия подачи. Зависимость получена при наладке с амплитудой $a^{*}=15$ мкм. Из графика видно, что с увеличением усилия подачи резонансная частота уходит в область более 
высоких частот. Следует отметить, что всем точкам графика соответствуют ударные режимы работы. Амплитуда силы удара в этих режимах в 2,5- 3 раза превышает усилие подачи.

Теперь можно оценить тот эффект, который дает подстройка частоты возбуждения. Так например, при работе на резонансной частоте холостого хода (рис. 12.9) режим с максимальной силой удара получается при $a^{*}=25$ мкм и $P=200 Н$. Как видно из рис. 12.10 и рис. 12.12 , а режимы со значительно большими силами удара можно получить при при меньших амплитудах предварительной настройки путем увеличения усилия подачи и регулировки частоты в соответствии с 12.12 .

Если вспомнить, что производительность станка определяется силой удара, а именно: объем разрушаемого материала пропорционален кубу амплитуды силы удара [111], и добавить к этому, что повышение производительности за счет увеличения мощности станка крайне не рационально, становится очевидной необходимость резонансной настройки станка под нагрузкой. Как было указано выше и подтверждается приведенными здесь результатами экспериментов, из-за сильных нелинейных искажений амплитудно-частотных характеристик такая настройка может быть реализована только в автоматическом режиме. Наиболее эффективные способы возбуждения и стабилизации резонансных колебаний ультразвуковых технологических систем под нагрузкой будут рассмотрены в Параграфе 14. 


\section{3. Нелинейная теория ультразвуковых концентраторов}

1. В предыдущем Параграфе рассмотрен способ резонансной настройки путем компенсации расстройки, вносимой технологическим процессом, выбором массы присоединенного инструмента. Близким к этому способу является рассматриваемый в настоящем параграфе способ резонансной настройки под нагрузкой путем соответствующего выбора геометрических размеров концентратора. В настоящем параграфе излагается теория ультразвукового концентратора, работающего на нелинейную упругодиссипативную нагрузку. Для процесса ультразвуковой обработки определяются зависимости скорости резания, упругой и диссипативной составляющих нагрузки от параметров колебаний инструмента. Приводятся примеры расчета экспоненциального и ступенчатого концентраторов.

Теория ультразвуковых концентраторов, предполагающая их идеальное согласование на холостом ходу, развита в работах $[146,147,191]$. Ниже рассматривается стержневой концентратор, обладающий внутренними потерями.

Сечение $x=0$ концентратора 1 (рис.13.1) связано с возбудителем 2 колебаний, а в сечении $x=l$ закреплен инструмент 3 , взаимодействующий с обрабатываемым изделием или средой 4.

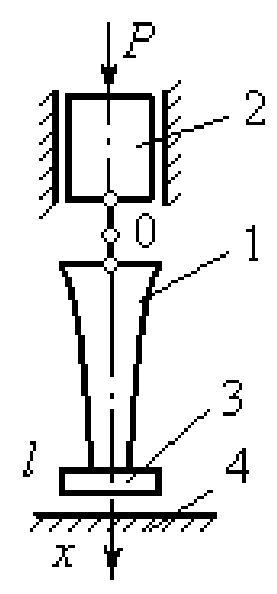

Pnc. 13.1 
Колебания сечения $x$ концентратора будем описывать функцией

$$
u_{x}(t)=a_{x} \exp j\left(\omega t-\varphi_{x}\right)
$$

где $a_{x}, \omega, \varphi_{x}$ - амплитуда, круговая частота и начальная фаза колебаний.

Как было показано в Параграфе 5, в технологических ультразвуковых процессах нагрузка на колебательную систему со стороны изделия носит, .как правило, нелинейный характер и может быть представлена в виде силовой реакции

$$
f\left(u_{l}\right)=\left[k\left(a_{l}\right)+j \omega \beta\left(a_{l}\right)\right] u_{l}
$$

Нелинейность нагрузки проявляется в том, что ее упругая $k\left(a_{l}\right)$ и дисси-пативная $\beta\left(a_{1}\right)$ составляющие оказываются зависящими от амплитуды колебаний инструмента. Такая зависимость неоднократно наблюдалась экспериментально [171].

Взаимное влияние концентратора и возбудителя колебаний будем оценивать силой, действующей в месте их стыка

$$
f_{0}(t)=F_{0} \exp j \omega t
$$

Движение концентратора по-прежнему определим с помощью величины динамической податливости $L_{s}(x, j \omega), \quad$ связывающей перемещение $u_{x}$ сечения $x$ с реакциями (13.2), (13.3) и инерционной силой $-M \omega^{2} u_{l}$ от присоединенного инструмента с массой $M$, действующими в сечениях $s=0, I$ :

$$
u_{x}(t)=L_{0 x}(j \omega) F_{0} \exp j \omega t-L_{0 x}(j \omega)\left[k\left(a_{l}\right)+\beta\left(a_{l}\right)-M \omega^{2}\right] u_{l}(t)
$$

Выразив, согласно формуле (1), величины $\exp j \omega t=\frac{u_{l}}{a_{l}} \exp j \varphi_{l}$, $u_{x}=u_{l} \frac{a_{x}}{a_{l}} \exp j\left(\varphi_{l}-\varphi_{x}\right)$ и подставив их в уравнение (4), получим при $x=0$ и $x=l$

$$
\begin{gathered}
a_{0}\left\{W_{l 0}(j \omega)+\frac{a_{l}}{a_{0}}\left[k\left(a_{l}\right)-M \omega^{2}+j \omega \beta\left(a_{l}\right)\right] \mathrm{e}^{j\left(\varphi_{0}-\varphi_{l}\right)}\right\}=\frac{W_{l 0}(j \omega)}{W_{00}(j \omega)} F_{0} \mathrm{e}^{j \varphi_{0}} \\
a_{l} W\left(a_{l}, j \omega\right)=F_{0} \mathrm{e}^{j \varphi_{l}}
\end{gathered}
$$

где $\quad W\left(a_{l}, j \omega\right)=\left[W_{l l}(j \omega)+k\left(a_{l}\right)-M \omega^{2}+j \omega \beta\left(a_{l}\right)\right] \frac{W_{0 l}(j \omega)}{W_{l l}(j \omega)} \quad ; \quad W_{s x}(j \omega)$ динамическая жесткость концентратора $(s, x=0, l)$. 
После разделения в формуле (6) действительной и мнимой составляющих найдем выражения для амплитуды $a_{l}$ и фазы $\varphi_{l}$ движения сечения $x=1$ концентратора

$$
\begin{gathered}
a_{l}=\frac{F_{0}}{\left|W\left(a_{l}, j \omega\right)\right|}=\frac{F_{0}}{\sqrt{\left[\operatorname{Re} W\left(a_{l}, j \omega\right)\right]^{2}+\left[\operatorname{Im} W\left(a_{l}, j \omega\right)\right]^{2}}} \\
\cos \varphi_{l}=\frac{a_{l}}{F_{0}} \operatorname{Re} W\left(a_{l}, j \omega\right), \quad \sin \varphi_{l}=\frac{a_{l}}{F_{0}} \operatorname{Im} W\left(a_{l}, j \omega\right)
\end{gathered}
$$

Воспользуемся для динамической жесткости формой (5.42)

$$
W_{s x}(j \omega)=U_{s x}(\omega)+j V_{s x}(\omega)
$$

В дальнейшем величины $V_{s x}(\omega)$ и $\beta\left(a_{l}\right)$, характеризующие диссипативные потери в концентраторе и в нагрузке, полагаются малыми, и все вычисления ограничиваются малыми первого порядка. Как показано в П.9.1, уравнение (13.7) определяет амплитудно-частотную характеристику концентратора при действии на конце $\mathrm{x}=0$ гармонической силы (13.3). Максимальная амплитуда al инструмента достигается при условии $\operatorname{Re} W\left(a_{l}, j \omega\right)=0$, которое с учетом формулы (13.8) приводится к виду

$$
U_{l l}(\omega)+k\left(a_{l}\right)-M \omega^{2}=0
$$

При этом, естественно, любая заданная амплитуда $a_{l}$ обеспечивается минимальной силой $F_{0}$, и, следовательно, соотношение (13.9) является условием наилучшего согласования возбудителя колебаний с концентратором, несущим инструмент массой $M$ и работающим на нелинейную технологическую нагрузку.

Исключая величину $F_{0}$ из уравнений (13.5), (13.6) и принимая во внимание соотношения (13.8), (13.9), получим

$$
K=\frac{a_{1}}{a_{0}}=\left|\frac{U_{00}(\omega)}{U_{01}(\omega)}\right|
$$

По аналогии с $[83,84]$ и П.5.5 величину $K=a_{l} / a_{0}$ характеризующую степень трансформации уровня колебаний, передаваемых от возбудителя к инструменту, будем называть коэффициентом усиления концентратора. 
Заметим, что выражение (13.10) по форме совпадает с полученным ранее равенством (5.48), определяющим коэффициент усиления ненагруженного концентратора. При заданной величине амплитуды $a_{0}$ возбудителя колебаний соотношение (13.10) сразу определяет амплитуду рабочего торца концентратора, удовлетворяющего условию (13.9) наилучшего согласования под нагрузкой.

2. Присоединение концентратора приводит к нагружению возбудителя. Пусть ненагруженный со стороны концентратора возбудитель совершает колебания $u_{0}^{*}(t)=a_{0}^{*} \exp j(\omega t-\varphi)$. В точке присоединения концентратора на возбудитель действует сила $-f_{0}(t)$. Его движение под нагрузкой опишем следующим уравнением:

$$
u_{0}(t)=u_{0}^{*}(t)-\frac{f_{0}(t)}{W_{n}(j \omega)}
$$

где $W_{n}(j \omega)=U_{n}(\omega)+j V_{n}(\omega)$ - динамическая жесткость возбудителя в точке присоединения концентратора.

С помощью соотношений (13.6), (13.8)-(13.10) величину реакции (13.3) представим в виде

$$
f_{0}(t)=j u_{0}(t) K^{2}\left[V_{l l}(\omega)+\omega \beta\left(a_{l}\right)\right]
$$

Выше в Параграфе 7 было показано, что в ультразвуковых технологических установках в качестве возбудителей колебаний используются, как правило, резонансные электроакустические преобразователи. Условие их резонансной настройки имеет вид $U_{n}(\omega)=0$. Теперь для нагруженного преобразователя из (13.11), (13.12) имеем

$$
u_{0}(t)=u_{0}^{*}(t)\left[1+\frac{V_{l l}(\omega)+\omega \beta\left(a_{l}\right)}{V_{n}(\omega)} K^{2}\right]^{-1}
$$

Отсюда следует, что присоединение согласованного концентратора не изменяет резонансной настройки преобразователя, а изменение уровня его колебаний определяется соотношением диссипативных параметров преобразователя, концентратора и нагрузки. Из соотношения (13.10) с 
учетом формулы (13.13) находим амплитуду колебаний инструмента в рабочем режиме

$$
a_{l}=a_{0}^{*} K\left[1+\frac{V_{l l}(\omega)+\omega \beta\left(a_{l}\right)}{V_{I}(\omega)} K^{2}\right]^{-1}
$$

3. Рассмотрим ситуацию, возникающую при ультразвуковой обработке хрупких материалов. В предыдущем параграфе показано, что обработка происходит в результате высокочастотных виброударных взаимодействий инструмента с изделием при их сжатии статической силой $P$. Там же на основе реологической модели процесса резания получены следующие выражения для упругой и диссипативной составляющих нагрузки (13.2):

$$
k\left(a_{l}\right)=\frac{D}{\pi a_{l}} \sin \frac{2 \pi P}{D} ; \quad \beta\left(a_{l}\right)=\frac{2 D}{\pi \omega a_{l}} \sin ^{2} \frac{\pi P}{D}
$$

где $D$ - величина, пропорциональная площади инструмента и зависящая от материала изделия и вида абразивной суспензии.

После подстановки выражения (13.15) в формулы (13.9) и (13.14) получим после преобразований систему уравнений

$$
\begin{gathered}
a_{l}=\frac{2 P}{M \omega^{2}-U_{I l}(\omega)} \\
a_{l}=a_{0}^{*} K \frac{1-K \frac{\pi P^{2}}{D V_{I I}(\omega)}}{1+K^{2} \frac{V_{I I}(\omega)}{V_{I}(\omega)}}
\end{gathered}
$$

решение которой определяет параметры и. амплитуду колебаний концентратора, согласованного под нагрузкой.

Ниже даны примеры расчета наиболее распространенных типов ультразвуковых концентраторов. Своим концом $x=0$ концентратор 1 (рис.13.1) присоединен к выходу возбудителя 2 колебаний. Динамические податливости концентраторов различных типов определены в Параграфе 5 путем решения уравнений продольных колебаний стержней переменного сечения. 
Рассмотрим экспоненциальный концентратор (см. П.5.5.), площадь $S_{x}$ поперечного сечения $x$ которого изменяется по закону $S_{x}=S_{0} \exp (2 \gamma x / l)$, где $\gamma=1 / 2 \ln \left(S_{l} / S_{0}\right)$.

Выражения для величин динамических податливостей экспоненциального волновода получены в П.5.6. и для оператора $L_{l x}(j \omega)$ имеет вид

$$
L_{l x}(j \omega)=\frac{\gamma \operatorname{sh} \lambda x-\lambda l \operatorname{ch} \lambda x}{\omega^{2} \rho S_{0} l \operatorname{sh} \lambda} e^{\gamma\left(1+\frac{x}{l}\right)}
$$

где $\lambda=\frac{\omega}{c}\left(j \frac{c^{2}}{c_{\phi}^{2}}+\frac{\psi}{2 \pi}\right)^{1 / 2} ; \quad c_{\phi}=\frac{c}{\sqrt{1-(\gamma c / l \omega)^{2}}}-$ фазовая скорость звука в экспоненциальном волноводе; $c=\sqrt{E / \rho}$ - скорость звука в материале концентратора; $E, \rho$ - модуль упругости и плотность материала концентратора.

Полагая коэффициент поглощения $\psi$ малым и ограничиваясь в формуле (13.18) линейными членами по малому параметру, найдем выражение для составляющих динамической жесткости экспоненциального концентратора

$$
\begin{gathered}
U_{l x}(\omega)=-\frac{E S_{0}}{l} \frac{\left(\xi^{2}+\gamma^{2}\right) \exp [\gamma(1+x / l)]}{\xi \cos \xi x / l+\gamma \sin \xi x / l} \\
V_{l x}(\omega)=\frac{\psi E S_{0}}{4 \pi l \xi} \frac{\left(\xi^{2}+\gamma^{2}\right)^{2} \exp [\gamma(1+x / l)]}{\xi \cos \xi x / l+\gamma \sin \xi x / l} \times \\
\times\left[\cos \xi-\frac{(1+\gamma x / l) \cos \xi x / l-(\xi x / l) \sin \xi x / l}{\xi \cos \xi x / l+\gamma \sin \xi x / l} \sin \xi\right]
\end{gathered}
$$

где $\xi=\omega l / c_{\phi}$.

С учетом выражения (13.19) соотношение (13.10) для коэффициента усиления нагруженного концентратора принимает вид

$$
K=\frac{a_{l}}{a_{0}}=\left|\frac{\gamma \cos \xi+\xi \sin \xi}{\xi}\right| \sqrt{\frac{S_{0}}{S_{l}}}
$$

Для свободного ненагруженного концентратора $(M=0, P=0)$, согласно формуле (13.16), имеем $U_{l l}=0$, что достигается при $\xi=\pi n$ 
$(n=1,2, \ldots)$, и выражение (21) дает величину коэффициента усиления $K_{0}=\sqrt{S_{0} / S_{I}}$, совпадающую с найденной в П.5.6. При наличии нагрузки согласование концентратора с возбудителем достигается при ином значении $\xi$. Для его отыскания используем выражение (13.16) после подстановки в него величины (13.19) при $x=l$

$$
a_{l}=\frac{2 P}{\omega W_{l}}\left(\mu+\frac{\sqrt{1+(\gamma / \xi)^{2}}}{\cos \xi+(\gamma / \xi) \sin \xi}\right)^{-1}
$$

где $\mu=M \omega / w_{l} ; w_{l}=S_{l} \sqrt{E \rho}$ - волновое сопротивление на выходном конце концентратора.

Совместное решение уравнений (13.21) и (13.22) определяет искомые величины $\xi$ и $a_{l}$ согласованного под нагрузкой экспоненциального концентратора при заданной величине амплитуды на входном конце концентратора. Такое решение удобно выполнить графическим способом, как это показано на рис.13.2. После этого длина концентратора вычисляется по формуле

$$
I=\frac{C}{\omega} \sqrt{\xi^{2}+\gamma^{2}}
$$

которая при отсутствии нагрузки $\xi=\pi n$ совпадает с полученными в [191] и П.5.6.

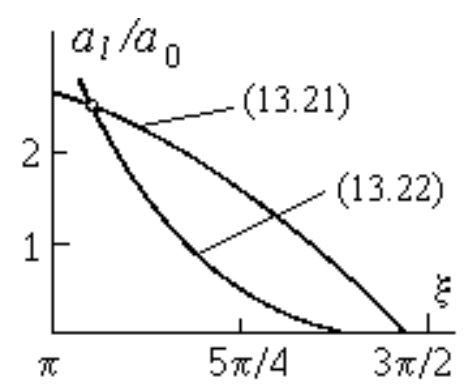

Рис. 13.2

На рис.13.3,а приведены построенные в результате решения зависимости длины $l$ согласованного под нагрузкой концентратора от силы $P$ прижима при различных значениях массы $M$ присоединенного инструмента. Здесь: $l_{0}$ - длина свободного ненагруженного концентратора, определяемая по формуле (13.23) при $\xi=\pi n$. При расчетах приняты 
следующие параметры системы: частота колебаний $f=\omega / 2 \pi=20$ кГц; $K_{0}=4 ; \quad w_{l}=3,14 \cdot 10^{3} \mathrm{H} \cdot \mathrm{c} / \mathrm{m} ; \quad \psi=0,01 ; \quad D=5 \cdot 10^{4} \mathrm{H}$. Кривые на pис.13.3,a построены при следующих значениях параметра $\mu$ : $1-\mu=0 ; 2-\mu=0,2$; $3-\mu=0,4 ; 4-\mu=0,6 ; 5-\mu=0,8 ; 6-\mu=1$.
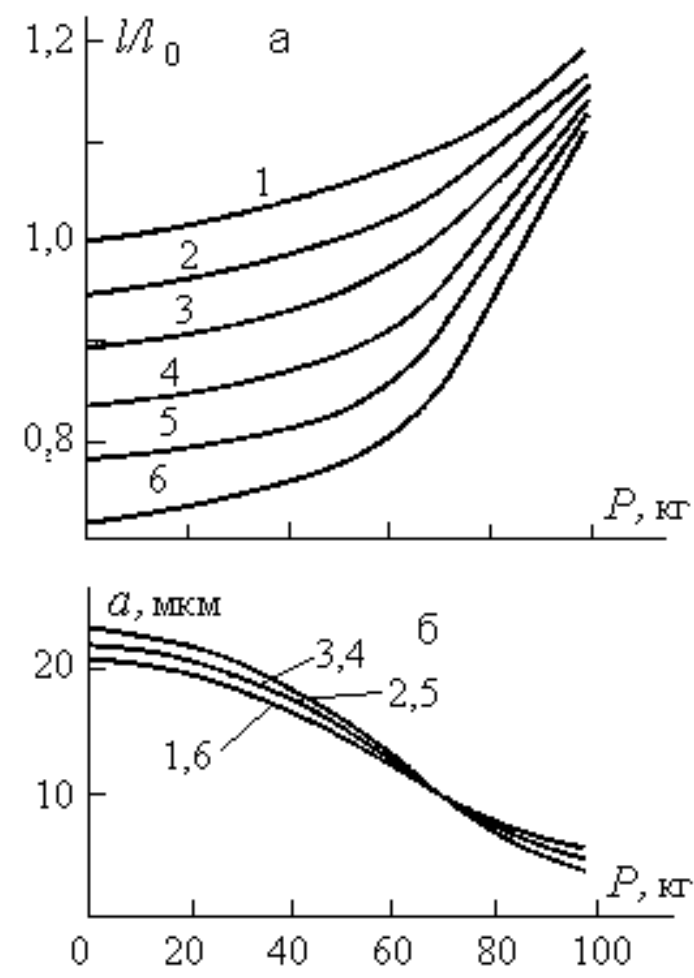

Pис. 13.3

На этом решение задачи было бы завершено, если бы присоединение концентратора не оказывало влияния на амплитуду колебаний электроакустического преобразователя. Однако, как видно из формулы (13.17), амплитуда колебаний на выходном конце концентратора зависит от соотношения диссипативных потерь в преобразователе и концентраторе.

Примем для определенности, что преобразователь представляет собой однородный стержень длины $l_{n} c$ сечением $S_{n}$. Его динамическую жесткость при резонансной настройке на частоту первой формы собственных колебаний найдем из формулы (13.20), положив $\gamma=0$; $\xi=\pi$; $x=l$ :

$$
V_{\Pi}=\psi_{\Pi} \omega W_{\Pi} / 4
$$


где $w_{n}=S_{g} \sqrt{E_{n} \rho_{n}}-$ волновое сопротивление преобразователя в точке присоединения концентратора; $E_{n}, \rho_{n}, \psi_{n}$ - модуль упругости, плотность и коэффициент поглощения материала преобразователя.

Присоединение даже ненагруженного $(P=0, M=0)$ концентратора к резонансному возбудителю изменяет его амплитуду, которая находится из формул (13.17), (13.20), (13.24)

$$
a_{l}^{*}=a_{0}^{*} K_{0}\left[1+\frac{\psi w_{l} K_{0}^{2}\left(1+\gamma^{2} / n^{2} \pi^{2}\right)}{\psi_{n} W_{I}}\right]^{-1}
$$

Из последнего выражения видно, что амплитуда колебаний концентратора с внутренними потерями уменьшается по сравнению с идеальным тем существеннее, чем больше величина этих потерь, причем изменение амплитуды на выходе концентратора зависит от его коэффициента усиления $K_{0}$.

На рис.13.4 по формуле (13.25) при $\psi_{\pi} w_{a} / \psi w_{l}=65$ построена зависимость (кривая 1) амплитуды колебаний свободного торца экспоненциального концентратора от коэффициента усиления $K_{0}$.

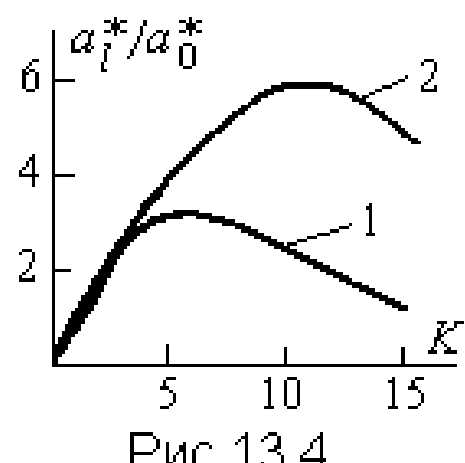

Отсюда видно существование коэффициента усиления $K_{0}$, при котором амплитуда колебаний выходного конца концентратора максимальна.

Амплитуда колебаний концентратора под нагрузкой находится по обшей формуле (13.17) с учетом найденных выше (см. рис.13.2) значений $K_{0}$ и $\xi$. На рис.13.3,б построены зависимости амплитуды колебаний согласованного под нагрузкой экспоненциального концентратора от силы 
прижима при различных значениях массы присоединенного инструмента. Графики построены при тех же параметрах, что и рис.13.3,a. В дополнение к этому принято: $\omega_{n}=4,084 \cdot 104 \mathrm{H} \cdot \mathrm{c} / \mathrm{M} ; \psi=0,05$.

Амплитуда колебаний концентратора под нагрузкой может быть найдена и непосредственным решением системы уравнений (13.16), (13.17), которое выполняется аналогично и дает, конечно, те же окончательные результаты.

4. Расчет концентраторов других типов выполняется аналогично. Здесь для примера рассмотрим случай концентратора, составленного из двух цилиндрических стержней длиной $l_{1} u l_{2}$ и площадью $S_{1}$ и $S_{2}$. Его динамическая податливость найдена в П.5.11. Там же построена теория ступенчатого концентратора без потерь. Здесь, используя развитую выше общую методику, найдем характеристики концентратора с внутренним трением в его материале при работе на инерционную и нелинейную упруго-диссипативную нагрузку.

Запишем выражение (5.118) для динамической податливости

$$
L_{l x}(j \omega)= \begin{cases}-\frac{\lambda}{\rho \cdot \omega^{2}} \cdot \frac{\operatorname{ch} \lambda x}{S_{1} \operatorname{sh} \lambda I_{1} \operatorname{ch} \lambda I_{2}+S_{2} \operatorname{sh} \lambda I_{2} \operatorname{ch} \lambda I_{1}} & 0 \leq x \leq I_{1} \\ -\frac{\lambda}{S_{2} \rho \cdot \omega^{2}} \cdot \frac{S_{1} \operatorname{sh} \lambda\left(x-l_{1}\right) \operatorname{sh} \lambda I_{1}+S_{2} \operatorname{ch} \lambda\left(x-l_{1}\right) \operatorname{ch} \lambda I_{1}}{S_{1} \operatorname{sh} \lambda l_{1} \operatorname{ch} \lambda I_{2}+S_{2} \operatorname{sh} \lambda I_{2} \operatorname{ch} \lambda I_{1}} & I_{1} \leq x \leq I\end{cases}
$$

где $l=l_{1}+l_{2}$; величина $\lambda$ имеет тот же смысл, что и в формуле (13.18) при $c_{\phi}=c,(\gamma=0)$.

Полагая по-прежнему коэффициент поглощения $\psi$ малой величиной для динамических жесткостей получим из (13.26)

$$
\begin{gathered}
W_{l 0}(j \omega)=-\frac{E S_{2}}{l_{2}} \xi_{2} \sin \xi_{1} \sin \xi_{2}\left(A_{1}+j \frac{\psi}{4 \pi} \Phi_{1}\right) \\
W_{l l}(j \omega)=-\frac{E S_{2}}{l_{2}} \xi_{2} A_{2}^{-2}\left(A_{1} A_{2}+j \frac{\psi}{4 \pi} \Phi_{2}\right)
\end{gathered}
$$

где $\xi_{i}=\frac{\omega l_{i}}{c}(i=1,2) ; A_{1}=\operatorname{ctg} \xi_{1}+\frac{S_{1}}{S_{2}} \operatorname{ctg} \xi_{2} ; A_{2}=\operatorname{ctg} \xi_{1} \operatorname{ctg} \xi_{2}-\frac{S_{1}}{S_{2}} ;$ 


$$
\begin{gathered}
\Phi_{1}=\xi_{1}+\operatorname{ctg} \xi_{1}\left(1-\xi_{2} \operatorname{ctg} \xi_{2}\right)+\frac{S_{1}}{S_{2}}\left[\xi_{2}+\operatorname{ctg} \xi_{2}\left(1-\xi_{1} \operatorname{ctg} \xi_{1}\right)\right] \\
\Phi_{2}=A_{1} A_{2}-\frac{S_{1}}{S_{2}} \frac{\xi_{1}}{\sin ^{2} \xi_{1} \sin ^{2} \xi_{2}}-\frac{\xi_{2}}{\sin ^{2} \xi_{2}}\left(\operatorname{ctg}^{2} \xi_{1}+\frac{S_{1}^{2}}{S_{2}^{2}}\right)
\end{gathered}
$$

С учетом (13.27), (13.28) выражение для коэффициента усиления (13.10) принимает вид

$$
K=\frac{a_{l}}{a_{0}}=\left|\cos \xi_{1} \cos \xi_{2}-\left(S_{1} / S_{2}\right) \sin \xi_{1} \sin \xi_{2}\right|
$$

совпадающее с полученной ранее формулой (5.122).

В П.5.11. было показано, что свободный ненагруженный концентратор $(M=0, P=0)$ обладает максимальным усилением $K_{0}=S_{1} / S_{2}$ при $\xi_{1}=\xi_{2}=\pi n / 2(n=1,2, \ldots)$, т.е. при равной длине ступеней $l_{1}=l_{2}=\pi n c / 2 \omega_{n}$.

При наличии нагрузки условие согласования концентратора с возбудителем отыскиваются решением системы уравнений (13.29) и (13.16), которое с учетом (13.28) принимает вид

$$
a_{l}=\frac{2 P}{\omega w_{2}}\left(\mu+\frac{\sin \xi_{2} \cos \xi_{1}+K_{0} \sin \xi_{1} \cos \xi_{2}}{\cos \xi_{1} \cos \xi_{2}-K_{0} \sin \xi_{11} \sin \xi_{2}}\right)^{-1}
$$

Решение уравнений (13.29), (13.30) определяет искомые величины $\xi_{i}$ и $a_{l}$ согласованного под нагрузкой ступенчатого концентратора при заданной величине амплитуды $a_{0}$ на входном конце $x=0$ концентратора. После этого длины ступеней концентратора вычисляются по формуле

$$
l_{i}=\frac{c \xi_{i}}{\omega}
$$

На рис.13.5,a приведены построенные в результате решения зависимости длины $l$ согласованного под нагрузкой концентратора от силы $P$ прижима при различных значениях массы $M$ присоединенного инструмента. Расчеты проведены для концентратора со ступенями равной длины $\left(l_{1}=l_{2}, \quad \xi_{1}=\xi_{2}\right)$. Здесь: $l_{0}$ - длина свободного ненагруженного концентратора, определяемая по формуле (13.31) при $\xi=\pi n ; \mu=M \omega / w_{l}$, где $w_{l}=S_{l} \sqrt{E \rho}$ - волновое сопротивление на выходном конце концентратора. 
При расчетах приняты следующие параметры системы: частота колебаний $f=\omega / 2 \pi=20 \kappa \Gamma ц ; K_{0}=4 ; w_{l}=3,14 \cdot 10^{3} \mathrm{H} \cdot \mathrm{c} / \mathrm{M} ; \psi=0,01 ; D=5 \cdot 10^{4} \mathrm{H}$.

Кривые на рис.13.5,a построены при следующих значениях параметра $\mu$ : $\mu=0 ; 2-\mu=0,2 ; 3-\mu=0,4 ; 4-\mu=0,6$; $5-\mu=0,8 ; 6-\mu=1$.
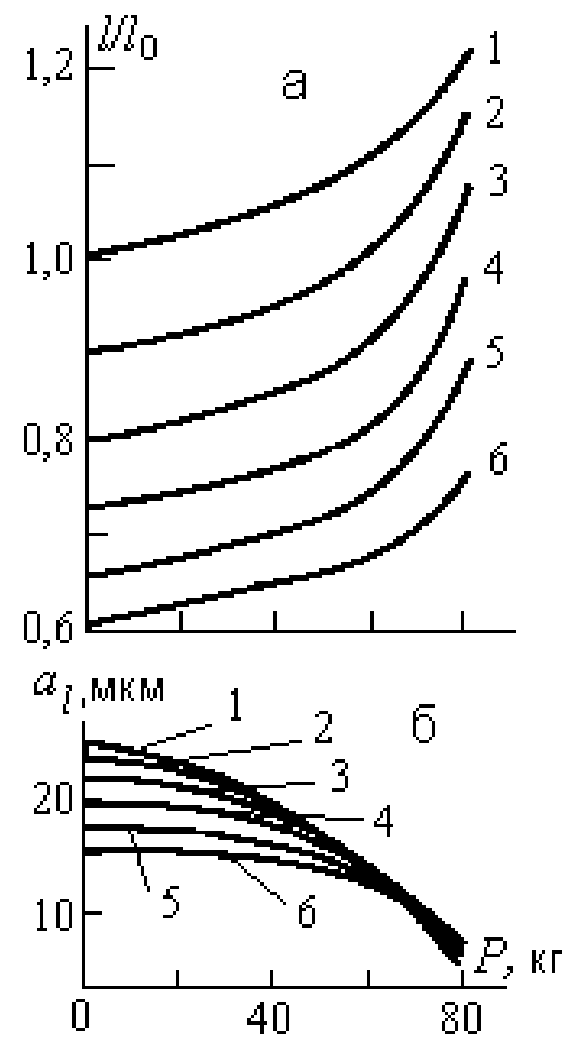

Рис. 13.5

Амплитуда колебаний ненагруженного концентратора, присоединенного к резонансному возбудителю, определяется из формул (13.14), (13.24), (13.28)

$$
a_{l}^{*}=a_{0}^{*} K_{0}\left[1+\frac{\psi w_{l} K_{0}\left(1+K_{0}\right)}{\psi_{I} W_{I}}\right]^{-1}
$$

Из выражения (13.32) видно, что, как и в рассмотренном в предыдущем пункте случае экспоненциального концентратора, амплитуда колебаний ступенчатого концентратора с внутренними потерями уменьшается по сравнению с идеальным тем существеннее, чем больше величина этих потерь, причем изменение амплитуды на выходе концентратора зависит от его коэффициента усиления $K_{0}$. На рис.13.4 по формуле (13.32) при $\psi_{\pi} w_{a} / \psi w_{l}=65$ построена зависимость (кривая 2) 
амплитуды колебаний свободного торца ступенчатого концентратора от коэффициента усиления $K_{0}$. Отсюда видно существование коэффициента усиления $K_{0}$, при котором амплитуда колебаний выходного конца концентратора максимальна.

Амплитуда колебаний ступенчатого концентратора под нагрузкой находится по обшей формуле (13.17) с учетом найденных (рис.13.2) значений $K_{0}$ и $\xi$. На рис.13.5,б построены зависимости амплитуды колебаний согласованного под нагрузкой ступенчатого концентратора от силы прижима при различных значениях массы присоединенного инструмента. Графики построены при тех же параметрах, что и рис.13.5,a. В дополнение к этому принято: $w_{n}=4,084 \mathrm{H} \cdot \mathrm{c} / \mathrm{m} ; \psi_{n}=0,05$.

5. В заключение оценим эффективность согласования концентратора с возбудителем колебаний и технологической нагрузкой, используя соотношение (12.7), связывающее скорость $v$ ультразвуковой обработки с основными параметрами процесса

$$
V=2 a_{l} f \sin ^{2} \frac{\pi P}{D}
$$

где $f=\omega / 2 \pi-$ частота колебаний инструмента.

На рис.13.6, приведены зависимости скорости ультразвуковой обработки от усилия прижима, построенные с помощью данных рис.13.3,б и 13.5,б. На рис.6 кривые 1 и 2 относятся к ступенчатому концентратору при $\mu=0$ и $\mu=1$ соответственно, а кривая 3 к экспоненциальному концентратору при $\mu=0,2$.

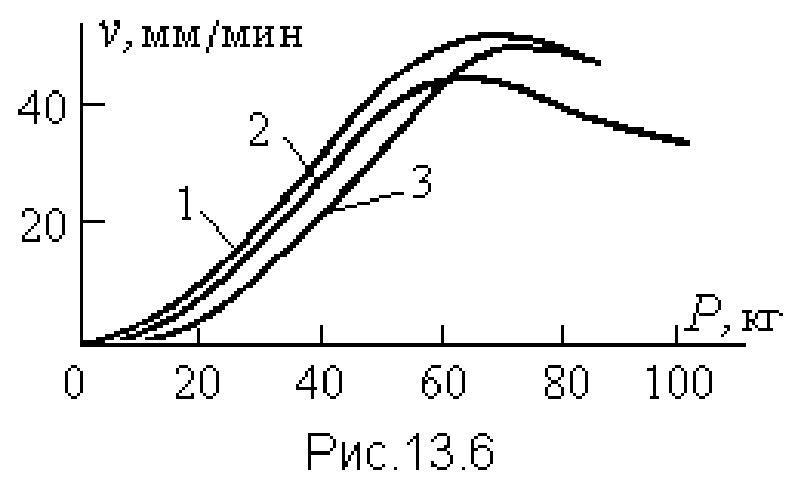

Для сравнения найдем скорости обработки для традиционно применяемых концентраторов, рассчитанных из условий согласования 
идеального концентратора без потерь в режиме холостого хода. Выше, в Параграфе 12, было показано, что в этом случае максимальная скорость обработки

$$
V_{0}=0,3 a_{l}^{* 2} \omega\left(\frac{V_{0}}{D}\right)^{2}
$$

достигается при силе прижима

$$
P_{0}=a_{1}^{*} V_{0} / \sqrt{6}
$$

где $V_{0}=V_{l l}+V_{n} / K_{0}^{2}$

Для системы с принятыми ранее параметрами с экспоненциальным концентратором $V_{0}=46300 \mathrm{H} / \mathrm{cm}$, а со ступенчатым - $V_{0}=52900 \mathrm{H} / \mathrm{cm}$, и расчет по формулам (13.34), (13.35) дает соответственно $P_{0}=45,8 \mathrm{H}, v_{0}=2,4$ мм/мин и $P_{0}=46,6 \mathrm{H}, v_{0}=2,8$ мм/мин. Эти величины, так же как и исходные данные, соответствуют серийно выпускаемому ультразвуковому станку модели 4770.

Из сопоставления полученных данных следует, что выбор концентратора из условий его согласования с резонансным возбудителем колебаний с учетом нелинейной технологической нагрузки позволяет в 15 - 20 раз увеличить производительность ультразвукового станка. Эти величины имеют тот же порядок, что и при резонансной настройке всей колебательной системы путем изменения частоты возбуждения (см. Параграф 12). Это и не удивительно, поскольку рассмотренный прием по сути также является способом резонансной настройки всей колебательной системы под нагрузкой путем выбора геометрических параметров ее элементов, а, следовательно, и их собственных частотных свойств. 


\section{4. Авторезонансное возбуждение}

\section{ультразвуковых технологических машин}

1. Выше в Параграфе 12 при исследовании колебательных систем ультразвуковых станков было показано, что существенное увеличение их производительности при значительном снижении энергетических затрат может быть достигнуто за счет повышения добротности колебательной системы и резонансной настройки под нагрузкой. Однако свойственные такой системе нелинейные искажения динамических характеристик вследствие взаимодействия инструмента с обрабатываемым изделием создают определенные трудности при реализации резонансной настройки.

Принципиальную возможность полного использования динамических свойств машины может дать только создание системы автоматической подстройки частоты, осуществляющей поддержание резонансного состояния путем перестройки частоты возбуждения. Следует, однако, заметить, что обычные поисковые системы автоматического регулирования, изменяющие частоту внешнего вынуждающего воздействия так, чтобы некоторый показатель эффективности, например амплитуда колебаний, стремился к максимуму, в ультразвуковых технологических машинах трудно реализуемы и мало эффективны в силу нелинейности нагрузки и вызываемых ею нелинейных искажений амплитудно-частотных характеристик.

Указанных трудностей при возбуждении и стабилизации резонансных режимов удается избежать переходом к автоколебательной схеме возбуждения, реализуемой введением контура положительной обратной связи, который осуществляет формирование вынуждающего воздействия путем нелинейного преобразования сигнала, пропорционального движению рабочего органа машины. Сказанное в 
полной мере относится ко всем видам ультразвуковых технологических процессов и оборудования, рассмотренных в Параграфах 1 и 4.

В настоящем Параграфе рассматриваются вопросы синтеза контура обратной связи, исследуется динамика автоколебательной системы возбуждения ультразвуковых технологических машин, проводится анализ условий самовозбуждения колебаний, исследуются вопросы существования и устойчивости периодических режимов, изучается поведение системы при изменении параметров колебательной системы, цепи обратной связи и нелинейной технологической нагрузки в широких пределах. Приводятся результаты экспериментов.

Схема автоколебательной системы возбуждения ультразвуковой технологической машины приведена на рис.14.1,a.

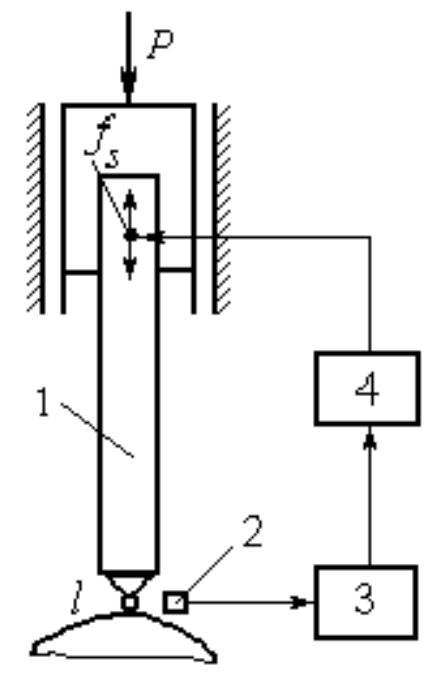

Рис.14.1

Здесь условно показана колебательная система 1, движение $u_{x}(t)$ произвольного элемента $x$ которой

$$
u_{x}(t)=m_{x}+u_{x}^{0}(t)=m_{x}+a_{x} \exp j\left(\omega t-\varphi_{x}\right)
$$

где $m_{x}$ - постоянная составляющая; $a_{x}, \omega, \phi$ - амплитуда, круговая частота и фаза гармонической составляющей $u_{x}^{0}(t)$ движения.

Рабочий орган $l$ машины взаимодействует $\mathrm{c}$ технологической нагрузкой, описываемой нелинейной динамической жарактеристикой

$$
f_{l}\left(u_{l}, j \omega u_{l}\right) \approx f_{0}\left(m_{l}, a_{l}\right)+\left[k\left(m_{l}, a_{l}\right)+j \omega b\left(m_{l}, a_{l}\right)\right] u_{l}^{0}
$$


где $f_{0}\left(m_{l}, a_{l}\right)$ - постоянная составляющая; $k\left(m_{l}, a_{l}\right), b\left(m_{l}, a_{l}\right)-$ упругая и диссипативная составляющие технологической нагрузки. Эти величины определяются по методикам, описанным в Параграфе 8.

Сигнал с датчика 2 обратной связи, пропор-циональный перемешению $u_{l}$ рабочеого органа, через фазосдвигающий элемент 3 поступает со сдвигом фазы $\phi$ в блок нелинейного преобразователя 4, формиру-ющего силу возбуждения $f_{s}=f_{s}\left(u_{l}\right)$, действующую в точке $x=s$ колебательной системы. Учитывая (14.1), запишем полную характеристику цепи обратной связи

$$
f_{s}=f_{s}\left(u_{l}^{0}\right) \exp (j \varphi) \approx Z\left(a_{l}\right) u_{l}^{0} \exp (j \varphi)
$$

где $Z\left(a_{l}\right)$ - гармонический коэффициент усиления нелинейного преобразователя.

Здесь предполагается, что цепь обратной связи отрабатывает лишь периодическую составляющую движения элемента $l, \quad$ причем максимальное усилие, развиваемое возбудителем колебаний считается ограниченным

$$
f_{s}\left(u_{J b}^{0}\right) \leq G
$$

Учитывая, что ограниченная уровнем $G$ периодическая сила $F_{s}$ возбуждения колебаний не может иметь амплитуду первой гармоники больше $4 G / \pi$, получаем из (14.4)

$$
a_{l} Z\left(a_{l}\right) \leq \frac{4 G}{\pi}
$$

Смещения сечений стержня определим при помощи оператора динамической податливости $L_{s x}(j \omega)$, связывающего движение $u_{x}$ элемента $x$ с силами, действующими на элемент $s$

$$
u_{x}(t)=L_{s x}(j \omega) f_{s}\left(u_{l}^{0}\right)-L_{l x}(j \omega) f_{l}\left(u_{l}, j \omega l\right)
$$

Подставляя (14.2), (14.3) в (14.6) и разделяя постоянные и периодические составляющие, имеем при $x=l$

$$
m_{l}=-L_{l l}(0) f_{0}\left(m_{l}, a_{l}\right)
$$




$$
\begin{aligned}
& 1+L_{l l}(j \omega)\left[k\left(m_{l}, a_{l}\right)+j \omega b\left(m_{l}, a_{l}\right)\right]- \\
& -L_{s l}(j \omega) Z\left(a_{l}\right) \exp (j \varphi)=Q\left(m_{l}, a_{l} . j \omega\right)=0
\end{aligned}
$$

Приравнивая нулю вещественную и мнимую части характеристического уравнения (14.8), найдём условия существования автоколебаний

$$
\begin{aligned}
& Z\left(a_{l}\right) \cos \varphi=U\left(\omega, m_{l}, a_{l}\right) \\
& Z\left(a_{l}\right) \sin \varphi=V\left(\omega, m_{l}, a_{l}\right)
\end{aligned}
$$

где $U\left(\omega, m_{l}, a_{l}\right)=\operatorname{Re} W\left(j \omega, m_{l}, a_{l}\right), \quad V\left(\omega, m_{l}, a_{l}\right)=\operatorname{Im} W\left(j \omega, m_{l}, a_{l}\right)$,

$$
W\left(j \omega, m_{l}, a_{l}\right)=\left\{1+L_{l l}(j \omega)\left[k\left(m_{l}, a_{l}\right)+j \omega b\left(m_{l}, a_{l}\right)\right]\right\} / L_{s l}(j \omega)
$$

Исключая из (14.9), (14.10) тригонометрические функции, получаем

$$
Z\left(a_{l}\right)=\sqrt{U^{2}\left(\omega, m_{l}, a_{l}\right)+V^{2}\left(\omega, m_{l}, a_{l}\right)}=\left|W\left(j \omega, m_{l}, a_{l}\right)\right|
$$

Перепишем это соотношение в виде

$$
a_{l}=\frac{a_{l} Z\left(a_{l}\right)}{\sqrt{U^{2}\left(\omega, m_{l}, a_{l}\right)+V^{2}\left(\omega, m_{l}, a_{l}\right)}}
$$

Сравнение выражений (14.12) и (14.5) показывает, что на всех частотах максимальное значение амплитуды автоколебаний достигается при выполнении равенства $a_{l} Z\left(a_{l}\right)=\frac{4 G}{\pi}$, откуда имеем

$$
Z\left(a_{l}\right)=\frac{4 G}{\pi a_{l}}
$$

Соотношение (14.13) может быть реализовано при использовании релейной характеристики $F_{s}=F_{s}\left(u_{\partial}\right)$ преобразователя контура возбуждения [1] (рис.14.2). При такой характеристике цепи обратной связи амплитуда колебаний определяется выражением

$$
a_{l}=\frac{4 G / \pi}{\sqrt{U^{2}\left(\omega, m_{l}, a_{l}\right)+V^{2}\left(\omega, m_{l}, a_{l}\right)}}
$$

Ранее в П.9,1 было получено выражение (9.15) для амплитудночастотной характеристики той же системы при вынужденных колебаниях 
под действием периодической силы $F_{s}=F_{0} \exp j(\omega t-\varphi)$. Это выражение имеет вид

$$
a_{l}=\frac{F_{s}}{\sqrt{U^{2}\left(\omega, m_{l}, a_{l}\right)+V^{2}\left(\omega, m_{l}, a_{l}\right)}}=\frac{F_{s}}{\left|W\left(j \omega, m_{l}, a_{l}\right)\right|}
$$

Сравнивая соотношения (14.14) и (14.15), видим, что при $G=\pi F_{0} / 4$ совокупность амплитуд и частот автоколебаний, получаемых при различных величинах фазы, соответствует точкам резонансных кривых рабочего органа $l$ машины. При этом частота автоколебаний определяется фазо-частотной характеристикой системы, описываемой уравнениями (14.9), (14.10).

Величина $V\left(\omega, m_{l}, a_{l}\right)$ характеризует приведенные диссипативные свойства колебательной системы. Полагая диссипацию достаточно малой, естественно принять, что наибольшая амплитуда $a_{l}^{*}$ колебаний достигается на частотах $\omega^{*}$, удовлетворяющих условию

$$
U\left(\omega^{*}, m_{l}^{*}, a_{l}^{*}\right)=0
$$

При этом в соответствии с (14.9), (14.10) величина фазы $\phi$ обеспечивающей возбуждение резонансных колебаний с максимальной амплитудой

$$
\mathrm{U}\left(\omega^{*}, \mathrm{~m}_{l}^{*}, a_{l}^{*}\right)=0
$$

С учётом (14.16) из (14.15) находим максимальную амплитуду автоколебаний

$$
a_{l}^{*}=\frac{4 G}{\pi V\left(\omega^{*}, m_{l}^{*}, a_{l}^{*}\right)}
$$

Система уравнений (14.16), (14.18) с учетом связи (14.7) определяет величины частоты $\omega^{*}$ и амплитуды $a_{l}^{*}$ автоколебаний. Заметим, что эти уравнения совпадают с полученными в П.9.1 уравнениями, определяющими резонансное состояние системы при вынужденных колебаниях. Уравнение (14.16) определяет скелетную кривую, а уравнение 
(14.18) - линию предельных амплитуд.

Таким образом, при фазе (14.17) в цепи обратной связи при любых изменениях параметров колебательной системы и технологической нагрузки устанавливается режим автоколебаний с максимально достижимой амплитудой, принадлежащей линии предельных амплитуд, т.е в системе автоматически поддерживается наиболее эффективное резонансное состояние. Именно такое возбуждение и называется авторезонансным [7].

Для исследования устойчивости найденных авторезонансных режимов воспользуемся приёмом, изложенным в П.9.1. Полагаем, что в окрестности установившегося периодического решения (14.1) колебания рабочего органа машины имеют вид

$$
\breve{u}_{l}(t)=\breve{m}_{l}(t)+\breve{a}_{l}(t) \exp j \breve{\omega}(t) t
$$

где $\breve{m}_{l}(t), \breve{a}_{l}(t), \breve{\omega}(t)-$ медленно меняющиеся функции Рассматривая равенство (14.10) как приведенное условие баланса энергии диссипативных и возмущающих сил на исследуемом стационарном движении, сформулируем условие устойчивости этого движения по отношению к малым отклонениям амплитуды в виде

$$
\left(\frac{d \operatorname{Im} Q}{d \breve{a}_{l}}\right)_{\widetilde{a}_{l}=a_{l}}>0
$$

Неравенство (14.20) означает, что при отклонении амплитуды $\breve{a}_{l}$ от стационарного значения $a_{l}=$ const баланс энергии нарушается таким образом, чтобы скомпенсировать это отклонение. С учетом принятых обозначений Это условие приводится к следующему:

$$
\frac{d}{d \breve{a}_{l}}\left[\omega b\left(\breve{a}_{l}\right)-K(\omega) Z\left(\breve{a}_{l}\right) \sin \varphi\right]_{\breve{a}_{l}=a_{l}}>0
$$

где $K(\omega)=U_{s l}(\omega) / U_{l l}(\omega)$ - коэффициент, зависящий от расположения места приложения силы возбуждения, причем

$$
\operatorname{sgn} K(\omega) \sin \varphi \geq 0
$$


Применим условие (14.21) для исследования устойчивости некоторых частных случаев. Прежде всего заметим, что согласно (14.13)

$$
\left[\frac{\partial Z}{\partial \breve{a}_{l}}\right]_{\bar{a}_{l}=a_{l}}<0
$$

Если колебательная система работает в режиме холостого хода $(b=0)$ или на технологическую нагрузку, диссипативная составляющая которой линейна ( $\left.b=b_{0}=\mathrm{const}\right)$, условие устойчивости (14.21) выполняется для всех режимов во всем диапазоне существования амплитудно-частотных характеристик. Это в частности означает, что изменением фазы обратной связи в автоколебательном режиме можно реализовать все ветви резонансных кривых, в том числе и неустойчивые при вынужденных колебаниях (см. Параграфы 9 и 12).

Рассмотрим некоторые случаи работы на нелинейную нагрузку.

Пусть на рабочий орган действует сила сухого трения $f_{l}\left(\dot{u}_{l}\right)=Q \operatorname{sgn} \dot{u}_{l}$. Эквивалентный коэффициент (8.61) сопротивления такой нагрузки найден в П.8.6 $b\left(a_{l}\right)=4 Q / \pi \omega a_{l}$, и условие устойчивости (14.21) при $s=l$ $(K(\omega)=1)$ принимает вид $G \sin \varphi>Q$. Легко показать, что это условие совпадает с условием существования вынужденных колебаний в системах с сухим трением [192]. В частности, в авторезонансных режимах колебания существуют при $G>Q$.

Пусть нагрузка на рабочий орган описывается характеристикой жесткопластического материала. При относительно малых силах подачи диссипативная составляющая технологической нагрузки задается выражением (8.72) $b\left(a_{l}\right)=\frac{2 \pi P^{2}}{\omega a_{l} D}$, и условие устойчивости режимов принимает вид $G \sin \varphi>\frac{(\pi P)^{2}}{2 D}$. При $\varphi=\pi / 2$ это условие совпадает с энергетическим условием существования резонансных виброударных режимов при данном уровне возбуждения. При выполнении этого условия 
в автоколебательных режимах изменением фазы обратной связи могут быть реализованы обе ветви амплитудно-частотной характеристики в частотном диапазоне определяемым данным неравенством.

Реализация автоколебаний наиболее просто обеспечивается при выполнении условий самовозбуждения колебаний системы. Самовозбуждение происходит, если теряет устойчивость состояние статического равновесия. Найдём условия устойчивости статического равновесия методом D-разбиения [150]. C этой целью проведем линеаризацию функций $(14.2),(14.3)$ в окрестности равновесного состояния

$$
f\left(u_{l}, p u_{l}\right) \approx f_{0}+\left(k_{0}+p \beta_{0}\right) u_{l}^{0}, F_{s}\left(u_{l}^{0}\right) \approx Z_{0} u_{l}^{0}
$$

где $Z_{0}, k_{0}, \beta_{0}$ - постоянные коэффициенты при первых членах разложения функций (14.2),(14.3) в ряды Тейлора.

По аналогии с уравнением (14.8) имеем

$$
\left[1+L_{l l}(p)\left(k_{0}+p \beta_{0}\right)-L_{s l}(p) Z_{0} \exp (p \varphi / \omega)\right] u_{l}^{0}=Q(p) u_{l}^{0}=0
$$

Потере устойчивости линейной системы (14.25) соответствует появление нулевого или пары чисто мнимых корней еe характеристического уравнения $Q(p)=0$. Подставляя в последнее $p=0$ и $p=j \omega$, получим следующие параметрические уравнения границ $D$ разбиения плоскости параметров $Z_{0}, \phi$

$$
Z_{0}=\left[1+k_{0} L_{l l}(0)\right] / L_{s l}(0) ; Z_{0}=U_{0}(\omega) / \cos \varphi, Z_{0}=V_{0}(\omega) / \sin \varphi
$$

где $U_{0}(\omega)=\operatorname{Re} W_{0}(j \omega), V_{0}(\omega)=\operatorname{Im} W_{0}(j \omega), W_{0}(j \omega)=\left[1+L_{l l}(j \omega)\left(k_{0}+j \omega \beta_{0}\right)\right] / L_{s l}(j \omega)$.

Последние два соотношения в (14.26) можно получить непосредственно из (14.9), (14.10), заменив коэффициенты гармонической линеаризации значениями (14.24). Используя частоту $\omega$ в качестве параметра, для каждого его значения из двух уравнений (14.26) находятся величины $Z_{0}$ и $\phi$, принадлежащие границе самовозбуждения. В силу (14.17) правые части (14.26) являются конечными величинами, поэтому 
синтезированная релейная характеристика, для которой $Z_{0}=\infty$, обеспечивает самовозбуждение и выход системы на периодический режим. Другие случаи характеристик цепей обратной связи будут рассмотрены ниже.

2. Выше в Главе 2 было показано, что исследование колебательных систем ряда ультразвуковых технологических машин под нагрузкой приводит к рассмотрению продольных колебаний вязкоупругого стержня с ограничителем. На основе этой модели рассмотрим основные свойства и возможности авторезонансного возбуждения колебаний таких машин.

Динамическая модель системы показана на рис.14.2,a.
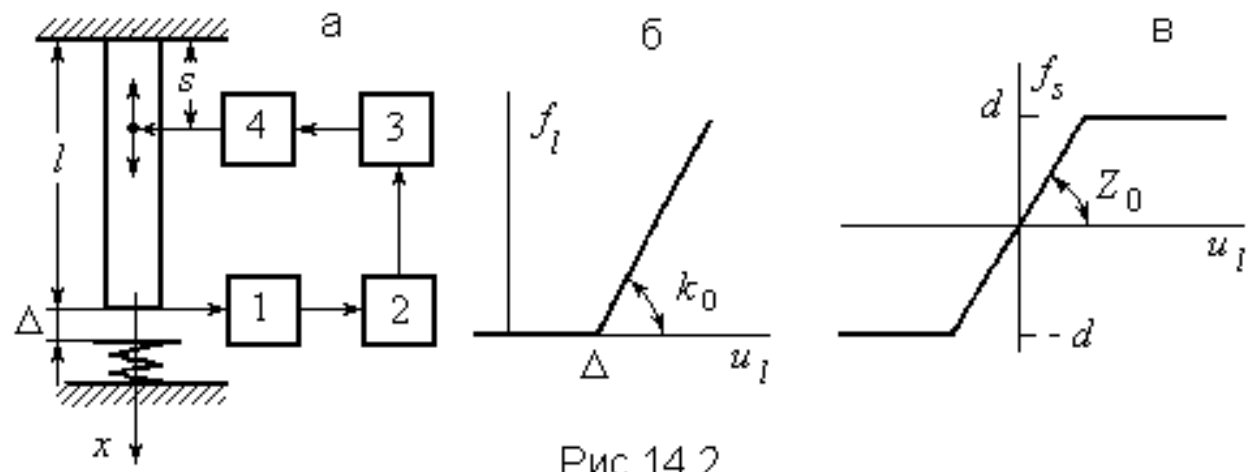

Вязкоупругий стержень длиной $l$ совершает продольные колебания под действием силы $F_{s}$, приложенной в некотором сечении $x=s$. Верхний конец $x=0$ стержня жестко закреплен, а нижний $x=I$ взаимодействует с упругим ограничителем, установленным с зазором $\Delta$ по отношению к положению статического равновесия стержня (отрицательное значение $\Delta$ соответствует натягу). Возбуждающая сила создается при помощи охватывающей стержень цепи обратной связи. Она содержит датчик 1 , регистрирующий перемещение сечения $x=l$, усилитель 2 , фазовращатель 3 и преобразователь 4, создающий силовое воздействие.

Найдем условия самовозбуждения и параметры периодических движений стержня, взаимодействующего через зазор (натяг) $\Delta$ с упругим ограничителем жесткости $k_{0}$ (рис.14.2,б). Пусть сила возбуждения приложена к нижнему сечению $(s=l)$ стержня. Предполагается, что цепь 
обратной связи имеет характеристику линейного усилителя с насыщением (рис.14.2,в).

Используя приведенные в П.9.2 выражения (9.22), (9.23), получим для рассматриваемого случая

$$
W(j \omega)=\frac{E S}{l}\left[\xi \cdot \operatorname{ctg} \xi+q+j \frac{\psi \cdot \xi}{4 \pi} \cdot \frac{(\xi+0,5 \sin 2 \xi)}{\sin ^{2} \xi}\right]
$$

где $\xi=\omega l / c ; c=\sqrt{E / \rho}-$ скорость распространения звука в материале стержня; $E, \rho-$ модуль упругости и плотность материала стержня; $S-$ площадь его поперечного сечения; $\psi$ - коэффициент поглощения; $q=k l / E S$.

Подставив соответствующие выражения из (14.27) в соотношения (14.26), получим следующие параметрические уравнения границ $D$ разбиения:

$$
\begin{gathered}
z_{0}=1+Q_{0} \\
z_{0} \cos \varphi=\xi \operatorname{ctg} \xi+Q_{0} \quad z_{0} \sin \varphi=\psi \xi(\xi+0,5 \sin 2 \xi) /\left(4 \pi \sin ^{2} \xi\right)
\end{gathered}
$$

где $z_{0}=Z_{0} l / E S ; Q_{0}=0$ при $\Delta>0$ и $Q_{0}=q_{0}$ при $\Delta<0 q_{0}=k_{0} l / E S$.

На рис.14.3,а показана картина D-разбиения в плоскости параметров $z_{0}, \varphi$ для системы с зазором $(\Delta>0)$ при изменении $\xi$ от 0 до 2.

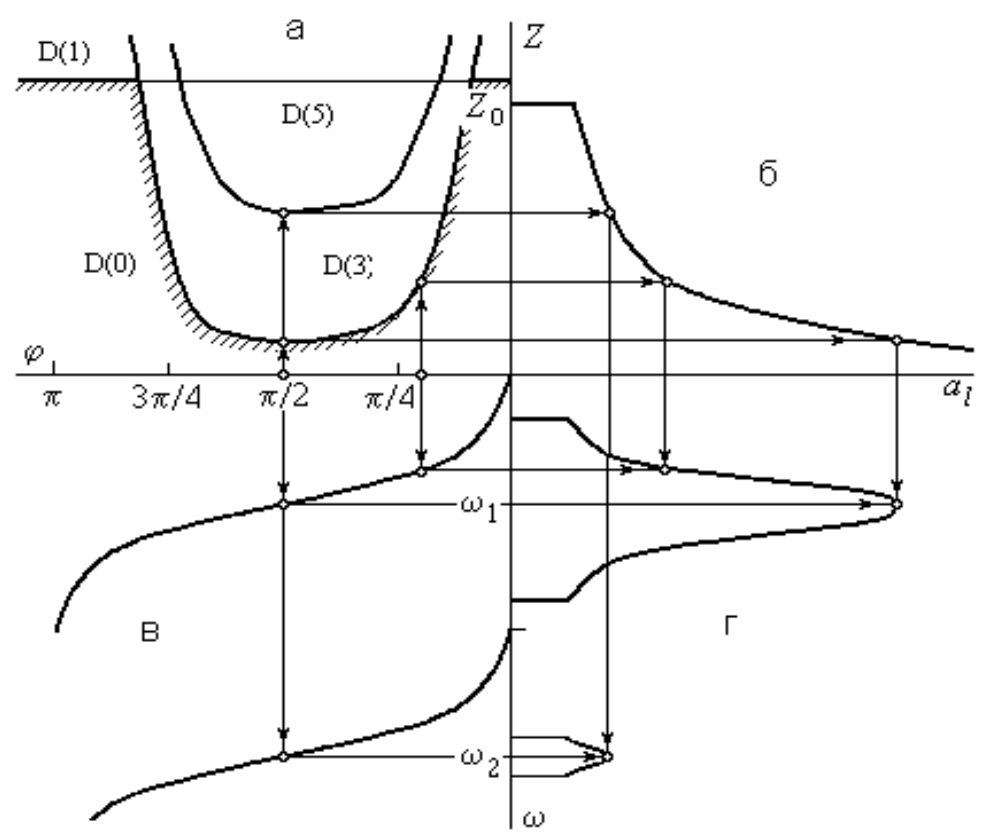

Рис. 14.3 
Штриховкой отмечена область устойчивости равновесного состояния, в которой характеристическое уравнение не имеет корней с положительной действительной частью.

При переходе через границу (14.28) D-разбиения в сторону увеличения $z_{0}$ появляется один положительный корень характеристического уравнения, а через границу (14.29) - пара комплексных сопряженных корней с положительной действительной частью. Область пространства параметров, в которой характеристическое уравнение имеет $i$ корней с положительной действительной частью, обозначена через $\mathrm{D}(i)$. В областях $i>1$ происходит самовозбуждение колебаний с нарастающей амплитудой. При этом ограничение амплитуд и установление периодических колебаний происходит за счет нелинейности цепи обратной связи.

Найдем параметры установившихся движений, амплитуды которых не выходят за пределы линейной зоны статической характеристики ограничителя (рис. $14,2, б)$, т.е. $a_{l}<\Delta$ при $\Delta>0$ и $a_{l}<-\Delta /\left(1+q_{0}\right)$ при $\Delta<0$.

Используя соотношения (14.9), (14.10) с учетом (14.27), получим

$$
z\left(a_{l}\right) \cos \varphi=\xi \operatorname{ctg} \xi+\mathrm{Q}_{0}, \quad z\left(a_{l}\right) \sin \varphi=\psi \xi(\xi+0,5 \sin 2 \xi) /\left(4 \pi \sin ^{2} \xi\right)
$$

Гармонической коэффициент усиления цепи обратной связи с нелинейной характеристикой (рис.14.2,в) при $a_{l} \geq G / Z_{0}$ имеет вид

$$
Z\left(a_{l}\right)=\frac{2 G}{\pi a_{l}}\left(\frac{a_{a} Z_{0}}{G} \arcsin \frac{G}{a_{a} Z_{0}}+\sqrt{1-\left(\frac{G}{a_{a} Z_{0}}\right)^{2}}\right)
$$

Эта зависимость построена на рис.14.3,б.

Из уравнений (14.30) найдем следующее соотношение, определяющее частоту периодического режима:

$$
\operatorname{tg} \varphi=\frac{\psi \xi(\xi+0,5 \sin 2 \xi)}{4 \pi \sin ^{2} 2 \xi\left(\xi \operatorname{ctg} \xi+Q_{0}\right)}
$$


С учетом этого на рис.14.3,в построена зависимости $\xi(\varphi)$ о выражению (14.32). Из рисунка видно, что частота $\omega$ установившихся автоколебаний в окрестности каждой собственной формы однозначно определяется величиной фазы.

Заметим, что существование одночастотного периодического режима возможно только на границе области устойчивости $\mathrm{D}(0)$ на рис.14.3,a. С учетом этого с помощью построений, показанных на рис.14.3 стрелками, находятся частота и амплитуда автоколебаний. С этой целью для заданного значения фазы $\phi$ из рис 14.3, в находим частоту $\xi$, а из рис.14.3,a величину коэффициента усиления $Z$, отвечающего условию существования периодического режима, и затем соответствующую амплитуду $a_{l}$ (рис.14.3,б). Изменяя фазу $\phi$, можно получить всю совокупность параметров $\xi, a_{l}$, реализуемых периодических режимов, которую удобно представить в виде амплитудно-частотной характеристики, показанной на рис.14.3,г. В предыдущем параграфе было показано, что при релейной характеристике цепи обратной связи она совпадает с резонансной кривой системы в режиме вынужденных колебаний при вынуждающей силе $F_{l}=4 G / \pi$. Поскольку в областях выполнения условий самовозбуждения гармонические коэффициенты усиления рассматриваемой и релейной характеристик близки между собой, то близки и соответствующие им амплитудно-частотные характеристики. Поэтому при отыскании и анализе периодических движений целесообразно рассматривать наиболее простой и наглядный случай релейной характеристики усилителя.

Рис.14.3 позволяет получить отчетливое представление о процессе развития колебаний при плавном изменении фазы. Каждому значению $\varphi$ соответствует единственная точка амплитудно-частотной характеристики (рис.14.3,г). Изменением запаздывания можно реализовать ветвь 
резонансной кривой, соответствующую первой форме колебаний (на рис. 2 они показаны для двух низших форм). При уменьшении коэффициента усиления $Z_{0}$ частотная область периодических движений все более сужается в окрестности резонансной частоты. Использование усилителя с большим коэффициентом усиления приводит к расширению частотного диапазона главным образом в область высоких частот.

Все сказанное выше в равной степени справедливо и для системы, установленной с натягом $(\Delta<0)$, при еe колебаниях в пределах предварительной деформации ограничителя $a_{l}<-\Delta /\left(1+q_{0}\right)$.

Здесь уместно заметить, что увеличение Начального коэффициента усиления $Z_{0}$ приводит к возможности возникновения многочастотных режимов. Для их исключения при возбуждении автоколебаний в окрестности первой собственной частоты $\omega_{1}$ можно уменьшать коэффициент $Z_{0}$, а это в свою очередь приводит к уменьшению частотного диапазона возбуждаемых колебаний. С другой стороны в практике построения ультразвуковых технологических систем часто возникает необходимость возбуждения высших форм колебаний, для реализации которых необходимо увеличивать коэффициент усиления $Z_{0}$ в контуре обратной связи. Поэтому для выделения нужной формы колебаний в цепь обратной связи приходится вводить полосовой фильтр, который может существенно исказить фазо-частотную характеристику системы и ухудшить ее свойства, как системы стабилизации резонансных режимов. И хотя эта проблема вполне решается с помощью средств современной электроники, такой путь приводит к некоторому усложнению электрической части системы и ее настройки.

3. Задачу выделения необходимой формы колебаний в ряде случаев удается решить иным путем, если в качестве фазовращателя в контуре обратной связи использовать звено временного запаздывания. Это звено 
естественным образом организуется путем применения в качестве датчика обратной связи микрофона, регистрирующего излучение звука одним из элементов колебательной системы. Запаздывание $t_{0}$ в цепи обратной связи определяется временем прохождения звуковой волной расстояния от излучающего элемента до принимающего звуковой сигнал микрофона.

В этом случае задача сводится к исследованию автоколебаний распределенных нелинейных систем с запаздыванием в цепи обратной связи. Автоколебания линейного осциллятора при действии запаздывающей силы рассмотрены в $[8,96,162,174]$. В рассматриваемых в данном Параграфе задачах оказываются справедливыми все полученные выше соотношения, в которых следует положить $\varphi=-\omega t_{0}$.

На рис.14.4,a показана картина D-разбиения в плоскости параметров $Z_{0}, \tau$ $\left(\tau=t_{0} c / l\right)$ для системы с зазором $(\Delta>0)$ при изменении частоты $\xi$ от 0 до $2 \pi$.

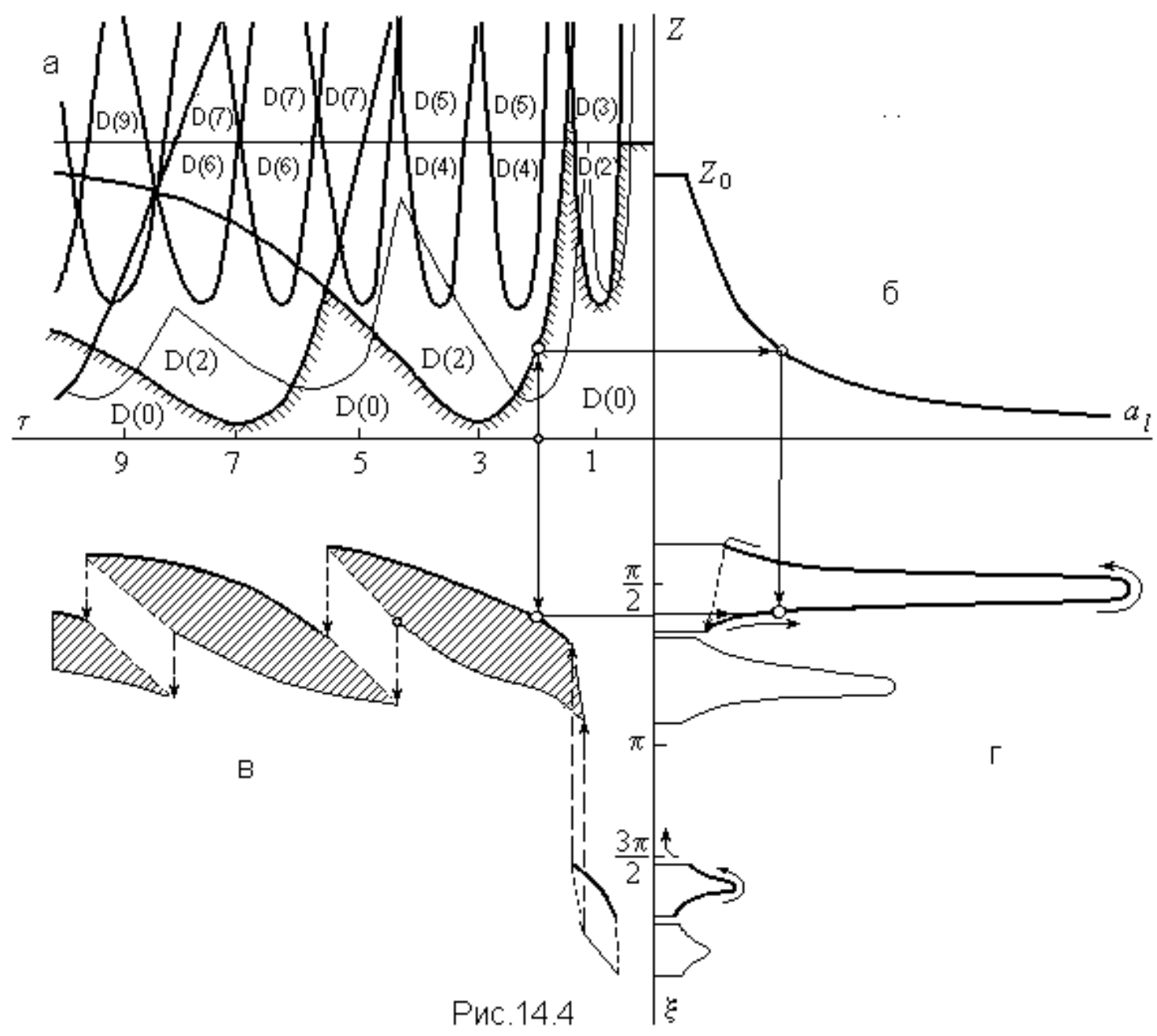


Штриховкой отмечена область устойчивости равновесного состояния, в которой характеристическое уравнение не имеет корней с положительной действительной частью. При переходе этой границы в сторону увеличения коэффициента $Z_{0}$ появляется пара комплексно сопряженных корней характеристического уравнения с положительной вещественной частью. В областях $\mathrm{D}(\mathrm{i})$, где $i$ - количество таких корней в данной области, происходит самовозбуждение колебаний с нарастающей амплитудой, которая ограничивается за счет нелинейности усилителя.

На рис.14.4.б приведена зависимость гармонического коэффициента усиления цепи обратной связи от амплитуды $a_{l}$ колебаний рабочего органа.

Существование одночастотного периодического режима возможно лишь на границе области устойчивости. С учетом этого на рис.14.4,в нанесены участки зависимости $\xi(\tau)$ по выражениям (14.29), отвечающие указанному условию существования. Таким образом, частота $\xi$ однозначно определяется величиной запаздывания $\tau$ при всех значениях кроме бифуркационных, в которых частота изменяется скачкообразно. Амплитуда колебаний определяется при помощи построений, показанных на рис.14.4 стрелками: для заданного значения $\tau_{0}$ находятся величины $Z_{0}$ (рис.14.4,a) и $\xi \quad$ (рис.14.4,в), а затем амплитуда колебаний $a$ (рис.14.4.б) и соответствующая точка амплитудно-частотной характеристики (рис.14.4,г).

Как видно из приведенных построений, изменением запаздывания можно реализовать ветви резонансных кривых, соответствующие различным формам колебаний (на рис.14.4,г они показаны для двух низших форм). По мере возрастания $\tau$ колебания возбуждаются во все более сужающейся окрестности первой собственной частоты. При достаточно малых значениях коэффициента усиления $Z_{0}$ зоны периодических движений могут чередоваться с областями статической 
устойчивости. $\mathrm{C}$ другой стороны при значительных $Z_{0}$ в прохождения бифуркационных значений $\tau$ происходят срывы колебаний либо с одной формы на другую, либо в пределах одной формы, как показано пунктирными стрелками на рис.14.4,в,г. Использование усилителя с с большим коэффициентом усиления приводит к расширению частотного диапазона главным образом в область высоких частот. Наибольший частотный диапазон реализуется в случае усилителя с релейной характеристикой.

Bсе сказанное справедливо и для системы с натягом $(\Delta<0)$ при колебаниях в пределах предварительной деформации ограничителя $\left(a_{l} \leq-\Delta /\left(1+q_{0}\right)\right)$. Для нее на рис.14.4 тонкими линиями показаны граница самовозбуждения (рис.14.4,a), зависимость частоты периодических колебаний от запаздывания (рис.14.4,в), и амплитудно-частотная характеристика (рис.14.4,г). Штриховкой на рис.14.4,в отмечены области (рис.14.4,в), существования периодических режимов при изменении жесткости ограничителя от 0 до q. Штриховые линии, ограничивающие эти области являются бифуркационными границами.

4. Перейдем к отысканию параметров периодических режимов, при которых нижнее сечение стержня совершает колебания с отрывами от ограничителя. При рассмотрении ограничимся случаем релейной характеристики усилителя цепи обратной связи. Уравнения (14.9), (14.10) с учетом выражений для гармонического коэффициента усиления (14.13) и приведенной динамической жесткости (14.27) принимают вид

$$
\begin{gathered}
a_{l}=\frac{\delta \cos \xi \tau}{\xi \operatorname{ctg} \xi+q} \\
a_{l}=-\frac{4 \pi \delta}{\psi \xi} \frac{\sin ^{2} \xi \sin \xi \tau}{\xi+0,5 \sin 2 \xi}
\end{gathered}
$$

где $\delta=4 G l / \pi E S$.

Из (14.33), (14.34) находим амплитуду автоколебаний 


$$
a_{l}=\delta\left\{(\xi \operatorname{ctg} \xi+q)^{2}+\left[\frac{\psi \xi}{4 \pi} \cdot \frac{(\xi+0,5 \sin 2 \xi)}{\sin ^{2} \xi}\right]^{2}\right\}^{-1 / 2}
$$

Выражение (14.35) совпадает с уравнением (9.25) амплитудночастотной характеристики стержня $\mathrm{c}$ ограничителем под действием гармонической силы $F_{l}=4 G / \pi$, приложенной в нижнем сечении стержня. На рис.14.5,a амплитудно-частотные характеристики построены для различных способов установки ограничителя по методике, изложенной в Параграфе 9.

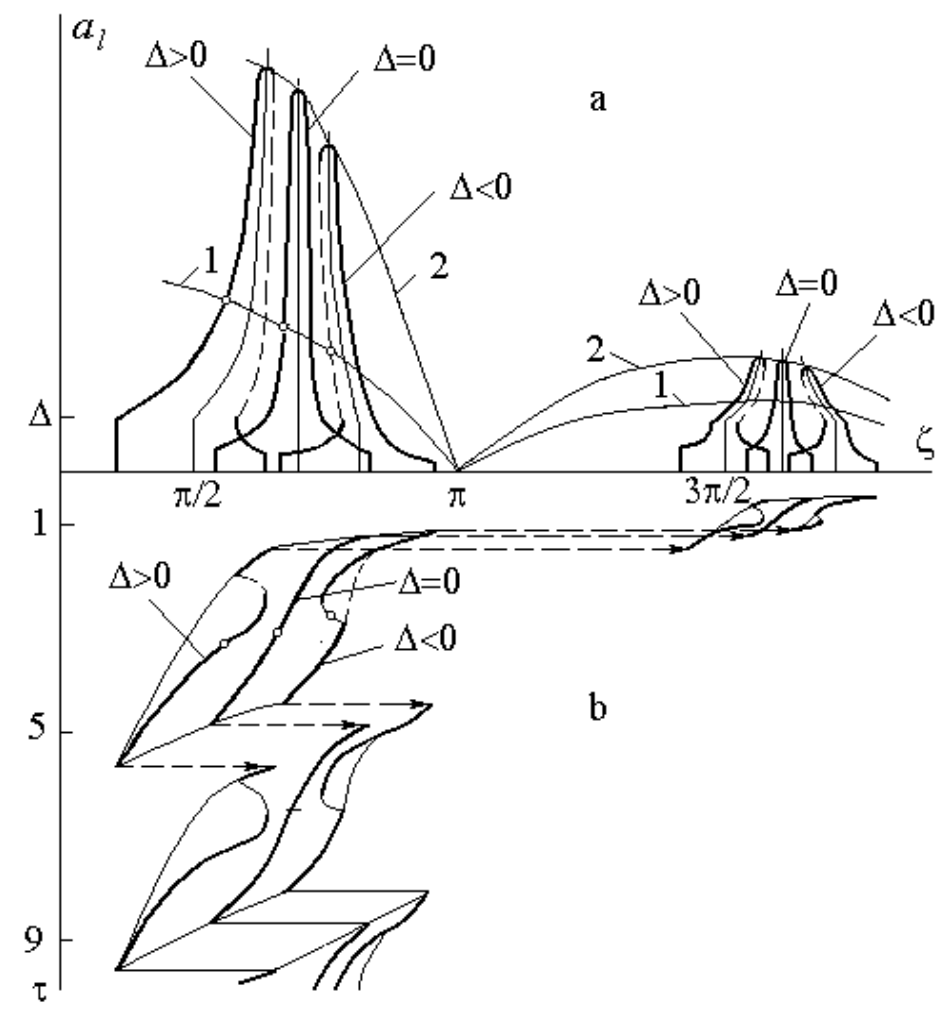

Рис.14.5

Зависимость частоты $\xi$ автоколебаний от запаздывания $\tau$ находится совместным решением уравнений (14.34) и (14.35). Заметим, что равенство $\xi \operatorname{ctg} \xi+q=0$, согласно (14.35), определяет конфигурацию скелетных кривых, показанных на рис.14.4,а штрихпунктирными линиями. Используя (14.33), (14.34), можно показать, что значениям $\pi \leq \xi \tau \leq 3 \pi / 2$ соответствуют ветви резонансных кривых, расположенные справа, а значениям $3 \pi / 2 \leq \xi \tau \leq 2 \pi-$ слева от скелетных кривых. С учетом этого обстоятельства точки пересечения (рис.14.5,a) резонансных кривых с линией 1 , построенной по 
зависимости (14.34) при $\xi \tau=$ const, определяют соответствующее данной фазе значение частоты $\xi$ автоколебаний. Найденная таким образом зависимость частоты $\xi$ автоколебаний от запаздывания $\tau$ в цепи обратной связи показана на рис.14.5,б.

При 2 из (14.36) получим уравнение кривой 2 предельных амплитуд

$$
a_{l}=\frac{4 \pi \delta}{\psi \xi} \frac{\sin ^{2} \xi}{\xi+0,5 \sin 2 \xi},
$$

пересечение которой со скелетными линиями дает, согласно (14.35), высшие точки амплитудно-частотных характеристик. Следует отметить, что величина коэффициента гармонической линеаризации $q$ находится в пределах $0 \leq q \leq q_{0}$. Поэтому системой реализуются лишь движения, отвечающие найденным в конце предыдущего пункта условиям существования. Области существования, отмеченные на рис.14.4.в штриховкой, перенесены на рис.14.5,б.

Из рис.14.5 видно, что и в этом случае плавным изменением запаздывания можно реализовать участки резонансных кривых, соответствующие различным формам колебаний. При этом возможны срывы колебаний с одной формы на другую или в пределах одной из форм. Такие перескоки происходят на найденных выше бифуркационных границах.

5. Системы авторезонансного возбуждения, выполненные по схеме рис.14.1, реализованы на экспериментальных установках для ультразвуковой обработки хрупких материалов и ультразвукового точения (см. Параграф 15).

На рис.14.6,a показана схема колебательной системы ультразвукового станка. 

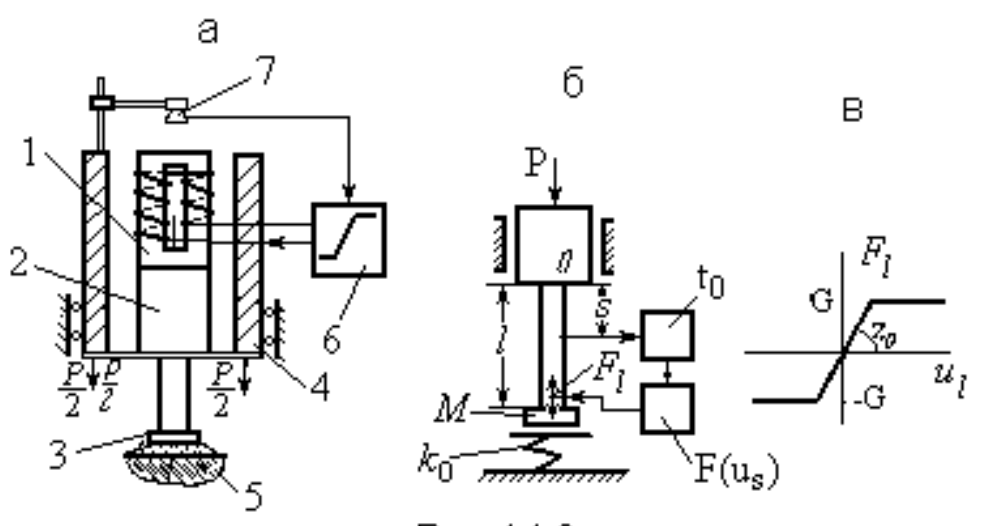

Pис. 14.6

Магнитострикционный преобразователь 1 через ступенчатый концентратор 2 сообщает колебания инструменту 3. Концентратор связан с кареткой 4 в узле колебаний и поджимается к обрабатываемому изделию 5 постоянной силой $P$. Питание преобразователя организуется цепью запаздывающей обратной связи. В этом случае напряжение питания обмоток магнитостриктора формируется усилителем 6 , имеющим характеристику с насыщением и преобразующим сигнал с датчика обратной связи 7. В качестве датчика используется микрофон, установленный на некотором расстоянии от свободного торца магнитостриктора. Время прохождения звуковой волны от излучающего торца до датчика 7 определяет запаздывание в цепи обратной связи. Изменяя уровень насыщения усилителя и время запаздывания, можно регулировать амплитуду и частоту колебаний, а при определенных их соотношениях обеспечить стабилизацию резонансных режимов при изменении нагрузки, определяемой массой инструмента и усилием подачи.

Динамику системы можно описать с помощью динамической модели (рис.14.6,б), представляющей собой однородный стержень длиной $l$, равной длине выходной ступени концентратора, с массой $M$ на конце. Колебания стержня возбуждаются приведенной к его нижнему сечению $x=l$ силой $F_{l}$, которая формируется цепью обратной связи в результате нелинейного преобразования $F_{l}=F_{l}\left(u_{r}\right)$ сигнала, пропорционального смещению сечения $x=r$ и подаваемого с запаздыванием $t_{0}$ в точку 
приложения силы. Для этой модели справедливы все общие соотношения, полученные в этом Параграфе.

На рис.14.7,a по формулам (14.15), (14.27) построены амплитудночастотные характеристики системы для двух низших форм колебаний при различных значениях силы прижима $P$. Здесь для примера принято, что жесткость ограничителя $k_{0} \rightarrow \infty$, т.е. рассмотрен случай, когда его влияние на характер колебаний проявляется наиболее сильно, а коэффициент гармонической линеаризации $k(a)=2 P / a_{l}$. На рисунке штрихпунктирными линиями показаны скелетные кривые, связывающие частоты и амплитуды свободных колебаний и разделяющие ветви резонансных кривых, и а индексом 1 обозначена линия предельных амплитуд, ограничивающая резонансные кривые сверху.

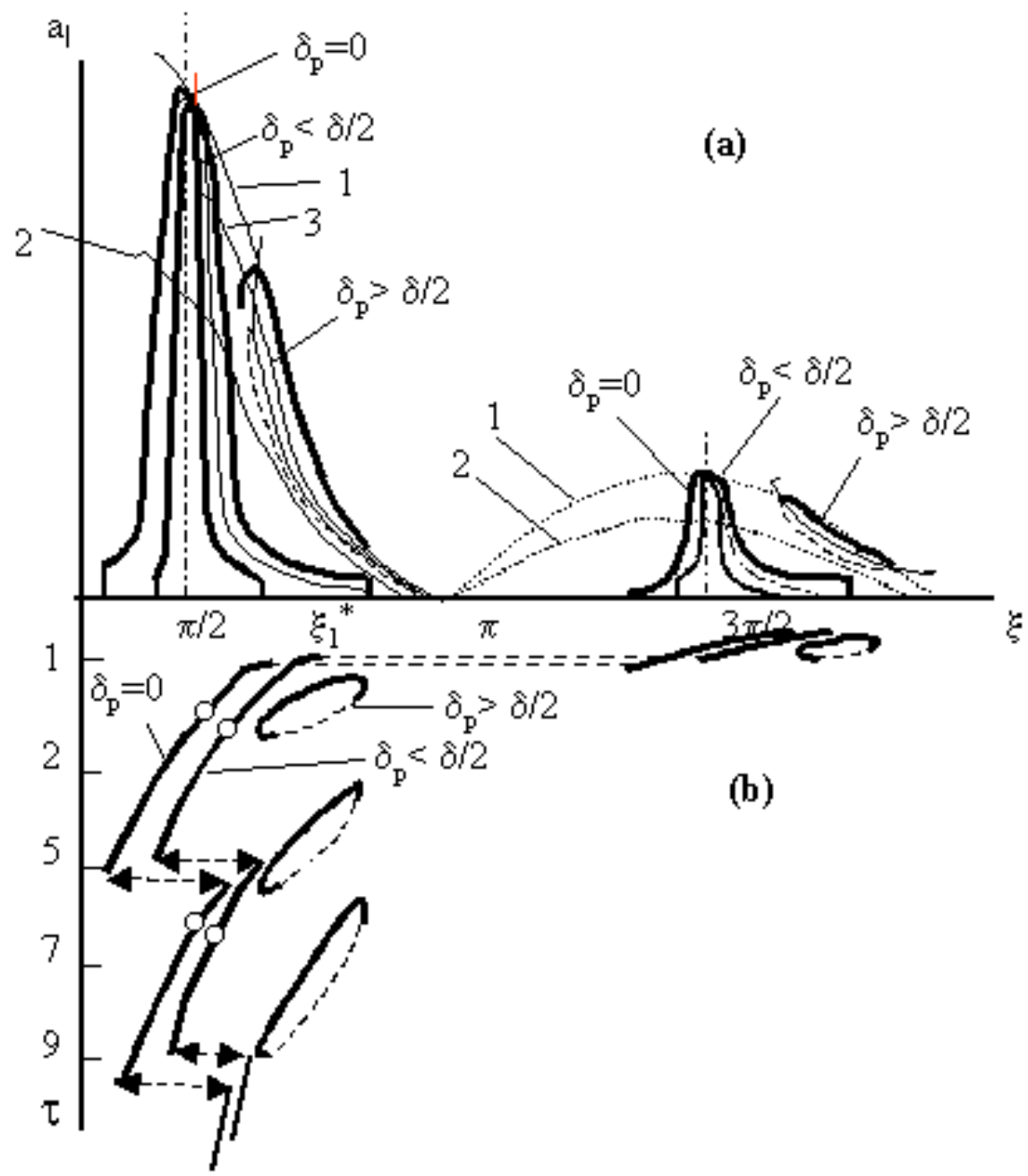

Рис.14.7 
Из рис.14.7,а видно, что при $\delta_{P}=P l / E S=0$ система линейна, и ограничитель не оказывает влияния на ее колебания.

Приложение постоянной силы $P$ смещает максимум резонансной кривой в область более высоких частот, причем при значениях $\delta_{P}>\delta=F l / E S$ существенно изменяется еe характер: она становится двузначной, и с возрастанием $\delta_{P}$ сужается ее частотный диапазон.

Связь между резонансной частотой и постоянной силой определяется выражением:

$$
\frac{\delta_{P}}{\delta}=\frac{2 \pi}{\psi} \frac{\left(\sin 2 \xi-\mu \xi \sin ^{2} \xi\right)}{(2 \xi+\sin 2 \xi)}
$$

Максимумам функции (14.37) соответствуют предельные значения, при которых резонансные кривые вырождаются в точки. При $\mu=0$, например, эти значения для двух первых форм колебаний будут: $\xi_{1}=0,71 \pi$, $\delta_{P 1}=1,75 \delta / \psi ; \xi_{2}=1,74 \pi, \delta_{P 1}=0,76 \delta / \psi$.

Выше было показано, что параметры автоколебаний соответствуют точкам пересечения резонансных кривых с линией 2, определяемой выражением (14.34). На рис.14.7,б построена зависимость частоты автоколебаний от запаздывания. Следует иметь в виду, что значениям $\pi(n-1) \leq \xi \tau<\pi(2 n-1) / 2 \quad(n=1,2, \ldots)$ отвечают ветви резонансных кривых, расположенные справа, а значениям $\pi(2 n-1) / 2<\xi \tau \leq \pi n)-$ слева от скелетных линий, причем четные значения $n$. принимаются при прямом $(\Delta>0)$, а нечетные - при инверсном $(\Delta<0)$ включении усилителя в цепи обратной связи.

В ультразвуковых технологических установках, как правило, необходимо получение резонансных колебаний с максимальными амплитудами. Резонансный режим автоколебаний частоты $\omega^{*}$ (авторезонанс), реализуется при условии $\left|\sin \omega^{*} t_{0}\right|=1$ или

$$
\tau=(2 n-1) \pi / 2 \xi^{*}
$$


Изменение параметров системы (массы инструмента, силы прижима, свойств среды) при постоянном запаздывании вызывает расстройку по частоте и приводит к отклонению амплитуды от ее резонансного значения $a^{*}$. Это отклонение будет минимальным, если из всех значений (14.38) выбрать наименьшее. Полагая $n=1$ и принимая предельное значение резонансной частоты $\xi^{*}=\xi_{1}=0,71 \pi$, найдем из (14.38) настроечное значение $\Delta=0,71$. На рис.14.7,а по уравнению (14.34) построена кривая 3, определяющая амплитуду автоколебаний при данной настройке. Во всем рабочем диапазоне эта настройка обеспечивает отклонение амплитуды от резонансного значения $a^{*}$ не более $10 \%$. Таким образом, рассматриваемая система может быть эффективно использована для стабилизации резонансных режимов при изменении внешней нагрузки в широких пределах.

6. В экспериментах на холостом ходу с помощью пьезодатчика, приклеенного к торцу концентратора, регистрировалась его амплитуда. В результате исследования колебательной системы в режиме вынужденных колебаний была построена амплитудно-частотная характеристика, по которой найдены резонансная частота $f=20,4$ кГц и коэффициент поглощения $\psi=2 \pi / Q$, где $Q=126$ - добротность системы.

Для динамической модели (рис.14.6,б) резонансная частота и амплитуда холостого хода определяются выражениями $f=c / 4 l$, $a_{l}=16 F l / \pi \psi E S, F=4 G / \pi$, приведенная длина стержня составляет $l=63,5$ мм. Все дальнейшие эксперименты проводились при постоянном уровне возбуждения магнитостриктора, при котором амплитуда в резонансном режиме холостого хода $a=10$ мкм, а потребляемая магнитостриктором мощность составляла 60 Вт. Приведенная к нижнему сечению стержня возбуждающая сила $F=10$ кг, а уровень насыщения характеристики цепи 
обратной связи $G=7,9$ кг. (В расчетах приняты следующие значения: $c=5,17 * 10^{5} \mathrm{~cm} /$ сек; $E=2 * 10^{6} \mathrm{\kappa} \Gamma / \mathrm{cm}^{2} ; S=3,14 \mathrm{~cm}^{2}$.)

В автоколебательном режиме на холостом ходу исследовались условия самовозбуждения и параметры установившихся колебаний при изменении запаздывания в широких пределах. Результаты исследования показаны на рис.14.8, где точками представлены экспериментальные данные, наложенные на расчетные кривые.

На рис.14.8,a построена граница самовозбуждения при характеристике с насыщением, подобной изображенной на рис.14.6,в.

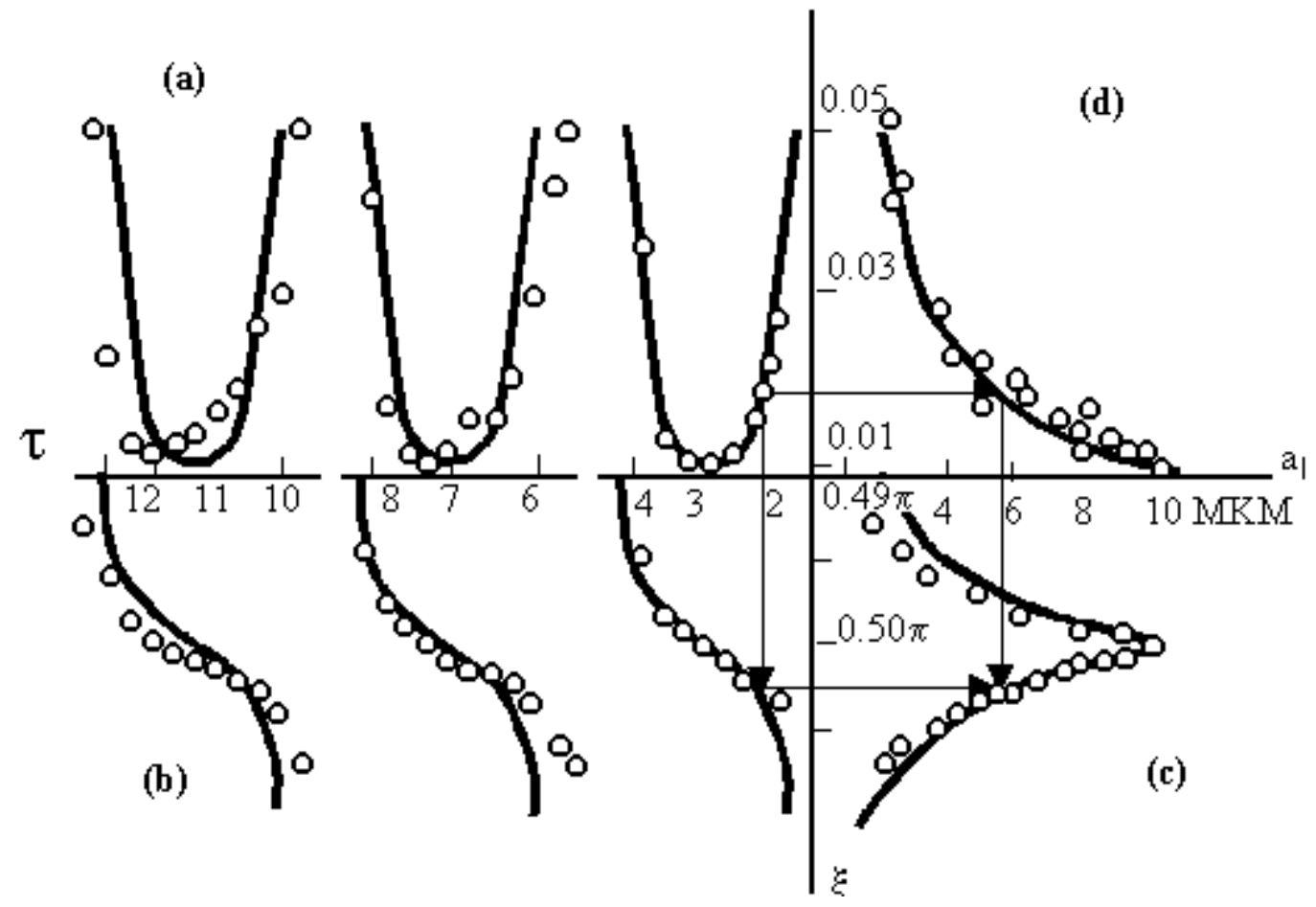

Рис. 14.8

Параметрические уравнения этой границы имеют вид (14.29) при $\varphi=-\xi \tau$. Исключая из этих уравнений величину $Z_{0}$, получим выражение (14.32), определяющее частоту установившихся автоколебаний. На рис.14.8,б приведена зависимость частоты $\xi$ автоколебаний от величины запаздывания $\tau$. Реализуемые при этом амплитуды показаны на рис.14.8,в. Они строго соответствуют амплитудно-частотной характеристике, полученной в режиме вынужденных колебаний. Теоретическая 
резонансная кривая на построена по уравнениям (14.15) и (14.27) при $q=0$. На рис.14.8,в представлен график гармонического коэффициента усиления (14.31) и даны экспериментальные значения $Z=k U_{\mathrm{m}} / U_{\text {д }}$, где $U_{\text {м }}$ и $U_{\text {д }}$ - соответственно напряжение на обмотке магнитостриктора и напряжение, снимаемое $\mathrm{c}$ датчика обратной связи; $k=2 \cdot 10^{-6}$ постоянный коэффициент, вычисленный по значениям, соответствующим авторезонансу. Приведенные на рис.14.8 кривые связаны построениями, показанными стрелками.

В процессе ультразвукового резания при нагружении колебательной

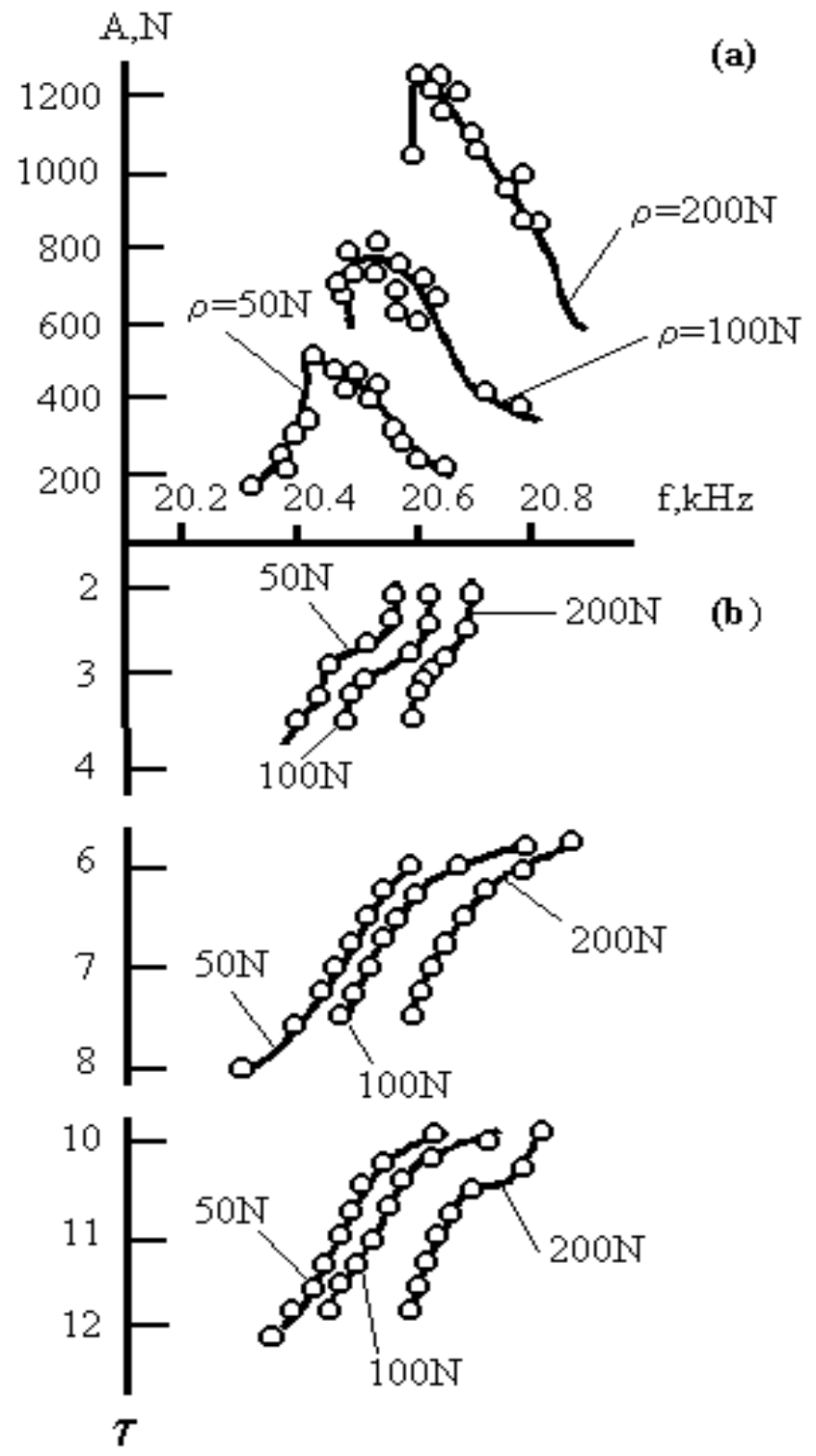

Рис.14.9 системы постоянной силой $P$ регистрировалась сила взаимодействия между инструментом и обрабатываемым изделием. В качестве датчика был использован пьезоэлемент, вклеенный между массивным основанием и обрабатываемой стеклянной пластиной, помещенной в абразивную суспензию (см. Параграф 12).

Результаты эксперимента, полученные при различных значениях силы Р, представлены на рис.14.9, иллюстрирующем характер изменения амплитуды $A$ силы ударного взаимодействия 
(рис.14.9,a) и частоты автоколебаний $f$ (рис.14.9,б) при плавном изменении величины запаздывания.

Следует отметить, что в экспериментах как на холостом ходу, так и под нагрузкой, подтвердился теоретический вывод о соответствии амплитуд и частот автоколебаний резонансным кривым, получаемым в режиме вынужденных колебаний. При этом наблюдались описанные выше нелинейные эффекты. Значение силы $P=5$ кг при заданном уровне возбуждения $F=10$ кг является тем граничным значением, при котором резонансная кривая (рис.14.7,a) еще однозначна во всем диапазоне существования. Превышение этого значения приводит к появлению второй ветви и срывам колебаний на определенных частотах.

Резонансные колебания, соответствующие максимумам амплитудночастотных характеристик, были реализованы при постоянном запаздывании $\tau=3$. Экспериментальные зависимости частоты автоколебаний и амплитуды силы удара от постоянной силы показаны на (рис.14.10).

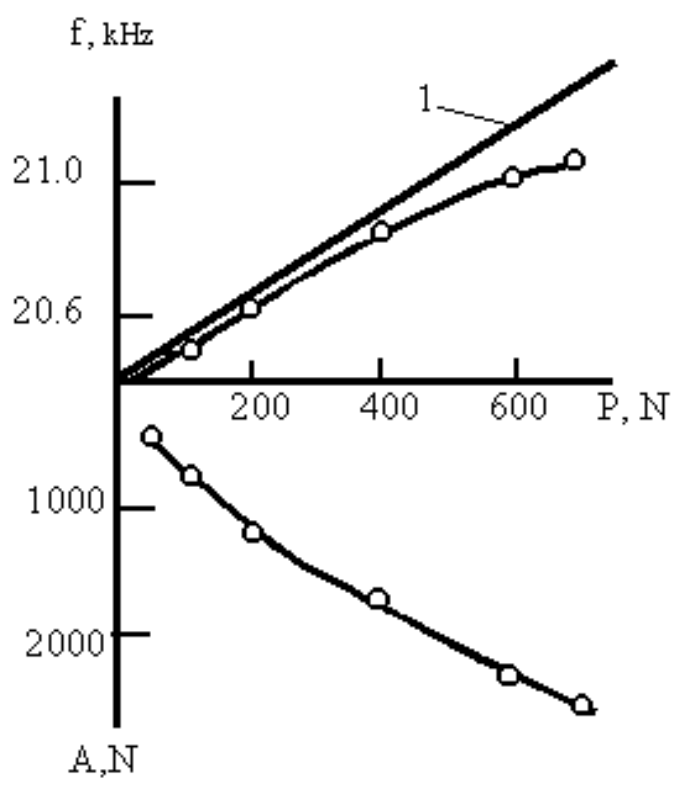

Рис. 14.10

Здесь же для сравнения дан результат расчета по формуле (14.37) при $\mu=0$ (линия 1 ).

Следует подчеркнуть, что система самовозбуждается и выходит на 
резонансный режим без какой-либо дополнительной подстройки при всех указанных на графиках значениях постоянной силы. Достигаемый при это эффект показан в столбце АР Таблицы 12.1. Испытания показали, что предложенная система возбуждения и автоматической стабилизации резонансных режимов эффективно работает и при изменении других параметров: массы присоединенного инструмента, жесткости и размеров изделия, температуры и пр. 


\section{5. Ультразвуковое резание как нелинейный (виброударный) процесс}

1. Многие современные материалы, такие как высокопрочные, жаропрочные и нержавеющие стали и сплавы, титан, керамические и другие неметаллические материалы и т.л., трудно поддаются обработке традиционными методами. Обработка деталей из этих материалов на обычных металлорежущих станках приводит к скалыванию или разрушению поверхностного слоя детали и не позволяет получить необходимое качество поверхности. С другой стороны, известно, что при обработке резанием таких мягких материалов как чистые медь и алюминий происходит налипание материала и образование нароста на режущей кромке инструмента, приводящее к ухудшению качества поверхности

Улучшение качества обработки на станке может быть достигнуто путем преобразования процесса резания за счет возбуждения высокочастотной (ультразвуковой) вибрации режущей кромки инструмента. Именно такой процесс и называется ультразвуковым [141143] (или вибрационным [130]) резанием. Схема процесса ультразвукового резания и некоторые экспериментально обнаруженные эффекты при его реализации были описаны в П.1.4. Одним из таких эффектов является снижение силы резания при воздействии вибрации. Этот эффект наиболее сильно проявляется в том случае, когда направление вибрации совпадает с направлением скорости резания. Здесь, как и ранее речь идет, конечно о постоянной составляющей силы резания о которой судят по величине момента $M$, развиваемого приводом.

В настоящем параграфе эти эффекты объясняются C помощью предлагаемой реологической модели процесса ультразвукового резания. Устанавливается, что под влиянием высокочастотной вибрации, как и в других рассмотренных ранее процессах, происходит феноменологическое преобразование упругопластических характеристик материала в 
вязкопластические и сухого трения в вязкое. Строятся динамические характеристики преобразованного таким образом процесса резания. Выявляется особая роль виброударных процессов в зоне резания, как наиболее эффективных. Исследуется динамика устройства для ультразвукового резания, и строятся его амплитудно-частотные характеристики. Приводятся результаты экспериментальных исследований. Дается описание авторезонансной системы возбуждения колебаний устройства.

На рис.15.1 показана схема устройства, реализующего процесс резания с наложением ультразвуковых колебаний.

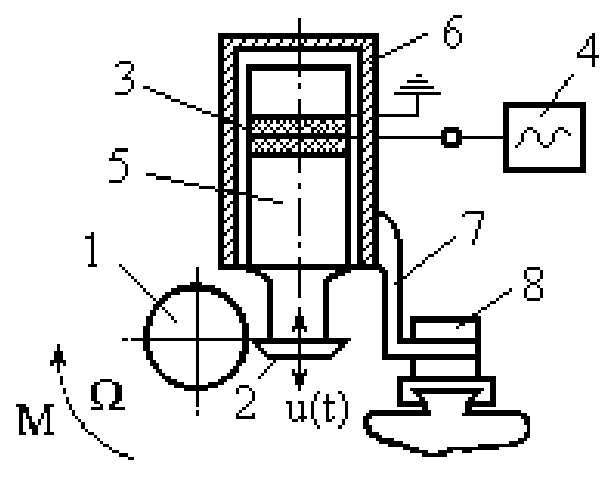

Рис.15.1

Обрабатываемая деталь 1 получает вращение с постоянной угловой скоростью $\Omega$ от привода токарного станка, развивающего постоянный крутящий момент $M$. Обработка детали производится резцом 2 , которому с помощью ультразвуковой колебательной системы сообщаются колебания $u^{0}(t)=a \cos \omega t \quad$ с угловой частотой $\omega$ и амплитудой $a$. При питании пьезоэлектрического (или магнитострикционного) преобразователя 3 от генератора 4 переменного возбуждаются механические колебания, которые через концентратор 5 передаются резцу с увеличением амплитуды. Колебательная система закреплена в корпусе 6 и с помощью кронштейна 7 установлена в резцедержателе 8 токарного станка.

Для достижения максимального эффекта от наложения вибрации на процесс резания необходимо обеспечить резонансную настройку колебательной системы. Однако, как и в рассмотренных ранее процессах, 
резонансные частоты системы существенно зависят от параметров технологического процесса и изменяются в зависимости от режимов резания. Резонансная настройка значительно осложняется в виду того, что технологическая нагрузка на колебательную систему в процессе вибрационного резания оказывается существенно нелинейной. Ниже будет показано, что общая динамическая картина поведения системы в условиях вибрационного резания становится еще более насыщенной нелинейными эффектами, а вызываемые технологической нагрузкой нелинейные искажения амплитудно-частотных характеристик - еще более экзотическими.

2. Силы резания при токарной обработке создаются главным образом за счет пластической деформации срезаемого слоя материала заготовки и преодоления сил трения на рабочих поверхностях резца [97]. При колебаниях резца на его режущих кромках возникает переменная сила резания $F$, направленная в сторону, противоположную скорости $\dot{u}(t)$ относительного движения резца

$$
u(t)=v t+u^{0}(t)=v t+a \cos \omega t
$$

где $v=\Omega r$ - скорость резания; $r-$ радиус точения.

Переменную силу ультразвукового резания опишем с помощью динамической характеристики контактного взаимодействия $f=f(u, \dot{u})$, которую можно представить в виде суммы двух составляющих

$$
f(u, \dot{u})=f_{1}(u, \dot{u})+f_{2}(u, \dot{u})
$$

Первая составляющая $f_{1}(u, \dot{u})$ зависит от упругопластических свойств обрабатываемого материала. При традиционном точении без вибрации эта составляющая силы резания определяется величиной сил пластического деформирования. Рассмотрение процесса вибрационного резания требует учета цикличности нагружения и введения в динамическую характеристику зоны упругого деформирования материала в контактной области. При построении динамической характеристики, как и ранее, будем считать, что при каждом значении скорости резания $v$ устанавливается периодический режим $u^{0}(t)$ колебаний резца, и поэтому 
можно ограничиться рассмотрением взаимодействия резца с заготовкой в течение одного периода колебаний.

Для построения динамической характеристики проведем следующий мысленный эксперимент: при неподвижном инструменте, медленно увеличивая крутящий момент $M$, будем следить за вращением изделия. Если момент $M$ достаточно мал, изделие повернется на некоторый угол и займет положение динамического равновесия, а скорость вращения окажется равной $v=0$. Это означает, что деформация в области контакта не выходит за пределы упругой зоны характеристики материала. В этом случае составляющую $f_{1}(u, \dot{u})$ динамической характеристики можно представить в виде

$$
f_{1}(u, \dot{u})=f_{1}(u)=k_{0}(u-\Delta) \eta(u-\Delta)
$$

где $k_{0}$ - статическая жесткость в зоне контакта резца с заготовкой; $\Delta$ относительная координата режущей кромки в момент начала контактного взаимодействия; $\eta(u)$ - единичная функция.

Эта составляющая не зависит от скорости деформирования и в пределах упругой зоны описывает как цикл нагружения, так и цикл разгрузки.

При увеличении момента $M$ наступает такая ситуация, при которой деформация в контактной области начинает выходить в зону пластической деформации материала. Принимая упругопластическую модель материала, для составляющей $F_{1}(u, \dot{u})$ силы ультразвукового резания запишем в этом случае

$$
f_{1}(u, \dot{u})=k_{0}\left\{\begin{array}{l}
\eta(\dot{u})[(u-\Delta) \eta(u-\Delta)-(u-\Delta-d) \eta(u-\Delta-d)]+ \\
+\eta(-\dot{u})\left(u+d-u_{m}\right) \eta\left(u+d-u_{m}\right)
\end{array}\right\}
$$

или в иной записи

$$
f_{1}(u, \dot{u})=\left\{\begin{array}{lll}
0 & u \leq \Delta & \dot{u} \geq 0 \\
k_{0}(u-\Delta) & \Delta \leq u \leq \Delta+d & \dot{u} \geq 0 \\
D_{0} & \Delta+d \leq u \leq u_{m} & \dot{u} \geq 0 \\
D_{0}+k_{0}\left(u-u_{m}\right) & u_{m}-d \leq u \leq u_{m} & \dot{u} \leq 0 \\
0 & u \leq u_{m}-d & \dot{u} \leq 0
\end{array}\right.
$$


где $D_{0}$ - сила в зоне контакта, соответствующая пределу текучести материала; $d=D_{0} / k_{0} ; u_{m}=a\left\lfloor\sqrt{1-(v / a \omega)^{2}}+(v / a \omega\} \arcsin (v / a \omega)\right\rfloor$ - максимальное в течение одного периода колебаний значение функции (15.1).

Составляющая (15.4) динамической характеристики процесса ультразвукового резания показана тонкой линией 1 на рис.15.2.

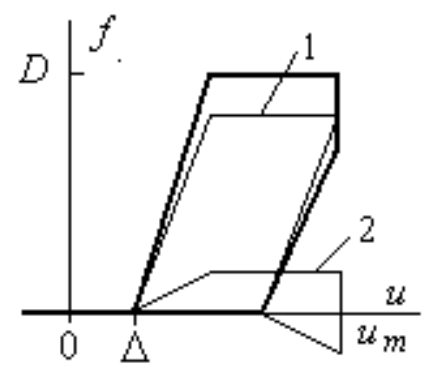

РИс. 15.2

Как видим, она абсолютно аналогична характеристике процесса пластического деформирования, подробно рассмотренного в Параграфе 2.

Вторая составляющая $f_{2}(u, \dot{u})$ динамической характеристики (15.2) определяется силами трения, сопровождающими процесс резания. Предполагается, что эта составляющая пропорциональна $F_{1}(u, \dot{u})$ и направлена противоположно скорости относительного движения резца и изделия. Поэтому эта составляющая описывается следующей зависимостью:

$$
f(u, \dot{u})=f_{1}(u, \dot{u})(1+k \operatorname{sgn} \dot{u})
$$

где $\kappa$ - коэффициент пропорциональности.

На рис.15.2 эта составляющая показана тонкой линией 2.

Суммарную характеристику (15.2) (жирная линия на рис.15.2) взаимодействия можно представить теперь следующим образом:

$$
f_{2}(u, \dot{u})=k f_{1}(u, \dot{u}) \operatorname{sgn} \dot{u}
$$

Характеристика такого вида обсуждалась в П.8.5.

Циклическое взаимодействие резца с деталью происходит следующим образом. При движении инструмента вверх (см. рис.15.1), сила взаимодействия отсутствует до тех пор, пока вершина резца не достигнет 
обрабатываемой детали в точке когда $u=\Delta$. Затем происходит линейное нагружение в зоне $\Delta \leq u \leq \Delta+d$, где материал в контактной области подчиняется закону Гука. После достижения предела текучести, начинается пластическое деформирование материала, т.е. происходит процесс резания с образованием стружки (резание в его общепринятом понимании), который продолжается до момента изменения направления относительного движения, т.е. изменения знака скорости $\dot{u}(t)$. В этот момент перемещение $u(t)$ достигает своего максимального значения $u_{m}$ (см. (15.2)). В течение следующего этапа сила взаимодействия резца с деталью остается постоянной и равной силе резания $f=D_{0}(1+\kappa)$ при традиционном точении без ультразвуковых колебаний. По окончании этого этапа происходит упругая разгрузка до прерывания контакта резца с изделием $(f=0)$.

3. Теперь можно оценить влияние ультразвуковых колебаний на процесс резания. Принимая во внимание, что вследствие периодичности движения инструмента сила взаимодействия $F(t)=f[u(t), \dot{u}(t)]$ является периодической функцией времени с периодом $T=2 \pi / \omega$, с помощью теоремы импульсов запишем, как это было сделано в Параграфах 2 и 3, соотношение, связывающее постоянную силу $P$ с параметрами движения резца и упруго-диссипативными характеристиками процесса

$$
P=\frac{1}{T} \int_{0}^{T} F(t) \mathrm{d} t=\frac{1}{T} \int_{0}^{T} f[u(t), u(t)] \mathrm{d} t
$$

Из (15.4) - (15.7) следует, что при традиционном точении без вибрации $(a=0)$, а также при вибрационном резании со скоростью $v$, превышающей вибрационную скорость $(v \geq a \omega)$, сила взаимодействия $F(t)=D_{0}(1+\kappa)=D$, и, согласно (15.8), для ее преодоления необходима постоянная сила $P=D$. Лри возбуждении колебаний резца, таких что $a \omega>v$, в интервале $0 \leq t \leq T$ сила взаимодействия $F(t) \leq D$, и необходимая 
для проведения процесса резания постоянная сила $P<D$, т.е. происходит наблюдаемое в экспериментах снижение силы резания.

Найдем зависимость статической силы $P$ при вибрационном резании от параметров колебаний инструмента и скорости резания. Подставив (15.5) - (15.7) в (15.8), получим после интегрирования

$$
P=\frac{1}{2 \pi}\left\{\begin{array}{l}
k_{0}(1+\kappa)\left[\frac{v}{2 \omega}\left(\tau_{2}^{2}-\tau_{1}^{2}\right)-\Delta\left(\tau_{2}-\tau_{1}\right)+a\left(\sin \tau_{2}-\sin \tau_{1}\right)\right]+ \\
+k_{0}(1-\kappa)\left[\frac{v}{2 \omega}\left(\tau_{4}^{2}-\tau_{3}^{2}\right)-(\Delta+\delta)\left(\tau_{4}-\tau_{3}\right)+a\left(\sin \tau_{4}-\sin \tau_{3}\right)\right]+D\left(\tau_{3}-\tau_{2}\right)
\end{array}\right\}
$$

где согласно рис. 15.3

$$
\delta=u_{m}-\Delta-d
$$

- длина дуги на поверхности, обработанной в течение одного периода колебаний ; $\tau_{i}=\omega t_{i}, \quad(i=1, \ldots, 4) ; t_{i}$ - моменты переключения участков характеристики (15.5), определяемые следующими уравнениями:

$$
\begin{aligned}
\cos \tau_{1} & =\frac{1}{a}\left(\Delta-\frac{v}{\omega} \tau_{1}\right), \cos \tau_{2}=\frac{1}{a}\left(\Delta+d-\frac{v}{\omega} \tau_{2}\right) \\
\tau_{3} & =\arcsin \frac{v}{a \cdot \omega}, \cos \tau_{4}=\frac{1}{a}\left(\Delta+\delta-\frac{v}{\omega} \tau_{4}\right)
\end{aligned}
$$

причем $\tau_{3} \in[0, \pi / 2], \tau_{1,2} \in\left[-\left(\pi+\tau_{3}\right), \tau_{3}\right], \tau_{4} \in\left[\tau_{3},\left(\pi-\tau_{3}\right)\right]$.

Средняя скорость резания рассчитывается теперь по формуле

$$
v=\frac{\delta}{T}=\frac{\omega}{2 \pi}\left(u_{m}-\Delta-d\right)
$$

Из (15.12) находим координату начала взаимодействия

$$
\Delta=u_{m}-d-2 \pi \frac{v}{\omega}
$$

Равенство (15.9) после подстановки решений уравнений (15.11) с учетом (15.13) определяет статическую силу $P$ в режимах, в которых контакт резца с изделием периодически прерывается, а сила взаимодействия носит характер периодической последовательности импульсов. Такие процессы будем называть виброударными. 
С увеличением скорости $v$ резания время контакта инструмента с обрабатываемой деталью возрастает, и, начиная с некоторого значения скорости резания, виброударные режимы трансформируются в режимы непрерывного вибрационного резания, в которых контакт инструмента с изделием сохраняется в течение всего процесса. Сила резания в режимах непрерывного резания описывается тем же выражением (15.9), в котором следует принять предельные значения моментов переключения. Это соответствует следующим выражениям для моментов перелючения в уравнениях (15.11):

$$
\tau_{1}=-\left(\pi+\arcsin \frac{v}{a \omega}\right), \tau_{4}=\tau_{1}+2 \pi=\pi-\arcsin \frac{v}{a \omega}
$$

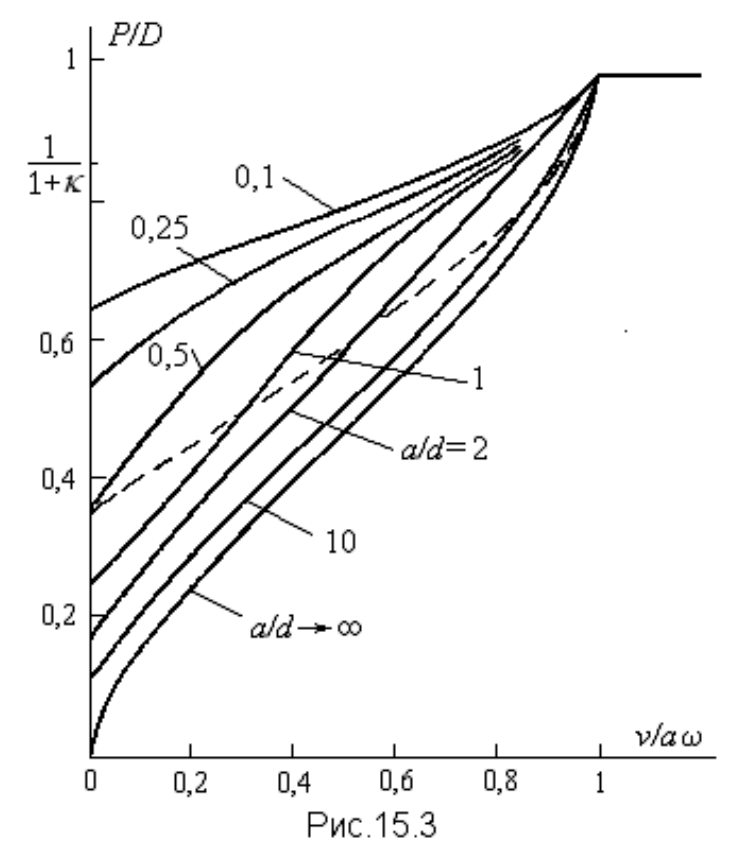

На рис.15.3 приведены зависимости статической силы $P$ от скорости $v$ резания, построенные при различных значениях величины $a / d$.

Штриховая линия разделяет области режимов непрерывного (верхняя) и виброударного (нижняя область) резания. Из рисунка видно, что с увеличением скорости резания сила резания возрастает, достигая значения $P=D$ при скоростях $V \geq a \omega$.

Полученные результаты вполне согласуются с данными экспериментов [35, 104, 126, 137-139], в которых исследовался эффект снижения силы резания при наложении ультразвуковой вибрации на 
режущий инструмент. Это видно из сопоставления рис.15.3 с экспериментальной кривой на рис.1.5, заимствованной из статьи [104].

Наибольший эффект снижения статической силы резания достигается при обработке жесткопластического материала $\left(k_{0} \rightarrow \infty\right)$, которому на рис. 15.3 соответствует кривая, отмеченная индексом $a / d \rightarrow \infty$. В этом случае статическая сила описывается выражением

$$
P=\frac{D}{2 \pi}\left(\tau_{3}-\tau_{1}\right)
$$

в котором момент переключения $\tau_{3}=\arcsin \frac{v}{a \cdot \omega}$, а $\tau_{1}$ - корень уравнения $\cos \tau_{1}=\frac{1}{a}\left(u_{m}-\frac{v}{\omega}\left(2 \pi+\tau_{1}\right)\right)$

Из рис.15.3 видно, что при заданной скорости $v$ сила $P$ уменьшается с увеличением амплитуды $а$ колебаний. При малых амплитудах колебаний $a \leq v / \omega$ сила резания $P=D$, и ультразвук не оказывает влияния на процесс резания. На это обстоятельство обращается внимание во всех известных экспериментах [35,104, 126, 137-139].

Заметим, что при $v=0$ возникает ситуация когда колебания резца происходят в пределах упругой зоны динамической характеристики (15.5), не выходя в зону пластических деформаций, и устанавливается режим динамического равновесия. В граничном случае, очевидно, $u_{m}=a$ и при $v=0$ из (15.11), (15.13) находим для виброударных режимов

$$
\tau_{1}=-\arccos \frac{a-d}{a}, \tau_{2}=\tau_{3}=0, \tau_{4}=-\tau_{1}=\arccos \frac{a-d}{a}
$$

и для непрерывных режимов

$$
\tau_{1}=-\pi, \tau_{2}=\tau_{3}=0, \tau_{4}=-\tau_{1}=\pi
$$

Подстановка значений (15.15), (15.16) в(15.9) выражение приводит к следующему соотношению:

$$
P=\left\{\begin{array}{lr}
D_{0}\left(1-\frac{a}{d}\right) & a \leq d / 2 \\
\frac{D_{0} a}{\pi d}\left[\sqrt{1-\left(1-\frac{d}{a}\right)^{2}}-\left(1-\frac{d}{a}\right) \arccos \left(1-\frac{d}{a}\right)\right] & a \geq d / 2
\end{array}\right.
$$


Выражение (15.17) определяет величину статической силы, превышение которой вызывает появление остаточных пластических деформаций и, следовательно, знаменует начало процесса резания. Зависимость граничной силы (15.17) от амплитуды $a$ колебаний резца показана на рис.15.4.

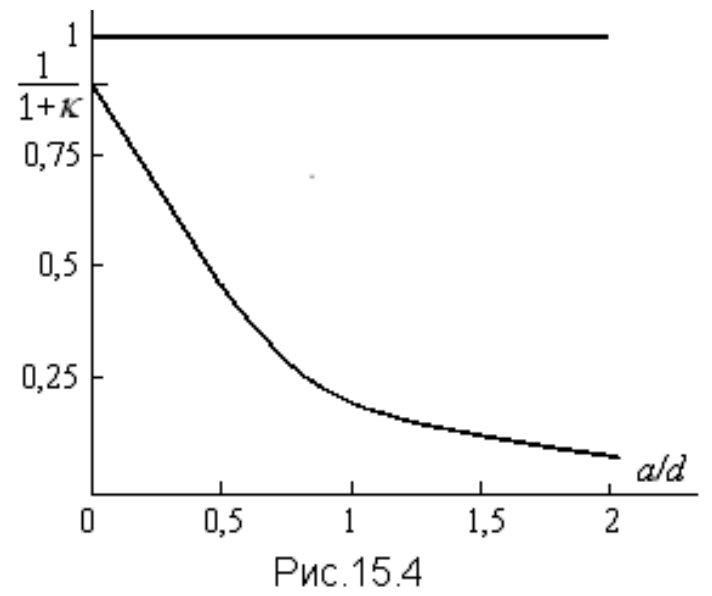

Обратим внимание на то, что при $a \rightarrow 0$ статическая сила $P$ изменяется скачком от значения $P=D_{0}$ до значения $P=D=(1+\kappa) D_{0}$ Это объясняется тем, что любая сколь угодно малая вибрация устраняет «зависание», вызванное сухим трением, преобразуя его в вязкое при малой скорости относительного движения (см. Параграф 3).

4. Как и во всех рассмотренных выше ультразвуковых технологических машинах, при ультразвуковом резании силовое взаимодействие инструмента с обрабатываемым изделием создает технологическую нагрузку на колебательную систему устройства, показанного на рис.15.1. Методы отыскания параметров технологической нагрузки были подробно рассмотрены в Параграфе 8. Учитывая периодический характер рассматриваемых движений, запишем динамическую характеристику в виде

$$
f(u, \dot{u}) \cong P(v, a)+k(v, a) u^{0}+b(v, a) \dot{u}^{0}
$$

где $P(v, a), \quad k(v, a), \quad b(v, a), \quad$ - постоянная составляющая, упругая и диссипативная составляющие технологической нагрузки. 
Эти величины отыскиваются по методике, изложенной в П.8.6, с помощью соотношений

$$
\begin{gathered}
P(v, a)=\frac{1}{T} \int_{0}^{T} F[u(t), \dot{u}(t)] \mathrm{d} t \\
k(v, a)=\frac{2}{T a} \int_{0}^{T} F[u(t), \dot{u}(t)] \cos \omega t \mathrm{~d} t \\
b(v, a)=-\frac{1}{T a} \int_{0}^{T} F[u(t), \dot{u}(t)] \sin \omega t \mathrm{~d} t
\end{gathered}
$$

где $T=2 \pi / \omega$ - период колебаний инструмента.

Отметим, что первая из формул (15.19) совпадает с (15.8) и описывает влияние колебаний на постоянную составляющую силы резания, которое подробно исследовано в предыдущем пункте. Из полученных результатов следует, что вибрация оказывает наибольшее влияние при обработке жесткопластического материала $k_{0} \rightarrow \infty$. С другой стороны, именно в этом случае взаимодействие инструмента с изделием оказывает наиболее сильное влияние на динамику колебательной системы.

Для такого случая динамическая характеристика контактного взаимодействия записывается следующим образом:

$$
F(u, \dot{u})=D_{0} \eta(u-\Delta)(1 \div k \operatorname{sgn} \dot{u})
$$

Напомним, что характеристика такого вида рассматривалась нами ранее при исследовании систем для ультразвуковой обработки хрупких материалов (см. Параграфы 12 и 13). Рассматриваемый здесь случай ультразвукового резания имеет два существенных отличия. Первое заключается в том, что скорость резания не может считаться малой по сравнению с амплитудой вибрационной скорости инструмента. Второе отличие состоит в том, что в данном случае резание осуществляется при заданной скорости резания, а не при заданном усилии подачи. Другими словами, мы имеем дело не с силовой, как ранее, а с кинематической 
подачей. Ниже будет показано, что эти отличия значительно обогащают общую картину динамического поведения системы.

После подстановки выражения (15.20) в формулы (15.19) для коэффициентов $k$ и $b$ и интегрирования имеем

$$
\begin{gathered}
k(v, a)=\frac{D}{\pi a}\left(\sin \tau_{3}-\sin \tau_{1}\right) \\
b(v, a)=\frac{D}{\pi a \omega}\left(\cos \tau_{3}-\cos \tau_{1}\right)
\end{gathered}
$$

где $\tau_{1}, \tau_{3}$ - моменты переключения, определяемые уравнениями в (15.14).

Уравнения (15.21), (15.22) и соотношения, определяющие моменты переключения, должны быть дополнены условием, отражающим равенство величины срезаемого слоя материала и перемещения обрабатываемой детали в течение одного периода колебаний: т.е

$$
u_{m}-\Delta=2 \pi v / \omega
$$

где $u_{m}=u\left(\tau_{3}\right)=\left(v \tau_{3} / \omega\right)+a \cos \tau_{3}$. Из (15.23) после преобразований находим

$$
\frac{\Delta}{a}=\sqrt{1-\left(\frac{V}{a \omega}\right)^{2}}-\frac{V}{a \omega}\left(\pi-\arcsin \frac{V}{a \omega}\right)
$$

Подстановка в (15.21), (15.22) значений $\tau_{1}, \tau_{3}$ с учетом (15.24) дает величины коэффициентов $k$ и $b$, которые можно представить в виде:

$$
k(v, a)=\frac{D \omega}{\pi \cdot v} K\left(\frac{v}{a \omega}\right), b(v, a)=\frac{D}{\pi \cdot v} B\left(\frac{v}{a \omega}\right),
$$

где $K(\nu / a \omega)=(\nu / a \omega)\left(\sin \tau_{3}-\sin \tau_{1}\right), B(\nu / a \omega)=(\nu / a \omega)\left(\cos \tau_{3}-\cos \tau_{1}\right)$

На рис.15.5 построены графики функций $K(v / a \omega)$ и $B(v / a \omega)$, определяющих упругую и диссипативную составляющие технологической нагрузки. Как видно, обе составляющие технологической нагрузки являются немонотонными функциями, которые обращаются в нуль при $v / a \omega=0, \quad v / a \omega \geq 1$ и $a \omega \rightarrow \infty$ и достигают максимума при $v / a \omega=0,5$ и $v / a \omega=0,8$ соответственно. Эти особенности технологической нагрузки определяют специфику поведения устройства для ультразвукового резания. 

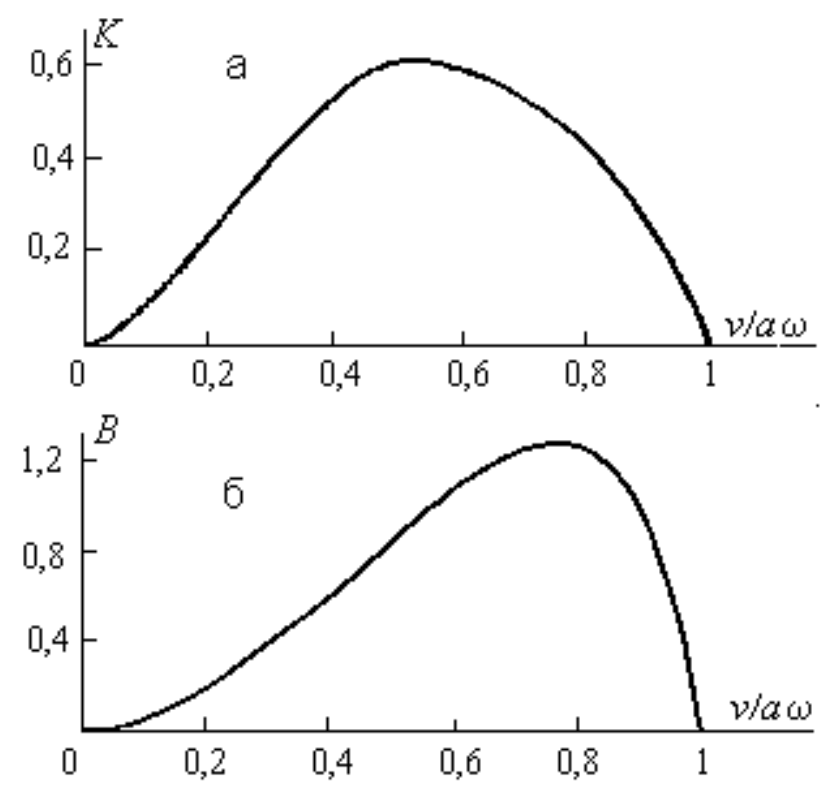

Рис. 15.5

Как было показано в Параграфе 4, и при анализе рассмотренных выше устройств, динамика устройства для ультразвукового резания может быть рассмотрена с помощью обобщенной динамической модели, в которой все действующие силы и динамические жесткости приводятся к одному элементу, В данном случае в качестве такого элемента естественно выбрать резец. Его колебания описываются уравнением

$$
u(t)=W^{-1}(j \omega)\left[f_{0}(j \omega) e^{j \omega t}-f(u, \dot{u})\right]
$$

где $f_{0}(j \omega)$ - комплексная амплитуда приведенной к инструменту силы возбуждения; $W(j \omega)$ - приведенная динамическая жесткость колебательной системы.

После подстановки в уравнение (15.26) линеаризованной характеристики (15.18) находим комплексную амплитуду периодической составляющей движения резца

$$
\tilde{a}=\frac{f_{0}(j \omega)}{W(j \omega)+k(v, a)+j \omega b(v, a)}
$$

Принимая во внимание, что коэффициенты (15.25) технологической нагрузки зависят от вибрационной скорости аю, из (15.27) получим следующее уравнение. 


$$
a \omega=\left|\frac{\omega \cdot f_{0}(j \omega)}{U(\omega)+k(v, a \omega)+j \omega[V(\omega)+b(v, a \omega)]}\right|
$$

где $U(\omega)=\operatorname{Re} W(j \omega), V(\omega)=\omega^{-1} \operatorname{Im} W(j \omega)$.

Решение трансцендентного уравнения (15.28) проводится с помощью следующего графического приема (рис.15.6).

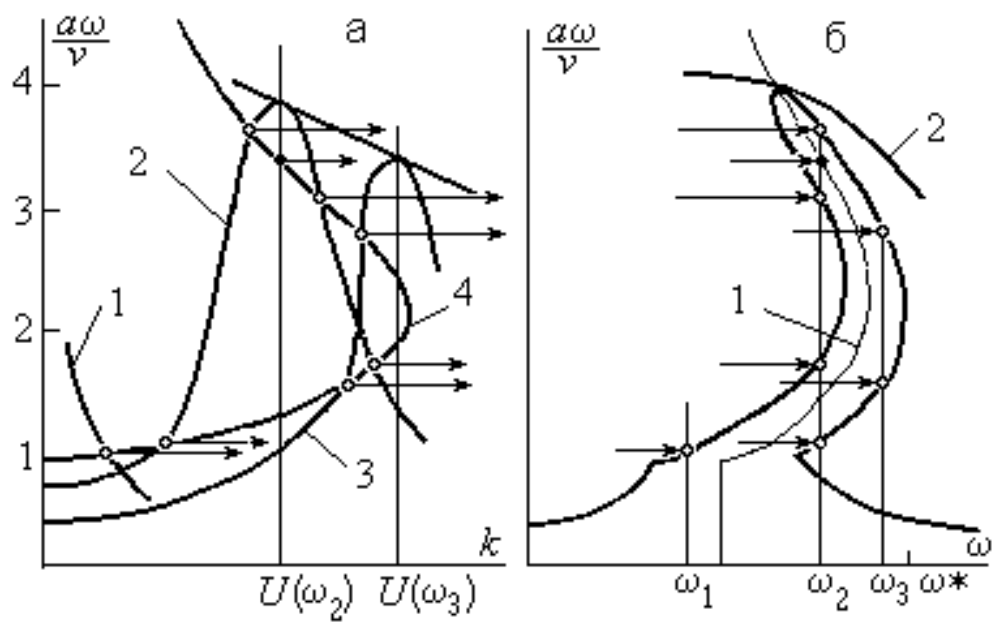

Рис.15.6

Полагая $k$ независимой переменной, по уравнению (15.28) для фиксированных значений частоты $\omega$ строятся графики зависимости аю от $k$. На рис.15.6,а эти кривые, обозначенные цифрами 1, 2, 3, построены для трех значений частоты $\omega_{1}<\omega_{2}<\omega_{3}$. Кривые вида 1 соответствуют дорезонансным частотам линейной части системы, для которых $U(\omega) \geq 0$. Кривые вида 2, 3 получаются для частот, расположенных в зарезонансной области, в которой $U(\omega)<0$. При построении этих кривых каждому значению $k$ необходимо сопоставлять соответствующее значение $b$ согласно зависимостям на рис.15.5.

Точки пересечения упомянутых кривых с графиком 4 обратной функции $\omega a(k)$, построенным с помощью рис. 15.5 , дают искомые решения.

Описанная процедура позволяет построить амплитудно-частотную характеристику колебаний инструмента при наличии технологической нагрузки, как это показано жирной линией на рис.15.6,б. Из рис 15.6 видно, что исследуемая система уравнений при различных частотах и определенных сочетаниях параметров может иметь одно, три или пять 
решений, принадлежащих различным ветвям резонансной кривой. Если вибрационные скорости резца достаточно малы, так что $a \omega \leq v$, реализуются участки резонансной кривой линейной системы, которым отвечают точки пересечения кривых (15.28) с вертикальной прямой $k=0$ на рис.15.5.

Учитывая высокую добротность ультразвуковой колебательной системы, которая предполагает малость диссипативных потерь, определяемых величинами $V(\omega)$ и $b(v, a \omega)$, можно считать, что максимумы кривых 2 и 3 на рис.15.6,a и соответственно резонансной кривой на

рис.15.6.б достигаются при выполнении условия

$$
U(\omega)+k(v, a \omega)=0
$$

и, следовательно, определяются выражением (см. уравнение (15.28)):

$$
a \omega=\frac{|f(j \omega)|}{V(\omega)+b(v, a \omega)}
$$

Уравнение (15.29) определяет скелетную кривую 1 амплитудночастотной характеристики (рис.15.6,б). Построение скелетной кривой показано на рис.15.6 затемненными точками. Как видно, резонансная кривая при $a \omega>v$ охватывает скелетную. Уравнение (15.30) определяет линию 2 предельных амплитуд, которая пересекает скелетную кривую в тех же точках, что и амплитудно- частотная характеристика.

Скелетная кривая представляет зависимость собственных частот ультразвуковой системы с нелинейной нагрузкой. Как видно из рис. 15.6,б, собственная частота равняется собственной частоте линейной системы $\omega_{0}=$ const, когда интенсивность ультразвуковых колебаний мала: $a \omega \leq v$. С увеличением интенсивности колебаний собственная частота сначала взрастает до значения $\omega^{*}$ при $v<a \omega \leq a^{*} \omega^{*}$, а затем уменьшается, причем $\omega \rightarrow \omega_{0}$ при $a \omega \rightarrow \infty$. Таким образом, система изохронна при $a \omega \leq v$, жестко анизохронна при $v<a \omega<a^{*} \omega^{*}$ и мягко анизохронна при $a \omega>a^{*} \omega^{*}$. 
Сложность собственных свойств колебательной системы с нелинейной нагрузкой определяет разнообразие возможных конфигураций резонансных кривых, конкретный вид которых зависит от скорости резания, соотношения между упругими и диссипативными параметрами колебательной системы и технологической нагрузки, уровня внешнего возбуждения. На рис.15.7 показана картина трансформации резонансных кривых по мере увеличения скорости резания $v$ от $v=0$ до значений, превышающих вибрационную скорость резца в резонансном режиме холостого хода $\left(v>a_{0} \omega_{0}\right)$.
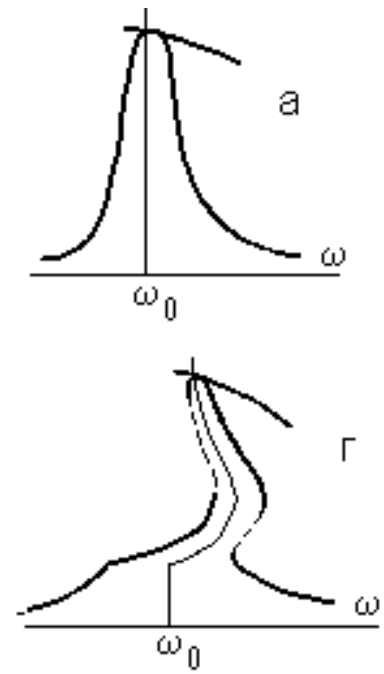
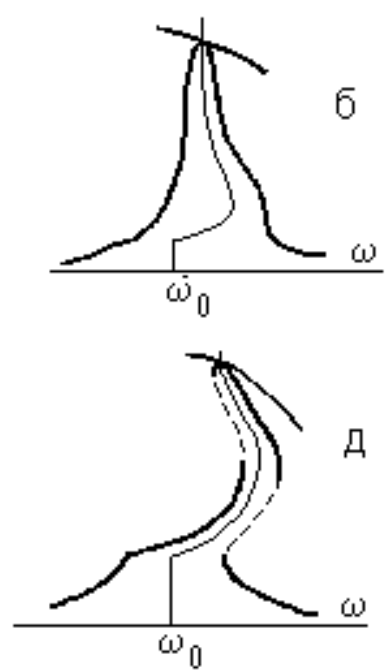

Рис. 15.7
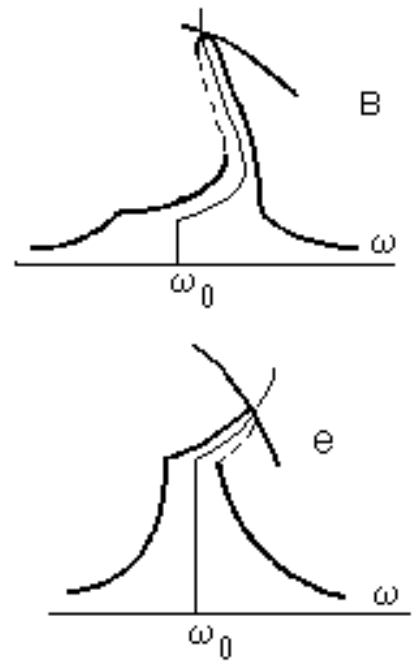

На рис.15.7,a приведена амплитудно-частотная характеристика системы на холостом ходу. Резонансная кривая имеет типичный для линейных систем вид. Максимальная амплитуда достигается на резонансной частоте $\omega=\omega_{0}$ холостого хода.

При малой скорости резания $v<<a_{0} \omega_{0}$ вид резонансной кривой (рис.15.7,б) изменяется несущественно, но резонансная частота смещается в область более высоких частот. С увеличением скорости резания дорезонансная ветвь становится все более крутой, а зарезонансная - более пологой, огибая скелетную кривую.

Дальнейшее увеличение скорости меняет характер резонансной кривой (рис.15.7,в), которая принимает вид, характерный для нелинейных 
систем с мягкой нелинейностью. Появляется область частот с тремя ветвями резонансной кривой, средняя из которых, показанная штриховой линией, соответствует неустойчивым решениям и физически нереализуема. Выход на наиболее эффективный резонансный режим с максимальной амплитудой осуществляется затягиванием колебаний по частоте из области высоких частот. При этом переход с одной ветви резонансной кривой на другую сопровождается скачкообразным изменением (срывом) амплитуды колебаний. При амплитудах вибрационной скорости $a \omega \leq v$ реализуются также ветви резонансных кривых линейной системы.

Следующее качественное изменение амплитудно-частотной характеристики имеет место, когда точка $a \omega=v$ зарезонансной линейной ветви попадает в частотную область существования скелетной кривой (рис.15.7,г). В этом случае амплитудно-частотная характеристика имеет две области существования трех ветвей. Ветви, показанные штриховыми линиями неустойчивы. При плавном изменении частоты возбуждения переход с одной ветви на другую сопровождается срывами амплитуд колебаний, а выход на резонансный режим может быть выполнен или затягиванием колебаний из области высоких частот, или увеличением частоты до срыва на верхнюю ветвь с последующим затягиванием вдоль вехней ветви путем уменьшения частоты.

Ситуация, показанная на рис,15.7.д, отличается от предыдущей тем, что между двумя областями, в которых существуют по три ветви амплитудно-частотной характеристики, появляется диапазон существования пяти ветвей. В этом случае выход на резонансный режим с максимальной амплитудой может быть выполнен только путем сначала увеличения, а после срыва на верхнюю ветвь уменышения частоты, т.е затягивания колебаний в режим с максимальной интенсивностью.

При скорости резания близкой к критической $\left(v=a_{0} \omega_{0}\right)$ амплитудночастотная характеристика принимает вид (рис.15.7,ж), присущий 
нелинейной системе с жесткой нелинейностью. В этом случае выход на резонансный режим выполняется затягиванием колебаний из области низких частот. Здесь также существуют области неоднозначности, неустойчивая ветвь и скачкообразные изменения амплитуд колебаний при изменении частоты возбуждения.

По мере дальнейшего увеличения скорости резания и ее приближения к критической частотная область неоднозначности резонансной кривой все более сужается, и наконец при скоростях $v \geq a_{0} \omega_{0}$ амплитудно-частотная характеристика вновь принимает обычный для линейной системы вид, показанный на рис.15.7,а.

Таким образом, мы видим, что указанные выше особенности колебательной системы устройства для вибрационного резания приводят к тому, что при различных условиях резания она может вести себя подобно виброударным системам с предварительным зазором, предварительным натягом или с силовым замыканием, a иногда обладает комбинированными свойствами.

5. В П. 15.3 было показано, что для эффективного проведения процесса ультразвукового резания необходимы режимы колебаний резца с максимально возможными амплитудами. Реализация таких режимов требует возбуждения и поддержания резонансных колебаний под нагрузкой. Из приведенной общей картины изменения амплитудночастотных характеристик очевидны те трудности, которые необходимо преодолеть при возбуждении резонансных режимов внешним вынуждающим воздействием. Кратко они сводятся к следующим: сложность и неоднозначность тактики выхода на резонанс изменением частоты возбуждения; высокая чувствительность системы к изменению технологической нагрузки; опасность срыва резонансных колебаний из-за непредсказуемых изменений нагрузки. По-видимому именно эти причины являются препятствием для широкого практического распространения процесса ультразвукового резания. 
Наиболее эффективно задача возбуждения и стабилизации резонансных колебаний устройства для ультразвукового резания решается переходом к авторезонансному возбуждению, основанному на возбуждении автоколебаний за счет положительной обратной связи. Основные принципы и возможности такого способа возбуждения колебаний ультразвуковых технологических систем рассмотрены в предыдущем Параграфе. На рис.15.8 показана схема устройства для ультразвукового резания с системой авторезонансного возбуждения.

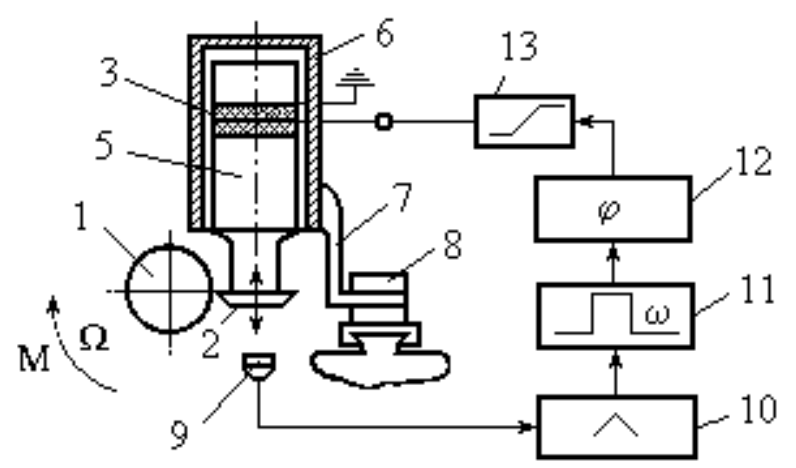

Pис. 15.8

Здесь в дополнение к рис.15.1:

9 - датчик обратной связи;

10 - предварительный усилитель;

11 - полосовой фильтр;

12 - фазосдвигающий элемент;

13 - нелинейный усилитель.

В качестве датчика обратной связи в системе использован микрофон. Усилитель имеет характеристику с насыщением и линейным начальным участком.

Авторезонансная система решает следующие задачи:

1) обеспечивает самовозбуждение колебаний при любых условиях технологического нагружения устройства;

2) автоматически поддерживает резонансные режимы при изменении технологической нагрузки в широких пределах; 
3) дает возможность регулировки амплитуды колебаний резца в заданных пределах, определяемых из технологических и конструктивных соображений.

Первая задача решается выбором начального коэффициента усиления цепи обратной связи. Выше было показано, что условия самовозбуждения всегда выполняются при использовании усилителя с релейной или близкой к ней характеристикой. Здесь, однако, следует иметь в виду, что в сложной колебательной системе, имеющей множество собственных частот, в режиме автоколебаний может возбудиться режим с нежелательной частотой. Как правило, это режим с наибольшей амплитудой сигнала с датчика обратной связи, который зависит, в том числе, и от положения датчика. [92]. Для выделения диапазона рабочих частот в цепи обратной связи описываемой авторезонансной системы используется полосовой фильтр 11. Коэффициент усиления цепи обратной связи выбирается в соответствии с рекомендациями, данными в предыдущем Параграфе.

Решение второй задачи реализации резонансных режимов при изменении технологической нагрузки обеспечивается согласованием фазовых характеристик привода, колебательной системы и цепи обратной связи. Эту функцию выполняет фазосдвигающий элемент 12. При правильном выборе фазовых характеристик авторезонансная система обеспечивает поддержание режимов, соответствующих линии предельных амплитуд (см. п.15.4), являющейся огибающей всевозможных резонансных кривых. Таким образом, автоматически реализуются наиболее эффективные резонансные колебания при изменении скорости, подачи и глубины резания, температуры колебательной системы, массы резца из-за его износа и т.д. В разработанной системе изменение сдвига фазы может выполняться перемещением микрофона относительно излучающего звук элемента, т.е. регулированием запаздывания сигнала в цепи обратной связи. 
И, наконец, регулирование амплитуды колебаний выполняется изменением уровня насыщения нелинейной характеристики усилителя 5 , которое приводит к изменению гармонического коэффициента усиления сигала в цепи обратной связи.

6. Приведем некоторые результаты экспериментальных исследований процесса ультразвукового резания. Устройство для ультразвукового резания, выполненное по схемам на рис.15.1 и рис.15.8, устанавливалось в резцедержателе токарного станка. Колебания резца возбуждались либо в режиме вынужденных колебаний от внешнего генератора, либо в режиме автоколебаний с помощью цепи положительной обратной связи $\mathrm{B}$ процессе экспериментов тензометрическими датчиками, наклеенными на кронштейн 7 (рис.15.8), регистрировалась постоянная составляющая силы резания. С установленного под резцом измерительного микрофона 1 снимался сигнал, пропорциональный скорости колебаний резца.

На рис.15.9 приведена осциллограмма силы резания при точении стального прутка диаметром $\varnothing 29$ мм с глубиной резания 0,5 мм и подачей 0,25мм/об при различных скоростях вращения.

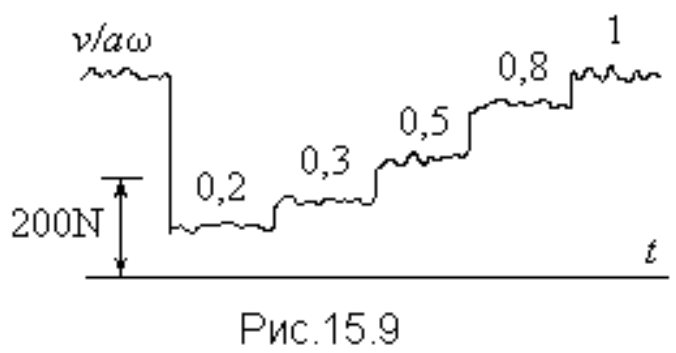

Параметры наложенных ультразвуковых колебаний резца с частотами в окрестности 20 кГц и амплитудами 10 мкм в процессе этой серии экспериментов возбуждались и поддерживались авторезонансной системой возбуждения.

Первый участок осциллограммы получен при традиционном точении без ультразвука. Числа над остальными участками указывают величину отношения скорости $v$ резания к вибрационной скорости $a \omega$ резца. Из 
осциллограммы видно, что наложение вибрации на процесс точения снижает силу резания, причем степень уменьшения силы резания зависит от соотношения между скоростью $v$ резания и вибрационной скоростью $a \omega$ , а при $v>a \omega$ вибрация не оказывает влияния на силу резания.

На рис.15.10 по результатам экспериментов построена зависимость силы $P$ резания от скорости $v$ резания.

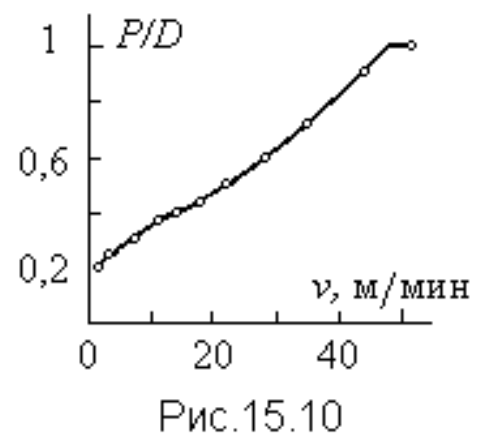

Эта зависимость аналогична построенной на рис.15.3 кривой, отмеченной индексом $\left(a k_{0} / D_{0}\right)=5$. Отметим, что эта кривая близка к характеристике процесса для жесткопластического материала $\left(k_{0} \rightarrow \infty\right)$, Поэтому принятые выше предпосылки относительно модели процесса при теоретическом анализе динамики устройства под нагрузкой вполне оправданы.

В экспериментах в режимах вынужденных колебаний снимались амплитудно-частотные характеристики колебательной системы под нагрузкой. Характеристики снимались при точении стального образца диаметром 30 мм с глубиной резания 0,25 мм и подачей 0,06 мм/об при различных скоростяж вращения шпинделя. Примеры резонансных кривых приведены на рис.15.11. Резонансная частота системы на холостом ходу (рис.15.11,a) $f_{0}=19030$ Гц. С увеличением скорости резания резонансная частота сначала возрастает до 19125 Гц (рис.15.11,б), и при этом форма резонансной кривой изменяется так, что ее зарезонансная ветвь становится более крутой, а дорезонансная - более пологой.

Дальнейшее увеличение скорости резания уменьшает резонансную частоту (рис.15.11,в), и при $v \geq a \omega$ резонансная кривая вновь возвращается к виду, показанному на рис.15.11,a. 

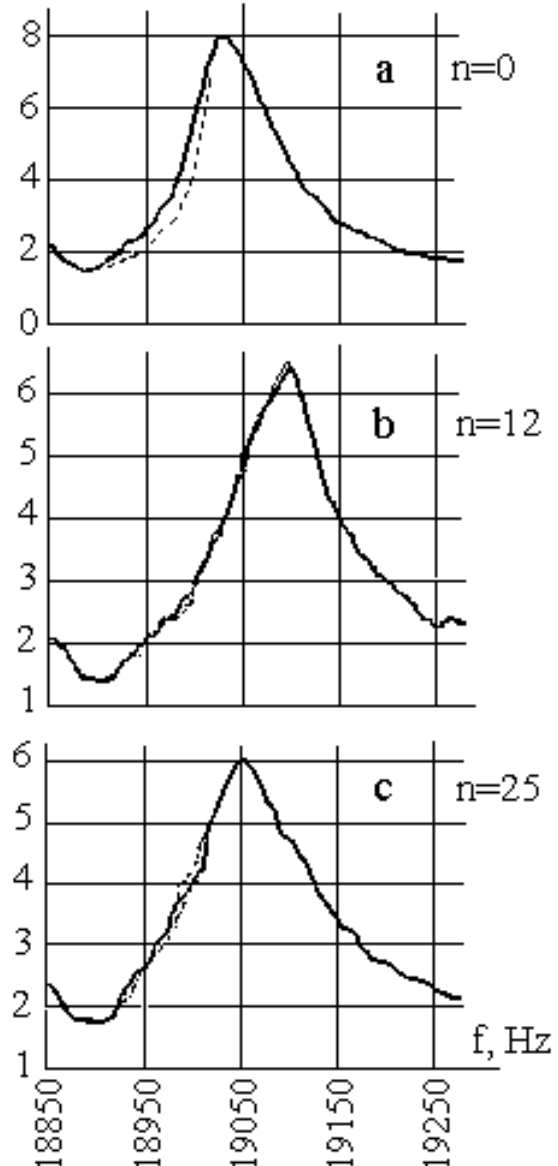

Рис. 15.11

По результатам экспериментов на рис. 15.12 построена зависимость, связывающая резонансные частоты системы под нагрузкой с величиной отношения вибрационной скорости $а \omega$ к скорости резания v. Эта зависимость представляет собой скелетную кривую, вид которой аналогичен теоретической скелетной кривой, построенной выше на рис.15.6.

Таким образом, экспериментально полученные эффекты достаточно хорошо согласуются с результатами анализа динамической модели процесса ультразвукового резания, проведенного в П.15.3, и динамики устройства, рассмотренной в П.15.4.

7. В заключение отметим некоторые характерные технологические особенности процесса ультразвукового резания, которые наблюдались в экспериментах при обработке различных материалов.

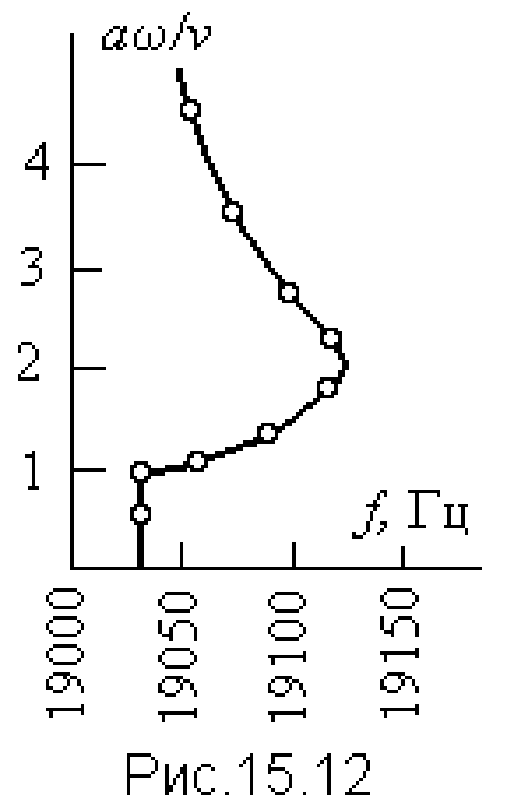

Снижение сил резания при скоростях $v<a \omega$ имело место при точении всех металлов: алюминия, меди, бронзы, латуни, углеродистых, нержавеющих и жаропрочных сталей, титана и др.

При ультразвуковом резании существенно меняется характер процесса, Так например, при традиционном точении алюминия и меди на поверхностях резца образуются наросты материала. Наложение ультразвуковых колебаний полностью исключает это явление. 
Ультразвуковая вибрация существенно изменяет характер снимаемой стружки. Даже при обработке материалов, обычное точение которых сопровождается образованием ломкой стружки, при ультразвуковом резании образуется сливная пластичная стружка без заусенцев и неровностей. Как результат, наложение ультразвука позволяет существенно уменьшить величину минимально возможного срезаемого слоя. Так при обработке углеродистой и нержавеющей сталей удалось провести точение с подачей 0,05 мм/об и глубиной резания 0,015 мм.

При ультразвуковом резании устраняется склонность системы «Станок - Инструмент - Деталь» к возбуждению автоколебаний $[127,128]$. Это отчетливо наблюдалось при точении длинного тонкого валика диаметром 30 мм и длиной 600 мм. При точении валика с глубиной резания 1 мм и подачей 0,1 мм при скоростях вращения, превышающих 200 об/мин, при прохождении некоторых участков наблюдалось периодическое возбуждение автоколебаний, которые сказывались на качестве обрабатываемой поверхности. Включение ультразвука, проводимое в авторезонансном режиме, полностью устраняло автоколебания и позволяло получать совершенно однородную поверхность на всей длине вала. Здесь уместно заметить, что вследствие снижения силы резания ультразвуковое резание позволяет обрабатывать технологически нежесткие изделия без применения промежуточных опор и люнетов.

Ультразвуковое резание радикально изменяет качество обработанной поверхности. При рассмотрении в микроскоп поверхности после традиционного точения напоминает грубо вспаханное поле. Поверхность после ультразвукового точения подобна культивированному полю со строго регулярной мелкоячеистой структурой поверхности. Это находит отражение на профилограммах поверхностей, полученных в результате традиционного (рис.15.13,a) и ультразвукового (рис.15.13,б) точения стальной заготовки диаметром 56 мм. В обоих случаях обработка производилось с подачей 0,1 мм/об и глубиной резания 1 мм. 

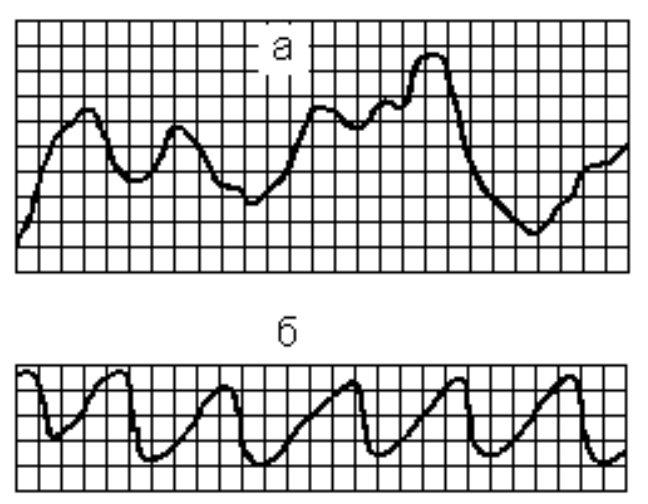

Рис.15.13

Во всех приведенных случаях ультразувуковое точение образцов проводилось в авторезонансных режимах возбуждения колебаний.

Полученные в настоящем Параграфе теоретические и экспериментальные результаты хорошо коррелируют с опытными данными, описанными во многих работах по исследованию ультразвукового резания [106,130,141-144,224,225].

Отметим, что полученные здесь результаты в основном справедливы и для ряда других ультразвуковых технологических процессов, например, для процесса волочения с наложением ультразвука, который описывается той же динамической моделью. 


\section{СПИСОК ЛИТЕРАТУРЫ}

1. Абрамов О.В. Кристаллизация металлов в ультразвуковом поле. М.: Металлургия, 1972. с с.

2. Аверьянова В.Г., Дьяченко П.Е., Мизрохи Ю.Н. Исследование диспергирования твердых тел при воздействии ультразвука. - В кн.: Трение и износ в машинах. Вып. 15. М.: Изд-во АН СССР, 1962.

3. Аверьянова В.Г., Миловидов . . Исследование процесса обработки материала ультразвуком. - Успехи научной фотографии, 1964, т. 9.

4. Акинфиев Т.С., Бабицкий В.И., Боровков Б.А., Гольдштейн Б.Г., Пятов В.Л. Резонансные режимы электромеханического компрессионно-вакуумного молотка. - Машиноведение, 1981, № 5, с.

5. [Ананьев И.В. Справочник по расчету собственных колебаний упругих систем. М. - Л.: Гостехиздат, 1946. 223 с.

6. Анго А. Математика для электро- и радиоинженеров. М.: Наука, $1965.780 \mathrm{c}$.

7. Андронов А.А., Витт А.А., Хайкин С.Э. Теория колебаний. М.: Физматгиз, 1959.

8. Андронов А.А., Майер А.Г. Простейшие линейные системы с запаздыванием - Автоматика и телемеханика, 1946, т. VII, №2-3.

9. Андронов В.В. О кажущемся превращении сухого трения в вязкое. Инж. журн., МТТ, 1967, № 2.

10. Андронов В.В. Исследование некоторых случаев вибрационного и статического внедрения твердого деформатора в сыпучую среду. Инж. журн., МТТ, 1967, № 3.

11. Андронов В.В. Об одном методе определения силы и коэффициента трения . - Машиноведение, 1970, № 4.

12. Андронов В.В. Вынужденные колебания в системе с преобразованным сухим трением . - Машиноведение, 1975, № 5. 
13. Андронов В.В., Нагаев Р.Ф. Вибрационное перемещение вдоль плоскости, колеблющейся перпендикулярно линии наибольшего ската. - Изв. АН СССР, МТТ, 1976, № 1.

14. Архангельский М.Е. О превращении ультразвуковых колебаний поверхности во вращательное и поступательное движение тела. - Акустический журнал, 1963. Т. 9, № 3, с. 275 - 278.

15. Архангельский М.Е. Уменьшение сухого динамического трения посредством ультразвуковых колебаний. - Известия АН СССР, МТТ, 1969, № 2, c.43 - 45 .

16. Асташев В.К. Периодические движения упругого стержня с ограничителем. - В кн.: Динамика машин с учетом упругости и переменности масс. М.: Наука, 1965, с. 128 - 134.

17. Асташев В.К. Исследование динамики виброударной системы с распределенными параметрами. - В кн.: Механика машин, вып. 17 - 18. М.: Наука, 1968, с. $14-22$.

18. Асташев В.К. К динамике осциллятора, ударяющегося об ограничитель. - Машиноведение, 1971, № 2, с. 5 - 9.

19. Асташев В.К. Настройка ультразвуковых станков под нагрузкой. Станки и инструмент, 1972, №10, с. 32 - 34.

20. Асташев В.К. О согласовании колебательной системы с приводом и нелинейной нагрузкой. - Машиноведение, 1978, № 3, с. 9 - 16.

21. Асташев В.К. Теория стержневых концентраторов для высокочастотных виброударных систем. - Труды Николаевского кораблестроительного института, 1980, вып. 169, с. 71 - 78.

22. Асташев В.К. О влиянии ультразвука на процессы механической обработки. - В кн.: Прочность и пластичность материалов в ультразвуковом поле. (Тез. докл. ІІІ Всесоюзн. конф.). Алма-Ата, 1980, с.5961. 
23. Асташев В.К. Расчет стержневых концентраторов с нелинейной нагрузкой. - Акустический журнал, 1981, т. 27, № 6, с.

24. Асташев В.К. О влиянии ультразвука на процессы пластического деформирования. - Машиноведение, 1983, № 2, с. 3 - 12.

25. Асташев В.К. Влияние ультразвуковых колебаний резца на процесс резания. - Проблемы машиностроения и надежности машин, 1992, №3, c.81-86

26. Асташев В.К. Авторезонансные системы возбуждения вибрационных машин. - Наука производству, 1998, № 10, с. 30 - 34.

27. Асташев В.К., Бабицкий В.И. Резонансные колебания вязкоупругого стержня с ограничителем. - Известия АН СССР, МТТ, 1972, № 4, с. $176-182$.

28. Асташев В.К., Бабицкий В.И. Виброударное взаимодействие вязкоупругих стержней. - Машиноведение, 1974, № 5, с. 3 - 9.

29. Асташев В.К., Бабицкий В.И. Виброударное взаимодействие упругих систем. - В кн.: Mehanica cvrstog deforma binog tela. XIII Jugoslovenski kongres racionalne i primerjene mechanike. Saraevo, 1976.

30. Асташев В.К., Бабицкий В.И. Эффективность резонансной настройки систем для ультразвуковой обработки. - Машиноведение, 1981, № 6 , c. $3-9$.

31. Асташев В.К., Бабицкий В.И. Методы повышения эффективности ультразвуковых станков. - Станки и инструмент, 1982, № 3, с. 25 27.

32. Асташев В.К., Бабицкий В.И. Способы настройки резонансных машин. - Машиноведение, 1982, №5, с. 3 - 9.

33. Асташев В.К., Бабицкий В.И. Динамика резонансных машин. - В кн.: Динамика машин и управление машинами. М.: Машиностроение, 1988, с. 168 - 209. 
34. Асташев В.К., Бабицкий В.И., Герц М.Е. К синтезу авторезонансных систем. - Научн. тр. ВУЗов Лит.ССР, Вибротехника, 1973, № 3 (20), c. $253-259$.

35. Асташев В.К., Бабицкий В.И., Герц М.Е. Виброударные процессы в вязкоупругом стержне. - Proceedings of the VIII Conference Dynamics of Machines. Praha - Liblice, 1973, p. 11 - 17.

36. Асташев В.К., Бабицкий В.И., Герц М.Е. Адаптивная система управления ультразвуковым станком. - Приборы и системы управления, 1977, № 2, c. 11 - 12.

37. Асташев В.К., Бабицкий В.И., Герц М.Е. Способ ультразвуковой обработки. Авторское свидетельство № 546390. - Бюлл. Изобретений, 1977, № 6 .

38. Асташев В.К., Бабицкий В.И., Герц М.Е., Кожин В.Д. Способ автоматической настройки ультразвуковых станков. Авторское свидетельство № 536850. - Бюлл. Изобретений, 1976, № 44.

39. Асташев В.К., Бабицкий В.И., Герц М.Е., Полунов Ю.Л. Способ измерения силы. - Авторское свидетельство № 877368. - Бюлл. Изобретений, 1981, № 40.

40. Асташев В.К., Бабицкий В.И., Герц М.Е., Полунов Ю.Л. измерение сил с использованием виброударной системы. - Измерительна техника, 1986, №7, с. 33 - 35.

41. Асташев В.К., Бабицкий В.И., Кобринский А.Е., Тывес Л.И. Способ уменьшения трения при перемещении объекта. Авторское свидетельство № 218610. - Бюлл. Изобретений, 1968, № 17.

42. Асташев В.К., Бабицкий В.И., Соколов И.Я. Авторезонансное вибровозбуждение синхронным электродвигателем. - - Проблемы машиностроения и надежности машин, 1990, №4, с.41-46 
43. Асташев В.К., Герц М.Е. Автоколебания вязкоупругого стержня с ограничителем при действии запаздывающей силы. - Машиноведение, 1973, № 5, с. 3 - 11.

44. Асташев В.К., Герц М.Е. Возбуждение и стабилизация резонансных колебаний ультразвуковых стержневых систем. - Акустический журнал, 1976, т. 22, № 2, с. $192-200$.

45. Асташев В.К., Герц М.Е. Система автоматической настройки ультразвуковых станков. - Электрофизические и электрохимичекие методы обработки, 1976, № 6, с. 15 -21.

46. Асташев В.К., Герц М.Е. Виброперемещение вязкоупругого стержня в среде с сухим трением. - В кн.: IX Всесоюзная Акустическая конференция. Доклады. Секция М. М.: Наука, 1977, с. 147 - 150.

47. Асташев В.К., Герц М.Е. К теории вибрационного перемещения.Известия АН СССР, Механика твердого тела, 1978, №1, С. 40-44

48. Асташев В.К., Герц М.Е., Тресвятский А.Н. Использование фокусированного ультразвука для бесконтактных измерений.- Акустический журнал, 1981, т.27, №5, с.

49. Асташев В.К., Кобринский А.Е., Кожин В.Д., Степаненко Ю.А. Способ оптимизации режима работы колебательной системы ультразвукового станка. Авторское свидетельство №215633.- Бюлл. Изобретений, 1968, №13

50. Асташев В.К., Кобринский А.Е., Корендясев А.И. Датчики для экспериментальных исследований станков.- Станки и инструменты, 1968, № 8, c.

51. Асташев В.К., Кобринский А.Е., Корендясев А.И., Сакаян А.Р., Тывес Л.И. Устройство для автоматической поднастройки частоты генератора ультразвукового станка. Авторское свидетельство №483148.- Бюлл. Изобретений, 11975, № 33 
52. Асташев В.К., Крупенин В.Л. Волны в распределенных и дискретных виброударных системах и сильно нелинейных средах - Проблемы машиностроения и надежности машин, 1998, №5, с.13-30.

53. Асташев В.К., Сакаян А.Р. Экспериментальное исследование динамики колебательной системы ультразвукового станка.- Машиноведение, 1967, №4, с.3-7

54. Асташев В.К., Семенова Е.Б. Устройство для волочения изделий с использованием ультразвуковых колебаний. Авторское свидетельство № 1731334.- Бюлл. Изобретений, 1992, №17

55. Асташев В.К., Тресвятский А.Н. Об изгибно-угловых колебаниях виброударной системы с распределенными параметрами.- Машиноведение, 1980, №5, с.10-19

56. Асташев В.К., Тресвятский А.Н. Виброударные режимы при изгибно-угловых колебаниях балки с присоединенным телом. - Труды Николаевского кораблестроительного институты, 1981, вып. 182, с. $43-$ 51.

57. Асташев В.К., Турич В.В. Качество поверхности деталей, обработанных даформирующим протягиванием с наложением ультразвука. - В кн.: Повышение эффективности протгивания. Рига: РПИ, 1986, с. 131-136.

58. Бабаков И.М., Теория колебаний. М.: Наука, 1968, 560 с.

59. Бабицкий В.И. Параметрические колебания виброударных систем. Машиноведение, 1971, №1, с.

60. Бабицкий В.И. Теория виброударных систем. М.: Наука, 1978, 352 с.

61. Бабицкий В.И., Крупенин В.Л. Колебания в сильно нелинейных стстемах. М.: Наука, 1985, 320 с.

62. Бабицкий В.И., Тресвятский А.Н. Вынужденные колебания балки с вязкоупругой диссипацией энергии и массой на конце, расположенной между ограничителями.- Машиноведение, 1976, №2, с.16-23 
63. Бансявичус Р.Ю., Рагульских К.М. Вибродвигатели. Вильнюс: Мокслас, 1981, 193 с.

64. Бауман В.А., Быховский И.И. Вибрационные машины и процессы в строительстве. М.: Высшая школа, 1977, 256 с.

65. Березина С.И., Лямов В.Е., Солодов И.Ю. Вестник МГУ, сер. Физика, астрономия, 1977, т.18, №1, с.3-18

66. Беспалова Л.В. К теории виброударного механизма. - Изв. АН СССР, ОTH, 1957, №5

67. Беспечный В.И., Викторов Е.Д. Распространение колебаний в стержнях, имеющих зазор в сочленениях. - Изв. АН СССР, Механика твердого тела. 1970, №4

68. Бидерман В.Л. Прикладная теория механических колебаний. М.: Высшая школа, 1972. 416 с.

69. Блехман И.И. Синхронизация динамических систем. М.: Наука, $1971,894 \mathrm{c}$.

70. Блехман И.И. Действие вибрации на механические системы. - Научные труды высш. уч. завед. Лит. ССР, Вибротехника, 1973, №3 (20), c.369-374

71. Блехман И.И. Метод прямого разделения движений в задачах о действии вибрации на нелинейные механические системы. - Известия АН СССР, Механика твердого тела, 1976, №6, с.13-27

72. Блехман И.И. Синхронизация в природе и технике. М.: Наука, 1981, $352 \mathrm{c}$.

73. Блехман И.И. Вибрационная механика. М.: Наука, 1994, 400 с.

74. Блехман И.И., Джанелидзе Г.Ю. Вибрационное перемещение. М.: Наука, 1964, 410 с.

75. Богомолов С.И., Симсон Э.А. Оптимальное проектирование концентраторов ультразвуковых колебаний. Акустический журнал, 1981, т.27, №4, с.491-499 
76. Бутковский А.Г. Методы управления системами с распределенными параметрами. М.: Наука, 1975, 320 с.

77. Бутковский А.Г. Структурная теория распределенных систем. М.: Наука, 1977, 320 с.

78. Бутковский А.Г. Характеристики систем с распределенными параметрами. М: Наука, 1979, 224 с.

79. Быховский И.И. Основы теории вибрационной техники. М: Машиностроение, 1969, 364 с.

80. Вагапов И.К. Нелинейные эффекты в ультразвуковой обработке. Минск: Наука и техника. 1987. - 159 с.

81. Вагапов И.К. Резонансные характеристики двухстержневой колебательной системы под нагрузкой. Проблемы машиностроения и надежности машин, 1994, №3. С. 17-25.

82. Вейц В.Л. Исследование трения покоя в направляющих скольжения при низкочастотных направленных микроколебаниях.- В кн.: Новое в теории трения. М.: Наука, 1966, с.60-81

83. Вибрации в технике. Справочник, т.1. Колебания линейных систем. М.: Машиностроение, 1978. 352 с.

84. Вибрации в технике. Справочник, т.2. Колебания нелинейных систем. М.: Машиностроение, 1979. 351с.

85. Вибрации в технике. Справочник, т.3. Вибрационные процессы и машины. М.: Машиностроение, 1981. 509 с.

86. Вибрации в технике. Справочник, т.6. Защита от вибрации и ударов. М.: Машиностроение, 1995. 456 с.

87. Вульфсон И.И., Коловский М.З. Нелинейные задачи динамики машин. Л.: Машиностроение, 1968, с.

88. Ганева Л.И., Голямина И.П., Марголин В.С. Механическое сопротивление нагрузки при ультразвуковом резании хрупких материалов - Акустический журнал, 1973, т.XIX, №4, с.524-530 
89. Ганиев Р.Ф., Кобаско Н.И., Кулик В.В. и др. Колебательные явления в многофазных средах и их использование в технологии. Киев.: Техника, 1980.-142 с.

90. Ганиев Р.Ф., Украинский Л.Е. Динамика частиц при воздействии вибрации. Киев: Наукова думка, 1975, 168 с.

91. Ганиев Р.Ф., Украинский Л.Е. О движении твердых частиц, взвешенных в несжимаемой жидкости, при вибрационных воздействиях. - Прикладная механика, 1975, т.11, №1, с.47-54

92. Герц М.Е. Авторезонансное возбуждение высших форм колебаний механических систем. - Машиноведение, 1979, №6. С. 3-11.

93. Гершгал Д.А., Фридман В.М. Ультразвуковая технологическая аппаратура. М.: Энергия, 1976, 320 с.

94. Голямина И.П., Астахова Р.Н., Ганева Л.И. Применение высокодобротных колебательных систем для сварки синтетических тканей. Труды МВТУ им. Баумана, 1970, №10, с.68-75.

95. Голямина И.П., Исакович М.А. Частотная характеристика механического гармонического осциллятора, погруженного в среду. - Акустический журнал, 1981, т27, №5, с.730-735

96. Гоноровский Н.С. К теории высокочастотных автогенераторов с запаздывающей обратной связью. - Радиотехника, 1958, т. 13, № 5

97. Грановский Г.И., Грановский В.Г. Резание металлов. М.: Высшая школа, 1985, 304 с.

98. Ден-Гартог Дж.П. Механические колебания. М.: Физматгиз, 1960, $580 \mathrm{c}$.

99. Дондошанский В.К. Расчет колебаний упругих систем на электронных вычислительных машинах. М.: Машиностроение, 1965, 368 с.

100. Донской А.В., Келлер О.К., Кратыш Г.С. Ультразвуковые электротехнологические установки. - Л.: Энергия, 1968. 276 с. 
101. Дьяченко П.Е., Мизрохи Ю.Н., Аверьянова В.Г. Некоторые вопросы ультразвуковой обработки металлов. - В кн.: Применение ультразвука в промышленности. М.: Машгиз, 1959, с. 149 - 152.

102. Егоров В.П. Зубодолбление мелкомодульных зубчатых колес с применением ультразвуковых колебаний - В кн.: Прочность и пластичность материалов в ультразвуковом поле. Алма-Ата, Изд-во КазПТИ, 1980 , c. $78-79$.

103. Ивович В.А. Переходные матрицы в динамике упругих систем. - М.: Машиностроение, 1969, с. 199

104. Израилович М.Я,. Оптимальное управление периодическими режимами гармонически линеаризуемых механических систем. - Проблемы машиностроения и надежности машин, 1993, №6. С. 76-83.

105. Израилович М.Я,. Управление вынужденными колебаниями гармонически линеаризуемых механических систем. - Проблемы машиностроения и надежности машин, 1994, №5. С. 18-27.

106. Исаев А.И., Анохин В.С. Применение ультразвуковых колебаний инструмента при резании металлов - Вестник машиностроения, 1961, № 5, c.56- 62

107. Исакович М.А. Общая акустика - М.: Наука, 1973. 495 с.

108. Ишлинский А.Ю., Соколов Б.Н., Черноусько Ф.Л. О движении плоских тел при наличии сухого трения. - Изв. АН СССР, МТТ, 1981, № 4 , c. $17-28$.

109. [Казанцев В.Ф. Зависимость напряжений в материале при ультразвуковой обработке от амплитуды колебаний и силы прижима. - Акустический журнал, 1963, т. 9, № 1, с. 120 - 122.

110. Казанцев В.Ф. Зависимость производительности ультразвуковой обработки от режима резания. - Станки и инструмент, 1963, № 3. 
111. Казанцев В.Ф., Мечетнер Б.Х., Розенберг Л.Д. Пути повышения производительности и точности ультразвуковой обработки. - Станки и инструмент, 1966, № 4, с. 23 - 27.

112. Катковник В.Я. Виброударное возбуждение высокочастотных упругих колебаний. - Инж. журнал, МТТ, 1968, № 5.

113. Кемпнер М.Л. Методы динамических податливостей и жесткостей для расчета изгибных колебаний упругих систем со многими степенями свободы. - В кн.: Поперечные колебания и критические скорости. М.: Изд-во АН СССР, 1951, с. 78 - 130.

114. Клубович В.В., Буцукин В.С., Рубаник В.В. Волочение проволоки из свинцово-оловянистого припоя с наложением продольных ультразвуковых колебаний. - В кн.: Прочность и пластичность материалов в ультразвуковом поле. Алма-Ата, 1980, с. 5 - 6.

115. Клубович В.В., Вагапов И.К. Модель пластического деформирования упрочняющегося материала с наложением ультразвука. - Докл. АН БССР, 1991, т. 35, № 4. С. 338-341.

116. Клубович В.В., Сакевич В.Н. Виброударное взаимодействие стержней. - Проблемы машиностроения и надежности машин, 1992, №6. C. 17-24.

117. Кобринский А.Е. Механизмы с упругими связями. - М.: Наука, 1964. $392 \mathrm{c}$.

118. Кобринский А.Е., Кобринский А.А. Виброударные системы. - М.: Наука, 1973. 592 с.

119. Колешко В.М. Ультразвуковая микросварка. - Минск: Наука и техника, 1977, 328 с.

120. Коловский М.3. Нелинейная теория виброзащитных систем. - М.: Наука, 1966. 320 с.

121. Коловский М.3. Автоматическое управление виброзащитными системами. - М.: Наука, 1976. 320 с. 
122. Красовский А.А. О вибрационном способе линеаризации некоторых нелинейных систем. - Автоматика и телемеханика, 1948, т. 9, № 1.

123. Крюков Б.И. Динамика вибрационных машин резонансного типа. Киев: Наукова думка, 1967, 210 с.

124. Крюков Б.И., Литвин Л.М., Логвиненко М.С. Новые резонансные виброплощадки. - Бетон и железобетон, 1976, № 7, с. 25 - 27.

125. Крюков Б.И., Логвиненко Е.А., Литвин Л.М., Малинин М.С. Вибростенд для уплотнения жестких бетонных смесей. - Механизация строительства, 1971, № 4, с. 20 - 22.

126. Крюков Б.И., Середович Г.И. Влияние амплитуды внешнего возмущения на характер проявления сложных резонансов в существенно нелинейных колеблющихся системах. - Машиноведение, 1982, №6, c. 26-30.

127. Кудинов В.А. Динамика станков. М.: Машиностроение, 1967, 363 с.

128. Кудинов В.А. Колебания в станках. - В кн.: Колебания машин, конструкций и их элементов. Вибрации в технике. Т.3. М.: Машиностроение, 1980, с.118-130.

129. Кузнецов В.Л., Лощилов В.И. Ультразвуковая сварка металлов и пластмасс. М.: Машиностроение, 1972. - 75 с.

130. Кумабэ Д. Вибрационное резание. - М.: Машиностроение, 1985. $424 \mathrm{c}$.

131. Ланда П.С. Автоколебательные системы с конечным числом степеней свободы. - М.: Наука, 1980. - 360 с.

132. Ланда П.С. Автоколебания в распределенных системах. - М.: Наука, 1983. - $320 \mathrm{c}$.

133. Ланда П.С. Нелинейные колебания и волны. - М.: Наука, 1997. - 496 c.

134. Лепендин Л.Ф. Акустика. - М.: Высшая школа, 1978. - 448 с. 
135. Лившиц П.С. К вопросу о вынужденных колебаниях системы, ударяющейся об ограничитель. - Журн. техн. физ., 1952, вып.6.

136. Логинов К.В. Гидроакустические поисковые приборы. - М.: Машиностроение, 1964.

137. Лойцянский Л.Г., Лурье А.И. Курс теоретической механики. Т.II. М.: Наука, 1983. - 640 с.

138. Лурье А.И. Крутильные колебания в дизельных установках. - М.-Л.: Военмориздат, 1940. 219 с.

139. Максимов А.Д. К теории вибрационного сглаживания нелинейных характеристик систем автоматического управления при помощи вынужденных колебаний. - В кн.: Автоматическое управление и вычислительная техника. Вып.2. М.: Машгиз, 1959.

140. Мандельштам Л.И. Лекции по теории колебаний. - М.: Наука, 1972. $470 \mathrm{c}$.

141. Марков А.И. Резание труднообрабатываемых материалов при помощи ультразвуковых и звуковых колебаний. - М.: Машгиз, 1962. $332 \mathrm{c}$.

142. Марков А.И. Ультразвуковое резание труднообрабатываемых материалов. - М.: Машиностроение, 1968, 367 с.

143. Марков А.И. Ультразвуковая обработка материалов. - М.: Машиностроение, 1980. - 237 с.

144. Марков А.И., Озерова М.А., Устинов И.Д. Применение ультразвука при алмазном выглаживании деталей. - Вестник машиностроения, 1973, № 9, с. 57-61.

145. Мейз Дж.Е. Теория и задачи механики сплошных сред. - М.: Мир, 1974. $-318 \mathrm{c}$.

146. Меркулов Л.Г. Теория ультразвуковых концентраторов. - Акустический журнал, 1957, т. 3, № 3, с. 230 - 238. 
147. Меркулов Л.Г., Харитонов А.В. Теория и расчет составных концентраторов. - Акустический журнал, 1959, т. 5, № 2, с. 183 - 190.

148. Мицкевич А.М., Пугачев С.И. Ультразвуковая сварка и металлизация. - М.: Машиностроение, 1979. - 58c.

149. Нагаев Р.Ф. Периодические режимы вибрационного премещения. М.: Наука, 1978. - 160 с.

150. Неймарк Ю.И. Устойчивость линеаризованных систем. Изд-во ЛКВВИА, 1949.

151. Неймарк Ю.И. Метод точечных отображений в теории нелинейных колебаний. М.: Наука, 1972.

152. Никитин Л.В. Продольные колебания упругих стержней при наличии сухого трения. - Изв. АН СССР. МТТ, 1978, №6, с. 137-145.

153. Николаев Г.А. Использование ультразвука в сварочной технике. - В кн.: Ультразвук в строительной технике. М.: Госстройиздат, 1962, с. $163-164$.

154. Николаев Г.А., Ольшанский Н.А. Новые методы сварки металлов и пластмасс. - М.: Машиностроение, 1966. - 179 с.

155. Ноздрев В.Ф. Федорищенко Н.В. Молекулярная акустика. - М.: Высшая школа, 1974. - 288 с.

156. Носаль В.В., Рымша О.М. Снижение тяговых усилий и определение технологических параметров процесса волочения труб с использованием ультразвуковых колебаний волоки. - Сталь, 1966, № 2, с. 159162.

157. Пальмов В.А. Колебания упругопластических тел. - М.: Наука, 1976. $-328 \mathrm{c}$.

158. Панов С.В., Пискунов Ю.Ф. Влияние ультразвуковых колебаний на процесс волочения. - Ультразвуковая техника, 1966, № 3, с. 42 - 44.

159. Пановко Я.Г. Внутреннее трение при колебаниях упругих систем. М.: Физматгиз, 1960. 193 с. 
160. Пановко Я.Г. Основы прикладной теории упругих колебаний. - М.: Машиностроение, 1967. 316 с.

161. Пановко Я.Г. Введение в теорию механического удара. - М.: Наука, 1977. $224 \mathrm{c}$.

162. Перепелятник П.А. Автоколебания в автогенераторе с запаздыванием. - Радиотехника и электроника, 1961, т. 6, №10.

163. Петруха П.Г., Марков А.И., Устинов И.Д. Ультразвуковое сверление глубоких отверстий. - Вестник машиностроения, 1970, № 10, с. 54 57.

164. Петухов В.И. Воздействие ультразвуковых колебаний на процессы обработки металлов давлением. - В кн.: Прочность и пластичность материалов в ультразвуковом поле. Алма-Ата, Изд-во КазПТИ, 1980, c.3.

165. Писаревский М.М. Расчет переходных стержней для магнитострикционных вибраторов. - В кн.: Расчет магнитострикционных преобразователей. МДНТП. 1957, с. 29 - 41.

166. Писаренко Г.С. Колебания механических систем с учетом несовершенной упругости материала. - Киев: Изд-во АН УССР, 1970. 379 с.

167. Попов Е.П., Пальтов И.П. Приближенные методы исследования нелинейных автоматических систем. - М.: Физматгиз, 1960.

168. Работнов Ю.Н. Механика деформируемого твердого тела. - М.: Наука, 1979. 744 с.

169. Рейнер М. Реология. - М.: Наука, 1965. - 254 с.

170. Римский-Корсаков А.В. Электроакустика. - М.: Связь, 1973.- 272 с.

171. Розенберг Л.Д., Казанцев В.Ф. Исследование механизма ультразвукового резания при помощи высокоскоростной киносъемки. - Станки и инструмент, 1959, № 5, с. 20 - 22. 
172. Розенберг Л.Д., Казанцев В.Ф. О физике ультразвуковой обработки твердых материалов. - Доклады АН СССР. 1959, т. 124, № 1, с. 79 82.

173. Розенберг Л.Д., Казанцев В.Ф., Макаров Л.О., Яхимович Д.Ф. Ультразвуковое резание. - М.: Изд-во АН СССР, 1962. - 251 с.

174. Рубаник В.П. Колебания квазилинейных систем с запаздыванием. М.: Наука, 1969.

175. Русаков И.Г., Харкевич А.А. Вынужденные колебания системы, ударяющейся об ограничитель. - Журн. техн. физ., 1942, вып.11-12.

176. Рэлей Дж. Теория звука. Т.1. - М.: Физматгиз, 1955. 504 с.

177. Светлицкий В.А. Механика стержней. Ч.ІІ. Динамика. - М.: Высшая школа, 1987. 304 с.

178. Северденко В.П., Клубович В.В., Степаненко А.В. Обработка металлов давлением с ультразвуком. - Минск: Наука и техника, 1973. 286 c.

179. Северденко В.П., Клубович В.В., Степаненко А.В. Ультразвук и пластичность.- Минск: Наука и техника, 1976. 446 с.

180. Семенов А.П. Схватывание металлов. - М.: Машгиз, 1958.

181. Силин Л.Л., Баландин Г.Ф., Коган М.Г. Ультразвуковая сварка. Соединение металлов в твердом состоянии и улучшение качества сварных швов. - М.: Машгиз, 1962. - 252 с.

182. Скучик Е. Основы акустики. Т.1.- М.: Мир, 1976. - 520 с.

183. Скучик Е. Основы акустики. Т.2.- М.: Мир, 1976. - 542 с.

184. Сорокин Е.С. К теории внутреннего трения при колебаниях упругих систем. - М.: Госстройиздат, 1966. - 134 с.

185. Степаненко А.В., Логачев М.В., Хан Дык Ким. Влияние продольных ультразвуковых колебаний на процесс безоправочного волочения труб. - В кн.: Прочность и пластичность материалов в ультразвуковом поле. Алма-Ата, Изд-во КазПТИ, 1980, с. 13 - 14. 
186. Стрелков С.П. Введение в теорию колебаний. - М.-Л.: ГТТИ, 1950. $344 \mathrm{c}$.

187. Сумкин В.В., Куприянов Н.С., Тушев Н.А., Солодов В.А. Волочение тонкостенных труб с применением ультразвуковых колебаний фильеры. - В кн.: Прочность и пластичность материалов в ультразвуковом поле. Алма-Ата, Изд-во КазПТИ, 1980, с. 31 - 32.

188. Тарханов Г.В. Распространение упругой волны по упругому полубесконечному стержню при наличии сухого трения. - В кн.: Виброакустическая активность механизмов с зубчатыми передачами. М.: Наука, 1971, с. 184-187.

189. Тарханов Г.В. Влияние предварительного смещения на распространение упругой волны по стержню при наличии сухого трения. - Изв. AH CCCP. MTT, 1982, №2, с. 173-178.

190. Терских В.П. Метод цепных дробей в применении к исследованию колебаний механических систем. - Л.: Судпромгиз, 1955. - 708 с.

191. Теумин И.И. Ультразвуковые колебательные системы. - М.: Машгиз, 1959. - $332 \mathrm{c}$.

192. Тимошенко С.П. Колебания в инженерном деле. - М.: Наука, 1967. $444 \mathrm{c}$.

193. Тимошенко С.П. Прочность и колебания элементов конструкций. М.: Наука, 1975. - 704 с.

194. Тресвятский А.Н. Экспериментальное исследование виброударных режимов консольной балки с массой, расположенной между ограничителями. - Машиноведение, 1979, № 6.

195. Ультразвук. Маленькая энциклопедия. / Под ред. И.П.Голяминой. М.: Советская энциклопедия, 1979. - 400 с.

196. Ультразвуковая технология. / Под ред. Б.А.Аграната. - М.: Металлургия, 1974. - 504 с. 
197. Ультразвуковые преобразователи. / Под ред. Е.Кикучи. - М.: Мир, 1972. $-424 \mathrm{c}$.

198. Фаерман В.Т. Соединение текстильных материалов ультразвуком. М.: Легкая промышленность, 1977. - 88 с.

199. Фейгин М.И. Вынужденные колебания систем с разрывными нелинейностями. - М.: Наука, 1994. - 288 с.

200. Феодосьев В.И. Сопротивление материалов. - М.: Наука, 1974. - 605 c.

201. Фролов К.В. Теория механизмов и машин. - М.: Высшая школа, 1987. $-496 \mathrm{c}$.

202. Харитонович М.В., Эскин Г.И. Ультразвук в процессах пластической деформации металлов и сплавов. Обзор отечественной и зарубежной литературы 1955-1969 гг. - М.: ВИЛС, 1970. - 112 с.

203. Харкевич А.А. Теория электроакустических преобразователей. - В кн.: Избранные труды. Т.1. М.: Наука, 1973, с. 33 - 217.

204. Холопов Ю.В. Ультразвуковая сварка. - Л.: Машиностроение, 1972. $-152 \mathrm{c}$.

205. Чечина Л.Г., Яхимович Д.Ф. Ультразвуковая обработка алмазным инструментом. - Электрофизические и электрохимические методы обработки, 1974, № 2, с. 17 - 21.

206. Чечина Л.Г., Яхимович Д.Ф. Ультразвуковая размерная обработка алмазным инструментом. - Электрофизические и электрохимические методы обработки, 1974, № 3, с. 18 - 26.

207. Яворский Б.М., Детлаф А.А. Справочник по физике - М.: Наука, 1979. 942c.

208. Яхимович Д.Ф., Блитштейн Н.И., Глазов Г.И. Ультразвуковой станок мод. 4770. - Бюлл. технико-экономической информации, ВИНИТИ, 1959, № 1 . 
209. Astashev V.K., Babitsky V.I. Ultrasonic cutting as a nonlinear (vibroimpact) process - Ultrasonics, 1998, №6, p. 89 - 96.

210. Balamuth L. Mechanical impedance transformers in relation to ultrasonics machining. - Transactions of the IRE, 1954, N 2, p. 23 - 34.

211. Blank D. Glasbearbeitung durch Stosslappen bei Ultraschallfrequenz. Glastechn. Bear, 1961, v. 34, N 11, p. 534 - 544.

212. Chen Y. On the vibration of beams or rods carrying a concentrated mass. - Journ. Appl. Mech., 1963, v. 30, N2.

213. Daniels H. Ultrasonic Welding. - Ultrasonics, 1965, N 3.

214. Drews P. Untersuchungen zum Ultraschallpunktschweissen von Metallen. - Schweissen und Schneiden, 1970, v. 22, N 2.

215. Evan-Iwanowski R.M. Resonance oscillations in Mechanical Systems. Amsterdam - Oxford - New York: Elsevier Scientific Publishing Company, 1976. 292 p.

216. Farrer J.O. Method of Abrading. - Patent of Great Britain N 602801, 1948.

217. Fridman H.D., Levensque P. Reduction of Static Friction by Sonic Vibrations. - Journal of Applied Physics, 1959, v. 30, N 10.

218. Goetze D. Effect of Pressure between Tool, Tip and Workpiece on the Rate of Ultrasonic Machining in Ketoes Tool Steel. - Journ. Acoust. Sos. Amer., 1957, v. 23, N 4.

219. Goetze D. Effect of Vibration Amplitude, Frequency and Composition of Abrasive Slurry on the Rate of Ultrasonic Machining in Ketoes Tool Steel. - Journ. Acoust. Sos. Amer., 1956, v. 28, N 6, p. 1033 - 1037.

220. Grant D.A. The effect of rotary inertia and shear deformation on frequency and normal mode equations of uniform beams carrying a concentrated mass. - Journ. Sound and Vibration, 1978, v. 57, N 3.

221. Hartley M.S. Ultrasonic Machining of Brittle Materials. - Electronics, 1956, N 1. 
222. Izumi O., Oyama K., Suzuki Y. On the Superimposing of Ultrasonic Vibration during Compressive Deformation of Metals. - Transactions of the Japan Institute of Metals, 1966, v. 7, N 3, p. 158 - 162.

223. Izumi O., Oyama K., Suzuki Y. Effects of Superimposed Ultrasonic Vibration on Compressive Deformation of Metals. - Transactions of the Japan Institute of Metals, 1966, v. 7, N 3, p. 162 - 167.

224. Komaraiah M., Manan M., Reddy Narashimha P., Victor S. Investigation of surface roughness and accuracy in ultrasonic machining - Precision Engineering, 1988, N 10, p. 59-63.

225. Kubota M., Tamura I., Shimamura N. Ultrasonic machining with diamond impregnated tool - Bull. of Japanese Society of Precision Engineering, 1977, N11, p. 127-131.

226. Langenecker E. Effects of Ultrasound on Deformation Characteristics of Metals.-IEEE Transactions on Sonics and Ultrasonics, 1966, v.SU-13, N1, p.1-8.

227. Langenecker E. Ultrasonic Treatment of Specimens in the Electron Microscope. - The Review of Scientific Instruments, 1966, v. 37, N 1, p. $103-106$.

228. Legge P. Machining without abrasive slurry. - Ultrasonics, 1966, Jully, p.157-162.

229. Maezava S. Steady forced vibration of unsymmetrical piecewise-linear system. - Bull. JSME, 1961, v. 4, N14.

230. Markov A.I., Ustinov I.D. A study of the ultrasonic diamond drilling Industrial Diamond Review, 1972, March, p. 97-99.

231. Mason W.P. A dynamic measurement of the elastic, electric and piezoelectric constants of Rochelle salt. - Phys. Rev., 1939, v. 55, p. 755 - 761.

232. Neilson R.D., Player M.A., Wiercigroch M. A dynamic model of ultrasonic drilling - Machine Vibration, 1993, N 12, p. 136-143. 
233. Neppiras E.A. Report on ultrasonic machining - - Metalworking production, 1956, v. 100, N 29, p. 1377-1382.

234. Neppiras E.A. Very high energy ultrasonics. - British Journal of Applied Physics, 1960, v. 11, N 4, p. 143 -150.

235. Nishimura G., Shimakawa S. Ultrasonic mechanical machining (Part IV). - Journ. Fac. Eng. Univ. Tokyo, 1959, v. 26, N 1, p. 13 - 35.

236. Nishimura G., Inagishima K., Shina T. Ultrasonic mechanical machining (Part VII). - Journ. Fac. Eng. Univ. Tokyo, 1959, v. 26, N 1, p. 53 - 68.

237. Nomoto A. Ultrasonic Machining by Low Power Vibration. - Journ. Acoust. Sos. Amer., 1954, v. 26, N 6.

238. Pahlitzsch G., Blank D. Fortchritte beim Stosslappen mit Ultraschallfrequenz. - Werkstatstechnik und Maschinenbau, 1960, v. 50.

239. Pan H.H. Transverse vibration of an Euler beam carrying a system of heavy bodies. - Journ. Appl. Mech., 1965, v. 32, N 2.

240. Pohlman R., Lehfeldt E. Influence of Ultrasonic Vibration on Metallic Friction. - Ultrasonics, 1966, N 4, p. 178 - 185.

241. Robinson A.T. The application of ultrasonic energy to metal wire drawing. - Wire and Wire Production, 1964, v. 39, N 12, p. 1925 - 1931.

242. Tsujino J. Studies on ultrasonic: welding with two longitudinal systems. Jup. J. Appl. Phis. 1985, V. 24, №1. P. 172-174.

243. Weare N.E., Antonevich J.N., Monrol R.E. Fundamental Studies of Ultrasonic Welding. - Welding Journal, 1960, v. 39, N 8. 


\section{ПРИЛОЖЕНИЕ}

\section{АВТОРЕЗОНАНСНАЯ УЛЬТРАЗВУКОВАЯ ОБРАБОТКА МАТЕРИАЛОВ И НАНОСТКТУРИРОВАНИЕ ПРИПОВЕРХНОСТНЫХ СЛОЕВ}

Помещенный в Приложение материал требует от читателя дополнительных знаний в области основ материаловедения и нанотехнологий. По стилю изложению этот материал ближе к развернутой научной статье, чем к учебнику. Поэтому, в частности, Приложение снабжено самостоятельным и автономным списком литературы.

1. Многочисленными экспериментальными исследованиями установлено, что наложение ультразвуковых колебаний приводит к существенному снижению статических сил, необходимых для проведения технологического процесса. Эти эффекты получили объяснение $[1,2]$ на основе анализа нелинейных реологических моделей технологических процессов.

Наряду с этим в работах [3-5] показано, что обработка поверхностей методами ультразвукового пластического деформирования, улучшая качество поверхности, создает наноструктурированный поверхностный слой с такими улучшенными механическими характеристиками, как предел текучести, предел прочности и твердость поверхности.

Однако, следует отметить, что получение стабильных и предсказуемых результатов ультразвуковой обработки возможно лишь при реализации наиболее эффективных резонансных режимов в условиях меняющейся технологической нагрузки на ультразвуковую систему со стороны обрабатываемого изделия. Возникающие при этом трудности обусловлены нелинейностью технологической нагрузки, вызывающей специфические искажения амплитудно-частотных характеристик колебательной системы [6, 7]. Этих трудностей при возбуждении и стабилизации резонансных режимов удается избежать переходом к 
автоколебательной схеме возбуждения, реализуемой введением контура положительной обратной связи, который осуществляет формирование вынуждающего воздействия путем нелинейного преобразования сигнала, пропорционального движению рабочего органа машины. При определенной настройке, называемой авторезонансной, при любых изменениях параметров колебательной системы и технологической нагрузки устанавливается режим автоколебаний с максимально возможной амплитудой, т.е. в системе автоматически поддерживается наиболее эффективное резонансное состояние [8].

Использование авторезонансных систем, благодаря высокой стабильности рабочих режимов, открывает новые широкие возможности ультразвуковых технологий, которые с полным правом можно назвать авторезонансными [9, 10]. В данном Приложении дается описание результатов авторезонансной ультразвуковой обработки (АРУЗО) различных материалов и проводится систематизация возможностей этой технологии [8-14].

2. Рассмотрим авторезонансное устройство для ультразвукового резания (токарной обработки). Оно предназначено для снижения сил резания, точения труднообрабатываемых материалов, повышения чистоты и качества поверхности обрабатываемых изделий, а также получения наноструктурированной поверхности при обработке деталей на токарных станках. Основные потребители устройства - предприятия, производственная деятельность которых связана с использованием различных токарных станков при получении высокого качества поверхности обрабатываемых изделий.

На рис. П-1 приведена схема устройства [8]: 1 - обрабатываемое изделие; 2 - резец; 3 - волновод-концентратор, передающий колебания от пьезокерамических элементов 4 резцу. Концентратор закреплен в корпусе 5 , который с помощью кронштейна 6 устанавливается в резцедержателе 7 токарного станка. Питание пьезокерамических элементов производится 
через цепь обратной связи, содержащей датчик 8, регистрирующий колебания одного из элементов колебательной системы, фазовращатель $\Phi$ и нелинейный усилитель 9. При достаточно большом коэффициенте усиления на начальном участке характеристики усилителя происходит самовозбуждение колебаний, а уровень ограничения выходного напряжения определяет амплитуду установившихся колебаний.

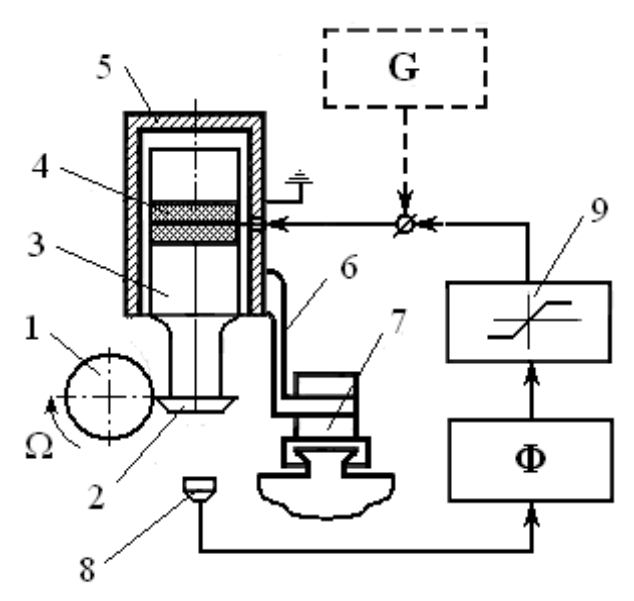

Рис.П-1

При определенной фазе сигнала обратной связи, устанавливаемой фазовращателем $\Phi$, возбуждаются резонансные колебания, частота которых зависит, в частности, и от технологической нагрузки. Если фазовый сдвиг выбран так, что разность фаз между колебаниями резца и создаваемой вибровозбудителем силой возбуждения соответствует резонансной, то устройство обеспечит реализацию резонансных колебаний при изменении технологической нагрузки и параметров колебательной системы в широких пределах. Именно такая система возбуждения колебаний с резонансной настройкой фазы в цепи обратной связи и является авторезонансной.

В традиционных ультразвуковых технологических системах питание осуществляется от генератора G c независимым возбуждением, показанного на рис. 1 штриховыми линиями. В этом случае ультразвуковая система даже при наличии подстраивающих внутренних обратных связей в 
генераторе работоспособна лишь при малых технологических нагрузках, не вызывающих появление неустойчивых ветвей резонансных кривых.

Следует отметить важную особенность такой системы возбуждения колебаний. Она заключается в том, что изменением фазы обратной связи можно реализовать всю амплитудно-частотную характеристику нелинейной колебательной системы, в том числе и нереализуемые при вынужденных колебаниях неустойчивые ветви. Поэтому в авторезонансной системе не происходит срывов амплитуд колебаний при отклонениях фазы и частоты колебаний от резонансных. Отмеченная особенность делает авторезонансную систему устойчивой по отношению к значительным изменениям параметров машины и нелинейной технологической нагрузки, что существенно расширяет технологические возможности ультразвуковой системы.

Работа авторезонансного устройства показала, что его эксплуатация не требует наличия специальных знаний или навыков. Оно стандартным образом устанавливается, например, в резцедержатель токарного станка, резец подводится к обрабатываемому изделию, и обработка изделия при включенном устройстве ничем не отличается от традиционной. Естественно, такие устройства можно устанавливать и на станки с ЧПУ без каких-либо изменений конструкции станка. Работа проводится при скорости резания 60- 80 м/мин и глубине до 2 мм.

На рис. П-2. Показано семейство устройств, обеспечивающих различные типы АРУЗО: здесь показаны ультразвуковые генераторы и семейство сменных приспособлений, позволяющих проводить обработку изделий (резание и выглаживание) на различных типах станочного оборудования. 


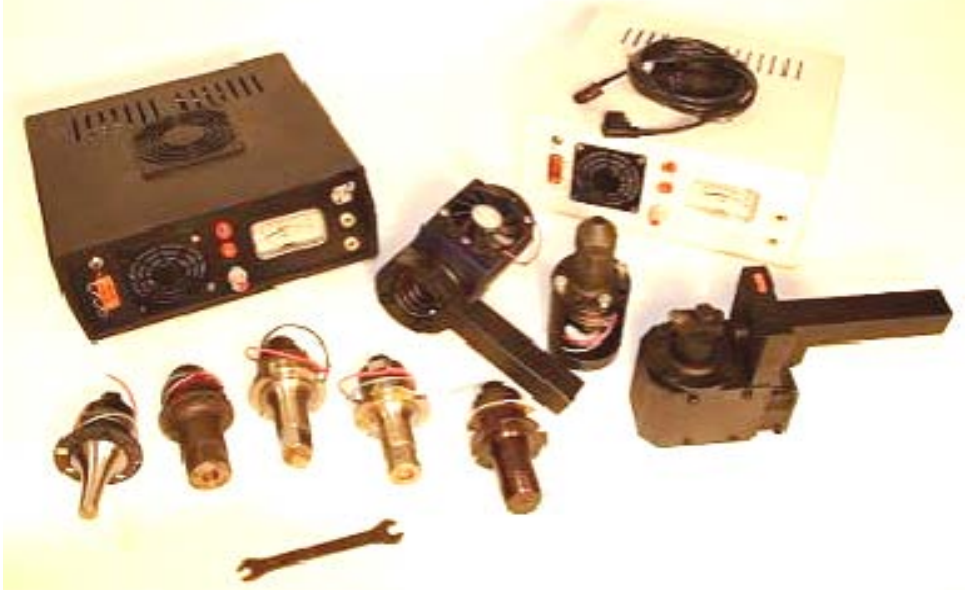

Рис.П-2

3. Рассмотрим некоторые динамические и технологические эффекты, наблюдаемые при использовании устройств для АРУЗО.

Снижение сил резания имеет место при точении практически всех конструкционных металлов: алюминия, меди, бронзы, латуни; углеродистых, нержавеющих и жаропрочных сталей, титана и др. При ультразвуковом резании снижение сил резания происходит при скоростях резания $v<a \omega$, где $a, \omega-$ амплитуда и частота ультразвуковых колебаний инструмента. Причем снижение тем больше, чем больше разница значений в указанном неравенстве. Причина уменьшения силы резания заключается в изменении характера процесса резания. Ультразвуковое резание представляет собой периодический с частотой процесс [8, 9], в котором взаимодействие резца и детали сопровождается периодическими разрывами контакта резца и обрабатываемого изделия. При этом амплитуда возникающих импульсных сил равна величине силы традиционного резания, а длительность импульсов зависит от соотношения скорости резания $v$ и амплитуды ањ вибрационной скорости резца. Измеряемая в экспериментах сила резания - это среднее за период значение импульсных сил.

Вследствие снижения силы резания с помощью авторезонансного ультразвукового резания можно обрабатывать технологически нежесткие 
изделия без применения промежуточных опор, люнетов и других дополнительных станочных приспособлений.

Ультразвуковая вибрация инструмента полностью исключает возможность возбуждения автоколебаний при резании, которые являются одной из главных причин ухудшения качества поверхности деталей из труднообрабатываемых материалов.

Ультразвуковая вибрация существенно изменяет характер снимаемой стружки. Даже при обработке материалов, обычное точение которых сопровождается образованием ломкой стружки, при ультразвуковом резании образуется сливная пластичная стружка без заусенцев и неровностей. На рис. П-3 показаны фотографии стружки в процессе традиционного (a) и авторезонансного ультразвукового точения (б) стальной заготовки.

a

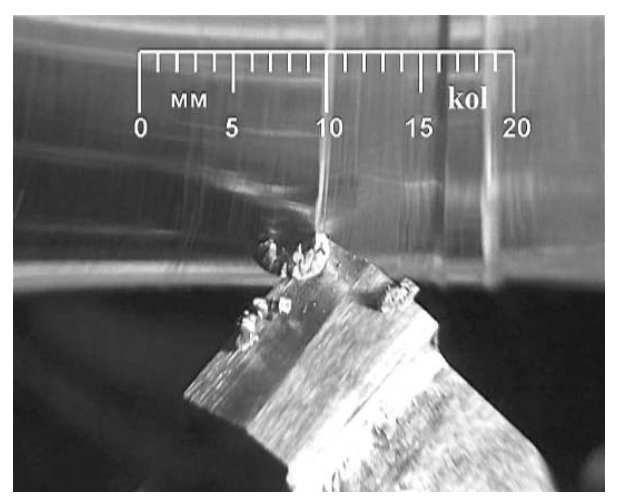

6

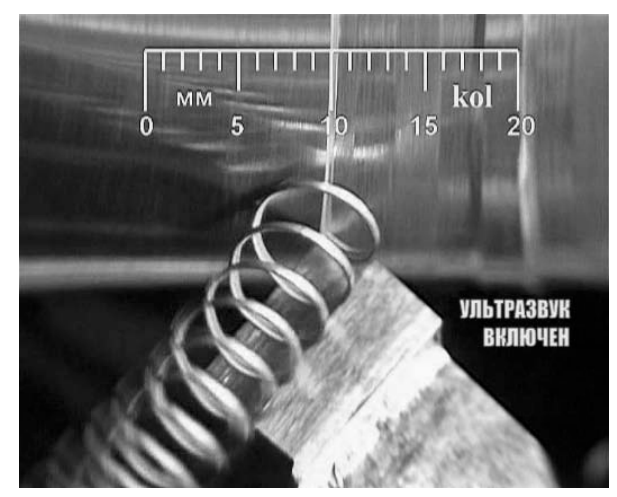

Рис.П-3

Видно, что при традиционном точении образуется дробленая, элементная стружка с ярко выраженными следами поверхностей сдвига и характерными зубцами. При ультразвуковом точении образуется винтовая гладкая непрерывная стружка. Стружка при ультразвуковом точении сходит существенно менее нагретой и пластичной, в то время как при традиционном точении она имеет цвета побежалости и оказывается хрупкой. Видимо поэтому наложение ультразвука позволяет существенно уменьшить величину минимально возможного срезаемого слоя. Так при 
обработке углеродистой и нержавеющей сталей удалось провести точение с подачей 0,05 мм/об и глубиной резания 0,015 мм.

Изменение характера процесса резания сказывается еще и в том, что наложение ультразвуковых колебаний полностью исключает явление образования наростов материала на гранях резца, что особенно важно при точении пластичных материалов, например, алюминия и меди. Это происходит, благодаря преобразованию сухого трения, которое является основной причиной образования наростов, в эквивалентное вязкое при действии высокочастотной вибрации [2].

4. Совершенно естественно, что изменение характера резания при наложении ультразвуковых колебаний приводит к изменению качества обрабатываемой поверхности, что видно на рис. П-3.

На рис. П-4, а, б приведены фотографии поверхностей детали из закаленной стали, обработанной традиционным (левые фрагменты) и ультразвуковым точением (правые фрагменты) при скоростях резания порядка 10 м/мин (a), 60 м/мин (б) и подаче 0,05 мм/об. Видно радикальное различие качества поверхностей. Поверхность, полученная ультразвуковым точением, имеет строго регулярную структуру. Это подтверждается профилограммой поверхности (рис. П-4, в), правая часть которой получена традиционным, а левая - ультразвуковым точением. Периодические впадины с шагом подачи 0,05 мм, которые прослеживаются на обеих частях профилограммы - это след вершины резца. На рис. П-4, б на участке ультразвукового точения отчетливо видны периодические отпечатки, вследствие периодических с частотой 20 кГц соударений резца с обрабатываемым изделием. Подобные отпечатки при большем увеличении наблюдаются и на поверхности рис. П-4, $a$, но здесь они не просматриваются, так как их шаг значительно меньше из-за малой скорости резания. 

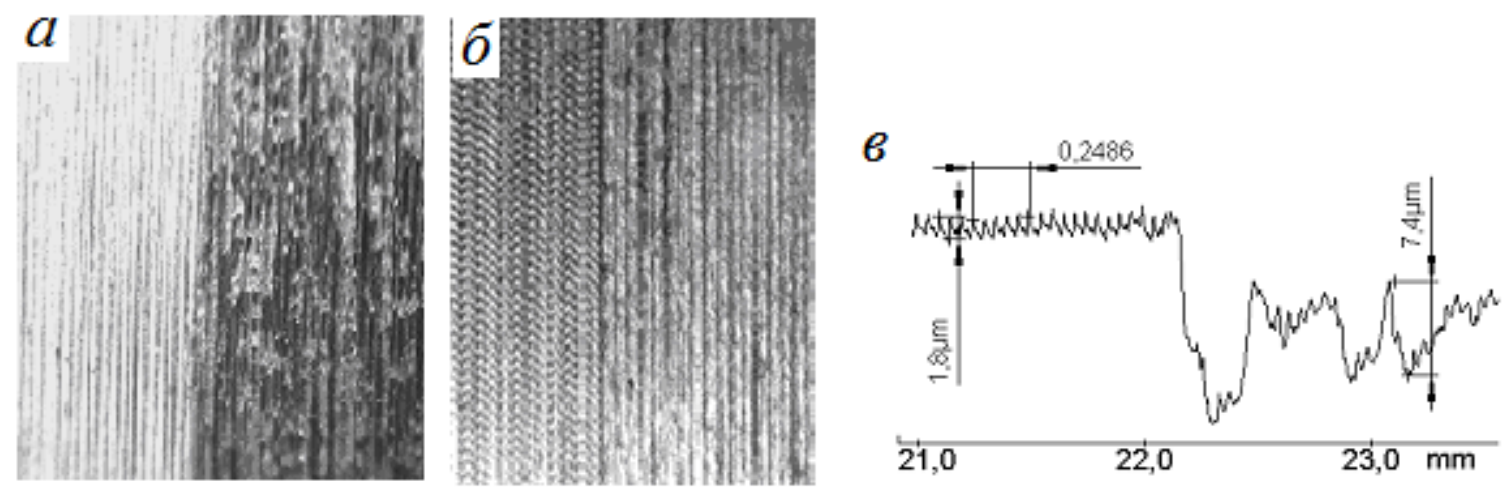

Рис. П-4

Таким образом, высокая стабильность режимов авторезонансной ультразвуковой обработки открывает возможность получения поверхностей с заданными геометрическими характеристиками. Такие поверхности могут обладать улучшенными трибологическими свойствами. Подбирая форму режущих кромок резцов, можно получать поверхности с чистой, приближающейся к полученной шлифованием.

Причина такого различия результатов резания заключается в изменения режима резания. При традиционном резании при непрерывном взаимодействии резца и детали зона пластической деформации, расположенная в окрестности режущих кромок, распространяется со скоростью резания. Эта зона насыщена микротрещинами, распределенными случайным образом. Рост той или иной микротрещины так же происходит случайным образом. Поэтому получаемая в результате резания поверхность имеет не регулярное распределение микронеровностей. Ультразвуковое резание происходит в результате периодических микроударов, следующих с высокой частотой. При этом каждый цикл нагружения начинается при одних и тех же начальных условиях. Вследствие высокой частоты следования и кратковременности импульсов зона пластической деформации сосредоточена в малой окрестности вершины резца и не насыщается микротрещинами, которые не успевают расти за столь короткий промежуток времени. Поэтому при ультразвуковом резании со стабильным режимом колебаний, который 
обеспечивается авторезонансной системой возбуждения, след резца на поверхности детали - это фактически отпечаток его формы, что видно на рис. П- 4.

Большую роль в формировании структуры материала в поверхностных слоях играют тепловые процессы в зоне резания. При одной и той же скорости резания за одинаковый достаточно продолжительный промежуток времени в процессе традиционного и ультразвукового резания затрачивается одинаковая энергия на пластическую деформацию и трение и, следовательно, производится одинаковое количество теплоты.

Разница состоит в том, что в первом случае теплота выделяется непрерывно и температура в рабочей зоне устанавливается при балансе скоростей поступления и отвода тепла в любой момент времени, причем отвод тепла происходит непрерывно и равномерно в обрабатываемую деталь, в резец и в окружающую среду.

В случае ультразвукового резания выделение теплоты происходит импульсами во время действия импульсов сил резания. При этом происходит импульсное повышение температур обрабатываемого материала в зоне резания и материала резца в окрестности режущих кромок. В каждый момент времени температура изделия и резца определяется двумя процессами: нагревом, определяемым удельной теплоемкостью материалов, и охлаждением, определяемым коэффициентами их теплопроводности и градиентом температур. В промежутках между импульсами происходит процесс охлаждения нагретых зон детали и резца. В результате средняя температура в зоне резания при ультразвуковой обработке оказывается значительно ниже, чем при традиционной. Иначе протекают процессы фазовых переходов и изменения структуры поверхностного слоя. Это подтверждают проведенные эксперименты и результаты конечноэлементного моделирования процессов резания [15].

5. Появление наноструктур отмечено при ультразвуковой обработке практически всех обработанных металлов. Проблема получения 
наноматериалов, предназначенных для различных областей техники, давно является предметом обсуждений [16] Было показано, что наноструктурирование поверхностного слоя металлических материалов может обеспечить повышение прочностных характеристик при одновременном увеличении их пластичности. Достижение подобного эффекта помимо формирования наноразмерных структур в поверхностном слое обеспечивается за счет создания так называемого барьерного подслоя, который представляет собой материал с полосовой дислокационной субструктурой, препятствующей проникновению дефектов с поверхности в объем нагружаемого материала [3].

Одним из методов создания наноструктурного состояния в поверхностном слое и обеспечения градиентного изменения характеристик прочности по глубине обработанного материала как раз и является ультразвуковая обработка поверхности. Например, было показано [4], что характерной особенностью структуры поверхностного слоя холоднокатаного титана, подвергнутого ультразвуковой обработке, является наличие полосовой дислокационной субструктуры в виде параллельных дислокационных субграниц. В работе [5] использовали ультразвуковую финишную обработку (УФО) для повышения эффективности ионного азотирования стали 40X13, что связывали с повышением интенсивности протекания диффузионных процессов при увеличении степени дефектности металлических сплавов. При этом были выявлены температурные режимы, обеспечивающие максимальное увеличение микротвердости в упрочненном поверхностном слое, а также показано, что предварительное безабразивное ультразвуковое выглаживание поверхности при последующем ионном азотировании оказывает влияние на увеличение толщины модифицированного слоя на глубину до 25-30 мкм. Также, как правило, ультразвуковая (финишная) обработка сталей, сопровождается увеличением износостойкости обработанных поверхностей. 
6. За счет высочайшей эффективности авторезонансных способов организации обработки, именно использование технологий АРУЗО оказывается одним из самых перспективных способов проведения УФО [6, 7, 8 -14]. Появление структур и регулярность поверхностного воздействия при авторезонансном резании металлов хорошо видны на рисунках П-5, П-6, П-7.

На рис П-5, а показан результат обработки меди М2p без ультразвуковых колебаний резца. Четкое структурирование материала показано на рис.П-5, б, где была использована АРУЗО. Наиболее четко видны упрочняющие наноструктуры после проведения травления образцов.
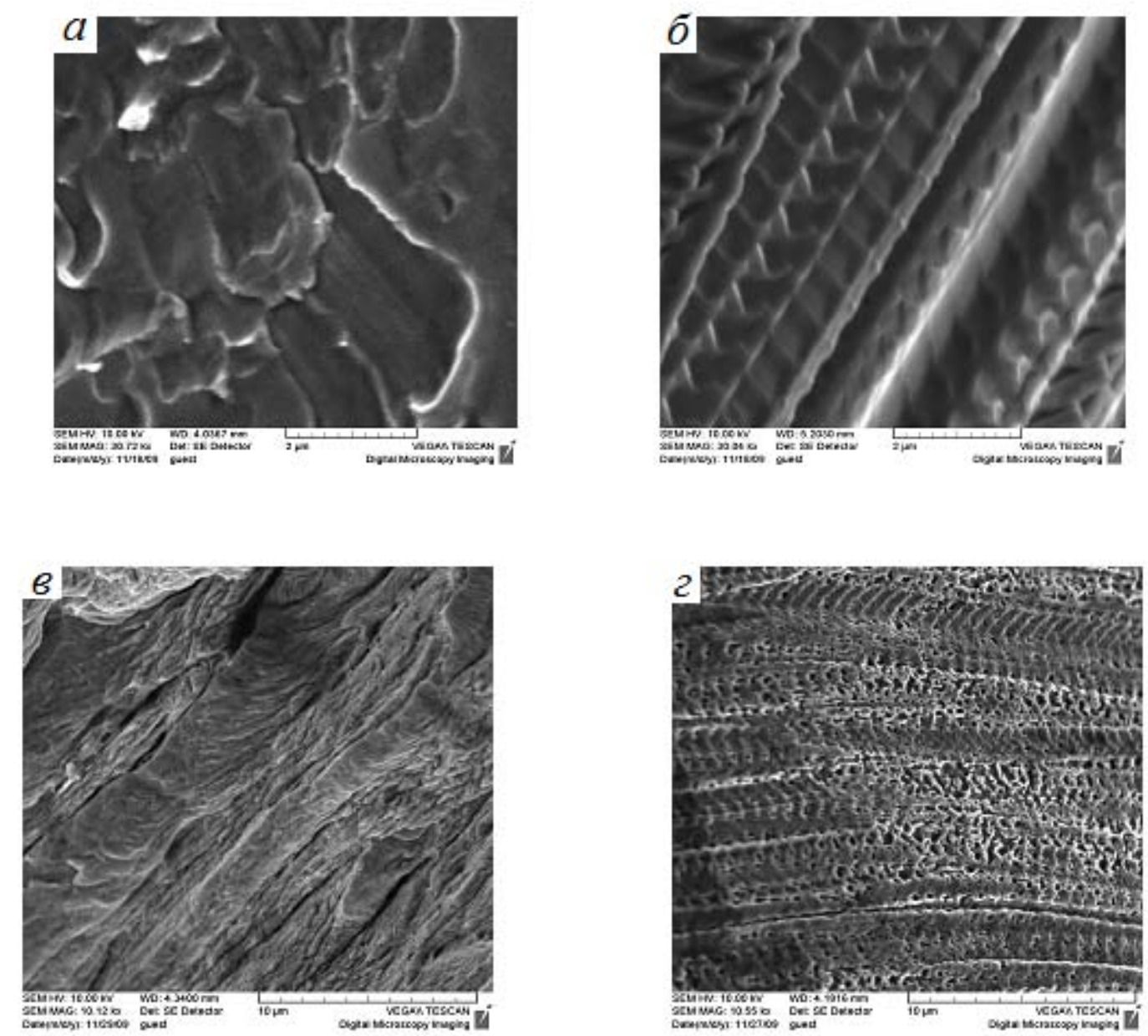

Рис.П-5

На рис. П-5, в показан результат обработки без применения ультразвука (время травления - 10 мин.); протравленный слой при использовании ультразвукового резания (время травления - то же) дан на 
рис. П-5, г. Хорошо видны получившиеся в результате упрочняющие поверхность наноструктуры.
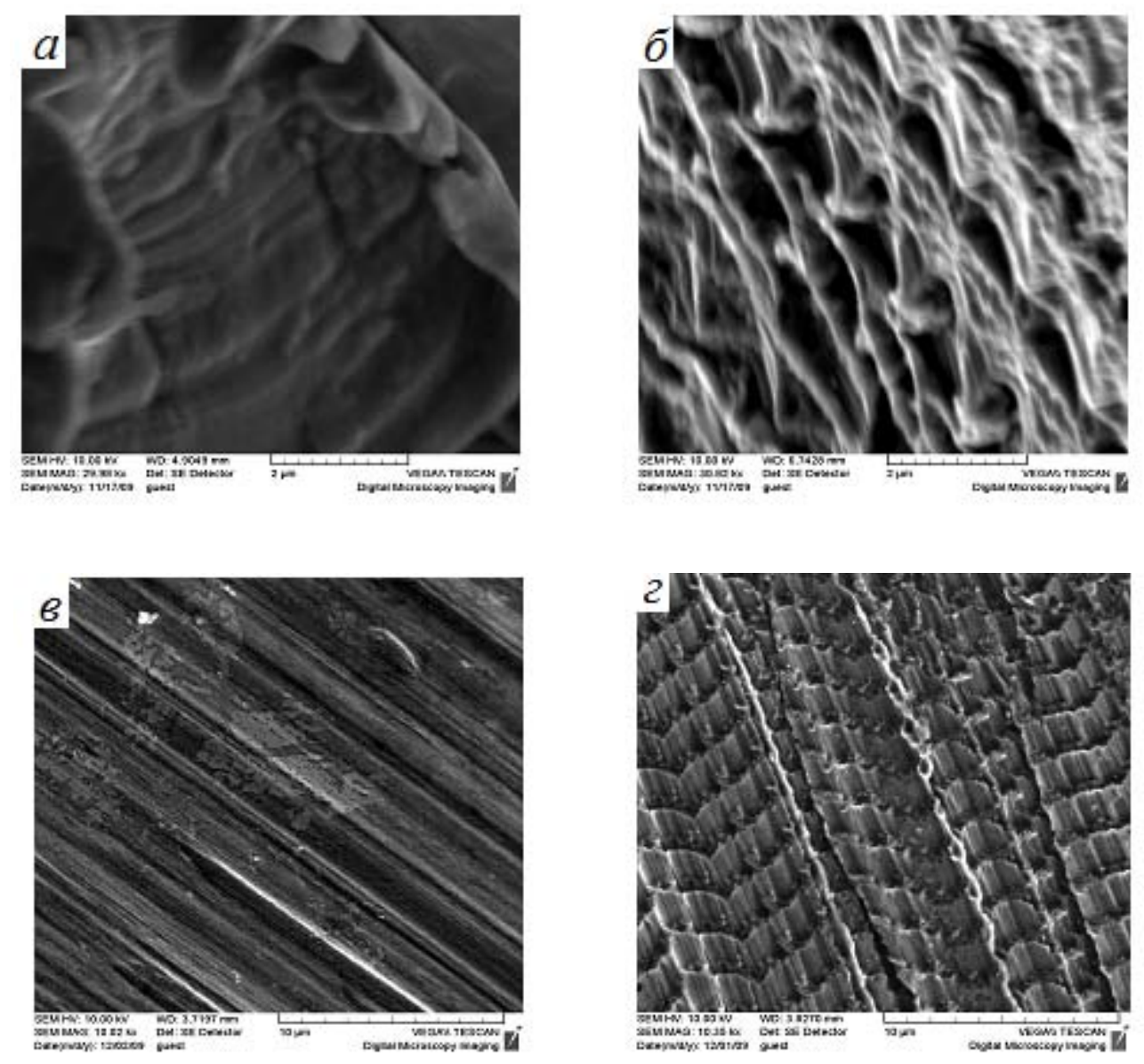

Рис.П-6.

На рис. П-6 показаны аналогичные результаты, полученные при изучении латунной стружки ЛС59-1: (а) - резание без ультразвука, (б)- режим АРУЗО; (в), (г) - аналогичные результаты с травлением (продолжительность 10 мин.).

Схожие результаты были получены и для других металлов. Например, на рис. П-7 показано: (а) - результат стандартной токарной обработки, подкаленной стали Ст45, (б) - токарная обработка в присутствии ультразвука, на рис. (в) - дана картина упрочняющих наноструктур структур, возникающих после АРУЗО. 

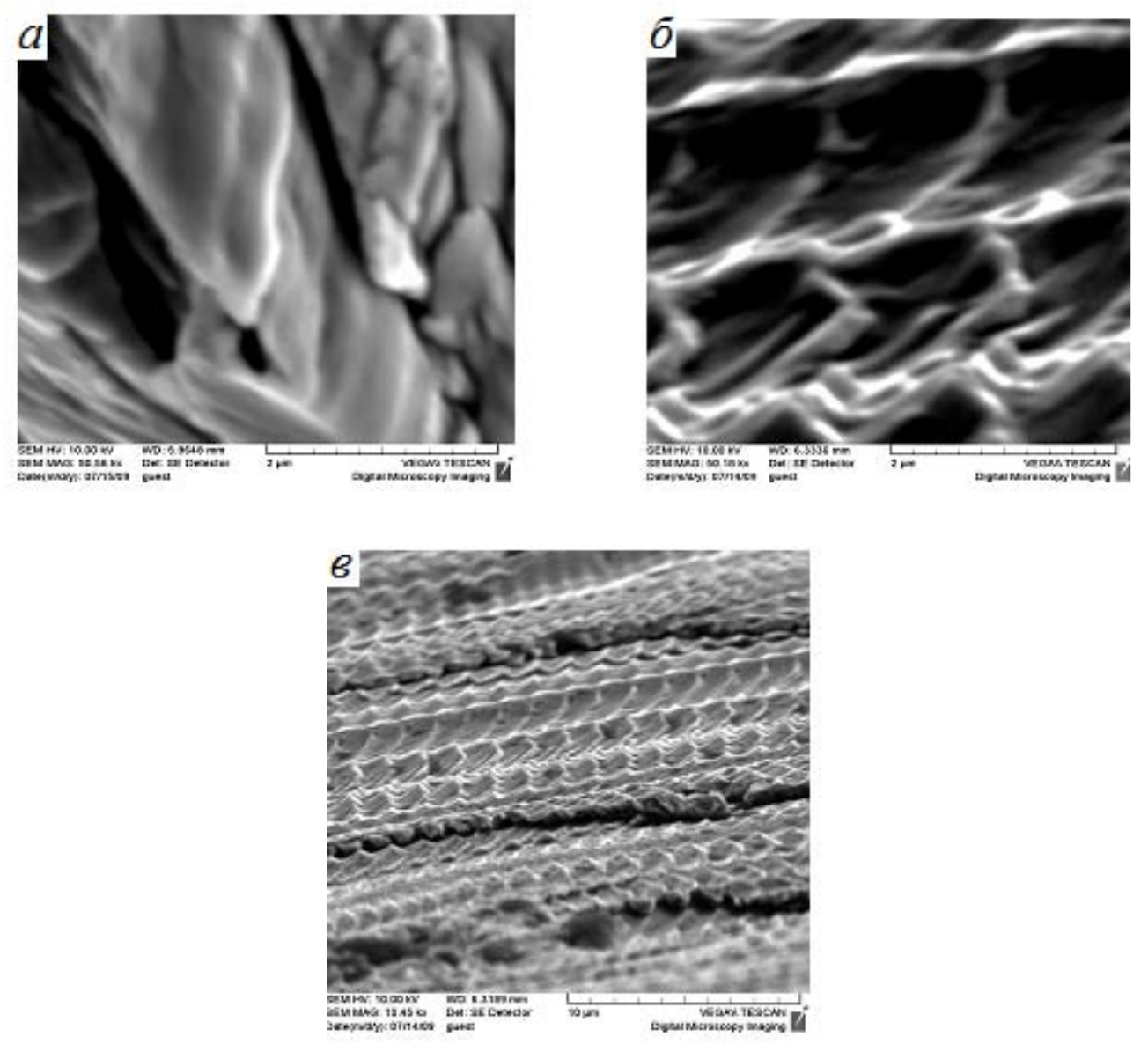

Рис. П-7

7. Для получения информации по структурированию и состоянию поверхности после авторезонансной ультразвуковой токарной обработки были изготовлены шлифы и сделаны их оптические (Neofot-32) и электронномикроскопические (РЭМ Tescan) фотографии. С помощью растрового электронного РЭМ Tescan были проведены фрактографические исследования. (Фрактография - это методы исследования изломов или, если говорить совсем обще - «наука об изломах».)

На рентгеновском дифрактометре Дрон-3М были измерены размеры областей когерентного рассеяния.

На снимках видны растравленные (и потому тупые) трещины, скачки деформации, «вторая» фаза. Однако зёренная структура при травлении не проявилась. Возможно, это вызвано тем, что при образовании стружки в кристаллической решётке зёрен возникло столько дефектов, что их 
суммарная упругая энергия оказалась сравнима с энергией границ. В результате и зёрна и границы травятся с одинаковыми скоростями.

Фрактограммы стружки после УЗ воздействия свидетельствуют об увеличении вязкости материала (площадь, которую можно характеризовать как ямочное разрушении значительно возросла), а также об уменьшении количества трещин.

Измерение размеров областей когерентного рассеяния показало, что их размер колеблется от 50 нм до 0,3 мкм и более. Разброс размеров на порядок обусловлен следующим обстоятельством: вблизи свободных поверхностей, образованных движением трещин, упругие поля напряжений от мезодефектов, ответственных за протекание деформационного измельчения структуры, отрелаксировали, в результате чего в этих областях, повидимому, сохранилась зёренная структура исходного материала с размером зёрен порядка 1 мкм. В других же областях, где деформация протекала с сохранением сплошности, зёренная структура дополнительно измельчилась вплоть до нанокристаллической.

Измерение и расчет микротвердости образцов, после авторезонансного резания приведены в таблице 1. Видно, что все образцы, подвергнутые АРУЗО имеют прирост микротвердости от 10 до 80\%.

Таблица 1

\begin{tabular}{|c|c|c|c|c|}
\hline & \multicolumn{2}{|c|}{ Ребро после АРУЗО } & \multicolumn{2}{|c|}{ Середина боковой плоскости } \\
\hline & $\begin{array}{c}\text { Микротвёрдость } \\
\text { Н } \mu, \text { МПа. }\end{array}$ & $\begin{array}{c}\text { абсол. } \\
\text { ошибка } \\
\Delta \text { Н } \mu, \text { МПа }\end{array}$ & $\begin{array}{c}\text { Микротвёрдость } \\
\text { Н } \mu \text {,МПа. }\end{array}$ & 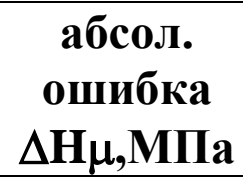 \\
\hline Лат. 13 & 3245 & 218 & 2826 & 122 \\
\hline Лат. 14 & 3013 & 124 & 2480 & 161 \\
\hline $\begin{array}{c}\text { Нерж. } \\
31 \\
\end{array}$ & 6140 & 318 & 3733 & 285 \\
\hline $\begin{array}{c}\text { Нерж. } \\
33\end{array}$ & 6140 & 209 & 3924 & 94 \\
\hline $\begin{array}{c}\text { Сталь } \\
41\end{array}$ & 6094 & 584 & 4791 & 351 \\
\hline $\begin{array}{c}\text { Сталь } \\
44\end{array}$ & 4847 & 94 & 4015 & 201 \\
\hline
\end{tabular}


Испытание микротвердости методом вдавливания производилось на приборе ПМТ-3 и стандартизовано (ГОСТ 9450-60). В качестве индентора при измерении микротвердости использовалась правильная четырехгранная алмазная пирамида с углом при вершине $136^{0}$. Эта пирамида плавно вдавливается в образец при нагрузке от 2 до 200 г. Число микротвердости $H$ определяется по формуле:

$$
H=1854 \cdot \frac{P}{d^{2}}\left[\frac{H}{M^{2}}\right],
$$

где $P$ - нагрузка, в $H$; $d$-диагональ отпечатка, в $M$;

Метод используется для определения твердости деталей малой толщины и тонких поверхностных слоев, имеющих высокую твердость. Чем тоньше материал, тем меньше должна быть нагрузка. Главное его назначение - оценка твердости отдельных фаз или структурных составляющих сплавов, а также разницы в твердости разных участков этих составляющих.

Микротвердость измеряют на металлографических шлифах, приготовленных специальным образом. Глубина вдавливания индентора при определении микротвердости $d / 7$-составляет несколько микрон и соизмерима с глубиной получаемого в результате механической шлифовки и полировки наклепанного поверхностного слоя. Поэтому очень важно правильно подготовить поверхностный слой образца. Все поверхностные дефекты (окалина, выбоины, вмятины, грубые ризки) должны быть удалены. При измерении микротвердости расстояние между центрами соседних отпечатков должно быть не менее двух длин диагонали большого отпечатка. Таким же должно быть расстояние от центра отпечатка до края образца. Длина диагонали отпечатка должна быть не более полуторной толщины образца.

Способ ультразвукового выглаживания и резания используемый для того, чтобы добиваться снижения шероховатости, повышения микротвердости и формирования сжимающих остаточных напряжений известен и обсуждается специалистами несколько десятилетий. Однако 
эффективных теоретических моделей упрочнения материалов при авторезонансном резании, опирающихся на современное состояние исследований в этой области создано не было.

Среди механических свойств нанокластеров и наноструктур необходимо отметить высокую твердость и высокую пластичность. Прежде всего, твердость наноструктуры должна возрастать с уменьшением размера кластера.

С другой стороны, при нанометровом размере большое значение имеет диффузионное скольжение нанокристаллитов, и скорость деформации значительно возрастает. Таким образом, прочностные свойства наноматериала определяются соотношением между пределом текучести (прочностью) и скоростью деформации. Еще одним фактором увеличения скорости деформации следует считать возрастание коэффициента диффузии при уменьшении размера кластера.

Классические технологии ультразвуковой обработки материалов позволяют получать поверхностные и приповерхностные наноструктуры, но только авторезонансные технологии могут обеспечить стабильность структурированности и заданные свойства поверхности после обработки, получение нанокристаллических структур с определенными параметрами.

Получение при авторезонансном ультразвуковом резании стали, меди или латуни специфических высокоэффективных, с точки зрения физикомеханических свойств нанокристаллических структур указывает на важность изучения и развития метода ультразвукового резания и выглаживания материалов.

Технологии АРУЗО, естественно, позволяют обрабатывать не только металлы, но и другие трудно обрабатываемые материалы, такие, например, как стекло. На рис. П-8, а показан фрагмент цилиндрической поверхности стеклянного изделия, обработанного резцом при помощи технологии АРУЗО (слева) и той же поверхности после выключения ультразвука (справа). Разница очевидна. 


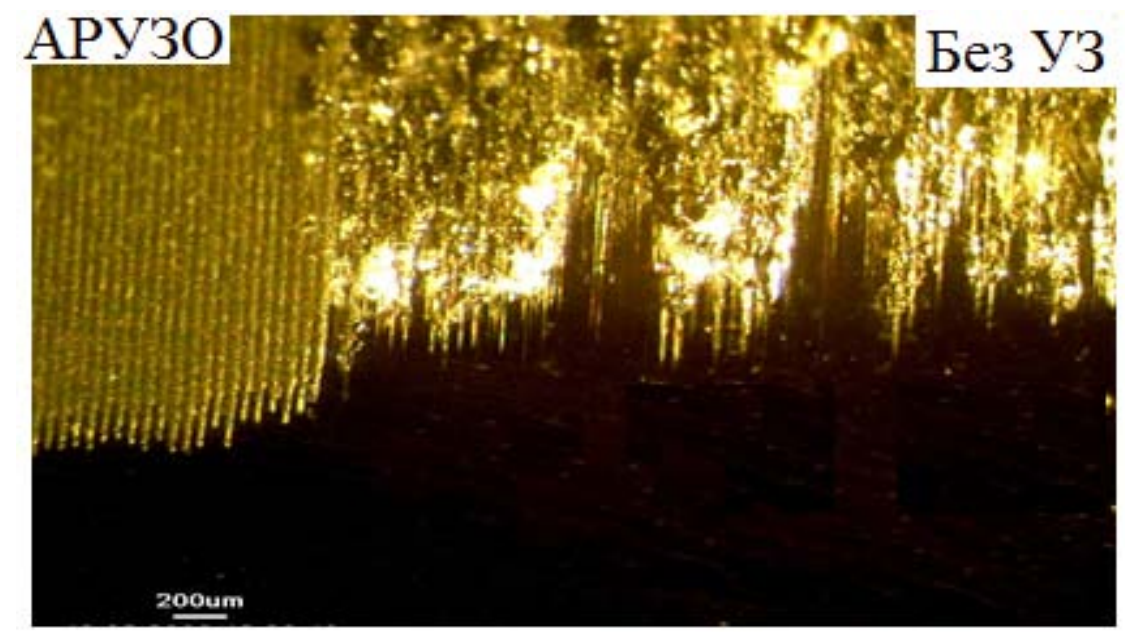

Рис. П-8

На рис. П-9 показан результат обработки специального (ниобиевого) стекла, характеризующего повышенной твердостью.

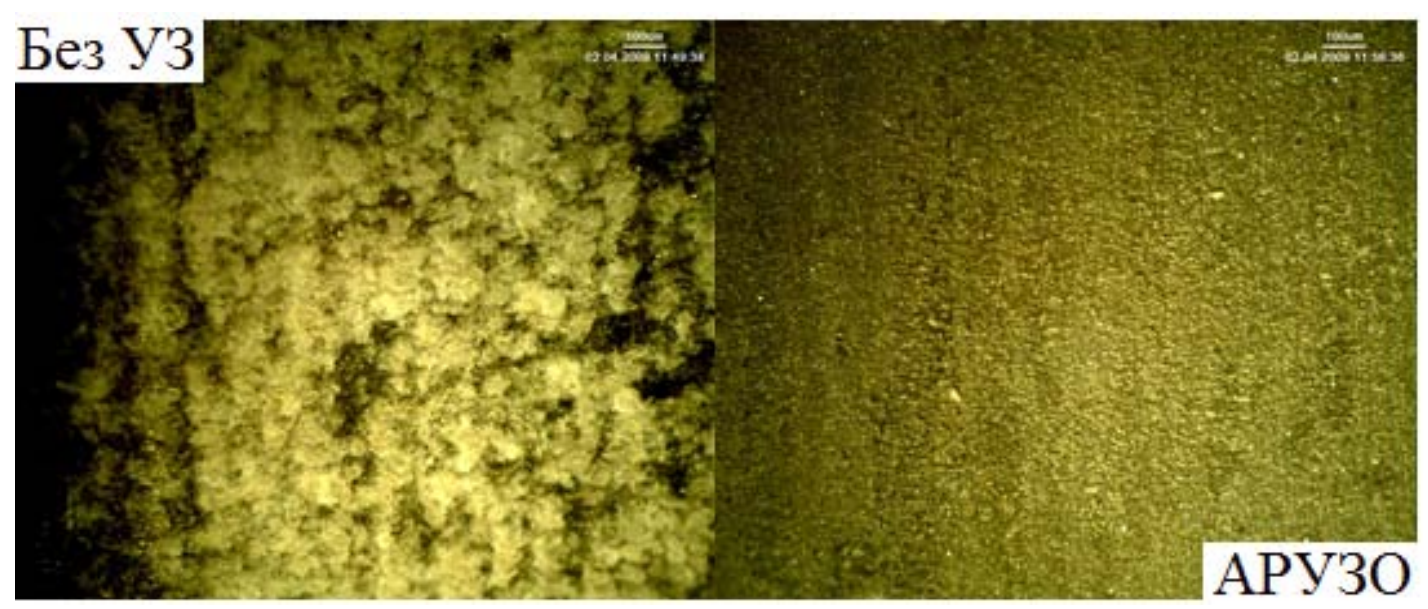

Рис. П-9

Здесь также различие представляется очевидным.

Как указывалось, вопрос о построении теоретических моделей рассмотренных здесь явлений, вообще говоря, представляет собой самостоятельную проблему [12]. 


\section{СПИСОК ЛИТЕРАТУРЫ К ПРИЛОЖЕНИЮ}

1. Асташев B.K. О влиянии ультразвука на процессы пластичного деформирования // Машиностроение. 1983. № 2. С. 3-12.

2. Асташев B.К. Влияние ультразвуковых колебаний резца на процесс резания // Проблемы машиностроения и надежности машин. 1992. № 3. C. $81-89$.

3. Панин B.E., Панин Л.E. Масштабные уровни гомеостаза в деформируемом твердом теле // Физическая мезомеханика. 2004. Т. 7. № 4. C. 5-23.

4. Панин А.В., Панин В.Е., Почивалов Ю.И. $и$ др. Особенности локализации деформации и механического поведения титана ВТ1-0 в различных структурных состояниях // Физическая мезомеханика. 2002. T. 5. № 4. C. 73-84.

5. Клименов В.А., Ковалевска Ж.Г., Уваркин П.В. и др. Ультразвуковое модифицирование поверхности и его влияние на свойства покрытий // Физическая мезомеханика. 2004. Т. 7. Специальный выпуск. Ч. 2. С. $157-160$.

6. Astashev V.K., Babitsky V.I. Ultrasonic cutting as a nonlinear (vibro-impact) process // Ultrasonics. 1998. № 6. P. 89-96.

7. Astashev V.K., Babitsky V.I. Ultrasonic Processes and Machines. Dynamics, Control and Applications. Springer. 2007. 330 p.

8. Асташев В.К., Андрианов Н.А., Крупенин В.Л. Устройство для возбуждения и автоматической стабилизации резонансных колебаний ультразвуковых систем // Патент RU 2350405 C2. Бюлл. изобретений. 2009. № 9.

9. Асташев В.К., Крупенин В.Л. Инновационные авторезонансные вибротехнологии // Современные наукоемкие технологии. 2008. № 7. C. $84-85$.

10.Крупенин В.Л., Колик Л.В. Об авторезонансных нанотехнологиях // Современные наукоемкие технологии. 2009. № 4. С. 63-64. 
11.Асташев В.К., Крупенин В.Л. Инновационные авторезонасные вибротехнологии // Современные наукоемкие технологии. 2008. № 7. C. 84-85.

12.В.К. Асташев, В.Л. Крупенин, В.Н. Перевезениев, Л.В. Колик, Н.А.Андрианов $\mathrm{K}$ изучению свойств наноструктурированных материалов, полученных в результате авторезонансной ультразвуковой обработки // интернет-журнал. Вестник научно-технического развития. № 11 (39). 2010. С. 3-12.

13. Асташев В.К., Андрианов Н.А., Козочкин М.П., Колик Л.В, Крупенин В.Л., Солис-Пинарготе Н.В. К реализации авторезонансных ультразвуковых технологий // Проблемы машиностроения и надежности машин. №6. 2009. С. 52-58.

14.Асташев В.К., Андрианов Н.А., Крупенин В.Л., Привезенцев В.Н., Колик Л.В., Свойства поверхностных слоев, наноструктурированных в результате авторезонансного ультразвукового точения // Проблемы машиностроения и надежности машин. 2011. № 5. С. 68-72.

15.Асташев B.K, Разинкин А.B. Моделирование термомеханических процессов при ультразвуковом резании методом конечных элементов // Проблемы машиностроения и надежности машин. 2008. № 3. С. 68-74.

16.Гусев А.И., Ремпель А.А. Нанокристаллические материалы. М., Физматлит, 2000. 223 с.

17. В.К. Астамев, Н.А. Андрианов, Л.В. Колик, В.Л. Крупенин. Авторезонансная ультразвуковая технология резания // Интернет журнал. Вестник научно-технического развития. № 1 (29). 2010 г. С.3 - 10 
Учебное издание

Асташев Владимир Константинович

Крупенин Виталий Львович

\title{
НЕЛИНЕЙНАЯ ДИНАМИКА УЛЬТРАЗВУКОВЫХ ТЕХНОЛОГИЧЕСКИХ ПРОЦЕССОВ
}

\author{
Учебник
}

Подписано в печать 16.06.16. Формат 60×84/16.

Бумага офсетная. Печать на ризографе. Усл. печ. л. 21,62.

Тираж 50 экз. Заказ № 179.

Московский государственный университет печати имени Ивана Федорова.

127550, Москва, ул. Прянишникова, д. 2А.

Отпечатано в Издательстве МГУП имени Ивана Федорова

с макета, подготовленного авторами 